\title{
THE EFFECT OF REWARD AND PUNISHMENT ON INHIBITORY CONTROL IN
}

\section{ANXIETY}

\author{
Bianca Levis
}

ORCID Identifier: 0000-0002-0254-8286

Master of Psychology (Clinical Neuropsychology)/Doctor of Philosophy

July 2017

Melbourne School of Psychological Sciences, University of Melbourne

This thesis is being submitted in total fulfilment of the requirements of the degree of Master of Psychology (Clinical Neuropsychology)/Doctor of Philosophy 


\begin{abstract}
Anxiety is associated with behavioural avoidance and inhibition that has been shown to influence cognitive control performance; however, it is not clear whether highly trait anxious individuals are less sensitive to reward or more sensitive to punishment when exerting selfcontrol. Trait anxiety is commonly measured in both clinical and research settings using the State Trait Anxiety Inventory trait anxiety scale (STAI T-Anxiety scale). Despite its popularity, there remain unanswered questions surrounding the factor structure and convergent and discriminant validity of this scale. In addition, studies that have investigated whether high trait anxiety as measured by the STAI T-Anxiety scale is associated with a general inhibitory control deficit have reported mixed results. The broad aims of the current research project were to 1) investigate the factor structure of the STAI T-Anxiety scale and examine its convergent and discriminant validity, 2) use a stop signal task to examine whether individuals high in trait anxiety show inhibitory control (action cancellation) deficits, and 3) use a novel monetary incentive control task (MICT) to examine the effect of presenting cues and feedback indicating reward and punishment on inhibitory control in individuals high in trait anxiety. To examine factor structure, 947 healthy participants completed the STAI T-Anxiety scale and an exploratory factor analysis was performed. Convergent and discriminant validity were investigated using the Pearson product-moment correlation coefficient. In a second study, 76 participants who were either low or high in trait anxiety completed a stop signal task and independent samples $t$-tests were used to analyse differences in stop signal task performance between the groups. Ninety-nine participants then completed an online version of the stop signal task and the Pearson product-moment correlation coefficient was used to investigate whether trait anxiety was associated with stop signal task performance. In a final study 48 participants high and low in trait anxiety completed the MICT while they underwent functional magnetic resonance imaging. Results
\end{abstract}


indicated: 1) the STAI T-Anxiety scale was comprised of two method factors but could also be represented as a single dimension. The scale had limited discriminant validity and failed to adequately measure somatic aspects of anxiety. 2) There was no significant difference in stop signal task performance between individuals low and high in trait anxiety, and no significant correlation between stop signal performance and trait anxiety measured continuously. 3) Individuals high in trait anxiety slowed reaction times to go stimuli during the MICT. There were no significant group differences in networks involved in inhibitory control, cognitive control, or reward and punishment during the anticipation, stopping or feedback epochs of the MICT. During anticipation there were group differences in posterior default mode areas, and during feedback there were group differences in right superior/middle frontal gyrus and right insula activity. These results suggest that 1) the STAI T-Anxiety scale may benefit from revision, 2) there appears to be no general inhibitory control (action cancellation) deficit in high trait anxiety, and 3) high compared to low trait anxious individuals apply greater effort and demonstrate dysfunction in brain structures that are part of important networks, including the default mode network, the ventral attention network and the cingulo-opercular network. 


\section{Declarations}

I declare:

i. that this thesis comprises only my original work toward the Master of Psychology (Clinical Neuropsychology)/Doctor of Philosophy;

ii. due acknowledgement has been made in the text to all other material used; and

iii. the thesis is fewer than the maximum word limit in length, exclusive of tables, maps, bibliographies and appendices as approved by the Research Higher Degrees Committee.

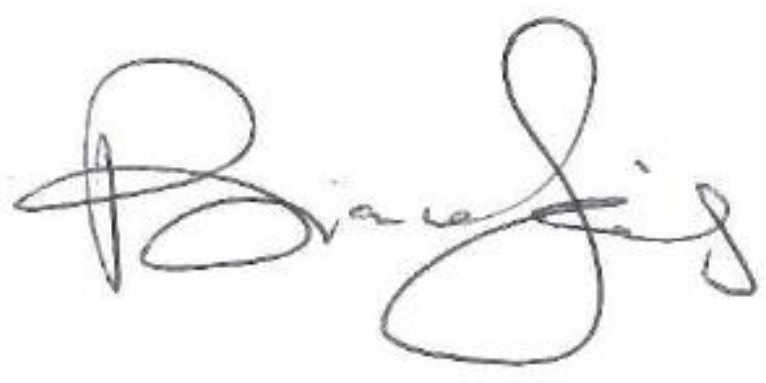

Bianca Levis 


\section{Preface}

This thesis was made possible by the following grants:

- National Health and Medical Research Council grant number: 1050766

- Australian Research Council Future Fellowship grant number: FT110100088 


\section{Acknowledgements}

My deepest thank you to my supervisors Prof Rob Hester and Dr Luke Smillie. Prof Hester agreed to supervise me when I needed a new project. He taught me to always stay calm and that nothing is unfixable. Without his limitless patience, wisdom, guidance and generosity, I could never have completed this PhD. I am also grateful for Dr Luke Smillie's supervision. He provided invaluable knowledge in the area of personality and factor analysis, and always made time to meet with me to discuss ideas and thoroughly read through and comment on manuscripts.

I would like to thank the Royal Children's Hospital and the Murdoch Children's Research Institute for the use of their magnetic resonance imaging scanner, and Mike Kean for his help setting up sequences. Also thank you to Michael Fox who programmed the online version of the stop signal task.

Thank you to all my colleagues and friends in Prof Hester's lab, including Evelyn Chen, Antoinette Poulton, Christina Van Heer, Kathleen Charles-Walsh, and Dr Dan Upton, and my officemate and friend Shaheed Azaad. Thank you to Stanton Kroenert for fuelling my desire to finish. A special and huge thank you to my wonderful friend Dr Francis Puccio for his warmth, advice, humour, and endless ability to listen and care.

Finally I would like to thank my family, especially my mother Helen and father William who worried about me, supported me and never stopped loving me, and hopefully can now see that undertaking a $\mathrm{PhD}$ really is the same as having a baby. 


\section{Table of Contents}

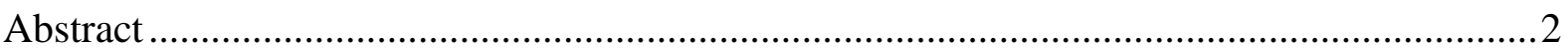

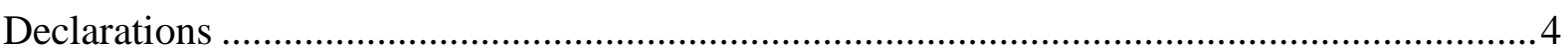

Preface

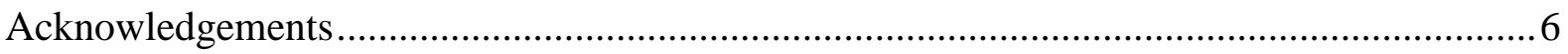

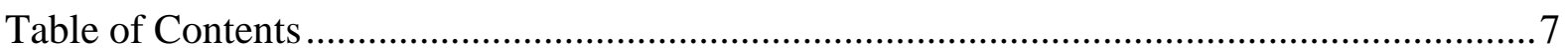

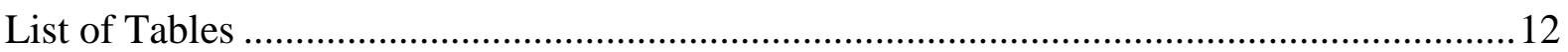

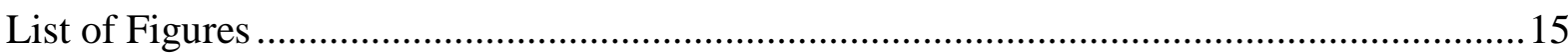

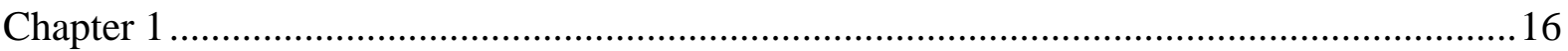

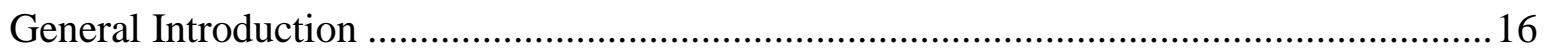

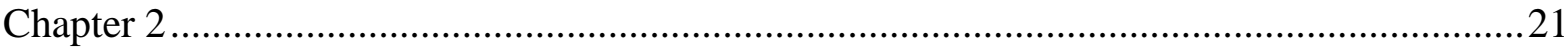

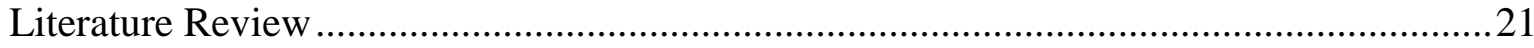

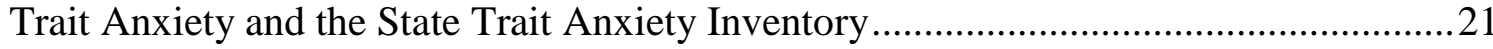

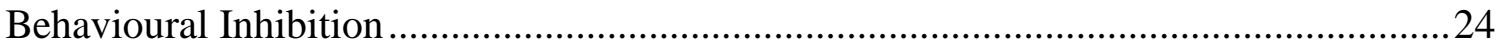

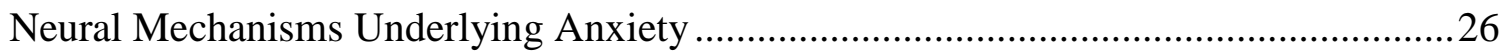

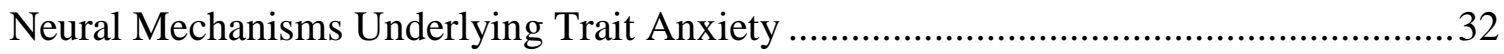

Cognitive Models of Anxiety, Threat and Attentional Bias ...........................................40

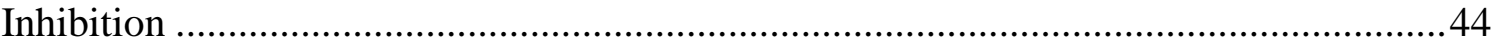

Neural Structures Involved in Response Inhibition in the Stop Signal Task ...................46

Processing of Rewards and Punishments in Healthy Populations ..................................50

Rewards and Punishments in the Stop Signal Task...................................................53 
Inhibitory Control, Anxiety and the Stop Signal Task

Motivational Factors, Inhibitory Control and Anxiety

Effect of Anticipation/Cues Relating to Motivational Factors on Inhibitory Control in

Anxiety and Behavioural Inhibition

Effect of Feedback Indicating Reward and/or Punishment on Inhibitory Control in

Anxiety and Behavioural Inhibition

The Current Research

Chapter 3

Factor Analysis of the STAI T-Anxiety Scale

Exploratory Factor Analysis of the State-Trait Anxiety Inventory

Confirmatory Factor Analysis of the State-Trait Anxiety Inventory

Dimensions of Anxiety Measured by the State-Trait Anxiety Inventory Trait Anxiety

Scale

Present Aims

Study 1

Method .85

Participants .85

Measures .86

Data Analysis. .94

Results .95

Reliability and Factor Analysis .95

Convergent and Discriminant Validity...... 104 
Study 2 .

Method

Results

Discussion

Chapter 4

The Effect of Trait Anxiety on Stop Signal Task Performance

Study 1

Method

Participants

Procedure and Stimuli

Results

Study 2

Method

Participants

Procedure and Stimuli

Results

Discussion

Chapter 5

The Effect of Reward and Punishment on Inhibitory Control in Anxiety - the Monetary

Incentive Control Task

Method 
Materials and Procedure

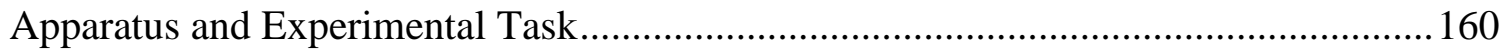

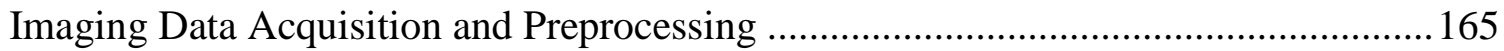

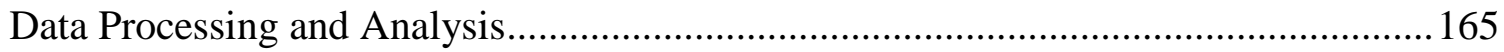

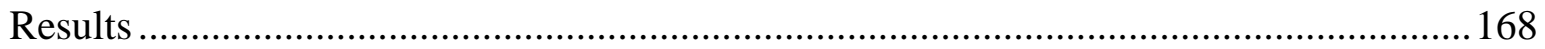

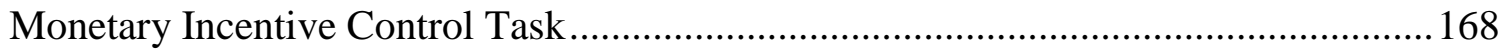

Functional Magnetic Resonance Imaging BOLD Activity ....................................... 170

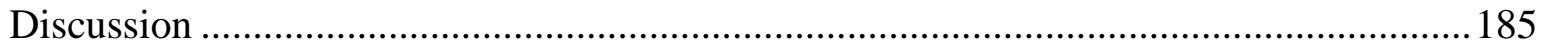

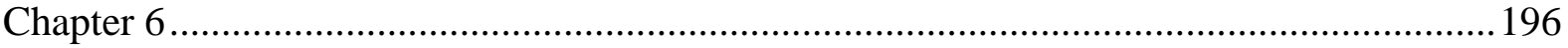

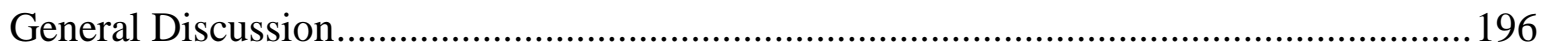

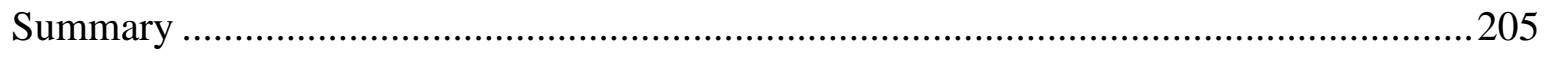

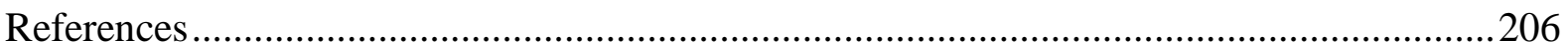

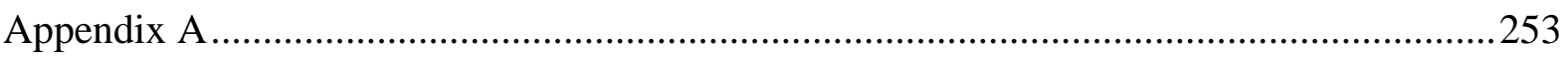

Published Measures Used in the Research Project.........................................................2 253

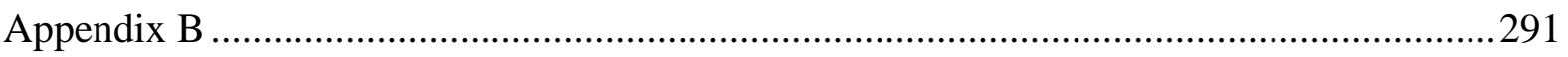

Non-Published Measures Used in the Research Project ................................................291

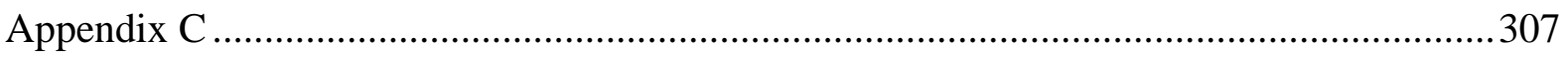

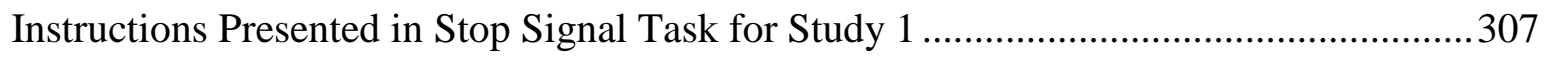

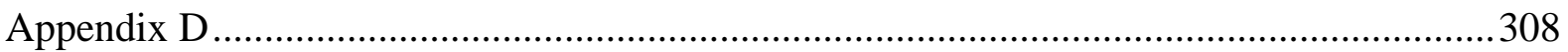

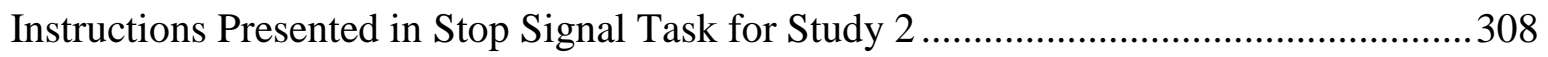

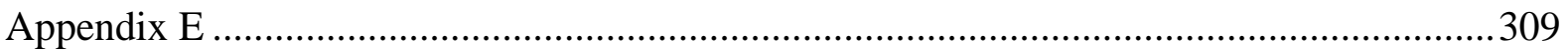


Stimulus Timing and Feedback Provided in Stop Signal Task for Study 2

Appendix F

Sequence of Go and Stop Trials for Practice Versions 1 and 2.

Appendix G

Task Design for Practice Version 1 and Practice Version 2

Appendix $\mathrm{H}$

Means and Significance Tests for All Main and Interaction Effects During the Cue Period

Appendix I

Means and Significance Tests for All Main and Interaction Effects During the Stop

Stimulus Period 381

Appendix $\mathbf{J}$

Means and Significance Tests for All Main and Interaction Effects During the Feedback

Period 422 


\section{List of Tables}

Table 1. Brain regions and Potential Cognitive/Behavioural Outcomes of Activation in Anxiety During an Affectively Neutral Cognitive Control Task

Table 2. Means, SD and Cronbach's Alpha if Item Deleted for State Trait Anxiety Inventory T-Anxiety Scale Items $(N=947)$

Table 3. Inter Item Correlation Matrix for Items in State Trait Anxiety Inventory T-Anxiety Scale 89

Table 4. Internal Reliabilities of Each Instrument and/or Scale 93

Table 5. Variance Explained in State Trait Anxiety Inventory T-Anxiety Scale Items Using Principal Axis Factoring .96

Table 6. Parallel Analysis for State Trait Anxiety Inventory T-Anxiety Scale..... .99

Table 7. Pattern Matrix, Structure Matrix and Communalities for Principal Axis Factoring with Oblimin Rotation of Two Factor Solution of State Trait Anxiety Inventory T-Anxiety Scale Items .

Table 8. Comparison of Factor Loadings Obtained via Exploratory Factor Analysis with Findings from Studies by Bieling et al. (1998) and Spielberger et al. (1983) 101

Table 9. Factor Matrix for Principal Axis Factoring of One Factor Solution for State Trait Anxiety Inventory T-Anxiety Scale Items

Table 10. Correlations of the State Trait Anxiety Inventory T-Anxiety Scale with Other Measures of Anxiety, Mood and Personality.

Table 11. Fisher's z-Scores for Correlations between the STAI T-Anxiety Scale and Other Measures of Anxiety, Mood and Personality 106

Table 12. Correlations Between Measures of Deactivated Negative Affect, Activated Negative Affect, and General Negative Affectivity 108 
Table 13. Internal Reliabilities of Each of the Questionnaires in the Smaller Sample $(N=48)$ 111

Table 14. Mean Demographic and Questionnaire Data for Low Trait Anxiety and High Trait Anxiety Groups 113

Table 15. Group Statistics, $T$-values, Significance Tests, 95\% Confidence Intervals and Effect Sizes for Measures of Anxiety, Mood and Personality in Participants Low and High in Trait Anxiety. 114

Table 16. Mean Demographic and Questionnaire Data for Whole Sample and Low Trait Anxiety and High Trait Anxiety Groups ........ 134

Table 17. Means and Standard Deviations for Stop-Signal Reaction Time, Stop-Signal Accuracy, Mean Correct Go Reaction Time and Stop Signal Delay for Low $(n=34)$ and High $(n=27)$ Trait Anxiety Groups 135

Table 18. Mean Demographic and Questionnaire Data for Sample Included in Analysis $(N=$ 99). 139

Table 19. Means and Standard Deviations for Stop-Signal Reaction Time, Stop-Signal Accuracy, Mean Correct Go Reaction Time, and Stop Signal Delay...... 139

Table 20. Correlations Between Stop Signal Reaction Time and Other Measures of Anxiety, Mood and Personality 142

Table 21. Descriptive Statistics for Low Trait Anxiety and High Trait Anxiety Groups..... 158 Table 22. Feedback Provided to Participants in the MICT in Each of the Reward, Punishment and Neutral Conditions Following Go and Stop Trials 162

Table 23. Means and Standard Deviations for Go Trial Accuracy, Stop Trial Accuracy, and Go Trial Reaction Times (Correct and Error) for the Low and High Trait Anxiety Groups. 168 Table 24. Regions of Event-Related BOLD Activity During Cue Period and Regions Demonstrating Significant Effects 172 
Table 25. Regions of Event-Related BOLD Activity During Stop Stimulus Period and Regions Demonstrating Significant Effects.

Table 26. Regions of Event-Related BOLD Activity During Feedback Period and Regions

Demonstrating Significant Effects 


\section{List of Figures}

Figure 1. Scree plot showing eigenvalues plotted against factors. .98

Figure 2. Schematic representation of a MICT reward condition go trial that has been accurately performed in the Main Version. Numbers representing the time are in milliseconds

Figure 3. Schematic representation of a MICT punishment condition stop trial where the response has not been withheld in the Main Version. Numbers representing the time are in milliseconds.

Figure 4. Mean stop accuracy for high trait anxiety and low trait anxiety groups in each condition.

Figure 5. Activation map for a) the left posterior cingulate cortex (PCC) (dark blue) showing significant deactivation i) in the low trait anxiety group during the neutral and punishment conditions compared to the reward condition during the anticipation event, and ii) in the neutral condition for the low trait anxiety group compared to the high trait anxiety group during the anticipation event; and b) the left dorsal posterior cingulate (dPCC) (light blue) showing significant deactivation in the high trait anxiety group during the reward compared to the neutral condition during the anticipation event, overlaid on a sagittal brain section (MNI template; using AFNI software).

Figure 6. (A) Activation maps for the right superior/middle frontal gyrus showing significant activity in both groups during reward compared to punishment and neutral trials and in the high trait anxiety group during punishment compared to neutral trials during the feedback event, overlaid on sagittal and axial brain sections (MNI template; using AFNI software). (B) Activation maps for the right insula showing significant activity in the low trait anxiety group during the feedback event following a correct response compared to an error, overlaid on sagittal and axial brain sections (MNI template; using AFNI software). 


\section{Chapter 1}

\section{General Introduction}

Anxiety disorders are one of the most common class of psychological disorder with a 12 month prevalence of $18 \%$ and a lifetime prevalence of $28.8 \%$ (Kessler et al., 2005). Given the high prevalence of anxiety, understanding the behavioural and neural mechanisms involved in its etiology and maintenance has the potential to change lives and reduce its associated burden through development of improved management and interventions.

Anxiety disorders are characterised by chronic avoidance of what are perceived to be aversive or threatening situations, even though these situations may involve no threat or may actually be positive and rewarding (see discussion in Grupe \& Nitschke, 2013). Despite the high prevalence of both anxiety disorders and sub-clinical symptoms of anxiety in the general population, the behavioural processes and neural mechanisms involved in such avoidance remain unclear (e.g., Bardeen, Tull, Stevens, \& Gratz, 2014; Maack, Tull, \& Gratz, 2012; Sheynin et al., 2014). Specifically, do individuals who experience high levels of anxiety (either clinically diagnosed anxiety or a tendency to experience symptoms of anxiety) avoid situations because of factors that include being less sensitive to positive signals, more sensitive to negative signals, or both (Sheynin, Moustafa, Beck, Servatius, \& Myers, 2015)? Positive and negative signals may be encountered either before a behaviour has been performed, i.e., they can act as cues that signal the possibility of a positive (rewarding) or negative (punishing) outcome based on the subsequent behaviour performed by an individual, or they can be received after a behaviour has been performed, i.e., they are an outcome that is fed back to the individual who performed the behaviour and, through a process of learning, make the behaviour more or less likely to occur in the future.

Currently there are substantial gaps in our understanding of the behavioural effect of positive and negative cues and positive and negative feedback in anxiety. For the purposes of 
this research, discussion of the behavioural effects of positive and negative cues and positive and negative feedback will be limited to the way in which these cues and feedback may influence the ability of individuals to control their responses; this is also called inhibitory control or response inhibition (see Inhibition below for a definition and more detailed discussion of these concepts). For discussion of the ways in which positive and negative cues and feedback influence other behaviours in anxiety see, for example, Cavanagh, Frank, and Allen (2011) (learning), de Visser et al., (2010), Giorgetta et al., (2012), Miu, Heilman, and Houser (2008) (decision-making), Frewen, Dozois, Joanisse, and Neufeld (2008), and Shechner et al., (2012) (attention). In relation to positive cues and feedback that indicates reward, it remains unclear whether, when compared to individuals who do not experience anxiety, individuals who experience anxiety show reduced, heightened, or no difference in their behavioural response when anticipating and receiving a reward. Similarly, it is unclear whether individuals who experience anxiety demonstrate increased or decreased control over their behavioural responses when anticipating and receiving a reward. The effect of negative cues that indicate potential punishment and negative feedback remains equally unclear.

Along with the behavioural effects of positive and negative cues and positive and negative feedback in anxiety, the neural networks underlying potential behavioural effects remain of interest. It is important to understand neural activity underlying behavioural effects in anxiety because understanding these networks may assist in the identification and development of treatments for anxiety. It is currently unclear whether potential insensitivity to reward in individuals with anxiety may be associated with reduced activation in structures involved in reward processing, including subcortical structures such as the striatum and cortical structures such as the medial orbitofrontal cortex (mOFC). It is also unclear whether potential hypersensitivity to punishment may be associated with increased activation in neural structures that are sensitive to aversive outcomes such as the amygdala and the insular 
cortex, as well as dysfunctional activation in neural structures that modulate other neural systems through top down executive control, such as the dorsolateral prefrontal cortex (DLPFC).

The current research sought to investigate these issues by administering a task called a monetary incentive control task (MICT) to participants low in trait anxiety and high in trait anxiety. The task was administered in a functional magnetic resonance imaging (fMRI) scanner that recorded brain activity associated with performing the task. Trait anxiety was measured using the trait anxiety scale of the State Trait Anxiety Inventory (STAI T-Anxiety scale) (Spielberger, Gorsuch, Lushene, Vagg, \& Jacobs, 1983). The MICT is a novel task designed to measure the influence of motivational factors (cues and feedback indicating reward and punishment) on inhibitory control. Previous studies show that, when performing this task, individuals who are reward sensitive (i.e., have a heightened sensitivity to the rewarding properties of stimuli) experience difficulty stopping their response on stop trials (Charles-Walsh, Upton, \& Hester, 2016; Rossiter, Thompson, \& Hester, 2012). Similarly, individuals who are punishment sensitive should not be influenced by the potential for reward on stop trials and show greater inhibition when an incorrect response could be punished.

To measure individual differences in anxiety and in order to be able to compare our findings with those in the existing literature, we used the STAI T-Anxiety scale. Given our use of the STAI T-Anxiety scale to index trait anxiety, we were interested in the scale's psychometric properties including whether it is a valid measure of anxiety. We therefore examined its factor structure as well as its convergent and discriminant validity. We administered the STAI T-Anxiety scale and a range of other instruments measuring anxiety, mood and personality to a large sample of 947 healthy participants. We performed an exploratory factor analysis on the data and correlated scores on the STAI T-Anxiety scale with scores on the other measures of anxiety, mood and personality. In neuroimaging 
research, financial and logistic constraints often preclude the collection of large samples of data. Due to these constraints, researchers need to recruit smaller groups of participants who are low and high on a variable of interest. Given that we intended to use groups low and high in trait anxiety for a subsequent neuroimaging study, we were interested in exploring how groups formed on the basis of low and high scores on the STAI T-Anxiety scale may differ with respect to related constructs such as anxiety, mood and personality traits. Therefore, from the larger sample of 947 participants, we extracted data from participants who achieved the lowest STAI T-Anxiety scale score and participants who achieved the highest STAI TAnxiety scale score and compared each group's STAI T-Anxiety scale score to the group's score on other instruments.

Given our interest in the role of inhibitory control in anxiety, before we administered the MICT we wanted to investigate whether high trait anxiety is associated with a general inhibitory control benefit or deficit. For example, do individuals high in trait anxiety perform better or worse on an affectively neutral response inhibition task when compared with individuals low in trait anxiety? We performed two studies to examine this question. The first study used the STAI T-Anxiety scale total score as a dichotomous measure and administered a stop signal task to 34 individuals low in trait anxiety and 27 individuals high in trait anxiety. The second study administered a web-based stop signal task to a larger group of 99 participants. In this second study we used the STAI T-Anxiety scale total score as a continuous measure and correlated participants' scores on the STAI T-Anxiety scale with dependent variables from the stop signal task. We also used a correlational design to investigate whether there was any relationship between stop signal task performance and other measures of anxiety, mood and personality in this sample.

In the final study we administered the MICT in an fMRI scanner to 24 participants low in trait anxiety and 24 participants high in trait anxiety. The MICT contains three types 
of interleaved conditions; reward, punishment and neutral. Our aim was to examine whether participants high in trait anxiety demonstrated enhanced inhibitory control on punishment trials. We also examined neural activity during anticipation of, performance on, and feedback for performance on go and stop trials during reward, punishment and neutral conditions. The aim was to examine if group differences were evident in relevant neural networks (e.g., cognitive control networks, inhibitory control networks, and reward/punishment networks) between the conditions.

Please note that in the chapters that follow, there is some replication of information across chapters. This is because each chapter was intended to be, and is in the process of being, submitted to peer-reviewed journals for publication. 


\section{Chapter 2}

\section{Literature Review}

\section{Trait Anxiety and the State Trait Anxiety Inventory}

The Diagnostic and Statistical Manual of Mental Disorders (5th ed.; DSM-5;

American Psychiatric Association, 2013a) identifies and defines a range of discrete anxiety disorders. These disorders include generalised anxiety disorder (GAD), social anxiety disorder, and panic disorder. Despite this categorical conceptualisation in the DSM-5, it is widely acknowledged that anxiety exists on a continuum and is found in varying degrees in non-clinical samples (e.g., American Psychiatric Association, 2013b; Marcus, Sawaqdeh, \& Kwon, 2014). Anxiety in clinical and non-clinical samples is often measured using selfreport questionnaires that are able to gauge levels of trait anxiety, which refers to a stable state of apprehension and hypervigilance across situations (Sylvers, Lilienfeld, \& LaPrairie, 2011), and state anxiety, which is a transient emotional condition involving feeling anxious at a particular moment in time (Gerstorf, Siedlecki, Tucker-Drob, \& Salthouse, 2009). Trait anxiety is elevated in individuals who meet criteria for anxiety disorders (Bieling, Antony, \& Swinson, 1998; J. A. Chambers, Power, \& Durham, 2004) and predicts future anxiety (Plehn \& Peterson, 2002) as well as affective disorder diagnosis (Sandi \& Richter-Levin, 2009). A commonly used instrument for measuring state and trait anxiety is the State Trait Anxiety Inventory (STAI) (Spielberger et al., 1983). It consists of two 20-item subscales that measure how you feel right now, that is, at this moment (i.e., state anxiety) and how you generally feel (i.e., trait anxiety).

The STAI trait anxiety scale (STAI T-Anxiety scale) is one of the oldest and most widely used measures of trait anxiety (M. Balsamo et al., 2013; Grös, Antony, Simms, \& McCabe, 2007). A number of studies have used factor analytic techniques to examine the psychometric structure of the scale. These studies have found that, rather than measuring 
relatively stable differences in anxiety-proneness - i.e., trait anxiety (Spielberger et al., 1983) - the STAI T-Anxiety scale appears to contain a general factor that has been called 'general negative affect' or 'vulnerability to psychological disorders' (Bados, Gómez-Benito, \& Balaguer, 2010; M. Balsamo et al., 2013) and two specific domain factors, one that evaluates characteristics such as low positive affect and negative self-appraisal (called 'depression'), and one that evaluates characteristics such as worry, tension and disturbing thoughts (called 'anxiety') (Bados et al., 2010). Despite findings that the STAI T-Anxiety scale measures general negative affect and is not a unidimensional measure of trait anxiety, the STAI TAnxiety scale continues to be widely used as a measure of trait anxiety, both in clinical and research settings.

Another issue surrounding the STAI T-Anxiety scale relates to precisely which dimensions of anxiety it measures. It has been argued that self-reported symptoms of anxiety can be decomposed into two distinct dimensions; anxious arousal which is characterised by somatic tension and physiologic hyperarousal, and anxious apprehension which entails worry and verbal rumination, typically about possible negative future outcomes (Burdwood et al., 2016; Moser, Moran, \& Jendrusina, 2012; Nitschke, Heller, Imig, McDonald, \& Miller, 2001; Nitschke, Heller, Palmieri, \& Miller, 1999; P. B. Sharp, Miller, \& Heller, 2014). As mentioned, the STAI T-Anxiety scale appears to evaluate aspects of anxiety such as worry, tension and disturbing thoughts which correspond more closely to anxious apprehension than anxious arousal; indeed, the STAI T-Anxiety scale has been criticised for not including items that measure somatic and behavioural aspects of anxiety (e.g., Bados et al., 2010; M. Balsamo et al., 2013). Somatic or physiological aspects of anxiety include, amongst other things, a pounding heart and sweating, and behavioural aspects include avoiding situations and inability to relax. Including somatic and behavioural aspects of anxiety in a measure like the STAI T-Anxiety scale would better reflect the heterogeneous nature of anxiety as 
conceptualised in Clark and Watson's (1991) influential tripartite model (Clark \& Watson, 1991). It would also better reflect transdiagnostic constructs shown to have neurobiological underpinnings (P. B. Sharp et al., 2014), and symptoms of DSM-5 anxiety disorders such as panic disorder and agoraphobia. Although the STAI T-Anxiety scale does not appear to adequately measure anxious apprehension and anxious arousal, the scale remains one of the most popular measures for assessment of trait anxiety in both healthy and clinical populations (M. Balsamo et al., 2013).

A third issue relating to the STAI is that studies that use the instrument to measure trait anxiety often do not control for state anxiety. The authors of the studies do not usually mention why they do not control for state anxiety but some behavioural and genetic studies show that trait and state anxiety appear to be related (Horikawa \& Yagi, 2012; J. Y. F. Lau, Eley, \& Stevenson, 2006; Ping, Subramaniam, \& Krishnaswamy, 2008; Spielberger et al., 1983). For example, when placed under pressure, individuals who are high in trait anxiety score significantly higher on measures of state anxiety than individuals who are not high in trait anxiety (Horikawa \& Yagi, 2012; Ping et al., 2008), and the genes that influence state and trait anxiety appear to be the same (J. Y. F. Lau et al., 2006). It has been suggested that people high in trait anxiety tend to exhibit higher state anxiety than people low in trait anxiety because they interpret a wider range of situations as threatening (Spielberger et al., 1983). Given that state anxiety and trait anxiety appear to be correlated, attempting to control for state anxiety would cause problems with multicollinearity (Blalock, 1963). In addition, state anxiety has been shown to have relatively low test-retest reliability presumably because it is influenced by situational factors at time of testing (Spielberger et al., 1983). Depending on the statistics used, attempting to control for such an unreliable variable would lead to larger standard errors or biased, inconsistent parameter estimates with inflated test statistics (Westfall \& Yarkoni, 2016). 
Finally, in many studies that use trait anxiety as the independent variable, participants are allocated to one of two groups either low or high in trait anxiety and group performance on a dependent variable is compared. It is well known that levels of arousal that are too low or too high affect performance in a U-shaped manner (Yerkes \& Dodson, 1908). Despite possible concerns that, when using groups low and high in trait anxiety, the effect of arousal may distort the pattern of effects because arousal may affect the two groups differently, the $U$ shaped effect of low, moderate or high levels of arousal on performance is related primarily to state anxiety. Level of arousal should not directly influence levels of trait anxiety, which is stable across situations.

\section{Behavioural Inhibition}

A concept closely related to trait anxiety is behavioural inhibition. Behavioural inhibition is a childhood temperament that describes the style in which a child responds to unfamiliar stimuli. Infants and children high on behavioural inhibition react fearfully and with caution, restraint and withdrawal when presented with novel or unfamiliar situations, events, people and objects (N. A. Fox, Henderson, Marshall, Nichols, \& Ghera, 2005; Hirshfeld-Becker et al., 2008). Children who are high on behavioural inhibition have a greater risk of developing anxiety and anxiety disorders - especially social anxiety disorder later in life (e.g., Chronis-Tuscano et al., 2009; Hirshfeld-Becker et al., 2008; S. L. Johnson, Turner, \& Iwata, 2003). The behavioural inhibition system (BIS) that underpins behavioural inhibition is thought to be a conflict detection system that is activated by goal-conflicts such as when a threatening stimulus must be approached (Gray \& McNaughton, 2000; Smillie, 2008). The BIS increases attention to resolve conflict by inhibiting both approach and withdrawal behaviour (Berkman, Lieberman, \& Gable, 2009; Wacker, Chavanon, Leue, \& Stemmler, 2010). The 'inhibition' part of behavioural inhibition does not refer to the intentional process of inhibitory control but to the stopping/interruption of behaviour in 
response to an expected or unexpected stimulus (Amodio, Master, Yee, \& Taylor, 2008). The BIS is sensitive to cues of punishment, non-reward and novelty (Gray \& McNaughton, 2000; Amodio et al., 2008) and activates inhibition, orienting, attention, risk analysis and avoidance as well as feelings of arousal and anxiety (Hundt, Nelson-Gray, Kimbrel, Mitchell, \& Kwapil, 2007; S. L. Johnson et al., 2003).

Despite extensive research into the conceptualisation and assessment of the BIS and its relationship to psychological disorders such as anxiety, the neurobiological substrates of the BIS have not yet been well defined (Kennis, Rademaker, \& Geuze, 2013). It is thought that the BIS is associated with activity of the septal-hippocampal system which includes the hippocampal formation, prefrontal cortex, cingulate cortex, striatum, thalamus, amygdala, hypothalamus, periadqueductal gray, and the lateral and medial septum (Aluja \& Blanch, 2011; Canteras, Resstel., Bertoglio, de Padua Carobrez, \& Guimaraes, 2010; J. A. Gray, 1982; J. A. Gray \& McNaughton, 2000; McNaughton \& Corr, 2004). BIS-related personality traits have been associated with hyperactivity of the amygdala (Pérez-Edgar et al., 2007; Schwartz, Wright, Shin, Kagan, \& Rauch, 2003; Smillie, 2008), cingulate activity, and decreased functional connectivity of the amygdala with the prefrontal cortex, anterior cingulate cortex (ACC) and hippocampus (Kennis et al., 2013).

The BIS is commonly measured using the Behavioural Inhibition System/Behavioural Activation System scales (BIS/BAS Scales) developed by Carver and White (1994). High scores on measures of behavioural inhibition are associated with higher scores on measures of anxiety (Hundt et al., 2007) and confirmatory factor analysis has been used to show that the STAI T-Anxiety scale is correlated with scores on the behavioral inhibition subscale (BIS subscale) of the BIS/BAS Scales (Cooper, Perkins, \& Corr, 2007). Researchers have been interested in and investigated the association between behavioural inhibition and reward and punishment sensitivity using a range of experimental paradigms (e.g., Bar-Haim et al., 2009; 
Guyer et al., 2006; Hahn et al., 2010; Helfinstein et al., 2011; Simon et al., 2010), presumably because Reinforcement Sensitivity Theory (J. A. Gray, 1973; J. A. Gray \& McNaughton, 2000) proposes that the BIS mediates reactions to aversive stimuli and is, therefore, associated with sensitivity to signals of punishment and omission/termination of reward (Corr, 2002a, 2002b; McNaughton \& Corr, 2004; Smillie, 2008). In contrast, relatively little research has been conducted into the association between trait anxiety and sensitivity to reward and punishment. A small number of studies have investigated the relationship between reward and punishment processing and anxiety disorders such as posttraumatic stress disorder, social anxiety disorder, and GAD (Admon et al., 2013; Cremers et al., 2010; Manning et al., 2015; Teng et al., 2016). This review will therefore use literature pertaining to the BIS and its relationship to rewarding and punishing cues and feedback to inform hypotheses about what we would expect to observe in trait anxiety.

\section{Neural Mechanisms Underlying Anxiety}

It has been argued that each anxiety disorder listed in DSM-5 has a unique constellation of behavioural, physiological and neural correlates (Kent \& Rauch, 2003; Shin \& Liberzon, 2010). Despite this heterogeneity, studies have identified dysfunction within neural structures and networks that appears to be common across anxiety disorders as well as to trait anxiety (see Shin \& Liberzon, 2010, for review). Anxiety is associated with hyperactivity of the amygdala and extended amygdala (bed nucleus of the stria terminalis) (Bishop, Duncan, \& Lawrence, 2004; Dickie \& Armony, 2008; Etkin et al., 2004; Ewbank et al., 2009; Rosen \& Schulkin, 1998) (for review see both Grupe \& Nitschke, 2013; Taylor \& Whalen, 2015). The amygdala is involved in a range of processes that include detecting aversive environmental stimuli and situations, producing emotional or behavioural states to respond to potential threats, assigning valence (positive or negative) or salience (intensity of a stimulus) to stimuli (Paulus, 2008), and learning from both positive and negative outcomes 
(Janak \& Tye, 2015). The amygdala shares reciprocal connections with the midline and orbital prefrontal cortices as well as with the hippocampus and primary sensory and sensory association areas, and has unidirectional efferent connections with the striatum, in particular the nucleus accumbens and bed nucleus of the stria terminalis (Janak \& Tye, 2015).

The prefrontal cortex (PFC) is thought to exert top down control to reduce amygdala activation when a fearful response is no longer required (M. Justin Kim et al., 2011; Taylor \& Whalen, 2015). The ventromedial prefrontal cortex (vmPFC) (which includes the subgenual anterior cingulate, ventromedial prefrontal and medial orbitofrontal cortex (M. Justin Kim et al., 2011) is reciprocally connected to the amygdala and forms a vmPFC-amygdala circuit that learns about and responds to safety signals in threatening situations (Grupe \& Nitschke, 2013). Although it is commonly thought that the vmPFC inhibits amygdala output and therefore suppresses negative affect, it has also been argued that activation of the vmPFC may itself result in the experience of negative affect (Myers-Schulz \& Koenigs, 2012). The vmPFC and amygdala have altered function and connectivity in individuals with anxiety disorders (Grupe \& Nitschke, 2013; Patel, Spreng, Shin, \& Girard, 2012; Rauch, Shin, \& Phelps, 2006) as well as in individuals who are high in trait anxiety (M. J. Kim, Gee, Loucks, Davis, \& Whalen, 2011; M. J. Kim \& Whalen, 2009) and harm avoidance, which is a construct linked to anxiety (Pezawas et al., 2005). The decreased activation of the vmPFC and inability of the vmPFC to downregulate activity of the amygdala in individuals with anxiety when engaged in tasks (e.g., Pezawas et al., 2005) as well as at rest (M. J. Kim et al., 2011; M. J. Kim \& Whalen, 2009) may be why individuals with anxiety experience reduced fear extinction (Etkin \& Wager, 2007; Taylor \& Whalen, 2015), exaggerated autonomic responses (Taylor \& Whalen, 2015), and generally poorer functional outcomes than individuals without anxiety (M. J. Kim \& Whalen, 2009). 
Anxiety disorders and anxiety proneness are associated with hyperactivity of the insula (Alvarez et al., 2015; Dillon et al., 2014; Etkin \& Wager, 2007; Paulus \& Stein, 2006; Shin \& Liberzon, 2010), which processes stimulus salience (Menon \& Uddin, 2010) including uncertain, aversive events (Sarinopoulos et al., 2010). The insula also contributes to the regulation of the autonomic nervous system (Oppenheimer, Gelb, Girvin, \& Hachinski, 1992) and is involved in cognitive and affective processes during learning (Paulus \& Stein, 2006), subjective feeling states, interoception (i.e., how the body feels) and predictive interoception (i.e., how the body may feel given a particular stimulus) (Paulus, 2008). Increased activation of the insula in anxiety may reflect over-monitoring of the internal environment for threats (Dillon et al., 2014) or increased activation of a network that generates fear responses (Etkin \& Wager, 2007). It may also reflect a heightened “interoceptive prediction signal" (Paulus \& Stein, 2006); these signals indicate a difference between an observed and expected bodily state. It has been proposed that individuals with anxiety experience an increased signalling of this difference and, in an attempt to reduce the perceived difference, engage in affective, cognitive and behavioural components of anxiety (Paulus \& Stein, 2006). The insula is highly interconnected with other brain regions including the amygdala, PFC and other limbic structures (Taylor \& Whalen, 2015). There appears to be reduced resting state connectivity between bilateral anterior and posterior insula in anxiety-prone individuals (Bijsterbosch, Smith, Forster, John, \& Bishop, 2014). Resting state connectivity refers to an imaging technique that measures the level of co-activation between brain regions when an individual is relaxing or 'at rest'; the co-activation between these regions is thought to reflect functional communication between them (van den Heuvel \& Hulshoff Pol, 2010). Anxiety proneness is also associated with reduced resting state connectivity between the insula and the amygdala-hippocampal networks, and physiological/somatic anxiety is associated with reduced resting state connectivity between 
the insula and both medial prefrontal and orbitofrontal cortex-subcortical networks (Bijsterbosch et al., 2014). Reduced connectivity of the insula may reflect a reduced ability to activate and regulate responses to changes in internal and external states/events, which manifests as emotion dysregulation and the physiological expression of anxiety (e.g., racing heart, sweating and so on) (Bijsterbosch et al., 2014; Paulus, 2008).

There is also evidence to suggest the structure, volume, function and connectivity of the dorsal anterior cingulate cortex (dACC) (also sometimes referred to as the anterior midcingulate cortex (aMCC)) is altered in anxiety. Using electroencephalogram (EEG) and functional magnetic resonance imaging (fMRI), studies have found that individuals with anxiety demonstrate increased $\mathrm{dACC}$ activation when errors are made in speeded response time tasks (Aarts \& Pourtois, 2010; Hajcak, McDonald, \& Simons, 2003; Moran, Taylor, \& Moser, 2012; Moser et al., 2012; Moser, Moran, Schroder, Donnellan, \& Yeung, 2013; Paulus, Feinstein, Simmons, \& Stein, 2004; Weinberg, Klein, \& Hajcak, 2012; Weinberg, Olvet, \& Hajcak, 2010; Zambrano-Vazquez \& Allen, 2014). Abnormal ACC functioning has been observed in a range of anxiety disorders including post-traumatic stress disorder (PTSD) and specific phobia (e.g., Bystritsky et al., 2001; Rauch et al., 1995; Rauch et al., 1996; Shin et al., 2001), and the induction of anxiety has been shown to increase ACC activity (Kimbrell et al., 1999). Trait anxiety is also associated with altered neurochemistry in the ACC (Shilpi Modi, Rana, Kaur, Rani, \& Khushu, 2014).

The dACC is connected to, amongst other areas, the insula (Cauda et al., 2011; Yu et al., 2011), the lateral basal nucleus of the amygdala (Roy et al., 2009), and frontoparietal regions involved in cognitive control and maintenance of goals (e.g., dorsolateral prefrontal cortex (DLPFC)) (Shackman et al., 2011; Yu et al., 2011). The dACC is thought to be involved in regulating autonomic activity, performance monitoring, cognitive control, and processing negative affect and pain, as well as synthesizing information about learned and 
unlearned reinforcers with current goals (Seeley et al., 2007; Shackman et al., 2011; Van Veen \& Carter, 2002). Shackman et al. (2011) outline what they have called an "adaptive control hypothesis" of the dACC whereby negative affect, pain and cognitive control all share the common core function of determining the best course of action in situations of uncertainty. They propose that the dACC integrates information about potential punishment in order to bias and optimize responding in uncertain situations. If this hypothesis is correct, individuals high in anxiety who demonstrate increased dACC activation under conditions of uncertainty (e.g., Paulus et al., 2004) may also demonstrate increased cognitive control, be more sensitive to cues of punishment, and may respond to cues of potential punishment and feedback indicating punishment in a more punishment-averse manner. Indeed, high compared to low trait anxious individuals demonstrate an enhanced dACC response when errors are punished (Riesel, Weinberg, Endrass, Kathmann, \& Hajcak, 2012). Beyond these findings, it is not clear precisely how dACC dysfunction influences cognitive control in the presence of punishment and how both dACC dysfunction and cognitive control are more broadly involved in the maladaptive behavioural, cognitive and emotional responses observed in anxiety. For example, dACC dysfunction might be implicated in difficulties resolving conflict during processing of emotional content (Amir et al., 2005; Taylor \& Whalen, 2015) and/or difficulty executing adaptive responses in situations involving uncertainty (Grupe \& Nitschke, 2013) in individuals high in anxiety.

The above discussion of brain regions and networks relates to a pattern of neural dysfunction that has been observed in anxiety generally, i.e., across anxiety disorders, subclinical anxiety and/or anxiety proneness. It is beyond the scope of the current review to discuss the specific pattern of neural dysfunction in each anxiety disorder but it should be noted that each disorder appears to be characterised by a particular pattern of neural 
dysfunction involving a range of structures including the amygdala, rostral ACC, dACC, hippocampus and insular cortex (see Shin \& Liberzon, 2010, for review). 


\section{Neural Mechanisms Underlying Trait Anxiety}

The purpose of the current review is to examine neural dysfunction relevant and specific to trait anxiety. Structural and network dysfunction specific to trait anxiety has been identified using resting state fMRI as well as fMRI in conjunction with affective and emotionally neutral experimental paradigms. Resting state studies have found altered resting state connectivity in participants high in trait anxiety in limbic and prefrontal/cortical structures (He, Xu, Zhang, \& Zuo, 2016; M. J. Kim et al., 2011), as well as in numerous networks including the default mode network, the medial visual network, the auditory network and the temporo-parietal network (Coutinho et al., 2016; He et al., 2016; S. Modi, Kumar, Kumar, \& Khushu, 2015).

Studies that have used tasks with affective or emotional stimuli have found that high trait anxiety is associated with dysfunction in subcortical structures responsible for processing threat related stimuli. Compared to healthy controls, individuals high in trait anxiety demonstrate increased bilateral anterior insular cortex activation, increased bilateral amygdala activation, and decreased functional connectivity of the vmPFC with the bilateral insula and left amygdala when matching images of faces conveying emotional expressions (Stein, Simmons, Feinstein, \& Paulus, 2007). Trait anxiety has also been associated with activation of the basolateral amygdala to unconsciously processed fearful faces (Etkin et al., 2004), suggestive of neural correlates underlying unconscious processing biases in trait anxiety. High trait anxious individuals demonstrate greater amygdala and subgenual ACC activation during extinction of a conditioned stimulus (Barrett \& Armony, 2009). High trait anxiety has also been associated with increased ACC activation but reduced functional connectivity between the ACC and the right lateral PFC when high trait anxious individuals process emotional conflict (Comte et al., 2015). 
In summary, individuals high in trait anxiety display abnormal neural responses when presented with threating or emotionally-relevant stimuli during experimental tasks. Much research has investigated this threat-related processing bias in anxiety (Bar-Haim, Lamy, Pergamin, Bakermans-Kranenburg, \& Van Ijzendoorn, 2007). Interestingly, trait anxiety also appears to be associated with impaired activation of brain structures and networks when no apparent threat is present. Studies have used emotionally neutral stimuli to look at these impairments. When presented with affectively neutral colour-word Stroop stimuli, compared to individuals who are low in trait anxiety, individuals high in trait anxiety show stronger activation in the right DLPFC when responding to incongruent compared to congruent trials but weaker functional connectivity of the right DLPFC with the inferior frontal junction area (IFJ) bilaterally, the dACC and the left fusiform gyrus during these trials (Basten, Stelzel, \& Fiebach, 2011). Individuals who are high in anxiety also make more errors on these trials than individuals who are low in anxiety (Basten et al., 2011). Given that the DLPFC is thought to be responsible for adaptive implementation of cognitive control, the stronger DLPFC activation may reflect the additional effort made by high trait anxious individuals to compensate for weaker connectivity of the DLPFC within the task-relevant inhibitory control network (Basten et al., 2011) (for relevance of IFJ and dACC in inhibitory control see Neural Structures Involved in Response Inhibition in the Stop Signal Task below). Alternatively, the stronger DLPFC activation may merely represent greater 'noise' within the DLPFC caused by the lack of efficient connectivity within the network (see Comte et al., 2015 for similar discussion in relation to ACC). Individuals high in trait anxiety also demonstrate greater transient and less sustained activation in cognitive control regions during tasks involving working memory than individuals low in trait anxiety (Fales et al., 2008). Such patterns of activation suggest that these individuals are working hard to increase reactive control 
(transient, stimulus bound control), perhaps to compensate for reductions in proactive (goalrelated) control (Fales et al., 2008).

In contrast to findings of increased activation in structures such as the DLPFC and associated networks involved in cognitive control in individuals high in trait anxiety (Basten et al., 2011; Fales et al., 2008), high trait anxiety has been associated with less DLPFC activation to response conflict (Bishop, 2009). The decreased DLPFC activation has been observed under conditions of low perceptual load (e.g., a string of the same letters therefore low attentional search requirement), not high perceptual load (e.g., a string of different letters therefore high attentional search requirement). These findings suggest that individuals with anxiety experience difficulty with trial-to-trial alterations in attentional control when a task does not fully occupy attentional resources. When a task is, for example, easy to perform such that it does not fully occupy attention, high trait anxious individuals may be distracted from the task at hand by salient stimuli - including internal states/stimuli such as rumination and worrying thoughts - so that they do not engage neural networks needed for efficient cognitive control. In support of this proposition, Forster, Nunez Elizalde, Castle, and Bishop (2015) found that, when inhibiting responses to infrequent No-Go stimuli during a sustained attention to response task, individuals with trait anxiety demonstrated less DLPFC and dACC activity than individuals without trait anxiety.

In summary, findings from studies that have used tasks with non-threat-related, neutral stimuli indicate that, if a task does not fully occupy attention, individuals high in trait anxiety show decreased reactive/transient activation of regions responsible for goal directed attention (e.g., DLPFC) (Bishop, 2009; Forster, Nunez Elizalde, Castle, \& Bishop, 2015) as well as decreased proactive control in these tasks (e.g., decreased functional connectivity between DLPFC and other regions during errorless Go trials in a sustained attention to response task (Forster et al., 2015)). When task difficulty increases, individuals high in trait 
anxiety increase transient, trial-dependent activation of cognitive control networks (Basten et al., 2011; Fales et al., 2008), perhaps to compensate for decreased sustained, goal-directed activation of these networks (Fales et al., 2008). On the whole, these findings demonstrate that individuals high in anxiety experience difficulties implementing cognitive control, perhaps due to interference by task-irrelevant thoughts or weak connectivity in cognitive control networks.

Worry, rumination and off-task self-referent thought is a prominent feature of anxiety. The tendency to engage in and/or attempt to counter the automaticity of these processes during tasks that require cognitive control has been observed at a neurobiological level. In healthy control participants, regions in the default mode network are more active during rest than during attention-demanding tasks (Greicius, Krasnow, Reiss, \& Menon, 2003; Gusnard \& Raichle, 2001; Raichle et al., 2001). The default mode network is active during mindwandering (Christoff, Gordon, Smallwood, Smith, \& Schooler, 2009), personal introspection, autobiographical memories and future thoughts (Whitfield-Gabrieli \& Ford, 2012), and becomes less active during focused task performance (Drevets \& Raichle, 1998; M. D. Fox \& Raichle, 2007; Greicius et al., 2003). It has been proposed that deactivation of the default mode network may act as an inhibitory mechanism that suspends spontaneous mental activity such as imagery or rumination; this suspension is necessary for efficient cognitive processing and task performance (Fales et al., 2008). Somewhat counterintuitively, high trait anxious individuals have shown less sustained activation in the default mode network during a n-back working memory task than low trait anxious individuals without any observed difference in behavioural measures such as accuracy or reaction time (Fales et al., 2008).

It has been suggested that the default mode deactivation seen in high trait anxious individuals may be an indicator that they are using greater cognitive effort to perform the task (in healthy control participants default mode deactivation usually increases with task 
difficulty (McKiernan, Kaufman, Kucera-Thompson, \& Binder, 2003)) and/or may be implicitly reducing their awareness of emotional arousal or rumination (Fales et al., 2008). Individuals higher compared to those lower in trait anxiety also show increased DLPFC activity and increased connectivity between the right DLPFC and default mode regions implicated in self-referent processing (bilateral precuneus and posterior cingulate) in sustained attention to response tasks on go trials where errors are made, with no association between brain activation and reaction times (Forster et al., 2015). Increased connectivity between the DLPFC and the default mode network in these individuals may reflect the use of explicit regulation strategies employed to downregulate negative emotions (Campbell-Sills et al., 2011; Hartley \& Phelps, 2010; Sylvester et al., 2012). Indeed, individuals who have anxiety disorders show greater activity than healthy participants in cognitive control areas including the left DLPFC, bilateral ventrolateral prefrontal cortex (vlPFC), dorsomedial prefrontal cortex (dmPFC) and subgenual ACC during down-regulation of emotions using cognitive reappraisal strategies, suggesting they require increased engagement of this network to achieve successful emotional down-regulation measured behaviourally via selfreport (Campbell-Sills et al., 2011). In summary, it is possible that individuals high in trait anxiety experience increased off-task thought and rumination when performing tasks that require cognitive control and, due to or perhaps independently of these internal distractions, have to work harder to maintain task performance. These individuals may also need to use more explicit strategies to downregulate these task-irrelevant thoughts and negative emotions. Sylvester et al. (2012) have proposed a model of network dysfunction in anxiety that involves a specific pattern of activation across four networks. The model relates to processing of emotionally neutral stimuli, that is, processing that does not involve threat-related, fearrelated or emotion-specific stimuli. Sylvester et al. suggest that anxiety is characterised by increased activation of a cingulo-opercular network that is comprised of the bilateral dACC, 
anterior insula, anterior PFC and anterior thalamus. This network is thought to detect errors and conflict and signals the need for increased cognitive control. It also processes pain and negative affect. The increased activity of this network may mean individuals with anxiety are oversensitive to errors, negative outcomes, punishment or response conflict. The frontoparietal network is comprised of anterior portions of the DLPFC, inferior parietal lobule, portions of the middle cingulate gyrus and portions of the precuneus. This network is involved in executive control, enabling the system to make adjustments in processing. The fronto-parietal network is thought to have decreased functioning in anxiety which results in impairments in top-down attentional control and impaired regulation of other neural structures and networks such as the amygdala and the cingulo-opercular network. In the model proposed by Sylvester et al., anxiety is also associated with increased activation of the ventral attention network which is comprised of the vlPFC, temporo-parietal junction (TPJ), and portions of the middle and superior temporal gyri. This network is responsible for automatic orientation of stimulus-driven attention. The overactivity of this network suggests that individuals with anxiety may have increased stimulus-driven attention for both external and internal events/stimuli. Finally, Sylvester et al. suggest that anxiety is associated with decreased functioning of the default mode network which is comprised of portions of the subgenual ACC, posterior cingulate cortex (PCC), precuneus, lateral parietal cortex, medial PFC, inferior temporal gyrus, parahippocampal gyrus and frontal pole/superior frontal cortex (for studies that have found decreased default mode network activation in anxiety see Modi Kumar, Kumar, \& Khushu (2015) and Fales et al. (2008), discussed above). As mentioned, the default mode network is responsible for functions such as self-referential activities, future planning, self-introspection and emotion regulation including extinction and the cognitive regulation needed to diminish a fear response. In performing these functions, the default mode network interacts with other networks. Sylvester et al. suggest decreased functioning of 
the default mode network in anxiety may result in difficulty using cognitive strategies to regulate emotions. In sum, the pattern of network dysfunction identified by Sylvester et al. may result in individuals with anxiety demonstrating increased error sensitivity and stimulusdriven attention while having decreased top-down attentional control and emotion regulation.

Table 1 summarises brain regions affected in anxiety and potential cognitive/behavioural outcomes of hyperactivation and hypoactivation of these regions during an affectively neutral task requiring cognitive control. 
Table 1

Brain regions and Potential Cognitive/Behavioural Outcomes of Activation in Anxiety During an Affectively Neutral Cognitive Control Task

\begin{tabular}{|c|c|c|c|c|c|c|c|c|}
\hline \multicolumn{5}{|c|}{ Structures } & \multicolumn{4}{|c|}{ Networks (Sylvester et al., 2012) } \\
\hline Amygdala & vmPFC & Insula & $\mathrm{dACC}$ & DLPFC & Default mode & $\begin{array}{c}\text { Cingulo- } \\
\text { opercular (error } \\
\text { monitoring) }\end{array}$ & $\begin{array}{c}\text { Fronto-parietal } \\
\text { (executive } \\
\text { control) }\end{array}$ & $\begin{array}{c}\text { Ventral } \\
\text { attention } \\
\text { (stimulus } \\
\text { based) }\end{array}$ \\
\hline $\begin{array}{l}\text { Increased } \\
\text { activation. } \\
\text { Detects aversive } \\
\text { environment } \\
\text { (punishment and } \\
\text { fear of } \\
\text { failure/not } \\
\text { performing well } \\
\text { may be } \\
\text { considered } \\
\text { aversive). }\end{array}$ & $\begin{array}{l}\text { Decreased } \\
\text { activation. Not } \\
\text { down-regulating } \\
\text { amygdala. } \\
\text { Reduced } \\
\text { connectivity. } \\
\text { Results in reduced } \\
\text { fear extinction, } \\
\text { exaggerated } \\
\text { autonomic } \\
\text { responses and } \\
\text { poorer functional } \\
\text { outcomes. }\end{array}$ & $\begin{array}{l}\text { Increased } \\
\text { activation. } \\
\text { Over- } \\
\text { monitoring of } \\
\text { internal threats. } \\
\text { Reduced } \\
\text { connectivity. } \\
\text { Reduced } \\
\text { emotion } \\
\text { regulation and } \\
\text { detection of } \\
\text { salience. }\end{array}$ & $\begin{array}{l}\text { Increased } \\
\text { activation. } \\
\text { Difficulty } \\
\text { resolving } \\
\text { conflict and } \\
\text { abnormal } \\
\text { response } \\
\text { monitoring. } \\
\text { Possible } \\
\text { increased } \\
\text { sensitivity } \\
\text { to } \\
\text { punishment. }\end{array}$ & $\begin{array}{l}\text { Increased } \\
\text { activation. } \\
\text { Individual } \\
\text { "trying harder" } \\
\text { because of } \\
\text { reduced } \\
\text { functional } \\
\text { connectivity and } \\
\text { need for greater } \\
\text { reactive control } \\
\text { due to decreased } \\
\text { proactive } \\
\text { control. } \\
\text { If task is easy, } \\
\text { may show } \\
\text { reduced DLPFC } \\
\text { response } \\
\text { because } \\
\text { distracted by } \\
\text { internal stimuli. }\end{array}$ & $\begin{array}{l}\text { Decreased activation. } \\
\text { Difficulty using cognitive } \\
\text { strategies to regulate } \\
\text { emotions. Also applying } \\
\text { more cognitive effort to } \\
\text { facilitate task performance } \\
\text { therefore default mode } \\
\text { network is more deactivated. } \\
\text { May represent implicit } \\
\text { reduction of awareness of } \\
\text { increased emotional arousal. } \\
\text { If there is increased } \\
\text { activation the individual } \\
\text { may be using explicit } \\
\text { regulation strategies to } \\
\text { downregulate negative } \\
\text { emotions. }\end{array}$ & $\begin{array}{l}\text { Increased } \\
\text { activation. } \\
\text { Oversensitive to } \\
\text { errors and } \\
\text { punishment - } \\
\text { this will } \\
\text { increase anxiety } \\
\text { (amygdala and } \\
\text { insula } \\
\text { response). }\end{array}$ & $\begin{array}{l}\text { Decreased } \\
\text { activation. } \\
\text { Decreased top- } \\
\text { down attentional } \\
\text { control - not } \\
\text { able to } \\
\text { downregulate } \\
\text { other structures } \\
\text { such as } \\
\text { amygdala and } \\
\text { insula, and } \\
\text { cingulo- } \\
\text { opercular } \\
\text { network. }\end{array}$ & $\begin{array}{l}\text { Increased } \\
\text { activation. } \\
\text { Increased } \\
\text { attention to } \\
\text { external and } \\
\text { internal stimuli } \\
\text { which may } \\
\text { increase } \\
\text { anxiety around } \\
\text { errors and } \\
\text { worrying } \\
\text { thoughts. }\end{array}$ \\
\hline
\end{tabular}




\section{Cognitive Models of Anxiety, Threat and Attentional Bias}

As mentioned above, anxiety is characterised by an attentional bias toward and preferential processing of threatening sources of information (Cisler \& Koster, 2010; Teachman, Joormann, Steinman, \& Gotlib, 2012). Cognitive models of anxiety propose that these biases in information processing may both cause and maintain symptoms of anxiety via selective attention to, interpretation of, judgment of and memory for cues that are relevant to anxious schemas (Teachman et al., 2012). There are a number of theories that seek to elucidate the cognitive mechanisms underlying attentional biases in anxiety and, while these theories differ in relation to their consideration of the role and timing of these biases, they all agree that anxiety involves an attentional bias for threat-related information (for discussion see Bar-Haim et al., 2007 and; Cisler \& Koster, 2010). This bias should lead to abnormal patterns of behaviour as well as abnormal activation in neural structures and circuits during tasks that require the making or withholding of a response during potentially threatening events or situations. Many studies have looked at the effect of threat related processing biases on attention using emotional stimuli such as threatening words or angry faces in response inhibition tasks such as the emotional Stroop or antisaccade task (for summary see Bar-Haim et al., 2007; Cisler \& Koster, 2010). These studies have found that highly anxious individuals have difficulty inhibiting threat-related task-irrelevant information (Bar-Haim et al., 2007; Derakshan, Ansari, Hansard, Shoker, \& Eysenck, 2009). The mechanisms underlying these biases are debated but it is thought that anxious individuals may have facilitated attention toward threat-related stimuli, and/or they may experience difficulty disengaging attention from threat-related stimuli, and/or they may engage in attentional avoidance of threat-related stimuli (Cisler \& Koster, 2010). There has been substantially less research conducted in relation to whether there is a general inhibitory control deficit in anxiety; that is, whether individuals with anxiety demonstrate inhibitory control deficits when no obvious threat is 
present. Across a range of neutrally valenced tasks (e.g., stop signal tasks, go/no-go tasks, Stroop tasks, flanker tasks and antisaccade tasks) and heterogeneous participant groups suffering from anxiety (e.g., groups that have a single anxiety disorder or a range of anxiety disorders and/or trait anxiety, which is measured using different instruments across different studies), results have been mixed. Some studies have found individuals high in anxiety have impairments in inhibitory control (Tahereh L. Ansari \& Derakshan, 2011b; Hallion, Tolin, Assaf, Goethe, \& Diefenbach, 2017), some have found better inhibitory control (Sehlmeyer et al., 2010), and some have found no difference (Epstein, Johnson, Varia, \& Conners, 2001; Hallion et al., 2017) when the performance of individuals high in anxiety is compared with that of healthy individuals or individuals low in anxiety. (See Inhibitory Control, Anxiety and the Stop Signal Task below for a more detailed discussion of inhibitory control in trait anxiety using the stop signal task.)

A prominent theory that seeks to clarify the cognitive mechanisms responsible for the effect of anxiety on behavioural performance - especially in tasks that place demands on attentional resources - is attentional control theory that developed from earlier processing efficiency theory (see Derakshan \& Eysenck, 2009, for review). Currently, attentional control theory appears to be one of the only theories to provide a targeted explanation of the potential effect of anxiety on inhibitory control. It is described in detail in a handful of articles (Derakshan \& Eysenck, 2009; M. W. Eysenck \& Derakshan, 2011; M. W. Eysenck, Derakshan, Santos, \& Calvo, 2007) and, although other studies have tested the theory and used it to support their findings (e.g., Derakshan et al., 2009; Fales et al., 2008; Savostyanov et al., 2009; Savostyanov et al., 2011) or used their findings to challenge its predictions (e.g., Bishop, 2009; Forster et al., 2015), no one has yet proposed an amendment or comprehensive alternative. According to attentional control theory, anxiety disrupts the balance between two attentional systems - the goal-directed attentional system and the stimulus-driven attentional 
system - so that the goal-directed system has decreased influence and the stimulus-driven attentional system has increased influence, and this effect is pronounced when anxiety levels are high. More specifically, attentional control theory suggests that anxiety occurs when a current goal is threatened. Threat to a current goal causes attention to be allocated to detecting the source of the threat (an increase in reactive control) and deciding on a response (M. W. Eysenck et al., 2007). Threat-related stimuli can include external stimuli such as threatening task-irrelevant distractors or internal stimuli such as worrying thoughts and selfpreoccupation (M. W. Eysenck et al., 2007). Due to increased allocation of attention to these threat-related stimuli and to deciding how to respond, attentional focus on the current task (proactive control) is reduced. Further, attentional control theory assumes anxiety impairs attentional focus even when no threat-related stimuli are actually present since, if an individual with anxiety believes that a potential threat may exist, the best strategy is to allocate attentional resources widely. The diffused allocation leads to a reduction in attentional focus (proactive control) in relation to any ongoing task (M. W. Eysenck et al., 2007). By reducing attentional focus on task demands, anxiety affects attentional control (Derakshan \& Eysenck, 2009). Attentional control is required by a number of cognitive processes including inhibition; inhibition involves the ability to inhibit a dominant, automatic or prepotent response (Miyake et al., 2000). When attempting to inhibit a response, an individual must use attentional control to ensure that attention is not allocated to taskirrelevant stimuli and internal responses (Derakshan \& Eysenck, 2009; M. W. Eysenck et al., 2007). If, as proposed by attentional control theory, anxiety negatively impacts attentional control, anxiety should also negatively impact response inhibition during cognitive tasks that use neutral stimuli.

Attentional control theory makes a distinction between performance effectiveness and processing efficiency. Performance effectiveness refers to quality of performance (e.g., 
accuracy rates). Processing efficiency refers to the relationship between performance effectiveness and the amount of effort/resources used to attain that level of performance (Derakshan \& Eysenck, 2009). In tasks that involve inhibition, i.e., the tasks require using attentional control in a restraining way, attentional control theory proposes that anxiety will impair processing efficiency and this may be manifest in, for example, longer reaction times and increased effort (M. W. Eysenck et al., 2007). Anxiety will not necessarily lead to decrements in performance effectiveness (e.g., accuracy), because individuals high in anxiety use compensatory strategies such as enhanced effort and use of processing resources (M. W. Eysenck et al., 2007). However, as overall task demands increase adverse effects of anxiety on performance become greater. Also, because they are devoting more processing resources to performance on a task, individuals high in anxiety have less residual processing capacity and will perform a simultaneous task less efficiently than non-anxious individuals (M. W. Eysenck et al., 2007). Attentional control theory does not specify what may happen when anxious individuals engage in compensatory processes over time. It is possible that engaging in compensatory strategies is taxing such that, over time, performance effectiveness deteriorates or the individual begins to suffer cognitive fatigue.

The impairments in attentional control suggest that individuals high in anxiety should demonstrate reductions in inhibitory control during inhibitory control tasks that use affectively neutral stimuli (although perhaps not as much as during tasks that use threat related stimuli - see Eysenck et al. 2007). Further, based on assumptions of attentional control theory, any cues that signal the potential for punishment during these tasks should be interpreted by individuals high in anxiety as an external threat. These threat signals should increase the probability that threat-related internal events, such as worrying or selfpreoccupation, will occur. The presence of external and internal threat-related stimuli/events may lead to an increase in allocation of attention to the threats. The re-allocation of attention 
may lead to a reduction in task-related attentional focus, attentional control, and in turn, impaired inhibitory control. The latter may manifest as either decrements in processing efficiency (e.g., slower reaction time and/or increased neural activity) and, if the task is sufficiently challenging, performance effectiveness (e.g., increased error rates) during the entire task or perhaps only on trials where cues signalling potential punishment are presented.

\section{Inhibition}

In the wider literature on cognitive control, the term inhibition is used to describe many different processes including inhibition of return, social inhibition, inhibitory reflexes and reactive inhibition (Bari \& Robbins, 2013). It has also been divided into a number of distinct forms such as voluntary/active/willed inhibition versus automatic inhibition (Aron, 2007). As the name implies, automatic inhibition involves automatic inhibitory responses; examples include latent inhibition and inhibition of return (Bari \& Robbins, 2013). In contrast, voluntary inhibition (also called active or willed inhibition) is the active suppression of a response, stimulus or memory (Aron, 2007; Aron, Robbins, \& Poldrack, 2014a). Voluntary inhibition of a physical or motor response (or 'response inhibition') is a subcomponent of cognitive control - the ability to coordinate sensory and motor processes toward a common goal (Miller \& Cohen, 2001) - which is part of the 'executive system' (Bari \& Robbins, 2013). Response inhibition may involve withholding (or 'restraint'), postponing (waiting) or cancelling (stopping) an action (Bari \& Robbins, 2013). It is also thought that response inhibition can be divided into two different types of inhibitory control; reactive and proactive. As mentioned briefly above, reactive control occurs when an individual stops a response because they are instructed to do so by an external signal. In contrast, proactive control involves a preparation for stopping that occurs before the response is triggered, is developed according to the goals of the individual and is manifest by, for example, slower response times (Aron, 2011). 
Tasks that measure response inhibition include stop signal tasks (cancellation or behavioural inhibition) go/no-go tasks (withholding or behavioural inhibition), and flanker tasks (interference control) (see Nigg, 2000). All these tasks require an action, such as a button press, to be performed or inhibited. Inhibition can also be measured using tasks that do not require a button press response, such as antisaccade (oculomotor inhibition) and Stroop tasks (interference control) (see Nigg, 2000). Due to the size of the literature and the lack of overlap between different task requirements and research findings, this review will focus on inhibition that involves stopping or cancelling an already initiated response and will consider the way in which this type of inhibition is impaired in or facilitated by high trait anxiety. Inhibition that involves stopping or cancelling an already initiated response is measured using the stop signal task.

The stop signal task is one of the most commonly used tasks for measuring inhibitory control (Criaud \& Boulinguez, 2013). Stop signal tasks involve a go and a stop task. In the go task, the participant must discriminate two stimuli from each other (e.g., an X from an O) usually via a button press mapped to each of the stimuli. In the stop task, which usually occurs on $25 \%$ of trials, the go signal is presented but is shortly followed by a stop signal. When presented with the stop signal, the participant must withhold their go task response. The stop signal task differs from the go/no-go task in that, because the go cue always precedes the stop-signal, the stop signal task involves the inhibition of an already started action, or 'action cancellation', whereas the go/no-go task involves stopping a planned but not yet initiated response (Bari \& Robbins, 2013; Wright, Lipszyc, Dupuis, Thayapararajah, \& Schachar, 2014). Whether inhibition occurs in the stop signal task depends on what has been called a race between the stop task and the go task. If an individual initiates the stop process before the go response has been finalised, the go response will be inhibited. Poor inhibitory control can result from either responding too quickly to the go signal or too slowly 
to the stop signal (Logan, Schachar, \& Tannock, 1997). The probability of inhibition is high when the stop-signal is presented in the period near the start of the go response and decreases as the delay between the go cue and stop-signal becomes longer (Bari \& Robbins, 2013). Individuals with poorer inhibitory control require a shorter period between the go and the stop signal in order to successfully inhibit their response.

The dependent variable that indexes inhibitory control in the stop signal task is stop signal reaction time (SSRT). The SSRT measures the temporal period between stop signal presentation and the stop process finish (F. Verbruggen \& Logan, 2009), i.e., the time required to cancel the movement after seeing the stop signal (see descriptions in Duann, Ide, Luo, \& Li, 2009; Eagle, Bari, \& Robbins, 2008). The SSRT cannot be measured directly but is estimated using several different methods employed in the literature (see F. Verbruggen \& Logan, 2009). Better inhibitory control is indexed by shorter SSRTs. In any stop trial, the probability of responding is dependent on three factors; the stop signal delay (SSD), the go reaction time and the SSRT. If the SSD (the period between the presentation of the go stimulus and the presentation of the stop stimulus) is increased, the probability of responding will be increased. If the go reaction time increases (i.e., participants respond more slowly to the go signal), the probability of responding will be decreased (and therefore the probability of successful inhibition will be increased, because the stop process will more likely finish before the go process). Finally, if the SSRT increases, the probability of responding will increase because the likelihood that the stop process will finish after the go process increases (i.e., it takes longer for the stop process to finish) (Frederick Verbruggen \& Logan, 2008; F. Verbruggen \& Logan, 2009).

\section{Neural Structures Involved in Response Inhibition in the Stop Signal Task}

It is thought that one of the most crucial cortical regions for stopping - and the area that may generate the stopping command - is the right inferior frontal cortex (rIFC) (also 
known as vlPFC) (Aron, Robbins, \& Poldrack, 2004). The rIFC includes pars triangularis, pars opercularis and part of pars orbitalis (corresponding to Brodmann areas (BA) 44, 45 and 47, respectively) (Aron, 2011), although pars opercularis may be the most important region for inhibition (Aron et al., 2014a). It has been proposed that one part of the rIFC, the right IFJ, which is located at the border of the inferior frontal sulcus and the precentral sulcus, may be responsible for directing stimulus-driven attention (Chikazoe, 2010) while a more ventral part (posterior inferior frontal gyrus) may implement inhibitory control (Aron, 2011; Bari \& Robbins, 2013; Chikazoe, 2010; Levy \& Wagner, 2011). Based on fMRI data some authors have suggested the entire region of the right inferior frontal gyrus (rIFG)/insula is involved in attentional processing of a low frequency unexpected event, i.e., the stop signal, rather than being involved in response inhibition per se (D. J. Sharp et al., 2010). In response, other authors have argued that the rIFC is involved specifically in response inhibition because it may actually act as a brake that is activated in response to an unexpected event or endogenous rule, and may slow or completely stop a response (Aron et al., 2014a; Aron, Robbins, \& Poldrack, 2014b). According to these authors, any salient or infrequent or unexpected stimulus will recruit inhibitory control and, because attention and inhibition are inherent properties of the network, it may ultimately be impossible to disentangle the respective roles of attention and inhibition in rIFC function (Aron et al., 2014a).

Although many researchers believe the rIFC is a crucial node in the stopping network, recent evidence does suggest that it may not be specialised for response inhibition. The areas of the rIFC that activate during response inhibition tasks also activate during other tasks that do not require inhibitory control and the rIFC has been shown to activate less than other areas, such as the anterior insula/inferior frontal operculum, during tasks requiring inhibition of a response (Erika-Florence, Leech, \& Hampshire, 2014; Hampshire, 2015). As a consequence, it may be that the rIFC is responsible for detecting task relevant stimuli or 
processing internal plans or executing motor responses, and response inhibition arises as a consequence of interactions across of a number of domain general fronto-parietal networks (Erika-Florence et al., 2014; Hampshire, 2015).

The rIFC is connected to the pre-supplementary motor area (pre-SMA) (Aron, 2011; Duann et al., 2009). Activation of the pre-SMA has been associated with SSRT (Chao, Luo, Chang, \& Li, 2009; C. S. R. Li, C. Huang, R. T. Constable, \& R. Sinha, 2006) and it is believed that the pre-SMA may generate a control signal that is then implemented by the rIFC (Aron, 2011). Some authors have proposed that the pre-SMA and not the rIFC is the critical region involved in response inhibition (D. J. Sharp et al., 2010). The pre-SMA is functionally connected to the striatum and the subthalamic nucleus (Duann et al., 2009). During action cancellation - when an individual must stop an already initiated response - it is thought that the go signal may be intercepted by a signal that travels via the subthalamic nucleus to the globus palladus/substantia nigra. The globus palladus/substantia nigra inhibits the thalamus and suppresses excitatory projections to cortical motor areas (primary motor cortex) so that the voluntary go response is not executed (Aron, 2011; Aron \& Poldrack, 2006; C. D. Chambers, Garavan, \& Bellgrove, 2009). It has also been suggested that the striatum may be involved in preparing to stop and in proactive stopping rather than reactive stopping (Aron, 2011).

Another critical node in the stopping network may be the bilateral anterior insula, activation of which has been observed to extend into the IFC and pre-SMA during action cancellation (Boehler, Appelbaum, Krebs, Hopf, \& Woldorff, 2010; Cieslik, Mueller, Eickhoff, Langner, \& Eickhoff, 2015; Dambacher et al., 2014; Swick, Ashley, \& Turken, 2011). As discussed above, although it has long been thought that the rIFC is the most crucial node in the network responsible for action cancellation, there is now some disagreement surrounding whether the most crucial region for stopping is the rIFC or the anterior insula 
(Dambacher et al., 2014). Swick, Ashley, and Turkin (2011) suggest two reasons why this disagreement may have arisen. Firstly, it is possible that regions of activation that include the insula are accidentally interpreted as activation of the inferior frontal gyrus (IFG). Secondly, spatial smoothing methods used when analysing fMRI data can blur functionally distinct regions and cause mislocalisation of activation. Nevertheless, lesion studies performed using non-human primates (e.g., Iversen \& Mishkin, 1970) do indeed suggest that the rIFC is an important node in the stopping network. It is also possible that the insula may be responsible for general cognitive control functions such as maintaining task rules and readiness rather than playing a specific role in response inhibition per se (Swick et al., 2011).

In addition to regions mentioned above (i.e., bilateral anterior insula, right posterior IFC (especially pars opercularis), pre-SMA, rIFJ, and bilateral thalamus) a recent metaanalysis reported activation in the right dorsal premotor cortex, right TPJ, aMCC and PCC during stop signal tasks (Cieslik et al., 2015) (see also Swick et al. (2011) for findings relating to the PCC and right inferior parietal lobe in action cancellation). Activation of the right TPJ and the right frontal cortex may be due to the redirection of attention to behaviourally relevant stimuli or contextual updating when the stop signal is noticed, which then indicates a need to inhibit the motor response (Cieslik et al., 2015). During the stop signal task, the dorsal PCC may mediate attentional focus in order to integrate the stop signal with the current task set (Cieslik et al., 2015). It should be noted that the nodes and networks discussed above have been identified using relatively simple stop signal task paradigms.

Although the neural mechanisms underlying action cancellation in simple stop signal paradigms have been of interest to researchers for over a decade, less research has looked at whether motivational factors - such as the provision of rewards - enhance or impair response inhibition in the stop signal task (e.g., Locke \& Braver, 2008). The issue of whether factors like reward influence inhibitory control is important because many clinical conditions 
featuring inhibitory control deficits are characterised by sensitivity to reward (e.g., attentiondeficit/hyperactivity disorder and substance use disorders) (e.g., Monterosso, Aron, Cordova, Xu, \& London, 2005; Solanto et al., 2001). Punishment also has the potential to influence inhibitory control as people are more likely to inhibit responses to aversive stimuli (GuitartMasip et al., 2011; Locke \& Braver, 2008; Stürmer, Nigbur, Schacht, \& Sommer, 2011). The aversive nature of punishment may also hyper-arouse and/or distract individuals from the task at hand, leading to reductions in performance such as reaction time slowing (e.g., Braem, Duthoo, \& Notebaert, 2013; Stürmer et al., 2011). Before reviewing studies that have investigated the influence of reward and punishment on inhibitory control in the stop signal task, the following is a brief review relating to networks involved in reward and punishment generally.

\section{Processing of Rewards and Punishments in Healthy Populations}

Imaging, lesion techniques, and experimental paradigms that manipulate motivational factors have been used to identify structures and networks involved in reward and punishment processing. Rewards are stimuli that positively reinforce behaviour or make the behaviour more likely to occur in the future (McClure, York, \& Montague, 2004); they are appetitive events that an organism will work to obtain (Seymour, Singer, \& Dolan, 2007). Punishments are stimuli that make behaviours less likely to occur in the future; they are aversive events that an organism will work to avoid or reduce the likelihood of occurring (Seymour et al., 2007).

Since the seminal study on rats' brains by Olds and Milner in 1954 (Olds \& Milner, 1954), the reward network has been investigated extensively using both animal and human subjects (see Haber \& Knutson, 2010; Pujara \& Koenigs, 2014, for review). The network is comprised of a number of cortical and subcortical structures that are functionally connected in a cortico-basal ganglia network. Briefly, this network is comprised of the ventral striatum, 
which includes the nucleus accumbens, ventral caudate nucleus and ventral putamen (Diekhof, Kaps, Falkai, \& Gruber, 2012; Liu, Hairston, Schrier, \& Fan, 2011). The ventral striatum appears to represent the value of expected outcomes (e.g., greater activation in the presence of rewarding versus punishing stimuli), is sensitive to unexpected rewards and the magnitude of such rewards (Diekhof et al., 2012), and fulfils motivational/behavioural functions in that it responds to both rewards and punishments that motivate the organism (e.g., to run toward or away from the stimulus) (Bissonette, Gentry, Padmala, Pessoa, \& Roesch, 2014). The ventral striatum receives input from the orbitofrontal cortex (OFC) that codes stimulus reward value (O'Doherty, 2004) and expectations about future outcomes that guide learning (Bissonette et al., 2014). The ventral striatum also receives input from the ACC, which is implicated in attentional control. In addition, the ventral striatum receives dopamine from midbrain structures such as the substantia nigra and ventral tegmental area (Haber \& Knutson, 2010; Pujara \& Koenigs, 2014; Schultz, 1998). The ventral striatum projects to the ventral pallidum and the ventral tegmental area/substantia nigra (Schultz, 1998). The ventral pallidum and ventral tegmental area/substantia nigra project back to the prefrontal cortex via the medial dorsal nucleus of the thalamus (Haber \& Knutson, 2010). Other structures that regulate this central circuit include the amygdala, which is involved in signalling attention, uncertainty and stimulus intensity as well as stimulus valence (Bissonette et al., 2014), hippocampus, lateral habenular nucleus and other brainstem structures (Haber \& Knutson, 2010). A meta-analysis of 142 neuroimaging studies found that the core reward network also included the dmPFC and lateral frontoparietal areas (Liu et al., 2011).

A distinction is often made between reward anticipation and reward outcome/delivery. Reward anticipation and reward outcome have been found to activate different neural structures and circuits. The bilateral anterior insula, ACC, supplementary motor area, inferior parietal lobule and brainstem appear to show more consistent activation 
when a reward or punishment is anticipated compared to when it is received, and the ventral striatum, medial OFC and amygdala are preferentially activated during reward/punishment outcome compared to anticipation (Liu et al., 2011). Some studies have, however, consistently found the ventral striatum to be activated during anticipation of reward compared to reward receipt (e.g., Dillon et al., 2008; Knutson \& Cooper, 2005; Knutson, Fong, Adams, Varner, \& Hommer, 2001; Rademacher et al., 2010), or both anticipation and consumption of rewards (Diekhof et al., 2012). The nucleus accumbens is activated during both anticipation and receipt of reward/punishment; this area may track reward/punishment signals and use them to modulate learning (Liu et al., 2011). The medial PFC (middle frontal gyrus of the medial wall anterior to the genu of the corpus callosum) appears to be activated during reward anticipation and outcome, suggesting it may integrate the value of stimuli across different stimuli (Haber \& Knutson, 2010). In addition, the vmPFC appears to be activated during reward receipt (Diekhof et al., 2012; Dillon et al., 2008; Knutson et al., 2001) and may evaluate reward magnitude during the consumption phase (Diekhof et al., 2012).

Punishing or aversive stimuli have been associated with activation in the lateral OFC, anterior insula, amygdala and ventral striatum (Seymour et al., 2007). A recent study found that anticipation of potential monetary punishment during a reward and avoidance of loss learning task activated the dorsal striatum, bilateral anterior insula, right lateral OFC, posterior medial frontal cortex and posterior cingulate region (S. H. Kim, Yoon, Kim, \& Hamann, 2014). Receipt (as opposed to anticipation) of monetary punishment activated the lateral OFC and posterior medial frontal cortex (S. H. Kim et al., 2014). A meta-analysis has also found the ventral striatum, anterior insular cortex, amygdala, ACC, and DLPFC are activated when processing punishment/loss (Liu et al., 2011).

Given that many structures in the 'reward' network described above also process aversive stimuli and vice versa, some authors have asked whether it makes sense to 
distinguish between structures that process appetitive and aversive information (e.g., Kobayashi, 2012). One suggestion is that some of the structures in the 'reward' and 'punishment' networks may actually encode stimulus intensity and activation of motivational/behavioural responses rather than whether the stimulus is rewarding or punishing per se (Bissonette et al., 2014; H. Kim, Shimojo, \& O'Doherty, 2006).

\section{Rewards and Punishments in the Stop Signal Task}

A number of studies using behavioural, electrophysiological and imaging methods have used reward to investigate the influence of motivation on inhibitory control. Some studies using the stop signal task have rewarded only successful stop trials. Behaviourally, rewards improved inhibitory control, with shorter SSRT for rewarded stop trials when compared to non-rewarded stop trials (Boehler, Hopf, Stoppel, \& Krebs, 2012; Boehler, Schevernels, Hopf, Stoppel, \& Krebs, 2014; Schevernels et al., 2015). Using this paradigm with EEG, one study demonstrated that successful reward-related stop trials were associated with a larger P3 compared to successful reward-unrelated stop trials which, the authors suggested, may indicate enhanced reactive control during reward-related trials (Schevernels et al., 2015). In addition, reward-related compared to reward-unrelated stop stimuli were associated with a larger N1 component, which may reflect enhanced top-down attention (proactive control) that may aid later inhibitory processes (Schevernels et al., 2015). Functional MRI blood oxygenated level-dependent (BOLD) measures indicate that during rewarded compared to unrewarded stop trials, regardless of whether stopping is successful or not, activity levels are higher in the right anterior insula/inferior frontal gyrus, the dACC/preSMA and the pregenual ACC at stimulus onset (Boehler et al., 2014). With the exception of the pregenual ACC, these areas are also implicated in response inhibition generally.

The enhancing effect of reward on the inhibitory control network has prompted the hypothesis that reward may improve inhibitory control by reactively increasing activity in 
areas that are important for inhibitory control (Boehler et al., 2014). In contrast, when only go (rather than stop) trials are rewarded in a stop signal task (i.e., a reward is provided for any correct go response), inhibition appears to become more difficult. For example, when only go trials are rewarded, participants exhibit longer SSRT without any corresponding difference in go reaction times in reward compared to no reward conditions (Padmala \& Pessoa, 2010). Furthermore, there is reduced activation at stimulus onset in the bilateral IFG, the left precentral gyrus, regions in the parietal cortex such as the bilateral intraparietal sulcus and right inferior parietal lobe, and the bilateral putamen for successful compared to unsuccessful stop trials in reward compared to no reward conditions (Padmala \& Pessoa, 2010). When both stop and go trials are rewarded using cues, participants display improved response inhibition indexed by shorter SSRT to rewarded stop trials (Rosell-Negre et al., 2014). Reaction times for rewarded go trials are also faster. Correct responses on reward compared to no reward stop trials result in increased activation in brain regions including the right cingulate gyrus, right precentral gyrus (supplementary motor area), right pre-SMA/ACC, right IFG, right medial frontal gyrus, left superior frontal gyrus, bilateral insula, right inferior and superior parietal lobules, and the left putamen, although it is not clear whether this activation occurs during anticipation, stimulus presentation or feedback (Rosell-Negre et al., 2014). In sum, findings from studies that have looked at the effect of reward on inhibitory control suggest that that both reactive (Boehler et al., 2012; Boehler et al., 2014; Schevernels et al., 2015) and proactive (Rosell-Negre et al., 2014; Schevernels et al., 2015) control can be improved by rewarding stop trials regardless of whether or not go trials are also rewarded, but inhibitory control is reduced when only go trials are rewarded (Padmala \& Pessoa, 2010).

To date, no studies have investigated the effect of punishing erroneous go and/or stop responses on response inhibition in healthy populations using a stop signal task. A number of studies have used a stop signal task with feedback indicating reward and punishment for 
correct and incorrect responses in individuals scoring high and low on psychopathy (Masui \& Nomura, 2011) and high and low on impulsivity (Rodríguez-Fornells, Lorenzo-Seva, \& Andrés-Pueyo, 2002). Unfortunately these studies did not look at the separate effects of rewards and punishments on inhibitory control.

\section{Inhibitory Control, Anxiety and the Stop Signal Task}

Problems with inhibitory control characterise conditions that are also associated with sensitivity to reward, such as attention-deficit/hyperactivity disorder and substance use disorders (see Bari \& Robbins, 2013; C. D. Chambers et al., 2009, for review). The stop signal task has been used to document inhibitory control deficits in these populations. Relatively less research has examined how inhibitory control in disorders that involve sensitivity to punishment, such as depression (Pinto-Meza et al., 2006) and anxiety (e.g., Corr, 2004), might be influenced by motivational factors. Functionally, disorders such as anxiety are characterised by what appears to be excessive inhibitory control as evidenced by avoidance behaviours seen in, for example, social anxiety disorder and agoraphobia. Despite this, there has been little formal scientific investigation into whether anxiety is associated with general inhibitory control improvements or deficits. Attentional control theory assumes that, generally, reductions in attentional focus in anxiety lead to reductions in inhibitory control. These reductions in inhibitory control should manifest as impaired processing efficiency (e.g., increased reaction time and neural effort) but not necessarily reduced performance effectiveness (e.g., accuracy) during affectively-neutral cognitive tasks.

Few studies have used the stop signal task to test the attentional control theory hypothesis that individuals high in anxiety will have a general inhibitory control deficit. Inconsistent with the hypothesis are findings of no difference in measures that index processing efficiency (e.g., SSRT) between healthy controls and participants high on trait anxiety or who have anxiety disorders (Avila \& Parcet, 2001; Epstein et al., 2001; M. A. Lau, 
Christensen, Hawley, Gemar, \& Segal, 2007). One recent study found no association between behavioural inhibition and SSRT, but longer SSRT was associated with a decrease in go accuracy for individuals higher in behavioural inhibition; this relationship was not observed in individuals lower in behavioural inhibition (Eriksson, Jansson, Lisspers, \& Sundin, 2016). This finding suggests that individuals higher in behavioural inhibition perform the stop signal task differently to those lower in behavioural inhibition but the reason for this difference is not clear.

The inconsistency in studies that have looked more specifically at anxiety may in part be due to methodological issues that warrant discussion. A study by Avila and Parcet (2001) found no correlation between SSRT and scores on the STAI T-Anxiety scale in a healthy female undergraduate population. In contrast, they found a significant positive correlation between the STAI T-Anxiety scale and the sensitivity to punishment scale of the Sensitivity to Punishment and Sensitivity to Reward Questionnaire (SPSRQ; Torrubia, Ávila, Moltó, \& Caseras, 2001), a significant negative correlation between SSRT and sensitivity to punishment, as well as a significant positive correlation between SSRT and sensitivity to reward as measured by the SPSRQ. In explaining their mixed results, Avila and Parcet concluded that lower anxiety (underactivity of the BIS) is associated with poorer inhibitory control. Such a hypothesis appears inconsistent with the absence of a relationship between the STAI T-Anxiety scale and SSRT. It is possible that the STAI T-Anxiety scale measures a different aspect or form of 'anxiety' from that of the sensitivity to punishment scale of the SPSRQ, i.e., a form of anxiety that does not have any association with inhibitory control. The significant correlation between the STAI T-Anxiety scale and the sensitivity to punishment scale may also be due to shared variance; the correlation between two questionnaires will often be higher than between a questionnaire and a measure obtained from an experimental task due to common method variance between the questionnaires (Podsakoff, MacKenzie, 
Lee, \& Podsakoff, 2003; Tepper \& Tepper, 1993). The study by Avila and Parcet also used the Form X version of the STAI (Spielberger, Gorsuch, \& Lushene, 1970), which includes more items related to depression than the more recent Form Y version (Spielberger et al., 1983). These items may have influenced the findings because depression does not appear to be associated with differences in stop signal performance (M. A. Lau et al., 2007) or impulsivity (Jakubczyk et al., 2012). Using the revised Form Y version of the STAI may give different results because a number of items related to depression were removed from the Form X version to create the Form Y version (Spielberger et al., 1983).

Epstein, Johnson, Varia, and Conners (2001) did use the revised Form Y version of the STAI in their study examining stop signal performance in patients with attentiondeficit/hyperactivity disorder, patients with both anxiety disorders and high trait anxiety, and healthy controls. The study found no group differences in SSRT but sample sizes were small. Also, it is difficult to draw any conclusions about the effect of trait anxiety on inhibitory control as the measure of trait anxiety (high $t$-score on STAI T-Anxiety scale) was confounded with psychiatric diagnosis. Another study has found no difference in SSRT for participants with a range of anxiety disorders compared to healthy controls (M. A. Lau et al., 2007) but it is possible that inhibitory control differs across anxiety disorders and that heterogeneity increases noise, making significant results less likely. As well as using small sample sizes and recruiting participants with a range of psychiatric diagnoses, studies that have found no inhibitory control improvement or deficit in anxious individuals have sometimes failed to report indices of inhibitory control such as mean go reaction time and accuracy (e.g., M. A. Lau et al., 2007). It is important to know mean go reaction time and accuracy because participants may slow their go reaction time in order to improve stop performance (accuracy). Other studies have used children rather than adults (Korenblum, 
Chen, Manassis, \& Schachar, 2007) or a mix of valenced (negative and positive) and neutral stimuli rather than only neutral stimuli (M. A. Lau et al., 2007).

None of the abovementioned studies found evidence for a moderating effect of anxiety on behavioural indices in the stop signal task. Attentional control theory suggests that, despite the lack of behavioural differences, individuals with anxiety may still be performing the stop signal task differently to healthy controls because they may be using compensatory strategies, such as drawing on more cognitive/neural resources and applying enhanced effort, in order to perform at the same level (i.e., maintain performance effectiveness). In attentional control theory, reaction time can be a measure of processing efficiency while accuracy rate is a measure of performance effectiveness (M. W. Eysenck et al., 2007). Although SSRT is a reaction time measure and therefore should be affected by anxiety, there appear to be no consistent differences in SSRT between anxious and nonanxious groups. In addition, the studies mentioned above that reported mean go reaction time found no group differences in go reaction time (Avila \& Parcet, 2001; Epstein et al., 2001; Korenblum et al., 2007). Despite the lack of group differences in reaction time measures, individuals with anxiety may still be experiencing reductions in processing efficiency that may be reflected in patterns of neural activation. Anxiety is associated with particular patterns of neural activation such as hyper-responsivity of the amygdala and hyporesponsivity of prefrontal structures (Bishop, 2007; Garner, Mohler, Stein, Mueggler, \& Baldwin, 2009) (see discussion above in Neural Mechanisms Underlying Anxiety), and genetic risk factors are believed to modulate these neural mechanisms (Garner et al., 2009). It has been suggested that, given their apparent heritability and association with particular gene variations, activation patterns of individual structures as well as whole networks have the potential to act as neurobiological intermediate phenotypes of anxiety disorders (Domschke \& Dannlowski, 2010). 
Few studies have investigated neural patterns associated with inhibitory control in trait anxiety using the stop signal task. One study measured brain oscillations (i.e., changes in synchrony of cell populations resulting from activation and deactivation of neural networks (Pfurtscheller, 2006; Pfurtscheller \& Lopes Da Silva, 1999)) in individuals high and low in trait anxiety using the STAI T-Anxiety scale (Savostyanov et al., 2009). Participants high in trait anxiety had desynchronisation in the alpha band $(8-13 \mathrm{~Hz}) 0-600 \mathrm{~ms}$ after the stop signal onset on successful trials; this was not present in low trait anxiety subjects. Alpha band desynchronization is thought to increase with increasing task demands and is associated with perception, attention and semantic memory demands (Doppelmayr, Klimesch, Pachinger, \& Ripper, 1998).

Another study examined responses to both successful and unsuccessful go and stop trials and found that participants with high trait anxiety (STAI T-Anxiety scale) had a higher and longer lasting level of alpha/beta desynchronization than participants with low trait anxiety. High trait anxiety participants also had higher amplitude delta slow wave synchronisation (1-4Hz) in successful go and stop conditions (Savostyanov et al., 2011). Oscillations in the delta band are believed to be associated with reward-related motivational structures (Savostyanov et al., 2011). Low trait anxiety participants had stronger and earlier high-frequency synchronisation $(12-25 \mathrm{~Hz})$ in both successful conditions. The authors suggested that elevated activity in the alpha and beta bands in high anxiety may indicate an increased readiness in conditions of uncertainty. The higher frequency beta synchronisation ('beta rebound') in low anxiety participants may indicate a more positive evaluation of a situation that enables more "relaxation" (Savostyanov et al., 2011, p. 25). Together, the studies that have measured brain oscillations suggest that individuals with high trait anxiety attempt to increase cognitive control during response inhibition, have increased vigilance/readiness in situations involving uncertainty, and evaluate situations less positively. 
Using fMRI, abnormal activity in structures and networks involved in response inhibition may be reflected in greater readiness to respond and negative evaluation in high trait anxious individuals.

\section{Motivational Factors, Inhibitory Control and Anxiety}

As discussed above, the stop signal task has been used to examine the effect of motivational factors on inhibitory control as well as the effect of anxiety on inhibitory control but no study has yet investigated the effect of motivational factors on inhibitory control in individuals high in trait anxiety. Attentional control theory supposes that the attentional systems of individuals who are anxious are directed toward stimuli rather than goals. In anxious individuals, motivational factors such as rewarding or punishing cues and feedback may capture attention. This may decrease goal-related attention, leading to poorer inhibitory control and increased need for and use of compensatory strategies, including abnormal recruitment of structures and networks involved in inhibitory control. In addition, studies have shown that anxiety is associated with dysfunctional structure, function and connectivity of, amongst other things, the vmPFC, DLPFC, dACC, amygdala, and insula. Signals of reward and punishment also activate these regions. It is therefore possible that, when responding to cues and feedback indicating reward and punishment, individuals with anxiety will show abnormal patterns of activation in these structures and their associated networks and may also demonstrate alterations in behavioural performance.

In summary, it is possible that individuals with anxiety will display insufficient inhibitory control when performing inhibitory control tasks if the task itself or any failure on the task is perceived as threatening. They may also display altered patterns of behaviour and neural activation in structures that have been shown to function differently in anxiety but that are required for processing rewards and punishments as well as for effective inhibitory 
control. These structures include (but are not limited to) the prefrontal cortex, the insular cortex, and the cingulate cortex.

\section{Effect of Anticipation/Cues Relating to Motivational Factors on Inhibitory Control in Anxiety and Behavioural Inhibition}

Compared to literature on the neural substrates of reward and punishment processing in healthy people, there has been relatively little research into how neural mechanisms involved in processing rewards and punishments may differ in individuals who suffer from trait anxiety or anxiety disorders (but see Admon et al., 2013; Cremers, Veer, Spinhoven, Rombouts, \& Roelofs, 2015; Manning et al., 2015; Teng et al., 2016). It has been suggested that different dimensions of anxiety may be associated with different reward-processing abnormalities (Kessel, Kujawa, Hajcak Proudfit, \& Klein, 2015), which makes comparing and interpreting findings difficult. There have been a number of studies that have looked at processing of rewards and punishments in individuals who score high on measures of behavioural inhibition. This literature may help to inform hypotheses about effect of rewards and punishments in trait anxiety because scores on measures of behavioural inhibition are associated with scores on measures of anxiety (Hundt et al., 2007) and the STAI-T-Anxiety scale correlates with scores on the BIS subscale of the BIS/BAS Scales (Cooper et al., 2007).

This review will focus on two stages of reward and punishment processing. First, it will examine the effect of anticipating rewards and punishments on inhibitory control. Second, it will examine at the effect of receiving rewarding and punishing outcomes/feedback on inhibitory control. In relation to anticipation of rewards and punishments, it is currently unclear whether individuals high in anxiety demonstrate better or poorer inhibitory control when anticipating reward and/or punishment than healthy controls or individuals low in anxiety. No studies have specifically looked at trait anxiety in adults, but adults high in test anxiety have greater inhibitory control in that they have been found to 
make more errors of omission, fewer errors of commission and fewer total responses in a go/no-go discrimination task than adults low in test anxiety (Hagopian \& Ollendick, 1994). They also demonstrate greater inhibitory control when anticipating the possible absence of reward and show the same increase in inhibitory control when anticipating punishment as participants low in test anxiety (Hagopian \& Ollendick, 1994). Although these findings suggest that individuals high in anxiety have better inhibitory control generally, as well as when they are aware that they could either be punished or not obtain a reward, results may be different when using other tasks such as the stop signal task that measure action cancellation rather than action restraint or withholding.

Only two other studies have investigated the effect of motivational factors on inhibitory control in participants with anxiety. Both administered an antisaccade task to adolescents with anxiety disorders. Generalising results from adolescents to adults is problematic due to differences in development and function of the frontal lobes, a part of the brain crucial for successful inhibition (S. B. Johnson, Blum, \& Giedd, 2009). In addition, adolescents appear to be more sensitive than adults to both rewarding and aversive stimuli (Galván \& McGlennen, 2013). Generalising findings from antisaccade tasks to inhibitory control in general is problematic because it is still not yet clear which aspects of inhibition antisaccade tasks actually measure (see Hutton, 2008; Hutton \& Ettinger, 2006, for review). Nevertheless, because there is little research on the effects of reward and punishment on inhibitory control in anxious populations, these studies warrant brief mention. One study administered an antisaccade task to adolescents with GAD and found they had a longer incorrect antisaccade latency than healthy controls when anticipating reward and punishment, suggesting a weaker capacity to inhibit a saccade in the presence of incentives and therefore less efficient cognitive control (Jazbec, McClure, Hardin, Pine, \& Ernst, 2005). However, using a slightly different task another study has shown adolescents with a range of anxiety 
disorders have shorter incorrect antisaccade latency than healthy controls when anticipating reward and punishment (Hardin, Schroth, Pine, \& Ernst, 2007).

In summary, there have only been three studies that have looked at the effect of motivational factors on inhibitory control in anxiety. One of them used a go/no-go task with adult participants high on test rather than trait or clinical anxiety (Hagopian \& Ollendick, 1994) and two used adolescent participants with a dependent variable (incorrect antisaccade latency) that is challenging to interpret (Hardin et al., 2007; Jazbec et al., 2005). It is therefore difficult to draw firm conclusions in relation to the effect of motivational factors on inhibitory control in adults with anxiety. Nevertheless, findings in the study by Hagopian and Ollendick (1994) suggest that adults with anxiety may demonstrate increased inhibitory control both generally and when anticipating non-reward as well as punishment.

Although there is a paucity of literature on the effect of reward and punishment on inhibitory control in individuals with trait anxiety or clinical anxiety disorders, studies have used other types of tasks to look at behavioural and neural responses in individuals high on personality traits related to anxiety (e.g., behavioural inhibition) when anticipating reward and punishment. During the monetary incentive delay task participants are presented with one of three cues that signal potential monetary rewards, monetary losses or no incentive. Participants then respond to a stimulus with a button press. Based on time taken to respond (which must be below a speed threshold), they receive feedback indicating a gain, a loss or no money. Anticipation of reward during the monetary incentive delay task activates the caudate, putamen, medial prefrontal cortex and left motor cortex (Knutson, Westdorp, Kaiser, \& Hommer, 2000). Anticipation of punishment during the monetary incentive delay task has been shown to active the same regions as for reward with the addition of the thalamus and anterior cingulate (Knutson et al., 2000). 
Generally, adolescents who score high on measures of behavioural inhibition demonstrate increased striatal activation when anticipating reward and punishment. They have greater striatal activation with increasing magnitude of anticipated reward and punishment compared to adolescents low on behavioural inhibition, even when behavioural performance is the same between the groups (Guyer et al., 2006). When the reward is feedback from peers, adolescents high in behavioural inhibition have increased putamen activation when anticipating feedback from selected compared to not-selected peers (Guyer et al., 2014). When an anticipated reward is contingent on their own action, adolescents high in behavioural inhibition show greater activation in the left nucleus accumbens compared to adolescents low in behavioural inhibition (Bar-Haim et al., 2009). Further, when the anticipated reward is contingent on their own action and is high in magnitude compared to low in magnitude, adolescents high in behavioural inhibition show increased right striatal activation compared to adolescents low in behavioural inhibition (Benson, Guyer, Nelson, Pine, \& Ernst, 2014). The latter finding suggests that adolescents high in behavioural inhibition are not only more sensitive to reward than adolescents low in behavioural inhibition, but they are also sensitive to the magnitude of that reward. Similar findings have been reported for adolescents with GAD, who demonstrate greater putamen activation during potential gain (reward) compared to loss (punishment) trials and greater nucleus accumbens activation for gains compared to losses for incentives that are both medium and large (compared to small) (Guyer et al., 2012). The reason for the increased striatal response of adolescents high in behavioural inhibition and adolescents with GAD is unclear. It may be somehow linked to concern about making errors, especially as stakes increase with larger incentives (Guyer et al., 2006), differences in action-preparatory responses (Benson et al., 2014), or increased emotional sensitivity, especially to social evaluation, in adolescents who 
are high in behavioural inhibition or who have been diagnosed with GAD (Guyer et al., 2014).

Abnormal responses have also been found in structures other than the striatum when adolescents high in behavioural inhibition process potential rewards and punishments. During anticipation of large and medium (compared to small) rewards and punishments, there is increased activation in the primary motor cortex (BA4) in adolescents high in behavioural inhibition compared to adolescents low in behavioural inhibition (Guyer et al., 2006). Studies have not found increased amygdala activation in adolescents or adults high in behavioural inhibition during anticipation of rewards of differing magnitudes (Bar-Haim et al., 2009; Simon et al., 2010). One study failed to find amygdala activation in adolescents high in behavioural inhibition during anticipation of punishment (Guyer et al., 2006). This is somewhat surprising given the role of the amygdala in processing aversive stimuli (Janak \& Tye, 2015).

The studies mentioned above all used adolescent participants who were classified as either high in behavioural inhibition or diagnosed with GAD. As mentioned earlier, due to developmental differences in the adolescent brain (S. B. Johnson et al., 2009), it is difficult to generalise findings from adolescent to adult populations. Added to that, although behavioural inhibition is associated with an increased risk for anxiety disorders and is correlated with scores on the STAI T-Anxiety scale, behavioural inhibition is not exactly the same as anxiety or trait anxiety. Therefore, while findings from these studies may help to guide hypotheses relating to behavioural and neural outcomes in adults who score high on measures of anxiety, more studies that use adult populations that are either high either in trait anxiety or have clinically diagnosed anxiety disorders are needed. 


\section{Effect of Feedback Indicating Reward and/or Punishment on Inhibitory Control in}

\section{Anxiety and Behavioural Inhibition}

Along with anticipation of reward and punishment during the anticipation (cue) phase of a trial, research has focused on the behavioural and neural effects of feedback indicating reward or punishment during the outcome (feedback) phase of a trial. When adults high on behavioural inhibition are punished for responding too slowly during an inhibitory control (flanker) task, they demonstrate slower responses following punished compared to nonpunished trials (M. A. S. Boksem, Tops, Kostermans, \& De Cremer, 2008; Braem et al., 2013). This slowing suggests a reduction in cognitive control following punishment. The reduction may be due to a perception of punishment as more aversive in these individuals, which increases the likelihood that they will be distracted by and/or have arousal levels that are too high to modulate subsequent cognitive control processes (Braem et al., 2013).

Adults who score high on measures of behavioural inhibition have larger electrophysiological responses to errors made during inhibitory control tasks. They have larger error related negativity or error negativity (ERN/Ne) amplitudes following punishment compared to reward omission during flanker tasks (M. A. S. Boksem et al., 2008), larger feedback related negativity (FRN) amplitudes and latency for no-go loss (punishment) trials during go/no-go tasks (De Pascalis, Varriale, \& D'Antuono, 2010), and a more negative N2 component following aversive feedback compared to individuals scoring lower on measures of behavioural inhibition (Leue, Lange, \& Beauducel, 2012). These event-related potentials (ERPs) are thought to be generated in the ACC (Van Veen \& Carter, 2002) and the increased amplitude observed in individuals who are sensitive to punishment may be due to enhanced processing of signals indicating penalty (De Pascalis et al., 2010) and/or more pronounced conflict monitoring (Leue et al., 2012). It has been suggested that increased ERP amplitudes in response to punishing feedback is contrary to what would be predicted by the 
reinforcement learning model (M. A. S. Boksem et al., 2008). According to the reinforcement learning model, when individuals who are more likely to expect punishment (i.e., people who are high in behavioural inhibition) receive punishment, they will have a smaller prediction error and therefore a smaller amplitude ERP than individuals who are low in behavioural inhibition. It is, however, possible that the amplitude of the ERP is dependent on the subjective value of the prediction error, and the amplitude depends mostly on how concerned individuals are with making mistakes (M. A. S. Boksem et al., 2008). If this is the case, when punished for committing errors, individuals high in punishment sensitivity should experience errors as more aversive than individuals low in punishment sensitivity and therefore have larger amplitude ERPs. Even if no punishment is involved, merely making a mistake may be perceived as punishing due to concerns about negative evaluation (M. A. S. Boksem et al., 2008). Indeed, individuals who have high levels of behavioural inhibition have shown increased ERN/Ne amplitude in response to errors during a flanker task that did not involve rewarding or punishing feedback (Maarten A. S. Boksem, Tops, Wester, Meijman, \& Lorist, 2006).

Imaging studies have focused primarily on patterns of activation in the striatum during receipt of rewarding and punishing feedback. When receiving rewards, adults who have high levels of behavioural inhibition have reduced activation in striatal structures such as the ventral striatum (Simon et al., 2010). Adolescents who have high levels of behavioural inhibition do not show the usual caudate activation to positive feedback (when compared with rejection) from selected peers (Guyer et al., 2014). When monetary rewards are contingent on individuals' own actions, adolescents high in behavioural inhibition have increased caudate activation in response to the absence of reward when compared to reward receipt, whereas adolescents low in behavioural inhibition show caudate activation to reward receipt compared to the absence of reward (Helfinstein et al., 2011). Since, in healthy 
adolescents, more overtly negative events are needed to elicit striatal activation (Galván \& McGlennen, 2013), the enhanced caudate activation in response to the absence of reward may be due to the increased tendency of individuals high in behavioural inhibition to encode even mildly negative events as aversive (Helfinstein et al., 2011). When given rewards of different magnitudes, adolescents diagnosed with anxiety disorders show a trend for greater putamen activation upon receipt of higher rather than lower rewards only when the outcome is contingent on their own action, whereas healthy controls show no difference in putamen activation (Benson et al., 2014). Overall, it appears that individuals high in behavioural inhibition demonstrate reductions in striatal activation upon receipt of reward. So far, no effect of punishment on striatal activation has been observed in individuals high in behavioural inhibition. The overall reduction in striatal activation in the presence of rewarding feedback suggests that individuals who score high on measures of behavioural inhibition have decreased sensitivity to reward.

In relation to areas other than the striatum, there appears to be no correlation between behavioural inhibition and amygdala activation or behavioural inhibition and medial OFC activation during receipt of and omission of rewards in healthy adults (Simon et al., 2010). In the presence of feedback indicating reward or omission of reward, adolescents low in behavioural inhibition show vmPFC activation to receipt of reward and deactivation to the omission of reward, whereas adolescents high in behavioural inhibition show little vmPFC response in either of these conditions (Helfinstein et al., 2011). The absence of vmPFC activity may mean these individuals have difficulty discriminating reward values of stimuli and, because the vmPFC modulates the striatum, the absence of vmPFC activity may result in reduced striatal responses to positive feedback (Helfinstein et al., 2011). Receipt of monetary loss during a learning task has been negatively associated with right lateral OFC activation in adults with high levels of behavioural inhibition (S. H. Kim et al., 2014). It has been 
proposed that the unexpected finding of reduced activity in the lateral OFC, which is involved in the representations of punishment/loss during reinforcement learning, may be because the punishing feedback was not perceived as personally significant to these individuals (S. H. Kim et al., 2014).

\section{The Current Research}

The current research seeks to a) examine the structure of the STAI T-Anxiety scale by performing an exploratory factor analysis (EFA) and examining the scale's convergent and discriminant validity b) investigate whether there is a general inhibitory control deficit in trait anxiety using two stop signal tasks and c) investigate effects of rewards and punishments on behavioural and neural indices of inhibitory control in trait anxiety using a monetary incentive control task (MICT).

In relation to EFA of the STAI T-Anxiety scale, it was anticipated that the factor structure of the STAI T-Anxiety scale would be similar to that observed in previous studies using healthy, non-clinical populations (Bados et al., 2010; M. Balsamo et al., 2013; Vigneau \& Cormier, 2008). Namely, the STAI T-Anxiety scale would contain two method factors; a factor that corresponds to forward-scored items (positive polarity) and a factor that corresponds to reverse-scored items (negative polarity). In relation to the convergent and discriminant validity of the STAI T-Anxiety scale, based on findings from previous studies it was predicted that the STAI T-Anxiety scale total score would correlate more strongly with measures of depression than with measures of anxiety (Bados et al., 2010; M. Balsamo et al., 2013).

In relation to whether there is a general inhibitory control deficit in trait anxiety, we administered a stop signal task to two different samples of participants. There have been methodological issues in previous studies that have used stop signal tasks (Avila \& Parcet, 2001; Epstein et al., 2001; Korenblum et al., 2007; M. A. Lau et al., 2007), therefore we 
administered the current version of the STAI T-Anxiety scale (Form Y version) to adults and used it to define two groups; one high and one low in trait anxiety. In a second study we recruited a larger sample of participants using the STAI T-Anxiety scale total score as a continuous measure and performed a correlational analysis. Hypotheses were informed by attentional control theory that assumes the disruption in the balance between goal-directed and stimulus-driven attentional systems in anxiety reduces inhibitory control. Reduced inhibitory control should be observed as a reduction in processing efficiency but not performance effectiveness. Therefore it was hypothesised that high trait anxiety would be associated with a longer SSRT. It is, however, possible that individuals high in trait anxiety use compensatory strategies effectively such that there may be no evidence of a relationship between trait anxiety and SSRT. If this is the case, enhanced effort may be observed in patterns of neural activity.

We also used fMRI to measure neural activity in low and high trait anxious individuals while they performed the MICT. The MICT is a variant of the stop signal task that is combined with three incentive conditions (reward, punishment and neutral) from the monetary incentive delay task. The MICT is similar to a stop signal task in that participants must respond to frequent go trials and cancel an already initiated response on less frequent stop trials. As with the monetary incentive delay task, it contains three types of interleaved trials - reward, punishment and neutral. On each trial participants are presented with a cue indicating the type of trial that will be presented and, following a response, they are given feedback informing whether they were correct and how much money they won or lost on that trial. In order for participants to learn that the go stimulus is associated with reward, in reward and punishment conditions correct responses on go trials are rewarded with 20c. When a stop trial is presented, participants are forced to cancel a response to these stimuli. Correct stopping does not lead to monetary gain or loss but failing to stop results in either 
reward, punishment or the absence of reward/punishment. Individuals who are reward sensitive should experience difficulty stopping on stop trials, while individuals who are punishment sensitive should not be influenced by the potential for reward during reward stop trials and may exhibit increased control during punishment stop trials. Only neural activation associated with stop trials was analysed. 


\section{Chapter 3}

\section{Factor Analysis of the STAI T-Anxiety Scale}

Anxiety disorders are one of the most common types of mental disorder (Kessler et al., 2005). Globally, at any one time at least $7.3 \%$ of the population or one out of 14 people experience an anxiety disorder (Baxter, Scott, Vos, \& Whiteford, 2013). Given the high prevalence of anxiety disorders it is not surprising that a substantial amount of research has been undertaken in order to understand their aetiology and maintenance. Among the many predisposing and maintaining factors, the role of personality is well-recognised (Elwood, Wolitzky-Taylor, \& Olatunji, 2012). One commonly studied personality characteristic associated with anxiety disorders is trait anxiety, which has been defined as a relatively stable and enduring difference among individuals to perceive stressful situations as dangerous or threatening (Spielberger et al., 1983). It is often measured alongside and contrasted with state anxiety, which is a transient emotional response to a stressful situation (Spielberger et al., 1983) though they are directly related; trait anxiety reflects individual differences in the susceptibility to anxiety states.

\section{Exploratory Factor Analysis of the State-Trait Anxiety Inventory}

A number of instruments have been developed to measure trait anxiety in both clinical (applied) and research (basic) settings. One of the most widely used measures is the State Trait Anxiety Inventory (STAI; Spielberger et al., 1983), a questionnaire comprised of 40 items measuring state anxiety (STAI state anxiety scale (STAI S-Anxiety scale), 20 items) and trait anxiety (STAI trait anxiety scale (STAI T-Anxiety scale), 20 items). The STAI was originally developed in 1970 (STAI Form X version; Spielberger et al., 1970) and, following a major revision in 1979, the STAI Form Y version was released in 1983 (Spielberger et al., 1983). The STAI Form Y version remains one of the most popular measures of state and trait 
anxiety and is used extensively in both research and clinical practice (M. Balsamo et al., 2013; Grös et al., 2007; Spielberger et al., 1983).

The revision of the STAI from the Form X to the Form Y version was undertaken in order to make the instrument a more pure measure of anxiety - this meant replacing items that appeared to be related to depression with items better related to anxiety - as well as to improve the factor structure of the STAI T-Anxiety scale by having a more equal balance of anxiety-present (i.e., forward-scored) and anxiety-absent (i.e., reverse-scored) items; in its current form, 9 of the 20 items in the STAI T-Anxiety scale measure the absence of anxiety (e.g., I feel pleasant) and need to be reverse-scored when calculating the STAI T-Anxiety scale total score (Spielberger et al., 1983). In the original factor analysis, the STAI-Form Y version was administered to year 10 high school students and air force recruits (Spielberger et al., 1983). Using exploratory factor analysis (EFA), a two factor and a four factor solution were found to be most interpretable. Spielberger, Gorsuch, Lushene, Vagg, and Jacobs (1983) called the two factors in the two factor solution S-Anxiety and T-Anxiety (i.e., state and trait anxiety). For the four factor solution, Spielberger et al. called the four factors state anxiety present, state anxiety absent, trait anxiety present and trait anxiety absent. The state anxiety present factor was comprised mostly of items reflecting the experience of anxious feelings in the moment, such as I feel frightened and I feel nervous. Similarly, state anxiety absent was comprised mostly of items reflecting the absence of anxious feelings in the moment, such as $I$ feel pleasant and Ifeel satisfied with myself. Trait anxiety present was comprised mostly of items reflecting the experience of anxious feelings in general, such as I have disturbing thoughts and Some unimportant thought runs through my mind and bothers me, and trait anxiety absent was comprised mostly of items reflecting the absence of anxious feelings in general, such as I am "calm, cool, and collected" and I am happy. It is not clear precisely what the anxiety-present, anxiety-absent structure reflects. It may reflect opposite ends of a 
unidimensional psychopathological construct (e.g., anxiety) such that if an individual scores high on anxiety-present they would also score low on anxiety-absent. Alternatively, it may reflect two orthogonal dimensions or constructs, more than one psychopathological construct (e.g., anxiety and depression), or two different dimensions of one psychopathological construct (e.g., presence of cognitive symptoms of anxiety and absence of somatic symptoms of anxiety).

Since the original factor analysis of the STAI Form Y version, a number of studies have used factor analysis to further investigate the structure of the STAI T-Anxiety scale. Only one study has undertaken an EFA on the STAI Form Y version (Bieling et al., 1998). In this study the authors administered the STAI T-Anxiety scale to 212 participants who had been diagnosed with a range of anxiety disorders. Using a principal components extraction, Bieling, Antony, and Swinston (1998) identified a two factor solution that they suggested appeared to map, for the most part, onto what Spielberger et al. (1983) called 'anxiety present' and 'anxiety absent' items. Although Bieling et al. did not offer any further interpretation of these factors, they are most likely method factors reflecting forward (anxiety present) and reversed (anxiety absent) item keying. An orthogonal Varimax rotation was used to assist interpretation but, given the possibility that factors may be correlated, oblique rotation may have been more appropriate. To date, it appears that no study has performed EFA on the STAI T-Anxiety scale (Form Y) using data from a healthy population.

\section{Confirmatory Factor Analysis of the State-Trait Anxiety Inventory}

Compared to EFA, confirmatory factor analysis (CFA) has been more commonly used to investigate the factor structure of the STAI T-Anxiety scale. CFA involves testing the fit of a set of hypothesised latent factors for a data set (Tabachnick \& Fidell, 2007). It is undertaken with a priori knowledge of the number of factors that will be extracted and which variables load onto them. In contrast, EFA is a technique where the factors are not known a priori. It 
involves analysing communalities in a covariance matrix. Using CFA, a number of studies have identified a factor structure that suggests the STAI T-Anxiety scale measures negative affect rather than trait anxiety (Bados et al., 2010; M. Balsamo et al., 2013; Bieling et al., 1998).

In the influential tripartite model of anxiety and depression (Clark \& Watson, 1991), negative affect refers to feeling upset or unpleasantly engaged as opposed to peaceful, and includes states such as being upset, angry, guilty, afraid, sad and worried. Positive affect is independent of negative affect and includes feelings of energy, activity, excitement, interest and enthusiasm, while its absence is characterised by feelings of fatigue and languor. According to the tripartite model, anxiety is a state of high negative affect mixed with physiological hyperarousal, whereas depression is a mixture of high negative affect and low positive affect. This means that high negative affect reflects the general presence of anxious and depressive symptoms, while the absence of positive affect is more specific to depression (Clark \& Watson, 1991). In the original factor analysis of the STAI T-Anxiety scale performed by Spielberger et al. (1983), the authors did not consider these dimensions in relation to the factors they named 'anxiety-present' and 'anxiety-absent'. Although later studies have described a higher order or general factor authors have called negative affect (Bados et al., 2010; M. Balsamo et al., 2013; Bieling et al., 1998), neither the anxiety-present nor the anxiety-absent factors described by Spielberger et al. appear to be comprised exclusively of items relating to negative affect or positive affect.

As well as a general factor that has been labelled negative affect, studies that have used CFA on the STAI T-Anxiety scale have found that the scale appears to contain two other factors, one that has been called 'anxiety' and one that has been called 'depression'. Authors have described the anxiety factor as being comprised of items that evaluate worry, tension and disturbing thoughts (Bados et al., 2010; M. Balsamo et al., 2013; Bieling et al., 
1998) while the depression factor is comprised of items that tap positive affect and happiness, relaxation, dysphoric mood, worry and statements of self-appraisal (Bados et al., 2010; M. Balsamo et al., 2013; Bieling et al., 1998). When the items that load onto each of these factors are inspected, the factors roughly fit the anxiety-present (anxiety factor) and anxietyabsent (depression factor) factors described by Spielberger et al. (1983) (Bados et al., 2010; M. Balsamo et al., 2013; Bieling et al., 1998) although, across studies, patterns of convergent and discriminant validity suggest that neither the anxiety-present and anxiety-absent factors nor the anxiety and depression factors accurately measure the dimensions suggested by the factor names.

Using CFA, the study by Bieling et al. (1989) found a hierarchical model comprised of two lower order factors that loaded on a higher order factor. Bieling et al. noted that items that loaded on one lower order factor assessed rumination, worry and disturbing thoughts, while items that loaded on the other lower order factor involved dysphoric mood and negative self-appraisal. They called these factors 'anxiety' and 'depression', respectively. The authors suggested that the factor Spielberger et al. (1983) identified as representing 'trait anxiety absent' did not represent the absence of anxiety because some of the items loading on this factor were related to low mood and self-deprecation rather than a lack of fear, worry or rumination (i.e., 'anxiety absent' traits). These items were also more strongly related to other measures of depression rather than being negatively correlated with other measures of anxiety, as would be expected if they were measuring the absence of this construct (Bieling et al., 1998). In relation to convergent and discriminant validity, the STAI T-Anxiety scale total score appeared to correlate more strongly with measures of depression (Beck Depression Inventory (BDI; Beck, Rush, Shaw, \& Emery, 1979) and the depression scale of the Depression Anxiety Stress Scales (DASS; Lovibond \& Lovibond, 1995)) than anxiety (Beck Anxiety Inventory (BAI; Beck \& Steer, 1990) and the anxiety scale and stress scale of the 
DASS); however, the differences between these correlations were not tested for significance. Although both the depression and anxiety subscales of the STAI T-Anxiety scale correlated with other measures of depression and anxiety respectively, the correlations and therefore the convergent validity of the anxiety subscale generally appeared to be weaker than the convergent validity of the depression subscale, but this was not tested for significance. Interestingly, the anxiety subscale appeared to correlate as strongly with the BDI as the depression subscale correlated with the BDI (Bieling et al., 1998).

A later study by Vigneau \& Cormier (2008) used CFA with data from healthy undergraduates and found that two different models best fit the data. The first model was a correlated version of the four factor model (state anxiety present, state anxiety absent, trait anxiety present, trait anxiety absent). The second model was a two construct (state content factor and trait content factor), two method (positive polarity method factor and negative polarity method factor) model, where the construct factors were kept independent to the method factors and the method factors were kept orthogonal to each other. The possibility that the factors identified in earlier reports (e.g., Bieling et al., 1998; Spielberger et al., 1983) may have merely reflected item keying was not mentioned by the authors of those earlier studies. In their study, Vigneau et al. preferred the two-construct, two-method model due to what they believed to be a number of interpretational advantages including compatibility with conceptualisations of state and trait anxiety and the use of each of the subscales, and enabling estimation of the size of the polarity effect (Vigneau \& Cormier, 2008).

Bados, Gómez-Benito, and Balaguer (2010) administered a Spanish version of the STAI T-Anxiety scale to healthy undergraduates and, consistent with findings from the study by Vigneau \& Cormier (2008), found that a one-construct, two-method model fit the data. They also found that a bifactor model with two specific factors they called depression and anxiety and one general factor they called negative affect fit the data. They identified overlap 
between the two models insofar as seven of the 11 positive polarity items formed the anxiety subscale and nine of the negative polarity items together with the remaining four positive polarity items formed the depression subscale. Although the authors called the first factor 'anxiety', they suggested that some of the items loading on this factor may actually be evaluating negative affect more generally rather than anxiety specifically. When Bados et al. examined the convergent and discriminant validity of the STAI T-Anxiety scale, they found that the STAI T-Anxiety scale total score and the two method subscales were significantly more strongly correlated with measures of depression (BDI, depression scale of the Depression and Anxiety Stress Scales 21 item version (DASS-21; Lovibond \& Lovibond, 1995), and the depression scale of the Symptom Checklish-90-R (SCL-90-R; Derogatis, 1983)) than anxiety (BAI, anxiety scale of the DASS-21, and the anxiety scale of the SCL90-R). In the bifactor model the depression subscale showed good convergent validity but, as in the study by Bieling et al. (1998), the convergent validity for the anxiety subscale did not appear to be as strong; again these correlations were not tested for significance. The anxiety subscale also had poor discriminant validity as it correlated similarly with a measure of depression and anxiety (depression scale and anxiety scale of the DASS-21) or significantly higher with measures of depression (BDI and depression scale of the SCL-90-R) than with measures of anxiety (BAI and anxiety scale of the SCL-90-R) (Bados et al., 2010).

In a study using 877 healthy participants and 1124 participants diagnosed with a range of Axis I disorders listed in the Diagnostic and Statistical Manual of Mental Disorders (4th ed.; DSM-IV-TR; American Psychiatric Association, 2000), Balsamo, Romanelli, Innamorati, Ciccarese, Carlucci, and Saggino (2013) also found a one-construct, two-method model as well as a bifactor model comprising two uncorrelated factors ('anxiety' and 'depression') and a general factor (negative affect). Similar to findings in studies by Bieling et al. (1998) and Bados et al. (2010), the STAI T-Anxiety scale total score correlated more strongly with 
measures of depression (Beck Depression Inventory-II (BDI-II; Beck, Steer, \& Brown, 1996) and Teate Depression Inventory (TDI; M. Balsamo, 2006)) than with a measure of anxiety (BAI), but only the relationship with the BDI-II was tested for significance. With the exception of the anxiety present factor in the non-clinical sample, both method factors appeared to be more strongly correlated with measures of depression (BDI-II and TDI) than anxiety (BAI), but these relationships were not tested for significance. The anxiety present factor was significantly more strongly correlated with the BAI than the anxiety absent factor, anxiety absent was significantly more strongly correlated with the TDI than anxiety present, and correlations between BDI-II and anxiety present and anxiety absent were similar. Although the correlations were not tested for significance, both the depression and anxiety subscales of the bifactor model appeared to correlate similarly or more strongly with measures of depression (BDI-II and TDI) than anxiety (BAI), supporting findings in the study by Bados et al. (2010).

In summary, studies that have performed CFA on the STAI Form Y version have generally supported the findings of the EFA conducted by Spielberger et al. (1983). These studies have found that a one construct, two method model (Bados et al., 2010; M. Balsamo et al., 2013) or, using combined STAI S-Anxiety scale data (Vigneau \& Cormier, 2008), two construct, two method model, provides the best fit to the data. In either model, the method factors distinguish items that are scored in same the direction as the total scale score (positive polarity) from items that need to be reverse scored (negative polarity). Individual differences and/or psychological variables (e.g., response styles) that may underlie these polarity effects have not yet been identified (Vigneau \& Cormier, 2008). In addition, studies have found a bifactor model with two factors that have been called depression and anxiety, and a general factor that has been called negative affect (Bados et al., 2010; M. Balsamo et al., 2013; Bieling et al., 1998) also fits the data. Although these findings suggest that there are a number 
of ways to conceptualise the STAI T-Anxiety scale (i.e., comprised of method factors or psychopathological factors), there is some - but not complete - overlap in the factors because many items that load on the anxiety and depression factors also load on the positive polarity and negative polarity factors, respectively. When correlated with other instruments, all four factors - as well as the STAI T-Anxiety scale total score - have either similar (anxiety factor) or higher correlations with measures of depression than with measures of anxiety (Bados et al., 2010; M. Balsamo et al., 2013). This raises the question of whether the STAI T-Anxiety scale should continue to be used as a measure of pure anxiety as distinct from depression, and the manner in which high scores on the instrument should be interpreted in both clinical and research settings.

Although the abovementioned studies found the STAI T-Anxiety scale total score correlated more strongly with measures of depression than anxiety, results from other studies have suggested otherwise. Kohn, Kantor, DeCicco, \& Beck (2008) used 191 healthy undergraduates and found that the STAI T-Anxiety scale total score appeared to correlate similarly with a measure of trait anxiety (Beck Anxiety Inventory - Trait (BAIT; Kohn, Kantor, DeCicco, \& Beck, 2008)) and a measure of depression (Depression Proneness Rating Scale (DPRS; Zemore, Fischer, Garratt, \& Miller, 1990), but a significance test was not conducted. In a study using 121 healthy participants and 22 participants with depression, Fountoulakis et al. (2006) also found what appeared to be similar correlations between the STAI T-Anxiety scale total score and measures of anxiety (anxiety scale of the Symptoms Rating Scale for Depression and Anxiety (SRSDA; Fountoulakis et al., 2003)), depression (BDI) and neuroticism (Eysenck Personality Questionnaire Revised, EPQ-R; H. J. Eysenck \& Eysenck, 1975). Again, the authors of this study did not conduct a significance test.

In contrast, as discussed above, in participants with anxiety disorders the STAI TAnxiety scale total score appears to correlate more strongly with the BDI and the depression 
scale of the DASS than the BAI and the stress and anxiety scales of the DASS (Bieling et al., 1998). Similarly, in participants with anxiety and mood disorders, the STAI T-Anxiety scale total score correlates significantly more strongly with the depression scale of the DASS than the anxiety scale of the DASS (Grös et al., 2007). Using a healthy and a clinical sample, Balsamo et al. (2013) found the STAI T-Anxiety scale total score correlated significantly more strongly with the BDI-II than with the BAI and appeared to correlate more strongly with the TDI than with the BAI. Using a healthy undergraduate sample, Bados et al. (2010) found significantly higher correlations between the STAI T-Anxiety scale and measures of depression (BDI, Depression scale of the DASS-21, and the depression scale of the SCL-90R) than anxiety (BAI, anxiety scale of the DASS-21, and the anxiety scale of the SCL-90-R). Similarly, using a healthy undergraduate sample Nitschke, Heller, Imig, McDonald, and Miller (2001) found what appeared to be higher correlations between the STAI T-Anxiety scale and measures of depression (BDI, the general depression scale of the Mood and Anxiety Symptom Questionnaire (MASQ; Watson et al., 1995a, 1995b), and the anhedonic depression scale of the MASQ) than measures of anxiety (BAI, anxious arousal scale of the MASQ, and the general anxiety scale of the MASQ), but the correlations were not tested for significance. In summary it appears that, overall, the STAI T-Anxiety scale correlates more strongly with measures of depression than anxiety and, although the results of convergent and discriminant validity analyses depend somewhat on the instruments used and some of the relationships have not yet been tested for statistical significance, at the very least these results suggest that caution should be exercised when using and interpreting scores on the STAI TAnxiety scale as an index of anxiety. 


\section{Dimensions of Anxiety Measured by the State-Trait Anxiety Inventory Trait Anxiety}

\section{Scale}

Authors of studies that have investigated the factor structure and/or convergent and discriminant validity of the STAI T-Anxiety scale have commented not only on the adequacy of the scale's measurement of trait anxiety but also on how well it may measure what have been recognised as different dimensions of anxiety (Bados et al., 2010; M. Balsamo et al., 2013; Grös et al., 2007; Nitschke et al., 2001). Anxiety has commonly been described as having two distinct dimensions (Nitschke et al., 1999). One dimension is called anxious arousal and is characterised by somatic tension and physiological hyperarousal such as a pounding heart and inability to relax. The anxious arousal scale of the MASQ is commonly used to measure this dimension of anxiety (e.g., Moser et al., 2012; Nitschke et al., 2001; Sass et al., 2010). The BAI also appears to assess anxious arousal rather than anxious apprehension (Nitschke et al., 2001). Unlike the anxious arousal scale of the MASQ, the BAI has not been marketed as a measure of anxious arousal; however, the original study by Beck and Steer (1990) found 15 of the 21 items in the BAI measured neurophysiological, panic and autonomic symptoms, and total scores from the BAI are higher in populations with panic disorder than those with social phobia or generalised anxiety disorder (Beck \& Steer, 1990). The second dimension of anxiety is called anxious apprehension and is comprised of worry and verbal rumination (Burdwood et al., 2016; Moser et al., 2012; Nitschke et al., 2001; P. B. Sharp et al., 2014). The Penn State Worry Questionnaire (PSWQ; Meyer, Miller, Metzger, \& Borkovec, 1990) is often used to measure this dimension of anxiety (e.g., Burdwood et al., 2016; Moser et al., 2012; Nitschke et al., 2001; Nitschke et al., 1999; Sass et al., 2010). Another instrument that contains items relating to anxious apprehension is the Generalized Anxiety Disorder 7-Item Scale (GAD-7; Spitzer, Kroenke, Williams, \& Löwe, 2006). The GAD -7 is a brief screen for generalized anxiety disorder (GAD), which is a disorder 
characterised by excessive worry (American Psychiatric Association, 2013a). In clinical samples who have received treatment for GAD, the GAD-7 has been shown to correlate highly with the PSWQ (Dear et al., 2011) and, in clinical samples diagnosed with GAD, the GAD-7 correlates highly with the BAI (Spitzer et al., 2006). Studies that have used CFA have found the GAD-7 is unidimensional (Löwe et al., 2008), which suggests the GAD-7 may measure anxiety generally rather than anxious apprehension or anxious arousal specifically. The high correlation between the GAD-7 and both the PSWQ and BAI in clinical populations implies that the GAD-7 may measure both anxious apprehension and anxious arousal, although the concurrent and discriminant validity of the instrument using a range of other measures in healthy as opposed to clinical samples has not been well established.

It has been proposed that the STAI T-Anxiety scale may measure anxious apprehension better than it measures anxious arousal (Nitschke et al., 2001). In support of this, the STAI T-Anxiety scale appears to correlate more highly with the PSWQ than with the BAI, the anxious arousal scale of the MASQ or the general distress - anxiety scale of the MASQ (Nitschke et al., 2001), but these correlations have not been tested for significance. It also appears to correlate more highly with the cognitive trait scale of the State-Trait Inventory for Cognitive and Somatic Anxiety (STICSA; Ree, MacLeod, French, \& Locke, 2000, November) than the somatic trait scale of the STICSA (Grös et al., 2007) but again, these correlations have not been tested for significance. These findings tentatively suggest that the STAI T-Anxiety scale may measure cognitive or worry-related dimensions of anxiety better than somatic or physiological dimensions of anxiety.

\section{Present Aims}

The STAI T-Anxiety scale is often used in research to identify participants high and low on trait anxiety in order to investigate trait anxiety's association with or effect on other psychological constructs. Due to the widespread use of this scale in samples drawn from 
different populations and the common assumption that it measures a unitary construct called trait anxiety in all those samples, it is important to understand its psychometric structure across different populations. Therefore the current study sought to examine the factor structure of the STAI T-Anxiety scale using EFA in a large, healthy sample. Although previous studies have examined the factor structure of the STAI T-Anxiety scale using EFA, those studies used air-force recruits and Year 10 high school students (Spielberger et al., 1983), and participants with a range of anxiety disorders rather than a healthy adult sample (Bieling et al., 1998). Based on results in previous studies it was predicted that the STAI TAnxiety scale may contain two method factors; a factor that corresponds to forward-scored items (positive polarity) and a factor that corresponds to reverse-scored items (negative polarity). A second aim of the study was to investigate the convergent and discriminant validity of the STAI T-Anxiety scale in the same sample. Although previous studies have looked at the relationship between the STAI T-Anxiety scale and other measures of anxiety, depression and personality traits, many of these studies did not test the significance of correlations that suggested the STAI T-Anxiety scale may have poor convergent and discriminant validity (Bados et al., 2010; M. Balsamo et al., 2013; Bieling et al., 1998; Nitschke et al., 2001) or adequate convergent and discriminant validity (Fountoulakis et al., 2006; Kohn et al., 2008). Based on previous findings we anticipated that the STAI T-Anxiety scale total score would correlate more strongly with measures of depression than with measures of anxiety. In addition, because no previous study had tested the significance of correlations that suggest the STAI T-Anxiety scale may correlate more strongly with measures of anxious apprehension than anxious arousal, we sought to explore the relationship between the STAI T-Anxiety scale and these two dimensions of anxiety. We predicted that the STAI T-Anxiety scale would correlate more strongly with a measure of anxious apprehension (i.e., the PSWQ) than with instruments that measure symptoms of anxious 
arousal (i.e., the BAI and the anxious arousal scale of the MASQ). We were also interested in exploring the relationship of the STAI T-Anxiety scale with other measures of personality related to anxiety, such as neuroticism and behavioural inhibition. Sometimes in research, financial or logistic constraints prevent collection of large participant samples. This is often the case in studies that use imaging techniques such as functional magnetic resonance imaging. In these situations, the STAI T-Anxiety scale total score is used to create and compare two smaller groups, one low in trait anxiety and one high in trait anxiety. Due to the widespread use of this research design, we aimed to explore how groups low and high in trait anxiety may differ on related constructs such as anxiety, mood and personality traits. We also used this two-group sample to explore the convergent and discriminant validity of the STAI T-Anxiety scale with another well-recognised measure of anxious arousal (i.e., anxious arousal scale of the MASQ) and other measures of personality and executive function (i.e., impulsivity and attention control).

\section{Study 1}

\section{Method}

\section{Participants}

Participants were 947 English speaking University of Melbourne students and members of the public ( 287 males, 652 females, 2 other and 6 non-identified; mean age $=$ 21.69 years, $\mathrm{SD}=4.81$, age range 16.5 to 45.45 years) recruited through a University of Melbourne first year research program, advertisements on careers websites and advertisements on the Gumtree website throughout the period August 2014 to September 2016. All participants provided written informed consent and the experiment was approved by the human research ethics committee at the University of Melbourne. Participants were either paid an honorarium for completing the study or received course credit. Measures were completed online via the Qualtrics Research Suite on the university campus or at an off- 
campus location. All participants completed the STAI, the Behavioral Inhibition System/Behavioral Activation System Scales (BIS/BAS Scales; Carver \& White, 1994), a demographic information questionnaire and a magnetic resonance imaging (MRI) eligibility information questionnaire; part of the sample also completed the BAI, the GAD-7, the PSWQ, the BDI-II and the Big Five Aspects Scales (BFAS; DeYoung, Quilty, \& Peterson, 2007) $(n=677 ; 187$ males, 483 females, 2 other, 5 non-identified; mean age $=20.27$ years, $\mathrm{SD}=3.59$, age range 16.50 to 44.92 years), the Inventory of Depressive Symptomatology Self Report (IDS-SR; John Rush et al., 1986) ( $n=566$; 161 males, 399 females, 2 other, 4 non-identified; mean age $=20.30$ years, $\mathrm{SD}=3.52$, age range 16.50 to 44.92 years), the Personality Inventory for DSM-5 Brief Form - Adult (PID-5-BF - Adult; Krueger, Derringer, Markon, Watson, \& Skodol, 2013) and the MASQ ( $n=200 ; 61$ males, 137 females, 1 other, 1 non-identified; mean age $=21.38$ years, $\mathrm{SD}=4.71$, age range 18.08 to 44.92 years), and the Sensitivity to Punishment and Sensitivity to Reward Questionnaire (SPSRQ; Torrubia et al., 2001) $(n=111 ; 26$ males, 84 females, 1 non-identified; mean age $=20.09$ years, $\mathrm{SD}=3.95$, age range 18.10 to 42.84 years). Non-copyrighted measures are reproduced in Appendix A and Appendix B.

\section{Measures}

State-Trait Anxiety Inventory, Form Y (STAI: Spielberger et al., 1983). The STAI is comprised of 20 items that measure state anxiety (STAI S-Anxiety scale) and 20 items that measure trait anxiety (STAI T-Anxiety scale). The STAI S-Anxiety scale asks respondents to indicate how they feel right now, that is, at this moment and the STAI TAnxiety scale asks respondents to indicate how you generally feel. Each item is scored on a four point scale (1 to 4), from not at all to very much so for the STAI S-Anxiety scale, and from almost never to almost always for the STAI T-Anxiety scale. The STAI S-Anxiety scale contains 10 positive polarity and 10 negative polarity items, and the STAI T-Anxiety scale 
contains 11 positive polarity and 9 negative polarity items. Negative polarity items are reverse scored and scores from all items in the scale are added to give a final score for each scale ranging from 20 to 80 , with higher scores indicating greater anxiety. In our sample the mean for the STAI S-Anxiety scale $(M=36.10)$ was significantly lower than the mean for the female college sample in the original scale development study by Spielberger et al. (1983) $\left(M_{\text {males }}=36.47, M_{\text {females }}=38.76\right)$ and not significantly different from male sample or the normative Australian sample aged 18-24 years in the study by Crawford, Cayley, Lovibond, Wilson, \& Hartley (2011) $(M=36.85)$. The mean for the STAI T-Anxiety scale in our sample $(M=42.87)$ was significantly higher than those reported by Spielberger $\left(M_{\text {males }}=38.30\right.$, $\left.M_{\text {females }}=40.40\right)$ and Crawford et al. $(M=40.39)$. Our scale reliability for the STAI SAnxiety scale $(\alpha=.93)$ was similar to that reported by Spielberger et al. $\left(\alpha_{\text {males }}=.91, \alpha_{\text {females }}=\right.$ $.93)$ and our reliability for the STAI T-Anxiety scale $(\alpha=.93)$ was slightly higher than the alpha reported by Spielberger et al. $\left(\alpha_{\text {males }}=.90, \alpha_{\text {females }}=.91\right)$. Table 2 shows item statistics for the STAI T-Anxiety scale including means, standard deviations, and Cronbach's alpha if item deleted. Table 3 contains the inter item correlation matrix.

Table 2

Means, SD and Cronbach's Alpha if Item Deleted for State Trait Anxiety Inventory T-Anxiety Scale Items $(N=947)$

\begin{tabular}{|c|c|c|c|}
\hline Item & $M$ & $S D$ & $\begin{array}{c}\text { Alpha if } \\
\text { item deleted }\end{array}$ \\
\hline 21. I feel pleasant & 2.06 & .68 & .93 \\
\hline 22. I feel nervous and restless & 2.11 & .67 & .93 \\
\hline 23. I feel satisfied with myself & 2.33 & .80 & .93 \\
\hline 24. I wish I could be as happy as others seem to be & 2.18 & .94 & .93 \\
\hline 25. I feel like a failure & 1.69 & .73 & .93 \\
\hline 26. I feel rested & 2.63 & .77 & .93 \\
\hline 27. I am "calm, cool and collected" & 2.34 & .81 & .93 \\
\hline
\end{tabular}




\begin{tabular}{|c|c|c|c|}
\hline Item & $M$ & $S D$ & $\begin{array}{c}\text { Alpha if } \\
\text { item deleted }\end{array}$ \\
\hline 28. I feel that difficulties are piling up so that I cannot overcome them & 1.95 & .79 & .93 \\
\hline 29. I worry too much over something that really doesn't matter & 2.35 & .93 & .93 \\
\hline 30. I am happy & 1.95 & .74 & .93 \\
\hline 31. I have disturbing thoughts & 1.71 & .83 & .93 \\
\hline 32. I lack self-confidence & 2.19 & .83 & .93 \\
\hline 33. I feel secure & 2.11 & .79 & .93 \\
\hline 34. I make decisions easily & 2.72 & .86 & .93 \\
\hline 35. I feel inadequate & 1.82 & .75 & .93 \\
\hline 36. I am content & 2.11 & .77 & .93 \\
\hline 37. Some unimportant thought runs through my mind and bothers me & 2.21 & .85 & .93 \\
\hline 38. I take disappointments so keenly that I can't put them out of my mind & 2.16 & .93 & .93 \\
\hline 39. I am a steady person & 2.16 & .78 & .93 \\
\hline $\begin{array}{l}\text { 40. I get in a state of tension or turmoil as I think over my recent concerns and } \\
\text { interests }\end{array}$ & 2.10 & .88 & .93 \\
\hline
\end{tabular}

Note. Item numbers range from 21 to 40. Items 1 to 20 are from the State Trait Anxiety Inventory state anxiety scale and are not shown in this table. 
Table 3

Inter Item Correlation Matrix for Items in State Trait Anxiety Inventory T-Anxiety Scale

\begin{tabular}{|c|c|c|c|c|c|c|c|c|c|c|c|c|c|c|c|c|c|c|c|c|}
\hline Item & 21 & 22 & 23 & 24 & 25 & 26 & 27 & 28 & 29 & 30 & 31 & 32 & 33 & 34 & 35 & 36 & 37 & 38 & 39 & 40 \\
\hline 21 & 1.00 & .43 & .58 & .47 & .40 & .44 & .51 & .37 & .32 & .66 & .36 & .39 & .56 & .35 & .39 & .61 & .36 & .38 & .57 & .41 \\
\hline 22 & .43 & 1.00 & .40 & .39 & .43 & .35 & .42 & .44 & .47 & .41 & .39 & .43 & .42 & .32 & .40 & .41 & .38 & .45 & .43 & .50 \\
\hline 23 & .58 & .40 & 1.00 & .46 & .48 & .44 & .51 & .40 & .34 & .62 & .33 & .52 & .56 & .37 & .46 & .59 & .30 & .36 & .54 & .39 \\
\hline 24 & .47 & .39 & .46 & 1.00 & .47 & .34 & .33 & .44 & .40 & .49 & .34 & .44 & .40 & .26 & .43 & .45 & .33 & .43 & .39 & .43 \\
\hline 25 & .40 & .43 & .48 & .47 & 1.00 & .30 & .35 & .46 & .34 & .46 & .41 & .53 & .42 & .26 & .58 & .40 & .32 & .42 & .40 & .45 \\
\hline 26 & .44 & .35 & .44 & .34 & .30 & 1.00 & .48 & .39 & .37 & .44 & .26 & .31 & .46 & .28 & .29 & .47 & .30 & .37 & .42 & .39 \\
\hline 27 & .51 & .42 & .51 & .33 & .35 & .48 & 1.00 & .39 & .40 & .48 & .23 & .42 & .49 & .40 & .34 & .48 & .32 & .36 & .60 & .40 \\
\hline 28 & .37 & .44 & .40 & .44 & .46 & .39 & .39 & 1.00 & .49 & .36 & .36 & .38 & .39 & .29 & .39 & .39 & .37 & .44 & .41 & .47 \\
\hline 29 & .32 & .47 & .34 & .40 & .34 & .37 & .40 & .49 & 1.00 & .27 & .32 & .43 & .32 & .35 & .35 & .34 & .59 & .56 & .40 & .51 \\
\hline 30 & .66 & .41 & .62 & .49 & .46 & .44 & .48 & .36 & .27 & 1.00 & .35 & .41 & .58 & .32 & .41 & .63 & .27 & .33 & .53 & .38 \\
\hline 31 & .36 & .39 & .33 & .34 & .41 & .26 & .23 & .36 & .32 & .35 & 1.00 & .29 & .35 & .15 & .41 & .33 & .42 & .42 & .34 & .40 \\
\hline 32 & .39 & .43 & .52 & .44 & .53 & .31 & .42 & .38 & .43 & .41 & .29 & 1.00 & .44 & .35 & .52 & .37 & .34 & .44 & .41 & .42 \\
\hline 33 & .56 & .42 & .56 & .40 & .42 & .46 & .49 & .39 & .32 & .58 & .35 & .44 & 1.00 & .40 & .43 & .60 & .32 & .39 & .57 & .42 \\
\hline 34 & .35 & .32 & .37 & .26 & .26 & .28 & .40 & .29 & .35 & .32 & .15 & .35 & .40 & 1.00 & .25 & .33 & .29 & .29 & .45 & .34 \\
\hline 35 & .39 & .40 & .46 & .43 & .58 & .29 & .34 & .39 & .35 & .41 & .41 & .52 & .43 & .25 & 1.00 & .39 & .34 & .39 & .40 & .40 \\
\hline 36 & .61 & .41 & .59 & .45 & .40 & .47 & .48 & .39 & .34 & .63 & .33 & .37 & .60 & .33 & .39 & 1.00 & .31 & .37 & .58 & .43 \\
\hline 37 & .36 & .38 & .30 & .33 & .32 & .30 & .32 & .37 & .59 & .27 & .42 & .34 & .32 & .29 & .34 & .31 & 1.00 & .56 & .37 & .46 \\
\hline 38 & .38 & .45 & .36 & .43 & .42 & .37 & .36 & .44 & .56 & .33 & .42 & .44 & .39 & .29 & .39 & .37 & .56 & 1.00 & .42 & .58 \\
\hline 39 & .57 & .43 & .54 & .39 & .40 & .42 & .60 & .41 & .40 & .53 & .34 & .41 & .57 & .45 & .40 & .58 & .37 & .42 & 1.00 & .45 \\
\hline 40 & .41 & .50 & .39 & .43 & .45 & .39 & .40 & .47 & .51 & .38 & .40 & .42 & .42 & .34 & .40 & .43 & .46 & .58 & .45 & 1.00 \\
\hline
\end{tabular}

Note. Item numbers range from 21 to 40. Items 1 to 20 are from the State Trait Anxiety Inventory state anxiety scale and are not shown in this table. 
Beck Anxiety Inventory (BAI: Beck \& Steer, 1990). The BAI was designed to measure symptoms of anxiety not shared with depression (Beck \& Steer, 1990). It is comprised of 21 items that the instrument describes as common symptoms of anxiety, such as Numbness or tingling and Heart pounding or racing. Respondents are asked to rate each item on a 4 point scale ranging from 0 (not at all) to 3 (severely; I could barely stand it) based on how much they have been bothered by each symptom during the past week. The total score ranges from 0 to 63, with higher scores indicating greater anxiety. The internal reliability for the BAI and for each of the other instruments used and/or scales from each instrument used in our sample is shown in Table 4.

Generalised Anxiety Disorder 7-Item Scale (GAD-7: Spitzer et al., 2006). The GAD-7 is a measure comprised of 7 items designed to identify generalised anxiety disorder and assess symptom severity (Spitzer et al., 2006). Each item asks respondents to indicate how often they have been bothered by a range of problems over the last two weeks, and is scored on a scale of 0 to 3 where 0 is not at all and 3 is nearly every day. Total scores range from 0 to 21 with higher scores indicating greater anxiety.

Penn State Worry Questionnaire (PSWQ: Meyer et al., 1990). The PSWQ is a 16 item measure that assesses the trait of worry, a cognitive aspect of anxiety comprised of negative thoughts and an anxious and depressive emotional experience (Meyer et al., 1990). The PSWQ includes 11 forward-scored items such as My worries overwhelm me and When I am under pressure I worry a lot and five reversed-scored items such as I do not tend to worry about things. It asks respondents to rate statements on a scale of 1 (not at all typical of me) to 5 (very typical of me). Total scores range from 16 to 80, with higher scores indicating more worry.

Beck Depression Inventory-II (BDI-II: Beck et al., 1996). The BDI-II is comprised of 21 items that measure severity of depressive symptoms. Each item asks respondents to 
describe the way they have been feeling during the past two weeks. Items are rated on a scale from 0 to 3 and the total score ranges from 0 to 63 with higher scores indicating greater severity of depressive symptoms.

\section{Inventory of Depressive Symptomatology - Self Report (IDS-SR: John Rush et}

al., 1986). The IDS-SR measures signs and symptoms of depression (John Rush et al., 1986). It contains 30 items rated on a scale of 0 to 3 . Twenty eight of the 30 items are included in the total score (only one of items 11 and 12 and one of items 13 and 14 are counted in the total score because each set of questions asks about the same construct but are reverse worded, e.g., item 11 relates to decreased appetite and item 12 relates to increased appetite). The total score ranges from 0 to 84 with higher scores indicating more signs and symptoms depression.

\section{Mood and Anxiety Symptom Questionnaire (MASQ: Watson et al., 1995a,}

1995b). The MASQ is a 90 item questionnaire that was developed to measure the tripartite model of anxiety and depression (Clark \& Watson, 1991), and measures symptoms of depression and anxiety (Watson et al., 1995a, 1995b). The MASQ consists of five symptom scales: anhedonic depression, anxious arousal, general distress - mixed, general distress depression and general distress - anxiety. Each item asks respondents to respond on a scale from 1 (not at all) to 5 (extremely) based on how much they have felt or experienced things during the past week. Items are averaged to create scores for each of the symptom scales and higher scores on each scale indicate greater levels of the construct measured by that scale.

The Big Five Aspects Scales (BFAS: DeYoung et al., 2007). The BFAS was developed to assess the five broad domains of personality (Neuroticism, Agreeableness, Conscientiousness, Extraversion and Openness/Intellect) which can each be divided into two narrower sub-traits, yielding 10 trait aspects (Volatility, Withdrawal, Compassion, Politeness, Industriousness, Orderliness, Enthusiasm, Assertiveness, Intellect, and Openness) (DeYoung et al., 2007). The BFAS is comprised of 100 items and respondents are asked to indicate the 
extent to which they agree or disagree with each item on a Likert-type scale from 1 (strongly disagree) to 5 (strongly agree). Each of the Big Five domains contains 20 items and each of the Big Five aspects contains 10 items, with approximately half of the items reverse scored. Items are averaged to create scores for each of the 10 aspects, and these scores are averaged across two aspects of each domain to create Big Five domain scores. Total scores for each aspect range from 1 to 5, with higher scores indicating higher levels of the aspect.

\section{Behavioral Inhibition System/Behavioral Activation System Scales (BIS/BAS}

Scales: Carver \& White, 1994). The BIS/BAS Scales were developed to measure sensitivity to signals of punishment, nonreward and novelty (underpinned by the biologically-based behavioural inhibition system (BIS) (J. A. Gray \& McNaughton, 2000), and sensitivity to signals of reward, nonpunishment, and escape from punishment (underpinned by the biologically-based behavioural activation system (BAS) (Carver \& White, 1994). The BIS/BAS Scales contain 24 items and respondents are asked to indicate how much they agree or disagree with what each item says on a 4-point scale ranging from 1 (very true for me) to 4 (very false for me). All but two items are reverse scored and four items are fillers.

\section{Sensitivity to Punishment and Sensitivity to Reward Questionnaire (SPSRQ:}

Torrubia et al., 2001). The SPSRQ is a questionnaire that measures individual differences in punishment and reward sensitivity, underpinned by the BIS and the impulsivity dimension of the BAS, respectively, as described in Gray's model of personality (Torrubia et al., 2001). The questionnaire is comprised of 48 items with yes/no responses (e.g., Are you often afraid of new or unexpected situations? Yes/No). Half of the items are reverse scored.

Personality Inventory for DSM-5 Brief Form - Adult (PID-5-BF - Adult:

Krueger et al., 2013). The PID-5-BF - Adult was designed to measure five personality trait domains (negative affect, detachment, antagonism, disinhibition, and psychoticism). Five items measure each trait and each item is rated on a 4 point scale from 0 (very false or often 
false) to 3 (very true or often true). Total scores for each trait domain range from 0 to 15 with higher scores indicating greater dysfunction in the trait domain.

Table 4

Internal Reliabilities of Each Instrument and/or Scale

\begin{tabular}{|c|c|}
\hline Scale & Alpha \\
\hline STAI S-Anxiety Scale & .93 \\
\hline STAI T-Anxiety Scale & .93 \\
\hline BAI & .93 \\
\hline GAD-7 & .87 \\
\hline PSWQ & .94 \\
\hline BDI-II & .91 \\
\hline IDS-SR & .88 \\
\hline MASQ & -- \\
\hline GM & .91 \\
\hline GA & .84 \\
\hline AA & .91 \\
\hline GD & .94 \\
\hline $\mathrm{AD}$ & .94 \\
\hline BFAS & -- \\
\hline 10 Aspects & -- \\
\hline Volatility & .89 \\
\hline Withdrawal & .86 \\
\hline Compassion & .86 \\
\hline Politeness & .74 \\
\hline Industriousness & .85 \\
\hline Orderliness & .76 \\
\hline Enthusiasm & .85 \\
\hline Assertiveness & .86 \\
\hline Intellect & .83 \\
\hline Openness & .76 \\
\hline 5 Domains & -- \\
\hline
\end{tabular}




\begin{tabular}{cc}
\hline \multicolumn{1}{c}{ Scale } & Alpha \\
\hline Neuroticism & .92 \\
Agreeableness & .85 \\
Conscientiousness & .84 \\
Extraversion & .89 \\
Openness/Intellect & .82 \\
BIS/BAS Scales & -- \\
BIS & .81 \\
BAS Drive & .76 \\
BAS Fun Seeking & .72 \\
BAS Reward & .64 \\
Responsiveness & -- \\
SPSRQ & .86 \\
SP & .77 \\
SR & -8 \\
PID-5-BF - Adult & .81 \\
Negative affect & .82 \\
Detachment & .74 \\
Antagonism & .89 \\
Disinhibition & \\
Psychoticism &
\end{tabular}

Note. STAI = State Trait Anxiety Inventory; STAI S-Anxiety Scale = state anxiety scale; STAI T-Anxiety Scale = trait anxiety scale; BAI = Beck Anxiety Inventory; GAD-7 = Generalised Anxiety Disorder 7-Item Scale; PSWQ = Penn State Worry Questionnaire; BDI-II = Beck Depression Inventory-II; IDS-SR = Inventory of Depressive Symptomatology - Self Report; MASQ = Mood and Anxiety Symptom Questionnaire; GM = general distress - mixed scale; GA = general distress - anxiety scale; $\mathrm{AA}=$ anxious arousal scale; $\mathrm{GD}=$ general distress - depression scale; $\mathrm{AD}=$ anhedonic depression scale; $\mathrm{BFAS}=$ Big Five Aspects Scales; BIS/BAS Scales = Behavioral Inhibition System/Behavioral Activation System Scales; BAS = behavioral activation subscales; BIS = behavioral inhibition subscale; SPSRQ = Sensitivity to Punishment and Sensitivity to Reward Questionnaire; SP = sensitivity to punishment scale; SR = sensitivity to reward scale; PID-5-BF - Adult = Personality Inventory for DSM-5 Brief Form - Adult.

\section{Data Analysis}

All analyses were conducted using the Statistical Package for the Social Sciences (SPSS) 18.0.0. An EFA was conducted on the correlation matrix of the 20 items of the STAI T-Anxiety scale using principal axis factoring with Oblimin rotation. Principal axis factoring was chosen because it is widely used and understood (Tabachnick \& Fidell, 2007) and is 
preferred when the assumption of normality is violated (Costello \& Osborne, 2005; Yong \& Pearce, 2013). The Pearson product-moment correlation coefficient was used to investigate the convergent and discriminant validity of the STAI T-Anxiety scale with the BAI, GAD-7, PSWQ, BDI-II, IDS-SR, MASQ, BFAS, BIS/BAS Scales, SPSRQ, and PID-5- BF - Adult. Fisher's $r$-to- $z$ transformation was used to investigate whether there were significant differences between pairs of correlations.

\section{Results}

\section{Reliability and Factor Analysis}

The STAI T-Anxiety scale had good internal consistency with a Cronbach alpha coefficient $(\alpha)$ of .93. The suitability of the data for factor analysis was assessed by inspecting the total sample size and the correlation coefficient matrix (the correlation coefficient matrix is displayed in Table 3, above). The sample size of 947 was above commonly accepted guidelines for EFA (Costello \& Osborne, 2005; Hogarty, Hines, Kromrey, Perron, \& Mumford, 2005; MacCallum, Widaman, Zhang, \& Hong, 1999; Tabachnick \& Fidell, 2007; Yong \& Pearce, 2013).

Preliminary analysis of skewness, kurtosis, linearity and homoscedasticity showed there were violations of the assumptions of normality but linearity and homoscedasticity appeared to be adequate. Although skewness and kurtosis for a number of items were statistically significant, in large samples statistically significant skewness and kurtosis does not make a substantive difference to the analysis (Tabachnick \& Fidell, 2007) and, with widely used scales, transformation of scores to overcome statistically significant skewness or kurtosis can hinder interpretation (Tabachnick \& Fidell, 2007). Scores were therefore not transformed.

Most coefficients in the correlation matrix were at .30 or above, suggesting the data was suitable for factor analysis. The Kaiser-Meyer-Olkin measure of sampling adequacy was 
.96 , which exceeded the recommended value of .60 (Kaiser, 1970, 1974; Tabachnick \& Fidell, 2007) and suggested the strength of the relationships among variables was high. Bartlett's Test of Sphericity (Bartlett, 1954) was significant, approx. $\chi^{2}(190)=9310.40, p$ $<.001$. Both tests indicated significant correlations and low partial correlations among variables and supported the suitability of the data for factor analysis.

We initially applied Kaiser's criterion which is a rule of thumb that retains all factors with eigenvalues above one (Kaiser, 1960). Principal axis factoring indicated three components had eigenvalues exceeding one and each accounted for $44.27 \%, 7.86 \%$, and $5.63 \%$ of the variance, respectively. Table 5 shows eigenvalues for all factors.

Table 5

Variance Explained in State Trait Anxiety Inventory T-Anxiety Scale Items Using Principal Axis Factoring

\begin{tabular}{cccc}
\hline Factor & Total variance & Percent of variance & Cumulative percent \\
\hline 1 & 8.85 & 44.27 & 44.27 \\
2 & 1.57 & 7.86 & 52.13 \\
3 & 1.13 & 5.63 & 57.76 \\
4 & .89 & 4.44 & 62.20 \\
5 & .74 & 3.68 & 65.89 \\
6 & .66 & 3.29 & 69.17 \\
7 & .63 & 3.15 & 72.32 \\
8 & .59 & 2.94 & 75.27 \\
9 & .55 & 2.77 & 78.03 \\
10 & .52 & 2.57 & 80.61 \\
11 & .49 & 2.42 & 83.03 \\
12 & .46 & 2.31 & 85.34 \\
13 & .44 & 2.22 & 87.56 \\
14 & .41 & 2.06 & 89.61 \\
15 & .38 & 1.92 & 91.53 \\
16 & .37 & 1.86 & 93.39 \\
\hline
\end{tabular}




\begin{tabular}{cccc}
\hline Factor & Total variance & Percent of variance & Cumulative percent \\
\hline 17 & .35 & 1.76 & 95.15 \\
18 & .34 & 1.72 & 96.87 \\
19 & .32 & 1.62 & 98.49 \\
20 & .30 & 1.51 & 100.00 \\
\hline
\end{tabular}

Kaiser's criterion has been criticised for overestimating the number of factors extracted (Costello \& Osborne, 2005) and it has been suggested that inspection of the scree plot should be used with Kaiser's criterion in order to more accurately determine the number of factors to retain (Yong \& Pearce, 2013). We therefore applied Cattel's (1966) scree test of eigenvalues plotted against factors (Cattel, 1966; Tabachnick \& Fidell, 2007). Results of the scree plot are more reliable when sample size is large, communalities are high and each factor has a number of variables with high loadings (Tabachnick \& Fidell, 2007). Our data fulfilled these criteria therefore the results of the scree test were considered reliable. The scree plot is shown in Figure 1. 


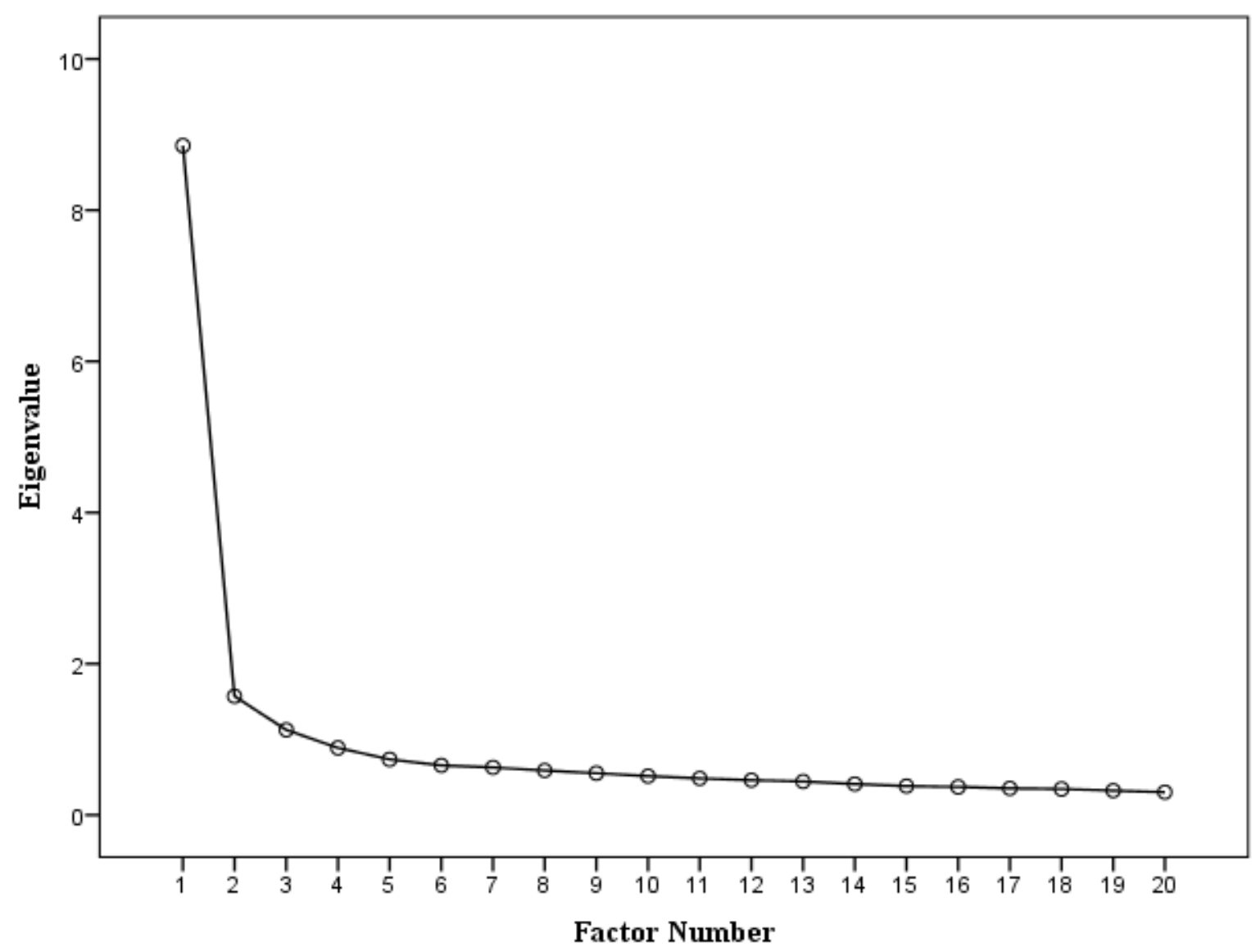

Figure 1. Scree plot showing eigenvalues plotted against factors.

Inspection of the scree plot showed a break after the second component, suggesting a two factor solution. Parallel analysis is an alternative method used to determine the number of factors that should be retained. It involves the extraction of eigenvalues from random sets of data with the same number of cases and variables as the actual data. The eigenvalues derived from the random data are compared to the eigenvalues derived from the actual data and factors that have actual data eigenvalues that are greater than the random data eigenvalues are retained (O'Connor, 2000). A parallel analysis using 1000 random data sets and eigenvalues that corresponded to the $80^{\text {th }}$ percentile of the distribution of random data eigenvalues was conducted. Results of the parallel analysis are displayed in Table 6. 
Table 6

Parallel Analysis for State Trait Anxiety Inventory T-Anxiety Scale

\begin{tabular}{|c|c|c|c|}
\hline $\begin{array}{c}\text { Component } \\
\text { number }\end{array}$ & $\begin{array}{l}\text { Actual eigenvalue } \\
\text { from factor analysis }\end{array}$ & $\begin{array}{l}\text { Eigenvalue from } \\
\text { random data set }\end{array}$ & $\begin{array}{c}\text { Criterion value from } \\
\text { parallel analysis }\end{array}$ \\
\hline 1 & 8.85 & 1.26 & 1.28 \\
\hline 2 & 1.57 & 1.22 & 1.23 \\
\hline 3 & 1.13 & 1.18 & 1.20 \\
\hline 4 & .89 & 1.15 & 1.16 \\
\hline 5 & .74 & 1.12 & 1.14 \\
\hline 6 & .66 & 1.10 & 1.11 \\
\hline 7 & .63 & 1.07 & 1.08 \\
\hline 8 & .59 & 1.05 & 1.06 \\
\hline 9 & .55 & 1.03 & 1.04 \\
\hline 10 & .52 & 1.01 & 1.01 \\
\hline 11 & .49 & .98 & .99 \\
\hline 12 & .46 & .96 & .97 \\
\hline 13 & .44 & .94 & .95 \\
\hline 14 & .41 & .92 & .93 \\
\hline 15 & .38 & .90 & .91 \\
\hline 16 & .37 & .87 & .88 \\
\hline 17 & .35 & .85 & .86 \\
\hline 18 & .34 & .83 & .84 \\
\hline 19 & .32 & .80 & .81 \\
\hline 20 & .30 & .76 & .78 \\
\hline
\end{tabular}

The results of the parallel analysis showed that only the first two factors in the actual data had higher eigenvalues than the corresponding eigenvalues from random data. Based on the results of the scree test and the parallel analysis, the decision was made to extract two factors. Further investigation using a two factor solution revealed that the two factors explained a total of $52.13 \%$ of the variance, with factor 1 accounting for $44.27 \%$ and factor 2 contributing $7.86 \%$ of the variance, respectively. Oblimin rotation was used because factors 
were highly correlated $(r=.68)$ (Fabrigar, MacCallum, Wegener, \& Strahan, 1999) and it has been argued that, when using oblique rotation methods, there is no single preferred method of rotation (Costello \& Osborne, 2005). Table 7 contains the pattern matrix, structure matrix and item communalities for the two factor solution using Oblimin rotation.

\section{Table 7}

Pattern Matrix, Structure Matrix and Communalities for Principal Axis Factoring with Oblimin Rotation of Two Factor Solution of State Trait Anxiety Inventory T-Anxiety Scale Items

\begin{tabular}{|c|c|c|c|c|c|c|}
\hline \multirow{2}{*}{ Item } & \multicolumn{2}{|c|}{ Pattern matrix } & \multicolumn{2}{|c|}{ Structure matrix } & \multicolumn{2}{|c|}{ Communalities } \\
\hline & Factor 1 & Factor 2 & Factor 1 & Factor 2 & Initial & Extraction \\
\hline 30. I am happy & .89 & -.14 & .80 & .46 & .60 & .64 \\
\hline 36. I am content & .80 & -.05 & .77 & .49 & .57 & .60 \\
\hline 21. I feel pleasant & .79 & -.03 & .77 & .51 & .57 & .60 \\
\hline 23. I feel satisfied with myself & .77 & .00 & .77 & .52 & .56 & .59 \\
\hline 33. I feel secure & .74 & .02 & .75 & .52 & .53 & .57 \\
\hline 39. I am a steady person & .65 & .13 & .73 & .56 & .55 & .54 \\
\hline 27. I am "calm, cool and collected" & .58 & .12 & .66 & .51 & .49 & .45 \\
\hline 26. I feel rested & .48 & .15 & .58 & .47 & .37 & .35 \\
\hline 24. I wish I could be as happy as others seem to be & .35 & .33 & .58 & .57 & .42 & .40 \\
\hline 34. I make decisions easily & .34 & .19 & .47 & .42 & .29 & .24 \\
\hline $\begin{array}{l}\text { 29. I worry too much over something that really doesn't } \\
\text { matter }\end{array}$ & -.11 & .81 & .44 & .74 & .53 & .56 \\
\hline $\begin{array}{l}\text { 38. I take disappointments so keenly that I can't put } \\
\text { them out of my mind }\end{array}$ & -.05 & .79 & .49 & .76 & .51 & .57 \\
\hline $\begin{array}{l}\text { 37. Some unimportant thought runs through my mind } \\
\text { and bothers me }\end{array}$ & -.10 & .75 & .40 & .68 & .46 & .47 \\
\hline $\begin{array}{l}\text { 40. I get in a state of tension or turmoil as I think over } \\
\text { my recent concerns and interests }\end{array}$ & .11 & .64 & .55 & .72 & .49 & .52 \\
\hline $\begin{array}{l}\text { 28. I feel that difficulties are piling up so that I cannot } \\
\text { overcome them }\end{array}$ & .18 & .51 & .52 & .63 & .41 & .42 \\
\hline 22. I feel nervous and restless & .23 & .48 & .54 & .64 & .41 & .43 \\
\hline 31. I have disturbing thoughts & .13 & .45 & .44 & .54 & .35 & .30 \\
\hline 32. I lack self-confidence & .31 & .40 & .57 & .60 & .47 & .42 \\
\hline
\end{tabular}




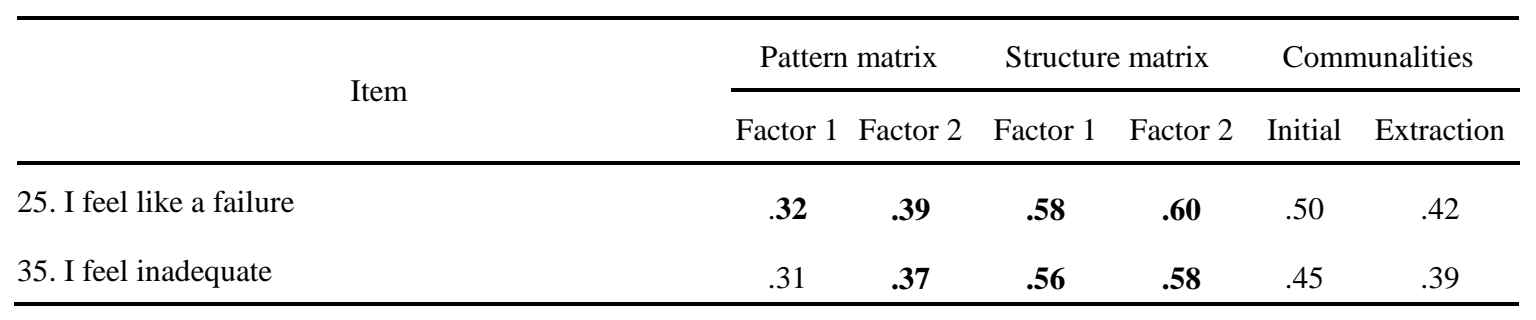

Note. Loadings over 32 for each item are indicated in bold. Item numbers range from 21 to 40. Items 1 to 20 are from the State Trait Anxiety Inventory state anxiety scale and are not shown in this table.

Only items with loadings of .32 and above were interpreted (Tabachnick \& Fidell, 2007); these items are shown in bold typeface in Table 7. Only two items (items 24 and 25) cross loaded at or slightly above .32 on each factor (Costello \& Osborne, 2005; Yong \& Pearce, 2013). We decided to retain these items when interpreting the solution as the size of the cross loadings was low (Yong \& Pearce, 2013). As can be seen in Table 7, 11 items loaded on each factor. Table 8 compares factor loadings from our two factor solution with results presented by Spielberger et al. (1983) for air force recruits and Bieling et al. (1998).

Table 8

Comparison of Factor Loadings Obtained via Exploratory Factor Analysis with Findings from Studies by Bieling et al. (1998) and Spielberger et al. (1983)

\begin{tabular}{|c|c|c|c|c|c|c|}
\hline \multirow{2}{*}{ Item } & \multicolumn{2}{|c|}{ Current study } & \multicolumn{2}{|c|}{ Bieling et al. (1998) } & \multicolumn{2}{|c|}{$\begin{array}{c}\text { Spielberger et al. } \\
(1983) \\
\end{array}$} \\
\hline & Factor 1 & Factor 2 & Factor 1 & Factor 2 & Factor 1 & Factor 2 \\
\hline 30. I am happy &.$\underline{89}$ & -.14 &.$\underline{76}$ & .04 &.$\underline{73}$ & \\
\hline 36. I am content & $\underline{80}$ & -.05 &.$\underline{76}$ & -.01 &.$\underline{60}$ & \\
\hline 21. I feel pleasant &.$\underline{79}$ & -.03 &.$\underline{70}$ & .10 &.$\underline{63}$ & \\
\hline 23. I feel satisfied with myself &.$\underline{77}$ & .00 &.$\underline{71}$ & .23 &.$\underline{59}$ & \\
\hline 33. I feel secure &.$\underline{74}$ & .02 &.$\underline{69}$ & .24 &.$\underline{67}$ & \\
\hline 39. I am a steady person &.$\underline{65}$ & .13 &.$\underline{.57}$ & .30 &.$\underline{49}$ & \\
\hline 27. I am "calm, cool and collected" &.$\underline{58}$ & .12 &.$\underline{47}$ & .35 &.$\underline{66}$ & \\
\hline 26. I feel rested &.$\underline{48}$ & .15 &.$\underline{.36}$ & .32 &.$\underline{56}$ & \\
\hline 24. I wish I could be as happy as others seem to be &.$\underline{35}$ & .33 &.$\underline{56}$ & .36 & .28 & \\
\hline 34. I make decisions easily &.$\underline{.34}$ & .19 &.$\underline{55}$ & .16 & .45 & \\
\hline
\end{tabular}




\begin{tabular}{|c|c|c|c|c|c|c|}
\hline \multirow[t]{2}{*}{ Item } & \multicolumn{2}{|c|}{ Current study } & \multicolumn{2}{|c|}{ Bieling et al. (1998) } & \multicolumn{2}{|c|}{$\begin{array}{c}\text { Spielberger et al. } \\
\text { (1983) } \\
\end{array}$} \\
\hline & Factor 1 & Factor 2 & Factor 1 & Factor 2 & Factor 1 & Factor 2 \\
\hline $\begin{array}{l}\text { 29. I worry too much over something that really doesn't } \\
\text { matter }\end{array}$ & -.11 &.$\underline{81}$ & .05 &.$\underline{74}$ & &.$\underline{51}$ \\
\hline $\begin{array}{l}\text { 38. I take disappointments so keenly that I can't put } \\
\text { them out of my mind }\end{array}$ & -.05 &.$\underline{79}$ & .29 & $\underline{.56}$ & &.$\underline{43}$ \\
\hline $\begin{array}{l}\text { 37. Some unimportant thought runs through my mind } \\
\text { and bothers me }\end{array}$ & -.10 &.$\underline{75}$ & .01 &.$\underline{79}$ & &.$\underline{59}$ \\
\hline $\begin{array}{l}\text { 40. I get in a state of tension or turmoil as I think over } \\
\text { my recent concerns and interests }\end{array}$ & .11 &.$\underline{64}$ & .16 &.$\underline{65}$ & &.$\underline{47}$ \\
\hline $\begin{array}{l}\text { 28. I feel that difficulties are piling up so that I cannot } \\
\text { overcome them }\end{array}$ & .18 &.$\underline{51}$ & .32 &.$\underline{50}$ & &.$\underline{49}$ \\
\hline 22. I feel nervous and restless & .23 &.$\underline{48}$ & .34 &.$\underline{\mathbf{5 0}}$ & .45 & \\
\hline 31. I have disturbing thoughts & .13 &.$\underline{45}$ & .11 &.$\underline{59}$ & &.$\underline{62}$ \\
\hline 32. I lack self-confidence & .31 &.$\underline{40}$ & .69 & .16 & &.$\underline{38}$ \\
\hline 25. I feel like a failure &.$\underline{32}$ & .39 &.$\underline{66}$ & .24 &.$\underline{36}$ & .41 \\
\hline 35. I feel inadequate & .31 & $\underline{.37}$ & .69 & .19 & &.$\underline{43}$ \\
\hline
\end{tabular}

Note. Loadings over .32 for each item are indicated in bold. Item numbers range from 21 to 40 . Items 1 to 20 are from the State Trait Anxiety Inventory state anxiety scale and are not shown in this table. Items loading over.32 in the current study as well as in the studies by Bieling et al. (1998) and/or Spielberger et al. (1983) are underlined.

As can be seen from the data in Table 8 , there is substantial overlap between the solution derived from the current data and those from the studies by Spielberger et al. (1983) and Bieling et al. (1998). Inspection of the content of the items loading on each factor in our study suggested that the two factor solution was influenced by methodology. The first factor was comprised of items that contained positive characteristics such as I am happy and Ifeel secure with the exception of item 25 (I feel like a failure) and item 24 (I wish I could be as happy as others seem to be), both of which cross loaded on the second factor. The second factor was comprised entirely of items that contained negative characteristics such as I worry too much over something that really doesn't matter and I feel nervous and restless. The first and second factors therefore appeared to correspond to what Spielberger et al. and Bieling et al. called 'trait anxiety absent' and 'trait anxiety present' factors, respectively. Given that the 
two factors were highly correlated and the two factor solution appeared to simply reflect forward versus reversed item keying (thereby suggesting a method factor), we decided to explore a one factor solution. This single factor explained $44.27 \%$ of the variance and all items loaded well above .32 on this factor. The factor matrix is presented in Table 9.

Table 9

Factor Matrix for Principal Axis Factoring of One Factor Solution for State Trait Anxiety Inventory T-Anxiety Scale Items

\begin{tabular}{lcc}
\hline & Item & Factor \\
\hline 23. I feel satisfied with myself & & $\mathbf{. 7 2}$ \\
39. I am a steady person & & $\mathbf{. 7 2}$ \\
21. I feel pleasant & & $\mathbf{. 7 1}$
\end{tabular}

33. I feel secure $\quad \mathbf{. 7 1}$

36. I am content $\quad \mathbf{. 7 1}$

30. I am happy $\quad \mathbf{. 7 0}$

40. I get in a state of tension or turmoil as I think over my recent concerns $\quad \mathbf{. 6 7}$ and interests

25. I feel like a failure $\quad \mathbf{. 6 5}$

38. I take disappointments so keenly that I can't put them out of my mind $\mathbf{. 6 5}$

22. I feel nervous and restless $\quad \mathbf{. 6 4}$

32. I lack self-confidence $\quad \mathbf{6 4}$

24. I wish I could be as happy as others seem to be $\mathbf{. 6 3}$

28. I feel that difficulties are piling up so that I cannot overcome them $\mathbf{. 6 2}$

35. I feel inadequate $\quad \mathbf{. 6 2}$

29. I worry too much over something that really doesn't matter $\quad \mathbf{. 6 1}$

26. I feel rested $\quad \mathbf{. 5 8}$

37. Some unimportant thought runs through my mind and bothers me $\mathbf{. 5 6}$

31. I have disturbing thoughts $\quad \mathbf{. 5 3}$

34. I make decisions easily

Note. Loadings over .32 for each item are indicated in bold. Item numbers range from 21 to 40 . Items 1 to 20 are from the State Trait Anxiety Inventory state anxiety scale and are not shown in this table. 
Due to the relatively high loadings of all items on one factor, this solution suggested that the STAI T-Anxiety scale can be represented as a single dimension. To aid interpretation of this structure we investigated the convergent and discriminant validity of the STAI TAnxiety scale.

\section{Convergent and Discriminant Validity}

The convergent and discriminant validity of the STAI T-Anxiety scale was investigated using the Pearson product-moment correlation coefficient. Table 10 presents correlations between the STAI T-Anxiety scale and the convergent and discriminant measures used in this study.

Table 10

Correlations of the State Trait Anxiety Inventory T-Anxiety Scale with Other Measures of Anxiety, Mood and Personality

\begin{tabular}{lcc}
\hline \multicolumn{1}{c}{ Scale } & $N$ & $\begin{array}{c}\text { Pearson's correlation } \\
\text { coefficient }\end{array}$ \\
\hline BAI & 677 & $\mathbf{5 5}^{* *}$ \\
GAD-7 & 677 & $\mathbf{. 9}^{* *}$ \\
PSWQ & 677 & $\mathbf{. 7 1}^{* *}$ \\
BDI-II & 677 & $\mathbf{. 7 5}^{* *}$ \\
IDS-SR & 566 & $\mathbf{. 7}^{* *}$ \\
MASQ-GM & 200 & $\mathbf{. 1 1}^{* *}$ \\
MASQ-GA & 200 & $\mathbf{. 6 1}^{* *}$ \\
MASQ-AA & 200 & $\mathbf{. 5 3}^{* *}$ \\
MASQ-GD & 200 & $\mathbf{. 1 1}^{* *}$ \\
MASQ-AD & 200 & $\mathbf{. 7 4}^{* *}$ \\
BFAS Withdrawal & 677 & $\mathbf{. 8 1}^{* *}$ \\
BFAS Volatility & 677 & $\mathbf{5 6}^{* *}$ \\
BFAS Compassion & 677 & $-.15^{* *}$ \\
BFAS Politeness & 677 & $-.09^{* *}$ \\
BFAS Industriousness & 677 &.$- .59^{* *}$ \\
\hline
\end{tabular}




\begin{tabular}{|c|c|c|}
\hline BFAS Orderliness & 677 & -.02 \\
\hline BFAS Enthusiasm & 677 & $-.49^{* *}$ \\
\hline BFAS Assertiveness & 677 & $-.39^{* *}$ \\
\hline BFAS Intellect & 677 & $-.39^{* *}$ \\
\hline BFAS Openness & 677 & .04 \\
\hline BFAS Neuroticism & 677 & $.76^{* *}$ \\
\hline BFAS Agreeableness & 677 & $-.14^{* *}$ \\
\hline BFAS Conscientiousness & 677 & $-.39^{* *}$ \\
\hline BFAS Extraversion & 677 & $-.52^{* *}$ \\
\hline BFAS Openness/Intellect & 677 & $-.23^{* *}$ \\
\hline BIS/BAS Scales - BAS Drive & 947 & $-.19^{* *}$ \\
\hline BIS/BAS Scales - BAS Fun Seeking & 947 & $-.17^{* *}$ \\
\hline $\begin{array}{l}\text { BIS/BAS Scales - BAS Reward } \\
\text { Responsiveness }\end{array}$ & 947 & $-.17^{* *}$ \\
\hline BIS BAS Scales - BIS Scale & 947 & $.58^{* *}$ \\
\hline SPSRQ - SP & 111 & $.62^{* *}$ \\
\hline SPSRQ - SR & 111 & .12 \\
\hline PID-5 Overall & 200 & $.66^{* *}$ \\
\hline PID-5-BF Negative affect & 200 & $.73^{* *}$ \\
\hline PID-5-BF Detachment & 200 & $.58^{* *}$ \\
\hline PID-5-BF Antagonism & 200 & $.26^{* *}$ \\
\hline PID-5-BF Disinhibition & 200 & $.28^{* *}$ \\
\hline PID-5-BF Psychoticism & 200 & $.57^{* *}$ \\
\hline
\end{tabular}

Note. BAI = Beck Anxiety Inventory; GAD-7 = Generalised Anxiety Disorder 7-Item Scale; PSWQ = Penn State Worry Questionnaire; BDI-II = Beck Depression Inventory-II; IDS-SR = Inventory of Depressive Symptomatology - Self Report; MASQ = Mood and Anxiety Symptom Questionnaire; GM = general distress - mixed scale; GA = general distress - anxiety scale; $\mathrm{AA}=$ anxious arousal scale; $\mathrm{GD}=$ general distress - depression scale; $\mathrm{AD}=$ anhedonic depression scale; $\mathrm{BFAS}=\mathrm{Big}$ Five Aspects Scales; BIS/BAS Scales = Behavioral Inhibition System/Behavioral Activation System Scales; BAS = behavioral activation subscales; BIS Scale = behavioral inhibition subscale; SPSRQ = Sensitivity to Punishment and Sensitivity to Reward Questionnaire; $\mathrm{SP}=$ sensitivity to punishment scale; $\mathrm{SR}$ = sensitivity to reward scale; PID-5-BF = Personality Inventory for DSM-5 Brief Form - Adult.

Correlations larger than .30 are in bold.

${ }^{*} p<.05$, two tailed. $* * p<.01$, two tailed.

As can be seen in Table 10, all correlations between the STAI T-Anxiety scale and other measures were significant, with the exception of the orderliness factor and the openness factor of the BFAS, and the sensitivity to reward scale of the SRSPQ. Some effect sizes were small (J. Cohen, 1992) but even small correlations will reach threshold for significance at 
conventional levels (e.g., $p<.05)$ if the sample size is large enough. Given the large sample in this study, in order to meaningfully interpret the pattern of correlations, we focused on effect size rather than significance level. We interpreted correlations higher than .30 as constituting a medium to large effect (J. Cohen, 1992). These correlations are presented in bold font in Table 10. As can be seen from the correlations in Table 10, effect sizes were generally medium to large. Table 11 presents Fisher's $z$-scores for each pair of correlations.

\section{Table 11}

Fisher's z-Scores for Correlations between the STAI T-Anxiety Scale and Other Measures of Anxiety, Mood and Personality

\begin{tabular}{|c|c|c|c|c|c|c|c|c|c|c|c|}
\hline & BAI & GAD-7 & PSWQ & BDI-II & $\begin{array}{l}\text { IDS- } \\
\text { SR }\end{array}$ & $\begin{array}{l}\text { MASQ- } \\
\text { GM }\end{array}$ & $\begin{array}{l}\text { MASQ- } \\
\text { GA }\end{array}$ & $\begin{array}{l}\text { MASQ- } \\
\text { AA }\end{array}$ & $\begin{array}{l}\text { MASQ- } \\
\text { GD }\end{array}$ & $\begin{array}{l}\text { MASQ- } \\
\text { AD }\end{array}$ & $\begin{array}{l}\text { BFAS } \\
\text { Neuro }\end{array}$ \\
\hline \multicolumn{12}{|l|}{ BAI } \\
\hline GAD-7 & $6.29 * *$ & & & & & & & & & & \\
\hline PSWQ & $5.93^{* *}$ & 0.93 & & & & & & & & & \\
\hline BDI-II & $8.91^{* *}$ & $3.27 * *$ & 1.85 & & & & & & & & \\
\hline IDS-SR & $5.07 * *$ & 1.05 & 0.36 & 1.14 & & & & & & & \\
\hline $\begin{array}{l}\text { MASQ- } \\
\text { GM }\end{array}$ & $3.32 * *$ & 0.48 & 0.00 & 1.06 & 0.25 & & & & & & \\
\hline $\begin{array}{l}\text { MASQ- } \\
\text { GA }\end{array}$ & 1.12 & 1.72 & $2.20 *$ & $3.26^{* * *}$ & $2.40 *$ & $3.14 * *$ & & & & & \\
\hline $\begin{array}{l}\text { MASQ- } \\
\text { AA }\end{array}$ & 0.35 & $3.18 * *$ & $3.67 * *$ & $4.73 * *$ & $3.84 * *$ & $4.71 * *$ & $2.21 *$ & & & & \\
\hline $\begin{array}{l}\text { MASQ- } \\
\text { GD }\end{array}$ & $3.32 * *$ & 0.48 & 0.00 & 1.06 & 0.25 & 0.00 & $2.76^{* *}$ & $4.21 * *$ & & & \\
\hline $\begin{array}{l}\text { MASQ- } \\
\mathrm{AD}\end{array}$ & $4.10^{* *}$ & 1.27 & 0.78 & 0.28 & 0.74 & 0.85 & $3.01 * *$ & $4.37 * *$ & 0.91 & & \\
\hline $\begin{array}{l}\text { BFAS } \\
\text { Neuro }\end{array}$ & $8.50 * *$ & $3.39 * *$ & $2.81 * *$ & 0.54 & 1.55 & 1.35 & $3.55 * *$ & $5.01 * *$ & 1.35 & 0.56 & \\
\hline $\begin{array}{l}\text { PID-5- } \\
\text { BF Neg } \\
\text { Affect }\end{array}$ & $3.83^{* *}$ & 1.00 & 0.51 & 0.75 & 1.07 & 0.51 & $2.56^{*}$ & $3.88 * *$ & 0.50 & 0.26 & 0.83 \\
\hline
\end{tabular}

Note. Numbers indicate Fisher's $z$-scores. These scores indicate the size of the difference in correlation between the variables in the table when each was correlated with the STAI T-Anxiety scale score. BAI = Beck Anxiety Inventory; GAD-7 = Generalised Anxiety Disorder 7-Item Scale; PSWQ = Penn State Worry Questionnaire; BDI-II = Beck Depression Inventory-II; IDS-SR = Inventory of Depressive Symptomatology - Self Report; MASQ = Mood and Anxiety Symptom Questionnaire; GM = general distress - mixed scale; GA = general distress - anxiety scale; AA = anxious arousal scale; GD = general distress - depression scale; $\mathrm{AD}=$ anhedonic depression scale; BFAS Neuro = Big Five Aspects Scales Neuroticism Scale; PID-5-BF Neg Affect = Personality Inventory for DSM-5 Brief Form - Adult Negative Affect Scale.

$* p<.05$, two tailed. $* * p<.01$, two tailed. 
With the exception of the correlation between the STAI T-Anxiety scale and the BDIII, and the STAI T-Anxiety scale and the GAD-7, the correlations between the STAI TAnxiety scale and measures of depression (BDI-II, IDS-SR, MASQ general distress depression scale and MASQ anhedonic depression scale) were not significantly different to the correlations between the STAI T-Anxiety scale and measures of anxiety that contain items related to worry and anxious apprehension (PSWQ and GAD-7). However, the correlations of the STAI T-Anxiety scale with these measures of depression were significantly higher than the correlations between the STAI T-Anxiety scale and other measures of anxiety (MASQ general distress - anxiety scale), including those that contain items related mostly to somatic symptoms and anxious arousal (BAI and MASQ anxious arousal scale). In addition, correlations between the STAI T-Anxiety scale and measures of general distress (MASQ general distress - mixed scale, BFAS neuroticism scale, and PID-5BF - Adult negative affect scale) were high with large effect sizes (J. Cohen, 1992). The STAI T-Anxiety Scale also correlated significantly higher with measures of worry and anxious apprehension (PSWQ and GAD-7) than with other measures of anxiety that contain a greater number of items related to somatic symptoms and anxious arousal (BAI and MASQ anxious arousal scale).

In order to further characterise the structure of the STAI T-Anxiety scale, we used the circumplex structure of core affect proposed by Yik, Russel, and Steiger (2011) as a conceptual guide to separating the convergent and discriminant measures into those assessing deactivated negative affect (i.e., BDI-II, IDS-SR, MASQ general distress - depression scale, MASQ anhedonic depression scale), those that measure activated negative affect (BAI, GAD-7, PSWQ, MASQ general distress - anxiety scale, MASQ anxious arousal scale, SPSRQ sensitivity to punishment scale), and those that measure general negative affectivity (MASQ general distress - mixed scale, BFAS neuroticism scale, BIS/BAS Scales behavioral 
inhibition subscale, PID-5-BF - Adult negative affect scale). Correlations between the STAI T-Anxiety scale and these measures are displayed in Table 12, along with average correlations for each of the groups of measures.

Table 12

Correlations Between Measures of Deactivated Negative Affect, Activated Negative Affect, and General Negative Affectivity

Scale

Correlation with STAI Trait

Anxiety Scale

Deactivated negative affect

BDI-II

IDS-SR $\quad .72$

MASQ General Distress - Depression $\quad .71$

MASQ Anhedonic Depression $\quad .74$

$\begin{array}{ll}\text { Mean } & .73\end{array}$

Activated negative affect

BAI

GAD-7

$\begin{array}{ll}\text { PSWQ } & .71\end{array}$

MASQ General Distress - Anxiety

MASQ Anxious Arousal $\quad .53$

SPSRQ Sensitivity to Punishment $\quad .62$

Mean $\quad .61$

General negative affectivity

MASQ General Distress - Mixed $\quad .72$

BFAS Neuroticism $\quad .76$

BIS/BAS Scales - BIS Scale $\quad .58$

PID-5-BF Negative Affect $\quad .73$

Meancohe $\quad .70$

Note. STAI = State Trait Anxiety Inventory; BDI-II $=$ Beck Depression Inventory-II; IDS-SR $=$ Inventory of Depressive Symptomatology - Self Report; MASQ = Mood and Anxiety Symptom Questionnaire; BAI = Beck Anxiety Inventory; GAD-7 $=$ Generalised Anxiety Disorder 7-Item Scale; PSWQ = Penn State Worry Questionnaire; SPSRQ = Sensitivity to Punishment and Sensitivity to Reward Questionnaire; BFAS = Big Five Aspects Scales; BIS/BAS Scales = Behavioral Inhibition 
System/Behavioral Activation System Scales; BIS Scale = behavioral inhibition subscale; PID-5-BF = Personality Inventory for DSM-5 Brief Form - Adult.

All the mean correlations in Table 12 show large effect sizes (J. Cohen, 1992). Both the mean correlation between the STAI T-Anxiety scale and instruments that measure deactivated negative affect and the mean correlation between the STAI-T Anxiety Scale and instruments that measure general negative affectivity were higher than the mean correlation between the STAI T-Anxiety scale and instruments that measure activated negative affect but, because of variation in number of participants that completed each of the questionnaires, it was not possible to test these differences for significance.

\section{Study 2}

The STAI T-Anxiety scale is a continuous measure but researchers often define cutoffs for low and high anxiety and, on the basis of those cut-offs, separate participants into two groups. Although this method is highly contentious for conceptual and statistical reasons (Mar, Spreng, \& Deyoung, 2013), it is often essential when financial or logistic constraints preclude the collection of large samples. A common example of relevance to the present thesis is research involving a neuroimaging component. In such studies, participants in a low and high anxiety group undergo electroencephalogram or functional magnetic resonance imaging (passively, or while performing a behavioural task), and key neural dependent measures are compared between groups. Given that this research design is widely used, we were interested in exploring how groups formed on the basis of high and low scores on the STAI T-Anxiety scale differ with respect to related constructs such as anxiety, mood and personality traits. In order to investigate whether individuals who score low and high on the STAI T-Anxiety scale would also score low and high on other instruments, we used the data set from the factor analysis to select participants with low and high scores on the STAI TAnxiety scale, and compared each group's STAI T-Anxiety scale score to the group's score on other instruments. 


\section{Method}

From the data set of 947 participants, data from 24 participants who achieved the lowest STAI T-Anxiety scale total score and 24 participants who achieved the highest STAI T-Anxiety scale total score (17 males, 31 females; mean age $=22.30$ years, $\mathrm{SD}=4.61$, age range 18.15 to 35.39 years) was subjected to further analysis. Participant exclusion criteria included a history of neurological or psychiatric disorders, current use of psychoactive medication, and history of substance abuse. Alcohol use was measured using the Alcohol Use Disorders Identification Test (AUDIT; Babor, Higgins-Biddle, Saunders, \& Monteiro, 2001). Nicotine dependence was measured using the Fagerstrom Test for Nicotine Dependence (Fagerstrom, Heatherton, \& Kozlowski, 1990), and substance use was measured using the Orygen Substance Use Index and a structured face-to-face interview. These measures are reproduced in Appendix A and Appendix B. Approximately two weeks after completing the initial set of measures, the 48 participants were scanned using MRI for another study. On the day of scanning and before they entered the scanner, they completed additional questionnaires in paper form; the data from these questionnaires was used in the current study. The additional questionnaires are reproduced in Appendix A and were:

Barratt Impulsiveness Scale (Patton, Stanford, \& Barratt, 1995). The Barratt Impulsiveness Scale is comprised of 30 items and measures the construct of impulsivity. Respondents are asked to rate each item on a scale ranging from rarely/never to almost always/always. Items are scored on a 4-point scale from 1 to 4, with 11 items reverse scored. Total scores range from 30 to 120 with higher scores indicating greater impulsivity. Three second order factors (attentional, motor, and nonplanning), each comprised of two of six first order factors (attention, cognitive instability, motor, perseverance, self-control and cognitive complexity) can be calculated, with higher scores indicating greater levels of impulsive characteristics. 
Attentional Control Scale (ACS: Derryberry \& Reed, 2002). The ACS is a 20 item questionnaire that measures the ability to control attention, also called attentional control. Respondents rate each item on a scale from 1 (almost never) to 4 (always). Eleven items are reverse scored and the total score ranges from 20 to 80, with higher scores indicating greater attentional control. The instrument also enables measurement of attentional focusing and attentional shifting, with higher scores indicating greater levels of each construct. The internal reliability for the each of the questionnaires is displayed in Table 13.

Table 13

Internal Reliabilities of Each of the Questionnaires in the Smaller Sample $(N=48)$

\begin{tabular}{lc}
\hline \multicolumn{1}{c}{ Scale } & Cronbach's alpha $(\alpha)$ \\
\hline STAI T-Anxiety & .98 \\
BAI & .93 \\
GAD-7 & .93 \\
PSWQ & .97 \\
MASQ-AA & .87 \\
BDI-II & .96 \\
IDS-SR & .94 \\
BFAS Withdrawal & .93 \\
BFAS Volatility & .93 \\
BFAS Compassion & .90 \\
BFAS Politeness & .73 \\
BFAS Industriousness & .89 \\
BFAS Orderliness & .76 \\
BFAS Enthusiasm & .86 \\
BFAS Assertiveness & .91 \\
BFAS Intellect & .93 \\
BFAS Openness & .74 \\
BFAS Neuroticism & .95 \\
BFAS Agreeableness & .88
\end{tabular}




\begin{tabular}{|c|c|}
\hline Scale & Cronbach's alpha $(\alpha)$ \\
\hline BFAS Conscientiousness & .81 \\
\hline BFAS Extraversion & .92 \\
\hline BFAS Openness/Intellect & .87 \\
\hline BAS Drive & .77 \\
\hline BAS Fun Seeking & .70 \\
\hline BAS Reward Responsiveness & .70 \\
\hline BIS Scale & .89 \\
\hline ACS Total & .90 \\
\hline ACS Focused Attention & .83 \\
\hline ACS Attention Shifting & .85 \\
\hline BIS Attention & .82 \\
\hline BIS Cognitive Instability & .57 \\
\hline BIS Motor & .67 \\
\hline BIS Perseverance & .07 \\
\hline BIS Self control & .80 \\
\hline BIS Cognitive Complexity & .52 \\
\hline BIS Attentional ( $2^{\text {nd }}$ order $)$ & .81 \\
\hline BIS Motor $\left(2^{\text {nd }}\right.$ order $)$ & .60 \\
\hline BIS Nonplanning ( $2^{\text {nd }}$ order $)$ & .77 \\
\hline
\end{tabular}

Note. STAI T-Anxiety = State Trait Anxiety Inventory, trait anxiety scale; BAI = Beck Anxiety Inventory; GAD-7 = Generalised Anxiety Disorder 7-Item Scale; PSWQ = Penn State Worry Questionnaire; MASQ = Mood and Anxiety Symptom Questionnaire; AA = anxious arousal scale; BDI-II = Beck Depression Inventory-II; IDS-SR = Inventory of Depressive Symptomatology - Self Report; BFAS = Big Five Aspects Scales; BAS = Behavioral Inhibition System/Behavioral Activation System Scales behavioral activation subscale; BIS Scale = Behavioral Inhibition System/Behavioral Activation System Scales behavioral inhibition subscale; ACS = Attentional Control Scale; BIS = Barratt Impulsiveness Scale.

\section{Results}

Descriptive statistics for participants in the low and high trait anxiety groups are displayed in Table 14. 
Table 14

Mean Demographic and Questionnaire Data for Low Trait Anxiety and High Trait Anxiety Groups

\begin{tabular}{lcc}
\hline \multicolumn{1}{c}{ Variables } & Low trait anxiety $(n=24)$ & High trait anxiety $(n=24)$ \\
\hline Age (years) & $23.29(5.19)$ & $21.30(3.79)$ \\
Age range (years) & 18.17 to 35.39 & 18.15 to 30.80 \\
Gender (male/female) & $12 / 12$ & $5 / 19$ \\
STAI-T score & $25.54(2.47)$ & $57.88(6.38)$ \\
STAI-T score (range) & 20 to 29 & 49 to 75
\end{tabular}

Note. Except for gender which is a count, numbers represent means with standard deviation in parentheses.

Preliminary analysis of skewness, kurtosis, linearity and homoscedasticity showed that the assumption of normality was violated for some variables but linearity and homoscedasticity appeared to be adequate for all variables. When analysing variables that were not normally distributed, the Mann-Whitney $U$ test rather than an independent samples $t$-test was used and is reported in Table 15.

An independent samples $t$-test or Mann-Whitney $U$ test was conducted to compare scores for the low and high trait anxiety groups on each of the measures. For some scales the assumption of homogeneity of variance was violated; in these instances we used the $t$-scores and significance values following adjustment for unequal variance. Table 15 shows the means, standard deviations, mean difference, $t$-values or Mann-Whitney $U$ values, significance levels, $95 \%$ confidence intervals and effect sizes for the STAI T-Anxiety scale and for each of the other measures completed by participants low and high in trait anxiety. 
Table 15

Group Statistics, T-values, Significance Tests, 95\% Confidence Intervals and Effect Sizes for Measures of Anxiety, Mood and Personality in Participants Low and High in Trait Anxiety

\begin{tabular}{|c|c|c|c|c|c|c|c|c|}
\hline Scale & Anxiety level & $M$ & $S D$ & $M$ diff & $t / M W-U$ & $D f$ & $95 \% \mathrm{CI}$ & $E S$ \\
\hline \multirow{2}{*}{ STAI } & Low & 25.54 & 2.47 & \multirow{2}{*}{$-32.33 * *$} & \multirow{2}{*}{-23.17} & \multirow{2}{*}{29.74} & \multirow{2}{*}[-35.18,-29.48]{} & \multirow{2}{*}{.92} \\
\hline & High & 57.88 & 6.38 & & & & & \\
\hline \multirow[t]{2}{*}{ BAI } & Low & 3.33 & 3.44 & \multirow{2}{*}{$-14.08 * *$} & \multirow{2}{*}{-6.93} & \multirow{2}{*}{29.11} & \multirow{2}{*}[-18.24,-9.93]{} & \multirow{2}{*}{.51} \\
\hline & High & 17.42 & 9.34 & & & & & \\
\hline \multirow[t]{2}{*}{ GAD-7 } & Low & 1.17 & 1.49 & \multirow{2}{*}{$-9.58 * *$} & \multirow{2}{*}{4.5} & \multirow{2}{*}{--} & \multirow{2}{*}{--} & \multirow{2}{*}{.85} \\
\hline & High & 10.75 & 4.37 & & & & & \\
\hline \multirow{2}{*}{ PSWQ } & Low & 31.58 & 10.76 & \multirow{2}{*}{$-32.38 * *$} & \multirow{2}{*}{-11.43} & \multirow{2}{*}{46} & \multirow{2}{*}[-38.08,-26.68]{} & \multirow{2}{*}{.74} \\
\hline & High & 63.96 & 8.76 & & & & & \\
\hline \multirow{2}{*}{ MASQ-AA } & Low & 12.04 & 10.88 & \multirow{2}{*}{-3.33} & \multirow{2}{*}{253.00} & & & $11+2+2$ \\
\hline & High & 15.38 & 11.05 & & & -- & -- & .11 \\
\hline BDI-II & Low & 2.88 & 2.33 & $2050 * *$ & $8>0$ & 2476 & $50550 \quad 1541]$ & 60 \\
\hline & High & 23.38 & 11.89 & $-20.50^{n+n}$ & -8.29 & 24.10 & {$[-25.29,-15.41]$} & .00 \\
\hline IDS-SR & Low & 6.21 & 4.08 & $1042 * *$ & 3650 & & & $75,2>0$ \\
\hline & High & 25.63 & 13.90 & $-19.4 L^{\prime \prime}$ & 50.50 & -- & -- & .73 \\
\hline BFAS Withdrawal & Low & 1.94 & .58 & $170 * *$ & 1107 & 16 & [ 200 & $76+2>12$ \\
\hline & High & 3.72 & .44 & $-1 . / 8{ }^{n}$ & -11.91 & 40 & {$[-2.08,-1.48]$} & .10 \\
\hline BFAS Volatility & Low & 1.95 & .64 & $125 \% *$ & 772 & 16 & $5170-001$ & 57 \\
\hline & High & 3.30 & .57 & -1.35 w & -1.13 & 46 & {$[-1 . / 0,-.99]$} & .51 \\
\hline BFAS Compassion & Low & 4.02 & .75 & & 30 & 16 & $5 \quad 45 \quad 221$ & 00 \\
\hline & High & 4.08 & .57 & -.06 & -.30 & 46 & {$[-.45, .33]$} & .00 \\
\hline BFAS Politeness & Low & 3.95 & .54 & 10 & 70 & 16 & ז 10 & 01 \\
\hline & High & 3.83 & .54 & .12 & .10 & 40 & {$[-.19, . .4 J]$} & .01 \\
\hline BFAS & Low & 3.71 & .54 & & & & & \\
\hline Industriousness & High & 2.60 & .47 & $1.11 * *$ & 1.60 & 46 & {$[.82,1.41]$} & .56 \\
\hline BFAS Orderliness & Low & 3.32 & .57 & 16 & 22050 & & & $17+2,+2$ \\
\hline & High & 3.48 & .57 & .10 & 2.00 .00 & & & \\
\hline BFAS Enthusiasm & Low & 4.08 & .61 & $02 * *$ & 100 & 16 & {$\left[\begin{array}{lll}5 & 1 & 177\end{array}\right]$} & 35 \\
\hline & High & 3.25 & .54 & . 05 & 4.90 & 70 & {$[.00,1.11]$} & \\
\hline BFAS & Low & 3.71 & .53 & Ont" & 400 & 16 & & 00 \\
\hline Assertiveness & High & 2.84 & .69 & $.88^{* *}$ & 4.93 & 46 & {$[.52,1.23]$} & .35 \\
\hline BFAS Intellect & Low & 4.11 & .64 & $115 * *$ & 570 & 16 & {$\left[\begin{array}{lll}74 & 551\end{array}\right.$} & 41 \\
\hline & High & 2.96 & .75 & 1.15 & 3.10 & & & \\
\hline BFAS Openness & Low & 3.66 & .57 & 11 & 63 & 46 & {$\left[\begin{array}{cc}-23 & 45\end{array}\right]$} & 01 \\
\hline & High & 3.56 & .59 & & & & & \\
\hline BFAS Neuroticism & Low & 1.95 & .55 & $-1.57 * *$ & -11.98 & 37.95 & {$[-1.83,-1.30]$} & .76 \\
\hline
\end{tabular}




\begin{tabular}{|c|c|c|c|c|c|c|c|c|}
\hline Scale & Anxiety level & $M$ & $S D$ & $M$ diff & $t / M W-U$ & $D f$ & $95 \% \mathrm{CI}$ & $E S$ \\
\hline \multirow{3}{*}{$\begin{array}{l}\text { BFAS } \\
\text { Agreeableness }\end{array}$} & High & 3.51 & .33 & \multirow[b]{3}{*}{.03} & \multirow[b]{3}{*}{.20} & & & \\
\hline & Low & 3.98 & .55 & & & \multirow[b]{2}{*}{46} & \multirow{2}{*}[-.28,.34]{} & \multirow{2}{*}{.00} \\
\hline & High & 3.95 & .51 & & & & & \\
\hline \multirow{2}{*}{$\begin{array}{l}\text { BFAS } \\
\text { Conscientiousness }\end{array}$} & Low & 3.52 & .45 & \multirow[b]{2}{*}{$.47 * *$} & \multirow[b]{2}{*}{3.81} & \multirow[b]{2}{*}{46} & \multirow[b]{2}{*}[.22,.72]{} & \multirow[b]{2}{*}{.24} \\
\hline & High & 3.04 & .41 & & & & & \\
\hline \multirow{2}{*}{$\begin{array}{l}\text { BFAS } \\
\text { Extraversion }\end{array}$} & Low & 3.90 & .46 & \multirow{2}{*}{$.85^{* *}$} & \multirow{2}{*}{6.17} & \multirow[b]{2}{*}{46} & \multirow[b]{2}{*}[.58,1.13]{} & \multirow[b]{2}{*}{.45} \\
\hline & High & 3.04 & .50 & & & & & \\
\hline \multirow{2}{*}{$\begin{array}{l}\text { BFAS } \\
\text { Openness/Intellect }\end{array}$} & Low & 3.89 & .50 & \multirow{2}{*}{$.63^{* *}$} & \multirow{2}{*}{4.36} & \multirow{2}{*}{46} & \multirow{2}{*}[.34,.91]{} & \multirow{2}{*}{.29} \\
\hline & High & 3.26 & .50 & & & & & \\
\hline \multirow{2}{*}{ BAS Drive } & Low & 11.96 & 2.27 & 125 & 194 & 46 & {$\left[\begin{array}{llll}-0 & 2 & 5 & 5\end{array}\right]$} & 08 \\
\hline & High & 10.71 & 2.20 & 1.25 & 1.94 & 40 & {$[-.05,2.5 J]$} & \\
\hline BAS Fun Seeking & Low & 13.04 & 2.12 & 88 & 138 & 46 & {$\left[\begin{array}{llll}-40 & 2 & 15\end{array}\right]$} & 04 \\
\hline & High & 12.17 & 2.28 & . 88 & 1.38 & 40 & {$[-.40,2.15]$} & .04 \\
\hline BAS RR & Low & 17.88 & 1.62 & 50 & 88 & 46 & {$\left[\begin{array}{lll}-64 & 1 & 64]\end{array}\right]$} & 02 \\
\hline & High & 17.38 & 2.26 & . 50 & .88 & 40 & {$[-.04,1.04]$} & .02 \\
\hline BIS Scale & Low & 17.13 & 4.54 & $-704 * *$ & 5100 & -. & $\ldots$ & 71 \\
\hline & High & 24.17 & 2.14 & $-1.04 \cdot$ & 51.00 & -- & -- & .11 \\
\hline ACS & Low & 62.21 & 7.97 & $1417 * *$ & 640 & 46 & {$\left[\begin{array}{llll}0 & 77 & 18 & 56\end{array}\right.$} & 48 \\
\hline & High & 48.04 & 7.14 & $14.1 / 7.45$ & 0.49 & 40 & {$[9.11,10.50]$} & .48 \\
\hline ACS - Focused & Low & 27.25 & 4.33 & $570 * *$ & 461 & 46 & 13268321 & $32+>3$ \\
\hline attention & High & 21.46 & 4.38 & $5.19 \cdots$ & 4.61 & 46 & {$[3.26,8.32]$} & .32 \\
\hline ACS - Attention & Low & 34.96 & 4.50 & $838 * *$ & 601 & 46 & {$\left[\begin{array}{llll}5 & 04 & 10 & 821\end{array}\right.$} & $51>>>$ \\
\hline shifting & High & 26.58 & 3.88 & $8.38 \cdots$ & 6.91 & 46 & {$[5.94,10.82]$} & .31 \\
\hline BIS Total & Low & 57.54 & 10.73 & $013 * *$ & 305 & 16 & {$\left[\begin{array}{lllll}15 & 14 & 3 & 111\end{array}\right.$} & 17 \\
\hline & High & 66.67 & 9.96 & $-9.13^{m}$ & -3.05 & 40 & {$[-15.14,-3.11]$} & .11 \\
\hline BIS Attention $\left(1^{\mathrm{st}}\right.$ & Low & 8.08 & 2.64 & 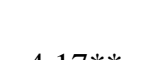 & 70009 & & & \\
\hline order) & High & 12.25 & 2.58 & $-4.17 * *$ & 78.00 & -- & -- & .63 \\
\hline BIS Cognitive & Low & 5.08 & 1.38 & & & & & \\
\hline $\begin{array}{l}\text { instability }\left(1^{\text {si }}\right. \\
\text { order })\end{array}$ & High & 7.00 & 1.53 & $-1.92 * *$ & 101.00 & -- & -- & .57 \\
\hline BIS Motor $\left(1^{\text {st }}\right.$ & Low & 15.67 & 3.17 & & & & & \\
\hline order) & High & 14.17 & 3.50 & 1.50 & 231.00 & -- & -- & .17 \\
\hline BIS Perseverance & Low & 7.13 & 1.48 & 08 & & & $\ldots$ & \\
\hline$\left(1^{\text {st }}\right.$ order $)$ & High & 7.21 & 1.79 & -.08 & 283.50 & -- & -- & .01 \\
\hline BIS Self-control & Low & 11.54 & 4.15 & $3 \Omega 4 * *$ & 15800 & & & 30 \\
\hline ( $1^{\text {st }}$ order $)$ & High & 14.58 & 3.39 & $-3.04 * *$ & 158.00 & -- & -- & .09 \\
\hline $\begin{array}{l}\text { BIS Cognitive } \\
\text { complexity }\left(1^{\text {st }}\right.\end{array}$ & Low & 10.04 & 2.94 & -142 & -191 & 46 & {$\left[\begin{array}{lll}2 & 9 & 01\end{array}\right.$} & 07 \\
\hline order) & High & 11.46 & 2.15 & -1.42 & -1.91 & 40 & {$[-2.91, .00]$} & \\
\hline BIS Attentional & Low & 13.17 & 3.16 & (n0\%** & 5000 & & & \\
\hline brder) & High & 19.25 & 3.38 & $-6.08^{* *}$ & 58.00 & -- & -- & .69 \\
\hline
\end{tabular}




\begin{tabular}{lcccccccc}
\hline \multicolumn{1}{c}{ Scale } & Anxiety level & $M$ & $S D$ & $M$ diff & $t / M W-U$ & $D f$ & $95 \%$ CI & $E S$ \\
\hline BIS Motor $\left(2^{\text {nd }}\right.$ & Low & 22.79 & 3.58 & & & & & \\
order $)$ & High & 21.38 & 4.38 & 1.42 & 1.23 & 46 & {$[-.91,3.74]$} & .03 \\
BIS Nonplanning & Low & 21.58 & 6.19 & & & & & \\
$\left(2^{\text {nd }}\right.$ order $)$ & High & 26.04 & 4.22 & $-4.46^{* *}$ & 156.50 & -- & -- & .39 \\
\hline
\end{tabular}

Note. $M W-U=$ Mann-Whitney $U$ test statistic; $E S=$ Effect size (for the independent samples $t$-test this value represents eta squared, for the Mann-Whitney $U$ test this value represents $r$ ); STAI = State Trait Anxiety Inventory, Trait Anxiety scale; BAI = Beck Anxiety Inventory; GAD-7 = Generalised Anxiety Disorder 7-Item Scale; PSWQ = Penn State Worry Questionnaire; MASQ = Mood and Anxiety Symptom Questionnaire; AA = anxious arousal scale; BDI-II = Beck Depression Inventory-II; IDS-SR = Inventory of Depressive Symptomatology Self Report; BFAS = Big Five Aspects Scales; BAS = Behavioral Inhibition System/Behavioral Activation System Scales behavioral activation subscale; RR = Reward Responsiveness; BIS Scale = Behavioral Inhibition System/Behavioral Activation System Scales behavioral inhibition subscale; ACS = Attentional Control Scale; BIS = Barratt Impulsiveness Scale.

Values derived from the Mann-Whitney $U$ test and effect sizes $(r)$ for the Mann-Whitney $U$ test are in italics.

Effect size values for the Mann-Whitney $U$ test $(r)$ are interpreted as $.1=$ small, $.3=$ medium, $.5=$ large; Effect size values for the independent samples $t$-test (eta-squared) are interpreted as $.01=$ small, $.06=$ moderate, $.14=$ large. Interpretation of effect sizes from Statistical power analysis for the behavioural sciences by J.W. Cohen (1988). Hillsdale, NJ: Lawrence Erlbaum Associates.

** $p<.01$, two tailed.

As can be seen from Table 15, the participants in the high trait anxiety group scored significantly higher than those in the low trait anxiety group on measures of anxiety (i.e., PSWQ, GAD-7, BAI) and depression (i.e., BDI-II, IDS-SR), as well as on measures of personality that have a close conceptual link with anxiety (i.e., BFAS neuroticism scale and BIS/BAS Scales behavioral inhibition subscale), and all the effect sizes were large. There was no significant difference between the groups on a measure of somatic anxiety symptoms (i.e., MASQ anxious arousal scale); this result is consistent with the results of the convergent and discriminant validity analysis of the STAI T-Anxiety scale in Study 1 described above. Interestingly, participants in the high trait anxiety group scored significantly higher on impulsivity than low trait anxiety participants (Barratt Impulsiveness Scale), with higher scores in domains relating to attention difficulties, cognitive instability and difficulties with self-control. In addition, participants in the high trait anxiety group also scored significantly lower on attentional control (ACS), including focused attention and attention shifting. With the exception of self-control (Barratt Impulsiveness Scale) which had a medium effect size, all these effect sizes were large. Taken together, these results suggest that participants who are high in trait anxiety experience impairments in executive function. In relation to other 
aspects of personality, participants in the high trait anxiety group scored significantly higher than the low trait anxiety group on neuroticism (BFAS) and, although there were no differences between the low and high trait anxiety groups on agreeableness (BFAS), participants in the low trait anxiety group scored significantly higher than those in the high trait anxiety group on conscientiousness (BFAS), extraversion (BFAS) and openness/intellect (BFAS). All the effect sizes associated with these significant mean differences were large. As both extraversion and openness/intellect tend to be negatively related to neuroticism, these latter group differences are unsurprising.

\section{Discussion}

Our aim in the current research was to examine the factor structure of the STAI TAnxiety scale using EFA in a large, healthy sample. EFA revealed two method factors that previous researchers have labelled 'anxiety absent' and 'anxiety present' (Bieling et al., 1998; Spielberger et al., 1983) but that may be better described as a negative polarity factor and a positive polarity factor. Forcing a one factor solution nevertheless produced a coherent solution with all items loading strongly on the single factor, demonstrating that the STAI TAnxiety scale can be represented as a single dimension (cf. comments on the unidimensionality of the STAI T-Anxiety scale by Balsamo et al. (2013)). In order to better understand the unidimensional structure of the STAI T-Anxiety scale, we investigated the scale's convergent and discriminant validity. Consistent with findings from previous research, the STAI T-Anxiety scale demonstrated less than ideal discriminant validity, correlating highly with measures of depression. We also found high correlations between the STAI T-Anxiety scale and measures of anxiety that contain items related to worry and anxious apprehension, as well as high correlations between the STAI T-Anxiety scale and measures that contain items related to general distress. 
The pattern of correlations is consistent with previous research that found the STAI TAnxiety scale total score correlated more strongly with measures of depression than anxiety in healthy populations (Bados et al., 2010; M. Balsamo et al., 2013; Nitschke et al., 2001). Authors have explained this finding in relation to the possibility that the STAI T-Anxiety scale may actually measure negative affect rather than anxiety in particular (Bados et al., 2010; Grös et al., 2007), or may measure a tendency to engage in extended processing with added dimensions of specific arousal response tendencies of anxiety and depression (M. Balsamo et al., 2013). According to the tripartite model of anxiety and depression (Clark \& Watson, 1991), if the STAI T-Anxiety scale measures negative affect it should also be measuring aspects of anxiety and depression since negative affect is common to both. In the model by Clark and Watson (1991), depression is distinguished from anxiety by the presence of low positive affect whereas anxiety is characterised by physiological tension and hyperarousal (Clark \& Watson, 1991). The higher correlations of the STAI T-Anxiety scale with measures of depression compared to the scale's correlations with other measures of anxiety suggest that the STAI T-Anxiety scale contains items that measure low positive affect more than physiological tension and hyperarousal. Indeed, the absence of items relating to physiological/somatic symptoms in the STAI T-Anxiety scale has been noted by several authors (Bados et al., 2010; M. Balsamo et al., 2013; Grös et al., 2007; Nitschke et al., 2001).

Our finding that the STAI T-Anxiety scale correlates significantly more strongly with measures of depression than with some measures of anxiety (i.e., MASQ general distress anxiety scale, BAI, and MASQ anxious arousal scale) in a healthy sample differs from previous studies that found the STAI T-Anxiety scale appeared to be correlated similarly with measures of depression and with measures of anxiety in healthy populations (Fountoulakis et al., 2006; Kohn et al., 2008). One difference between the current study and previous studies is that previous studies did not test these correlations to establish whether they were 
significantly different to each other. They also used measures of anxiety that were not administered here (e.g., the BAIT (Kohn et al., 2008), and the SRSDA (Fountoulakis et al., 2006)). An avenue for future research is to administer these instruments together with the PSWQ, GAD-7, BAI and MASQ, then calculate correlations between the STAI T-Anxiety scale and each of these instruments, as well as between each of the instruments, to better identify the nature of the construct measured by the STAI T-Anxiety scale. The current finding that the STAI T-Anxiety scale correlates as strongly with measures of depression as it does with measures that contain items relating to negative affect (e.g., BFAS neuroticism scale, MASQ general distress - mixed scale, PID-5-Adult negative affect scale) is also consistent with findings from previous studies that have used CFA to conclude that the STAI T-Anxiety scale is a general measure of negative affectivity rather than a specific measure of anxiety.

Consistent with previous research, the current study also found that the STAI TAnxiety scale correlated significantly more strongly with measures of worry and anxious apprehension such as the PSWQ than with other measures of anxiety, especially those that contain items related to physiological hyperarousal and somatic symptoms such as the BAI and the anxious arousal scale of the MASQ (e.g., see Bados et al., 2010; Bieling et al., 1998; Grös et al., 2007; Nitschke et al., 2001). This finding indicates that the STAI T-Anxiety scale may measure anxious apprehension better than it measures anxious arousal, as has been suggested by Nitschke et al. (2001) and Bados et al. (2010) and noted by Balsamo et al. (2013) and Grös et al. (2007). To the best of our knowledge the current study is the first to use the GAD-7 to examine the concurrent validity of the STAI T-Anxiety scale. Although the GAD-7 has previously been shown to correlate strongly with both the PSWQ and the BAI (Dear et al., 2011), in the current study the correlation between the GAD-7 and the STAI TAnxiety scale was as large as the correlation between the PSWQ and the STAI T-Anxiety 
scale, and significantly larger than the correlation between the BAI and the STAI T-Anxiety scale. This suggests the GAD-7, the PSWQ and the STAI T-Anxiety scale may all measure cognitive aspects of anxiety, including worry and rumination, better than the commonly used BAI.

The current study also aimed to explore whether participants who were identified as low and high in trait anxiety also scored low and high on other instruments that measure anxiety, mood and personality traits. Findings indicated that individuals high in trait anxiety as measured by the STAI T-Anxiety scale also score high on both general measures of anxiety (i.e., BAI) and more specific measures of anxious apprehension (i.e., PSWQ and GAD-7), as well as depression (i.e., BDI-II and IDS-SR). They also score high on instruments that measure personality traits associated with anxiety, such as neuroticism and behavioural inhibition. Findings that individuals who score low and high on the STAI TAnxiety scale also score low and high, respectively, on instruments measuring other types of psychopathology (e.g., depression) and personality traits (e.g., neuroticism) means that, when conducting research that separates individuals into groups of low and high trait anxiety in order to test the effect of trait anxiety on behavioural or physiological responses, researchers should take note that they may be measuring responses associated with other, related disorders and traits. As a consequence, they need to control for these conceptually relevant and potentially confounding variables. Interestingly, in the current study the MASQ anxious arousal scale, which is an instrument designed to measure somatic symptoms of anxiety, did not distinguish between participants low and high in trait anxiety. Similarly, in the larger sample, the MASQ anxious arousal scale demonstrated a smaller correlation with the STAI T-Anxiety scale than the STAI T-Anxiety scale demonstrated with most other measures of anxiety. As mentioned above, it is possible that the STAI T-Anxiety scale does not adequately measure physiological and somatic aspects of anxiety, meaning that individuals 
who score high on the STAI T-Anxiety scale may not score as high on instruments that contain a large number of items related to physiological/somatic symptoms.

In relation to personality traits less commonly associated with anxiety, we found that the high trait anxiety group was lower in attentional control and higher in impulsivity than the low trait anxiety group. The finding that individuals high in trait anxiety have more difficulty with attentional control is consistent with predictions made by attentional control theory $(\mathrm{M}$. W. Eysenck et al., 2007). Attentional control theory states that anxiety, both as a personality dimension and an emotional state, is associated with impairment on numerous tasks. This is because anxiety disrupts the balance between the stimulus-driven attentional system involved in the bottom-up control of attention, and the goal-directed attentional system involved in the top-down control of attention. Due to the increased influence of the stimulus-driven attentional system, highly anxious people allocate more attention to potential sources of threat (both external task-irrelevant stimuli and internal task-irrelevant stimuli such as worrying thoughts). This results in a decrease in attentional focus and, consequently, a decrease in attentional control (M. W. Eysenck et al., 2007). The finding that individuals high in trait anxiety are higher in impulsivity than individuals low in trait anxiety was unexpected insofar as impulsivity and anxiety are rarely investigated as related constructs in the literature on impulsivity. Indeed, the goal of early revisions of the Barratt Impulsiveness Scale was to identify a set of impulsiveness items that were orthogonal to anxiety items (Patton et al., 1995; Stanford et al., 2009). The finding in the current study suggests that impulsivity as measured by the Barratt Impulsiveness Scale is related to trait anxiety as measured by the STAI T-Anxiety scale. A closer inspection of the first order impulsivity factors endorsed by participants high in trait anxiety reveals that the factors contain items related to attentional difficulties and cognitive instability, both of which involve an inability to focus attention or concentrate (Stanford et al., 2009), and items related to self-control, which involves 
difficulties in planning and thinking. The finding that individuals high in anxiety also experience difficulty in the domains of focused attention, concentration, planning and thinking is generally consistent with their elevated scores on the Barratt Impulsiveness Scale as well as predictions from attentional control theory and research that has found highly anxious individuals display difficulties with executive function (e.g., for review see Derakshan \& Eysenck, 2009; M. W. Eysenck \& Derakshan, 2011).

One limitation of the current study is that, although this study used measures of general reactivity commonly associated with anxiety such as the BFAS neuroticism scale and the BIS/BAS Scales behavioral inhibition system scale, it did not include a specific measure of negative affectivity such as the negative affect scale of the Positive Affect Negative Affect Scale (Watson, Clark, \& Tellegen, 1988). Given that the STAI T-Anxiety scale appears to measure negative affect rather than or in addition to anxiety, any research that seeks to explore the convergent validity of the STAI T-Anxiety scale should include a well-validated measure of negative affect. In addition, due to time constraints associated with data collection it was not possible to administer all questionnaires to all 947 participants. We also did not administer the same questionnaires to participants in the larger and smaller samples. This meant that we were not able to comment on whether groups low and high in trait anxiety were also low and high in, for example, sensitivity to punishment as measured by the SPSRQ and then compare their scores on the SPSRQ with their scores on the BIS/BAS Scales behavioral inhibition system Scale, which has been associated with sensitivity to punishment (Avila, 1994). Finally, measurement of convergent and discriminant validity was limited to self-report questionnaires. Future research may consider using clinician rated scales to measure psychopathological constructs such as depression and anxiety, and behavioural tasks such as the stop signal task (Logan et al., 1997) to measure constructs such as impulsivity, and the attention network test (Fan, McCandliss, Sommer, Raz, \& Posner, 2002) to measure 
constructs such as attentional control. Strengths of the current study include the large sample of mostly undergraduate university students and the use of EFA with oblique rotation to replicate the factor structure of the STAI T-Anxiety scale found in previous studies that have used CFA and EFA with orthogonal rotation.

In conclusion, our examination of the STAI T-Anxiety scale using EFA suggests that the scale contains two method factors but may also be conceptualised as unidimensional. Convergent validity with other measures of anxious apprehension was relatively good but discriminant validity was low because the STAI T-Anxiety scale correlated highly with all measures of depression and many personality traits related to negative affect. These findings suggest that, as noted by previous authors (Bados et al., 2010; M. Balsamo et al., 2013; Bieling et al., 1998), the STAI T- Anxiety scale may actually measure negative affect more broadly rather than anxiety in particular. Future research may consider revising the STAI TAnxiety scale to include fewer items related to depression (i.e., low positive affect) and more items related to physiological hyperarousal and somatic symptoms of anxiety. 


\section{Chapter 4}

\section{The Effect of Trait Anxiety on Stop Signal Task Performance}

Key features of all anxiety disorders include muscle tension, vigilance, caution and avoidant behaviours (American Psychiatric Association, 2013a). Engaging in cautious and avoidant behaviour involves the ability to withhold, postpone or cancel an action, called inhibitory control (Bari \& Robbins, 2013). Experimentally, inhibitory control is measured using tasks such as the stop-signal task, the go/no-go task, the Stroop task, the antisaccade task and the flanker task (Eriksen \& Eriksen, 1974; Everling \& Fischer, 1998; Hallett, 1978; Jensen \& Rohwer Jr, 1966; Logan et al., 1997; Stroop, 1935; Swick et al., 2011; Takezawa \& Miyatani, 2005). In the literature relating to anxiety, some of these paradigms have been used with threatening or emotionally relevant stimuli in order to examine the effect of threat related processing biases on attention. Studies that have used these paradigms with highly anxious individuals have found that these individuals have difficulty inhibiting threat-related, task-irrelevant information (Bar-Haim et al., 2007; Derakshan et al., 2009).

The mechanisms underlying these threat-related processing biases are debated but it is thought that individuals with anxiety may have facilitated attention toward threat-related stimuli, may experience difficulty disengaging attention from threat-related stimuli, and/or may engage in attentional avoidance of threat-related stimuli (see Cisler \& Koster, 2010, for review). Substantially less research has investigated whether individuals high in anxiety have a more general inhibitory control deficit; that is, an inhibitory control deficit when no threatrelated information is present. Functionally, individuals with anxiety appear to be better at inhibiting responses (i.e., they engage in behavioural avoidance) when an event or situation is feared, even when that event or situation may hold the potential for reward. For example, individuals with social anxiety disorder avoid what others consider to be rewarding social contact and individuals with agoraphobia forfeit benefits of integration into the community. 
Although individuals with anxiety demonstrate impairments when inhibiting the processing of irrelevant threat-related information, the question remains whether task-relevant threat (such as, for example, the threat of failure), improves inhibitory control performance. For example, when the goal of a task is to inhibit a response but that task does not present any task-irrelevant threatening information, individuals with anxiety may demonstrate enhanced inhibitory control (e.g., Sehlmeyer et al., 2010).

One theory that addresses the issue of inhibitory control and anxiety is attentional control theory. According to attentional control theory, there are two attentional systems active at any given time; a goal-directed attentional system that is involved in the top down control of attention and a stimulus-driven attentional system that is involved in the bottom up control of attention (M. W. Eysenck et al., 2007). Anxiety disrupts the balance of these systems such that the stimulus-driven attentional system has increased influence. Attentional control theory assumes that in anxiety, attention is allocated to detecting sources of threat. Even when no threat exists, the best strategy to detect potential sources of threat is to allocate attentional resources widely. This, however, results in a reduction in attentional focus in relation to any ongoing task, which reduces the attentional control necessary to prevent allocation of attention to task-irrelevant stimuli and internal responses (Derakshan \& Eysenck, 2009; M. W. Eysenck et al., 2007). The reduction in attentional control consequently impairs inhibition. Inhibition is used to resist interference from task-irrelevant stimuli and responses and it requires attentional control (M. W. Eysenck \& Derakshan, 2011). Attentional control theory also proposes that anxiety has less impact on performance effectiveness (e.g., accuracy) than processing efficiency, which is the relationship between performance effectiveness and the resources or effort used to attain that level performance. Processing efficiency can be ascertained by looking at reaction time or neural activation (M. W. Eysenck \& Derakshan, 2011). Anxiety can create processing inefficiency without 
affecting performance effectiveness because anxious individuals use compensatory strategies such as increased effort and use of processing resources to avoid decrements in task performance (M. W. Eysenck et al., 2007).

To investigate whether anxious individuals experience deficiencies in inhibitory control in the absence of threat-related information, studies have used a range of inhibitory control tasks such as stop-signal tasks (e.g., Avila \& Parcet, 2001; Epstein et al., 2001), antisaccade tasks (T. L. Ansari \& Derakshan, 2010; Tahereh L. Ansari \& Derakshan, 2011a, 2011b; Derakshan et al., 2009), go/no-go tasks (Righi, Mecacci, \& Viggiano, 2009; Sehlmeyer et al., 2010), Stroop tasks (Basten et al., 2011; E. Fox, 1993, 1994) and flanker tasks, including the attention network test (Pacheco-Unguetti, Acosta, Callejas, \& Lupiáñez, 2010). These tasks have been administered to heterogeneous participant groups diagnosed with a range of anxiety and comorbid disorders, as well as participants high on non-clinical measures of anxiety such as trait anxiety (Spielberger et al., 1983). These studies have reported mixed results, including inhibitory control deficits (Tahereh L. Ansari \& Derakshan, 2011a, 2011b), better inhibitory control (Sehlmeyer et al., 2010), and no difference in performance (Epstein et al., 2001) for high anxious compared to low anxious individuals. The mixed findings are challenging to interpret; the heterogeneous nature of the tasks and participants used means that it is difficult to draw firm conclusions regarding the effect of anxiety on inhibitory control. Each task measures a different aspect of inhibitory control (e.g., stop signal tasks measure action cancellation whereas go/no-go tasks measure action withholding/restraint (Eagle et al., 2008)) that are partially independent and have, for example, been shown to have different neural correlates (Swick et al., 2011). It is also difficult to interpret findings within the same category of task. Studies examining anxiety and inhibitory control have used different experimental designs and methodology including stimulus type (e.g., words versus pictures), length of interstimulus intervals (ISI), and 
intermixed versus single stimulus presentations within blocks (see Bar-Haim et al., 2007 for similar criticisms).

Inhibitory control is a broad term that refers to a range of abilities; for example, in relation to action (as opposed to thought) inhibitory control can refer to action withholding/restraint, action postponing/waiting, or action cancellation/stopping (Bari \& Robbins, 2013). Different types of inhibitory control are measured by different types of tasks to different degrees. One form of inhibitory control involves stopping or cancelling an already initiated response (Frederick Verbruggen \& Logan, 2008). This type of inhibition is most commonly measured using the stop signal task, which involves a go task requiring discrimination of two stimuli (e.g., an $\mathrm{X}$ and an $\mathrm{O}$ ) via a button press mapped to each of the stimuli. It also involves a stop task that occurs on $25 \%$ of all trials, which is comprised of a stop signal (e.g., a red border) being presented shortly after the go signal. On presentation of the stop signal, participants must withhold their go task response. The variable that is used as an index of inhibitory control is the stop signal reaction time (SSRT), which measures the temporal period between the stop signal presentation and the stop process finish. Better inhibitory control is indexed by shorter SSRT.

In addition to clearly identifying the type of inhibition measured in an experimental task, studies need to recruit homogenous groups of anxious individuals without comorbid conditions. Comorbidities such as obsessive compulsive disorder and attentiondeficit/hyperactivity disorder have been shown to influence findings from inhibitory control tasks (Lipszyc \& Schachar, 2010). Trait anxiety is a relatively stable and enduring difference among individuals to perceive stressful situations as dangerous or threatening (Spielberger et al., 1983). It is elevated in individuals who meet criteria for anxiety disorders (Bieling et al., 1998; J. A. Chambers et al., 2004) and predicts future anxiety disorder diagnosis (Sandi \& Richter-Levin, 2009). Trait anxiety is most commonly measured using the State Trait Anxiety 
Inventory (STAI) trait anxiety scale (STAI T-Anxiety scale) Form Y version. The STAI TAnxiety scale is a self-report instrument that contains 20 questions measuring trait anxiety (Spielberger et al., 1983). Recruiting participants with elevated trait anxiety rather than a range of anxiety disorders improves interpretability of findings as it reduces the likelihood that unique characteristics associated with each anxiety disorder (e.g., Kampman, Viikki, Jarventausta, \& Leinonen, 2014; Kent \& Rauch, 2003; Shin \& Liberzon, 2010) will influence results.

From studies conducted to date, anxiety does not appear to have an effect on stop signal task performance. So far, no difference in SSRT has been found between individuals high and low in anxiety (Epstein et al., 2001; Korenblum et al., 2007; M. A. Lau et al., 2007; Savostyanov et al., 2009; Savostyanov et al., 2011) or when trait anxiety is used as a continuous variable (Avila \& Parcet, 2001). Highly anxious individuals have, however, been found to have greater electroencephalogram (EEG) desynchronization in both go and stop conditions of the stop signal task, suggesting they may use more resources to perform the task to the same standard as low anxious individuals, as proposed by attentional control theory (Savostyanov et al., 2009; Savostyanov et al., 2011).

Studies using the stop signal task to investigate the relationship between anxiety and inhibitory control have had numerous methodological issues. The issues include small sample sizes (Avila \& Parcet, 2001; Epstein et al., 2001; Korenblum et al., 2007), use of pediatric rather than adult participants (Korenblum et al., 2007), use of translated versions of the STAI (Savostyanov et al., 2009; Savostyanov et al., 2011) or the older Form X version of the STAI that included more items related to depression (Avila \& Parcet, 2001; Linkovski, Kalanthroff, Henik, \& Anholt, 2013). Depression is a disorder that does not appear to be associated with differences in stop signal task performance (Jakubczyk et al., 2012; M. A. Lau et al., 2007; Lyche, Jonassen, Stiles, Ulleberg, \& Landrø, 2010; Matthews et al., 2009). Studies have also 
confounded effects of trait anxiety on inhibitory control with effects of other anxiety disorders as they have recruited participants who may be high in trait anxiety but who also have a range of other anxiety disorders (Epstein et al., 2001; Korenblum et al., 2007; M. A. Lau et al., 2007; Sjoerds, Van Den Brink, Beekman, Penninx, \& Veltman, 2014). Studies have used valenced rather than neutral stimuli (M. A. Lau et al., 2007; Savostyanov et al., 2009; Savostyanov et al., 2011), which means these studies are unable to shed light on the question of whether individuals high in trait anxiety have an inhibitory control deficit when no threat-related information is present. Authors have failed to report important measures of stop signal performance such as SSRT (Savostyanov et al., 2009; Savostyanov et al., 2011) or mean go reaction time and accuracy (M. A. Lau et al., 2007). Mean go reaction time and accuracy help interpret findings of no difference in group comparisons of SSRT, because participants may slow their go reaction time to improve stop performance.

Contrary to previously mentioned null findings, a number of studies have found differences in stop signal task performance for individuals high and low on other traits related to trait anxiety. Behavioural inhibition is a personality trait related to trait anxiety. Higher scores on the behavioral inhibition subscale (BIS subscale) of the Behavioral Inhibition System/Behavioral Activation System Scales (BIS/BAS Scales; Carver \& White, 1994) are associated with higher scores on self-report measures of anxiety (Hundt et al., 2007), and the STAI T-Anxiety scale is correlated with scores on the BIS subscale (Cooper et al., 2007). Although studies using measures of behavioural inhibition such as the BIS/BAS Scales and the Gray-Wilson Personality Questionnaire (Wilson, Barrett, \& Gray, 1989) have found no association between behavioural inhibition and SSRT (Eriksson et al., 2016; Knyazev, Levin, \& Savostyanov, 2008), longer SSRT has been found to be associated with a decrease in go accuracy for individuals higher in behavioural inhibition; this relationship has not been observed in individuals lower in behavioural inhibition (Eriksson et al., 2016). This finding 
suggests that individuals higher in behavioural inhibition might perform the stop signal task differently to those lower in behavioural inhibition, but the exact reason for this difference is not clear. Sensitivity to punishment as measured by the sensitivity to punishment subscale of the Sensitivity to Punishment and Sensitivity to Reward Questionnaire (SPSRQ; Torrubia et al., 2001) has been found to be positively correlated with the STAI T-Anxiety scale (Avila \& Parcet, 2001) and other self-report measures of anxiety (Hundt et al., 2007). One study has found a negative correlation between SSRT and the sensitivity to punishment subscale of the SPSQR (Avila \& Parcet, 2001), suggesting that individuals high in sensitivity to punishment exhibit better inhibitory control.

Given the relatively small number of previous studies that have looked at the relationship between trait anxiety and stop signal task performance, and the methodological issues identified in those studies, the current research aimed to examine inhibitory control in trait anxiety using the English version of the STAI T-Anxiety scale Form Y version. Study 1 compared stop signal performance of a group of individuals high in trait anxiety with a group of individuals low in trait anxiety. None of the individuals had been diagnosed with an anxiety disorder or other comorbid disorder, and the stop signal task was modelled on the original task from Logan, Schachar, and Tannock (1997). In Study 1 participants were separated into two groups, high and low in trait anxiety. To replicate Study 1 findings and to assay the continuous distribution of trait anxiety, Study 2 recruited a larger group of participants across the trait anxiety spectrum and a correlational analysis was performed to investigate if trait anxiety was associated with stop signal task performance.

Based on predictions of attentional control theory it was hypothesised that if individuals high in trait anxiety perceive the possibility of poor performance on the stop signal task as a source of threat, the ability to successfully inhibit a prepotent go response will be poorer. This should be reflected in increased mean go reaction time and increased SSRT. 


\section{Study 1}

\section{Method}

\section{Participants}

Participants were 76 healthy, English speaking University of Melbourne students and members of the public recruited through a University of Melbourne first year psychology research program and advertisements on careers and other websites (e.g., Gumtree) throughout the period August 2014 to February 2016. Participants were either paid an honorarium for completing the study or received course credit. Exclusion criteria included a history of neurological or psychiatric disorders, current use of psychoactive medication, and history of substance abuse. All participants had normal or corrected-to-normal vision. Participants provided written informed consent and the experiment was approved by the human research ethics committee at the University of Melbourne.

\section{Procedure and Stimuli}

Participants were screened and selected for participation using the STAI T-Anxiety scale a number of days to weeks before completing the stop signal task. Eight hundred and thirty five individuals were initially screened for inclusion in the study. All completed the STAI online via the Qualtrics Research Suite either on the university campus or at an offcampus location. During the same session participants also completed the BIS/BAS Scales (Carver \& White, 1994); the Penn State Worry Questionnaire (PSWQ; Meyer et al., 1990), the Generalized Anxiety Disorder 7-Item Scale (GAD-7; Spitzer et al., 2006), the Beck Anxiety Inventory (BAI; Beck \& Steer, 1990), the Beck Depression Inventory-II (BDI-II; Beck et al., 1996), the Big Five Aspects Scales (BFAS; DeYoung et al., 2007), the Inventory of Depressive Symptomatology - Self Report (IDS-SR; John Rush et al., 1986), a demographic information questionnaire, a magnetic resonance imaging eligibility information questionnaire and a number of other measures that are not reported here. Alcohol use was 
measured using the Alcohol Use Disorders Identification Test (AUDIT; Babor et al., 2001). Nicotine dependence was measured using the Fagerstrom Test for Nicotine Dependence (Fagerstrom et al., 1990) and substance use was measured using the Orygen Substance Use Index. Non-copyrighted measures are reproduced in Appendix A and Appendix B. Participants were classified as low or high in trait anxiety if their STAI T-Anxiety scale score fell at least one standard deviation below or above either the Australian mean for the STAI TAnxiety scale (Crawford, Cayley, Lovibond, Wilson, \& Hartley, 2011) or the sample mean $\left(M_{\text {sample }}=42.51, S D=10.46\right)$. Participants who met criteria for inclusion in the study were invited to the university campus to complete the stop signal task. Narcotic use, psychiatric history and current medications were measured using self-report following completion of the stop signal task.

Participants completed the stop signal task (see below for description) in a quiet room on the university campus. Instructions and stimuli were presented using E-Prime ${ }^{\circledR} 2.0$ software (version 1.1, Psychology Software Tools, Inc. Pittsburgh, PA) running on an IBMcompatible PC. Each participant was presented with standardized instructions via the monitor and was verbally instructed to respond as quickly and as accurately as possible and to not slow down in an attempt to improve accuracy. See Appendix $C$ for written instructions. Prior to undertaking the stop signal task participants completed two practice blocks each containing 24 trials. At the end of each practice block feedback relating to stop accuracy, mean go reaction time and stop signal reaction time was provided.

The experimental task was comprised of three blocks each containing 100 trials. Feedback as per the practice task was provided at the end of each block. Go stimuli were the letters $\mathrm{X}$ and $\mathrm{O}$ and were mapped to keyboard buttons $\mathrm{X}$ and $\mathrm{O}$, respectively. Stimuli were presented centrally in black Arial font on a grey background. The stop signal was a red box that surrounded the go stimulus and appeared on one in every four trials. Stop signals were 
not presented on consecutive trials. During stop trials the time between the presentation of the go stimulus and the stop stimulus (the stop signal delay) was initially set at $250 \mathrm{~ms}$ and adjusted dynamically depending on the participant's response. If the participant successfully inhibited their response, the delay increased by $50 \mathrm{~ms}$ which made it more difficult to inhibit on the subsequent stop trial. If the participant failed to inhibit their response, the delay decreased by $50 \mathrm{~ms}$ which made it easier to inhibit on the subsequent stop trial. This staircase design ensured that, on average, each participant had a probability of successful inhibition approaching $50 \%$ by the end of the experiment. Stop signal reaction time was calculated as the mean reaction time to go stimuli minus the stop signal delay for the $50 \%$ inhibition threshold (Logan et al., 1997). Higher stop signal reaction times indicate poorer ability to inhibit a response. Each go trial began with a fixation cross for $500 \mathrm{~ms}$, which was followed by a go stimulus for $1000 \mathrm{~ms}$ and an intertrial interval (ITI) of $1000 \mathrm{~ms}$.

\section{Results}

Data was initially collected from 76 participants. Data from four participants was removed because stop accuracy on the stop signal task fell below $40 \%$ or over $60 \%$. SSRT is potentially not a valid measure when stop accuracy falls outside these values (Congdon et al., 2012). In addition, three participants were excluded due to current substance abuse, one was excluded due to a history of depression and current use of antidepressant medication, one was excluded due to thyroid dysfunction and medication, one was excluded because of difficulty understanding instructions and five were excluded because their STAI T-Anxiety scale score had increased or decreased on the day they completed the stop signal task such that they no longer fulfilled the inclusion criterion of low or high trait anxiety. When examining stop signal task performance between groups, including the above participants in the analysis did not change the significance of results. Descriptive statistics for the remaining participants in 
the whole sample as well as in the low and high trait anxiety groups are displayed in Table 16.

Table 16

Mean Demographic and Questionnaire Data for Whole Sample and Low Trait Anxiety and High Trait Anxiety Groups

\begin{tabular}{lccc}
\hline Variables & Whole sample $(n=61)$ & $\begin{array}{c}\text { Low trait anxiety } \\
(n=34)\end{array}$ & $\begin{array}{c}\text { High trait anxiety } \\
(n=27)\end{array}$ \\
\hline Age (years) & $22.93(4.76)$ & $23.65(5.30)$ & $22.02(3.88)$ \\
Age range (years) & 18.11 to 39.82 & 18.12 to 39.82 & 28.25 to 30.84 \\
$\begin{array}{l}\text { Gender } \\
\text { (male/female/other) }\end{array}$ & $19 / 41 / 1$ & $14 / 19 / 1$ & $5 / 22 / 0$ \\
$\begin{array}{l}\text { Education (years) } \\
\text { STAI-T score }\end{array}$ & $\begin{array}{l}14.87(2.59) \\
\text { (Time 1) }\end{array}$ & $15.09(2.52)$ & $14.59(2.69)$ \\
$\begin{array}{l}\text { STAI-T score } \\
\text { (Time 2) }\end{array}$ & $\begin{array}{r}37.37(14.4) \\
\text { range } 20 \text { to } 75\end{array}$ & $\begin{array}{c}25.76(2.4) \\
\text { range } 20 \text { to } 29\end{array}$ & $\begin{array}{c}56.26(7.10) \\
\text { range } 42 \text { to } 75\end{array}$ \\
\hline
\end{tabular}

Note. Except for gender which is a count, numbers represent means with standard deviation in parentheses. STAI $-\mathrm{T}$ score $=$ State Trait Anxiety Inventory trait anxiety scale score.

The high and low trait anxiety groups did not significantly differ on the variables of age $(t(59)=1.33, p=.19)$, years of education $(t(59)=.74, p=.46)$ or gender $\left(\chi^{2}(2)=4.74, p\right.$ $=.09)$. Independent samples $t$-tests were used to investigate differences between the groups in SSRT, percentage of successful inhibitions on stop trials (stop accuracy), mean correct go reaction time, and stop signal delay. Descriptive statistics for performance on the stop signal task for each of the high and low trait anxiety groups are presented in Table 17. 
Table 17

Means and Standard Deviations for Stop-Signal Reaction Time, Stop-Signal Accuracy, Mean Correct Go Reaction Time and Stop Signal Delay for Low $(n=34)$ and High $(n=27)$ Trait Anxiety Groups

\begin{tabular}{llcc}
\hline \multicolumn{1}{c}{ Task variable } & \multicolumn{1}{c}{ Group } & $M$ & $S D$ \\
\hline \multirow{2}{*}{ SSRT } & Low trait anxiety & 199.71 & 47.56 \\
& High trait anxiety & 192.33 & 52.44 \\
Stop accuracy & Low trait anxiety & 53.30 & 2.95 \\
& High trait anxiety & 52.64 & 2.97 \\
\multirow{2}{*}{ MGRT } & Low trait anxiety & 631.40 & 128.29 \\
& High trait anxiety & 596.17 & 131.99 \\
SSD & Low trait anxiety & 431.69 & 152.72 \\
& High trait anxiety & 403.80 & 153.89 \\
\hline
\end{tabular}

Note: SSRT = stop-signal reaction time; Stop accuracy = percentage of successful inhibitions on stop trials; MGRT = mean correct go reaction time; $\mathrm{SSD}=$ stop-signal delay. Means are provided, standard deviation is shown in parentheses. Times are given in milliseconds.

Preliminary analyses indicated there was no violation of the assumptions of normality, linearly or homoscedasticity. There were no significant differences between the groups in $\operatorname{SSRT}\left(t(59)=.58 ., p=.57, \eta^{2}=.01\right)$, stop accuracy $\left(t(56)=.86, p=.40 ., \eta^{2}=.01\right)$, mean correct go reaction time $\left(t(59)=1.05, p=.30, \eta^{2}=.02\right)$, or stop signal delay $(t(56)=.71, p=$ $\left..48, \eta^{2}=.01\right)$. A power analysis was performed to investigate the proportion of times a significant result would be achieved given the size of the effect obtained. An effect size of .01 was used in the analysis because effect sizes for all four dependent variables were at or close to .01 . With a sample size of 61 and alpha .05 , power was .06 , which means that a significant result would be achieved only $6 \%$ of the time. Power to detect a significant result was low because the sample size was too small for the small effect obtained.

\section{Study 2}

Given the failure to support the hypothesis of a significant relationship between anxiety and stop signal task performance in Study 1 which used a small sample separated into 
two groups, we performed another study with a larger continuous sample. The effect size data from Study 1 indicated group effects on stop signal task measures were small $\left(\eta^{2}=.01\right.$ to .02 ), which would require a group sample size of $n=500+$ to detect a significant difference. Because trait anxiety is a continuous rather than a dichotomous construct, we aimed to improve our sample size and potential statistical power by conducting a second study using a larger sample of participants and correlating their STAI T-Anxiety score with dependent variables from the stop signal task. We also administered other measures of anxiety, mood and personality to this larger sample of participants in Study 2 to examine whether any effects were specific to the STAI or were generalised across measures attempting to assay self-reported anxiety and other related constructs.

\section{Method}

\section{Participants}

Participants were 119 healthy, English speaking University of Melbourne students and members of the public recruited through a University of Melbourne first year research program and twitter throughout the period July 2016 to September 2016. Participants were excluded if they indicated current use of psychoactive medication by self-report. All participants had normal or corrected-to-normal vision and provided written informed consent. The experiment was approved by the human research ethics committee at the University of Melbourne. Participants either received course credit or volunteered for the study.

\section{Procedure and Stimuli}

Participants completed all questionnaires and the stop signal task online at a location of their choosing. Questionnaires were administered via the Qualtrics Research Suite and included the STAI, the BAI, the GAD-7, the PSWQ, the BDI-II, the Mood and Anxiety Symptom Questionnaire (MASQ; Watson et al., 1995a, 1995b), the BFAS, the BIS/BAS Scales, the SPSRQ (Torrubia et al., 2001), the Personality Inventory for DSM-5 Brief Form - 
Adult (PID-5-BF - Adult; Krueger et al., 2013), the AUDIT, a demographic information questionnaire and a number of other measures that are not reported here. Non-copyrighted measures are reproduced in Appendix A and Appendix B. Following completion of the questionnaires, participants were directed to the stop signal task. Instructions and stimuli were programmed using HTML version 5 and JavaScript client side. For data storage and management, PHP and My SQL were used server side. The task ran on all recent versions of major browsers. Each participant was presented with standardized instructions on the computer monitor in 20-point white sans-serif font on a black background. See Appendix D for instructions. Prior to undertaking the task participants completed one practice block of 32 trials. Feedback was provided at the end of each practice trial. At the end of the practice block participants were provided with feedback relating to number of incorrect responses to go stimuli, number of missed responses to go stimuli, mean reaction time to go stimuli, percentage of correctly stopped responses on stop trials, and seconds left to wait until the next block. See Appendix E for stimulus timing and feedback.

The experimental task was comprised of three blocks each containing 64 trials. Feedback as per the practice task was provided at the end of each block. Feedback to respond faster was given at the end of a trial if a participant had not responded to the previous three go stimuli and was presented in 25-point red serif font on a black background. Go stimuli were the letters $\mathrm{X}$ and $\mathrm{O}$ mapped to keyboard buttons $\mathrm{X}$ and $\mathrm{O}$, respectively, and were presented in 150-point serif font. Stimuli were preceded by a fixation cross presented in 100point serif font. The stop signal was a white box that surrounded the go stimulus and appeared on 25 per cent of trials. Stop signals were not presented on consecutive trials. On stop trials the stop signal delay was initially set at $250 \mathrm{~ms}$ and adjusted dynamically depending on the participant's response. If the participant successfully inhibited their response, the delay increased by $50 \mathrm{~ms}$ and if the participant failed to inhibit their response, 
the delay decreased by $50 \mathrm{~ms}$. This staircase design ensured that, on average, each participant had a probability of successful inhibition approaching 50\%. The minimum stop signal delay was set at $0 \mathrm{~ms}$ and the maximum stop signal delay was set at $950 \mathrm{~ms}$, and the stop signal delay was not reset at the start of each experimental block. Stop signal reaction time was calculated as the mean reaction time to go stimuli minus the stop signal delay for the $50 \%$ inhibition threshold (Logan et al., 1997). Each trial began with a fixation cross for 250ms, followed by a go (with or without a stop) stimulus for 1000ms and then either a blank screen for $1000 \mathrm{~ms}$ or, if three consecutive go trials had been missed, feedback for $750 \mathrm{~ms}$ and a blank screen for 250ms. See Appendix E for feedback provided.

\section{Results}

Data was initially collected from 119 participants, which were as many participants as could be recruited given resource constraints. Data from five participants was removed because stop accuracy on the stop signal task fell below $40 \%$ or over $60 \%$. In addition, data from one participant was removed because of incomplete questionnaire data, data from 10 participants was removed because of current use of psychoactive medication, and data from four participants was not able to be used because the participants did not undertake the stop signal task. Data from the remaining 99 participants was analysed. Descriptive statistics for these participants are displayed in Table 18. 
Table 18

Mean Demographic and Questionnaire Data for Sample Included in Analysis $(N=99)$

\begin{tabular}{lc}
\hline \multicolumn{1}{c}{ Variables } & Values \\
\hline Age (years) & $20.21(4.03)$ \\
Age range (years) & 18.10 to 39.60 \\
Gender (male/female) & $25 / 74$ \\
Education (years) & $13.37(1.51)$ \\
STAI-T score & $45.19(11.74)$ \\
STAI-T score range & 20 to 73 \\
\hline
\end{tabular}

Note. STAI $-\mathrm{T}$ score $=$ State Trait Anxiety Inventory trait anxiety scale score.

Except where indicated and for gender which is a count, numbers represent means with standard deviation in parentheses.

Descriptive statistics for stop signal performance are reported in Table 19.

Table 19

Means and Standard Deviations for Stop-Signal Reaction Time, Stop-Signal Accuracy, Mean Correct Go Reaction Time, and Stop Signal Delay

\begin{tabular}{lcc}
\hline \multicolumn{1}{c}{ Task variable } & $M$ & $S D$ \\
\hline SSRT & 269.80 & 47.88 \\
Stop accuracy & 48.91 & 2.58 \\
MGRT & 477.67 & 62.42 \\
SSD & 207.87 & 76.10 \\
\hline
\end{tabular}

Note $:$ SSRT $=$ stop-signal reaction time; Stop accuracy $=$ percentage of successful inhibitions on stop trials; MGRT = mean correct go reaction time; SSD = stop-signal delay. Means are provided, standard is shown in parentheses. Times are given in milliseconds.

The Pearson product-moment correlation coefficient was used to investigate relationships between trait anxiety and SSRT, percentage of successful inhibitions on stop trials (stop accuracy), mean correct go reaction time, and stop signal delay. Correlations between SSRT and scores on the BAI, GAD-7, PSWQ, BDI-II, MASQ, BFAS, BIS/BAS 
Scales (BIS Scale only), SRSPQ and PID-5-BF - Adult were also calculated using the Pearson product-moment correlation coefficient and, where the assumption of normality was violated, Spearman's rank correlation coefficient. Preliminary analysis of skewness and linearity showed that, for scores on some measures, there were violations of the assumptions of normality but linearity appeared to be adequate for scores on all measures.

There was no significant correlation between trait anxiety and SSRT $(r=-.11, p=$ $.30)$ or between trait anxiety and stop accuracy $(r=-.02, p=.88)$, mean correct go reaction time $(r=-.06, p=.53)$, or stop signal delay $(r=-.02, p=.89)$. As in Study 1 , a power analysis was performed. With a sample size of 99 and alpha of .05, power for the SSRT effect size of .11 was .29 , power for the mean correct go reaction time effect size of .06 was .15 , and power for the stop accuracy and stop signal delay effect size of .02 was .07. As can be seen from the low power obtained for each dependent variable in the second study, increasing the sample size to 99 did not increase power from that observed in Study 1 to a great extent.

Depression has been found to be correlated with trait anxiety (Bados et al., 2010; M. Balsamo et al., 2013), therefore hierarchical multiple regression was used to control for the effect of depression on SSRT, which is the dependent variable that indexes inhibitory control. Participants' score on the BDI-II was entered at step 1 and explained only .1\% of the variance in SSRT. After entry of trait anxiety at step 2, the total variance explained by the model as a whole was $1.7 \%, F(2,96)=.82, p=.44$. Trait anxiety explained an additional $1.6 \%$ of the variance in SSRT, after controlling for depression, $R$ squared change $=.02, F$ change $(1,96)$ $=1.56, p=.22$. In the final model none of the variables were statistically significant, with depression having a beta value of $.11, p=.46$ and trait anxiety having a beta value of $-.19, p$ $=.22$. Depression did not therefore appear to affect the relationship between trait anxiety and SSRT. 
In addition to depression, personality traits such as behavioural inhibition, neuroticism and negative affect appear to be associated with trait anxiety (Levis, 2017), therefore hierarchical multiple regression was used to control for the effect of these traits on SSRT. Participants' score on the BDI-II, the behavioural inhibition scale of the BIS-BAS Scales, the neuroticism scale of the BFAS, and the negative affect scale of the PID-5-BF were entered at step 1. Together these four variables explained $.6 \%$ of the variance in SSRT. After entry of trait anxiety at step 2, the total variance explained by the model as a whole was $2.5 \%, F(5$, $93)=.47, p=.80$. Trait anxiety explained an additional $1.9 \%$ of the variance in SSRT after controlling for depression, behavioural inhibition, neuroticism and negative affect, $R$ squared change $=.02, F$ change $(1,93)=1.83, p=.18$. In the final model none of the variables were statistically significant, with depression having a beta value of .08, $p=.61$, behavioural inhibition having a beta value of $-.03, p=.85$, negative affect having a beta value of $-.00, p=$ .99 , neuroticism having a beta value of $.16, p=.41$, and trait anxiety having a beta value of $.27, p=.18$. It therefore appeared that these variables did not affect the relationship between trait anxiety and SSRT.

Table 20 shows correlations between SSRT and scores on other measures of anxiety, mood and personality. Due to the number of correlations, multiple comparisons were controlled for using a more strict $\mathrm{p}$ value (i.e., $p<.01$ ). 
Table 20

Correlations Between Stop Signal Reaction Time and Other Measures of Anxiety, Mood and Personality

\begin{tabular}{|c|c|c|}
\hline Scale & $\begin{array}{c}\text { Pearson's Correlation } \\
\text { Coefficient } \\
\end{array}$ & $\begin{array}{c}\text { Spearman's } \\
\text { Correlation Coefficient }\end{array}$ \\
\hline BAI & - & .14 \\
\hline GAD-7 & - & .06 \\
\hline PSWQ & -.04 & - \\
\hline BDI-II & - & .07 \\
\hline MASQ-GM & - & .06 \\
\hline MASQ-GA & - & .02 \\
\hline MASQ-AA & - & .14 \\
\hline MASQ-GD & - & .08 \\
\hline MASQ-AD & -.04 & - \\
\hline BFAS Neuroticism & -.02 & - \\
\hline BFAS Agreeableness & - & -.04 \\
\hline BFAS Conscientiousness & .13 & - \\
\hline BFAS Extraversion & .05 & - \\
\hline BFAS Openness/Intellect & .14 & - \\
\hline BIS BAS Scales - BIS Scale & - & -.02 \\
\hline SPSRQ - SP & - & -.03 \\
\hline SPSRQ - SR & - & .11 \\
\hline PID-5 Overall & - & .00 \\
\hline PID-5-BF - Adult Negative affect & - & -.02 \\
\hline PID-5-BF - Adult Detachment & - & -.11 \\
\hline PID-5-BF - Adult Antagonism & - & .00 \\
\hline PID-5-BF - Adult Disinhibition & - & .00 \\
\hline PID-5-BF - Adult Psychoticism & - & .08 \\
\hline
\end{tabular}

Note. BAI = Beck Anxiety Inventory; GAD-7 = Generalised Anxiety Disorder 7-Item Scale; PSWQ = Penn State Worry Questionnaire; BDI-II = Beck Depression Inventory-II; IDS-SR = Inventory of Depressive Symptomatology - Self Report; MASQ = Mood and Anxiety Symptom Questionnaire; GM = general distress - mixed scale; GA = general distress - anxiety scale; $\mathrm{AA}=$ anxious arousal scale; $\mathrm{GD}=$ general distress - depression scale; $\mathrm{AD}=$ anhedonic depression scale; $\mathrm{BFAS}=\mathrm{Big}$ Five Aspects Scales; BIS/BAS Scales = Behavioral Inhibition System/Behavioral Activation System Scales; BIS Scale = behavioral inhibition subscale; SPSRQ = Sensitivity to Punishment and Sensitivity to Reward Questionnaire; SP = sensitivity to punishment scale; SR = sensitivity to reward scale; PID-5-BF - Adult = Personality Inventory for DSM-5 Brief Form Adult.

$* p<.01$, two tailed. 
As can been seen from the correlations presented in Table 20, there were no significant correlations between any of the variables. Given that Avila and Parcet (2001) found a significant correlation between SSRT and the sensitivity to punishment scale of the SPSRQ using only female participants, following Avila and Parcet we repeated the correlational analysis using SSRT and the sensitivity to punishment scale of the SPSRQ including only female participants in the analysis. There was no significant correlation between SSRT and the sensitivity to punishment scale of the SPSRQ $(n=74)(r=.04, p=$ .74)

\section{Discussion}

Study 1 aimed to overcome methodological problems identified in previous studies by administering a typical stop signal task design to participants high and low in trait anxiety who did not suffer from comorbid anxiety or other disorders. There were no group differences on the SSRT measure of inhibitory control, or other stop signal task dependent variables. Study 2 used an online version of the stop signal task administered to a larger sample of participants. Using a correlational design, Study 2 also found no association between the STAI trait anxiety measure and stop signal task performance. Similarly, no association between stop signal task performance and other self-report measures of anxiety, depression and personality was identified.

The absence of SSRT differences between individuals high and low in trait anxiety is consistent with previous research (Avila \& Parcet, 2001; Epstein et al., 2001; Savostyanov et al., 2009; Savostyanov et al., 2011). Given the consistency of findings, there does not appear to be support for the hypothesis, derived from attentional control theory, that individuals high in trait anxiety have deficient inhibitory control when compared to individuals low in trait anxiety. Attentional control theory also hypothesises that effects on inhibitory control might be obscured by compensatory strategies such as using increased effort and processing 
resources in order to perform the task. Use of compensatory strategies should be reflected in anxiety's effects on measures of processing efficiency such as reaction time. In the current study there were no differences in SSRT or mean go reaction time between the groups. Both measures of reaction time reflect processing efficiency, potentially capturing the speed/accuracy trade-off measured by the stop signal task and other speeded inhibitory control tasks. The absence of any group differences in SSRT or mean go reaction time might suggest these measures are not sensitive to detecting the hypothesised processing efficiency deficits. Alternatively it is possible that, contrary to the hypothesis proposed by attentional control theory, individuals high in trait anxiety do not need to use compensatory mechanisms because they do not experience difficulty performing the stop signal task. Attentional control theory proposes that, as overall task demands increase, it becomes more difficult for anxious individuals to compensate for impaired efficiency and decrements in performance become greater (M. W. Eysenck et al., 2007). If this is the case, designing and using more difficult stop signal tasks may elicit performance effectiveness decrements in individuals high in anxiety. In addition, instead of using SSRT as a measure of processing efficiency, it may be possible to observe processing efficiency deficits in patterns of neural activation. Altered patterns of neural activation in individuals high in anxiety have been observed in studies that have used stop signal tasks and other types of tasks (Tahereh L. Ansari \& Derakshan, 2011a, 2011b; Basten et al., 2011; Fales et al., 2008; Osinsky, Alexander, Gebhardt, \& Hennig, 2010; Osinsky, Gebhardt, Alexander, \& Hennig, 2012; Righi et al., 2009; Savostyanov et al., 2009; Savostyanov et al., 2011; Sehlmeyer et al., 2010), both in conjunction with behavioural task performance differences (Tahereh L. Ansari \& Derakshan, 2011a, 2011b; Basten et al., 2011; Sehlmeyer et al., 2010) and in the absence of any behavioural differences (Fales et al., 2008; Osinsky et al., 2010; Osinsky et al., 2012; Righi et al., 2009; Savostyanov et al., 2009; Savostyanov et al., 2011). Measuring these neural changes using EEG (e.g., Savostyanov et 
al., 2009; Savostyanov et al., 2011) or functional magnetic resonance imaging (e.g., Basten et al., 2011; Fales et al., 2008) during stop signal tasks is an avenue for future research.

Consistent with findings from Study 1, Study 2 failed to find an association between trait anxiety and stop signal task performance. In addition, consistent with findings from previous studies that failed to find an association between SSRT and traits related to anxiety such as behavioural inhibition (Eriksson et al., 2016; Knyazev et al., 2008), the current study failed to find an association between SSRT and any other self-report measure related to anxiety (e.g., behavioural inhibition and sensitivity to punishment). However, this finding is not consistent with results of a previous study that found an association between SSRT and sensitivity to punishment as measured by the SPSRQ (Avila \& Parcet, 2001).

Avila and Parcet (2001) found an association between SSRT and sensitivity to punishment and failed to find an association between SSRT and trait anxiety or between SSRT and the closely related personality trait of neuroticism, although both sensitivity to punishment and neuroticism were positively associated with trait anxiety. A comparison of the task and sample characteristics in Avila and Parcet with the task and sample characteristics in the current study does not clarify the source of the contrary findings. For example, although Avila and Parcet used only female participants, when we repeated our analysis using only female participants the results did not change. One possible explanation for the contrary findings is the sample size used in the study by Avila and Parcet. That study used forty five participants and the size of the correlation between SSRT and sensitivity to punishment was -.30. Forty five participants is a small sample for a correlational study. Small sample sizes result in low statistical power which leads to unreliable findings with inflated effect sizes that cannot be replicated (Button et al., 2013).

Limitations of the current study include the use of different platforms and methods of administration of the stop signal task in Studies 1 and 2. Study 1 was administered using E- 
Prime ${ }^{\circledR}$ software whereas Study 2 was administered using JavaScript. In addition, Study 1 was conducted in a laboratory setting whereas Study 2 was conducted as a web-based experiment at an off-site location of participants' choosing. There are a number of disadvantages of web-based compared to laboratory experiments; these include less experimental control, participants participating more than once, participant dropout, inability to clarify or confirm participant understanding, and variability in technology (e.g., computer speeds affecting reaction time measurement) (Birnbaum, n.d.). In order to ensure variability in technology - such as participants using different computers with different size processors did not increase error variance, we ran the experiment on various computers at different locations and found no significant difference in reaction time measurement across computers. Due to the inability to confirm participant understanding during the web-based administration, instructions were simplified to ensure understanding.

A further limitation of Study 1 was that it used a small sample. Using small samples leads to higher likelihood of obtaining false negative results and it reduces the probability that an observed effect actually reflects a true effect (Button et al., 2013). While it is possible that the small sample in Study 1 produced a false negative result, the absence of an association was again observed using a larger sample in Study 2. Although Study 2 used a larger sample, it may be argued that a sample of 99 is still relatively small for a correlational study. Considering the small effect sizes obtained, an unfeasibly large sample would have been required to adequately increase the power of the study. For example, with an effect size of .02 , to obtain power of .64 a sample size of 10,000 would be required. With an effect size of .11 , to obtain power of .80 a sample size of 500 would be required. Another potential limitation of Study 2 was that, when compared with Study 1, more generous inclusion criteria were used, i.e., participants with comorbid conditions (e.g., clinical anxiety, depression) were not excluded. Although using participants with comorbid conditions may increase error 
variance, it also increases generalizability of findings (e.g., Blanco et al., 2008; le Strat, Rehm, \& le Foll, 2011; Sharpe, 2002; Storbjörk, 2014). Trait anxiety is associated with a range of clinical conditions including anxiety disorders and depression (J. A. Chambers et al., 2004; Sandi \& Richter-Levin, 2009), so including participants with comorbidities increases the likelihood that results apply to individuals in the general community. Finally, although the hypotheses in these studies were derived from attentional control theory, we did not aim to or directly test mechanisms that may underlie this theory.

In conclusion it appears that trait anxiety is not associated with impairments in inhibitory control as measured by the stop signal task; however, future studies may wish to develop and use more difficult stop signal task designs. Using more difficult tasks may elicit behavioural performance deficits in highly anxious individuals. It remains to be seen whether differences in stop signal task performance can be detected using other measures of enhanced effort, such as neural activation (e.g., Savostyanov et al., 2009; Savostyanov et al., 2011). 


\section{Chapter 5}

\section{The Effect of Reward and Punishment on Inhibitory Control in Anxiety - the Monetary Incentive Control Task}

Anxiety disorders are the most common class of psychological disorder, affecting at least one out of nine adults in any given year (Baxter et al., 2013; Kessler et al., 2005). All anxiety disorders are characterised by avoidance behaviours (American Psychiatric Association, 2013a) that involve avoidance of what the individual perceives to be aversive or threatening situations, even though those situations may hold the potential to be rewarding. It is well-established that individuals who suffer from both anxiety disorders and subclinical or trait anxiety have an attentional bias toward threatening sources of information (see BarHaim et al., 2007; Cisler \& Koster, 2010, for review), possibly because their attention toward the threat is facilitated or because they experience difficulty disengaging attention from the threat. What is not yet well understood is whether the avoidance observed in anxiety is associated with reduced sensitivity to positive/rewarding signals, increased sensitivity to negative/punishing signals, or both.

Over the past 60 years, the human reward network has been extensively investigated (see Haber \& Knutson, 2010; Pujara \& Koenigs, 2014, for review). It is comprised of cortical (e.g., orbitofrontal cortex (OFC), anterior cingulate cortex (ACC), dorso-medial prefrontal cortex, lateral frontoparietal areas) and subcortical (e.g., ventral striatum, substantia nigra, ventral tegmental area, ventral pallidum, thalamus, amygdala, and hippocampus) structures that are functionally connected in a cortico-basal ganglia network. One task that is widely used with functional magnetic resonance imaging (fMRI) to investigate this network in humans is the monetary incentive delay task. During the task participants are presented with one of three cues that signal potential monetary rewards, monetary losses or no incentive. Participants respond to a stimulus with a button press and, based on time taken to respond, 
they receive feedback indicating a gain, a loss or no money. Anticipation of reward during the monetary incentive delay task activates the caudate, putamen, medial prefrontal cortex and left motor cortex (Knutson et al., 2000). In contrast to the reward network, the network that processes aversive signals or punishment has not been as thoroughly described but is thought to include the ACC, dorsolateral prefrontal cortex (DLPFC) (Liu et al., 2011), posterior medial frontal cortex (S. H. Kim et al., 2014), lateral OFC, anterior insula, amygdala, and ventral striatum (Seymour et al., 2007). Anticipation of punishment during the monetary incentive delay task has been shown to activate the same regions as anticipation of reward, with the addition of the thalamus and anterior cingulate (Knutson et al., 2000).

Anxiety is associated with dysfunction in a number of structures that are also involved in processing rewards and punishments. For example, anxiety is associated with hyperactivity of the amygdala (Bishop et al., 2004; Dickie \& Armony, 2008; Etkin et al., 2004; Ewbank et al., 2009; Rosen \& Schulkin, 1998), and insula (Alvarez et al., 2015; Dillon et al., 2014; Etkin \& Wager, 2007; Paulus \& Stein, 2006; Shin \& Liberzon, 2010), altered function and connectivity of the ventromedial prefrontal cortex (vmPFC) and amygdala (Grupe \& Nitschke, 2013; M. Justin Kim et al., 2011; M. J. Kim \& Whalen, 2009; Patel et al., 2012; Pezawas et al., 2005; Rauch et al., 2006) and altered structure, function, volume, connectivity of the dorsal anterior cingulate cortex (dACC) (Aarts \& Pourtois, 2010; Bystritsky et al., 2001; Hajcak et al., 2003; Kimbrell et al., 1999; Shilpi Modi et al., 2014; Rauch et al., 1995; Rauch et al., 1996; Shin et al., 2001). Given the overlap between neural structures and networks implicated in anxiety and incentive processing, it is perhaps not surprising that anxiety involves abnormal processing of rewards and punishments (e.g., Admon et al., 2013; Cremers et al., 2015; Manning et al., 2015; Teng et al., 2016). Processing of threat-related stimuli by individuals high in trait anxiety has been associated with increased insula, amygdala and ACC activation, and decreased functional connectivity between cortical and 
subcortical structures (Comte et al., 2015; Etkin et al., 2004; Stein et al., 2007). People high in trait anxiety also react faster when identifying neutral faces that are preceded by masked fearful faces compared to masked neutral faces (Etkin et al., 2004). A number of anxiety disorders have been associated with abnormal responses of the amygdala, nucleus accumbens (Admon et al., 2013) and putamen (Cremers et al., 2015), as well as abnormal connectivity between the striatum and prefrontal and posterior brain regions (Cremers et al., 2015; Manning et al., 2015) when participants are either at rest (Manning et al., 2015) or when processing rewards and punishments (Admon et al., 2013; Cremers et al., 2015). Behaviourally, people with symptoms of post-traumatic stress disorder (PTSD) appear to be more punishment sensitive because PTSD symptoms are associated with less risky choices (Admon et al., 2013), but people with generalized anxiety disorder (GAD) have been shown to be slower at learning to avoid punishing stimuli (Teng et al., 2016).

The behavioural inhibition system (BIS), a biologically-based system that underpins a trait known as behavioural inhibition, is sensitive to cues of punishment, non-reward and novelty (Amodio et al., 2008; Gray \& McNaughton, 2000), and is associated with higher scores on measures of anxiety (Cooper et al., 2007; Hundt et al., 2007). Individuals who score higher on measures of behavioural inhibition show increased striatal activation when anticipating reward and punishment compared to individuals who score lower on these measures (Bar-Haim et al., 2009; Benson et al., 2014; Guyer et al., 2014; Guyer et al., 2006). Compared to individuals who score lower on measures of behavioural inhibition, they also have larger electrophysiological responses to errors and punishing feedback during tasks where they are required to inhibit their response (M. A. S. Boksem et al., 2008; De Pascalis et al., 2010; Leue et al., 2012), reduced striatal activation when receiving rewarding feedback (Guyer et al., 2014; Helfinstein et al., 2011; Simon et al., 2010), and increased caudate activation in the absence of reward (Helfinstein et al., 2011). Behaviourally, they show 
slower responses following punished compared to non-punished trials during flanker tasks (M. A. S. Boksem et al., 2008; Braem et al., 2013). Taken together, these findings suggest individuals who are high in behavioural inhibition are more sensitive to punishment than individuals who are low in behavioural inhibition.

Behavioural avoidance of what could potentially be rewarding events and situations presumably involves an increase in inhibitory control, which is the ability to withhold, postpone or cancel an action (Bari \& Robbins, 2013). Voluntary inhibition of a physical or motor response is a sub-component of cognitive control - the ability to coordinate sensory and motor processes toward a common goal (Miller \& Cohen, 2001). Studies that have used inhibitory control tasks such as the stop signal task, antisaccade task, go/no-go task, Stroop task and variants of the flanker task (e.g., attention network test) to investigate whether anxiety is associated with improvements in inhibitory control have delivered mixed results. Some studies have found enhanced inhibitory control (e.g., Sehlmeyer et al., 2010) and others have found poorer inhibitory control (e.g., Tahereh L. Ansari \& Derakshan, 2011b; Hallion et al., 2017) or no difference in inhibitory control (e.g., Epstein et al., 2001; Hallion et al., 2017) in higher compared to lower anxious individuals.

Influential cognitive theories such as attentional control theory (Derakshan \& Eysenck, 2009; M. W. Eysenck \& Derakshan, 2011; M. W. Eysenck et al., 2007) suggest that individuals high in anxiety should experience impairment rather than enhancement of inhibitory control. According to attentional control theory, this impairment occurs because anxiety involves increased allocation of attention to potential sources of threat, which reduces attentional focus on a task. The reduction in attentional focus affects attentional control, which is required in order to successfully inhibit a task-irrelevant response. Such theories have not considered whether behavioural avoidance is associated with improved inhibitory control. It is possible that, because individuals with anxiety and traits closely related to 
anxiety (e.g., behavioural inhibition) appear to be more sensitive to punishment, when avoidance of a threat/punishment is the goal of the task, they may become focused on avoiding the threat and show enhanced inhibitory control. However, when the goal of the task is to inhibit a response and a threat is present but task-irrelevant, anxiety may lead to impaired inhibitory control due to facilitated attention toward and difficulty disengaging attention from the threat.

Situations that involve a perceived threat may be conceptualised as punishing; punishments are aversive events that individuals expand energy to avoid or to reduce the likelihood of occurring (Seymour et al., 2007). The effect of presenting cues and feedback signalling punishment and/or reward on inhibitory control in individuals who score high on measures of anxiety, such as trait anxiety, as well as in individuals who experience anxiety disorders is not yet clear. While numerous studies have investigated effects of cues and feedback indicating reward and punishment on the behaviour and neural activity of adults and adolescents high in personality traits of shyness and behavioural inhibition (Bar-Haim et al., 2009; Guyer et al., 2014; Guyer et al., 2006; Hardin et al., 2006; Helfinstein et al., 2011; S. H. Kim et al., 2014; Simon et al., 2010), few studies have used individuals high in trait anxiety or anxiety disorders (Benson et al., 2014; Guyer et al., 2012), and there appears to be only limited research examining rewards and punishments specifically with inhibitory control tasks (M. A. S. Boksem et al., 2008; Braem et al., 2013; De Pascalis et al., 2010; Hagopian \& Ollendick, 1994; Hardin et al., 2007; Jazbec et al., 2005; Leue et al., 2012). One of the most common tasks used to measure inhibitory control is the stop signal task. This task specifically measures cancellation of a response that has already started, or action cancellation. Stopping during the stop signal task has been shown to reliably activate the right inferior frontal cortex (rIFC) (Aron, 2011), the pre-supplementary motor area (preSMA) (Aron, 2011; Chao et al., 2009; C. S. R. Li et al., 2006; D. J. Sharp et al., 2010), and 
the anterior insula (Erika-Florence et al., 2014; Hampshire, 2015). Other areas that appear to be involved in action cancellation during the stop signal task include the right dorsal premotor cortex, the right temporo-parietal junction (TPJ), the anterior midcingulate cortex and the posterior cingulate cortex (PCC) (Cieslik et al., 2015; Swick et al., 2011). The few studies that have used a stop signal task to investigate the neural mechanisms of inhibitory control in anxious individuals have found that individuals high in trait anxiety appear to have greater electroencephalogram (EEG) desynchronization after stop signal onset on successful trials than individuals low in trait anxiety (Savostyanov et al., 2009; Savostyanov et al., 2011). This finding suggests that individuals high in trait anxiety may need to use more effort to successfully stop their response.

In other types of inhibitory control tasks such as the Stroop task, trait anxiety has been associated with higher DLPFC activation in incongruent compared to congruent trials but weaker functional connectivity of the right DLPFC with areas of the stopping network (Basten et al., 2011). Higher DLPFC activity may reflect additional effort needed to compensate for weaker connectivity of the DLPFC within the task-relevant network (Basten et al., 2011), or greater noise caused by lack of efficient connectivity. In contrast, trait anxiety has also been associated with reduced DLPFC activation during processing of response conflict under conditions of low but not high perceptual load (Bishop, 2009; see also Forster et al., 2015). Reconciling these contradictory findings, it is possible that anxious individuals experience decreased goal-related (proactive) control (Forster et al., 2015), as well as decreased transient activation of regions such as the DLPFC that are responsible for goal directed attention (i.e., reactive control), when a task does not fully occupy attentional resources (Bishop, 2009; Forster et al., 2015). When task difficulty increases, these individuals increase transient, trial-dependent activation of cognitive control networks (Basten et al., 2011; Fales et al., 2008), perhaps to compensate for decreased sustained, goal- 
directed activation of these networks (Fales et al., 2008). Overall, it appears that individuals high in anxiety may experience difficulties implementing cognitive control, perhaps due to interference by task-irrelevant thoughts and weak connectivity in cognitive control networks. The effect of presenting rewarding and punishing information on the operation of these networks is not yet clear.

The current study examines the effect of presenting cues and feedback indicating reward and punishment on inhibitory control in individuals high in trait anxiety using the monetary incentive control task (MICT). The MICT is a variant of the stop signal task combined with the rewarding, punishing and neutral conditions of the monetary incentive delay task. Trait anxiety is a relatively stable and enduring difference among individuals to perceive stressful situations as dangerous or threatening (Spielberger et al., 1983). It is elevated in individuals who meet criteria for anxiety disorders (Bieling et al., 1998; J. A. Chambers et al., 2004) and predicts future anxiety disorder diagnosis (Plehn \& Peterson, 2002). In the current study, trait anxiety was measured using the State Trait Anxiety Inventory, trait anxiety scale (STAI T-Anxiety scale) (Spielberger et al., 1983). The MICT assesses the ability of participants to stop a response under reward, punishment and neutral conditions. The task requires participants to control (i.e., stop) their response to a go stimulus that, throughout the task, is associated with reward. On stop trials, if the participant incorrectly makes a response, the task will provide monetary reward (reward condition), punishment (punishment condition), or no money (neutral condition). Individuals who are reward sensitive should experience difficulty stopping particularly in the reward condition, and individuals who are punishment sensitive should not be influenced by the potential for reward on stop trials and should show greater inhibitory control when an incorrect response could be punished (punishment condition). The task does not provide any trial-by-trial reward for correct stopping but delivers a large reward at the end of the task if participants are able to 
successfully stop their response on $60 \%$ of stop trials in the reward and punishment conditions. This delayed reward is included to incentivise participants to control their response to a rewarding stimulus.

In relation to hypotheses concerning effects of high trait anxiety on behavioural performance in the MICT, attentional control theory predicts that, due to increased allocation of attention to all sources of threat which includes any type of failure on the task, individuals high in anxiety should experience a reduction in attentional focus, attentional control and inhibitory control across all conditions. However, individuals high in behavioural inhibition a trait closely related to anxiety (Cooper et al., 2007; Hundt et al., 2007) - have been shown to be more sensitive to punishment (M. A. S. Boksem et al., 2008; Braem et al., 2013; De Pascalis et al., 2010; Leue et al., 2012). Given that behavioural inhibition is related to anxiety, it was predicted that individuals high in trait anxiety will also be sensitive to punishment and will therefore attempt to avoid punishment during punishment condition trials. This will result in increased inhibitory control (i.e., increased stop accuracy) on punishment trials compared to reward and neutral trials.

In relation to hypotheses concerning effects of high trait anxiety on neural activity during the MICT, during stop trials in all conditions individuals high in trait anxiety may have increased activation in cognitive control, goal-directed attentional networks (e.g., DLPFC and ACC) due to the need to compensate for decreased proactive control caused by increased attention to external and internal sources of threat (e.g., worry, rumination etc.) (Basten et al., 2011; Fales et al., 2008). Given that response inhibition as measured by the stop signal task is conceptualised as a sub-component of cognitive control and the neural networks that subserve these functions often overlap (Goghari \& MacDonald Iii, 2009; Levy $\&$ Wagner, 2011), the increase in reactive control may also result in increased activation in inhibitory control networks and structures generally activated during stop trials in the stop 
signal task (e.g., rIFC and pre-SMA). In relation to the anticipation epoch it is predicted that, because individuals high in trait anxiety may be sensitive to punishment, when they are anticipating potential punishment they will show increased activation in punishment-related networks including the striatum (e.g., Guyer et al., 2006) compared to individuals low in trait anxiety. In relation to the feedback epoch it is predicted that, when receiving feedback indicating punishment, individuals high in trait anxiety will show increased activation in punishment-related networks (e.g., Helfinstein et al., 2011) as well as in networks and structures involved in cognitive control, such as the ACC (e.g., M. A. S. Boksem et al., 2008; De Pascalis et al., 2010; Leue et al., 2012).

\section{Method}

\section{Participants}

A total of 49 (18 males, 31 females) participants were recruited through a University of Melbourne first year psychology research program and advertisements on careers and other (e.g., Gumtree) websites. Data from one participant was excluded from analysis due to MICT go reaction time three standard deviations above the group mean, therefore data from 48 participants ( 17 males, $M_{\text {age }}=22.52$ years, $S D=4.51$, age range 18.20 to 35.52 years) was analysed. All participants were right-handed as assessed by the Edinburgh Handedness Inventory (Oldfield, 1971), had normal or corrected-to-normal eyesight, were English speakers, had an IQ above 80 as measured by the Wechsler Test of Adult Reading, had no history of alcohol or substance abuse, no current use of psychoactive medication, no structural brain abnormalities or magnetic resonance imaging (MRI) contraindications, and no history of head injury, inpatient psychiatric care or neurological or psychiatric disorders. Based on STAI T-Anxiety scores, participants were assigned to the low trait anxiety or the high trait anxiety group (see details below). The groups did not significantly differ on the variables of age, years of education or IQ but did significantly differ on the variable of 
gender, $\chi^{2}(1)=4.463, p=.035$. Although the groups significantly differed in relation to gender, men and women do not typically differ in behavioural performance on stop signal tasks (Huster, Westerhausen, \& Herrmann, 2011; C. s R. Li, C. Huang, R. T. Constable, \& R. Sinha, 2006; Li et al., 2009; Mühlberg, Mathar, Villringer, Horstmann, \& Neumann, 2016) or in brain activation during response inhibition as indexed by stop signal reaction time (SSRT) (Li et al., 2009). In addition, there was no significant SSRT difference between males and females in the stop signal task in Study 1 of the current research project (described in the previous chapter $)\left(M_{\text {males }}=189.84 S D=52.35, M_{\text {females }}=200.29 S D=48.79, t(58)=-.75, p=\right.$ $.45)$ or in Study 2 of the current research project (described in the previous chapter) $\left(M_{\text {males }}=\right.$ $\left.280.58 S D=36.41, M_{\text {females }}=270.64 S D=51.57, t(97)=-.89, p=.38\right)$.

Descriptive statistics for each group are displayed in Table 21. 
Table 21

Descriptive Statistics for Low Trait Anxiety and High Trait Anxiety Groups

\begin{tabular}{lccc}
\hline \multicolumn{1}{c}{ Variables } & $\begin{array}{c}\text { Whole sample } \\
(N=48)\end{array}$ & $\begin{array}{c}\text { Low trait anxiety } \\
(n=24)\end{array}$ & $\begin{array}{c}\text { High trait anxiety } \\
(n=24)\end{array}$ \\
\hline Age (years) & $22.52(17.32)$ & $23.51(5.08)$ & $21.53(3.70)$ \\
Age range (years) & 18.20 to 35.52 & 18.20 to 35.52 & 18.42 to 30.84 \\
Gender (male/female) & $17 / 31$ & $12 / 12$ & $5 / 19$ \\
Education (years) & $14.51(2.59)$ & $14.65(2.42)$ & $14.38(2.78)$ \\
IQ (WTAR) & $108.60(6.14)$ & $109.13(5.41)$ & $108.08(6.88)$ \\
BDI-II & $13.24(13.27)$ & $2.88(2.33)$ & $23.2(11.67)$ \\
STAI T-Anxiety scale score & $41.71(17.02)$ & $25.54(2.47)$ & $57.88(6.38)$ \\
(Time 1) & range 20 to 75 & range 20 to 29 & range 49 to 75 \\
STAI T-Anxiety scale score & $38.85(15.34)$ & $24.54(2.89)$ & $53.17(6.72)$ \\
(Time 2) & range 20 to 72 & range 20 to 29 & range 44 to 72 \\
\hline
\end{tabular}

Note. Except for gender which is a count, numbers represent means with standard deviation in parentheses. WTAR, Wechsler Test of Adult Reading; BDI-II, Beck Depression Inventory-II; STAI T-Anxiety scale; State Trait Anxiety Inventory trait anxiety scale.

All participants provided written informed consent and the experiment was approved by the human research ethics committee at the University of Melbourne. Participants were paid an honorarium for completing the second session and the fMRI portion of the study. The University of Melbourne first year psychology research program students received course credit for completing the screening measures.

\section{Materials and Procedure}

Eight hundred and thirty five individuals were initially screened for inclusion in the study using the STAI T-Anxiety scale. It was necessary to screen a large number of participants because, in the population sampled, very few trait anxiety scores were low enough to fulfil the inclusion criterion of low trait anxiety (see details below), and many individuals who scored high on the STAI T-Anxiety scale did not fulfil other inclusion criteria (e.g., they were taking psychoactive medication or had a history of psychiatric disorders). Participants completed the screening measures using the Qualtrics Research Suite 
either on the University campus or at an off-campus location of their choosing. During the screening and subsequent sessions they completed a number of other instruments, including the Edinburgh Handedness Inventory (Oldfield, 1971), the Beck Depression Inventory-II (BDI-II; Beck et al., 1996), the Alcohol Use Disorders Identification Test (AUDIT; Babor et al., 2001), the Fagerstrom Test for Nicotine Dependence (Fagerstrom et al., 1990), a demographic information questionnaire and a MRI eligibility information questionnaire. Non-copyrighted questionnaires are reproduced in Appendix A and Appendix B. Forty-one participants who scored at least one standard deviation below either the Australian mean for the STAI T-Anxiety scale (Crawford et al., 2011) or the sample mean $\left(M_{\text {sample }}=42.51, S D=\right.$ 10.46) and 32 participants who scored at least one standard deviation above either the Australian mean for the STAI T-Anxiety scale or the sample mean were invited to participate in the next part of the study. In a second session, these participants attended the university campus to complete a pen-and-paper version of the STAI T-Anxiety scale to ensure their initial score had remained stable (e.g., Aarts \& Pourtois, 2012). They also completed a number of online questionnaires, a stop signal task, the Wechsler Test of Adult Reading to measure IQ, the Orygen Substance Use Index to measure substance use, a structured face-toface interview and one practice block of the MICT to ensure they understood and were able to perform the task in the MRI scanner. Non-copyrighted questionnaires are reproduced in Appendix A and Appendix B. Twenty four participants were excluded due to issues such as an increase or decrease in STAI T-Anxiety score that meant they no longer fulfilled the inclusion criterion of low or high trait anxiety, a history of substance use, current use of psychotropic medications, psychiatric disorder or MRI contraindications that had not been identified during initial screening. The remaining 49 participants eligible for fMRI were instructed to abstain from alcohol and illicit drug use for 48 hours prior to the MRI scan and not to consume nicotine or caffeine for three and one hour, respectively, prior to the MRI 
scan. Upon arrival at the MRI scanner participants completed the STAI S-Anxiety scale, other questionnaires and one practice block of the MICT. Following the MRI scan participants completed the STAI S-Anxiety scale and were thanked, paid and debriefed.

\section{Apparatus and Experimental Task}

Participants completed the MICT that was designed to investigate response inhibition under conditions of monetary reward and punishment. Participants completed one version of the task during a behavioural screening session (Practice Version 1), another version during a practice session immediately prior to entering the MRI scanner (Practice Version 2), and a third version during the MRI scanning session (Main Version).

For the Practice Version 1 and Practice Version 2, stimuli were presented in 24 bit colour on a laptop computer. For the Main Version stimuli were presented in 24 bit colour by either an overhead projector or on a computer monitor (method of presentation was changed mid-way through the study due to changes in set up at the scanning facility but these changes did not have any measurable effect on task performance). Participants viewed the screen/monitor via a mirror attached to the 32 channel head coil. Stimulus presentation was controlled by E-Prime ${ }^{\circledR} 2.0$ software (version 2.0, Psychology Software Tools, Inc. Pittsburgh, PA), running on an IBM-compatible PC. For the Main Version, the PC was interfaced with the MRI scanner during acquisition of MRI data. Responses were recorded using a magnetic resonance compatible two-button response box (Fibre-Optic response pad, Current Designs, Philadelphia, PA, USA). Stimuli were presented on a black background with all items appearing in white or red font. The stimuli appeared in the middle of the screen.

The MICT consisted of go trials and stop trials. Go trials involved presentation of the letter X or O centrally on a black background. Participants were asked to respond to go trials by pressing a corresponding button as quickly as possible upon stimulus presentation. In 
Practice Version 1 and Practice Version 2 the letters $\mathrm{X}$ and $\mathrm{O}$ were mapped to the keyboard buttons $\mathrm{X}$ and $\mathrm{O}$, respectively, while in the Main Version the letters $\mathrm{X}$ and $\mathrm{O}$ were mapped to left and right buttons on the button box, respectively. The stop stimulus was a white box that surrounded the go stimulus. During stop trials the stop stimulus indicated that the participant was to refrain from pressing a button. Three experimental conditions were administered: reward, punishment and neutral. Each condition was indicated at the commencement of a trial via presentation of a white dollar sign (\$) (reward condition), red dollar sign (\$) (punishment condition) or white dash (-) (neutral condition). In the reward condition, participants received a 20c reward for a button press while the stimulus was presented (a response time less than $400 \mathrm{~ms}$ ), regardless of whether the trial was a go trial (reward for correct button press) or stop trial (reward for any button press). In the punishment condition, participants received a 20c reward for an accurate button press during go trials, but received a 20c penalty for any button press during stop trials. Correct withholding of a response on stop trials in both the reward and punishment conditions did not incur a reward or penalty. In the neutral condition participants did not receive any reward or penalty during go or stop trials. Participants were instructed to attempt to withhold their response on all stop trials regardless of the condition (i.e., even though they would receive a 20c reward for a button press on a stop trial in the reward condition). Table 22 displays the feedback given in each condition. 
Table 22

Feedback Provided to Participants in the MICT in Each of the Reward, Punishment and Neutral Conditions Following Go and Stop Trials

\begin{tabular}{|c|c|c|c|c|}
\hline \multirow[t]{3}{*}{ Condition } & \multicolumn{2}{|c|}{ Go trial } & \multicolumn{2}{|c|}{ Stop trial } \\
\hline & \multicolumn{4}{|c|}{ Responses that precede feedback } \\
\hline & $\begin{array}{l}\text { Correct button } \\
\text { press in less } \\
\text { than } 400 \mathrm{~ms}\end{array}$ & $\begin{array}{l}\text { No response / } \\
\text { incorrect button } \\
\text { press in less } \\
\text { than } 400 \mathrm{~ms} / \\
\text { correct or } \\
\text { incorrect button } \\
\text { press in more } \\
\text { than } 400 \mathrm{~ms}\end{array}$ & $\begin{array}{l}\text { Press any button } \\
\text { while stimulus is } \\
\text { on screen }(500 \mathrm{~ms})\end{array}$ & $\begin{array}{l}\text { No response } \\
\text { (correct } \\
\text { withhold) }\end{array}$ \\
\hline & \multicolumn{4}{|c|}{ Feedback presented } \\
\hline Reward & Hit 20c & Miss 0c & Wrong 20c & Correct $0 \mathrm{c}$ \\
\hline Punishment & Hit 20c & Miss 0c & Wrong lose 20c & Correct 0c \\
\hline Neutral & Hit $0 \mathrm{c}$ & Miss 0c & Wrong 0c & Correct $0 \mathrm{c}$ \\
\hline
\end{tabular}

To incentivise inhibitory control, rather than making a response during all reward trials (as participants may have decided to do given that a button press during a stop trial in the reward condition resulted in a 20c reward), participants were informed that if they could successfully withhold their response on $60 \%$ of stop trials in the reward and punishment conditions, they would be rewarded with an extra $\$ 20$ at the end of the task. Following each block of trials in the Main Version, participants were given feedback showing overall go trial accuracy and amount of money won. At the end of the task participants were given feedback showing stop accuracy for each of the conditions, amount of money won on go trials and total amount of money won. Figures 1 and 2 show the sequence of go and stop trials for the Main Version. See Appendix F for sequence of go and stop trials for Practice Versions 1 and 2. 


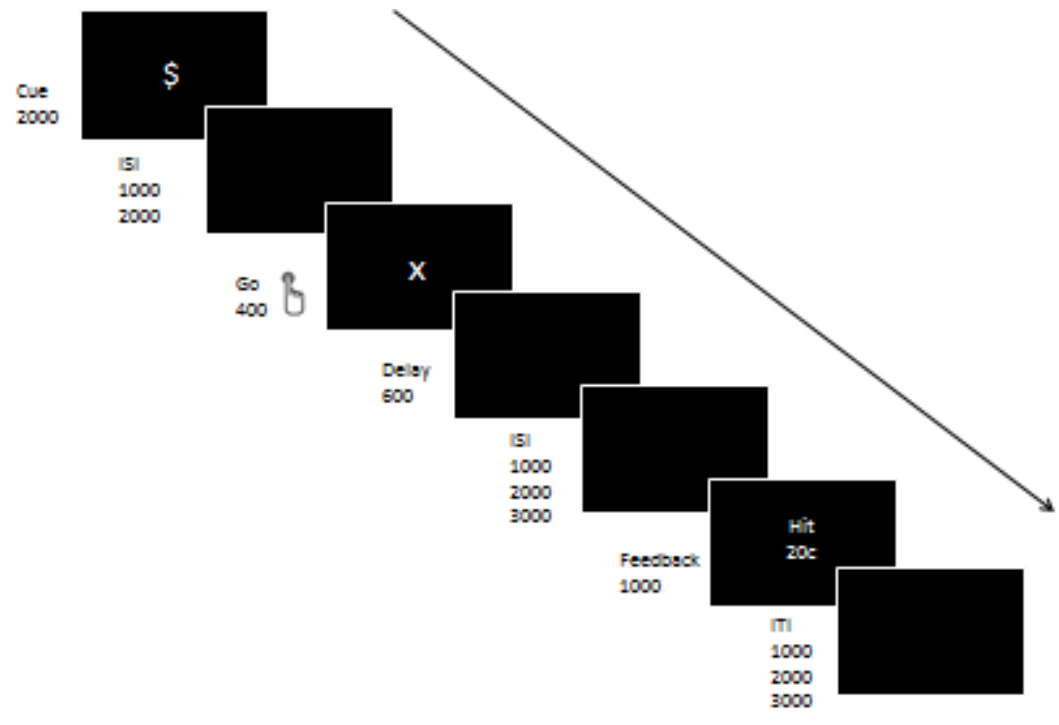

Figure 2. Schematic representation of a MICT reward condition go trial that has been accurately performed in the Main Version. Numbers representing the time are in milliseconds. 


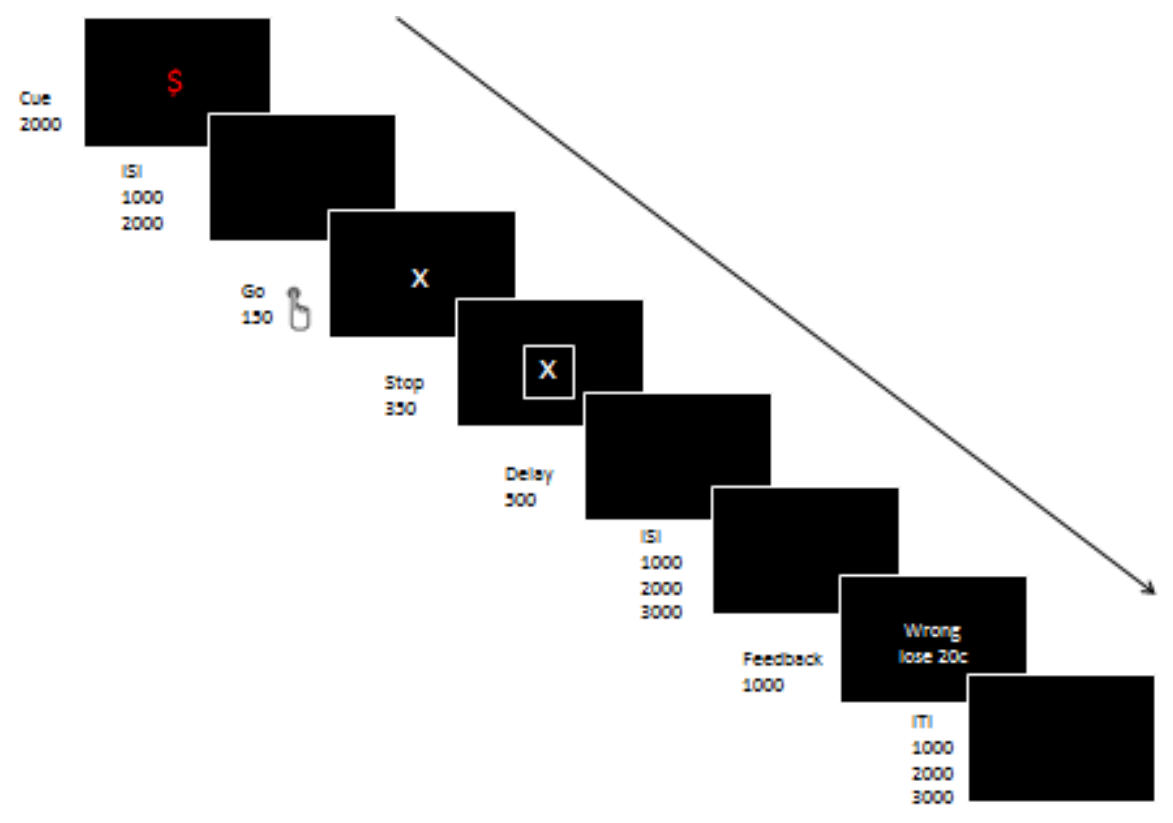

Figure 3. Schematic representation of a MICT punishment condition stop trial where the response has not been withheld in the Main Version. Numbers representing the time are in milliseconds.

In the Main Version, the variable interstimulus interval (ISI) and intertrial interval (ITI) had the effect of jittering the onset of each task epoch (cue period, stimulus period, feedback period) which is necessary for event-related fMRI designs in which blood oxygenated leveldependent (BOLD) changes are modelled for single trials (Hester, Murphy, Brown, \& Skilleter, 2010). The Main Version was comprised of seven blocks of 40 trials. Trial types and conditions were randomly presented using a ratio of 3 go trials to 1 stop trial. The high proportion of go trials established a prepotent 'go' response which required inhibition upon presentation of a stop trial (Ramautar, Kok, \& Ridderinkhof, 2004). Sixty nine reward, 70 punishment and 69 neutral trials were presented during the task. See Appendix G for task design of the Practice Versions. 


\section{Imaging Data Acquisition and Preprocessing}

Functional magnetic resonance images were acquired at the Murdoch Children's Research Institute, Royal Children's Hospital, Parkville, Australia using a Siemens 3T Trio Tim MRI scanner (Erlangan, Germany). One hundred and ninety four (gradient) echo-planar imaging (EPI) sequences providing T2* (time constant for loss of signal in sequence)weighted BOLD activity measurements were acquired for each functional run with the following parameters: repetition time (TR), 2000ms; echo time (TE), 35ms: flip angle (FA), $90^{\circ} ; 384 \mathrm{~mm} \times 384 \mathrm{~mm}$ field of view (FOV); and 32 contiguous slices of $4 \mathrm{~mm}$ slice thickness. Seven functional runs were collected for each participant. The first three volumes of each run were discarded prior to data analysis to account for equilibration effects in the magnetic field of the scanner. The task was an event-related design that allowed for analysis of BOLD response following specific events, e.g., successful inhibition to stop trials.

At completion of functional neuroimaging, high-resolution structural images were acquired using an MPRAGE T1-weighted sequence $[\mathrm{TR}=1900 \mathrm{~ms} ; \mathrm{TE}=2.63 \mathrm{~ms}, \mathrm{FA}=9$, slice thickness $=.80 \mathrm{~mm}$; in-plane resolution $=.9 \mathrm{~mm}$ isotropic $]$. During data processing, functional data were overlaid on the structural image for each participant to localize the pattern of physiological changes with the task time-series.

\section{Data Processing and Analysis}

Monetary incentive control task behavioural data. Behavioural data was assessed using Statistical Package for the Social Sciences (SPSS) 18.0.0. Analyses involved two independent variables, Condition (neutral v punishment v reward) and Group (low trait anxiety v high trait anxiety). Dependent variables were mean stop accuracy (correct withholding of response during a stop trial), mean go accuracy (correct button press within 1000ms after stimulus onset during a go trial), and mean go reaction time. Each dependent variable was subjected to a 3 (Condition: neutral v punishment v reward) x 2 (Group: low 
trait anxiety v high trait anxiety) mixed-design analysis of variance (ANOVA). Significance of effects (Bonferroni corrected for multiple comparisons) (Dunn, 1961) was tested using the conventional critical value, $\alpha<.05$. Where significant ANOVA results were obtained, posthoc $t$-tests were performed to determine any significant differences between means.

Functional magnetic resonance imaging data. Functional MRI data were processed using Analysis of Functional NeuroImages (AFNI) software (R. W. Cox, 1996). Following image reconstruction and concatenation of runs, the time-series data were time-shifted using Fourier interpolation to remove differences in slice acquisition times, aligned to corresponding anatomical data and warped to standard Talairach space. Activation outside the brain was removed using edge detection techniques. Motion was corrected twice using three-dimensional volume registration, the first time against the first image of each block and the second time against the middle image of the seven blocks. Volumes were blurred using a 4.1 mm full-width half max filter, each voxel was then scaled to a mean of 100 and values over 200 were clipped. To examine the influence of condition and group on inhibition performance, an event-related analysis was performed that estimated BOLD activity in anticipation of stop trials (cue period), during withholding of response (stop trial stimulus presentation), and during feedback on stop trials (stop trial feedback). Hemodynamic response functions were calculated using deconvolution for the cue period, stop trial stimulus presentation and stop trial feedback. Activity related to go trials and motion were modelled as additional regressors to avoid contamination of baseline and event-related data. TR pairs were censored where the Euclidian Norm of the motion derivative exceeded 1.0.

The baseline estimate was the mean activation recorded during the ongoing trial period (the ISI and delays in each trial), such that the activation observed during cue periods, stop trial stimulus presentation and stop trial feedback represent activation over and above that required for the ongoing trial period activity. The absence of collinearity between 
regressors within AFNI X-matrices was confirmed during deconvolution using xmat_tool.py. Event-related map voxels for each regressor of interest were extracted, resampled to anatomical data resolution $\left(1 \mathrm{~mm}^{3}\right)$, and masked using a group-averaged EPI mask dataset. Group activation maps were determined separately for the cue period, stop trial stimulus presentation and stop trial feedback via one-sample $t$-tests against the null hypothesis of zero event-related activation changes (i.e., no change relative to baseline). Significant voxels passed (a) a voxelwise statistical threshold ( $t=4.24, p \leq .001)$, and (b) a continuity threshold - part of a larger 176 microliter cluster of contiguous significant voxels. The combination of probability and cluster thresholding was used to maximize the power of the statistical test while holding the likelihood of false positives to a minimum. Simulation using the 3D ClustSim function in AFNI (AFNI Ver 16.2.11) and an uncorrected voxelwise threshold $p=$ .001 , indicated a minimum cluster size of 176 , given a threshold of $p=.01$ (Bonferroni corrected).

Activation clusters derived from this whole-brain analysis were used to compare activation across the three conditions (reward, punishment, neutral) and type of response (correct, error). The mean activation for clusters in this map was calculated for the purposes of a functionally-derived region of interest (ROI) analysis. Activation estimates between conditions were compared using a two-way mixed-design ANOVA, and corrected using a least squares difference (LSD) procedure for multiple comparisons. LSD rather than Bonferroni was used in order to balance the probability of type I and type II errors without overinflating the likelihood of type II errors (Saville, 1990). This ROI procedure was used with the cue-period epoch map to derive mean estimated activity for the cue period prior to successful and failed response inhibition across the three conditions. A similar procedure was used to calculate the stop-trial stimulus-period epoch map, which was then used to derive mean estimated activity for the response period during successful response inhibition across 
the three conditions. A feedback-period epoch map was calculated to derive mean estimated activity during feedback following successful and failed response inhibition across the three conditions.

\section{Results}

Data were examined for outliers and normality. Stop accuracy and go reaction time approximated a normal distribution for each condition but in all conditions go accuracy appeared to be negatively skewed. Data for go accuracy were transformed using a Log10 transformation but, as the transformation further skewed the data, analysis was performed on the original, untransformed data.

\section{Monetary Incentive Control Task}

Means and standard deviations for stop trial accuracy, go trial accuracy and go trial reaction times for correct and incorrect trials in each of the reward, punishment and neutral conditions for the low and high trait anxiety groups are listed in Table 23.

Table 23

Means and Standard Deviations for Go Trial Accuracy, Stop Trial Accuracy, and Go Trial Reaction Times (Correct and Error) for the Low and High Trait Anxiety Groups

\begin{tabular}{|c|c|c|c|c|}
\hline \multirow[t]{2}{*}{ Trial/Condition } & \multicolumn{2}{|c|}{ Low trait anxiety } & \multicolumn{2}{|c|}{ High trait anxiety } \\
\hline & $n$ & $M(S D)$ & $n$ & $M(S D)$ \\
\hline \multicolumn{5}{|l|}{ Go Accuracy } \\
\hline Reward & 24 & $.90(.10)$ & 24 & $.88(.13)$ \\
\hline Punishment & 24 & $.89(.10)$ & 24 & $.88(.14)$ \\
\hline Neutral & 24 & $.85(.13)$ & 24 & $.84(.20)$ \\
\hline \multicolumn{5}{|l|}{ Stop Accuracy } \\
\hline Reward & 24 & $.57(.24)$ & 24 & $.58(.25)$ \\
\hline Punishment & 24 & $.56(.22)$ & 24 & $.63(.22)$ \\
\hline Neutral & 24 & $.56(.21)$ & 24 & $.62(.27)$ \\
\hline Go Reaction Time Correct & & & & \\
\hline
\end{tabular}




\begin{tabular}{|c|c|c|c|c|}
\hline \multirow[t]{2}{*}{ Trial/Condition } & \multicolumn{2}{|c|}{ Low trait anxiety } & \multicolumn{2}{|c|}{ High trait anxiety } \\
\hline & $n$ & $M(S D)$ & $n$ & $M(S D)$ \\
\hline Reward & 24 & $374.22(26.40)$ & 24 & $396.75(34.00)$ \\
\hline Punishment & 24 & $376.34(23.01)$ & 24 & $398.89(31.25)$ \\
\hline Neutral & 24 & $379.29(31.01)$ & 24 & $409.63(51.89)$ \\
\hline \multicolumn{5}{|c|}{ Go Reaction Time Incorrect } \\
\hline Reward & 23 & $219.26(131.52)$ & 24 & $227.71(87.31)$ \\
\hline Punishment & 23 & $205.00(131.18)$ & 24 & $226.02(103.61)$ \\
\hline Neutral & 23 & $189.21(121.51)$ & 23 & $158.84(121.24)$ \\
\hline
\end{tabular}

Note: Accuracies are given as proportions, times are given in milliseconds.

Stop trial accuracy. In order to examine whether there were differences in stop trial accuracy between the two groups in each condition, stop trial accuracy was subjected to a 3 (Condition: reward v punishment $\mathrm{v}$ neutral) x 2 (Group: low trait anxiety v high trait anxiety) mixed-design ANOVA. There was no significant main effect of condition, $F(2,45)=0.74, p$ $=.49, \eta^{2}=.03$ or group, $F(1,46)=0.62, p=.44, \eta^{2}=.01$, and no significant interaction between condition and group, $F(2,45)=1.36, p=.27, \eta^{2}=.06$.

Go trial accuracy. In order to investigate whether there were differences in go trial accuracy between the two groups in each condition, go trial accuracy was subjected to the same mixed-design ANOVA. There was no significant main effect of condition, $F(2,45)=$ $2.39, p=.10, \eta^{2}=.10$ or group, $F(1,46)=0.25, p=.62, \eta^{2}=.01$, and no significant interaction between condition and group, $F(2,42)=.40, p=.67, \eta^{2}=.02$.

Go trial reaction time. Although Levene's test of equality of error variances showed that the variance for go reaction time in the neutral condition was heterogeneous $(F(1,46)=$ $6.00, p=.02)$, ANOVA is relatively robust against inequality of variance when sample sizes are equal and not extremely small (Glass, Peckham, \& Sanders, 1972) and, because transformations can reduce power and make interpretation of results difficult (Erceg-Hurn \& Mirosevich, 2008), the data was not transformed. In order to investigate whether the null 
group effect was due to a speed-accuracy trade off (i.e., slowing of go reaction time to achieve higher stop accuracy), go reaction time for correct trials was subjected to the same mixed-design ANOVA as stop trial accuracy and go trial accuracy. There was a significant main effect for group $F(2,46)=8.00, p=.01, \eta^{2}=.15$, with the high trait anxiety group demonstrating slower reaction times $\left(M_{\text {go reaction time }}=401.76, S E M=6.28\right)$ than the low trait anxiety group $\left(M_{\mathrm{go}}\right.$ reaction time $\left.=376.62, S E M=6.28\right)$. There was no significant main effect of condition, $F(2,45)=2.74, p=.08, \eta^{2}=.11$ or interaction between condition and group, $F(2$, $45)=.47, p=.63, \eta^{2}=.02$. Means and standard errors for the groups in each condition are displayed in Figure 4.

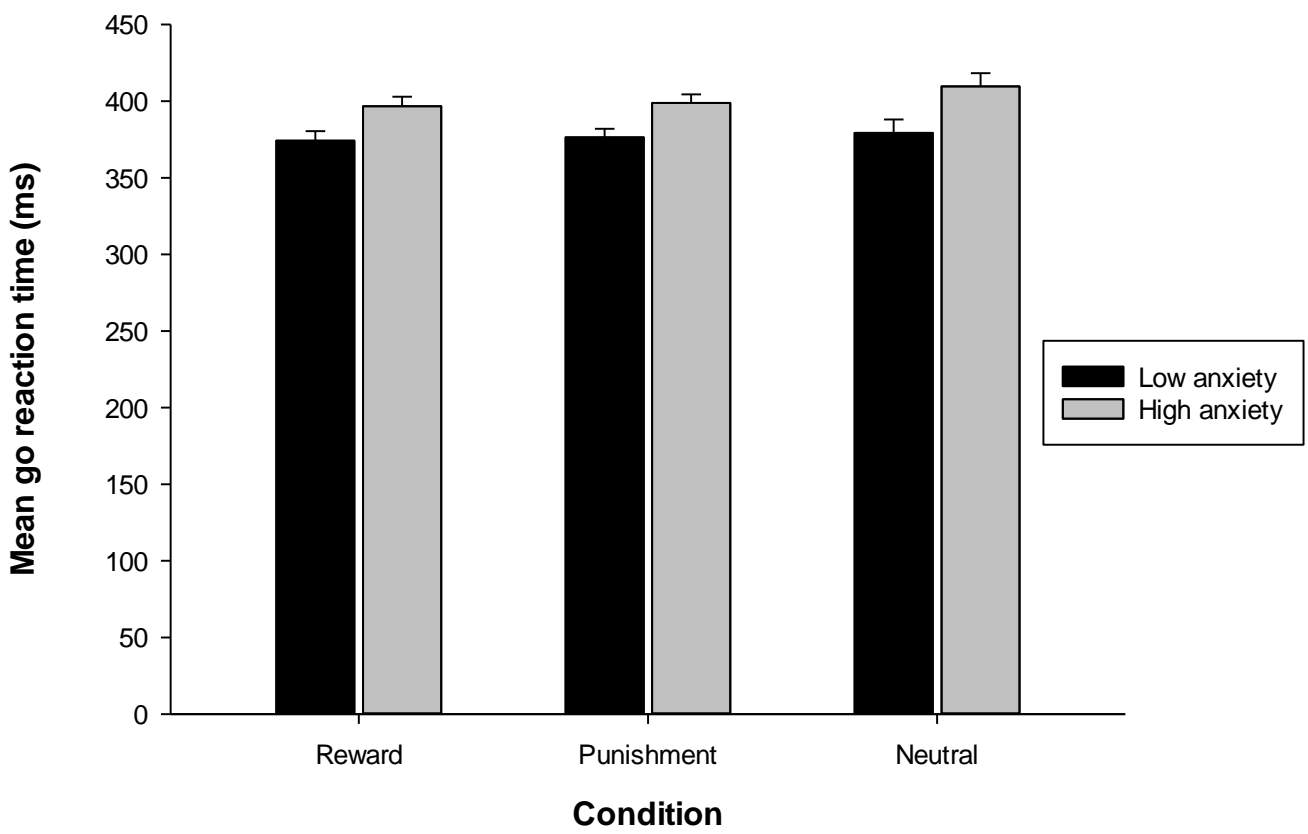

Figure 4. Mean stop accuracy for high trait anxiety and low trait anxiety groups in each condition.

\section{Functional Magnetic Resonance Imaging BOLD Activity}

Cue period (anticipation) activity. The period during presentation of the condition cue preceding stop trials was examined for BOLD activity greater than resting baseline. 
Thirteen functionally defined regions were associated with the cue-period. Table 24 lists regions and significant main and interaction effects. Means and significance tests for all main and interaction effects are presented in Appendix $\mathrm{H}$. 
Table 24

Regions of Event-Related BOLD Activity During Cue Period and Regions Demonstrating Significant Effects

\begin{tabular}{|c|c|c|c|c|c|c|c|c|c|c|c|}
\hline \multirow[t]{2}{*}{ Brain Region } & \multirow{2}{*}{$\begin{array}{l}\text { Vol } \\
(\mu \mathrm{l})\end{array}$} & \multicolumn{3}{|c|}{ MNI } & \multirow[t]{2}{*}{ Group } & \multirow[t]{2}{*}{ Incentive condition } & \multirow[t]{2}{*}{ Accuracy } & \multicolumn{2}{|c|}{ Group $\mathrm{x}$ incentive condition } & \multicolumn{2}{|c|}{ Group $x$ accuracy } \\
\hline & & $\mathrm{x}$ & $\mathrm{y}$ & $\mathrm{z}$ & & & & Low anxiety & High anxiety & Low anxiety & High anxiety \\
\hline L posterior cingulate & 39329 & -0.0 & 65.8 & 9.1 & -- & $\begin{array}{c}\mathrm{N}(\mathrm{d})>\mathrm{R}(\mathrm{d}) \\
\mathrm{P}(\mathrm{d})>\mathrm{R}(\mathrm{d}) \\
F(2,92)=8.25, p=.001\end{array}$ & -- & $\begin{array}{l}\mathrm{N}(\mathrm{d})>\mathrm{R}(\mathrm{d}) \\
\mathrm{P}(\mathrm{d})>\mathrm{R}(\mathrm{d}) \\
F(2,92)=3.52, p=.034 \\
\\
\text { In } \mathrm{N} \text { condition, there was a } \\
\text { significant difference } \\
\text { between groups (LA (d) }> \\
\mathrm{HA} \text { (d)) }\end{array}$ & $\begin{array}{l}\text { In N condition, there was a } \\
\text { significant difference } \\
\text { between groups (LA (d) > } \\
\text { HA (d)) }\end{array}$ & -- & -- \\
\hline $\mathrm{R}$ Inferior occipital gyrus & 5189 & -29.2 & 84.7 & -3.6 & -- & $\begin{array}{c}\mathrm{P}(\mathrm{a})>\mathrm{N}(\mathrm{a}) \\
\mathrm{R}(\mathrm{a})>\mathrm{N}(\mathrm{a}) \\
F(2,92)=73.61, p=.000\end{array}$ & -- & -- & - & -- & -- \\
\hline $\mathrm{R}$ Inferior frontal gyrus & 3538 & -47.8 & -21.3 & 16.8 & -- & -- & -- & -- & -- & -- & -- \\
\hline L Inferior occipital gyrus & 3390 & 27.2 & 86.3 & -4.8 & -- & $\begin{array}{c}\mathrm{P}(\mathrm{a})>\mathrm{N}(\mathrm{a}) \\
\mathrm{R}(\mathrm{a})>\mathrm{N}(\mathrm{a}) \\
F(2,92)=70.64, p=.000\end{array}$ & -- & -- & -- & -- & -- \\
\hline R Inferior parietal lobule & 1967 & -56.2 & 40.3 & 24.1 & -- & $\begin{array}{c}\mathrm{P}(\mathrm{d})>\mathrm{R}(\mathrm{d}) \\
F(2,92)=5.05, p=.008\end{array}$ & -- & -- & -- & -- & -- \\
\hline R Middle temporal gyrus & 1070 & -45.5 & 72.6 & 15.4 & -- & -- & -- & -- & -- & -- & -- \\
\hline L Middle occipital gyrus & 799 & 39.0 & 78.2 & 17.4 & $\begin{aligned} \mathrm{LA}(\mathrm{d}) & >\mathrm{HA}(\mathrm{d}) \\
F(1,46) & =5.88, p= \\
.019 & \end{aligned}$ & -- & -- & -- & -- & -- & -- \\
\hline L Uvula & 567 & 10.5 & 70.1 & -30.6 & -- & -- & -- & -- & -- & -- & -- \\
\hline L Supramarginal gyrus & 400 & 56.8 & 42.9 & 30.8 & -- & $\begin{array}{c}\mathrm{P}(\mathrm{d})>\mathrm{R}(\mathrm{d}) \\
F(2,92)=5.21, p=.007\end{array}$ & $\begin{array}{c}\mathrm{E}(\mathrm{d})>\mathrm{C}(\mathrm{d}) \\
F(1,46)=5.73, p \\
\quad=.021\end{array}$ & -- & -- & -- & -- \\
\hline $\begin{array}{l}\mathrm{R} \text { Precuneus / R superior } \\
\text { parietal lobule }\end{array}$ & 349 & -17.6 & 52.7 & 54.0 & -- & -- & -- & -- & -- & -- & -- \\
\hline $\begin{array}{l}\text { L dorsal posterior cingulate } \\
\text { (BA31) }\end{array}$ & 317 & 14.6 & 39.6 & 36.4 & -- & -- & -- & -- & $\begin{array}{c}\mathrm{R}(\mathrm{d})>\mathrm{N}(\mathrm{d}) \\
F(2,92)=5.56, p=.005\end{array}$ & -- & -- \\
\hline $\begin{array}{l}\text { L Postcentral gyrus near } \\
\text { Inferior parietal lobule / } \\
\text { Supramarginal gyrus }\end{array}$ & 239 & 56.2 & 25.3 & 35.2 & -- & $\begin{array}{c}\mathrm{N}(\mathrm{d})>\mathrm{R}(\mathrm{d}) \\
\mathrm{P}(\mathrm{d})>\mathrm{R}(\mathrm{d}) \\
F(2,92)=5.13, p=.008\end{array}$ & $\begin{array}{c}\mathrm{E}(\mathrm{d})>\mathrm{C}(\mathrm{d}) \\
F(1,46)=9.44, p \\
=.004\end{array}$ & -- & -- & -- & $\begin{array}{c}\mathrm{E}(\mathrm{d})>\mathrm{C}(\mathrm{d}) \\
F(1,46)= \\
4.26, p=.045\end{array}$ \\
\hline L Inferior semi-lunar lobule & 222 & 18.4 & 63.6 & -42.3 & $\begin{array}{c}\mathrm{LA}(\mathrm{d})>\mathrm{HA}(\mathrm{d}) \\
F(1,46)=4.78, p= \\
.034\end{array}$ & -- & - & -- & -- & -- & - \\
\hline
\end{tabular}

Note. $N=48 . \mathrm{Vol}=$ Volume; $\mu \mathrm{l}=$ Microliters; $\mathrm{MNI}=$ Montreal Neurological Institute Brain Atlas Anatomical Coordinates; Low anxiety $=$ Low anxiety group, High anxiety $=$ High trait anxiety group; LA = Low trait anxiety group; $\mathrm{HA}=$ High anxiety group; $\mathrm{R}=$ Right $\mathrm{L}=$ Left; $\mathrm{B}=\mathrm{Bilateral} ;(\mathrm{a})=$ Activated; $(\mathrm{d})=$ Deactivated; $\mathrm{R}=$ Reward condition; $\mathrm{P}=\mathrm{Punishment}$ condition; $\mathrm{N}=\mathrm{Neutral}$ condition; $\mathrm{C}=$ Correct; $\mathrm{E}=$ Error; $>=$ More than; -- = no significant main or interaction effect. 
Anticipation of the stimulus significantly activated visual regions, the right middle temporal gyrus and a number of posterior/parietal regions including the right inferior and superior parietal lobe, left postcentral and supramarginal gyrus, and the left posterior cingulate (see Table 24). A number of areas involved in inhibitory control and commonly activated during the stop signal task (right inferior frontal gyrus (IFG), right inferior parietal lobe and PCC (Cieslik et al., 2015) were significantly activated during this period.

Main effects. During anticipation of the stimulus, the bilateral inferior occipital gyrus was significantly more activated during anticipation of trials with motivational (punishment and reward) cues than during anticipation of trials with neutral cues. Posterior regions (the left posterior cingulate, right inferior parietal lobule, left supramarginal gyrus and left postcentral gyrus) showed significantly less activation than baseline (deactivation) during anticipation of punishment compared to reward trials. During anticipation of trials that subsequently resulted in an erroneous response, the left-sided inferior parietal region showed significantly greater deactivation than during anticipation of trials that resulted in a correct response (see Table 24).

Interaction effects. Differences in brain activation between the high and low trait anxiety groups in relation to condition and accuracy were seen in left-sided posterior regions. The low trait anxiety group showed significantly greater deactivation of the left posterior cingulate during anticipation of the neutral and punishment conditions compared to the reward condition; there were no significant differences between conditions in this region for the high trait anxiety group (see Figure 5). There was a significant difference between groups in deactivation of the left posterior cingulate in the neutral condition; the low trait anxiety group had significantly greater deactivation in this region than the high trait anxiety group. The high trait anxiety group showed significantly greater deactivation in the left dorsal posterior cingulate during anticipation of the reward condition compared to the neutral 
condition; this effect was not observed for the low trait anxiety group (see Figure 5). On trials where an error was subsequently committed compared to trials that were subsequently performed correctly, the high trait anxiety group demonstrated significantly greater deactivation in the left postcentral gyrus; this effect was not observed in the low trait anxiety group.

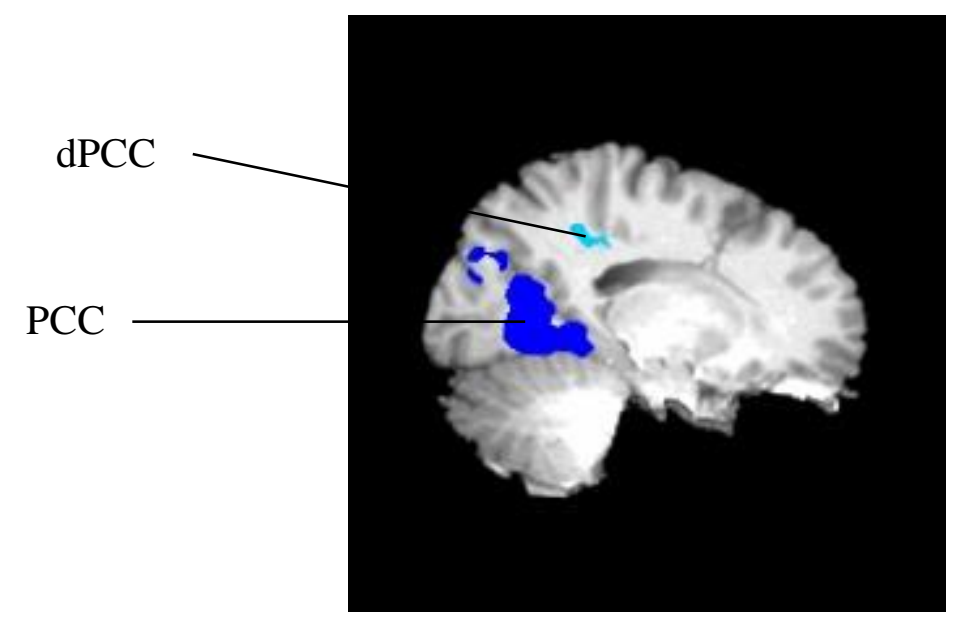

Figure 5. Activation map for a) the left posterior cingulate cortex (PCC) (dark blue) showing significant deactivation i) in the low trait anxiety group during the neutral and punishment conditions compared to the reward condition during the anticipation event, and ii) in the neutral condition for the low trait anxiety group compared to the high trait anxiety group during the anticipation event; and b) the left dorsal posterior cingulate (dPCC) (light blue) showing significant deactivation in the high trait anxiety group during the reward compared to the neutral condition during the anticipation event, overlaid on a sagittal brain section (MNI template; using AFNI software).

Stimulus period (withholding of response) activity. The period during presentation of the stimulus when participants successfully cancelled their response was examined for BOLD activity greater than resting baseline. Twenty five functionally defined regions were 
associated with this period. Table 25 lists regions and significant main and interaction effects. Means and significance tests for all main and interaction effects are presented in Appendix I. 
Table 25

Regions of Event-Related BOLD Activity During Stop Stimulus Period and Regions Demonstrating Significant Effects

\begin{tabular}{|c|c|c|c|c|c|c|c|c|}
\hline \multirow[t]{2}{*}{ Brain Region } & \multirow[t]{2}{*}{$\operatorname{Vol}(\mu l)$} & \multicolumn{3}{|c|}{ MNI } & \multirow[t]{2}{*}{ Group } & \multirow[t]{2}{*}{ Incentive condition } & \multicolumn{2}{|c|}{ Group $\mathrm{x}$ incentive condition } \\
\hline & & $\mathrm{x}$ & $\mathrm{y}$ & $\mathrm{z}$ & & & Low anxiety & High anxiety \\
\hline $\begin{array}{l}\text { R Cingulate gyrus / } \\
\text { B medial frontal gyrus / } \\
\text { R superior and middle frontal } \\
\text { Gyrus }\end{array}$ & 85337 & -11 & -12 & 27.1 & -- & -- & -- & -- \\
\hline R Supramarginal gyrus & 13982 & -44.8 & 49.5 & 37 & -- & -- & -- & -- \\
\hline R Lingual gyrus & 12822 & -30.6 & 72.7 & -2.4 & -- & -- & -- & -- \\
\hline L Lingual gyrus & 12626 & 29.9 & 75.4 & -0.8 & -- & -- & -- & -- \\
\hline $\begin{array}{l}\text { L Precentral gyrus / } \\
\text { L BA9 (DLPFC) }\end{array}$ & 11588 & 34 & -20.7 & 34.5 & -- & -- & -- & -- \\
\hline L Inferior parietal lobule & 5056 & 34.2 & 47.5 & 40.8 & -- & -- & -- & -- \\
\hline L Caudate (genu/tail) & 4462 & 26.9 & 40 & 12.7 & $\begin{array}{c}\mathrm{LA}(\mathrm{d})>\mathrm{HA}(\mathrm{d}) \\
F(1,46)=4.30, p=.044\end{array}$ & -- & -- & -- \\
\hline L Caudate (head) & 2789 & 5.1 & -23.4 & 6.7 & -- & -- & -- & -- \\
\hline $\begin{array}{l}\text { R Anterior cingulate / } \\
\text { B Anterior cingulate (ventral } \\
\text { or rostral region) }\end{array}$ & 2179 & -1.4 & -35.3 & -4.4 & -- & -- & -- & -- \\
\hline R Caudate (genu/tail) & 1632 & -25.8 & 39.9 & 12.4 & -- & -- & -- & -- \\
\hline L Culmen & 1581 & 30.4 & 50.9 & -25.5 & -- & $\begin{array}{c}\mathrm{P}(\mathrm{a})>\mathrm{N}(\mathrm{a}) \\
\mathrm{R}(\mathrm{a})>\mathrm{N}(\mathrm{a}) \\
F(2,92)=5.49, p=.006\end{array}$ & -- & -- \\
\hline $\begin{array}{l}\text { L Superior frontal gyrus } \\
\text { (and L Superior medial gyrus) }\end{array}$ & 1287 & 5.2 & -59.2 & 29.5 & $\begin{array}{c}\mathrm{LA}(\mathrm{d})>\mathrm{HA}(\mathrm{d}) \\
F(1,46)=4.60, p=.037\end{array}$ & -- & -- & -- \\
\hline $\begin{array}{l}\text { L Superior occipital gyrus / } \\
\text { L BA19 }\end{array}$ & 582 & 39.3 & 76.4 & 26.8 & -- & -- & $\begin{array}{c}\mathrm{R}(\mathrm{d})>\mathrm{N}(\mathrm{d}) \\
\mathrm{R}(\mathrm{d})>\mathrm{P}(\mathrm{d}) \\
F(2,92)=3.67, p=.029\end{array}$ & -- \\
\hline L Supramarginal gyrus & 550 & 52.7 & 52 & 37.4 & -- & -- & -- & -- \\
\hline $\mathrm{R}$ Middle temporal gyrus & 484 & -56.7 & 0.8 & -11.4 & -- & -- & -- & -- \\
\hline L Cuneus & 430 & 2.5 & 82.6 & 29.6 & $\begin{array}{c}\mathrm{LA}(\mathrm{d})>\mathrm{HA}(\mathrm{d}) \\
F(1,46)=4.29, p=.044\end{array}$ & -- & -- & -- \\
\hline $\begin{array}{l}\text { L Posterior cingulate / } \\
\text { L Precuneus }\end{array}$ & 411 & 6.4 & 51.4 & 12.2 & -- & -- & -- & -- \\
\hline
\end{tabular}




\begin{tabular}{|c|c|c|c|c|c|c|c|c|}
\hline \multirow[t]{2}{*}{ Brain Region } & \multirow[t]{2}{*}{$\operatorname{Vol}(\mu \mathrm{l})$} & \multicolumn{3}{|c|}{ MNI } & \multirow[t]{2}{*}{ Group } & \multirow[t]{2}{*}{ Incentive condition } & \multicolumn{2}{|c|}{ Group $\mathrm{x}$ incentive condition } \\
\hline & & $\mathrm{x}$ & $\mathrm{y}$ & $\mathrm{z}$ & & & Low anxiety & High anxiety \\
\hline L Middle temporal gyrus & 364 & 54.5 & 1.2 & -9.2 & -- & -- & -- & -- \\
\hline $\mathrm{R}$ Parahippocampal gyrus & 359 & -20.9 & 33.2 & 0 & -- & -- & -- & -- \\
\hline $\begin{array}{l}\text { R Medial Frontal Gyrus } \\
\text { / more B Medial frontal } \\
\text { Gyrus }\end{array}$ & 343 & -0.7 & -56.4 & 4.2 & -- & -- & -- & -- \\
\hline R Superior temporal gyrus & 323 & -46.3 & 21.2 & -3.8 & -- & -- & -- & -- \\
\hline R Brain (brainstem) & 241 & -1.7 & 24 & -33.4 & -- & -- & -- & -- \\
\hline $\begin{array}{l}\text { R Middle temporal gyrus / } \\
\text { R Middle occipital gyrus }\end{array}$ & 239 & -44.1 & 69.8 & 26.1 & -- & -- & -- & -- \\
\hline $\begin{array}{l}\text { R Superior temporal gyrus / } \\
\text { R middle temporal (on border } \\
\text { between them) }\end{array}$ & 203 & -46 & 39.3 & 10.2 & -- & -- & -- & -- \\
\hline L Uvula & 181 & 14.1 & 70.5 & -23.2 & -. & -. & .. & -. \\
\hline
\end{tabular}

Note $. N=48 . \mathrm{Vol}=$ Volume; $\mu \mathrm{l}=$ Microliters; $\mathrm{MNI}=$ Montreal Neurological Institute Brain Atlas Anatomical Coordinates; Low anxiety $=$ Low anxiety group, High anxiety $=$ High trait anxiety group; LA = Low trait anxiety group; $\mathrm{HA}=$ High anxiety group; $\mathrm{R}=$ Right $\mathrm{L}=$ Left $\mathrm{B}=$ Bilateral; $(\mathrm{a})=$ Activated; $(\mathrm{d})=$ Deactivated; $\mathrm{R}=$ Reward condition; $\mathrm{P}=\mathrm{Punishment}$ condition; $\mathrm{N}=\mathrm{Neutral}$ condition; $\mathrm{C}=$ Correct; $\mathrm{E}=$ Error; $>=$ More than; -- = no significant main or interaction effect. 
Cancellation of a response significantly activated structures in frontal, temporal, parietal and occipital regions (see Table 25). There was no activation observed in regions most commonly activated during action cancellation (e.g., bilateral anterior insula, right IFG, pre-SMA) but the right ACC, left PCC, right TPJ (i.e., right supramarginal gyrus), which have also been implicated in action cancellation, showed significantly increased activation (Cieslik et al., 2015).

Main effects. During successful cancellation of a response, the left culmen significantly increased activation during motivational (punishment and reward) trials compared to neutral trials. No other brain area showed a significant main effect of condition. Compared to the high trait anxiety group, during successful cancellation of a response the low trait anxiety group showed significant deactivation in left-sided cortical and subcortical regions (left superior frontal/medial gyrus, left cuneus and genu/tail of the left caudate nucleus).

Interaction effects. When successfully cancelling a response, the low trait anxiety group showed significant deactivation in the left superior occipital gyrus in the reward compared to the other conditions. This effect was not seen in the high trait anxiety group.

Feedback period activity. The period during presentation of erroneous feedback on stop trials (reward condition: 20c; punishment condition: lose 20c; neutral condition: 0c) and correct feedback (all conditions: 0c) was examined for BOLD activity greater than resting baseline. Twenty two functionally defined regions were associated with this period. Table 26 lists regions and significant main and interaction effects. Means and significance tests for all main and interaction effects are presented in Appendix $\mathbf{J}$. 
Table 26

Regions of Event-Related BOLD Activity During Feedback Period and Regions Demonstrating Significant Effects

\begin{tabular}{|c|c|c|c|c|c|c|c|c|c|c|c|c|c|}
\hline \multirow[t]{2}{*}{ Brain Region } & \multirow[t]{2}{*}{$\begin{array}{l}\text { Vol } \\
(\mu 1)\end{array}$} & \multicolumn{3}{|c|}{ MNI } & \multirow[t]{2}{*}{$\begin{array}{l}\text { Incentive } \\
\text { condition }\end{array}$} & \multirow[t]{2}{*}{ Accuracy } & \multicolumn{2}{|c|}{ Group $\mathrm{x}$ incentive condition } & \multicolumn{2}{|c|}{ Group x accuracy } & \multicolumn{3}{|c|}{ Incentive condition $\mathrm{x}$ accuracy } \\
\hline & & $\mathrm{x}$ & y & $\mathrm{z}$ & & & Low anxiety & High anxiety & Low anxiety & High anxiety & Reward & Punishment & Neutral \\
\hline $\begin{array}{l}\text { L Fusiform gyrus and } \\
\text { L lingual gyrus }\end{array}$ & 33901 & 31.5 & 64.4 & -8.4 & -- & -- & -- & -- & -- & -- & -- & -- & -- \\
\hline $\begin{array}{l}\mathrm{R} \text { Lingual gyrus and } \mathrm{R} \\
\text { Fusiform gyrus }\end{array}$ & 27554 & -28.3 & 70.3 & -2.5 & -- & -- & -- & -- & -- & -- & -- & -- & -- \\
\hline & & & & & & & & & & & $\begin{array}{l}\mathrm{C}(\mathrm{a})>\mathrm{E}(\mathrm{a}) \\
F(2,92)= \\
3.65, p= \\
.030\end{array}$ & $\begin{array}{l}\mathrm{C}(\mathrm{a})>\mathrm{E} \text { (a) } \\
F(2,92)= \\
3.65, p= \\
.030\end{array}$ & \\
\hline L Cingulate gyrus & 16821 & 23.7 & 1.6 & 26.4 & -- & $\begin{array}{c}\mathrm{C}(\mathrm{a})>\mathrm{E} \text { (a) } \\
F(1,46)= \\
10.44, p=.002\end{array}$ & -- & -- & -- & -- & $\begin{array}{l}\text { In C } \\
\text { condition } \\
\text { there was a } \\
\text { significant } \\
\text { difference } \\
\text { between } \\
\text { incentive } \\
\text { conditions } \\
\text { (P (a) }>\text { R } \\
\text { (a)) }\end{array}$ & $\begin{array}{l}\text { In C } \\
\text { condition } \\
\text { there was a } \\
\text { significant } \\
\text { difference } \\
\text { between } \\
\text { incentive } \\
\text { conditions } \\
\text { (P (a) }>\mathrm{R} \\
\text { (a)) }\end{array}$ & -- \\
\hline & & & & & & & & & & & $\begin{array}{l}\mathrm{C}(\mathrm{a})>\mathrm{E}(\mathrm{a}) \\
F(2,92)= \\
4.17, p= \\
.018\end{array}$ & $\begin{array}{l}\mathrm{C}(\mathrm{a}) \mid \mathrm{E}(\mathrm{d}) \\
F(2,92)= \\
4.17, p= \\
.018\end{array}$ & $\begin{array}{l}\mathrm{C}(\mathrm{a})>\mathrm{E}(\mathrm{a}) \\
F(2,92)= \\
4.17, p= \\
.018\end{array}$ \\
\hline $\mathrm{R}$ Cingulate gyrus & 15398 & -22.2 & -0.2 & 24.7 & -- & $\begin{array}{c}\mathrm{C}(\mathrm{a})>\mathrm{E}(\mathrm{a}) \\
F(1,46)= \\
22.65, p=.000\end{array}$ & -- & -- & -- & -- & $\begin{array}{l}\text { In C } \\
\text { condition } \\
\text { there was a } \\
\text { significant } \\
\text { difference } \\
\text { between } \\
\text { incentive } \\
\text { conditions } \\
\text { (P (a) }>\text { R } \\
\text { (a)) }\end{array}$ & $\begin{array}{l}\text { In C } \\
\text { condition } \\
\text { there was a } \\
\text { significant } \\
\text { difference } \\
\text { between } \\
\text { incentive } \\
\text { conditions } \\
\text { (P (a) }>\mathrm{R} \\
\text { (a)) }\end{array}$ & \\
\hline $\begin{array}{l}\text { L Medial frontal gyrus / } \\
\text { L BA6 / L SMA }\end{array}$ & 10263 & 2.2 & 4 & 54.1 & $\begin{array}{c}\mathrm{P}(\mathrm{d})>\mathrm{N}(\mathrm{d}) \\
\mathrm{R}(\mathrm{d})>\mathrm{N}(\mathrm{d}) \\
F(2,92)=7.37, p \\
\quad=.001\end{array}$ & $\begin{array}{c}\mathrm{E}(\mathrm{d})>\mathrm{C}(\mathrm{d}) \\
F(1,46)= \\
7.57, p=.008\end{array}$ & -- & -- & -- & -- & -- & -- & -- \\
\hline $\begin{array}{l}\text { R Middle frontal gyrus / } \\
\text { BA10 }\end{array}$ & 4260 & -43.1 & -41.3 & 13.8 & $\begin{aligned} \mathrm{P}(\mathrm{a}) & >\mathrm{N}(\mathrm{a}) \\
\mathrm{R}(\mathrm{a}) & >\mathrm{N}(\mathrm{a}) \\
F(2,92) & =6.91, p \\
& =.002\end{aligned}$ & -- & -- & -- & -- & -- & $\begin{array}{l}\mathrm{E}(\mathrm{a})>\mathrm{C}(\mathrm{a}) \\
F(2,92)= \\
3.19, p= \\
.046\end{array}$ & & \\
\hline
\end{tabular}




\begin{tabular}{|c|c|c|c|c|c|c|c|c|c|c|c|c|c|}
\hline \multirow[t]{2}{*}{ Brain Region } & \multirow[t]{2}{*}{$\begin{array}{l}\text { Vol } \\
(\mu \mathrm{l})\end{array}$} & \multicolumn{3}{|c|}{ MNI } & \multirow[t]{2}{*}{$\begin{array}{l}\text { Incentive } \\
\text { condition }\end{array}$} & \multirow[t]{2}{*}{ Accuracy } & \multicolumn{2}{|c|}{ Group $x$ incentive condition } & \multicolumn{2}{|c|}{ Group x accuracy } & \multicolumn{3}{|c|}{ Incentive condition $\mathrm{x}$ accuracy } \\
\hline & & $\mathrm{x}$ & y & $\mathrm{z}$ & & & Low anxiety & High anxiety & Low anxiety & High anxiety & Reward & Punishment & Neutral \\
\hline & & & & & & & & & & & $\begin{array}{l}\text { In E } \\
\text { condition } \\
\text { there was a } \\
\text { significant } \\
\text { difference } \\
\text { between } \\
\text { incentive } \\
\text { conditions } \\
\text { (R (a) }>\mathrm{N} \\
\text { (a)) }\end{array}$ & $\begin{array}{l}\text { In } \mathrm{E} \\
\text { condition } \\
\text { there was a } \\
\text { significant } \\
\text { difference } \\
\text { between } \\
\text { incentive } \\
\text { conditions } \\
\text { (P (a) }>\mathrm{N} \\
\text { (a)) }\end{array}$ & $\begin{array}{l}\text { In E } \\
\text { condition } \\
\text { there was a } \\
\text { significant } \\
\text { difference } \\
\text { between } \\
\text { incentive } \\
\text { conditions } \\
\text { (R (a) }>\mathrm{N} \\
\text { (a)) and (P } \\
\text { (a) }>\mathrm{N} \text { (a)) }\end{array}$ \\
\hline $\begin{array}{l}\text { R Inferior parietal lobule / } \\
\text { BA40 }\end{array}$ & 3688 & -46.6 & 46.1 & 46 & $\begin{array}{c}\mathrm{R}(\mathrm{a})>\mathrm{N}(\mathrm{a}) \\
\mathrm{R}(\mathrm{a})>\mathrm{P}(\mathrm{a}) \\
F(2,92)=10.36 \\
p=.000\end{array}$ & -- & -- & -- & -- & -- & -- & -- & -- \\
\hline $\begin{array}{l}\text { R Anterior cingulate / } \\
\text { R BA32 (dACC) }\end{array}$ & 2781 & -7.2 & -43 & 13.4 & $\begin{array}{c}\mathrm{R}(\mathrm{a})>\mathrm{N}(\mathrm{a}) \\
F(2,92)=5.02, p \\
=.009\end{array}$ & $\begin{array}{c}\mathrm{C}(\mathrm{a})>\mathrm{E}(\mathrm{a}) \\
F(1,46)= \\
5.17, p=.028\end{array}$ & -- & -- & -- & -- & -- & -- & -- \\
\hline $\begin{array}{l}\mathrm{R} \text { Medial frontal gyrus } \\
\text { / R superior frontal gyrus }\end{array}$ & 2162 & -14.8 & -35.2 & 42.1 & $\begin{aligned} \mathrm{R}(\mathrm{a}) & >\mathrm{N}(\mathrm{a}) \\
F(2,92) & =4.89, p \\
& =.010\end{aligned}$ & -- & -- & -- & -- & -- & -- & -- & -- \\
\hline $\begin{array}{l}\text { L Inferior frontal gyrus } \\
\text { (P. triangularis BA45) }\end{array}$ & 922 & 48.8 & -29.6 & 10.6 & -- & -- & -- & -- & -- & -- & -- & -- & -- \\
\hline $\mathrm{R}$ Insula & 746 & -42.9 & 27.2 & -1.1 & -- & -- & -- & -- & $\begin{array}{c}\mathrm{C}(\mathrm{a})>\mathrm{E}(\mathrm{a}) \\
F(1,46)= \\
7.43, p=.009\end{array}$ & -- & -- & -- & -- \\
\hline & & & & & & & & & $\begin{array}{c}\mathrm{C}(\mathrm{a})>\mathrm{E}(\mathrm{a}) \\
F(1,46)= \\
4.90, p=.032\end{array}$ & & & & \\
\hline R Cerebellar Tonsil & 659 & -36.2 & 55.9 & -36.6 & -- & -- & -- & -- & $\begin{array}{l}\text { In } \mathrm{C} \text { condition } \\
\text { there was a } \\
\text { significant } \\
\text { difference } \\
\text { between } \\
\text { groups (LA(a) } \\
>\text { HA(a)) }\end{array}$ & $\begin{array}{l}\text { In C condition } \\
\text { there was a } \\
\text { significant } \\
\text { difference } \\
\text { between } \\
\text { groups (LA(a) } \\
>\text { HA(a)) }\end{array}$ & -- & -- & -- \\
\hline $\mathrm{R}$ Thalamus & 532 & -8.9 & 12.5 & 0.9 & -- & -- & -- & -- & -- & -- & -- & -- & -- \\
\hline $\begin{array}{l}\mathrm{R} \text { Superior frontal gyrus / } \\
\mathrm{R} \text { middle frontal gyrus }\end{array}$ & 466 & -33.3 & -15.2 & 50.6 & $\begin{array}{c}\mathrm{R}(\mathrm{a})>\mathrm{N}(\mathrm{a}) \\
\mathrm{R}(\mathrm{a})>\mathrm{P}(\mathrm{a}) \\
F(2,92)=14.99 \\
p=.000\end{array}$ & -- & $\begin{array}{c}\mathrm{R}(\mathrm{a})>\mathrm{N}(\mathrm{a}) \\
\mathrm{R}(\mathrm{a})>\mathrm{P}(\mathrm{a}) \\
F(2,92)= \\
3.74, p=.027\end{array}$ & $\begin{array}{c}\mathrm{P} \text { (a) }>\mathrm{N} \text { (a) } \\
\mathrm{R} \text { (a) }>\mathrm{N} \text { (a) } \\
\mathrm{R}(\mathrm{a})>\mathrm{P} \text { (a) } \\
F(2,92)= \\
3.74, p=.027\end{array}$ & -- & -- & -- & -- & -- \\
\hline $\begin{array}{l}\text { L Thalamus - L Ventral } \\
\text { lateral nucleus }\end{array}$ & 398 & 9.5 & 11.6 & 3.7 & -- & -- & - & - & -- & -- & -- & -- & -- \\
\hline L Thalamus & 337 & 0 & 20 & 17.1 & -- & $\begin{array}{c}\mathrm{C}(\mathrm{a})>\mathrm{E}(\mathrm{a}) \\
F(1,46)= \\
5.59, p=.022\end{array}$ & -- & -- & -- & -- & -- & -- & -- \\
\hline
\end{tabular}




\begin{tabular}{|c|c|c|c|c|c|c|c|c|c|c|c|c|c|}
\hline \multirow[t]{2}{*}{ Brain Region } & \multirow[t]{2}{*}{$\begin{array}{l}\text { Vol } \\
(\mu \mathrm{l})\end{array}$} & \multicolumn{3}{|c|}{ MNI } & \multirow[t]{2}{*}{$\begin{array}{l}\text { Incentive } \\
\text { condition }\end{array}$} & \multirow[t]{2}{*}{ Accuracy } & \multicolumn{2}{|c|}{ Group $\mathrm{x}$ incentive condition } & \multicolumn{2}{|c|}{ Group x accuracy } & \multicolumn{3}{|c|}{ Incentive condition $\mathrm{x}$ accuracy } \\
\hline & & $\mathrm{x}$ & y & $\mathrm{z}$ & & & Low anxiety & High anxiety & Low anxiety & High anxiety & Reward & Punishment & Neutral \\
\hline $\begin{array}{l}\text { L Lentiform nucleus and } \\
\text { L Putamen }\end{array}$ & 327 & 28.2 & 10.4 & 8 & -- & -- & -- & -- & -- & -- & -- & - & -- \\
\hline R Culmen & 322 & -27.9 & 28.5 & -24.6 & -- & -- & -- & -- & -- & -- & -- & -- & -- \\
\hline $\begin{array}{l}\text { L Culmen / L fusiform } \\
\text { Gyrus }\end{array}$ & 275 & 24.6 & 29.7 & -21.9 & -- & -- & -- & -- & -- & -- & -- & -- & -- \\
\hline R Angular gyrus & 185 & -43.6 & 57.7 & 33.5 & $\begin{array}{c}\mathrm{R}(\mathrm{a})>\mathrm{N}(\mathrm{a}) \\
\mathrm{R}(\mathrm{a})>\mathrm{P}(\mathrm{a}) \\
F(2,92)=9.61, p \\
=.000\end{array}$ & -- & -- & -- & -- & -- & -- & -- & -- \\
\hline $\begin{array}{l}\text { L Precentral gyrus and L } \\
\text { BA4 (PMC) }\end{array}$ & 180 & 27.3 & 27.7 & 54.1 & $\begin{aligned} \mathrm{P}(\mathrm{d}) & >\mathrm{N}(\mathrm{d}) \\
\mathrm{R}(\mathrm{d}) & >\mathrm{N}(\mathrm{d}) \\
F(2,92) & =6.23, p \\
& =.003\end{aligned}$ & $\begin{array}{c}\mathrm{E}(\mathrm{d})>\mathrm{C}(\mathrm{d}) \\
F(1,46)= \\
9.52, p=.003\end{array}$ & -- & -- & -- & -- & -- & -- & -- \\
\hline $\begin{array}{l}\mathrm{R} \text { Cingulate gyrus / } \\
\mathrm{B} \text { Cingulate }\end{array}$ & 176 & -0.9 & 16.5 & 32.3 & $\begin{array}{c}\mathrm{R}(\mathrm{a})>\mathrm{N}(\mathrm{a}) \\
F(2,92)=3.16, p \\
=.047\end{array}$ & -- & -- & -- & -- & -- & -- & -- & -- \\
\hline
\end{tabular}

Note. $N=48 . \mathrm{Vol}=$ Volume; $\mu \mathrm{l}=$ Microliters; $\mathrm{MNI}=$ Montreal Neurological Institute Brain Atlas Anatomical Coordinates; Low anxiety $=$ Low anxiety group, High anxiety $=$ High trait anxiety group; LA $=$ Low trait anxiety group; HA = High anxiety group; $\mathrm{R}=$ Right $\mathrm{L}=$ Left $\mathrm{B}=$ Bilateral; $(\mathrm{a})=$ Activated; $(\mathrm{d})=$ Deactivated $\mathrm{R}=$ Reward condition; $\mathrm{P}=\mathrm{Punishment}$ condition; $\mathrm{N}=\mathrm{Neutral}$ condition; $\mathrm{C}=\mathrm{Correct} ; \mathrm{E}=$ Error; $>=$ More than; -- = no significant main or interaction effect. 
Receipt of feedback significantly activated medial frontal regions (bilateral medial frontal gyrus, left supplementary motor area (SMA), dACC) as well as other cortical (left middle, inferior frontal and precentral gyrus, inferior parietal lobe, right insula, bilateral fusiform gyrus) and subcortical regions (bilateral thalamus, bilateral cingulate gyrus, left lentiform nucleus).

Main effects. During feedback, right-sided frontal and posterior regions (right superior/middle frontal gyrus, right medial frontal gyrus, right anterior cingulate, right/bilateral cingulate gyrus, right inferior parietal lobule and right angular gyrus) showed significantly greater activation during motivational trials (reward - 20c or 0c and/or punishment - lose 20c or 0c) compared to neutral trials (0c). Three of these regions (right superior/middle frontal gyrus, right inferior parietal lobule, and right angular gyrus) also showed significantly greater activation during feedback on reward trials compared to punishment trials. Left sided regions (left medial frontal gyrus/SMA and the left precentral gyrus/primary motor cortex) demonstrated significant deactivation during feedback on reward and punishment trials compared to neutral trials. When compared to feedback indicating an error (20c, lose 20c, 0c), feedback indicating a correct response (0c) resulted in significantly increased activation in the bilateral cingulate gyrus (including the right anterior cingulate) and left thalamus. Compared to feedback indicating a correct response, feedback indicating an error resulted in significant deactivation in the same areas as feedback on motivational compared to neutral trials (left medial frontal gyrus/SMA and left precentral gyrus/primary motor cortex). There were no significant main effects of group.

Interaction effects. For both the high and low trait anxiety group, the right superior/middle frontal gyrus showed significantly greater activation during reward trials compared to punishment and neutral trials. The high trait anxiety group also showed 
significantly greater activation in this region during punishment compared to neutral trials (see Figure 6A). During feedback indicating a correct response compared to an error, the low trait anxiety group had significantly greater activation of the right insula; this effect was not observed in the high trait anxiety group (see Figure 6B).

(A)
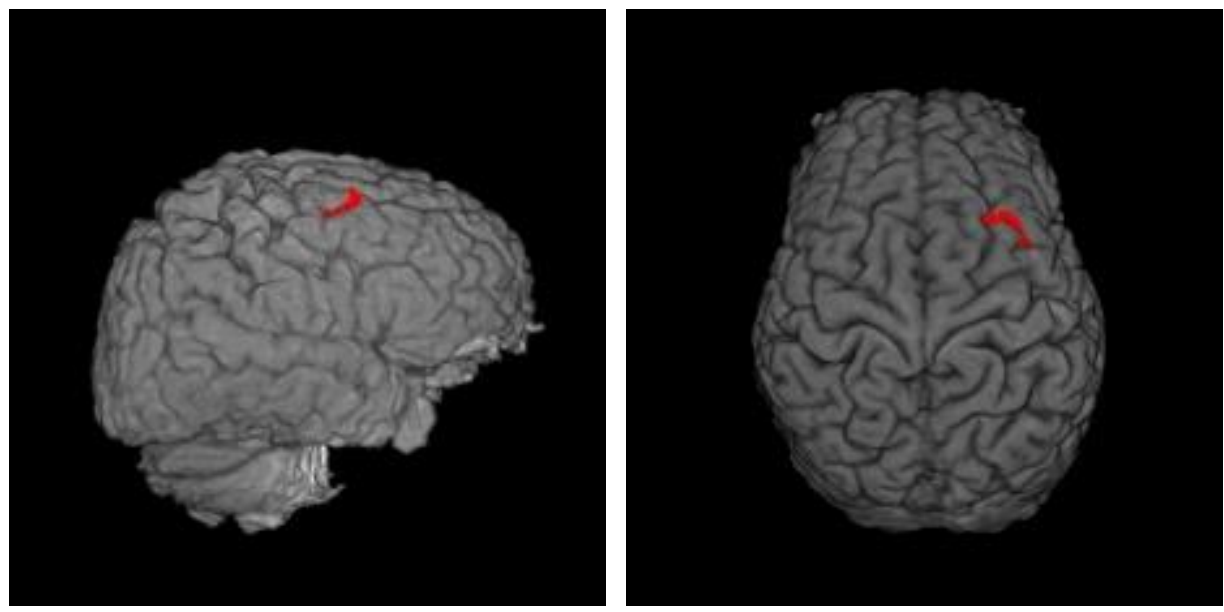

(B)
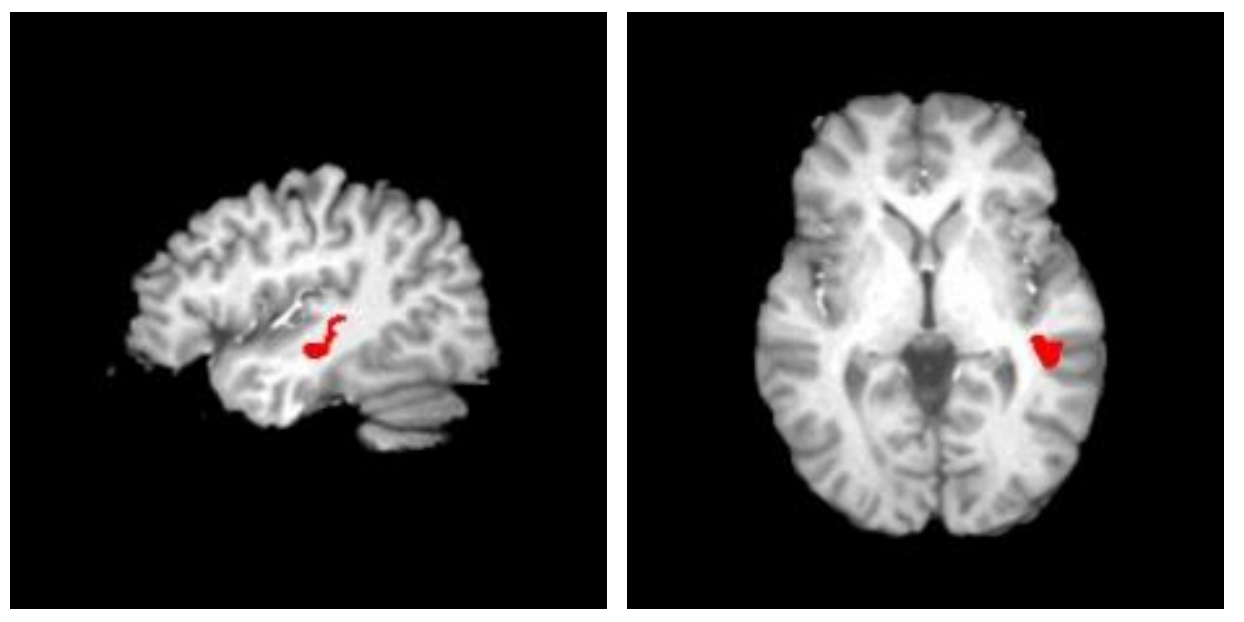

Figure 6. (A) Activation maps for the right superior/middle frontal gyrus showing significant activity in both groups during reward compared to punishment and neutral trials and in the high trait anxiety group during punishment compared to neutral trials during the feedback event, overlaid on sagittal and axial brain sections (MNI template; using AFNI software). (B) Activation maps for the right insula showing significant 
activity in the low trait anxiety group during the feedback event following a correct response compared to an error, overlaid on sagittal and axial brain sections (MNI template; using AFNI software).

When participants made an error during a stop trial they received feedback depending on condition. Receipt of 20c following an error (reward condition) could be construed as 'incongruous feedback' because reward following error is usually not expected. Similarly, loss of 20c following an error (punishment condition) could be construed as 'congruous feedback'. To compare brain activation during congruous versus incongruous feedback, we looked at brain activation in correct versus error trials across the three conditions. Compared to receiving neutral feedback, receiving both congruous (error in punishment condition) and incongruous (error in reward condition) feedback resulted in significant greater activation in the right middle frontal gyrus (BA10). The bilateral cingulate gyrus showed significantly different levels of activation for correct (absence of reward) compared to error trials across the three conditions (see Table 26 for more detail). 


\section{Discussion}

The current study sought to investigate the effect of reward and punishment on inhibitory control in trait anxiety. Contrary to expectations, individuals high in trait anxiety did not demonstrate increased inhibitory control in comparison to low trait anxiety participants, i.e., they did not have higher accuracy in the punishment condition. However, compared to individuals low in trait anxiety, individuals high in trait anxiety slowed their response speed on go trials across all conditions, which may have improved their accuracy on stop trials. Also contrary to expectations, no significant increase in BOLD activation was observed in cognitive control, goal-directed attentional networks or in inhibitory control networks when participants high in trait anxiety were correctly inhibiting their response. Compared to individuals low in trait anxiety, high trait anxiety individuals did not demonstrate a significant increase in activation in punishment-related brain regions during presentation of punishing cues or during receipt of punishing feedback following stop trials.

Although participants high in trait anxiety did not demonstrate increased accuracy on punishment condition stop trials and therefore did not appear to have better inhibitory control on these trials compared to reward or neutral condition stop trials, or compared to individuals low in trait anxiety, the slowing of go reaction time across all conditions is consistent with predictions made by attentional control theory (M. W. Eysenck et al., 2007). Attentional control theory proposes that anxiety increases the influence of the stimulus-driven attentional system and decreases the influence of the goal-directed attentional system. The increased allocation of attention to potential internal and external sources of threat reduces attentional focus, which in turn negatively affects attentional and inhibitory control. Attentional control theory further claims that inhibitory control deficits may not become apparent if individuals high in anxiety respond to processing 
inefficiencies by using compensatory strategies. These strategies include enhanced effort and use of processing resources which may be reflected in, for example, longer reaction times (M. W. Eysenck et al., 2007). It is possible that participants in the high trait anxiety group slowed their go reaction time in order to improve stop accuracy. The slowed go reaction times indicate reduced processing efficiency and suggest that individuals who are high in anxiety experience greater difficulty cancelling an already initiated response than those lower in anxiety.

Previous studies that have used the stop signal task have found that stopping activates a network of brain regions including right IFC (Aron, 2011), the pre-SMA (Aron, 2011; Chao et al., 2009; C. S. R. Li et al., 2006; D. J. Sharp et al., 2010), and the anterior insula (Erika-Florence et al., 2014; Hampshire, 2015). Despite using a variant of the stop signal task, the current study failed to find significant activation in these areas during successful stopping; however, the right IFG was activated during the anticipation period. Consistent with a recent meta-analysis by Cieslik et al. (2015), both groups showed increased activation in the right ACC, left PCC (Dambacher et al., 2014) and right TPJ (i.e., right supramarginal gyrus) during anticipation and/or successful stopping. The activation seen during both anticipation and successful stopping is consistent with studies that have found that anticipation of stopping activates the same regions as stopping itself (Chikazoe et al., 2009).

The absence of activation in structures commonly implicated in stopping, such as the right IFG, pre-SMA and anterior insula, during the stimulus presentation period (when participants were required to cancel their response) might have been influenced by anticipatory caution. Greater preparation to stop prior to a stop trial results in less activity in inhibitory control structures during the stop trial epoch (Chikazoe et al., 2009). In the current study, if participants used greater proactive control (i.e., they consciously decided 
to withhold a response before the stimulus appeared rather than reactively respond to the stop stimulus to cancel an already initiated response), this may have reduced the recruitment of the typical inhibitory control cortical network.

The finding that the high and low trait anxiety groups did not have different levels of activity in cognitive control structures during anticipation and successful stopping is not consistent with previous studies that have administered inhibitory control tasks. For example, high trait anxiety has been associated with increased (Basten et al., 2011; Fales et al., 2008) and decreased (Bishop, 2009; Forster et al., 2015) levels of activation in cognitive control regions such as the DLPFC. The inconsistency with previous studies may be related to differences in task paradigm. Basten et al. (2011) used a Stroop task, Bishop et al. (2009) used a letter search task with high and low perceptual load (reduced DLPFC recruitment to response conflict in high anxiety was found only under conditions of low, not high, perceptual load), Forster et al. (2015) used a sustained attention to response task, and Fales et al. (2008) used an $n$-back task. Some of these tasks, such as the letter search task and the $n$-back task, may contain a greater working memory component than the current task, which would place a greater load on areas such as the DLPFC. Some previous studies have also used less frequent stimuli to elicit cognitive control, which in turn promotes a greater BOLD response in prefrontal cortical regions due to attentional rather than cognitive control processes (Katsuki \& Constantinidis, 2012); for example, the task in the study by Forster et al. (2015) only included two or three no-go trials in each block of 28 trials.

In relation to reward and punishment networks activated in each of the conditions, examination of the anticipation epoch of the MICT across groups indicated anticipation of reward and punishment failed to significantly activate areas related to reward and punishment processing, respectively. Anticipation of punishment resulted in deactivation 
of posterior regions (left posterior cingulate, right inferior parietal lobule, left

supramarginal gyrus, left postcentral gyrus). The finding that anticipation of reward and punishment failed to significantly activate the same areas activated in studies that have used the monetary incentive delay task (e.g., Knutson et al., 2000) may be because in the monetary incentive delay task, participants are not required to inhibit a response. In that task, participants are merely asked to respond as quickly as possible to a stimulus that follows a cue stimulus indicating potential reward, punishment or no incentive. In the MICT, because participants must respond as quickly as possible to a go stimulus that follows a cue stimulus but they must also withhold their response on unexpected stop trials, they may have been more concerned with preparing to inhibit their response than responding as quickly as possible to the stimulus in order to gain reward or avoid punishment. This possibility is supported by our finding that regions implicated in inhibitory control - namely, the right IFG, right ACC, left PCC, and right TPJ - were activated during the anticipation period in the MICT.

Examination of the feedback epoch of the MICT across groups indicated receipt of reward following an error (incongruous feedback) and punishment following an error (congruous feedback) did not differentially activate reward or punishment-related structures, but receipt of rewarding (incongruous) and punishing (congruous) feedback following an error activated the right middle frontal gyrus (BA10). The absence of reward following a correct response (reward condition) also activated the right cingulate gyrus more than receipt of reward following an error (reward condition), and the absence of reward following a correct response (punishment condition) activated the left cingulate gyrus more than receipt of punishment following an error (punishment condition).

In relation to group (low and high trait anxiety)-specific differences in reward and punishment processing, we failed to find group differences in activity in reward-related 
or punishment-related brain regions during presentation of rewarding and punishing cues, presentation of the stop stimulus or during receipt of rewarding or punishing feedback. These findings are not consistent with previous studies that have shown that individuals high on measures of behavioural inhibition, a construct related to trait anxiety, have increased striatal activation when anticipating reward and punishment (Bar-Haim et al., 2009; Benson et al., 2014; Guyer et al., 2014; Guyer et al., 2006), larger

electrophysiological responses to error and punishment (M. A. S. Boksem et al., 2008; De Pascalis et al., 2010; Leue et al., 2012), and reduced striatal responses to rewarding feedback (Guyer et al., 2014; Helfinstein et al., 2011; Simon et al., 2010). The inconsistency between the current study and past studies examining reward and punishment responsiveness might be related to the MICT paradigm. The absence of condition specific effects on inhibition accuracy suggests that the manipulation of task contingencies, whereby immediate reward and punishment were levied, was insufficient to alter performance. In the absence of such effects, it remains possible that participants did not sufficiently process cues and/or feedback. Activation of structures in the reward network appear to be dependent on the conscious processing of stimuli (Bijleveld et al., 2014), and the same may be true of structures in the punishment network, particularly as processing rewards and punishments requires many of the same structures (e.g., Knutson et al., 2000).

During the period when participants were viewing the anticipatory cue, the low trait anxiety group demonstrated greater deactivation of the left PCC in the neutral and punishment conditions compared to the reward condition and the high trait anxiety group demonstrated greatest deactivation of the left dorsal posterior cingulate (BA31) in the reward condition with significantly greater deactivation of this region in the reward compared to the neutral condition. The posterior cingulate region is part of the default 
mode network. It is believed that this network activates when the brain is 'at rest' and deactivates during attentionally demanding tasks (Leech, Braga, \& Sharp, 2012; Leech \& Sharp, 2014). In particular, the dorsal posterior cingulate cortex (dorsal PCC) is thought to be strongly connected to other frontal and parietal regions involved in cognitive control (e.g., Vincent, Kahn, Snyder, Raichle, \& Buckner, 2008) and activation in the dorsal PCC is negatively correlated with activation in cognitive control regions such as the DLPFC, rostral ACC, pre-SMA, anterior insula and inferior parietal lobule (Leech et al., 2012; Leech \& Sharp, 2014). The greater deactivation of the left dorsal PCC in the high trait anxiety group when anticipating reward may be due to this group engaging in reduced internal processes such as distracting thoughts or rumination when anticipating reward, perhaps reflecting increased effort to enhance performance on these trials. Given the negative correlation between activation in the dorsal PCC and cognitive control areas, it has been proposed that deactivation of the dorsal PCC improves performance in the same way as increased activation of cognitive control areas (Fales et al., 2008). The hypothesised increase in effort to enhance performance by the high trait anxiety group in the current study is also consistent with our behavioural findings that the high trait anxiety group slowed their go reaction time across all conditions.

When anticipating reward, the low trait anxiety group had greater activation (less deactivation) in the left PCC compared to the neutral and punishment condition. The reduced deactivation in this region in the reward condition suggests that when anticipating reward, healthy individuals increase activation of the left PCC. Given that the left PCC is part of the default mode network, increased activation in the reward condition may be due to attentional lapses caused by distraction from the reward cue (Weissman, Roberts, Visscher, \& Woldorff, 2006) or enhanced attention toward the external environment caused by waiting for the go/stop stimulus in the reward condition 
(Gilbert, Dumontheil, Simons, Frith, \& Burgess, 2007). That this effect was not observed in the high trait anxiety group suggests that individuals high in trait anxiety might not process potential reward in the same way as individuals low in trait anxiety. The greater activation in the left PCC in the low trait anxiety group may also be due to the low trait anxiety level in this group, and further research using a healthy control group with average rather than low scores on the STAI T-Anxiety scale would help to clarify whether this finding is unique to individuals low in trait anxiety or whether it is generalizable to all individuals who are not high in trait anxiety. The finding that the low trait anxiety group had greater deactivation in the left PCC in the neutral condition than the high trait anxiety group also suggests that when there are no motivational incentives, individuals high in anxiety may experience greater difficulty maintaining attention on the task than individuals low in anxiety (Weissman et al., 2006).

When receiving feedback, both the high and low trait anxiety group showed greater activation in the right superior/middle frontal gyrus in the reward (correct: 0c, error: 20c) compared to the punishment (correct: 0c, error: lose 20c) and neutral (correct: 0c, error: 0c) condition. Compared to the punishment and neutral conditions, feedback in the reward condition could be considered most incongruous as there is an absence of reward for a correct response and the presence of a reward for an incorrect response. The high trait anxiety group also showed greater activation in this region during feedback in the punishment (correct: 0c, error: lose 20c) compared to the neutral (correct: 0c, error: 0c) condition. The right superior/middle frontal gyrus is thought to be part of a ventral frontoparietal network that activates in response to the detection of behaviourally relevant or unexpected, low frequency events (see Corbetta, Patel, \& Shulman, 2008; Corbetta \& Shulman, 2002, for review). In relation to unexpected outcomes, it has been suggested that activation in the right superior/middle frontal gyrus reflects the prediction error (i.e., 
when the actual outcome differs from the expected outcome) regardless of valence of the outcome (i.e., aversive or appetitive) (Gu et al., 2016). In the current study, if the right superior/middle frontal gyrus reflects prediction error, the unexpected nature of the absence of a reward for a correct response (0c) or presence of a reward for an incorrect response (20c) may explain why all participants showed greater activation in this region for correct feedback in the reward condition compared to correct feedback in the punishment condition or the neutral condition. Only participants high in trait anxiety showed increased activation in this region in the punishment condition, i.e., when they received absence of reward for a correct response (0c) or punishment for an incorrect response (lose 20c). There was also no interaction between accuracy of response and activation in this region, i.e., the right superior/middle frontal cortex was not activated only for what could be considered 'classic' punishment trials, i.e., punishment following an incorrect response (lose 20c). The absence of an interaction suggests that it was the expectation of punishment (established by the punishment cue that was consistent across all punishment trials) together with the feedback on punishment trials that was associated with the increased right superior/middle frontal cortex activity. In punishment sensitive individuals who are more likely to expect negative outcomes, absence of a reward for a correct response or presence of punishment for an incorrect response should result in a smaller, not a larger, prediction error. It has been suggested that, in individuals who are sensitive to punishment, increased activation in areas that code prediction errors following punishing feedback reflects the subjective value of the prediction error and how concerned those individuals are with making mistakes rather than the magnitude of the prediction error per se (M. A. S. Boksem et al., 2008). Activation in the right superior/middle frontal cortex in response to the absence of rewarding feedback or the presence of punishing feedback in the current study may reflect the subjective value of 
the prediction error for participants high in trait anxiety. As mentioned, it is also thought that this area is part of a ventral frontoparietal network that activates in response to the detection of behaviourally relevant events. It is therefore possible that punishment sensitive individuals who receive an anticipated punishment will better remember the aversive feedback and learn to avoid engaging in behaviours that caused it (Cavanagh, Bismark, Frank, \& Allen, 2011; Holroyd \& Coles, 2002). This may lead to chronic, lifelong avoidance of what could actually be rewarding situations and events.

The low trait anxiety group showed increased activity in the right insula for correct compared to error feedback. The insula is involved in cognitive and affective processes during learning (Paulus \& Stein, 2006), regulation of the autonomic nervous system (Oppenheimer et al., 1992), subjective feeling states including interoception and predictive interoception (Paulus, 2008), and tracking and encoding risk (Bossaerts, 2010). In people with anxiety, the insula has been shown to have reduced connectivity with medial prefrontal and orbitofrontal cortex-subcortical networks, which may reflect a reduced ability to activate and regulate responses to changes in internal and external states/events (Bijsterbosch et al., 2014). In the present study, increased right insula activation in the low trait anxiety group following correct 'stop' responses - that were all accompanied by the absence of monetary reward - may reflect the subjective feeling state and regulation of the autonomic response associated either with correct feedback ('Correct' $=$ positive affective state) or the associated absence of monetary reward ('0c' = negative affective state). The absence of increased right insula activation in individuals high in trait anxiety suggests these individuals experienced reduced autonomic activation and internal feeling states following a correct response.

A limitation of the current study was the complexity of the task used. The number of conditions and types of feedback in the MICT may have overwhelmed participants and 
caused them to simplify the way they performed the task. They may have focused specifically on the stimulus epoch in all conditions rather than paying attention to and responding specifically to rewarding and punishing cues and feedback. If participants ignored cues, we would find an absence of neural activity in reward and punishment networks. In order to elicit responses to incentivised cues in the MICT, future studies may need to better differentiate conditions by increasing the length of time of cue presentation and/or using a blocked design rather than interleaving trial types within the same block. In addition, the difficulty of the task may have resulted in participants responding more cautiously in all conditions. This possibility is supported by the significantly lower go accuracy rates (omission errors) in the current study compared with go accuracy rates in similar types of inhibitory control tasks such as go/no-go tasks (see accuracy rates in studies by Bokura, Yamaguchi, \& Kobayashi, 2001; Menon, Adleman, White, Glover, \& Reiss, 2001, for example). If participants responded more cautiously in all conditions and withheld rather than cancelled an already initiated response in the anticipatory epoch as well as during stop trials, we would find a reduction or absence of neural activity in cognitive control networks and inhibitory control networks. In order to increase activation in cognitive control and inhibitory control networks, future studies could potentially reduce participants' cautionary behaviour and their focus on correctly stopping on stop trials by providing incentives to increase response speed on go trials.

It should be noted that the findings of group differences in activity in the left PCC, the left dorsal PCC, the right superior/middle frontal gyrus, and right insula were not predicted a priori. This raises the possibility that these findings may be due to chance or alternative explanations to those presented here may apply. Further studies are needed in order to replicate these findings, as replication will increase the likelihood that the 
results observed here reflect a true effect. These studies may also wish to include variables that have been found to be associated with trait anxiety - such as depression (Bados et al., 2010; M. Balsamo et al., 2013) - as covariates when attempting to replicate these findings.

In conclusion, this study showed that individuals high in trait anxiety do not appear to be better at inhibiting their response to a rewarding stimulus relative to low trait anxious individuals, or to their own performance when inhibition failure resulted in punishment. High trait anxious participants did appear to use compensatory strategies such as slowing their go reaction time in order to perform at the same level as individuals low in trait anxiety. We found that when presented with cues signalling potential reward, individuals high in trait anxiety show greater deactivation in the left dorsal PCC and do not increase activation in the left PCC. These patterns suggest that when anticipating potential reward, individuals high in trait anxiety may experience reductions in internal processing and may need to apply increased cognitive effort to perform the task. When individuals high in trait anxiety anticipate potential punishment and receive an absence of reward or receive punishing feedback, greater activation of the right superior/middle frontal gyrus may represent the behavioural and/or emotional relevance of this type of feedback to punishment sensitive individuals. Absence of right insula activation for feedback indicating a correct response in the high trait anxiety group suggests that these individuals may experience reduced autonomic activation and internal feeling states following correct responses. Overall, our findings suggest that individuals who experience anxiety may experience difficulty exerting control during potentially rewarding situations. Under such conditions, they appear to have a reduced level of monitoring of affect associated with receipt of positive and negative outcomes. 


\section{Chapter 6}

\section{General Discussion}

The current research project aimed to investigate the effect of reward and punishment on behavioural and neural indices of inhibitory control in trait anxiety. We were interested in this question because all anxiety disorders are characterised by avoidance behaviours but the research literature to date has not clarified whether this avoidance is associated with reduced sensitivity to positive/rewarding signals, increased sensitivity to negative/punishing signals, or both. In order to examine the relationship between inhibitory control and motivational factors, we used the monetary incentive control task (MICT) that combines the anticipatory reward, punishment and neutral cues and feedback of a monetary incentive delay task with the go and stop trials of a stop signal task. We administered this task in a magnetic resonance imaging (MRI) scanner in order to observe brain activity associated with inhibitory control and motivational factors, and recruited participants based on their level of trait anxiety. Trait anxiety is related to anxiety disorders; it is elevated in individuals who meet criteria for anxiety disorders (Bieling et al., 1998; J. A. Chambers et al., 2004) and predicts future anxiety disorder diagnosis (Plehn \& Peterson, 2002). Trait anxiety was measured using the State Trait Anxiety Inventory (STAI) trait anxiety scale (STAI T-Anxiety scale), which is one of the most widely used instruments for measurement of trait anxiety (M. Balsamo et al., 2013; Grös et al., 2007). A number of studies have questioned the factor structure and convergent and discriminant validity of the STAI T-Anxiety scale, and there has been little research that has applied exploratory factor analysis (EFA) to data from a healthy population. Therefore, in the current research the factor structure and convergent and discriminant validity of the STAI T-Anxiety scale was examined using EFA with a large sample of 947 healthy individuals. 
EFA suggested that the STAI T-Anxiety scale is comprised of two method factors that have previously been called 'trait anxiety absent' and 'trait anxiety present' (Bieling et al., 1998; Spielberger et al., 1983). These two factors were highly correlated and appeared to reflect reversed versus forward item keying, thereby suggesting a method factor. A one factor solution revealed that the STAI T-Anxiety scale could be represented as a single dimension. To aid interpretation of this structure, the scale's convergent and discriminant validity was explored. The scale had less than ideal discriminant validity for anxiety because it correlated highly with measures of depression. This finding was consistent with previous research performed using both healthy and clinical populations (Bados et al., 2010; M. Balsamo et al., 2013; Bieling et al., 1998; Grös et al., 2007; Nitschke et al., 2001) and suggests that the STAI T-Anxiety scale may measure high negative affect and low positive affect rather than anxiety in particular. The STAI TAnxiety scale also correlated more highly with measures of anxiety that contain items related to worry and anxious apprehension (e.g., Penn State Worry Questionnaire (PSWQ) and Generalized Anxiety Disorder 7-Item Scale (GAD-7)) and measures that contain items related to general distress (e.g., Mood and Anxiety Symptom Questionnaire (MASQ) General Distress Mixed scale, Big Five Aspects Scales (BFAS) Neuroticism scale, Personality Inventory for DSM-5 Brief Form - Adult (PID-5-BF - Adult) Affect scale) compared to instruments that contain items related mostly to somatic symptoms and anxious arousal (e.g., Beck Anxiety Inventory (BAI) and MASQ anxious arousal scale). The absence of items measuring physiologic hyperarousal and somatic symptoms in the STAI T-Anxiety scale has been noted previously (Bados et al., 2010; M. Balsamo et al., 2013; Grös et al., 2007; Nitschke et al., 2001) and indicates that the STAI TAnxiety scale does not measure anxiety as it is conceptualised in, for example, the tripartite model proposed by Clark and Watson (1991). 
The STAI T-Anxiety scale has been used in thousands of studies (Grös et al., 2007). Given the scale's limited discriminant validity, when used in research the scale may mistakenly identify participants who have depressive symptoms as high in trait anxiety. In relation to the scale's convergent validity, it appears to measure cognitive aspects of anxiety such as worry and rumination adequately but does not contain items related to physiologic hyperarousal and somatic symptoms. When the scale is used to recruit participants in research, individuals who are highly anxious but experience somatic rather than cognitive symptoms (such as racing heart and sweating) may be overlooked. This reduces clinical relevance and generalisability of findings because theories such as the tripartite model of anxiety (Clark \& Watson, 1991) propose that a crucial component of anxiety is physiological hyperarousal, and anxiety disorders such as panic disorder, agoraphobia and social anxiety disorder include physiological hyperarousal and somatic symptoms.

For the functional magnetic resonance imaging (fMRI) study, we intended to allocate participants to one of two groups; one group low in trait anxiety and one group high in trait anxiety based on their STAI T-Anxiety scale total score. We were therefore interested in how groups low and high in trait anxiety may differ on related constructs such as anxiety, mood and personality traits. After performing the EFA, we extracted data from 24 participants who achieved the lowest STAI T-Anxiety scale total score and 24 participants who achieved the highest STAI T-Anxiety scale total score. We found that individuals who scored high on the STAI T-Anxiety scale also scored higher than individuals low in trait anxiety on other measures of anxiety (e.g., PSWQ, GAD-7 and BAI), depression (e.g., Beck Depression Inventory-II (BDI-II) and Inventory of Depressive Symptomatology - Self Report (IDS-SR)), measures of personality commonly associated with anxiety (e.g., BFAS Neuroticism scale and Behavioral 
Inhibition System/Behavioral Activation System Scales behavioral inhibition subscale (BIS subscale)), and some aspects of impulsivity (Barratt Impulsiveness Scale), and they scored lower on attentional control (Attentional Control Scale). We also administered a measure of purely somatic symptoms of anxiety (MASQ anxious arousal scale) and, consistent with findings obtained in the first study using the larger sample, the low and high trait anxiety groups did not obtain significantly different scores on this scale. This result further confirmed the initial finding that the STAI T-Anxiety scale contains relatively few items that measure physiologic hyperarousal or somatic symptoms of anxiety

Despite finding that the STAI T-Anxiety scale correlates highly with measures of depression and negative affect and contains few items relating to physiologic hyperarousal or somatic symptoms of anxiety, it has been used to measure trait anxiety in research and clinical settings for over 30 years. It also appears to measure cognitive aspects of anxiety (e.g., worry and rumination) adequately. We therefore used the STAI T-Anxiety scale to select participants for the fMRI study. In the MRI scanner, we administered the MICT that was developed to assess the effect of rewarding and punishing cues and feedback on inhibitory control. In the MICT, inhibitory control is measured using an adaptation of the stop signal task. Given our aim of examining the role of anxiety in controlling a response to a rewarding stimulus, we first examined the literature to find whether there was relationship between anxiety and inhibitory control. It became apparent that the research literature examining both stop signal task performance and other measures of inhibitory control (e.g., go/no-go tasks, Stroop tasks etc.), was mixed in relation to the effect of anxiety. The review of the literature suggested that differences in task type and design as well as use of heterogeneous groups of participants might have contributed to the inconsistency in findings. 
To address some of the potential limitations of the research literature examining the influence of anxiety on stop signal performance, we conducted two experiments that administered stop signal tasks to participants differing in levels of trait anxiety. First, we administered a stop signal task to participants we identified as being either low or high in trait anxiety using the STAI T-Anxiety scale. Contrary to expectations, we failed to find group differences in any of the dependent variables from the stop signal task (i.e., stop signal reaction time (SSRT), stop accuracy, mean correct go reaction time, and stop signal delay). In an attempt to improve the study design, we recruited a larger group of participants using trait anxiety as a continuous rather than a dichotomous variable. We administered the stop signal task to this larger group of participants via the internet and performed correlational analyses on the relationship between trait anxiety and inhibitory control performance. Consistent with results of the first experiment, we failed to find evidence of a correlation between trait anxiety and any dependent variable from the stop signal task. Given our attempts to address some of the issues with studies that have failed to find a significant relationship between anxiety and inhibitory control, for example by recruiting larger samples with greater variation in trait anxiety scores, our findings appeared consistent with findings in the literature of no significant relationship between stop signal performance and trait anxiety (Avila \& Parcet, 2001; Epstein et al., 2001; Korenblum et al., 2007; M. A. Lau et al., 2007; Savostyanov et al., 2009; Savostyanov et al., 2011). Given that the current research appeared to address many of the methodological issues identified in previous studies, the null finding appears to be robust.

The absence of a relationship between trait anxiety and inhibitory control may have been influenced by the structure of the STAI T-Anxiety scale. The STAI T-Anxiety scale total score correlates better with measures of depression than anxiety; specifically, the STAI T-Anxiety scale appears to measure high negative affect together with low 
positive affect (Bados et al., 2010; M. Balsamo et al., 2013; Bieling et al., 1998; Clark \& Watson, 1991). Although the literature is minimal and results have sometimes been inconsistent (Sjoerds et al., 2014), a number of studies have found that depression does not appear to be associated with differences in stop signal performance (Jakubczyk et al., 2012; M. A. Lau et al., 2007; Lyche et al., 2010; Matthews et al., 2009). The absence of a relationship between trait anxiety and stop signal performance may be confounded by depressive symptoms and/or symptoms relating to high negative affect influencing our measure of anxiety. Importantly, when we examined the relationship between anxiety and inhibitory control using other measures of anxiety (i.e., BAI, GAD-7, PSWQ, BIS subscale of the BIS/BAS Scales, sensitivity to punishment scale of the Sensitivity to Punishment and Sensitivity to Reward Questionnaire (SPSRQ) and the MASQ), some of which have been found to adequately discriminate anxiety from depression, (Brown, Antony, \& Barlow, 1992; B. J. Cox, Cohen, Direnfeld, \& Swinson, 1996; Creamer, Foran, \& Bell, 1995; Keogh \& Reidy, 2000) we again failed to find an association between anxiety and stop signal performance. This suggests that anxiety - regardless of whether or not it is measured together with symptoms of depression - does not appear to significantly influence stop signal task performance.

Attentional control theory proposes that individuals who are high in anxiety may use compensatory strategies such as increased effort and processing resources to improve performance on tasks that measure inhibitory control. Increased effort and use of processing resources may be observed in patterns of neural activation measured using electroencephalogram (EEG) (e.g., Savostyanov et al., 2009; Savostyanov et al., 2011) or imaging (e.g., Basten et al., 2011; Fales et al., 2008). Given this possibility, we performed fMRI on participants high and low in trait anxiety while they performed the MICT. 
The overarching aim of the current research was to examine the effect of reward and punishment on inhibitory control in anxiety. We administered the MICT to 24 individuals low in trait anxiety and 24 individuals high in trait anxiety while we measured the blood oxygenated level-dependent (BOLD) response following specific events such as anticipation preceding a go or stop trial, successful inhibition on stop trials, and feedback following stop trials. Contrary to our evidence-based hypotheses, individuals high in trait anxiety did not demonstrate increased inhibitory control (i.e., higher stop accuracy) in the punishment condition. Participants high in trait anxiety did, however, slow their response times on go trials across all conditions compared to participants low in trait anxiety, which may have improved their stop accuracy. According to attentional control theory, slower go reaction times indicate reduced processing efficiency. Although anxiety did not impair performance effectiveness (i.e., stop accuracy), the use of compensatory strategies such as slowing go reaction time suggests that individuals high in trait anxiety do experience inhibitory control deficits and may use compensatory strategies to overcome them.

We also examined neural activity associated with inhibitory control (i.e., cancellation of an already initiated response upon presentation of the stop signal), along with neural activity associated with anticipation of and feedback indicating reward and punishment. Together with behavioural findings, neural activity associated with these task periods may indicate sensitivity to reward and punishment. Although one region known to be involved in inhibitory control was activated during the anticipation period (right inferior frontal gyrus), we did not observe significant (or above-threshold) activation in other inhibitory control regions (e.g., pre-supplementary motor area and anterior insula) during the anticipation period or the stopping period. Contrary to expectations, we also did not observe any group differences in cognitive control, goal- 
directed attentional networks. This finding may be due to the nature of the MICT, which may place fewer demands on cognitive control regions such as the dorsolateral prefrontal cortex than other tasks, such as Stroop tasks, letter search tasks, $n$-back tasks, and sustained attention to response tasks with low frequency stop trials, that have been used in previous literature with significant results (Basten et al., 2011; Bishop, 2009; Fales et al., 2008; Forster et al., 2015).

We failed to find activity in structures or networks involved in reward and punishment processing during anticipation, stopping or feedback generally and we did not observe any group-related differences in activity in these structures/networks. These findings may have been due to the complexity of the task which meant that participants did not consciously process cues and feedback in each of the conditions.

Despite the non-significant findings, we did find a number of group differences in neural activation. First, during the anticipatory period we observed group differences in posterior default mode areas that may have been because the high trait anxiety group applied increased effort and/or increased cognitive control during reward trials, and experienced greater difficulty maintaining task-based attention in the absence of motivational incentives (i.e., during neutral trials). Second, during the feedback period we observed group differences in the right superior/middle frontal gyrus that suggested that, for individuals high in trait anxiety, the absence of rewarding feedback or the presence of punishing feedback when they are already expecting potential punishment has increased subjective value and salience. This may mean that individuals high in trait anxiety remember those outcomes more. Finally, we observed less right insula activity in the high compared to the low trait anxiety group for feedback indicating a correct response as opposed to an error. This finding suggests that individuals high in trait anxiety experience reduced autonomic activation and internal feeling states following correct responses. 
Taken together, these findings map onto the model of network dysfunction in anxiety proposed by Sylvester et al. (2012). Specifically, we identified dysfunction in structures involved in three of the four networks described by Sylvester et al., namely the cinguloopercular network (insula) involved in detecting errors and conflict and processing pain and negative affect, the ventral attention network (right superior/middle frontal cortex) involved in automatic orientation of stimulus-driven attention, and the default mode network (posterior cingulate cortex) involved in self-referential activities, selfintrospection and emotion regulation. Our imaging findings suggest that highly anxious individuals apply extra effort and therefore may find it more difficult to act in potentially rewarding situations (default mode network). Non-rewarding and punishing outcomes also appear more evocative when highly anxious individuals are anticipating punishment (ventral attention network), and they may not feel the usual affect associated with receipt of positive outcomes and the absence of reward (cingulo-opercular network). 


\section{Summary}

We were interested in investigating the effect of rewards and punishments on inhibitory control in anxiety. We used the State Trait Anxiety Inventory trait anxiety scale to measure trait anxiety. Although it may adequately measure cognitive aspects of anxiety (e.g., worry and rumination), the instrument would benefit from revision to improve its convergent and discriminant validity, as well as to include more items relating to physiological hyperarousal and somatic symptoms. There appears to be no evidence for a relationship between trait anxiety and inhibitory control as indexed by stop signal task performance. It is possible that the stop signal task commonly used to measure inhibitory control is not difficult enough to elicit processing efficiency deficits in individuals high on the construct of trait anxiety. When other inhibitory control tasks such as the monetary incentive control task (MICT) are administered, individuals high in trait anxiety do display reductions in processing efficiency as evidenced by slower reaction times on go trials. Unfortunately, although the MICT appeared to elicit processing efficiency deficits in individuals high in trait anxiety, performance was associated with an absence of activity in neural structures of interest; namely, structures involved in cognitive control, inhibitory control and reward and punishment processing. Nevertheless, we identified that individuals high in trait anxiety demonstrate abnormal activity in a number of neural structures that belong to networks of interest, both during anticipation of a stimulus and

during feedback following a response. The patterns of activation we observed provide the first glimpse of a neural mechanism that may be able to explain why individuals high in anxiety engage in behavioural avoidance, but further research is required to more clearly characterise it. 


\section{References}

Aarts, K., \& Pourtois, G. (2010). Anxiety not only increases, but also alters early errormonitoring functions. Cognitive, Affective and Behavioral Neuroscience, 10(4), 479-492.

Aarts, K., \& Pourtois, G. (2012). Anxiety disrupts the evaluative component of performance monitoring: An ERP study. Neuropsychologia, 50(7), 1286-1296.

Admon, R., Lubin, G., Rosenblatt, J. D., Stern, O., Kahn, I., Assaf, M., \& Hendler, T. (2013). Imbalanced neural responsivity to risk and reward indicates stress vulnerability in humans. Cerebral Cortex, 23(1), 28-35.

Aluja, A., \& Blanch, A. (2011). Neuropsychological Behavioral Inhibition System (BIS) and Behavioral Approach System (BAS) assessment: a shortened Sensitivity to Punishment and Sensitivity to Reward Questionnaire version (SPSRQ-20). Journal of Personality Assessment, 93(6), 628-636.

doi: $10.1080 / 00223891.2011 .608760$

Alvarez, R. P., Kirlic, N., Misaki, M., Bodurka, J., Rhudy, J. L., Paulus, M. P., \& Drevets, W. C. (2015). Increased anterior insula activity in anxious individuals is linked to diminished perceived control. Transl Psychiatry Psychiatry, 5, e591.

American Psychiatric Association. (2000). Diagnostic and statistical manual of mental disorders (4th ed.). Washington, DC: Author.

American Psychiatric Association. (2013a). Diagnostic and statistical manual of mental disorders (5th ed.). Washington, DC: Author.

American Psychiatric Association. (2013b). DSM-5's Integrated approach to diagnosis and classifications. Retrieved from 
https://www.psychiatry.org/File\%20Library/Psychiatrists/Practice/DSM/APA_DS

\section{M-5-Integrated-Approach.pdf}

Amir, N., Klumpp, H., Elias, J., Bedwell, J. S., Yanasak, N., \& Miller, L. S. (2005). Increased activation of the anterior cingulate cortex during processing of disgust faces in individuals with social phobia. Biological Psychiatry, 57(9), 975-981. doi:10.1016/j.biopsych.2005.01.044

Amodio, D. M., Master, S. L., Yee, C. M., \& Taylor, S. E. (2008). Neurocognitive components of the behavioral inhibition and activation systems: Implications for theories of self-regulation. Psychophysiology, 45(1), 11-19.

Ansari, T. L., \& Derakshan, N. (2010). Anxiety impairs inhibitory control but not volitional action control. Cognition and Emotion, 24(2), 241-254.

Ansari, T. L., \& Derakshan, N. (2011a). The neural correlates of cognitive effort in anxiety: Effects on processing efficiency. Biological Psychology, 86(3), 337-348. doi:http://dx.doi.org/10.1016/j.biopsycho.2010.12.013

Ansari, T. L., \& Derakshan, N. (2011b). The neural correlates of impaired inhibitory control in anxiety. Neuropsychologia, 49(5), 1146-1153. doi:http://dx.doi.org/10.1016/j.neuropsychologia.2011.01.019

Aron, A. R. (2007). The neural basis of inhibition in cognitive control. Neuroscientist, 13(3), 214-228.

Aron, A. R. (2011). From reactive to proactive and selective control: Developing a richer model for stopping inappropriate responses. Biological Psychiatry, 69(12), e55e68.

Aron, A. R., \& Poldrack, R. A. (2006). Cortical and subcortical contributions to stop signal response inhibition: Role of the subthalamic nucleus. Journal of Neuroscience, 26(9), 2424-2433. doi:10.1523/jneurosci.4682-05.2006 
Aron, A. R., Robbins, T. W., \& Poldrack, R. A. (2004). Inhibition and the right inferior frontal cortex. Trends in Cognitive Sciences, 8(4), 170-177. doi:10.1016/j.tics.2004.02.010

Aron, A. R., Robbins, T. W., \& Poldrack, R. A. (2014a). Inhibition and the right inferior frontal cortex: One decade on. Trends in Cognitive Sciences, 18(4), 177-185. doi:10.1016/j.tics.2013.12.003

Aron, A. R., Robbins, T. W., \& Poldrack, R. A. (2014b). Right inferior frontal cortex: Addressing the rebuttals. Frontiers in Human Neuroscience, 8(November). doi:10.3389/fnhum.2014.00905

Avila, C. (1994). Sensitivity to punishment and resistance to extinction: a test of Gray's behavioral inhibition system. Personality and Individual Differences, 17(6), 845847.

Avila, C., \& Parcet, M. A. (2001). Personality and inhibitory deficits in the stop-signal task: The mediating role of Gray's anxiety and impulsivity. Personality and Individual Differences, 31(6), 975-986.

Babor, T. F., Higgins-Biddle, J. C., Saunders, J. B., \& Monteiro, M. G. (2001). The alcohol use disorders identification test: Guidelines for use in primary care (2nd ed.). Geneva: World Health Organisation.

Bados, A., Gómez-Benito, J., \& Balaguer, G. (2010). The state-trait anxiety inventory, trait version: Does it really measure anxiety? Journal of Personality Assessment, 92(6), 560-567.

Balsamo, M. (2006). Costruzione di un nuovo strumento self-report di depressione [Construction of a new Italian depression self-report instrument]. Università "G. d'Annunzio" di Chieti-Pescara, Chieti. 
Balsamo, M., Romanelli, R., Innamorati, M., Ciccarese, G., Carlucci, L., \& Saggino, A. (2013). The state-trait anxiety inventory: Shadows and lights on its construct validity. Journal of Psychopathology and Behavioral Assessment, 35(4), 475-486.

Bar-Haim, Y., Fox, N. A., Benson, B., Guyer, A. E., Williams, A., Nelson, E. E., . . Ernst, M. (2009). Neural correlates of reward processing in adolescents with a history of inhibited temperament. Psychological Science, 20(8), 1009-1018.

Bar-Haim, Y., Lamy, D., Pergamin, L., Bakermans-Kranenburg, M. J., \& Van Ijzendoorn, M. H. (2007). Threat-related attentional bias in anxious and nonanxious individuals: A meta-analytic study. Psychological Bulletin, 133(1), 124.

Bardeen, J. R., Tull, M. T., Stevens, E. N., \& Gratz, K. L. (2014). Exploring the relationship between positive and negative emotional avoidance and anxiety symptom severity: the moderating role of attentional control. Journal of Behavior Therapy and Experimental Psychiatry, 45(3), 415-420. doi:10.1016/j.jbtep.2014.04.006

Bari, A., \& Robbins, T. W. (2013). Inhibition and impulsivity: Behavioral and neural basis of response control. Progress in Neurobiology, 108, 44-79.

Barrett, J., \& Armony, J. L. (2009). Influence of trait anxiety on brain activity during the acquisition and extinction of aversive conditioning. Psychological Medicine, 39(2), 255-265. doi:10.1017/s0033291708003516

Bartlett, M. S. (1954). A note on the multiplying factors for various chi square approximations. Journal of the Royal Statistical Society, 16(Series B), 296-298.

Basten, U., Stelzel, C., \& Fiebach, C. J. (2011). Trait anxiety modulates the neural efficiency of inhibitory control. Journal of Cognitive Neuroscience, 23(10), 31323145. 
Baxter, A. J., Scott, K. M., Vos, T., \& Whiteford, H. A. (2013). Global prevalence of anxiety disorders: A systematic review and meta-regression. Psychological Medicine, 43(5), 897-910.

Beck, A. T., Rush, A. J., Shaw, B. F., \& Emery, G. (1979). Cognitive therapy of depression: A treatment manual. New York: Guilford.

Beck, A. T., \& Steer, R. A. (1990). Beck Anxiety Inventory manual. San Antonio: The Psychological Corporation.

Beck, A. T., Steer, R. A., \& Brown, G. K. (1996). Manual for the Beck Depression Inventory-II. San Antonio: The Psychological Corporation.

Benson, B. E., Guyer, A. E., Nelson, E. E., Pine, D. S., \& Ernst, M. (2014). Role of contingency in striatal response to incentive in adolescents with anxiety. Cognitive, Affective, \& Behavioral Neuroscience.

Berkman, E. T., Lieberman, M. D., \& Gable, S. L. (2009). BIS, BAS, and response conflict: Testing predictions of the revised reinforcement sensitivity theory. Personality and Individual Differences, 46(5-6), 586-591.

Bieling, P. J., Antony, M. M., \& Swinson, R. P. (1998). The state-trait anxiety inventory, trait version: Structure and content re-examined. Behaviour Research and Therapy, 36(7-8), 777-788.

Bijleveld, E., Custers, R., Van der Stigchel, S., Aarts, H., Pas, P., \& Vink, M. (2014). Distinct neural responses to conscious versus unconscious monetary reward cues. Human Brain Mapping, 35(11), 5578-5586. doi:10.1002/hbm.22571

Bijsterbosch, J., Smith, S., Forster, S., John, O. P., \& Bishop, S. J. (2014). Resting state correlates of subdimensions of anxious affect. Journal of Cognitive Neuroscience, 26(4), 914-926. 
Birnbaum, M. H. E. (n.d.). Psychology Experiments on the Internet. The Web Experiment Method: Advantages, Disadvantages, and Solutions. Retrieved from http://citeseerx.ist.psu.edu/viewdoc/download?doi=10.1.1.471.85\&rep=rep1\&typ $\underline{\mathrm{e}=\mathrm{pdf}}$

Bishop, S. J. (2007). Neurocognitive mechanisms of anxiety: an integrative account. Trends in Cognitive Sciences, 11(7), 307-316.

Bishop, S. J. (2009). Trait anxiety and impoverished prefrontal control of attention. Nature Neuroscience, 12(1), 92-98.

Bishop, S. J., Duncan, J., \& Lawrence, A. D. (2004). State anxiety modulation of the amygdala response to unattended threat-related stimuli. Journal of Neuroscience, 24(46), 10364-10368. doi:10.1523/jneurosci.2550-04.2004

Bissonette, G. B., Gentry, R. N., Padmala, S., Pessoa, L., \& Roesch, M. R. (2014). Impact of appetitive and aversive outcomes on brain responses: Linking the animal and human literatures. Frontiers in Systems Neuroscience, 8(MAR).

Blalock, H. M. (1963). Correlated independent variables: The problem of multicollinearity. Social Forces, 42(2), 233-237.

Blanco, C., Olfson, M., Goodwin, R. D., Ogburn, E., Liebowitz, M. R., Nunes, E. V., \& Hasin, D. S. (2008). Generalizability of clinical trial results for major depression to community samples: Results from the National Epidemiologic Survey on Alcohol and Related Conditions. Journal of Clinical Psychiatry, 69(8), 12761280.

Boehler, C. N., Appelbaum, L. G., Krebs, R. M., Hopf, J. M., \& Woldorff, M. G. (2010). Pinning down response inhibition in the brain - Conjunction analyses of the Stopsignal task. NeuroImage, 52(4), 1621-1632. doi:10.1016/j.neuroimage.2010.04.276 
Boehler, C. N., Hopf, J. M., Stoppel, C. M., \& Krebs, R. M. (2012). Motivating inhibition - reward prospect speeds up response cancellation. Cognition, 125(3), 498-503. doi:10.1016/j.cognition.2012.07.018

Boehler, C. N., Schevernels, H., Hopf, J. M., Stoppel, C. M., \& Krebs, R. M. (2014). Reward prospect rapidly speeds up response inhibition via reactive control. Cognitive, Affective and Behavioral Neuroscience, 14(2), 593-609. doi:10.3758/s13415-014-0251-5

Boksem, M. A. S., Tops, M., Kostermans, E., \& De Cremer, D. (2008). Sensitivity to punishment and reward omission: Evidence from error-related ERP components. Biological Psychology, 79(2), 185-192.

Boksem, M. A. S., Tops, M., Wester, A. E., Meijman, T. F., \& Lorist, M. M. (2006). Error-related ERP components and individual differences in punishment and reward sensitivity. Brain Research, 1101(1), 92-101.

doi:10.1016/j.brainres.2006.05.004

Bokura, H., Yamaguchi, S., \& Kobayashi, S. (2001). Electrophysiological correlates for response inhibition in a Go/NoGo task. Clinical Neurophysiology, 112(12), 22242232. doi:10.1016/S1388-2457(01)00691-5

Bossaerts, P. (2010). Risk and risk prediction error signals in anterior insula. Brain structure \& function, 214(5-6), 645-653.

Braem, S., Duthoo, W., \& Notebaert, W. (2013). Punishment Sensitivity Predicts the Impact of Punishment on Cognitive Control. PLoS ONE, 8(9).

Brown, T. A., Antony, M. M., \& Barlow, D. H. (1992). Psychometric properties of the Penn state worry questionnaire in a clinical anxiety disorders sample. Behaviour Research and Therapy, 30(1), 33-37. doi:http://dx.doi.org/10.1016/00057967(92)90093-V 
Burdwood, E. N., Infantolino, Z. P., Crocker, L. D., Spielberg, J. M., Banich, M. T., Miller, G. A., \& Heller, W. (2016). Resting-state functional connectivity differentiates anxious apprehension and anxious arousal. Psychophysiology. doi:10.1111/psyp.12696

Button, K. S., Ioannidis, J. P. A., Mokrysz, C., Nosek, B. A., Flint, J., Robinson, E. S. J., \& Munafò, M. R. (2013). Power failure: Why small sample size undermines the reliability of neuroscience. Nature Reviews Neuroscience, 14(5), 365-376. doi:10.1038/nrn3475

Bystritsky, A., Pontillo, D., Powers, M., Sabb, F. W., Craske, M. G., \& Bookheimer, S. Y. (2001). Functional MRI changes during panic anticipation and imagery exposure. NeuroReport, 12(18), 3953-3957.

Campbell-Sills, L., Simmons, A. N., Lovero, K. L., Rochlin, A. A., Paulus, M. P., \& Stein, M. B. (2011). Functioning of neural systems supporting emotion regulation in anxiety-prone individuals. NeuroImage, 54(1), 689-696.

doi:10.1016/j.neuroimage.2010.07.041

Canteras, N. S., Resstel., L. B., Bertoglio, L. J., de Padua Carobrez, A., \& Guimaraes, F. S. (2010). Neuroanatomy of anxiety. In M. B. Stein \& T. Steckler (Eds.), Behavioral neurobiology of anxiety and its treatment (pp. 77-96). Berlin: Springer-Verlag.

Carver, C. S., \& White, T. L. (1994). Behavioral Inhibition, Behavioral Activation, and Affective Responses to Impending Reward and Punishment: The BIS/BAS Scales. Journal of Personality and Social Psychology, 67(2), 319-333.

Cattel, R. B. (1966). The scree test for number of factors. Multivariate Behavioral Research, 1(2), 245-276. 
Cauda, F., D'Agata, F., Sacco, K., Duca, S., Geminiani, G., \& Vercelli, A. (2011). Functional connectivity of the insula in the resting brain. NeuroImage, 55(1), 823. doi:10.1016/j.neuroimage.2010.11.049

Cavanagh, J. F., Bismark, A. J., Frank, M. J., \& Allen, J. J. B. (2011). Larger error signals in major depression are associated with better avoidance learning. Frontiers in Psychology, 2(NOV).

Cavanagh, J. F., Frank, M. J., \& Allen, J. J. B. (2011). Social stress reactivity alters reward and punishment learning. Social Cognitive and Affective Neuroscience, 6(3), 311-320.

Chambers, C. D., Garavan, H., \& Bellgrove, M. A. (2009). Insights into the neural basis of response inhibition from cognitive and clinical neuroscience. Neuroscience and Biobehavioral Reviews, 33(5), 631-646. doi:10.1016/j.neubiorev.2008.08.016

Chambers, J. A., Power, K. G., \& Durham, R. C. (2004). The relationship between trait vulnerability and anxiety and depressive diagnoses at long-term follow-up of Generalized Anxiety Disorder. Journal of Anxiety Disorders, 18(5), 587-607.

Chao, H. H., Luo, X., Chang, J. L., \& Li, C. S. (2009). Activation of the presupplementary motor area but not inferior prefrontal cortex in association with short stop signal reaction time--an intra-subject analysis. BMC neuroscience, 10 , 75. doi:10.1186/1471-2202-10-75

Charles-Walsh, K., Upton, D. J., \& Hester, R. (2016). Examining the interaction between cognitive control and reward sensitivity in substance use dependence. Drug and Alcohol Dependence, 166, 235-242. doi:10.1016/j.drugalcdep.2016.07.020

Chikazoe, J. (2010). Localizing Performance of go/no-go tasks to prefrontal cortical subregions. Current Opinion in Psychiatry, 23(3), 267-272. 
Chikazoe, J., Jimura, K., Hirose, S., Yamashita, K. I., Miyashita, Y., \& Konishi, S. (2009). Preparation to inhibit a response complements response inhibition during performance of a stop-signal task. Journal of Neuroscience, 29(50), 15870-15877. doi:10.1523/jneurosci.3645-09.2009

Christoff, K., Gordon, A. M., Smallwood, J., Smith, R., \& Schooler, J. W. (2009). Experience sampling during fMRI reveals default network and executive system contributions to mind wandering. Proceedings of the National Academy of Sciences of the United States of America, 106(21), 8719-8724. doi:10.1073/pnas.0900234106

Chronis-Tuscano, A., Degnan, K. A., Pine, D. S., Perez-Edgar, K., Henderson, H. A., Diaz, Y., . . Fox, N. A. (2009). Stable Early Maternal Report of Behavioral Inhibition Predicts Lifetime Social Anxiety Disorder in Adolescence. Journal of the American Academy of Child and Adolescent Psychiatry, 48(9), 928-935. doi:10.1097/CHI.0b013e3181ae09df

Cieslik, E. C., Mueller, V. I., Eickhoff, C. R., Langner, R., \& Eickhoff, S. B. (2015). Three key regions for supervisory attentional control: Evidence from neuroimaging meta-analyses. Neuroscience and Biobehavioral Reviews, 48, 2234.

Cisler, J. M., \& Koster, E. H. W. (2010). Mechanisms of attentional biases towards threat in anxiety disorders: An integrative review. Clinical Psychology Review, 30(2), 203-216.

Clark, L. A., \& Watson, D. (1991). Tripartite Model of Anxiety and Depression: Psychometric Evidence and Taxonomic Implications. Journal of Abnormal Psychology, 100(3), 316-336.

Cohen, J. (1992). A power primer. Psychological Bulletin, 112(1), 155-159. 
Cohen, J. W. (1988). Statistical power analysis for the behavioural sciences. Hillsdale, NJ: Lawrence Erlbaum Associates.

Comte, M., Cancel, A., Coull, J. T., Schon, D., Reynaud, E., Boukezzi, S., . . Fakra, E. (2015). Effect of trait anxiety on prefrontal control mechanisms during emotional conflict. Human Brain Mapping, 36(6), 2207-2214.

Congdon, E., Mumford, J. A., Cohen, J. R., Galvan, A., Canli, T., \& Poldrack, R. A. (2012). Measurement and reliability of response inhibition. Frontiers in Psychology, 3(FEB). doi:10.3389/fpsyg.2012.00037

Cooper, A. J., Perkins, A. M., \& Corr, P. J. (2007). A confirmatory factor analytic study of anxiety, fear, and behavioral inhibition system measures. Journal of Individual Differences, 28(4), 179-187.

Corbetta, M., Patel, G., \& Shulman, G. L. (2008). The Reorienting System of the Human Brain: From Environment to Theory of Mind. Neuron, 58(3), 306-324. doi:10.1016/j.neuron.2008.04.017

Corbetta, M., \& Shulman, G. L. (2002). Control of goal-directed and stimulus-driven attention in the brain. Nature Reviews Neuroscience, 3(3), 201-215.

Corr, P. J. (2002a). J. A. Gray's reinforcement sensitivity theory: Tests of the joint subsystems hypothesis of anxiety and impulsivity. Personality and Individual Differences, 33(4), 511-532.

Corr, P. J. (2002b). J.A. Gray's reinforcement sensitivity theory and frustrative nonreward: A theoretical note on expectancies in reactions to rewarding stimuli. Personality and Individual Differences, 32(7), 1247-1253.

Corr, P. J. (2004). Reinforcement sensitivity theory and personality. Neuroscience and Biobehavioral Reviews, 28(3), 317-332. 
Costello, A. B., \& Osborne, J. W. (2005). Best practices in exploratory factor analysis: Four recommendations for getting the most from your analysis. Practical Assessment, Research and Evaluation, 10(7).

Coutinho, J. F., Fernandesl, S. V., Soares, J. M., Maia, L., Gonçalves, Ó. F., \& Sampaio, A. (2016). Default mode network dissociation in depressive and anxiety states. Brain Imaging and Behavior, 10(1), 147-157. doi:10.1007/s11682-015-9375-7

Cox, B. J., Cohen, E., Direnfeld, D. M., \& Swinson, R. P. (1996). Does the beck anxiety inventory measure anything beyond panic attack symptoms? Behaviour Research and Therapy, 34(11-12), 949-954. doi:10.1016/S0005-7967(96)00037-X

Cox, R. W. (1996). Analysis of Functional NeuroImages. Retrieved from http://afni.nimh.nih.gov/afni/

Crawford, J., Cayley, C., Lovibond, P. F., Wilson, P. H., \& Hartley, C. (2011). Percentile Norms and Accompanying Interval Estimates from an Australian General Adult Population Sample for Self-Report Mood Scales (BAI, BDI, CRSD, CES-D, DASS, DASS-21, STAI-X, STAI-Y, SRDS, and SRAS). Australian Psychologist, 46(1), 3-14.

Creamer, M., Foran, J., \& Bell, R. (1995). The Beck Anxiety Inventory in a non-clinical sample. Behaviour Research \& Therapy, 33(4), 477-485.

Cremers, H. R., Demenescu, L. R., Aleman, A., Renken, R., van Tol, M. J., van der Wee, N. J. A., . . Roelofs, K. (2010). Neuroticism modulates amygdala-prefrontal connectivity in response to negative emotional facial expressions. NeuroImage, 49(1), 963-970.

Cremers, H. R., Veer, I. M., Spinhoven, P., Rombouts, S. A. R. B., \& Roelofs, K. (2015). Neural sensitivity to social reward and punishment anticipation in social anxiety 
disorder. Frontiers in Behavioral Neuroscience, 8(JAN).

doi:10.3389/fnbeh.2014.00439

Criaud, M., \& Boulinguez, P. (2013). Have we been asking the right questions when assessing response inhibition in go/no-go tasks with fMRI? A meta-analysis and critical review. Neuroscience and Biobehavioral Reviews, 37(1), 11-23.

Dambacher, F., Sack, A. T., Lobbestael, J., Arntz, A., Brugman, S., \& Schuhmann, T. (2014). A network approach to response inhibition: Dissociating functional connectivity of neural components involved in action restraint and action cancellation. European Journal of Neuroscience, 39(5), 821-831. doi:10.1111/ejn.12425

De Pascalis, V., Varriale, V., \& D'Antuono, L. (2010). Event-related components of the punishment and reward sensitivity. Clinical Neurophysiology, 121(1), 60-76.

de Visser, L., van der Knaap, L. J., van de Loo, A. J. A. E., van der Weerd, C. M. M., Ohl, F., \& van den Bos, R. (2010). Trait anxiety affects decision-making differently in healthy men and women: towards gender-specific endophenotypes of anxiety. Neuropsychologia, 48(6), 1598-1606.

Dear, B. F., Titov, N., Sunderland, M., McMillan, D., Anderson, T., Lorian, C., \& Robinson, E. (2011). Psychometric comparison of the Generalized Anxiety Disorder Scale-7 and the Penn State Worry Questionnaire for measuring response during treatment of Generalised Anxiety Disorder. Cognitive Behaviour Therapy, 40(3), 216-227.

Derakshan, N., Ansari, T. L., Hansard, M., Shoker, L., \& Eysenck, M. W. (2009). Anxiety, inhibition, efficiency, and effectiveness: An investigation using the Antisaccade task. Experimental Psychology, 56(1), 48-55. 
Derakshan, N., \& Eysenck, M. W. (2009). Anxiety, processing efficiency, and cognitive performance: New developments from attentional control theory. European Psychologist, 14(2), 168-176.

Derogatis, L. R. (Ed.) (1983) (2nd ed.). Baltimore, MD: Clinical Psychometric Research.

Derryberry, D., \& Reed, M. A. (2002). Anxiety-related attentional biases and their regulation by attentional control. Journal of Abnormal Psychology, 111(2), 225236. doi:10.1037/0021-843X.111.2.225

DeYoung, C. G., Quilty, L. C., \& Peterson, J. B. (2007). Between Facets and Domains: 10 Aspects of the Big Five. Journal of Personality and Social Psychology, 93(5), 880-896.

Dickie, E. W., \& Armony, J. L. (2008). Amygdala responses to unattended fearful faces: Interaction between sex and trait anxiety. Psychiatry Research - Neuroimaging, 162(1), 51-57. doi:10.1016/j.pscychresns.2007.08.002

Diekhof, E. K., Kaps, L., Falkai, P., \& Gruber, O. (2012). The role of the human ventral striatum and the medial orbitofrontal cortex in the representation of reward magnitude - An activation likelihood estimation meta-analysis of neuroimaging studies of passive reward expectancy and outcome processing. Neuropsychologia, 50(7), 1252-1266. doi:http://dx.doi.org/10.1016/j.neuropsychologia.2012.02.007

Dillon, D. G., Holmes, A. J., Jahn, A. L., Bogdan, R., Wald, L. L., \& Pizzagalli, D. A. (2008). Dissociation of neural regions associated with anticipatory versus consummatory phases of incentive processing. Psychophysiology, 45(1), 36-49. doi:10.1111/j.1469-8986.2007.00594.X

Dillon, D. G., Rosso, I. M., Pechtel, P., Killgore, W. D. S., Rauch, S. L., \& Pizzagalli, D. A. (2014). Peril and pleasure: An rdoc-inspired examination of threat responses 
and reward processing in anxiety and depression. Depression and Anxiety, 31(3), 233-249.

Domschke, K., \& Dannlowski, U. (2010). Imaging genetics of anxiety disorders. NeuroImage, 53(3), 822-831.

Doppelmayr, M., Klimesch, W., Pachinger, T., \& Ripper, B. (1998). Individual differences in brain dynamics: Important implications for the calculation of eventrelated band power. Biological Cybernetics, 79(1), 49-57.

Drevets, W. C., \& Raichle, M. E. (1998). Reciprocal Suppression of Regional Cerebral Blood Flow during Emotional versus Higher Cognitive Processes: Implications for Interactions between Emotion and Cognition. Cognition and Emotion, 12(3), 353-385.

Duann, J. R., Ide, J. S., Luo, X., \& Li, C. S. R. (2009). Functional connectivity delineates distinct roles of the inferior frontal cortex and presupplementary motor area in stop signal inhibition. Journal of Neuroscience, 29(32), 10171-10179. doi:10.1523/jneurosci.1300-09.2009

Dunn, O. J. (1961). Multiple comparisons among means. Journal of the American Statistical Association, 56(293), 52-64. doi:10.1080/01621459.1961.10482090

Eagle, D. M., Bari, A., \& Robbins, T. W. (2008). The neuropsychopharmacology of action inhibition: Cross-species translation of the stop-signal and go/no-go tasks. Psychopharmacology, 199(3), 439-456.

Elwood, L. S., Wolitzky-Taylor, K., \& Olatunji, B. O. (2012). Measurement of anxious traits: A contemporary review and synthesis. Anxiety, Stress and Coping, 25(6), 647-666. 
Epstein, J. N., Johnson, D. E., Varia, I. M., \& Conners, C. K. (2001). Neuropsychological assessment of response inhibition in adults with ADHD. Journal of Clinical and Experimental Neuropsychology, 23(3), 362-371.

Erceg-Hurn, D. M., \& Mirosevich, V. M. (2008). Modern Robust Statistical Methods: An Easy Way to Maximize the Accuracy and Power of Your Research. American Psychologist, 63(7), 591-601. doi:10.1037/0003-066X.63.7.591

Erika-Florence, M., Leech, R., \& Hampshire, A. (2014). A functional network perspective on response inhibition and attentional control. Nature Communications, 5. doi:10.1038/ncomms5073

Eriksen, B. A., \& Eriksen, C. W. (1974). Effects of noise letters upon the identification of a target letter in a nonsearch task. Perception \&amp; Psychophysics, 16(1), 143149. doi:10.3758/BF03203267

Eriksson, L. J. K., Jansson, B., Lisspers, J., \& Sundin, Ö. (2016). The interactive effect of the Behavioral Inhibition System (BIS) and response inhibition on accuracy in a modified stop-signal task. Personality and Individual Differences, 97, 198-202. doi:10.1016/j.paid.2016.03.057

Etkin, A., Klemenhagen, K. C., Dudman, J. T., Rogan, M. T., Hen, R., Kandel, E. R., \& Hirsch, J. (2004). Individual differences in trait anxiety predict the response of the basolateral amygdala to unconsciously processed fearful faces. Neuron, 44(6), 1043-1055. doi:10.1016/j.neuron.2004.12.006

Etkin, A., \& Wager, T. D. (2007). Functional neuroimaging of anxiety: A meta-ana lysis of emotional processing in PTSD, social anxiety disorder, and specific phobia. American Journal of Psychiatry, 164(10), 1476-1488. 
Everling, S., \& Fischer, B. (1998). The antisaccade: a review of basic research and clinical studies. Neuropsychologia, 36(9), 885-899. doi:http://dx.doi.org/10.1016/S0028-3932(98)00020-7

Ewbank, M. P., Lawrence, A. D., Passamonti, L., Keane, J., Peers, P. V., \& Calder, A. J. (2009). Anxiety predicts a differential neural response to attended and unattended facial signals of anger and fear. NeuroImage, 44(3), 1144-1151.

doi:10.1016/j.neuroimage.2008.09.056

Eysenck, H. J., \& Eysenck, S. B. G. (1975). Manual of the Eysenck Personality Questionnaire (Junior and Adult). Kent, UK: Hodder \& Stoughton.

Eysenck, M. W., \& Derakshan, N. (2011). New perspectives in attentional control theory. Personality and Individual Differences, 50(7), 955-960.

Eysenck, M. W., Derakshan, N., Santos, R., \& Calvo, M. G. (2007). Anxiety and cognitive performance: Attentional control theory. Emotion, 7(2), 336-353.

Fabrigar, L. R., MacCallum, R. C., Wegener, D. T., \& Strahan, E. J. (1999). Evaluating the use of exploratory factor analysis in psychological research. Psychological Methods, 4(3), 272-299. doi:10.1037/1082-989x.4.3.272

Fagerstrom, K. O., Heatherton, T. F., \& Kozlowski, L. T. (1990). Nicotine addiction and its assessment. Ear, Nose and Throat Journal, 69(11), 763-765+768.

Fales, C. L., Barch, D. M., Burgess, G. C., Schaefer, A., Mennin, D. S., Gray, J. R., \& Braver, T. S. (2008). Anxiety and cognitive efficiency: Differential modulation of transient and sustained neural activity during a working memory task. Cognitive, Affective and Behavioral Neuroscience, 8(3), 239-253.

Fan, J., McCandliss, B. D., Sommer, T., Raz, A., \& Posner, M. I. (2002). Testing the efficiency and independence of attentional networks. Journal of Cognitive Neuroscience, 14(3), 340-347. doi:10.1162/089892902317361886 
Forster, S., Nunez Elizalde, A. O., Castle, E., \& Bishop, S. J. (2015). Unraveling the anxious mind: anxiety, worry, and frontal engagement in sustained attention versus off-task processing. Cerebral Cortex, 25(3), 609-618.

Fountoulakis, K. N., Iacovides, A., Kleanthous, S., Samolis, S., Gougoulias, K., St Kaprinis, G., \& Bech, P. (2003). The greek translation of the symptoms rating scale for depression and anxiety: Preliminary results of the validation study. $B M C$ Psychiatry, 3. doi:10.1186/1471-244x-3-21

Fountoulakis, K. N., Papadopoulou, M., Kleanthous, S., Papadopoulou, A., Bizeli, V., Nimatoudis, I., .. . Kaprinis, G. S. (2006). Reliability and psychometric properties of the Greek translation of the State-Trait Anxiety Inventory form Y: Preliminary data. Annals of General Psychiatry, 5. doi:10.1186/1744-859x-5-2

Fox, E. (1993). Attentional bias in anxiety: Selective or not? Behaviour Research and Therapy, 31(5), 487-493.

Fox, E. (1994). Attentional Bias in Anxiety: A defective inhibition hypothesis. Cognition and Emotion, 8(2), 165-195. doi:10.1080/02699939408408934

Fox, M. D., \& Raichle, M. E. (2007). Spontaneous fluctuations in brain activity observed with functional magnetic resonance imaging. Nature Reviews Neuroscience, 8(9), 700-711. doi:10.1038/nrn2201

Fox, N. A., Henderson, H. A., Marshall, P. J., Nichols, K. E., \& Ghera, M. M. (2005) Behavioral inhibition: Linking biology and behavior within a developmental framework. Vol. 56. Annual Review of Psychology (pp. 235-262).

Frewen, P. A., Dozois, D. J., Joanisse, M. F., \& Neufeld, R. W. (2008). Selective attention to threat versus reward: meta-analysis and neural-network modeling of the dot-probe task. Clinical Psychology Review, 28(2), 307-337. 
Galván, A., \& McGlennen, K. M. (2013). Enhanced striatal sensitivity to aversive reinforcement in adolescents versus adults. Journal of Cognitive Neuroscience, 25(2), 284-296.

Garner, M., Mohler, H., Stein, D. J., Mueggler, T., \& Baldwin, D. S. (2009). Research in anxiety disorders: from the bench to the bedside. European Neuropsychopharmacology, 19(6), 381-390.

Gerstorf, D., Siedlecki, K. L., Tucker-Drob, E. M., \& Salthouse, T. A. (2009). Withinperson variability in state anxiety across adulthood: Magnitude and associations with between-person characteristics. International Journal of Behavioral Development, 33(1), 55-64.

Gilbert, S. J., Dumontheil, I., Simons, J. S., Frith, C. D., \& Burgess, P. W. (2007). Comment on "Wandering minds: the default network and stimulus-independent thought". Science (New York, N.Y.), 317(5834).

Giorgetta, C., Grecucci, A., Zuanon, S., Perini, L., Balestrieri, M., Bonini, N., . . . Brambilla, P. (2012). Reduced risk-taking behavior as a trait feature of anxiety. Emotion, 12(6), 1373-1383.

Glass, G. V., Peckham, P. D., \& Sanders, J. R. (1972). Consequences of Failure to Meet Assumptions Underlying the Fixed Effects Analyses of Variance and Covariance. Review of Educational Research, 42(3), 237-288. doi: $10.3102 / 00346543042003237$

Goghari, V. M., \& MacDonald Iii, A. W. (2009). The neural basis of cognitive control: Response selection and inhibition. Brain and Cognition, 71(2), 72-83. doi:10.1016/j.bandc.2009.04.004 
Gray, J. A. (1973). Causal models of personality and how to test them. In J. R. Royce (Ed.), Multivariate analysis and psychological theory (pp. 409-463). London: Academic Press.

Gray, J. A. (1982). The neurobiology of anxiety: An enquiry into the functions of the septo-hippocampal system. Oxford: Oxford University Press.

Gray, J. A., \& McNaughton, N. (2000). The neuropsychology of anxiety: An enquiry into the functions of the septo-hippocampal system. Oxford: Oxford University Press.

Greicius, M. D., Krasnow, B., Reiss, A. L., \& Menon, V. (2003). Functional connectivity in the resting brain: A network analysis of the default mode hypothesis. Proceedings of the National Academy of Sciences of the United States of America, 100(1), 253-258. doi:10.1073/pnas.0135058100

Grös, D. F., Antony, M. M., Simms, L. J., \& McCabe, R. E. (2007). Psychometric Properties of the State-Trait Inventory for Cognitive and Somatic Anxiety (STICSA): Comparison to the State-Trait Anxiety Inventory (STAI). Psychological Assessment, 19(4), 369-381.

Grupe, D. W., \& Nitschke, J. B. (2013). Uncertainty and anticipation in anxiety: An integrated neurobiological and psychological perspective. Nature Reviews Neuroscience, 14(7), 488-501. doi:10.1038/nrn3524

Gu, Y., Hu, X., Pan, W., Yang, C., Wang, L., Li, Y., \& Chen, A. (2016). Neural Activities Underlying the Feedback Express Salience Prediction Errors for Appetitive and Aversive Stimuli. Scientific Reports, 6. doi:10.1038/srep34032

Guitart-Masip, M., Fuentemilla, L., Bach, D. R., Huys, Q. J. M., Dayan, P., Dolan, R. J., \& Duzel, E. (2011). Action dominates valence in anticipatory representations in the human striatum and dopaminergic midbrain. Journal of Neuroscience, 31(21), 7867-7875. doi:10.1523/jneurosci.6376-10.2011 
Gusnard, D. A., \& Raichle, M. E. (2001). Searching for a baseline: Functional imaging and the resting human brain. Nature Reviews Neuroscience, 2(10), 685-694. doi: $10.1038 / 35094500$

Guyer, A. E., Benson, B., Choate, V. R., Bar-Haim, Y., Perez-Edgar, K., Jarcho, J. M., . . . Nelson, E. E. (2014). Lasting associations between early-childhood temperament and late-adolescent reward-circuitry response to peer feedback. Development and Psychopathology, 26(1), 229-243.

Guyer, A. E., Choate, V. R., Detloff, A., Benson, B., Nelson, E. E., Perez-Edgar, K., .. . Ernst, M. (2012). Striatal functional alteration during incentive anticipation in pediatric anxiety disorders. American Journal of Psychiatry, 169(2), 205-212.

Guyer, A. E., Nelson, E. E., Perez-Edgar, K., Hardin, M. G., Roberson-Nay, R., Monk, C. S., . . Ernst, M. (2006). Striatal functional alteration in adolescents characterized by early childhood behavioral inhibition. Journal of Neuroscience, 26(24), 63996405.

Haber, S. N., \& Knutson, B. (2010). The reward circuit: Linking primate anatomy and human imaging. Neuropsychopharmacology, 35(1), 4-26. doi:10.1038/npp.2009.129

Hagopian, L. P., \& Ollendick, T. H. (1994). Behavioral inhibition and test anxiety: An empirical investigation of Gray's theory. Personality and Individual Differences, 16(4), 597-604.

Hahn, T., Dresler, T., Plichta, M. M., Ehlis, A. C., Ernst, L. H., Markulin, F., . . . Fallgatter, A. J. (2010). Functional amygdala-hippocampus connectivity during anticipation of aversive events is associated with Gray's Trait "sensitivity to Punishment". Biological Psychiatry, 68(5), 459-464. doi:10.1016/j.biopsych.2010.04.033 
Hajcak, G., McDonald, N., \& Simons, R. F. (2003). Anxiety and error-related brain activity. Biological Psychology, 64(1-2), 77-90. doi:http://dx.doi.org/10.1016/S0301-0511(03)00103-0

Hallett, P. E. (1978). Primary and secondary saccades to goals defined by instructions. Vision Research, 18(10), 1279-1296. doi:10.1016/0042-6989(78)90218-3

Hallion, L. S., Tolin, D. F., Assaf, M., Goethe, J., \& Diefenbach, G. J. (2017). Cognitive Control in Generalized Anxiety Disorder: Relation of Inhibition Impairments to Worry and Anxiety Severity. Cognitive Therapy and Research, 1-9. doi:10.1007/s10608-017-9832-2

Hampshire, A. (2015). Putting the brakes on inhibitory models of frontal lobe function. NeuroImage, 113, 340-355. doi:10.1016/j.neuroimage.2015.03.053

Hardin, M. G., Perez-Edgar, K., Guyer, A. E., Pine, D. S., Fox, N. A., \& Ernst, M. (2006). Reward and punishment sensitivity in shy and non-shy adults: Relations between social and motivated behavior. Personality and Individual Differences, 40(4), 699-711.

Hardin, M. G., Schroth, E., Pine, D. S., \& Ernst, M. (2007). Incentive-related modulation of cognitive control in healthy, anxious, and depressed adolescents: Development and psychopathology related differences. Journal of Child Psychology and Psychiatry and Allied Disciplines, 48(5), 446-454.

Hartley, C. A., \& Phelps, E. A. (2010). Changing fear: The neurocircuitry of emotion regulation. Neuropsychopharmacology, 35(1), 136-146. doi:10.1038/npp.2009.121

He, Y., Xu, T., Zhang, W., \& Zuo, X.-N. (2016). Lifespan anxiety is reflected in human amygdala cortical connectivity. Human Brain Mapping, 37(3), 1178-1193. 
Helfinstein, S. M., Benson, B., Perez-Edgar, K., Bar-Haim, Y., Detloff, A., Pine, D. S., . . . Ernst, M. (2011). Striatal responses to negative monetary outcomes differ between temperamentally inhibited and non-inhibited adolescents.

Neuropsychologia, 49(3), 479-485.

Hester, R., Murphy, K., Brown, F. L., \& Skilleter, A. J. (2010). Punishing an error improves learning: The influence of punishment magnitude on error-related neural activity and subsequent learning. Journal of Neuroscience, 30(46), 1560015607. doi:10.1523/JNEUROSCI.2565-10.2010

Hirshfeld-Becker, D. R., Micco, J., Henin, A., Bloomfield, A., Biederman, J., \& Rosenbaum, J. (2008). Behavioral inhibition. Depression and Anxiety, 25(4), 357367.

Hogarty, K. Y., Hines, C. V., Kromrey, J. D., Perron, J. M., \& Mumford, A. K. R. (2005). The quality of factor solutions in exploratory factor analysis: The influence of sample size, communality, and overdetermination. Educational and Psychological Measurement, 65(2), 202-226. doi:10.1177/0013164404267287

Holroyd, C. B., \& Coles, M. G. H. (2002). The neural basis of human error processing: Reinforcement learning, dopamine, and the error-related negativity. Psychological Review, 109(4), 679-709.

Horikawa, M., \& Yagi, A. (2012). The relationships among trait anxiety, state anxiety and the goal performance of penalty shoot-out by university soccer players. PLoS ONE, 7(4).

Hundt, N. E., Nelson-Gray, R. O., Kimbrel, N. A., Mitchell, J. T., \& Kwapil, T. R. (2007). The interaction of reinforcement sensitivity and life events in the prediction of anhedonic depression and mixed anxiety-depression symptoms. Personality and Individual Differences, 43(5), 1001-1012. 
Huster, R. J., Westerhausen, R., \& Herrmann, C. S. (2011). Sex differences in cognitive control are associated with midcingulate and callosal morphology. Brain Structure and Function, 215(3-4), 225-235. doi:10.1007/s00429-010-0289-2

Hutton, S. B. (2008). Cognitive control of saccadic eye movements. Brain and Cognition, 68(3), 327-340.

Hutton, S. B., \& Ettinger, U. (2006). The antisaccade task as a research tool in psychopathology: A critical review. Psychophysiology, 43(3), 302-313.

Iversen, S. D., \& Mishkin, M. (1970). Perseverative interference in monkeys following selective lesions of the inferior prefrontal convexity. Experimental Brain Research, 11(4), 376-386. doi:10.1007/bf00237911

Jakubczyk, A., Klimkiewicz, A., Topolewska-Wochowska, A., Serafin, P., SadowskaMazuryk, J., Pupek-Pyzioł, J., . . . Wojnar, M. (2012). Relationships of impulsiveness and depressive symptoms in alcohol dependence. Journal of Affective Disorders, 136(3), 841-847. doi:10.1016/j.jad.2011.09.028

Janak, P. H., \& Tye, K. M. (2015). From circuits to behaviour in the amygdala. Nature, 517(7534), 284-292. doi:10.1038/nature14188

Jazbec, S., McClure, E., Hardin, M., Pine, D. S., \& Ernst, M. (2005). Cognitive control under contingencies in anxious and depressed adolescents: an antisaccade task. Biological Psychiatry, 58(8), 632-639.

Jensen, A. R., \& Rohwer Jr, W. D. (1966). The stroop color-word test: A review. Acta Psychologica, 25(C), 36-93.

John Rush, A., Giles, D. E., Schlesser, M. A., Fulton, C. L., Weissenburger, J., \& Burns, C. (1986). The inventory for depressive symptomatology (IDS): Preliminary findings. Psychiatry Research, 18(1), 65-87. doi:10.1016/0165-1781(86)90060-0 
Johnson, S. B., Blum, R. W., \& Giedd, J. N. (2009). Adolescent Maturity and the Brain: The Promise and Pitfalls of Neuroscience Research in Adolescent Health Policy. Journal of Adolescent Health, 45(3), 216-221.

doi:10.1016/j.jadohealth.2009.05.016

Johnson, S. L., Turner, R. J., \& Iwata, N. (2003). BIS/BAS levels and psychiatric disorder: An epidemiological study. Journal of Psychopathology and Behavioral Assessment, 25(1), 25-36. doi:10.1023/a:1022247919288

Kaiser, H. F. (1960). The application of electronic computers to factor analysis. Educational and Psychological Measurement, 20(1), 141-151. doi:10.1177/001316446002000116

Kaiser, H. F. (1970). A second generation little jiffy. Psychometrika, 35(4), 401-415. doi:10.1007/BF02291817

Kaiser, H. F. (1974). An index of factorial simplicity. Psychometrika, 39(1), 31-36. doi:10.1007/BF02291575

Kampman, O., Viikki, M., Jarventausta, K., \& Leinonen, E. (2014). Meta-analysis of anxiety disorders and temperament. Neuropsychobiology, 69(3), 175-186. doi: $10.1159 / 000360738$

Katsuki, F., \& Constantinidis, C. (2012). Unique and shared roles of the posterior parietal and dorsolateral prefrontal cortex in cognitive functions. Frontiers in Integrative Neuroscience(APRIL), 1-53. doi:10.3389/fnint.2012.00017

Kennis, M., Rademaker, A. R., \& Geuze, E. (2013). Neural correlates of personality: An integrative review. Neuroscience and Biobehavioral Reviews, 37(1), 73-95. doi:10.1016/j.neubiorev.2012.10.012

Kent, J. M., \& Rauch, S. L. (2003). Neurocircuitry of anxiety disorders. Current Psychiatry Reports, 5(4), 266-273. 
Keogh, E., \& Reidy, J. (2000). Exploring the factor structure of the mood and anxiety symptom questionnaire (MASQ). Journal of Personality Assessment, 74(1), 106125.

Kessel, E. M., Kujawa, A., Hajcak Proudfit, G., \& Klein, D. N. (2015). Neural reactivity to monetary rewards and losses differentiates social from generalized anxiety in children. Journal of Child Psychology and Psychiatry and Allied Disciplines, 56(7), 792-800. doi:10.1111/jcpp.12355

Kessler, R. C., Berglund, P., Demler, O., Jin, R., Merikangas, K. R., \& Walters, E. E. (2005). Lifetime prevalence and age-of-onset distributions of DSM-IV disorders in the national comorbidity survey replication. Archives of General Psychiatry, 62(6), 593-602.

Kim, H., Shimojo, S., \& O'Doherty, J. P. (2006). Is avoiding an aversive outcome rewarding? Neural substrates of avoidance learning in the human brain. PLoS Biology, 4(8), 1453-1461.

Kim, M. J., Gee, D. G., Loucks, R. A., Davis, F. C., \& Whalen, P. J. (2011). Anxiety Dissociates dorsal and ventral medial prefrontal cortex functional connectivity with the amygdala at rest. Cerebral Cortex, 21(7), 1667-1673. doi:10.1093/cercor/bhq237

Kim, M. J., Loucks, R. A., Palmer, A. L., Brown, A. C., Solomon, K. M., Marchante, A. N., \& Whalen, P. J. (2011). The structural and functional connectivity of the amygdala: From normal emotion to pathological anxiety. Behavioural Brain Research, 223(2), 403-410. doi:http://dx.doi.org/10.1016/j.bbr.2011.04.025

Kim, M. J., \& Whalen, P. J. (2009). The structural integrity of an amygdala-prefrontal pathway predicts trait anxiety. Journal of Neuroscience, 29(37), 11614-11618. doi:10.1523/jneurosci.2335-09.2009 
Kim, S. H., Yoon, H. S., Kim, H., \& Hamann, S. (2014). Individual differences in sensitivity to reward and punishment and neural activity during reward and avoidance learning. Social Cognitive and Affective Neuroscience, 10(9), 12191227. doi:10.1093/scan/nsv007

Kimbrell, T. A., George, M. S., Parekh, P. I., Ketter, T. A., Podell, D. M., Danielson, A. L., . . Post, R. M. (1999). Regional brain activity during transient self-induced anxiety and anger in healthy adults. Biological Psychiatry, 46(4), 454-465. doi:10.1016/s0006-3223(99)00103-1

Knutson, B., \& Cooper, J. C. (2005). Functional magnetic resonance imaging of reward prediction. Current Opinion in Neurology, 18(4), 411-417.

Knutson, B., Fong, G. W., Adams, C. M., Varner, J. L., \& Hommer, D. (2001). Dissociation of reward anticipation and outcome with event-related fMRI. NeuroReport, 12(17), 3683-3687.

Knutson, B., Westdorp, A., Kaiser, E., \& Hommer, D. (2000). FMRI visualization of brain activity during a monetary incentive delay task. NeuroImage, 12(1), 20-27.

Knyazev, G. G., Levin, E. A., \& Savostyanov, A. N. (2008). Impulsivity, anxiety, and individual differences in evoked and induced brain oscillations. International Journal of Psychophysiology, 68(3), 242-254.

Kobayashi, S. (2012). Organization of neural systems for aversive information processing: Pain, error, and punishment. Frontiers in Neuroscience(SEP).

Kohn, P. M., Kantor, L., DeCicco, T. L., \& Beck, A. T. (2008). The Beck Anxiety Inventory-Trait (BAIT): A measure of dispositional anxiety not contaminated by dispositional depression. Journal of Personality Assessment, 90(5), 499-506. doi:10.1080/00223890802248844 
Korenblum, C. B., Chen, S. X., Manassis, K., \& Schachar, R. J. (2007). Performance monitoring and response inhibition in anxiety disorders with and without comorbid ADHD. Depression and Anxiety, 24(4), 227-232.

Krueger, R. F., Derringer, J., Markon, K. E., Watson, D., \& Skodol, A. E. (2013). The Personality Inventory for DSM-5-Brief Form (PID-5-BF) - Adult: American Psychiatric Association.

Lau, J. Y. F., Eley, T. C., \& Stevenson, J. (2006). Examining the state-trait anxiety relationship: A behavioural genetic approach. Journal of Abnormal Child Psychology, 34(1), 19-27. doi:10.1007/s10802-005-9006-7

Lau, M. A., Christensen, B. K., Hawley, L. L., Gemar, M. S., \& Segal, Z. V. (2007). Inhibitory deficits for negative information in persons with major depressive disorder. Psychological Medicine, 37(9), 1249-1259.

le Strat, Y., Rehm, J., \& le Foll, B. (2011). How generalisable to community samples are clinical trial results for treatment of nicotine dependence: A comparison of common eligibility criteria with respondents of a large representative general population survey. Tobacco Control, 20(5), 338-343. doi:10.1136/tc.2010.038703

Leech, R., Braga, R., \& Sharp, D. J. (2012). Echoes of the brain within the posterior cingulate cortex. Journal of Neuroscience, 32(1), 215-222. doi:10.1523/jneurosci.3689-11.2012

Leech, R., \& Sharp, D. J. (2014). The role of the posterior cingulate cortex in cognition and disease. Brain, 137(1), 12-32. doi:10.1093/brain/awt162

Leue, A., Lange, S., \& Beauducel, A. (2012). Modulation of the conflict monitoring intensity: The role of aversive reinforcement, cognitive demand, and trait-BIS. Cognitive, Affective and Behavioral Neuroscience, 12(2), 287-307. doi:10.3758/s13415-012-0086-x 
Levis, B. (2017). The effect of reward and punishment on inhibitory control in anxiety, Unpublished manuscript, University of Melbourne.

Levy, B. J., \& Wagner, A. D. (2011) Cognitive control and right ventrolateral prefrontal cortex: Reflexive reorienting, motor inhibition, and action updating. Vol. 1224. Annals of the New York Academy of Sciences (pp. 40-62).

Li, C. s. R., Huang, C., Constable, R. T., \& Sinha, R. (2006). Gender differences in the neural correlates of response inhibition during a stop signal task. NeuroImage, 32(4), 1918-1929. doi:10.1016/j.neuroimage.2006.05.017

Li, C. S. R., Huang, C., Constable, R. T., \& Sinha, R. (2006). Imaging response inhibition in a stop-signal task: Neural correlates independent of signal monitoring and postresponse processing. Journal of Neuroscience, 26(1), 186-192.

Li, C. s. R., Zhang, S., Duann, J. R., Yan, P., Sinha, R., \& Mazure, C. M. (2009). Gender differences in cognitive control: An extended investigation of the stop signal task. Brain Imaging and Behavior, 3(3), 262-276. doi:10.1007/s11682-009-9068-1

Linkovski, O., Kalanthroff, E., Henik, A., \& Anholt, G. (2013). Did I turn off the stove? Good inhibitory control can protect from influences of repeated checking. Journal of Behavior Therapy and Experimental Psychiatry, 44(1), 30-36. doi:10.1016/j.jbtep.2012.07.002

Lipszyc, J., \& Schachar, R. (2010). Inhibitory control and psychopathology: A metaanalysis of studies using the stop signal task. Journal of the International Neuropsychological Society, 16(6), 1064-1076.

Liu, X., Hairston, J., Schrier, M., \& Fan, J. (2011). Common and distinct networks underlying reward valence and processing stages: A meta-analysis of functional neuroimaging studies. Neuroscience and Biobehavioral Reviews, 35(5), 12191236. 
Locke, H. S., \& Braver, T. S. (2008). Motivational influences on cognitive control: Behavior, brain activation, and individual differences. Cognitive, Affective and Behavioral Neuroscience, 8(1), 99-112. doi:10.3758/cabn.8.1.99

Logan, G. D., Schachar, R. J., \& Tannock, R. (1997). Impulsivity and inhibitory control. Psychological Science, 8(1), 60-64.

Lovibond, S. H., \& Lovibond, P. F. (1995). Manual for the Depression Anxiety Stress Scales (2nd ed.). Sydney, Australia: The Psychology Foundation of Australia

Löwe, B., Decker, O., Müller, S., Brähler, E., Schellberg, D., Herzog, W., \& Herzberg, P. Y. (2008). Validation and standardization of the generalized anxiety disorder screener (GAD-7) in the general population. Medical Care, 46(3), 266-274. doi:10.1097/MLR.0b013e318160d093

Lyche, P., Jonassen, R., Stiles, T. C., Ulleberg, P., \& Landrø, N. I. (2010). Cognitive control functions in unipolar major depression with and without co-morbid anxiety disorder. Frontiers in Psychiatry, 1(DEC). doi:10.3389/fpsyt.2010.00149

Maack, D. J., Tull, M. T., \& Gratz, K. L. (2012). Experiential avoidance mediates the association between behavioral inhibition and posttraumatic stress disorder. Cognitive Therapy and Research, 36(4), 407-416. doi:10.1007/s10608-011-93622

MacCallum, R. C., Widaman, K. F., Zhang, S., \& Hong, S. (1999). Sample size in factor analysis. Psychological Methods, 4(1), 84-99. doi:10.1037/1082-989x.4.1.84

Manning, J., Reynolds, G., Saygin, Z. M., Hofmann, S. G., Pollack, M., Gabrieli, J. D. E., \& Whitfield-Gabrieli, S. (2015). Altered resting-state functional connectivity of the frontal-striatal reward system in social anxiety disorder. PLoS ONE, 10(4). doi:10.1371/journal.pone.0125286 
Mar, R. A., Spreng, R. N., \& Deyoung, C. G. (2013). How to produce personality neuroscience research with high statistical power and low additional cost. Cognitive, Affective and Behavioral Neuroscience, 13(3), 674-685. doi:10.3758/s13415-013-0202-6

Marcus, D. K., Sawaqdeh, A., \& Kwon, P. (2014). The latent structure of generalized anxiety disorder in midlife adults. Psychiatry Research, 215(2), 366-371.

Masui, K., \& Nomura, M. (2011). The effects of reward and punishment on response inhibition in non-clinical psychopathy. Personality and Individual Differences, 50(1), 69-73. doi:10.1016/j.paid.2010.08.024

Matthews, S., Simmons, A., Strigo, I., Gianaros, P., Yang, T., \& Paulus, M. (2009). Inhibition-related activity in subgenual cingulate is associated with symptom severity in major depression. Psychiatry Research - Neuroimaging, 172(1), 1-6.

McClure, S. M., York, M. K., \& Montague, P. R. (2004). The neural substrates of reward processing in humans: The modern role of fMRI. Neuroscientist, 10(3), 260-268.

McKiernan, K. A., Kaufman, J. N., Kucera-Thompson, J., \& Binder, J. R. (2003). A parametric manipulation of factors affecting task-induced deactivation in functional neuroimaging. Journal of Cognitive Neuroscience, 15(3), 394-408. doi:10.1162/089892903321593117

McNaughton, N., \& Corr, P. J. (2004). A two-dimensional neuropsychology of defense: Fear/anxiety and defensive distance. Neuroscience and Biobehavioral Reviews, 28(3), 285-305. doi:10.1016/j.neubiorev.2004.03.005

Menon, V., Adleman, N. E., White, C. D., Glover, G. H., \& Reiss, A. L. (2001). Errorrelated brain activation during a Go/NoGo response inhibition task. Human Brain Mapping, 12(3), 131-143. doi:10.1002/1097-0193(200103)12:3\&1t;131::AIDHBM1010\&gt;3.0.CO;2-C 
Menon, V., \& Uddin, L. Q. (2010). Saliency, switching, attention and control: a network model of insula function. Brain structure \& function, 214(5-6), 655-667.

Meyer, T. J., Miller, M. L., Metzger, R. L., \& Borkovec, T. D. (1990). Development and validation of the penn state worry questionnaire. Behaviour Research and Therapy, 28(6), 487-495. doi:http://dx.doi.org/10.1016/0005-7967(90)90135-6

Miller, E. K., \& Cohen, J. D. (2001). An integrative theory of prefrontal cortex function. Annual review of neuroscience, 24, 167-202. doi:10.1146/annurev.neuro.24.1.167

Miu, A. C., Heilman, R. M., \& Houser, D. (2008). Anxiety impairs decision-making: Psychophysiological evidence from an Iowa Gambling Task. Biological Psychology, 77(3), 353-358.

Miyake, A., Friedman, N. P., Emerson, M. J., Witzki, A. H., Howerter, A., \& Wager, T. D. (2000). The Unity and Diversity of Executive Functions and Their Contributions to Complex "Frontal Lobe" Tasks: A Latent Variable Analysis. Cognitive Psychology, 41(1), 49-100.

Modi, S., Kumar, M., Kumar, P., \& Khushu, S. (2015). Aberrant functional connectivity of resting state networks associated with trait anxiety. Psychiatry Research Neuroimaging, 234(1), 25-34. doi:10.1016/j.pscychresns.2015.07.006

Modi, S., Rana, P., Kaur, P., Rani, N., \& Khushu, S. (2014). Glutamate level in anterior cingulate predicts anxiety in healthy humans: A magnetic resonance spectroscopy study. Psychiatry Research: Neuroimaging, 224(1), 34-41.

Monterosso, J. R., Aron, A. R., Cordova, X., Xu, J., \& London, E. D. (2005). Deficits in response inhibition associated with chronic methamphetamine abuse. Drug and Alcohol Dependence, 79(2), 273-277. doi:10.1016/j.drugalcdep.2005.02.002 
Moran, T. P., Taylor, D., \& Moser, J. S. (2012). Sex moderates the relationship between worry and performance monitoring brain activity in undergraduates. International Journal of Psychophysiology, 85(2), 188-194.

Moser, J. S., Moran, T. P., \& Jendrusina, A. A. (2012). Parsing relationships between dimensions of anxiety and action monitoring brain potentials in female undergraduates. Psychophysiology, 49(1), 3-10.

Moser, J. S., Moran, T. P., Schroder, H. S., Donnellan, M. B., \& Yeung, N. (2013). On the relationship between anxiety and error monitoring: A meta-analysis and conceptual framework. Frontiers in Human Neuroscience(AUG).

Mühlberg, C., Mathar, D., Villringer, A., Horstmann, A., \& Neumann, J. (2016). Stopping at the sight of food - How gender and obesity impact on response inhibition. Appetite, 107, 663-676. doi:10.1016/j.appet.2016.08.121

Myers-Schulz, B., \& Koenigs, M. (2012). Functional anatomy of ventromedial prefrontal cortex: Implications for mood and anxiety disorders. Molecular Psychiatry, 17(2), 132-141. doi:10.1038/mp.2011.88

Nigg, J. T. (2000). On Inhibition/Disinhibition in Developmental Psychopathology: Views from Cognitive and Personality Psychology and a Working Inhibition Taxonomy. Psychological Bulletin, 126(2), 220-246.

Nitschke, J. B., Heller, W., Imig, J. C., McDonald, R. P., \& Miller, G. A. (2001). Distinguishing Dimensions of Anxiety and Depression. Cognitive Therapy and Research, 25(1), 1-22.

Nitschke, J. B., Heller, W., Palmieri, P. A., \& Miller, G. A. (1999). Contrasting patterns of brain activity in anxious apprehension and anxious arousal. Psychophysiology, $36(5), 628-637$. 
O'Connor, B. P. (2000). SPSS and SAS programs for determining the number of components using parallel analysis and Velicer's MAP test. Behavior Research Methods, Instruments, and Computers, 32(3), 396-399.

O'Doherty, J. P. (2004). Reward representations and reward-related learning in the human brain: Insights from neuroimaging. Current Opinion in Neurobiology, 14(6), 769776.

Oldfield, R. C. (1971). The assessment and analysis of handedness: The Edinburgh inventory. Neuropsychologia, 9(1), 97-113.

Olds, J., \& Milner, P. (1954). Positive reinforcement produced by electrical stimulation of septal area and other regions of rat brain. Journal of Comparative and Physiological Psychology, 47(6), 419-427. doi:10.1037/h0058775

Oppenheimer, S. M., Gelb, A., Girvin, J. P., \& Hachinski, V. C. (1992). Cardiovascular effects of human insular cortex stimulation. Neurology, 42(9), 1727-1732.

Osinsky, R., Alexander, N., Gebhardt, H., \& Hennig, J. (2010). Trait anxiety and dynamic adjustments in conflict processing. Cognitive, Affective and Behavioral Neuroscience, 10(3), 372-381.

Osinsky, R., Gebhardt, H., Alexander, N., \& Hennig, J. (2012). Trait anxiety and the dynamics of attentional control. Biological Psychology, 89(1), 252-259.

Pacheco-Unguetti, A. P., Acosta, A., Callejas, A., \& Lupiáñez, J. (2010). Attention and anxiety: Different attentional functioning under state and trait anxiety. Psychological Science, 21(2), 298-304.

Padmala, S., \& Pessoa, L. (2010). Interactions between cognition and motivation during response inhibition. Neuropsychologia, 48(2), 558-565. doi:http://dx.doi.org/10.1016/j.neuropsychologia.2009.10.017 
Patel, R., Spreng, R. N., Shin, L. M., \& Girard, T. A. (2012). Neurocircuitry models of posttraumatic stress disorder and beyond: A meta-analysis of functional neuroimaging studies. Neuroscience and Biobehavioral Reviews, 36(9), 21302142. doi:10.1016/j.neubiorev.2012.06.003

Patton, J. H., Stanford, M. S., \& Barratt, E. S. (1995). Factor structure of the barratt impulsiveness scale. Journal of Clinical Psychology, 51(6), 768-774. doi:10.1002/1097-4679(199511)51:6\&lt;768::AIDJCLP2270510607\&gt;3.0.CO;2-1

Paulus, M. P. (2008). The role of neuroimaging for the diagnosis and treatment of anxiety disorders. Depression and Anxiety, 25(4), 348-356. doi:10.1002/da.20499

Paulus, M. P., Feinstein, J. S., Simmons, A., \& Stein, M. B. (2004). Anterior cingulate activation in high trait anxious subjects is related to altered error processing during decision making. Biological Psychiatry, 55(12), 1179-1187.

Paulus, M. P., \& Stein, M. B. (2006). An Insular View of Anxiety. Biological Psychiatry, $60(4), 383-387$.

Pérez-Edgar, K., Roberson-Nay, R., Hardin, M. G., Poeth, K., Guyer, A. E., Nelson, E. E., . . Ernst, M. (2007). Attention alters neural responses to evocative faces in behaviorally inhibited adolescents. NeuroImage, 35(4), 1538-1546. doi:10.1016/j.neuroimage.2007.02.006

Pezawas, L., Meyer-Lindenberg, A., Drabant, E. M., Verchinski, B. A., Munoz, K. E., Kolachana, B. S., . . . Weinberger, D. R. (2005). 5-HTTLPR polymorphism impacts human cingulate-amygdala interactions: A genetic susceptibility mechanism for depression. Nature Neuroscience, 8(6), 828-834. doi:10.1038/nn1463 
Pfurtscheller, G. (2006) Chapter 2 The cortical activation model (CAM). Vol. 159.

Progress in Brain Research (pp. 19-27).

Pfurtscheller, G., \& Lopes Da Silva, F. H. (1999). Event-related EEG/MEG

synchronization and desynchronization: Basic principles. Clinical

Neurophysiology, 110(11), 1842-1857. doi:10.1016/s1388-2457(99)00141-8

Ping, L. T., Subramaniam, K., \& Krishnaswamy, S. (2008). Test anxiety: State, trait and relationship with exam satisfaction. Malaysian Journal of Medical Sciences, $15(2), 18-23$.

Pinto-Meza, A., Caseras, X., Soler, J., Puigdemont, D., Pérez, V., \& Torrubia, R. (2006).

Behavioural inhibition and Behavioural Activation Systems in current and recovered major depression participants. Personality and Individual Differences, 40(2), 215-226. doi:10.1016/j.paid.2005.06.021

Plehn, K., \& Peterson, R. A. (2002). Anxiety sensitivity as a predictor of the development of panic symptoms, panic attacks, and panic disorder: A prospective study. Journal of Anxiety Disorders, 16(4), 455-474. doi:10.1016/s0887-6185(02)001299

Podsakoff, P. M., MacKenzie, S. B., Lee, J. Y., \& Podsakoff, N. P. (2003). Common Method Biases in Behavioral Research: A Critical Review of the Literature and Recommended Remedies. Journal of Applied Psychology, 88(5), 879-903. doi:10.1037/0021-9010.88.5.879

Pujara, M., \& Koenigs, M. (2014). Mechanisms of reward circuit dysfunction in psychiatric illness: Prefrontal-striatal interactions. Neuroscientist, 20(1), 82-95. doi: $10.1177 / 1073858413499407$

Rademacher, L., Krach, S., Kohls, G., Irmak, A., Gründer, G., \& Spreckelmeyer, K. N. (2010). Dissociation of neural networks for anticipation and consumption of 
monetary and social rewards. NeuroImage, 49(4), 3276-3285.

doi:10.1016/j.neuroimage.2009.10.089

Raichle, M. E., MacLeod, A. M., Snyder, A. Z., Powers, W. J., Gusnard, D. A., \& Shulman, G. L. (2001). A default mode of brain function. Proceedings of the National Academy of Sciences of the United States of America, 98(2), 676-682. doi:10.1073/pnas.98.2.676

Ramautar, J. R., Kok, A., \& Ridderinkhof, K. R. (2004). Effects of stop-signal probability in the stop-signal paradigm: The N2/P3 complex further validated. Brain and Cognition, 56(2 SPEC. ISS.), 234-252. doi:10.1016/j.bandc.2004.07.002

Rauch, S. L., Savage, C. R., Alpert, N. M., Miguel, E. C., Baer, L., Breiter, H. C., . . Jenike, M. A. (1995). A Positron Emission Tomographic Study of Simple Phobic Symptom Provocation. Archives of General Psychiatry, 52(1), 20-28. doi:10.1001/archpsyc.1995.03950130020003

Rauch, S. L., Shin, L. M., \& Phelps, E. A. (2006). Neurocircuitry Models of Posttraumatic Stress Disorder and Extinction: Human Neuroimaging ResearchPast, Present, and Future. Biological Psychiatry, 60(4), 376-382. doi:10.1016/j.biopsych.2006.06.004

Rauch, S. L., Van Der Kolk, B. A., Fisler, R. E., Alpert, N. M., Orr, S. P., Savage, C. R., . . Pitman, R. K. (1996). A symptom provocation study of posttraumatic stress disorder using positron emission tomography and script-driven imagery. Archives of General Psychiatry, 53(5), 380-387.

Ree, M. J., MacLeod, C., French, D., \& Locke, V. (2000, November). The State-Trait Inventory for Cognitive and Somatic Anxiety: Development and validation. Poster session presented at the annual meeting of the Association for the Advancement of Behavior Therapy. New Orleans, LA. 
Riesel, A., Weinberg, A., Endrass, T., Kathmann, N., \& Hajcak, G. (2012). Punishment has a lasting impact on error-related brain activity. Psychophysiology, 49(2), 239247.

Righi, S., Mecacci, L., \& Viggiano, M. P. (2009). Anxiety, cognitive self-evaluation and performance: ERP correlates. Journal of Anxiety Disorders, 23(8), 1132-1138.

Rodríguez-Fornells, A., Lorenzo-Seva, U., \& Andrés-Pueyo, A. (2002). Are highimpulsive and high risk-taking people more motor disinhibited in the presence of incentive? Personality and Individual Differences, 32(4), 661-683.

Rosell-Negre, P., Bustamante, J. C., Fuentes-Claramonte, P., Costumero, V., Benabarre, S., \& Barros-Loscertales, A. (2014). Reward anticipation enhances brain activation during response inhibition. Cognitive, Affective and Behavioral Neuroscience, 14(2), 621-634. doi:10.3758/s13415-014-0292-9

Rosen, J. B., \& Schulkin, J. (1998). From Normal Fear to Pathological Anxiety. Psychological Review, 105(2), 325-350.

Rossiter, S., Thompson, J., \& Hester, R. (2012). Improving control over the impulse for reward: Sensitivity of harmful alcohol drinkers to delayed reward but not immediate punishment. Drug and Alcohol Dependence, 125(1-2), 89-94. doi:10.1016/j.drugalcdep.2012.03.017

Roy, A. K., Shehzad, Z., Margulies, D. S., Kelly, A. M. C., Uddin, L. Q., Gotimer, K., . . . Milham, M. P. (2009). Functional connectivity of the human amygdala using resting state fMRI. NeuroImage, 45(2), 614-626. doi:10.1016/j.neuroimage.2008.11.030

Sandi, C., \& Richter-Levin, G. (2009). From high anxiety trait to depression: a neurocognitive hypothesis. Trends in Neurosciences, 32(6), 312-320. doi:10.1016/j.tins.2009.02.004 
Sarinopoulos, I., Grupe, D. W., Mackiewicz, K. L., Herrington, J. D., Lor, M., Steege, E. E., \& Nitschke, J. B. (2010). Uncertainty during anticipation modulates neural responses to aversion in human insula and amygdala. Cerebral Cortex, 20(4), 929-940. doi:10.1093/cercor/bhp155

Sass, S. M., Heller, W., Stewart, J. L., Silton, R. L., Edgar, J. C., Fisher, J. E., \& Miller, G. A. (2010). Time course of attentional bias in anxiety: Emotion and gender specificity. Psychophysiology, 47(2), 247-259.

Saville, D. J. (1990). Multiple comparison procedures: The practical solution. American Statistician, 44(2), 174-180. doi:10.1080/00031305.1990.10475712

Savostyanov, A. N., Tsai, A. C., Liou, M., Levin, E. A., Lee, J. D., Yurganov, A. V., \& Knyazev, G. G. (2009). EEG-correlates of trait anxiety in the stop-signal paradigm. Neuroscience Letters, 449(2), 112-116.

Savostyanov, A. N., Tsai, A. C., Zhigalov, A. Y., Levin, E. A., Lee, J. D., \& Liou, M. (2011). Trait anxiety and neurophysiology of executive control in the stop-signal paradigm Trait Anxiety (pp. 191-222).

Schevernels, H., Bombeke, K., Van der Borght, L., Hopf, J. M., Krebs, R. M., \& Boehler, C. N. (2015). Electrophysiological evidence for the involvement of proactive and reactive control in a rewarded stop-signal task. NeuroImage, 121, 115-125. doi:10.1016/j.neuroimage.2015.07.023

Schultz, W. (1998). Predictive reward signal of dopamine neurons. Journal of Neurophysiology, 80(1), 1-27.

Schwartz, C. E., Wright, C. I., Shin, L. M., Kagan, J., \& Rauch, S. L. (2003). Inhibited and uninhibited infants "grown up": Adult amygdalar response to novelty. Science, 300(5627), 1952-1953. doi:10.1126/science.1083703 
Seeley, W. W., Menon, V., Schatzberg, A. F., Keller, J., Glover, G. H., Kenna, H., . . Greicius, M. D. (2007). Dissociable intrinsic connectivity networks for salience processing and executive control. Journal of Neuroscience, 27(9), 2349-2356. doi:10.1523/jneurosci.5587-06.2007

Sehlmeyer, C., Konrad, C., Zwitserlood, P., Arolt, V., Falkenstein, M., \& Beste, C. (2010). ERP indices for response inhibition are related to anxiety-related personality traits. Neuropsychologia, 48(9), 2488-2495.

Seymour, B., Singer, T., \& Dolan, R. (2007). The neurobiology of punishment. Nature Reviews Neuroscience, 8(4), 300-311.

Shackman, A. J., Salomons, T. V., Slagter, H. A., Fox, A. S., Winter, J. J., \& Davidson, R. J. (2011). The integration of negative affect, pain and cognitive control in the cingulate cortex. Nature Reviews Neuroscience, 12(3), 154-167.

doi:10.1038/nrn2994

Sharp, D. J., Bonnelle, V., De Boissezon, X., Beckmann, C. F., James, S. G., Patel, M. C., \& Mehta, M. A. (2010). Distinct frontal systems for response inhibition, attentional capture, and error processing. Proceedings of the National Academy of Sciences of the United States of America, 107(13), 6106-6111.

Sharp, P. B., Miller, G. A., \& Heller, W. (2014). Transdiagnostic dimensions of anxiety: Neural mechanisms, executive functions, and new directions. International Journal of Psychophysiology. doi:10.1016/j.ijpsycho.2015.07.001

Sharpe, N. (2002). Clinical trials and the real world: Selection bias and generalisability of trial results. Cardiovascular Drugs and Therapy, 16(1), 75-77.

doi:10.1023/A:1015327801114 
Shechner, T., Britton, J. C., Pérez-Edgar, K., Bar-Haim, Y., Ernst, M., Fox, N. A., . . .

Pine, D. S. (2012). Attention biases, anxiety, and development: Toward or away from threats or rewards? Depression and Anxiety, 29(4), 282-294.

Sheynin, J., Beck, K. D., Pang, K. C. H., Servatius, R. J., Shikari, S., Ostovich, J., \& Myers, C. E. (2014). Behaviourally inhibited temperament and female sex, two vulnerability factors for anxiety disorders, facilitate conditioned avoidance (also) in humans. Behavioural Processes, 103, 228-235.

doi:10.1016/j.beproc.2014.01.003

Sheynin, J., Moustafa, A. A., Beck, K. D., Servatius, R. J., \& Myers, C. E. (2015).

Testing the role of reward and punishment sensitivity in avoidance behavior: A computational modeling approach. Behavioural Brain Research, 283, 121-138. doi:10.1016/j.bbr.2015.01.033

Shin, L. M., \& Liberzon, I. (2010). The neurocircuitry of fear, stress, and anxiety disorders. Neuropsychopharmacology, 35(1), 169-191.

Shin, L. M., Whalen, P. J., Pitman, R. K., Bush, G., Macklin, M. L., Lasko, N. B., . . Rauch, S. L. (2001). An fMRI study of anterior cingulate function in posttraumatic stress disorder. Biological Psychiatry, 50(12), 932-942. doi:10.1016/s0006-3223(01)01215-X

Simon, J. J., Walther, S., Fiebach, C. J., Friederich, H. C., Stippich, C., Weisbrod, M., \& Kaiser, S. (2010). Neural reward processing is modulated by approach- and avoidance-related personality traits. NeuroImage, 49(2), 1868-1874.

Sjoerds, Z., Van Den Brink, W., Beekman, A. T. F., Penninx, B. W. J. H., \& Veltman, D. J. (2014). Response inhibition in alcohol-dependent patients and patients with depression/anxiety: A functional magnetic resonance imaging study. Psychological Medicine, 44(8), 1713-1725. 
Smillie, L. D. (2008). What is reinforcement sensitivity? Neuroscience paradigms for approach-avoidance process theories of personality. European Journal of Personality, 22(5), 359-384.

Solanto, M. V., Abikoff, H., Sonuga-Barke, E., Schachar, R., Logan, G. D., Wigal, T., . . . Turkel, E. (2001). The ecological validity of delay aversion and response inhibition as measures of impulsivity in AD/HD: A supplement to the $\mathrm{NIMH}$ multimodal treatment study of AD/HD. Journal of Abnormal Child Psychology, 29(3), 215-228.

Spielberger, C. D., Gorsuch, R. L., \& Lushene, R. E. (1970). Manual for the State Trait Anxiety Inventory. Palo Alto: Consulting Psychologists Press.

Spielberger, C. D., Gorsuch, R. L., Lushene, R. E., Vagg, P. R., \& Jacobs, G. A. (1983). Manual for the State-Trait Anxiety Inventory STAI (Form Y). Redwood City, CA: Mind Garden.

Spitzer, R. L., Kroenke, K., Williams, J. B. W., \& Löwe, B. (2006). A brief measure for assessing generalized anxiety disorder: The GAD-7. Archives of Internal Medicine, 166(10), 1092-1097.

Stanford, M. S., Mathias, C. W., Dougherty, D. M., Lake, S. L., Anderson, N. E., \& Patton, J. H. (2009). Fifty years of the Barratt Impulsiveness Scale: An update and review. Personality and Individual Differences, 47(5), 385-395. doi:10.1016/j.paid.2009.04.008

Stein, M. B., Simmons, A. N., Feinstein, J. S., \& Paulus, M. P. (2007). Increased amygdala and insula activation during emotion processing in anxiety-prone subjects. American Journal of Psychiatry, 164(2), 318-327. 
Storbjörk, J. (2014). Implications of enrolment eligibility criteria in alcohol treatment outcome research: Generalisability and potential bias in 1- and 6-year outcomes. Drug and Alcohol Review, 33(6), 604-611. doi:10.1111/dar.12211

Stroop, J. R. (1935). Studies of interference in serial verbal reactions. Journal of Experimental Psychology, 18(6), 643-662. doi:10.1037/h0054651

Stürmer, B., Nigbur, R., Schacht, A., \& Sommer, W. (2011). Reward and punishment effects on error processing and conflict control. Frontiers in Psychology, 2(NOV).

Swick, D., Ashley, V., \& Turken, U. (2011). Are the neural correlates of stopping and not going identical? Quantitative meta-analysis of two response inhibition tasks. NeuroImage, 56(3), 1655-1665.

Sylvers, P., Lilienfeld, S. O., \& LaPrairie, J. L. (2011). Differences between trait fear and trait anxiety: Implications for psychopathology. Clinical Psychology Review, 31(1), 122-137.

Sylvester, C. M., Corbetta, M., Raichle, M. E., Rodebaugh, T. L., Schlaggar, B. L., Sheline, Y. I., . . Lenze, E. J. (2012). Functional network dysfunction in anxiety and anxiety disorders. Trends in Neurosciences, 35(9), 527-535.

Tabachnick, B. G., \& Fidell, L. S. (2007). Using Multivariate Statistics. Boston: Pearson. Takezawa, T., \& Miyatani, M. (2005). Quantitative relation between conflict and response inhibition in the flanker task. Psychological Reports, 97(2), 515-526.

Taylor, J. M., \& Whalen, P. J. (2015). Neuroimaging and Anxiety: the Neural Substrates of Pathological and Non-pathological Anxiety. Current Psychiatry Reports, 17(6). doi:10.1007/s11920-015-0586-9

Teachman, B. A., Joormann, J., Steinman, S. A., \& Gotlib, I. H. (2012). Automaticity in anxiety disorders and major depressive disorder. Clinical Psychology Review, 32(6), 575-603. 
Teng, C., Otero, M., Geraci, M., Blair, R. J. R., Pine, D. S., Grillon, C., \& Blair, K. S. (2016). Abnormal decision-making in generalized anxiety disorder: Aversion of risk or stimulus-reinforcement impairment? Psychiatry Research, 237, 351-356. doi:10.1016/j.psychres.2015.12.031

Tepper, B. J., \& Tepper, K. (1993). The effects of method variance within measures. Journal of Psychology: Interdisciplinary and Applied, 127(3), 293-302. doi:10.1080/00223980.1993.9915563

Torrubia, R., Ávila, C., Moltó, J., \& Caseras, X. (2001). The Sensitivity to Punishment and Sensitivity to Reward Questionnaire (SPSRQ) as a measure of Gray's anxiety and impulsivity dimensions. Personality and Individual Differences, 31(6), 837862.

van den Heuvel, M. P., \& Hulshoff Pol, H. E. (2010). Exploring the brain network: A review on resting-state fMRI functional connectivity. European Neuropsychopharmacology, 20(8), 519-534. doi:10.1016/j.euroneuro.2010.03.008 Van Veen, V., \& Carter, C. S. (2002). The anterior cingulate as a conflict monitor: FMRI and ERP studies. Physiology and Behavior, 77(4-5), 477-482.

Verbruggen, F., \& Logan, G. D. (2008). Response inhibition in the stop-signal paradigm. Trends in Cognitive Sciences, 12(11), 418-424. doi:http://dx.doi.org/10.1016/j.tics.2008.07.005

Verbruggen, F., \& Logan, G. D. (2009). Models of response inhibition in the stop-signal and stop-change paradigms. Neuroscience and Biobehavioral Reviews, 33(5), 647-661.

Vigneau, F., \& Cormier, S. (2008). The factor structure of the State-Trait Anxiety Inventory: an alternative view. Journal of Personality Assessment, 90(3), 280285. 
Vincent, J. L., Kahn, I., Snyder, A. Z., Raichle, M. E., \& Buckner, R. L. (2008). Evidence for a frontoparietal control system revealed by intrinsic functional connectivity. Journal of Neurophysiology, 100(6), 3328-3342. doi:10.1152/jn.90355.2008

Wacker, J., Chavanon, M. L., Leue, A., \& Stemmler, G. (2010). Trait BIS predicts alpha asymmetry and P300 in a Go/No-Go task. European Journal of Personality, 24(2), 85-105.

Watson, D., Clark, L. A., \& Tellegen, A. (1988). Development and Validation of Brief Measures of Positive and Negative Affect: The PANAS Scales. Journal of Personality and Social Psychology, 54(6), 1063-1070.

Watson, D., Clark, L. A., Weber, K., Assenheimer, J. S., Strauss, M. E., \& McCormick, R. A. (1995a). Testing a tripartite model: I. Evaluating the convergent and discriminant validity of anxiety and depression symptom scales. Journal of Abnormal Psychology, 104(1), 3-14. doi:10.1037/0021-843X.104.1.3

Watson, D., Clark, L. A., Weber, K., Assenheimer, J. S., Strauss, M. E., \& McCormick, R. A. (1995b). Testing a tripartite model: II. Exploring the symptom structure of anxiety and depression in student, adult, and patient samples. Journal of Abnormal Psychology, 104(1), 15-25. doi:10.1037/0021-843X.104.1.15

Weinberg, A., Klein, D. N., \& Hajcak, G. (2012). Increased error-related brain activity distinguishes generalized anxiety disorder with and without comorbid major depressive disorder. Journal of Abnormal Psychology, 121(4), 885-896.

Weinberg, A., Olvet, D. M., \& Hajcak, G. (2010). Increased error-related brain activity in generalized anxiety disorder. Biological Psychology, 85(3), 472-480. doi:10.1016/j.biopsycho.2010.09.011 
Weissman, D. H., Roberts, K. C., Visscher, K. M., \& Woldorff, M. G. (2006). The neural bases of momentary lapses in attention. Nature Neuroscience, 9(7), 971-978. doi: $10.1038 / \mathrm{nn} 1727$

Westfall, J., \& Yarkoni, T. (2016). Statistically controlling for confounding constructs is harder than you think. PLoS ONE, 11(3). doi:10.1371/journal.pone.0152719

Whitfield-Gabrieli, S., \& Ford, J. M. (2012) Default mode network activity and connectivity in psychopathology. Vol. 8. Annual Review of Clinical Psychology (pp. 49-76+C41-C49).

Wilson, G. D., Barrett, P. T., \& Gray, J. A. (1989). Human reactions to reward and punishment: a questionnaire examination of Gray's personality theory. The British journal of psychology, 80.

Wright, L., Lipszyc, J., Dupuis, A., Thayapararajah, S. W., \& Schachar, R. (2014). Response inhibition and psychopathology: A meta-analysis of Go/No-Go task performance. Journal of Abnormal Psychology, 123(2), 429-439.

Yerkes, R. M., \& Dodson, J. D. (1908). The relation of strength of stimulus to rapidity of the habit-formation. Journal of Comparative Neurology and Psychology, 18, 459482.

Yong, A. G., \& Pearce, S. (2013). A beginner's guide to factor analysis: Focusing on exploratory factor analysis. Tutorials in Quantitative Methods for Psychology, 9(2), 79-94.

Yu, C., Zhou, Y., Liu, Y., Jiang, T., Dong, H., Zhang, Y., \& Walter, M. (2011). Functional segregation of the human cingulate cortex is confirmed by functional connectivity based neuroanatomical parcellation. NeuroImage, 54(4), 2571-2581. doi:10.1016/j.neuroimage.2010.11.018 
Zambrano-Vazquez, L., \& Allen, J. J. B. (2014). Differential contributions of worry, anxiety, and obsessive compulsive symptoms to ERN amplitudes in response monitoring and reinforcement learning tasks. Neuropsychologia, 61(1), 197-209.

Zemore, R., Fischer, D. G., Garratt, L. S., \& Miller, C. (1990). The depression proneness rating scale: Reliability, validity, and factor structure. Current Psychology, 9(3), 255-263. doi:10.1007/BF02686863 


\section{Appendix A}

\section{Published Measures Used in the Research Project}

1. Alcohol Use Disorders Identification Test (Babor et al., 2001)

2. Attentional Control Scale (Derryberry \& Reed, 2002)

3. Behavioral Inhibition System/Behavioral Activation System Scales (Carver \& White, 1994)

4. Barratt Impulsiveness Scale (Patton et al., 1995)

5. The Big Five Aspects Scales (DeYoung et al., 2007)

6. Edinburgh Handedness Inventory (Oldfield, 1971)

7. Fagerstrom Test of Nicotine Dependence (Fagerstrom et al., 1990)

8. Generalised Anxiety Disorder 7-Item Scale (Spitzer et al., 2006)

9. Inventory of Depressive Symptomatology - Self Report (Rush et al., 1986)

10. Mood and Anxiety Symptom Questionnaire (Watson et al., 1995a, 1995b)

11. Orygen Substance Use Index

12. Penn State Worry Questionnaire (Meyer et al., 1990)

13. The Personality Inventory for DSM-5 - Brief Form (PID-5-BF) - Adult (Krueger et al., 2013)

14. The Sensitivity to Punishment and Sensitivity to Reward Questionnaire (Torrubia et al., 2001) 


\section{Alcohol Use Disorders Identification Test}

The following questions ask about your use of alcohol. Your answers will remain confidential so please be honest. Try to answer the questions in terms of 'standard drinks'. Standard drinks measure the amount of pure alcohol you are drinking. One standard drink equals approximately 10 grams of pure alcohol - see the diagram below.

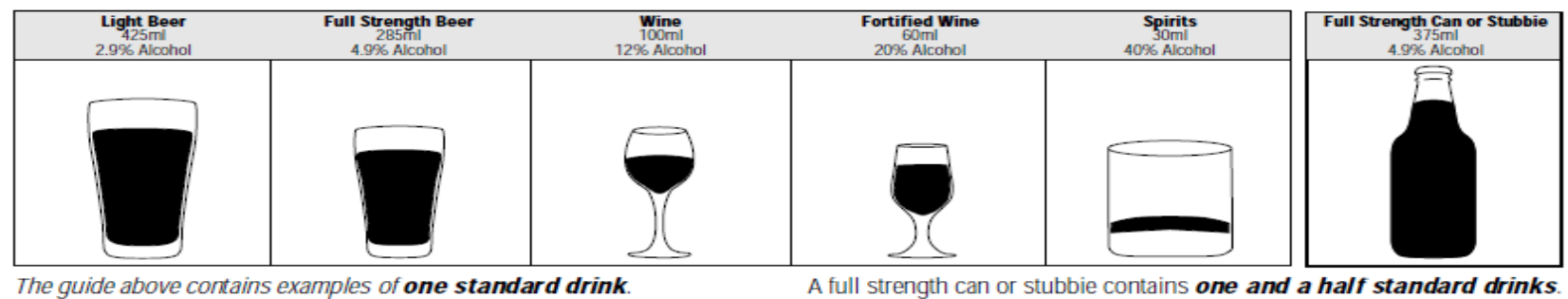

Mark the circle that best describes your answer to each question.

1 How often do you have a drink containing alcohol?

O Never

Monthly or less

O 2 - 4 times a month

O 2 - 3 times a week

O 4 or more times a week

2 How many drinks containing alcohol do you have on a typical day when you are drinking?

O 1 or 2

O 3 or 4

O 5 or 6

O 7 to 9

O 10 or more

3 How often do you have six or more drinks on one occasion?

O Never

Less than monthly

Monthly

Weekly

Daily or almost daily 
4 How often during the last year have you found that you were not able to stop drinking once you had started?

O Never

Less than monthly

Monthly

Weekly

Daily or almost daily

5 How often during the last year have you failed to do what was normally expected of you because of drinking?

O Never

Less than monthly

Monthly

Weekly

Daily or almost daily

6 How often during the last year have you needed a first drink in the morning to get yourself going after a heavy drinking session?

O Never

Less than monthly

Monthly

Weekly

Daily or almost daily

7 How often during the last year have you had a feeling of guilt or remorse after drinking?

O Never

Less than monthly

Monthly

Weekly

Daily or almost daily

8 How often during the last year have you been unable to remember what happened the night before because of your drinking?

O Never

Less than monthly

O Monthly

Weekly

O Daily or almost daily 
9 Have you or someone else been injured because of your drinking?

O No

Y Yes, but not in the last year

Yes, during the last year

10 Has a relative, friend, doctor, or other health care worker been concerned about your drinking or suggested you cut down?

O No

Y Yes, but not in the last year

$O$ Yes, during the last year 
Here are some different ways that people can feel about working and concentrating. Please indicate how strongly each statement applies to you.

$$
\begin{aligned}
& \mathbf{1}=\text { Almost never } \\
& \mathbf{2}=\text { Sometimes } \\
& \mathbf{3}=\text { Often } \\
& \mathbf{4}=\text { Always }
\end{aligned}
$$

1. It's very hard for me to concentrate on a difficult task when there are noises around.

2. When I need to concentrate and solve a problem, I have trouble focusing my attention.

3. When I am working hard on something, I still get distracted by events around me.

4. My concentration is good even if there is music in the room around me.

5. When concentrating, I can focus my attention so that I become unaware of what's going on in the room around me.

6. When I am reading or studying, I am easily distracted $\begin{array}{llll}1 & 2 & 3 & 4\end{array}$ if there are people talking in the same room.

7. When trying to focus my attention on something, I have difficulty blocking out distracting thoughts.

8. I have a hard time concentrating when

I'm excited about something.

9. When concentrating I ignore feelings of hunger or thirst.

10. I can quickly switch from one task to another.

11. It takes me a while to get really involved in a new task.

$\begin{array}{llll}1 & 2 & 3 & 4\end{array}$

12. It is difficult for me to coordinate my attention between

$\begin{array}{llll}1 & 2 & 3 & 4\end{array}$ the listening and writing required when taking notes during lectures. 
13. I can become interested in a new topic very quickly

$\begin{array}{llll}1 & 2 & 3 & 4\end{array}$ when I need to.

14. It is easy for me to read or write while I'm also talking on the phone.

15. I have trouble carrying on two conversations at once.

$\begin{array}{llll}1 & 2 & 3 & 4\end{array}$

16. I have a hard time coming up with new ideas quickly

$\begin{array}{llll}1 & 2 & 3 & 4\end{array}$

17. After being interrupted or distracted, I can easily $\begin{array}{llll}1 & 2 & 3 & 4\end{array}$ shift my attention back to what I was doing before.

18. When a distracting thought comes to mind, it is easy $\begin{array}{llll}1 & 2 & 3 & 4\end{array}$ for me to shift my attention away from it.

19. It is easy for me to alternate between two different tasks. $\begin{array}{lllll}1 & 2 & 3 & 4\end{array}$

20. It is hard for me to break from one way of thinking about $\quad \begin{array}{lllll}1 & 2 & 3 & 4\end{array}$ something and look at it from another point of view. 
Each item of this questionnaire is a statement that a person may either agree with or disagree with. For each item, indicate how much you agree or disagree with what the item says. Please respond to all the items; do not leave any blank. Choose only one response to each statement. Please be as accurate and honest as you can be. Respond to each item as if it were the only item. That is, don't worry about being "consistent" in your responses. Choose from the following four response options:

\section{$1=$ very true for me \\ 2 = somewhat true for me \\ 3 = somewhat false for me \\ 4 = very false for me}

1. A person's family is the most important thing in life.

2. Even if something bad is about to happen to me, I rarely experience fear or nervousness.

3. I go out of my way to get things I want.

4. When I'm doing well at something I love to keep at it.

5. I'm always willing to try something new if I think it will be fun.

6. How I dress is important to me.

7. When I get something I want, I feel excited and energized.

8. Criticism or scolding hurts me quite a bit.

9. When I want something I usually go all-out to get it.

10. I will often do things for no other reason than that they might be fun.

11. It's hard for me to find the time to do things such as get a haircut.

12. If I see a chance to get something I want I move on it right away.

13. I feel pretty worried or upset when I think or know somebody is angry at me.

14. When I see an opportunity for something I like I get excited right away.

15. I often act on the spur of the moment.

16. If I think something unpleasant is going to happen I usually get pretty "worked up."

17. I often wonder why people act the way they do.

18. When good things happen to me, it affects me strongly.

19. I feel worried when I think I have done poorly at something important.

20. I crave excitement and new sensations.

21. When I go after something I use a "no holds barred" approach. 
22. ___ I have very few fears compared to my friends.

23. ___ It would excite me to win a contest.

24. ___ I worry about making mistakes. 


\section{Barratt Impulsiveness Scale}

People differ in the ways they act and think in different situations. This is a test to measure some of the ways in which you act and think. Read each statement and put an X on the response that best describes you. Do not spend too much time on any statement. Answer quickly and honestly.

\section{Rarely/Never Occasionally Often Almost Always/Always}

1. I plan tasks carefully.

2. I do things without thinking.

3. I make-up my mind quickly.

4. I am happy-go-lucky.

5. I don't "pay attention".

6. I have "racing" thoughts.

7. I plan trips well ahead of time.

8. I am self-controlled.

9. I concentrate easily.

10. I save regularly.

11. I "squirm" at plays or lectures.

12. I am a careful thinker.

13. I plan for job security.

14. I say things without thinking.

15. I like to think about complex problems.
0

0

O

0

$\mathrm{O}$

0

$\mathrm{O}$

$\mathrm{O}$

0

0

$\mathrm{O}$

$\mathrm{O}$

0

O

0 


\section{Rarely/Never Occasionally Often Almost Always/Always}

16. I change jobs.

$\mathrm{O}$

0

0

0

17. I act "on impulse".

0

0

0

0

18. I get easily bored when solving thought problems.

0

0

0

0

19. I act on the spur of the moment.

$\mathrm{O}$

0

0

20. I am a steady thinker.

0

0

$\mathrm{O}$

O

21. I change residences.

0

0

0

0

22. I buy things on impulse.

0

0

0

0

23. I can only think about one thing at a time.

$\mathrm{O}$

0

0

0

24. I change hobbies.

0

$\mathrm{O}$

0

25. I spend or charge more than I earn.

0

0

0

26. I often have extraneous

thoughts when thinking.

0

0

0

27. I am more interested in the present than the future.

0

0

0

0

28. I am restless at the theatre or lectures

O

0

0

0

29. I like puzzles.

0

0

0

0

30. I am future oriented.

0

0

0

0 
Here are a number of characteristics that may or may not describe you. For example, do you agree that you seldom feel blue, compared to most other people? Please fill in the number that best indicates the extent to which you agree or disagree with each statement listed below. Be as honest as possible, but rely on your initial feeling and do not think too much about each item.

Use the following scale:

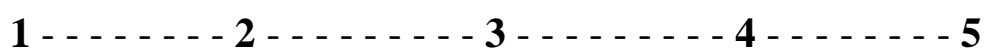

\section{Strongly \\ Neither Agree \\ Strongly \\ Disagree \\ Nor Disagree \\ Agree}

1. ___ Seldom feel blue.

2. ___ Am not interested in other people's problems.

3. ___ Carry out my plans.

4. ___ Make friends easily.

5. ___ Am quick to understand things.

6. ___ Get angry easily.

7. ___ Respect authority.

8. ___ Leave my belongings around.

9. ___ Take charge.

10. ___ Enjoy the beauty of nature.

11. ___ Am filled with doubts about things.

12. ___ Feel others' emotions.

13. ___ Waste my time.

14. ___ Am hard to get to know.

15. __ Have difficulty understanding abstract ideas.

16. ___ Rarely get irritated.

17. __ Believe that I am better than others.

18. ___ Like order.

19. ___ Have a strong personality.

20. __ Believe in the importance of art.

21. ___ Feel comfortable with myself.
22. ___ Inquire about others' well-being.

23. ___ Find it difficult to get down to work.

24. K__ Keep others at a distance.

25. ___ Can handle a lot of information.

26. __ Get upset easily.

27. ___ Hate to seem pushy.

28. __ Keep things tidy.

29. ___ Lack the talent for influencing people.

30. __ Love to reflect on things.

31. ___ Feel threatened easily.

32. ___ Can't be bothered with other's needs.

33. ___ Mess things up.

34. ___ Reveal little about myself.

35. L__ Like to solve complex problems.

36. K__ Keep my emotions under control.

37. __ Take advantage of others.

38. ___ Follow a schedule.

39. Kn Know how to captivate people.

40. __ Get deeply immersed in music.

41. __ Rarely feel depressed.

42. ___ Sympathize with others' feelings. 
43. __ Finish what I start.

44. _ Warm up quickly to others.

45. __ Avoid philosophical discussions.

46. ___ Change my mood a lot.

47. ___ Avoid imposing my will on others.

48. __ Am not bothered by messy people.

49. _ Wait for others to lead the way.

50. __ Do not like poetry.

51. __ Worry about things.

52. _ Am indifferent to the feelings of others.

53. _ Don't put my mind on the task at hand.

54. __ Rarely get caught up in the excitement.

55. __ Avoid difficult reading material.

56. __ Rarely lose my composure.

57. __ Rarely put people under pressure.

58. _ Want everything to be "just right."

59. __ See myself as a good leader.

60. __ Seldom notice the emotional aspects of paintings and pictures.

61. __ Am easily discouraged.

62. __ Take no time for others.

63. __ Get things done quickly.

64. __ Am not a very enthusiastic person.

65. __ Have a rich vocabulary.

66. __ Am a person whose moods go up and down easily.

67. __ Insult people.

68. __ Am not bothered by disorder.

69. _ Can talk others into doing things.

70. __ Need a creative outlet.

71. __ Am not embarrassed easily.

72. __ Take an interest in other people's lives.
73. _ Always know what I am doing.

74. __ Show my feelings when I'm happy.

75. _ Think quickly.

76. ___ Am not easily annoyed.

77. __ Seek conflict.

78. __ Dislike routine.

79. __ Hold back my opinions.

80. __ Seldom get lost in thought.

81. _ Become overwhelmed by events.

82. _ Don't have a soft side.

83. __ Postpone decisions.

84. _ Have a lot of fun.

85. __ Learn things slowly.

86. __ Get easily agitated.

87. __ Love a good fight.

88. __ See that rules are observed.

89. __ Am the first to act.

90. __ Seldom daydream.

91. __ Am afraid of many things.

92. __ Like to do things for others.

93. __ Am easily distracted.

94. __ Laugh a lot.

95. __ Formulate ideas clearly.

96. __ Can be stirred up easily.

97. __ Am out for my own personal gain.

98. __ Want every detail taken care of.

99. _ Do not have an assertive personality.

100. __ See beauty in things that others might not notice. 


\section{Edinburgh Handedness Inventory}

Please indicate which hand you prefer to use during the following activities by marking the appropriate circle. Where the preference is so strong that you would never try to use the other hand unless absolutely forced to, select 'Always'. If in any case you are really indifferent, select 'No preference'. Some of the activities require both hands. In these cases the part of the task, or object, for which the hand preference is wanted is indicated in brackets. Please try to answer all the questions, and only leave a blank if you have no experience at all of the object or task.

\begin{tabular}{|c|c|c|c|c|c|}
\hline & Always left & Prefer left & Prefer right & Always right & No preference \\
\hline Writing & 0 & 0 & 0 & 0 & $\mathrm{O}$ \\
\hline Drawing & 0 & 0 & 0 & 0 & 0 \\
\hline Throwing & 0 & 0 & 0 & 0 & 0 \\
\hline Scissors & 0 & 0 & 0 & 0 & 0 \\
\hline Toothbrush & 0 & 0 & 0 & 0 & 0 \\
\hline $\begin{array}{l}\text { Knife } \\
\text { (without } \\
\text { fork) }\end{array}$ & 0 & 0 & 0 & 0 & 0 \\
\hline Spoon & 0 & 0 & 0 & 0 & 0 \\
\hline $\begin{array}{l}\text { Broom } \\
\text { (upper hand) }\end{array}$ & 0 & 0 & 0 & 0 & 0 \\
\hline $\begin{array}{l}\text { Striking } \\
\text { match } \\
\text { (match) }\end{array}$ & 0 & 0 & 0 & 0 & 0 \\
\hline $\begin{array}{l}\text { Opening box } \\
\text { (lid) }\end{array}$ & 0 & 0 & 0 & 0 & 0 \\
\hline $\begin{array}{l}\text { Which foot } \\
\text { do you prefer } \\
\text { to kick with? }\end{array}$ & 0 & 0 & 0 & 0 & 0 \\
\hline $\begin{array}{l}\text { Which eye do } \\
\text { you use when } \\
\text { using only } \\
\text { one (e.g. } \\
\text { looking } \\
\text { through a } \\
\text { camera) }\end{array}$ & 0 & 0 & 0 & 0 & 0 \\
\hline
\end{tabular}


Are you a smoker or a non-smoker?

Smoker (i.e., smoke regularly, socially, occasionally, sometimes, or rarely)

O Non-smoker (i.e., never smoke)

1 How soon after you wake up do you smoke your first cigarette?

W Within 5 minutes

O 6 - 30 minutes

O 31 - 60 minutes

Ofter 60 minutes

2 Do you find it difficult to refrain from smoking in places where it is forbidden e.g. in church, at the library, in cinemas, etc.?

$\mathrm{O}$ Yes

O No

3 Which cigarette would you hate most to give up?

The first one in the morning

O All others

4 How many cigarettes a day do you smoke?

O 10 or less

○ $11-20$

○ $21-30$

O 31 or more

5 Do you smoke more frequently during the first hours after waking than during the rest of the day?

$\mathrm{O}$ Yes

O No

6 Do you smoke if you are so ill that you are in bed most of the day?

$\mathrm{O}$ Yes

O No 
Over the last 2 weeks, how often have you been bothered by the following problems? (Circle one option per question)

$\begin{array}{ccc}\text { Not at all } & \begin{array}{c}\text { Several } \\ \text { days }\end{array} & \begin{array}{c}\text { Over half } \\ \text { the days }\end{array} \\ & \text { every day }\end{array}$

1. Feeling nervous, anxious, or on edge

0

1

2

3

2. Not being able to stop or control worrying

0

1

2

3

3. Worrying too much about different things

0

1

2

4. Trouble relaxing

0

1

2

3

5. Being so restless that it is hard to sit still

0

1

2

3

6. Becoming easily annoyed or irritable

0

1

2

7. Feeling afraid as if something awful might

0

1

2

3 happen

If you checked off any problems, how difficult have these made it for you to do your work, take care of things at home, or get along with other people?

Not at all difficult

Somewhat difficult

Very difficult

Extremely difficult 


\section{Inventory of Depressive Symptomatology - Self Report}

Please select the one response to each item that best describes you for the past seven days.

1. Falling asleep:

I never take longer than 30 minutes to fall asleep.

I I take at least 30 minutes to fall asleep, some nights.

I take at least 30 minutes to fall asleep, most nights.

I take more than 60 minutes to fall asleep, most nights.

2. Sleep during the night:

O I do not wake up at night.

O I have a restless, light sleep with a few brief awakenings each night.

O I wake up at least once a night, but I go back to sleep easily.

I wake up more than once a night and stay awake for 20 minutes or more, most nights.

3. Waking up too early:

Most days, I wake up less than 30 minutes before I need to get up.

O Most days, I wake up more than 30 minutes before I need to get up.

I I almost always wake up at least one hour or so before I need to, but I go back to sleep eventually.

O I always wake up at least one hour before I need to, and can't go back to sleep.

4. Sleeping too much:

I sleep 7-8 hours/night or less, without napping during the day.

O I sleep 10 hours or less in a 24-hour period, including naps.

O I sleep 12 hours or less in a 24-hour period, including naps.

I sleep longer than 12 hours in a 24-hour period, including naps.

5. Feeling sad:

O I do not usually feel sad.

O I feel sad less than half the time.

O I feel sad more than half the time.

O I feel sad nearly all of the time.

6. Feeling irritable:

O I do not usually feel irritable.

O I feel irritable less than half the time.

O I feel irritable more than half the time.

I feel extremely irritable nearly all of the time. 
7. Feeling anxious or tense:

I do not usually feel anxious or tense.

O I feel anxious (tense) less than half the time.

O I feel anxious (tense) more than half the time.

I feel extremely anxious (tense) nearly all of the time.

8. Response of your mood to good or desired events:

When good events happen, my mood brightens for a few hours.

When good events happen, my mood brightens but I do not feel like my normal self.

Only when a particularly good thing happens does my mood brighten a little.

O My mood does not brighten at all, even when very good things happen in my life.

9. Mood in relation to the time of day:

I have not noticed a connection between my mood and the time of day.

My mood is connected to circumstances, such as being alone or working, rather than to the time of day.

In general, my mood is more related to the time of day than to circumstances.

O My mood is clearly and predictably better or worse at a particular time each day.

9A. Is your mood typically worse:

$\mathrm{O}$ in the morning.

$O$ afternoon.

$O$ night.

9B. Is your mood variation attributed to the environment?

O yes.

no.

10. The quality of your mood:

The mood (internal feelings) that I experience is very much a normal mood.

My mood is sad, but this sadness is pretty much like the sad mood I would feel if someone close to me died or left.

My mood is sad, but this sadness has a rather different quality to it than the sadness I would feel if someone close to me died or left.

My mood is sad, but this sadness is different from the type of sadness associated with grief or loss.

Please complete either 11 or 12 (not both) 
11. Decreased appetite:

There is no change in my usual appetite.

O I eat somewhat less often or lesser amounts of food than usual.

O I eat much less than usual and only if I make an effort.

I rarely eat within a 24-hour period, and only if I am determined to eat or others persuade me to eat.

12. Increased appetite:

There is no change from my usual appetite.

I feel a need to eat more frequently than usual.

I I regularly eat more often and/or greater amounts of food than usual.

O I feel driven to overeat both at mealtime and between meals.

Please complete either 13 or 14 (not both)

13. Within the last two weeks:

I have not had a change in my weight.

O I feel as if I've had a slight weight loss.

O I have lost 1 kilo or more.

I have lost 2.5 kilos or more.

14. Within the last two weeks:

I have not had a change in my weight.

I feel as if I've had a slight weight gain.

I I have gained 1 kilo or more.

I have gained 2.5 kilos or more.

15. Concentration/decision making:

There is no change in my usual capacity to concentrate or make decisions.

I occasionally feel indecisive or find that my attention wanders.

Most of the time, I struggle to focus my attention or to make decisions.

O I cannot concentrate well enough to read or cannot make even minor decisions.

16. View of myself:

O I see myself as just as worthwhile and deserving as other people.

I blame myself (for things that go wrong) more than usual.

I I believe that I cause a lot of problems for others.

I think almost constantly about my faults and the problems I cause. 
17. View of my future:

I have a positive (optimistic) view of my future.

I I am occasionally negative (pessimistic) about my future, but for the most part I believe things will get better.

I'm pretty certain that my immediate future (1-2 months) does not hold much promise of good things for me.

I see no hope of anything good happening to me any time in the future.

18. Thoughts of deaths or suicide:

I do not think of suicide or dying.

O I feel that life is empty or wonder if it's worth living.

I find myself thinking of suicide or dying several times a week.

I I keep thinking about suicide or dying several times a day in some detail, or I have made specific plans for suicide or have actually tried to take my life.

19. General interest:

There is no change from usual in how interested I am in other people or activities.

O I notice that I have been less interested in people or activities recently.

I find I have interest in only one or two of the activities I used to enjoy.

I have virtually no interest in the activities I used to enjoy.

20. Energy level:

There is no change in my usual energy level.

I get tired more easily than usual.

O I have to make a big effort to start or finish my usual daily activities (for example, shopping, homework, cooking or going to work).

I really cannot carry out most of my usual daily activities because I just don't have the energy.

21. Capacity for pleasure or enjoyment (excluding sex):

I enjoy pleasurable activities just as much as usual.

I do not get my usual sense of enjoyment from pleasurable activities.

I rarely get a feeling of pleasure from any activity.

O I am unable to get any pleasure or enjoyment from anything.

22. Interest in (or desire for) sex (please rate interest not activity):

I'm just as interested in sex as usual.

My interest in sex is somewhat less than usual or I do not get the same pleasure from sex as I used to.

O I have little desire for sex and/or get very little pleasure from it.

I have absolutely no interest in sex and/or get no pleasure from it at all. 
23. Feeling slowed down:

I think, speak, and move at my usual speed.

O I find that my thinking is slowed down or my voice sounds dull and/or flat.

It takes me several seconds to respond to most questions and I'm sure my thinking is slowed.

I am often unable to respond to questions without making a real effort.

24. Feeling restless:

O I do not feel restless.

I'm often fidgety, wring my hands, or need to move around in my seat.

O I feel I need to move around and am quite restless.

I am often unable to stay seated and need to pace around.

25. Aches and pains:

O I don't have any aches or pains.

Sometimes I get headaches or pains in my stomach, back or joints but these pains are only present sometimes and they don't stop me from doing what I need to do.

I have these sorts of pains most of the time.

These pains are so bad they force me to stop what I am doing.

26. Other bodily symptoms:

I do NOT have any of these symptoms: heart pounding fast, blurred vision, sweating, hot and cold flushes, chest pain, heart turning over in my chest, ringing in my ears, or shaking.

I I have some of these symptoms but they are mild and are present only sometimes.

O I have several of these symptoms and they bother me quite a bit.

O I have several of these symptoms and when they occur I have to stop doing whatever I am doing.

27. Panic/phobic symptoms:

I have no episodes of panic or specific fears or phobias (such as animals or heights).

I I have mild panic episodes or fears that do not usually change my behavior or stop me from doing things.

I have definite panic episodes or fears that make me change my behavior but do not stop me from doing things altogether.

I have panic episodes at least once a week or severe fears that stop me from carrying on my normal daily activities. 
28. Constipation/diarrhoea:

There is no change in my usual bowel habits.

O I have occasional, mild constipation or diarrhoea.

I have diarrhoea or constipation most of the time but it does not interfere with my day-today activities.

I have constipation or diarrhoea for which I take medicine or which interferes with my day-to-day activities.

29. Interpersonal sensitivity:

I do not feel easily rejected, slighted, criticised or hurt by others at all.

O I occasionally feel rejected, slighted, criticised or hurt by others.

I often feel rejected, slighted, criticised or hurt by others, but these feelings have had only slight effects on my relationships or work.

I feel so rejected, slighted, criticised or hurt by others that these feelings interfere with my relationships and work

30. Leaden paralysis/physical energy:

I do NOT have the physical sensation of feeling weighted down or lacking physical energy.

I I occasionally experience periods of feeling physically weighted down and lacking physical energy, but without a negative effect on work, school, or activity level.

I feel physically weighted down and lacking physical energy more than half the time.

I feel physically weighted down and lacking physical energy for long periods every day. 
Mood and Anxiety Symptom Questionnaire

Below is a list of feelings, sensations, problems, and experiences that people sometimes have. Read each item and then mark the appropriate choice in the space next to that item. Use the choice that best describes how much you have felt or experienced things this way during the past week, including today.

\begin{tabular}{|c|c|c|c|c|c|}
\hline & 1. Not at all & 2. A little bit & 3. Moderately & 4. Quite a bit & 5. Extremely \\
\hline $\begin{array}{l}\text { 1. Felt } \\
\text { cheerful }\end{array}$ & 0 & 0 & 0 & 0 & 0 \\
\hline 2. Felt afraid & 0 & 0 & 0 & 0 & 0 \\
\hline $\begin{array}{l}\text { 3. Startled } \\
\text { easily }\end{array}$ & 0 & 0 & 0 & O & 0 \\
\hline $\begin{array}{l}\text { 4. Felt } \\
\text { confused }\end{array}$ & 0 & $\mathrm{O}$ & $\mathrm{O}$ & $\mathrm{O}$ & O \\
\hline $\begin{array}{l}\text { 5. Slept very } \\
\text { well }\end{array}$ & 0 & 0 & 0 & 0 & 0 \\
\hline 6. Felt sad & $\mathrm{O}$ & O & O & O & $\mathrm{O}$ \\
\hline $\begin{array}{l}\text { 7. Felt very } \\
\text { alert }\end{array}$ & 0 & $\mathrm{O}$ & $\mathrm{O}$ & $\mathrm{O}$ & 0 \\
\hline $\begin{array}{l}\text { 8. Felt } \\
\text { discouraged }\end{array}$ & O & O & 0 & $\mathrm{O}$ & 0 \\
\hline $\begin{array}{l}\text { 9. Felt } \\
\text { nauseous }\end{array}$ & 0 & 0 & 0 & $\mathrm{O}$ & 0 \\
\hline $\begin{array}{l}10 . \text { Felt like } \\
\text { crying }\end{array}$ & $\mathrm{O}$ & $\mathrm{O}$ & 0 & $\mathrm{O}$ & 0 \\
\hline $\begin{array}{l}\text { 11. Felt } \\
\text { successful }\end{array}$ & 0 & 0 & O & 0 & 0 \\
\hline $\begin{array}{l}\text { 12. Had } \\
\text { diarrhea }\end{array}$ & 0 & O & $\mathrm{O}$ & O & 0 \\
\hline $\begin{array}{l}\text { 13. Felt } \\
\text { worthless }\end{array}$ & 0 & 0 & 0 & 0 & 0 \\
\hline $\begin{array}{l}\text { 14. Felt really } \\
\text { happy }\end{array}$ & 0 & 0 & $\mathrm{O}$ & 0 & 0 \\
\hline $\begin{array}{l}\text { 15. Felt } \\
\text { nervous }\end{array}$ & 0 & O & 0 & 0 & 0 \\
\hline $\begin{array}{l}\text { 16. Felt } \\
\text { depressed }\end{array}$ & $\mathrm{O}$ & O & $\mathrm{O}$ & $\mathrm{O}$ & O \\
\hline $\begin{array}{l}\text { 17. Felt } \\
\text { irritable }\end{array}$ & $\mathrm{O}$ & O & $\mathrm{O}$ & 0 & O \\
\hline $\begin{array}{l}\text { 18. Felt } \\
\text { optimistic }\end{array}$ & 0 & O & $\mathrm{O}$ & O & 0 \\
\hline 19. Felt faint & 0 & 0 & 0 & 0 & 0 \\
\hline
\end{tabular}




\begin{tabular}{|c|c|c|c|c|c|}
\hline $\begin{array}{l}\text { 20. Felt } \\
\text { uneasy }\end{array}$ & 0 & 0 & 0 & 0 & 0 \\
\hline $\begin{array}{l}21 . \text { Felt really } \\
\text { bored }\end{array}$ & 0 & 0 & 0 & 0 & 0 \\
\hline $\begin{array}{l}22 . \text { Felt } \\
\text { hopeless }\end{array}$ & 0 & 0 & 0 & 0 & 0 \\
\hline $\begin{array}{l}23 . \text { Felt like I } \\
\text { was having a } \\
\text { lot of fun }\end{array}$ & 0 & 0 & 0 & 0 & 0 \\
\hline $\begin{array}{l}\text { 24. Blamed } \\
\text { myself for a } \\
\text { lot of things }\end{array}$ & 0 & 0 & 0 & 0 & 0 \\
\hline $\begin{array}{l}25 . \text { Felt } \\
\text { numbness or } \\
\text { tingling in my } \\
\text { body }\end{array}$ & 0 & 0 & 0 & 0 & 0 \\
\hline $\begin{array}{l}\text { 26. Felt } \\
\text { withdrawn } \\
\text { from other } \\
\text { people }\end{array}$ & 0 & 0 & 0 & 0 & 0 \\
\hline $\begin{array}{l}\text { 27. Seemed } \\
\text { to move } \\
\text { quickly and } \\
\text { easily }\end{array}$ & 0 & 0 & 0 & 0 & 0 \\
\hline $\begin{array}{l}\text { 28. Was } \\
\text { afraid I was } \\
\text { going to lose } \\
\text { control }\end{array}$ & 0 & 0 & 0 & 0 & 0 \\
\hline $\begin{array}{l}\text { 29. Felt } \\
\text { dissatisfied } \\
\text { with } \\
\text { everything }\end{array}$ & 0 & 0 & 0 & 0 & 0 \\
\hline $\begin{array}{l}\text { 30. Looked } \\
\text { forward to } \\
\text { things with } \\
\text { enjoyment }\end{array}$ & 0 & 0 & 0 & 0 & 0 \\
\hline $\begin{array}{l}31 . \text { Had } \\
\text { trouble } \\
\text { remembering } \\
\text { things }\end{array}$ & 0 & 0 & 0 & 0 & 0 \\
\hline $\begin{array}{l}\text { 32. Felt like I } \\
\text { didn't need } \\
\text { much sleep }\end{array}$ & 0 & 0 & 0 & 0 & 0 \\
\hline $\begin{array}{l}\text { 33. Felt like } \\
\text { nothing was } \\
\text { very }\end{array}$ & 0 & 0 & 0 & 0 & 0 \\
\hline
\end{tabular}




\begin{tabular}{|c|c|c|c|c|c|}
\hline $\begin{array}{l}\text { enjoyable } \\
34 . \text { Felt like } \\
\text { something } \\
\text { awful was } \\
\text { going to } \\
\text { happen }\end{array}$ & 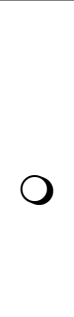 & 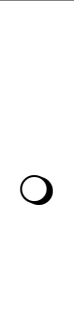 & $\mathrm{O}$ & 0 & O \\
\hline $\begin{array}{l}35 . \text { Felt like I } \\
\text { had } \\
\text { accomplished } \\
\text { a lot }\end{array}$ & $\mathrm{O}$ & 0 & 0 & 0 & 0 \\
\hline $\begin{array}{l}\text { 36. Felt like I } \\
\text { had a lot of } \\
\text { interesting } \\
\text { things to do }\end{array}$ & 0 & 0 & 0 & 0 & O \\
\hline $\begin{array}{l}37 . \text { Did not } \\
\text { have much of } \\
\text { an appetite }\end{array}$ & 0 & 0 & 0 & 0 & O \\
\hline $\begin{array}{l}\text { 38. Felt like } \\
\text { being with } \\
\text { other people }\end{array}$ & 0 & 0 & 0 & 0 & O \\
\hline $\begin{array}{l}\text { 39. Felt like it } \\
\text { took extra } \\
\text { effort to get } \\
\text { started }\end{array}$ & 0 & 0 & 0 & 0 & O \\
\hline $\begin{array}{l}\text { 40. Felt like I } \\
\text { had a lot to } \\
\text { look forward } \\
\text { to }\end{array}$ & 0 & 0 & 0 & 0 & O \\
\hline $\begin{array}{l}41 . \text { Thoughts } \\
\text { and ideas } \\
\text { came to me } \\
\text { very easily }\end{array}$ & 0 & 0 & 0 & 0 & 0 \\
\hline $\begin{array}{l}\text { 42. Felt } \\
\text { pessimistic } \\
\text { about the } \\
\text { future }\end{array}$ & 0 & 0 & 0 & 0 & O \\
\hline $\begin{array}{l}\text { 43. Felt like I } \\
\text { could do } \\
\text { everything I } \\
\text { needed to do }\end{array}$ & 0 & 0 & 0 & $\mathrm{O}$ & 0 \\
\hline $\begin{array}{l}\text { 44. Felt like } \\
\text { there wasn't } \\
\text { anything } \\
\text { interesting or } \\
\text { fun to do }\end{array}$ & 0 & 0 & 0 & 0 & $\mathrm{O}$ \\
\hline 45. Had pain & 0 & 0 & 0 & 0 & 0 \\
\hline
\end{tabular}




\begin{tabular}{|c|c|c|c|c|c|}
\hline in my chest & & & & & \\
\hline $\begin{array}{l}\text { 46. Felt really } \\
\text { talkative }\end{array}$ & $\bigcirc$ & $\mathrm{O}$ & 0 & $\mathrm{O}$ & 0 \\
\hline $\begin{array}{l}\text { 47. Felt like a } \\
\text { failure }\end{array}$ & $\bigcirc$ & $\mathrm{O}$ & $O$ & 0 & $\mathrm{O}$ \\
\hline $\begin{array}{l}48 . \text { Had hot } \\
\text { or cold spells }\end{array}$ & $\bigcirc$ & $\mathrm{O}$ & 0 & $\mathrm{O}$ & 0 \\
\hline $\begin{array}{l}\text { 49. Was } \\
\text { proud of } \\
\text { myself }\end{array}$ & $\bigcirc$ & $\mathrm{O}$ & 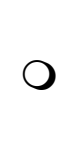 & $\mathrm{O}$ & O \\
\hline $\begin{array}{l}50 . \text { Felt very } \\
\text { restless }\end{array}$ & 0 & $\mathrm{O}$ & 0 & 0 & 0 \\
\hline $\begin{array}{l}51 . \text { Had } \\
\text { trouble } \\
\text { falling asleep }\end{array}$ & 0 & $\mathrm{O}$ & 0 & $\mathrm{O}$ & 0 \\
\hline $\begin{array}{l}52 . \text { Felt dizzy } \\
\text { or } \\
\text { lightheaded }\end{array}$ & $\bigcirc$ & $\mathrm{O}$ & 0 & $\mathrm{O}$ & 0 \\
\hline $\begin{array}{l}\text { 53. Felt } \\
\text { unattractive }\end{array}$ & 0 & $\mathrm{O}$ & 0 & $\mathrm{O}$ & 0 \\
\hline $\begin{array}{l}54 . \text { Felt very } \\
\text { clearheaded }\end{array}$ & $\bigcirc$ & $\mathrm{O}$ & 0 & $\mathrm{O}$ & 0 \\
\hline $\begin{array}{l}55 . \text { Was short } \\
\text { of breath }\end{array}$ & $\bigcirc$ & $\mathrm{O}$ & 0 & 0 & 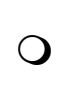 \\
\hline $\begin{array}{l}\text { 56. Felt } \\
\text { sluggish or } \\
\text { tired }\end{array}$ & $\bigcirc$ & $\mathrm{O}$ & 0 & $\mathrm{O}$ & 0 \\
\hline $\begin{array}{l}\text { 57. Hands } \\
\text { were shaky }\end{array}$ & $\bigcirc$ & $\mathrm{O}$ & 0 & $\mathrm{O}$ & 0 \\
\hline $\begin{array}{l}\text { 58. Felt really } \\
\text { "up" or lively }\end{array}$ & $\mathrm{O}$ & $\mathrm{O}$ & 0 & $\mathrm{O}$ & $\mathrm{O}$ \\
\hline $\begin{array}{l}59 . \text { Was } \\
\text { unable to } \\
\text { relax }\end{array}$ & $\mathrm{O}$ & 0 & 0 & 0 & $\mathrm{O}$ \\
\hline $\begin{array}{l}60 . \text { Felt like } \\
\text { being by } \\
\text { myself }\end{array}$ & $\bigcirc$ & $\mathrm{O}$ & 0 & $\mathrm{O}$ & 0 \\
\hline $\begin{array}{l}61 . \text { Felt like I } \\
\text { was choking }\end{array}$ & $\bigcirc$ & $\mathrm{O}$ & 0 & 0 & 0 \\
\hline $\begin{array}{l}\text { 62. Was } \\
\text { unable to } \\
\text { laugh easily }\end{array}$ & $\bigcirc$ & $\mathrm{O}$ & 0 & $\mathrm{O}$ & $\mathrm{O}$ \\
\hline $\begin{array}{l}\text { 63. Had an } \\
\text { upset }\end{array}$ & 0 & 0 & 0 & 0 & 0 \\
\hline
\end{tabular}




\begin{tabular}{|c|c|c|c|c|c|}
\hline stomach & & & & & \\
\hline $\begin{array}{l}64 \text {. Felt } \\
\text { inferior to } \\
\text { others }\end{array}$ & $\bigcirc$ & 0 & 0 & 0 & 0 \\
\hline $\begin{array}{l}65 . \text { Had a } \\
\text { lump in my } \\
\text { throat }\end{array}$ & 0 & $\bigcirc$ & 0 & $\mathrm{O}$ & 0 \\
\hline $\begin{array}{l}\text { 66. Felt really } \\
\text { slowed down }\end{array}$ & 0 & 0 & 0 & 0 & 0 \\
\hline $\begin{array}{l}67 . \text { Had a } \\
\text { very dry } \\
\text { mouth }\end{array}$ & $\bigcirc$ & 0 & 0 & $\mathrm{O}$ & 0 \\
\hline $\begin{array}{l}68 . \text { Felt } \\
\text { confident } \\
\text { about myself }\end{array}$ & 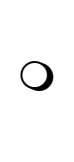 & $\bigcirc$ & 0 & $\mathrm{O}$ & 0 \\
\hline $\begin{array}{l}\text { 69. Muscles } \\
\text { twitched or } \\
\text { trembled }\end{array}$ & 0 & $\bigcirc$ & 0 & $\mathrm{O}$ & 0 \\
\hline $\begin{array}{l}70 . \text { Had } \\
\text { trouble } \\
\text { making } \\
\text { decisions }\end{array}$ & $\mathrm{O}$ & $\mathrm{O}$ & 0 & $\mathrm{O}$ & 0 \\
\hline $\begin{array}{l}71 . \text { Felt like I } \\
\text { was going } \\
\text { crazy }\end{array}$ & $\bigcirc$ & $\bigcirc$ & $O$ & $\mathrm{O}$ & $\mathrm{O}$ \\
\hline $\begin{array}{l}\text { 72. Felt like I } \\
\text { had a lot of } \\
\text { energy }\end{array}$ & $\mathrm{O}$ & $\bigcirc$ & 0 & $\mathrm{O}$ & 0 \\
\hline $\begin{array}{l}\text { 73. Was } \\
\text { afraid I was } \\
\text { going to die }\end{array}$ & $\bigcirc$ & 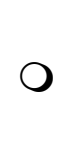 & 0 & $\mathrm{O}$ & 0 \\
\hline $\begin{array}{l}\text { 74. Was } \\
\text { disappointed } \\
\text { in myself }\end{array}$ & $\bigcirc$ & $\mathrm{O}$ & 0 & 0 & 0 \\
\hline $\begin{array}{l}75 . \text { Heart was } \\
\text { racing or } \\
\text { pounding }\end{array}$ & 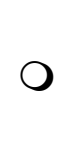 & $\bigcirc$ & 0 & $\mathrm{O}$ & 0 \\
\hline $\begin{array}{l}\text { 76. Had } \\
\text { trouble } \\
\text { concentrating }\end{array}$ & $\bigcirc$ & $\mathrm{O}$ & 0 & $\mathrm{O}$ & $\mathrm{O}$ \\
\hline $\begin{array}{l}\text { 77. Felt tense } \\
\text { or "high } \\
\text { strung" }\end{array}$ & $\bigcirc$ & 0 & $\mathrm{O}$ & $\mathrm{O}$ & $\mathrm{O}$ \\
\hline $\begin{array}{l}78 . \text { Felt } \\
\text { hopeful about }\end{array}$ & $\bigcirc$ & 0 & 0 & $\mathrm{O}$ & 0 \\
\hline
\end{tabular}




\begin{tabular}{|c|c|c|c|c|c|}
\hline the future & & & & & \\
\hline $\begin{array}{l}\text { 79. Was } \\
\text { trembling or } \\
\text { shaking }\end{array}$ & $\mathrm{O}$ & 0 & 0 & 0 & 0 \\
\hline $\begin{array}{l}80 . \text { Had } \\
\text { trouble } \\
\text { paying } \\
\text { attention }\end{array}$ & $\mathrm{O}$ & 0 & $\mathrm{O}$ & $\mathrm{O}$ & 0 \\
\hline $\begin{array}{l}\text { 81. Muscles } \\
\text { were tense or } \\
\text { sore }\end{array}$ & $\mathrm{O}$ & $\bigcirc$ & 0 & 0 & 0 \\
\hline $\begin{array}{l}\text { 82. Felt } \\
\text { keyed up, "on } \\
\text { edge" }\end{array}$ & $\mathrm{O}$ & $\bigcirc$ & $\mathrm{O}$ & $\mathrm{O}$ & 0 \\
\hline $\begin{array}{l}\text { 83. Had } \\
\text { trouble } \\
\text { staying asleep }\end{array}$ & 0 & 0 & 0 & 0 & 0 \\
\hline $\begin{array}{l}\text { 84. Worried a } \\
\text { lot about } \\
\text { things }\end{array}$ & 0 & $\mathrm{O}$ & 0 & 0 & 0 \\
\hline $\begin{array}{l}85 . \text { Had to } \\
\text { urinate } \\
\text { frequently }\end{array}$ & $\mathrm{O}$ & $\mathrm{O}$ & 0 & $\mathrm{O}$ & 0 \\
\hline $\begin{array}{l}\text { 86. Felt really } \\
\text { good about } \\
\text { myself }\end{array}$ & 0 & 0 & 0 & 0 & 0 \\
\hline $\begin{array}{l}\text { 87. Had } \\
\text { trouble } \\
\text { swallowing }\end{array}$ & 0 & 0 & 0 & 0 & 0 \\
\hline $\begin{array}{l}88 . \text { Hands } \\
\text { were cold or } \\
\text { sweaty }\end{array}$ & 0 & 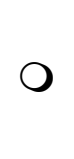 & 0 & $\mathrm{O}$ & 0 \\
\hline $\begin{array}{l}89 . \text { Thought } \\
\text { about death } \\
\text { or suicide }\end{array}$ & $\mathrm{O}$ & 0 & $\mathrm{O}$ & 0 & 0 \\
\hline $\begin{array}{l}90 . \text { Got tired } \\
\text { or fatigued } \\
\text { easily }\end{array}$ & 0 & 0 & 0 & 0 & 0 \\
\hline
\end{tabular}


Orygen Substance Use Index

\begin{tabular}{|c|c|c|c|c|c|c|c|c|}
\hline \multirow{2}{*}{$\begin{array}{l}\text { Have you ever tried } \\
\text { (drug)? } \\
\text { If Yes continue } \\
\text { If No go onto the } \\
\text { next drug }\end{array}$} & \multirow{2}{*}{$\begin{array}{l}\text { How old } \\
\text { were } \\
\text { you } \\
\text { when } \\
\text { you first } \\
\text { used } \\
\text { (drug)? }\end{array}$} & \multirow[b]{2}{*}{$\begin{array}{l}\text { How old } \\
\text { were you } \\
\text { when you } \\
\text { first used the } \\
\text { (drug) on a } \\
\text { regular } \\
\text { basis? } \\
\text { * Note that } \\
\text { regular use is } \\
\text { considered to } \\
\text { be weekly use }\end{array}$} & \multicolumn{3}{|l|}{ Over your lifetime... } & \multicolumn{3}{|c|}{ Over the past 3 months... } \\
\hline & & & $\begin{array}{l}\text { For how many years have } \\
\text { you used on a regular basis? } \\
\text { 5. > } 20 \text { years } \\
\text { 4. } 10-20 \text { years } \\
\text { 3. } 5-10 \text { years } \\
\text { 2. } 1-5 \text { years } \\
\text { 1. Less than } 1 \text { year } \\
\text { 0. Never } \\
\text { *Note: this total can be made up } \\
\text { of many smaller periods of use } \\
\text { broken up by periods of } \\
\text { abstinence } \\
\text { *Note what regular basis is } \\
\text { interpreted as by the subject }\end{array}$ & $\begin{array}{l}\begin{array}{l}\text { What's the most } \\
\text { frequently you have } \\
\text { ever used? }\end{array} \\
\text { 5. Daily or almost } \\
\text { daily } \\
\text { 4. Weekly } \\
\text { 3. 2-3 times a month } \\
\text { 2. Monthly } \\
\text { 1. Less than monthly }\end{array}$ & $\begin{array}{l}\text { What was the most you } \\
\text { used in a day for a } \\
\text { regular period? } \\
\text { Estimate units } \\
\text { How long did you use at } \\
\text { this rate? } \\
\text { 5. }>5 \mathrm{yrs} \\
4.2-5 \mathrm{yrs} \\
\text { 3. } 6-24 \mathrm{mths} \\
2.1-6 \mathrm{mths} \\
\text { 1. }<1 \mathrm{mth}\end{array}$ & $\begin{array}{l}\text { How often do you } \\
\text { usually use the } \\
\text { drug? } \\
\text { 5. Daily or almost } \\
\text { daily } \\
\text { 4. Weekly } \\
\text { 3. } 2-3 \text { times a } \\
\text { month } \\
\text { 2. Monthly } \\
\text { 1. Less than } \\
\text { monthly } \\
\text { 0. None in past } 3 \\
\text { months }\end{array}$ & $\begin{array}{l}\text { How much a day do } \\
\text { you use usually? } \\
\text { Estimate units }\end{array}$ & $\begin{array}{l}\text { How long has it } \\
\text { been since you } \\
\text { last used? } \\
\text { Write date } \\
\text { OR } \\
\text { No. of days, } \\
\text { weeks, months, } \\
\text { years ago }\end{array}$ \\
\hline $\begin{array}{l}\text { Tobacco } \\
\square \text { Yes } \square \text { No }\end{array}$ & & & & & __ number & & & \\
\hline $\begin{array}{l}\text { Alcohol } \\
\square \text { Yes } \square \text { No }\end{array}$ & & & & & Standard units & & Standard units & \\
\hline $\begin{array}{l}\text { Cannabis } \\
\text { (eg. marijuana, hash) } \\
\square \text { Yes } \square \text { No }\end{array}$ & & & & & & & $\begin{array}{r}\text { g/oz } \\
\text { Joints/Cones/Bongs } \\
\text { Leaf/Hydro/Buds/Hash }\end{array}$ & \\
\hline $\begin{array}{l}\text { Amphetamines } \\
\text { (eg. speed, ice) } \\
\square \text { Yes } \square \text { No } \\
\text { IV use ever? } \\
\square \text { Yes } \square \text { No }\end{array}$ & & & & & $g$ & & $\begin{array}{l}\text { _ }{ }^{-} \text {Hits } \\
\text { Oral Snorts IV }\end{array}$ & \\
\hline $\begin{array}{l}\text { Cocaine (eg. coke) } \\
\square \text { Yes } \square \text { No } \\
\text { IV use ever? } \\
\square \text { Yes } \square \text { No }\end{array}$ & & & & & & & $\begin{array}{l}{ }_{\text {Oral }} \quad \text { Snorts } \\
\text { IV }\end{array}$ & \\
\hline $\begin{array}{l}\text { Hallucinogens } \\
\text { (eg. LSD, mushrooms) }\end{array}$ & & & & & Tabs/Pills & & Tabs/Pills & \\
\hline
\end{tabular}




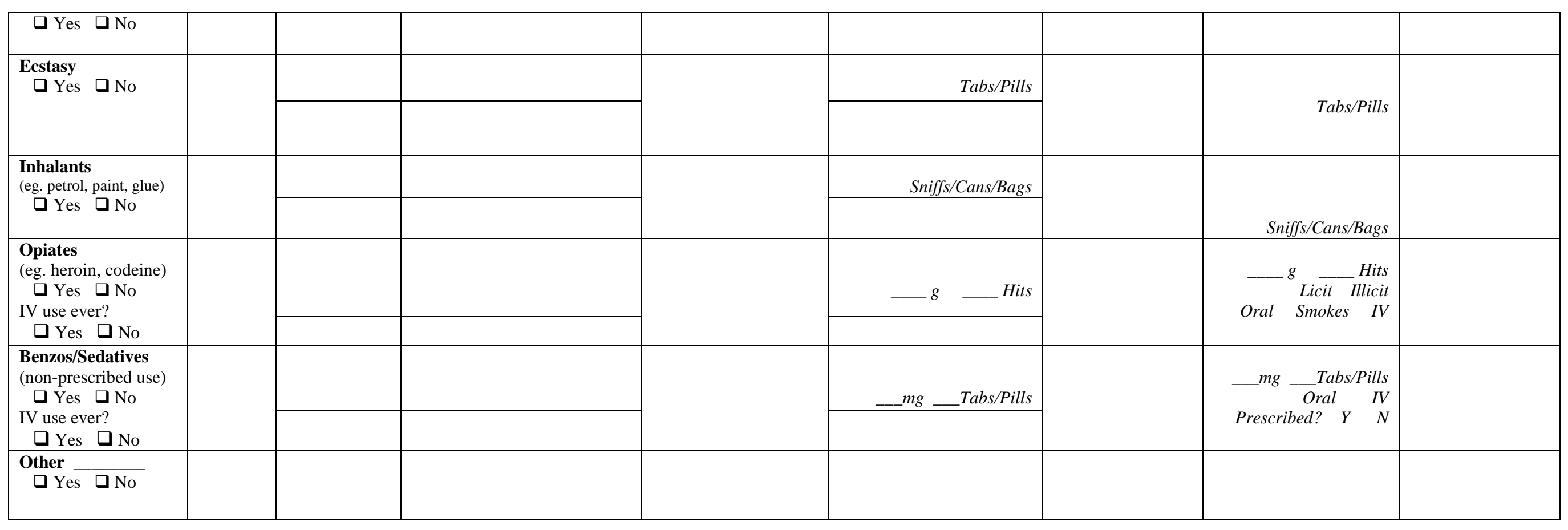




\section{Penn State Worry Questionnaire}

Rate each of the following statements on a scale of 1 ("not at all typical of me") to 5 ("very typical of me"). Please do not leave any items blank.

\begin{tabular}{|c|c|c|c|c|c|}
\hline & $\begin{array}{l}\text { 1. (not at all } \\
\text { typical of me) }\end{array}$ & 2. & 3. & 4. & $\begin{array}{l}\text { 5. (very typical } \\
\text { of me) }\end{array}$ \\
\hline $\begin{array}{l}\text { 1. If I do not } \\
\text { have enough } \\
\text { time to do } \\
\text { everything, I } \\
\text { do not worry } \\
\text { about it. }\end{array}$ & 0 & 0 & 0 & 0 & 0 \\
\hline $\begin{array}{l}\text { 2. My } \\
\text { worries } \\
\text { overwhelm } \\
\text { me. }\end{array}$ & 0 & 0 & 0 & 0 & 0 \\
\hline $\begin{array}{l}\text { 3. I do not } \\
\text { tend to worry } \\
\text { about things. }\end{array}$ & 0 & 0 & 0 & 0 & 0 \\
\hline $\begin{array}{l}\text { 4. Many } \\
\text { situations } \\
\text { make me } \\
\text { worry. }\end{array}$ & 0 & 0 & 0 & 0 & 0 \\
\hline $\begin{array}{l}\text { 5. I know I } \\
\text { should not } \\
\text { worry about } \\
\text { things, but I } \\
\text { just cannot } \\
\text { help it. }\end{array}$ & 0 & 0 & 0 & 0 & 0 \\
\hline $\begin{array}{l}\text { 6. When I am } \\
\text { under } \\
\text { pressure I } \\
\text { worry a lot. }\end{array}$ & 0 & 0 & 0 & 0 & 0 \\
\hline $\begin{array}{l}\text { 7. I am } \\
\text { always } \\
\text { worrying } \\
\text { about } \\
\text { something. }\end{array}$ & 0 & 0 & 0 & 0 & 0 \\
\hline $\begin{array}{l}\text { 8. I find it } \\
\text { easy to } \\
\text { dismiss } \\
\text { worrisome } \\
\text { thoughts }\end{array}$ & 0 & 0 & 0 & 0 & 0 \\
\hline $\begin{array}{l}\text { 9. As soon as } \\
\text { I finish one }\end{array}$ & 0 & 0 & 0 & 0 & 0 \\
\hline
\end{tabular}




\begin{tabular}{|c|c|c|c|c|c|}
\hline $\begin{array}{l}\text { task, I start to } \\
\text { worry about } \\
\text { everything } \\
\text { else I have to } \\
\text { do. }\end{array}$ & & & & & \\
\hline $\begin{array}{l}\text { 10. I never } \\
\text { worry about } \\
\text { anything. }\end{array}$ & 0 & 0 & 0 & 0 & 0 \\
\hline $\begin{array}{l}11 . \text { When } \\
\text { there is } \\
\text { nothing more } \\
\text { I can do } \\
\text { about a } \\
\text { concern, I do } \\
\text { not worry } \\
\text { about it any } \\
\text { more. }\end{array}$ & 0 & 0 & 0 & 0 & 0 \\
\hline $\begin{array}{l}\text { 12. I have } \\
\text { been a } \\
\text { worrier all } \\
\text { my life. }\end{array}$ & 0 & 0 & 0 & 0 & 0 \\
\hline $\begin{array}{l}\text { 13. I notice } \\
\text { that I have } \\
\text { been } \\
\text { worrying } \\
\text { about things. }\end{array}$ & 0 & 0 & 0 & 0 & 0 \\
\hline $\begin{array}{l}\text { 14. Once I } \\
\text { start } \\
\text { worrying, I } \\
\text { cannot stop. }\end{array}$ & 0 & 0 & 0 & 0 & 0 \\
\hline $\begin{array}{l}15 . \text { I worry } \\
\text { all the time. }\end{array}$ & 0 & 0 & 0 & 0 & 0 \\
\hline $\begin{array}{l}16 . \text { I worry } \\
\text { about } \\
\text { projects until } \\
\text { they are all } \\
\text { done. }\end{array}$ & 0 & 0 & 0 & 0 & 0 \\
\hline
\end{tabular}


This is a list of things different people might say about themselves. We are interested in how you would describe yourself. There are no right or wrong answers. We'd like you to take your time and read each statement carefully, selecting the response that best describes you.

\begin{tabular}{|c|c|c|c|c|}
\hline & $\begin{array}{c}\text { Very False or Often } \\
\text { False }\end{array}$ & $\begin{array}{l}\text { Sometimes or } \\
\text { Somewhat False }\end{array}$ & $\begin{array}{l}\text { Sometimes or } \\
\text { Somewhat True }\end{array}$ & $\begin{array}{c}\text { Very True or Often } \\
\text { True }\end{array}$ \\
\hline $\begin{array}{l}\text { 1. People would } \\
\text { describe me as } \\
\text { reckless. }\end{array}$ & 0 & 0 & 0 & 0 \\
\hline $\begin{array}{l}\text { 2. I feel like I act } \\
\text { totally on } \\
\text { impulse. }\end{array}$ & 0 & $\bigcirc$ & 0 & 0 \\
\hline $\begin{array}{l}\text { 3. Even though I } \\
\text { know better, I } \\
\text { can't stop } \\
\text { making rash } \\
\text { decisions. }\end{array}$ & 0 & 0 & $\bigcirc$ & 0 \\
\hline $\begin{array}{l}\text { 4. I often feel } \\
\text { like nothing I do } \\
\text { really matters. }\end{array}$ & 0 & 0 & 0 & 0 \\
\hline $\begin{array}{l}\text { 5. Others see me } \\
\text { as irresponsible. }\end{array}$ & 0 & 0 & 0 & 0 \\
\hline $\begin{array}{l}6 . \text { I'm not good } \\
\text { at planning } \\
\text { ahead. }\end{array}$ & 0 & 0 & 0 & 0 \\
\hline $\begin{array}{l}\text { 7. My thoughts } \\
\text { often don't make } \\
\text { sense to others. }\end{array}$ & 0 & 0 & 0 & 0 \\
\hline $\begin{array}{l}\text { 8. I worry about } \\
\text { almost } \\
\text { everything. }\end{array}$ & 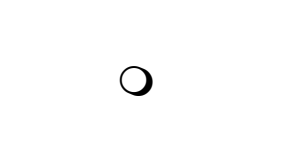 & 0 & 0 & 0 \\
\hline $\begin{array}{l}\text { 9. I get } \\
\text { emotional easily, } \\
\text { often for very } \\
\text { little reason. }\end{array}$ & 0 & 0 & 0 & 0 \\
\hline $\begin{array}{l}\text { 10. I fear being } \\
\text { alone in life } \\
\text { more than } \\
\text { anything else. }\end{array}$ & 0 & 0 & 0 & 0 \\
\hline $\begin{array}{l}11 . \text { I get stuck } \\
\text { on one way of } \\
\text { doing things, } \\
\text { even when it's } \\
\text { clear it won't }\end{array}$ & 0 & 0 & 0 & 0 \\
\hline
\end{tabular}




$$
\text { work. }
$$

12. I have seen things that weren't really there.

13. I steer clear of romantic relationships.

14. I'm not interested in making friends.

15. I get irritated easily by all sorts of things.

16. I don't like to get too close to people.

17. It's no big deal if I hurt other people's feelings.

18 . I rarely get enthusiastic about anything. 19. I crave attention.

20. I often have to deal with people who are less important than me.

21. I often have thoughts that make sense to me but that other people say are strange.

22. I use people to get what I want.

23. I often "zone out" and then suddenly come to and realize that a lot of time has passed. 


\begin{tabular}{|l|c|c|c|}
\hline $\begin{array}{l}\text { 24. Things } \\
\text { around me often } \\
\text { feel unreal, or } \\
\text { more real than }\end{array}$ & 0 & 0 & 0 \\
$\begin{array}{l}\text { usual. } \\
\begin{array}{l}\text { 25. It is easy for } \\
\text { me to take } \\
\text { advantage of } \\
\text { others. }\end{array}\end{array} \quad$ O & 0 & 0 & 0 \\
\hline
\end{tabular}


Please answer the following questions

\begin{tabular}{|c|c|c|}
\hline & Yes & No \\
\hline $\begin{array}{l}\text { 1. Do you often refrain from } \\
\text { doing something because you } \\
\text { are afraid of it being illegal? }\end{array}$ & O & $\mathrm{O}$ \\
\hline $\begin{array}{l}\text { 2. Does the good prospect of } \\
\text { obtaining money motivate } \\
\text { you strongly to do some } \\
\text { things? }\end{array}$ & O & $\mathrm{O}$ \\
\hline $\begin{array}{l}\text { 3. Do you prefer not to ask } \\
\text { for something when you are } \\
\text { not sure you will obtain it? }\end{array}$ & $\mathrm{O}$ & $\mathrm{O}$ \\
\hline $\begin{array}{l}\text { 4. Are you frequently } \\
\text { encouraged to act by the } \\
\text { possibility of being valued in } \\
\text { your work, in your studies, } \\
\text { with your friends or with } \\
\text { your family? }\end{array}$ & $\mathrm{O}$ & $\mathrm{O}$ \\
\hline $\begin{array}{l}\text { 5. Are you often afraid of } \\
\text { new or unexpected } \\
\text { situations? }\end{array}$ & $\mathrm{O}$ & $\mathrm{O}$ \\
\hline $\begin{array}{l}\text { 6. Do you often meet people } \\
\text { that you find physically } \\
\text { attractive? }\end{array}$ & $\mathrm{O}$ & $\mathrm{O}$ \\
\hline $\begin{array}{l}\text { 7. Is it difficult for you to } \\
\text { telephone someone you do } \\
\text { not know? }\end{array}$ & O & $\mathrm{O}$ \\
\hline $\begin{array}{l}\text { 8. Do you like to take some } \\
\text { drugs because of the pleasure } \\
\text { you get from them? }\end{array}$ & O & $\mathrm{O}$ \\
\hline $\begin{array}{l}\text { 9. Do you often renounce } \\
\text { your rights when you know } \\
\text { you can avoid a quarrel with } \\
\text { a person or an organisation? }\end{array}$ & $\mathrm{O}$ & O \\
\hline $\begin{array}{l}\text { 10. Do you often do things to } \\
\text { be praised? }\end{array}$ & O & $\mathrm{O}$ \\
\hline $\begin{array}{l}\text { 11. As a child, were you } \\
\text { troubled by punishments at } \\
\text { home or in school? }\end{array}$ & O & O \\
\hline $\begin{array}{l}\text { 12. Do you like being the } \\
\text { centre of attention at a party } \\
\text { or a social meeting? }\end{array}$ & O & $\mathrm{O}$ \\
\hline 13. In tasks that you are not & O & O \\
\hline
\end{tabular}


prepared for, do you attach great importance to the possibility of failure?

14. Do you spend a lot of your time on obtaining a good image?

15. Are you easily discouraged in difficult situations?

16. Do you need people to show their affection for you all the time?

17. Are you a shy person?

18. When you are in a group, do you try to make your opinions the most intelligent or the funniest?

19. Whenever possible, do you avoid demonstrating your skills for fear of being embarrassed?

20. Do you often take the opportunity to pick up people you find attractive?

21 . When you are with a group, do you have difficulties selecting a good topic to talk about?

22. As a child, did you do a lot of things to get people's approval?

23. Is it often difficult for you to fall asleep when you think about things you have done or must do?

24 . Does the possibility of social advancement move you to action, even if this involves not playing fair?

25 . Do you think a lot before complaining in a restaurant if your meal is not well prepared?

26. Do you generally give preference to those activities that imply an immediate 
gain?

27. Would you be bothered if you had to return to a store when you noticed you were given the wrong change?

28. Do you often have trouble resisting the temptation of doing forbidden things?

29. Whenever you can, do you avoid going to unknown places?

30. Do you like to compete and do everything you can to win?

31. Are you often worried by things that you said or did?

32. Is it easy for you to associate tastes and smells to very pleasant events?

33. Would it be difficult for you to ask your boss for a raise (salary increase)?

34. Are there a large number of objects or sensations that remind you of pleasant events?

35. Do you generally try to avoid speaking in public?

36. When you start to play with a slot machine, is it often difficult for you to stop?

37. Do you, on a regular basis, think that you could do more things if it was not for your insecurity or fear?

38. Do you sometimes do things for quick gains?

39. Comparing yourself to people you know, are you afraid of many things?

40. Does your attention easily stray from your work in the presence of an attractive stranger? 
41. Do you often find yourself worrying about things to the extent that performance in intellectual abilities is impaired?

42. Are you interested in money to the point of being able to do risky jobs?

43. Do you often refrain from doing something you like in order not to be rejected or disapproved of by others?

44. Do you like to put competitive ingredients in all of your activities?

45. Generally, do you pay more attention to threats than to pleasant events?

46. Would you like to be a socially powerful person?

47. Do you often refrain from doing something because of your fear of being embarrassed?

48. Do you like displaying your physical abilities even though this may involve danger? 


\section{Appendix B}

\section{Non-Published Measures Used in the Research Project}

1. Demographic Screening Information Questionnaire

2. Demographic Screening Information Questionnaire for Web-Based Stop Signal Task

3. Magnetic Resonance Imaging Screening Questionnaire

4. Structured Face-to-Face Interview 
Demographic Screening Information Questionnaire

Please read carefully and complete the following demographic information

1 What is your sex?

o Male

○ Female

o Other

2 Please estimate your current:

Height (in m):

Weight (in $\mathrm{kg}$ ):

3 What is your date of birth (DD/MM/YYYY)?

4 What is your country of birth?

5 What is your ethnic self-identification (e.g., Australian, Vietnamese, etc.)?

6 Is English your first language?

$\rightarrow$ Yes

$\rightarrow \mathrm{No}$

7 If English is not your first language, please specify your first language below

8 Are you right or left handed?

○ Right

$\circ$ Left

○ Ambidextrous

9 Marital status

○ Married/De Facto

O Separated

O Divorced

O Widowed

O Never married

10 Number of children 
11 Current accommodation arrangement

O None

○ Crisis Accommodation

○ Hotel/Supported or assisted housing

o Rented room

O Rented flat/house

O Own flat/house

o House/Flat with family of origin

O Institution

O Other

12 Person(s) currently living with

○ Alone

○ Spouse/De Facto

O Child(ren)

○ Spouse/De Facto + Child(ren)

○ Parent(s)

O Sibling(s)

$\bigcirc$ Other relative(s)

$\circ$ Friend(s)

$\bigcirc$ Institution/boarding

O Other

13 Years of education (primary, secondary and tertiary only) (To calculate: Highest year completed at school +1 for prep (If you are a student, do not include current year)E.g., completed Yr $9=10$ years of education; completed VCE + Bachelor degree $=16$ years of education)

14 Please list any other education (e.g., TAFE, adult education courses, etc.)

15 Current main work status

○ Unemployed

○ Full time

- Part time/Casual

○ Student

○ Home duties/housework

o Volunteer work

o Other 
16 Current main financial support
O Parents
O Other family/friends
○ Full time work
○ Part time work
○ Government benefits
○ Other

17 Current occupation (incl. student)

18 Personal income for past fortnight after tax (including government benefits)

19 Total household income for past fortnight after tax (if living alone, same as previous)

20 If you are a student, what is the name of your course and your current year level?

21 Health status: Please list any past or present medical conditions you have been affected by, including problems with your heart or lungs, any neurological conditions (e.g. epilepsy), infectious diseases (e.g. HIV, HEP C) or psychiatric disorders (e.g., depression, schizophrenia).

22 Please provide a list of any medical or psychiatric conditions that you are currently being treated for with medication. For each one, include details of what you are being treated for, the name of the medication, the current dose/frequency (e.g. $8 \mathrm{mg} /$ day) and the approximate date you started taking it.

\begin{tabular}{|l|l|l|l|l|}
\hline Condition(s) & Condition 1 & Condition 2 & Condition 3 & Condition 4 \\
being treated for & & & \\
Name of & & & \\
medication(s) & & & \\
Current & & & \\
dose/frequency & & & \\
Approximate & & & \\
date started & & & \\
taking & & & \\
\hline
\end{tabular}

23 Do you have a family history of psychiatric illness (e.g., do one or both parents / siblings / grandparents / aunties / uncles have depression, anxiety, schizophrenia, etc.)?

24 If you have a family history of psychiatric illness, please provide brief details. 
25 Have you ever been admitted as an inpatient for drug/alcohol rehabilitation, or received outpatient treatment?
○ Yes
○ No

26 How many times have you been admitted as an inpatient for drug/alcohol rehabilitation or received outpatient treatment?

27 Have you ever overdosed (i.e. lost consciousness as a result of taking drugs)?

$$
\begin{array}{ll}
\text { O } & \text { Yes } \\
\text { O No }
\end{array}
$$

28 How many times have you overdosed?

29 Have you ever attempted suicide?

○ Yes

○ No

30 How many times have you attempted suicide?

31 Have you ever been involved in an accident or incident that resulted in you receiving a head injury?
O Yes
○ No

32 How many times have you been involved in an accident or incident that resulted in you receiving a head injury? 
33 Please provide brief details about each head injury, including: the type of accident (e.g. car, fall, fight), length of loss of consciousness (LOC), whether you were hospitalized (and for how long), if you had a brain scan, as well as any functional/behavioral impairments or ongoing symptoms

\begin{tabular}{|l|l|l|l|l|}
\hline Type of accident & Head injury 1 & Head injury 2 & Head injury 3 & Head injury 4 \\
Length of LOC & & & & \\
Hospitalised & & & \\
(y/n) and length & & & \\
of hospitalisation & & & \\
Brain scan (y/n) & & & \\
and results & & & & \\
Impairments & & & \\
Symptoms & & & \\
\hline
\end{tabular}

34 Do you have any problems with your sight, hearing or smell?
○ Yes
○ No

35 Please provide details of issues with sight, hearing or smell

36 Do you have any visual problems? (E.g., glasses, contacts, colour blindness, astigmatism). If yes, please describe.

37 Have you ever had surgery?

○ Yes

○ No

38 Please enter brief details and approximate date of surgery/surgeries

39 Women who may be pregnant are advised to avoid MRI scanning unless essential. Female participants of child-bearing capacity, please confirm you are using a method of birth control.

O Not applicable to me

○ Yes, using birth control

No, not using birth control, there is a possibility that I may be pregnant

40 Did you have any difficulties learning how to read?
○ Yes
○ No 
41 Did you have any difficulties learning how to speak?

○ Yes

○ No

42 Have you ever had a brain scan?

$\circ$ Yes

○ No

43 If you have had a brain scan, what was the reason for the scan?

44 Do you smoke? If yes, how many cigarettes do you smoke per day/week? If no, how many cigarettes have you smoked in your lifetime?

Yes

No 
Demographic Screening Information Questionnaire for Web-Based Stop Signal Task

Please read carefully and complete the following demographic information

1 What is your sex?

o Male

○ Female

O Other

2 What is your date of birth (DD/MM/YYYY)?

3 What is your country of birth?

4 What is your ethnic self-identification (e.g., Australian, Vietnamese, etc.)?

5 Is English your first language?

$\rightarrow$ Yes

$\rightarrow \mathrm{No}$

6 If English is not your first language, please specify your first language below

7 Are you right or left handed?

○ Right

○ Left

O Ambidextrous (can use both hands)

8 Years of education (primary, secondary and tertiary only) (To calculate: Highest year completed at school +1 for prep (If you are a student, do not include current year)E.g., completed Yr 9 = 10 years of education; completed VCE + Bachelor degree $=16$ years of education)

9 Please list any other education (e.g., TAFE, adult education courses, etc.)

10 Current main work status

○ Unemployed

O Full time

- Part time/Casual

O Student

○ Home duties/housework

o Volunteer work

O Other 
11 Current occupation (incl. student)

12 If you are a student, what is the name of your course and your current year level?

13 Health status: Please list any past or present medical conditions you have been affected by, including problems with your heart or lungs, any neurological conditions (e.g. epilepsy), infectious diseases (e.g. HIV, HEP C) or psychiatric disorders (e.g., depression, schizophrenia).

14 Please provide a list of any medical or psychiatric conditions that you are currently being treated for with medication. For each one, include details of what you are being treated for, the name of the medication, the current dose/frequency (e.g. $8 \mathrm{mg} /$ day) and the approximate date you started taking it.

\begin{tabular}{|l|l|l|l|l|}
\hline Condition(s) & Condition 1 & Condition 2 & Condition 3 & Condition 4 \\
being treated for & & & & \\
Name of & & & \\
medication(s) & & & \\
Current & & & \\
dose/frequency & & & \\
Approximate & & & \\
date started & & & & \\
taking & & & \\
\hline
\end{tabular}

15 Have you ever been admitted as an inpatient for drug/alcohol rehabilitation, or received outpatient treatment?

$$
\begin{array}{ll}
\circ & \text { Yes } \\
\circ & \text { No }
\end{array}
$$

16 How many times have you been admitted as an inpatient for drug/alcohol rehabilitation or received outpatient treatment?

17 Have you ever been involved in an accident or incident that resulted in you receiving a head injury?

$$
\begin{array}{ll}
\circ & \text { Yes } \\
\circ & \text { No }
\end{array}
$$

18 How many times have you been involved in an accident or incident that resulted in you receiving a head injury? 
19 Please provide brief details about each head injury, including: the type of accident (e.g. car, fall, fight), length of loss of consciousness (LOC), whether you were hospitalized (and for how long), if you had a brain scan, as well as any functional/behavioral impairments or ongoing symptoms

\begin{tabular}{|l|l|l|l|l|}
\hline & Head injury 1 & Head injury 2 & Head injury 3 & Head injury 4 \\
\hline Lype of accident & & & & \\
Length of LOC & & & \\
(y/n) and length & & & \\
of hospitalisation & & & \\
Brain scan (y/n) & & & \\
and results & & & & \\
Impairments & & & \\
Symptoms & & & \\
\hline
\end{tabular}

20 Do you have any problems with your sight, hearing or smell?

○ Yes

○ No

21 Please provide details of issues with sight, hearing or smell

22 Do you have any visual problems? (E.g., glasses, contacts, colour blindness, astigmatism). If yes, please describe.

23 Do you smoke? If yes, how many cigarettes do you smoke per week?If no, how many cigarettes have you smoked in your lifetime?

Yes

No 
Many of our studies involve Magnetic Resonance Imaging (MRI) brain scans. This questionnaire helps us determine whether you are eligible for a scan. If you are unsure about any of the questions or procedures on the following page, please ask the experimenter to explain. Note, completing this questionnaire does not mean that you are obliged to participate in an MRI scan. There are some items that may interfere with the MRI and some that may be potentially hazardous. To help us determine your suitability for an MRI scan and to ensure your safety, please complete the following checklist carefully.

Not all people can have an MRI scan because the strong magnetic field may be hazardous to them. People with:

- Permanent pacemakers;

- Prosthetic heart valves;

- Implanted cardiac defibrillators;

- Certain types of vascular clips;

CANNOT have an MRI scan.

Do you have any of the following?

1 Cardiac pacemaker

O Yes

O No

2 Artificial cardiac valve

O Yes

O No

3 Vascular surgery clips

O Yes

$\mathrm{O}$ No

4 Inner ear implants

$\mathrm{O}$ Yes

O No

5 Embolization coils

O Yes

O No

6 Tattooed eyeliner or large, dark ink tattoos

O Yes

O No 
7 Intraventricular shunt

O Yes

$\mathrm{O}$ No

8 Neurostimulator

$\mathrm{O}$ Yes

O No

9 Renal shunt

O Yes

O No

10 Vena Cava filter

O Yes

$\mathrm{O}$ No

11 Orthopaedic devices

O Yes

O No

12 Dentures

O Yes

O No

13 Ocular prosthesis

O Yes

O No

14 Intrauterine device (IUD)

O Yes

O No

$15 \mathrm{Shrapnel} / \mathrm{bullet}$ fragments or wounds

O Yes

O No

16 Any other implanted devices (including piercings that cannot be removed)? If yes, please specify

17 Have you ever had any surgical procedures? If yes, what type, when and where? 
18 Have you ever had an injury to your eye involving metal fragments?

O Yes

O No

19 Are you claustrophobic (fear of confined or small spaces)?

O Yes

O No

20 Are you pregnant or suspect you may be pregnant?

$\mathrm{O}$ Yes

O No 


\section{Structured Face-to-Face Interview}

Tell the participant that they have already answered these but we want to check them because sometimes questions are missed. Tell them that they will be asked questions about their medical, psychiatric and drug histories and answering is completely voluntary. They do not have to answer if they do not want to. Also they should feel free to let you know if they feel uncomfortable answering any of the questions. Tell them that their answers will be completely confidential unless we feel that they are a danger to themselves or other people and then we may need to discuss their responses with other health professionals.

\section{Demographics}

1. Between the ages of 18 and 45 ? $\mathrm{Y} \quad \mathrm{N}$

2. Right handed? $\mathrm{Y}$ N

3. English as preferred language? $\mathrm{Y} \quad \mathrm{N}$

4. WTAR estimate IQ > 80? Y N

\section{Medical}

5. Currently on prescription medication or receiving treatment for any medical or psychiatric condition? [check for current use of respiratory, cardiovascular, anticonvulsant or psychoactive medications, and steroid use]. If yes, get details of 1) what being treated for 2) name of the medication 3) current dose/frequency (e.g., $8 \mathrm{mg} /$ day) and 4) approximate date they started taking it.

\section{Y N}

6. Any significant medical conditions past or present that may affect cognitive functioning [probe for thyroid impairment here]?

$$
\text { Y N }
$$

7. Have you ever been diagnosed with any mental health disorder (give them an example such as depression/anxiety), or has your mood changed significantly in the past 2-4 weeks?

$$
\text { Y N }
$$

8. History of psychosis?

$$
\text { Y N }
$$

9. Have you ever attempted suicide? If yes, how many times?

\section{Y N}

10. Ever had a serious head injury that resulted in trauma to the brain, or required surgery, prolonged hospitalization, and rehabilitation, and may have involved prolonged 
unconsciousness or concussion [If person can't recall head injuries prompt by asking whether they have ever had concussion or been unconscious -see below]? If yes to head injury, how many times. If yes to head injury, give details, i.e., type of accident, length of LOC, whether hospitalized (and for how long), did they have a brain scan, are there any functional/behavioural impairments or ongoing symptoms?

\section{Y N}

11. History of loss of consciousness (LOC) for longer than 30 minutes or LOC with any neurological sequelae?

$$
\text { Y N }
$$

12. Ever had any of the following: for confidentiality, not necessary to elaborate on the particular diagnosis that relates to person, just required to state 'yes' or 'no' after list completed - (a) fits, convulsions, epileptic seizures, (b) stroke, brain tumour, meningitis, encephalitis, multiple sclerosis, cerebral vascular disease, migraine treatment, movement disorder (c) positive for HIV or Hep C, (d) ECT? [probing for history of neurological disease here] e) coagulopathies, superficial or deep vein thrombosis, peripheral vascular diseases, problems with heart or lungs

$$
\text { Y N }
$$

13. Have you ever had surgery? If so, give general details (what and where) and approximate dates

$$
\text { Y N }
$$

14. Colour vision and acuity within normal (or corrected-to-normal) limits?

$$
\text { Y N }
$$

15. Do you have any metallic parts in your body - e.g., pacemaker, metal plates, pins, etc? [explain that this information is important in terms of their safety during MRI scan]

$$
\text { Y N }
$$

16. Participation requires that you be able to feel at ease in the MRI environment which is a little restricted and does involve being in an enclosed space. If you have a tendency towards being claustrophobic, it is recommended that you do not participate in this study.

Claustrophobic? [good indication in MRI-nä̈ve participants is a chronic history of anxiety; previous claustrophobic reactions during previous scans in participants who have already had an MRI scan].

$$
\text { Y N }
$$


17. Have you ever felt dependent on alcohol [often alcohol not considered a drug] or any other drug? [If yes, which substance and how long ago was that?]

\section{Y N}

18. Have you ever felt that you may have had a problem with or were using too much of any substance?

$$
\text { Y N }
$$

19. Have you ever used any substance on a regular basis? [take note of regularity, as defined by potential participant]. If yes, which substance and how long ago?

$$
\text { Y N }
$$

20. Have you ever been admitted as an inpatient for drug/alcohol rehab, or received outpatient treatment? If yes, how many times?

$$
\text { Y N }
$$

21. Have you ever overdosed (ie lost consciousness as a result of taking drugs)? If yes, how many times?

$$
\text { Y N }
$$




\section{Appendix C}

\section{Instructions Presented in Stop Signal Task for Study 1}

\section{Practice instructions:}

In this task, you will be presented with either the letter X or O. Please respond as quickly as possible to the $\mathrm{X}$ or $\mathrm{O}$ stimulus using the corresponding $\mathrm{x}$ or o key on the keyboard.

On some trials, a red border will appear around the letter. On these trials, do not press any key. These trials are stop trials.

Do not slow your response speed in an attempt to improve performance, as this will not improve your performance in the long term.

Press any key when you are ready to begin the practice trials

\section{Main task instructions:}

This is the experimental part of the task

Please respond as quickly as possible to the $\mathrm{X}$ or $\mathrm{O}$ stimulus using the corresponding $\mathrm{x}$ or $\mathrm{o}$ key on the keyboard.

Remember, on some trials, a red border will appear around the letter. On these trials, do not press any key. These trials are stop trials.

Do not slow your response speed in an attempt to improve performance, as this will not improve your performance in the long term.

Press any key when you are ready to begin the maintask 


\section{Appendix D}

\section{Instructions Presented in Stop Signal Task for Study 2}

\section{Display:}

Welcome to this Stop Signal experiment

Please enter your email address and press enter:

Display:

On every trial, you will see an $\mathrm{X}$ or an O. Your task is to respond as FAST and ACCURATELY as possible to these (go) stimuli:

Press the $\mathrm{X}$ key with your left index finger when you see an $\mathrm{X}$

Press the $\mathrm{O}$ key with your right index finger when you see an $\mathrm{O}$

Occasionally, a box will appear around the stimuli. This indicates it is a stop trial. You have to STOP your response on stop trials:

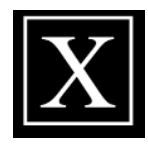

Do not press any key when a box appears around the $\mathrm{X}$ or $\mathrm{O}$

Press any key to continue.

\section{Display:}

On approximately half of the stop trials, the box will appear soon after the presentation of the stimuli and you will notice it is easy to stop your response. On the other half of the stop trials, the box will be presented rather late and it will become very difficult or even impossible to stop your response.

IMPORTANT: You must respond as FAST as possible to go stimuli. DO NOT WAIT for a stop signal to occur or the computer will wait with presenting the stop signals.

You will complete:

$1 \mathrm{x}$ practice block

$3 \mathrm{x}$ experimental blocks

You can rest between blocks. The whole task will take approximately 8 minutes.

Press any key to commence the Practice Block.

Display following practice block:

Press any key to commence Experimental Block 1 


\section{Appendix E}

\section{Stimulus Timing and Feedback Provided in Stop Signal Task for Study 2}

Table E1

Stimulus Timing, Participant Actions and Information Presented on Screen in Practice Block of Stop Signal Task Go Trials in Study 2

\begin{tabular}{lcl}
\hline \multicolumn{1}{c}{ Presented on screen } & Timing & Actions of participant during epoch \\
\hline Blank & 1000 & Wait \\
+ & 250 & Get ready \\
X or O & 1000 & Press X/O as fast as possible \\
Feedback & 2000 & Read feedback \\
$\quad$ Actual feedback received & & \\
$\quad$ Hit & & \\
$\quad$ Hit (but try to go faster) & & \\
$\quad$ Miss (you must go faster) & & \\
& Miss (incorrect keystroke) $)_{\mathrm{d}}$ & \\
WARNING: You MUST & & \\
respond to X/O go stimuli as \\
fast as possible
\end{tabular}

Note. Times are given in milliseconds.

$a=$ Correct go key press at $<500 \mathrm{~ms} ; b=$ Correct go key press at $500 \mathrm{~ms}-1000 \mathrm{~ms} ; c=$ Correct go key press $>1000 \mathrm{~ms} ; d=$ Incorrect go key press; $e=$ Three consecutive missed go key presses. 
Table E2

Stimulus Timing, Participant Actions and Information Presented on Screen in Practice Block of Stop Signal Task Stop Trials in Study 2

\begin{tabular}{lcl}
\hline \multicolumn{1}{c}{ Presented on screen } & Timing & Actions of participant during epoch \\
\hline Blank & 1000 & Wait \\
+ & 250 & Get ready \\
X or O enclosed in a box & 1000 & Press X/O as fast as possible \\
Feedback & 2000 & Read feedback \\
$\quad$ Actual feedback received & & \\
$\quad$ Successful stop - Well done ${ }_{\mathrm{a}}$ & & \\
$\quad \begin{array}{l}\text { Unsuccessful stop }- \text { try not to } \\
\text { respond to stop trials! } !_{\mathrm{b}}\end{array}$ & & \\
\hline
\end{tabular}

Note. Times are given in milliseconds.

$a=$ Successful inhibition; $b=$ Unsuccessful inhibition

Table E3

Stimulus Timing, Participant Actions and Information Presented on Screen in Experimental Block of Stop Signal Task Go Trials in Study 2

\begin{tabular}{lcl}
\hline \multicolumn{1}{c}{ Presented on screen } & Timing & Actions of participant during epoch \\
\hline $\begin{array}{l}\text { Blank or feedback } \\
\text { WARNING: You MUST respond }\end{array}$ & 1000 & Wait \\
to X/O go stimuli as fast as & & \\
possible & & \\
+ & 250 & Get ready \\
X or O & 1000 & Press X/O as fast as possible \\
\hline
\end{tabular}

Note. On the first trial in each block there is no feedback presented on screen during the first $1000 \mathrm{~ms}$. Feedback is presented for $750 \mathrm{~ms}$ if participants have not responded to the previous 3 go stimuli with blank screen for $250 \mathrm{~ms}$. Times are given in milliseconds. Actual feedback is presented in red font. 
Table E4

Stimulus Timing, Participant Actions and Information Presented on Screen in Experimental Block of Stop Signal Task Stop Trials in Study 2

\begin{tabular}{lcl}
\hline \multicolumn{1}{c}{ Presented on screen } & Timing & Actions of participant during epoch \\
\hline Blank or feedback & 1000 & Wait \\
+ & 250 & Get ready \\
X or O enclosed in a box & 1000 & Press X/O as fast as possible \\
\hline
\end{tabular}

Note. Times are given in milliseconds. 


\section{Appendix F}

Sequence of Go and Stop Trials for Practice Versions 1 and 2.

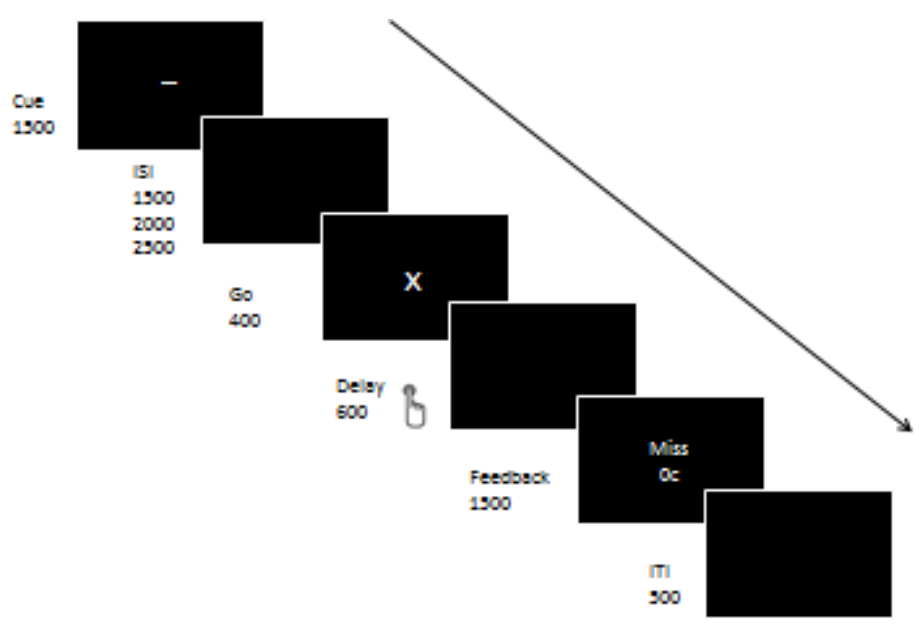

Figure 1A. Schematic representation of a MICT neutral condition go trial that has been inaccurately (too slow) performed in Practice Version 1 and Practice Version 2. Numbers representing the time are in milliseconds. 


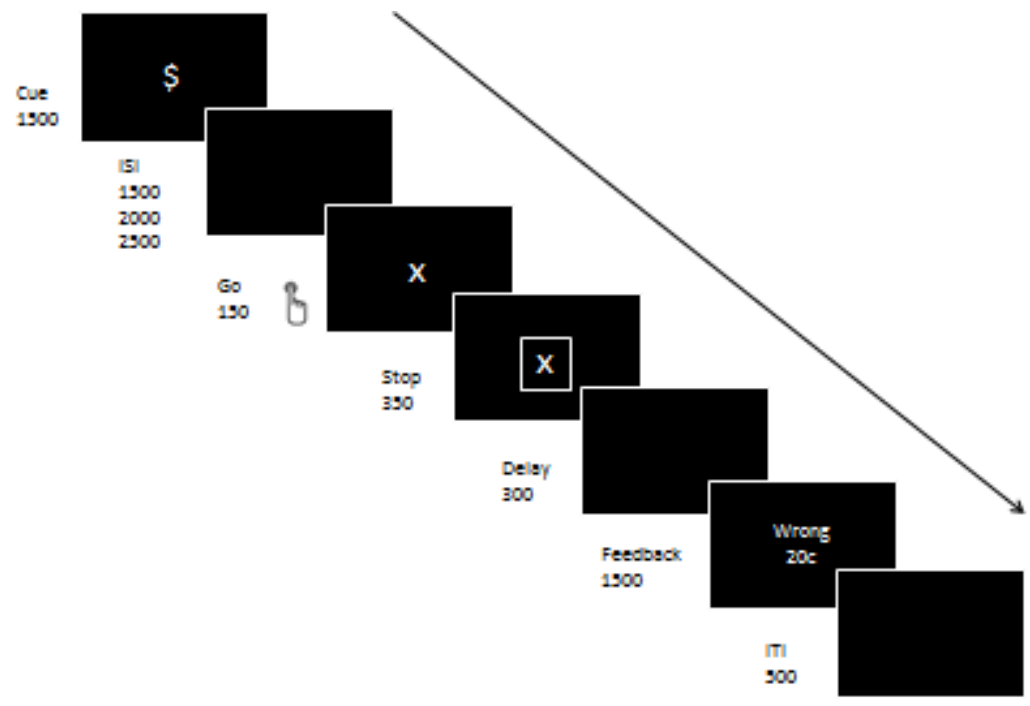

Figure 2A. Schematic representation of a MICT reward condition stop trial where the response has not been withheld in Practice Version 1 and Practice Version 2. Numbers representing the time are in milliseconds. 


\section{Appendix G}

\section{Task Design for Practice Version 1 and Practice Version 2}

Practice Version 1 and Practice Version 2 involved a practice task and a main task. For both versions the practice task was comprised of 12 randomly selected trials using a ratio of 3 go trials to 1 stop trial. The main task in Practice Version 1 was comprised of 72 trials and in Practice Version 2 it was comprised of 44 trials. Trials from each of the three conditions (reward, punishment and neutral) were randomly selected throughout the practice and main task in each version. During the main task in both versions, a stop stimulus appeared on one in every four trials. 


\section{Appendix H}

\section{Means and Significance Tests for All Main and Interaction Effects During the Cue}

\section{Period}

The tables in this Appendix contain names of brain regions and levels of variables that have been abbreviated for formatting purposes. The abbreviations and the brain regions, groups, level of accuracy and incentive conditions they represent are set out below.

Labels in the Tables and the Brain Regions They Represent

\begin{tabular}{ll}
\hline Label in table & Brain region \\
\hline Brain region 1 & Left posterior cingulate \\
Brain region 2 & Left inferior occipital gyrus \\
Brain region 3 & Right inferior frontal gyrus \\
Brain region 4 & Left inferior occipital gyrus \\
Brain region 5 & Right inferior parietal lobule \\
Brain region 6 & Right middle temporal gyrus \\
Brain region 7 & Left middle occipital gyrus \\
Brain region 8 & Left uvula \\
Brain region 9 & Left supramarginal gyrus \\
Brain region 10 & Right precuneus / Right superior parietal lobule \\
Brain region 11 & Left dorsal posterior cingulate (BA31) \\
Brain region 12 & Left postcentral gyrus near inferior parietal lobule / \\
Brain region 13 & Supramarginal gyrus \\
\hline
\end{tabular}


Labels in the Tables Referencing Low Trait Anxiety and High Trait Anxiety Groups

\begin{tabular}{cl}
\hline Group label & Group \\
\hline 1.00 & Low trait anxiety \\
2.00 & High trait anxiety \\
\hline
\end{tabular}

Labels in the Tables Referencing Accuracy

\begin{tabular}{cl}
\hline Accuracy label & Accuracy \\
\hline 1.00 & Correct \\
\hline 2.00 & Error \\
\hline
\end{tabular}

Labels in the Tables Referencing Incentive Condition

\begin{tabular}{cl}
\hline Incentive condition label & Incentive condition \\
\hline 1.00 & Neutral condition \\
2.00 & Punishment condition \\
3.00 & Reward condition \\
\hline
\end{tabular}

Note: In the tables that follow Incentive condition is abbreviated to Incentive cond 
Table H1

F-Values, Degrees of Freedom, and Levels of Significance for Regions of Event-Related BOLD Activity During Cue Period

\begin{tabular}{|c|c|c|c|c|c|}
\hline \multirow{2}{*}{$\begin{array}{l}\text { Condition } \\
\text { Group }\end{array}$} & \multirow{2}{*}{$\frac{\text { Brain region }}{\text { Brain region } 1}$} & \multicolumn{2}{|c|}{$\mathrm{df}$} & \multirow{2}{*}{$\frac{F}{3.780}$} & \multirow{2}{*}{$\frac{\text { Sig. }}{.058}$} \\
\hline & & 1 & 46 & & \\
\hline & Brain region 2 & 1 & 46 & 1.341 & .253 \\
\hline & Brain region 3 & 1 & 46 & 2.002 & .164 \\
\hline & Brain region 4 & 1 & 46 & .384 & .538 \\
\hline & Brain region 5 & 1 & 46 & .554 & .461 \\
\hline & Brain region 6 & 1 & 46 & 2.738 & .105 \\
\hline & Brain region 7 & 1 & 46 & 5.879 & .019 \\
\hline & Brain region 8 & 1 & 46 & .002 & .961 \\
\hline & Brain region 9 & 1 & 46 & .267 & .608 \\
\hline & Brain region 10 & 1 & 46 & 3.288 & .076 \\
\hline & Brain region 11 & 1 & 46 & .078 & .782 \\
\hline & Brain region 12 & 1 & 46 & .130 & .720 \\
\hline & Brain region 13 & 1 & 46 & 4.780 & .034 \\
\hline \multirow[t]{10}{*}{ Incentive cond } & Brain region 1 & 2 & 92 & 8.245 & .001 \\
\hline & Brain region 2 & 2 & 92 & 73.606 & .000 \\
\hline & Brain region 3 & 2 & 92 & 2.732 & .070 \\
\hline & Brain region 4 & 2 & 92 & 70.636 & .000 \\
\hline & Brain region 5 & 2 & 92 & 5.052 & .008 \\
\hline & Brain region 6 & 2 & 92 & 1.370 & .259 \\
\hline & Brain region 7 & 2 & 92 & 1.097 & .338 \\
\hline & Brain region 8 & 2 & 92 & 2.702 & .072 \\
\hline & Brain region 9 & 2 & 92 & 5.208 & .007 \\
\hline & Brain region 10 & 2 & 92 & .782 & .460 \\
\hline
\end{tabular}




\begin{tabular}{|c|c|c|c|c|c|}
\hline & Brain region 11 & 2 & 92 & .202 & .817 \\
\hline & Brain region 12 & 2 & 92 & 5.130 & .008 \\
\hline & Brain region 13 & 2 & 92 & 1.968 & .146 \\
\hline \multirow[t]{13}{*}{ Incentive cond $\mathrm{x}$ group } & Brain region 1 & 2 & 92 & 3.516 & .034 \\
\hline & Brain region 2 & 2 & 92 & .201 & .818 \\
\hline & Brain region 3 & 2 & 92 & .992 & .375 \\
\hline & Brain region 4 & 2 & 92 & 616 & .542 \\
\hline & Brain region 5 & 2 & 92 & .978 & .380 \\
\hline & Brain region 6 & 2 & 92 & 1.526 & .223 \\
\hline & Brain region 7 & 2 & 92 & 1.672 & .193 \\
\hline & Brain region 8 & 2 & 92 & 1.166 & .316 \\
\hline & Brain region 9 & 2 & 92 & 1.339 & .267 \\
\hline & Brain region 10 & 2 & 92 & .580 & .562 \\
\hline & Brain region 11 & 2 & 92 & 5.559 & .005 \\
\hline & Brain region 12 & 2 & 92 & 1.162 & .317 \\
\hline & Brain region 13 & 2 & 92 & 1.610 & .205 \\
\hline \multirow[t]{12}{*}{ Accuracy } & Brain region 1 & 1 & 46 & 1.234 & .272 \\
\hline & Brain region 2 & 1 & 46 & .000 & .984 \\
\hline & Brain region 3 & 1 & 46 & 2.083 & .156 \\
\hline & Brain region 4 & 1 & 46 & 269 & .606 \\
\hline & Brain region 5 & 1 & 46 & 627 & .433 \\
\hline & Brain region 6 & 1 & 46 & .096 & .758 \\
\hline & Brain region 7 & 1 & 46 & .661 & .420 \\
\hline & Brain region 8 & 1 & 46 & 3.556 & .066 \\
\hline & Brain region 9 & 1 & 46 & 5.725 & .021 \\
\hline & Brain region 10 & 1 & 46 & .001 & .974 \\
\hline & Brain region 11 & 1 & 46 & 2.732 & .105 \\
\hline & Brain region 12 & 1 & 46 & 9.439 & .004 \\
\hline
\end{tabular}




\begin{tabular}{|c|c|c|c|c|c|}
\hline & Brain region 13 & 1 & 46 & 3.655 & .062 \\
\hline \multirow[t]{13}{*}{ Accuracy $\mathrm{x}$ group } & Brain region 1 & 1 & 46 & .851 & .361 \\
\hline & Brain region 2 & 1 & 46 & 3.340 & .074 \\
\hline & Brain region 3 & 1 & 46 & .001 & .970 \\
\hline & Brain region 4 & 1 & 46 & 3.061 & .087 \\
\hline & Brain region 5 & 1 & 46 & .749 & .391 \\
\hline & Brain region 6 & 1 & 46 & 1.131 & .293 \\
\hline & Brain region 7 & 1 & 46 & 2.400 & .128 \\
\hline & Brain region 8 & 1 & 46 & 2.886 & .096 \\
\hline & Brain region 9 & 1 & 46 & 2.543 & .118 \\
\hline & Brain region 10 & 1 & 46 & 2.022 & .162 \\
\hline & Brain region 11 & 1 & 46 & .777 & .383 \\
\hline & Brain region 12 & 1 & 46 & 4.255 & .045 \\
\hline & Brain region 13 & 1 & 46 & .610 & .439 \\
\hline \multirow[t]{13}{*}{ Incentive cond $\mathrm{x}$ accuracy } & Brain region 1 & 2 & 92 & .675 & .512 \\
\hline & Brain region 2 & 2 & 92 & 1.381 & .256 \\
\hline & Brain region 3 & 2 & 92 & .735 & .483 \\
\hline & Brain region 4 & 2 & 92 & .937 & .395 \\
\hline & Brain region 5 & 2 & 92 & .642 & .529 \\
\hline & Brain region 6 & 2 & 92 & .261 & .771 \\
\hline & Brain region 7 & 2 & 92 & 1.956 & .147 \\
\hline & Brain region 8 & 2 & 92 & .187 & .830 \\
\hline & Brain region 9 & 2 & 92 & .137 & .873 \\
\hline & Brain region 10 & 2 & 92 & .912 & .405 \\
\hline & Brain region 11 & 2 & 92 & .302 & .740 \\
\hline & Brain region 12 & 2 & 92 & .157 & .855 \\
\hline & Brain region 13 & 2 & 92 & .781 & .461 \\
\hline Incentive cond $\mathrm{x}$ accuracy $\mathrm{x}$ & Brain region 1 & 2 & 92 & 241 & .787 \\
\hline
\end{tabular}




\begin{tabular}{llllll}
\hline group & & & & \\
& Brain region 2 & 2 & 92 & .823 & .442 \\
Brain region 3 & 2 & 92 & .381 & .684 \\
Brain region 4 & 2 & 92 & .400 & .671 \\
Brain region 5 & 2 & 92 & 1.335 & .268 \\
Brain region 6 & 2 & 92 & .914 & .404 \\
Brain region 7 & 2 & 92 & .252 & .778 \\
Brain region 8 & 2 & 92 & .646 & .526 \\
Brain region 9 & 2 & 92 & .662 & .518 \\
Brain region 10 & 2 & 92 & .102 & .903 \\
Brain region 11 & 2 & 92 & .111 & .895 \\
Brain region 12 & 2 & 92 & .028 & .972 \\
Brain region 13 & 2 & 92 & .788 & .458 \\
\hline
\end{tabular}




\section{Table H2}

Means, Standard Errors and 95\% Confidence Intervals for Group Effects

\begin{tabular}{|c|c|c|c|c|c|}
\hline \multirow[b]{2}{*}{ Brain region } & \multirow[b]{2}{*}{ Group } & \multirow[b]{2}{*}{ Mean } & \multirow[b]{2}{*}{ Std. Error } & \multicolumn{2}{|c|}{ 95\% Confidence Interval } \\
\hline & & & & Lower Bound & Upper Bound \\
\hline \multirow[t]{2}{*}{ Brain region 1} & 1.00 & -.248 & .035 & -.319 & -.177 \\
\hline & 2.00 & -.151 & .035 & -.222 & -.080 \\
\hline \multirow[t]{2}{*}{ Brain region 2} & 1.00 & .168 & .039 & .090 & .246 \\
\hline & 2.00 & .232 & .039 & .154 & .310 \\
\hline \multirow[t]{2}{*}{ Brain region 3} & 1.00 & -.171 & .026 & -.222 & -.119 \\
\hline & 2.00 & -.119 & .026 & -.171 & -.067 \\
\hline \multirow[t]{2}{*}{ Brain region 4} & 1.00 & .183 & .041 & .100 & .266 \\
\hline & 2.00 & .219 & .041 & .136 & .303 \\
\hline \multirow[t]{2}{*}{ Brain region 5} & 1.00 & -.157 & .028 & -.213 & -.101 \\
\hline & 2.00 & -.128 & .028 & -.184 & -.072 \\
\hline \multirow[t]{2}{*}{ Brain region 6} & 1.00 & -.133 & .022 & -.176 & -.089 \\
\hline & 2.00 & -.082 & .022 & -.126 & -.038 \\
\hline \multirow[t]{2}{*}{ Brain region 7} & 1.00 & -.150 & .025 & -.200 & -.099 \\
\hline & 2.00 & -.064 & .025 & -.114 & -.013 \\
\hline \multirow[t]{2}{*}{ Brain region 8} & 1.00 & -.109 & .027 & -.163 & -.056 \\
\hline & 2.00 & -.108 & 年. & -.161 & -.054 \\
\hline \multirow[t]{2}{*}{ Brain region 9} & 1.00 & -.142 & .032 & -.207 & -.077 \\
\hline & 2.00 & -.118 & .032 & -.183 & -.053 \\
\hline \multirow[t]{2}{*}{ Brain region 10} & 1.00 & -.136 & .023 & -.183 & -.089 \\
\hline & 2.00 & -.076 & .023 & -.123 & -.029 \\
\hline Brain region 11 & 1.00 & -.069 & .017 & -.103 & -.036 \\
\hline
\end{tabular}




\begin{tabular}{|c|c|c|c|c|c|}
\hline & 2.00 & -.063 & .017 & -096 & -.029 \\
\hline \multirow[t]{2}{*}{ Brain region 12} & 1.00 & -.146 & .034 & -.214 & -.078 \\
\hline & 2.00 & -.128 & .034 & -.196 & -.060 \\
\hline \multirow[t]{2}{*}{ Brain region 13} & 1.00 & -.129 & .021 & -.170 & -.087 \\
\hline & 2.00 & -.065 & .021 & -.106 & -.024 \\
\hline
\end{tabular}




\section{Table H3}

Mean Difference, Standard Error, P-Value and 95\% Confidence Interval for Low Trait Anxiety and High Trait Anxiety Groups for Each Effect

\begin{tabular}{|c|c|c|c|c|c|c|c|}
\hline \multirow[b]{2}{*}{ Brain region } & \multirow[b]{2}{*}{ (I) Group } & \multicolumn{2}{|c|}{ Mean Difference } & \multirow[b]{2}{*}{ Std. Error } & \multirow[b]{2}{*}{ Sig. ${ }^{a}$} & \multicolumn{2}{|c|}{ 95\% Confidence Interval for Difference ${ }^{a}$} \\
\hline & & (J) Group & $(\mathrm{I}-\mathrm{J})$ & & & Lower Bound & Upper Bound \\
\hline \multirow[t]{2}{*}{ Brain region 1} & 1.00 & 2.00 & -.097 & .050 & .058 & -.197 & .003 \\
\hline & 2.00 & 1.00 & .097 & .050 & .058 & -.003 & .197 \\
\hline \multirow{2}{*}{ Brain region 2} & 1.00 & 2.00 & -.063 & .055 & .253 & -.174 & .047 \\
\hline & 2.00 & 1.00 & .063 & .055 & .253 & -.047 & .174 \\
\hline \multirow[t]{2}{*}{ Brain region 3} & 1.00 & 2.00 & -.051 & .036 & .164 & -.125 & .022 \\
\hline & 2.00 & 1.00 & .051 & .036 & .164 & -.022 & .125 \\
\hline \multirow{2}{*}{ Brain region 4} & 1.00 & 2.00 & -.036 & .059 & .538 & -.154 & .082 \\
\hline & 2.00 & 1.00 & .036 & .059 & .538 & -.082 & .154 \\
\hline \multirow[t]{2}{*}{ Brain region 5} & 1.00 & 2.00 & -.029 & .039 & .461 & -.108 & .050 \\
\hline & 2.00 & 1.00 & .029 & .039 & .461 & -.050 & .108 \\
\hline \multirow[t]{2}{*}{ Brain region 6} & 1.00 & 2.00 & -.051 & .031 & .105 & -.113 & .011 \\
\hline & 2.00 & 1.00 & .051 & .031 & .105 & -.011 & .113 \\
\hline \multirow[t]{2}{*}{ Brain region 7} & 1.00 & 2.00 & $-.086^{*}$ & .035 & .019 & -.157 & -.015 \\
\hline & 2.00 & 1.00 & $.086^{*}$ & .035 & .019 & .015 & .157 \\
\hline \multirow[t]{2}{*}{ Brain region 8} & 1.00 & 2.00 & -.002 & .038 & .961 & -.078 & .074 \\
\hline & 2.00 & 1.00 & .002 & .038 & .961 & -.074 & .078 \\
\hline \multirow[t]{2}{*}{ Brain region 9} & 1.00 & 2.00 & -.024 & .046 & .608 & -.116 & .068 \\
\hline & 2.00 & 1.00 & .024 & .046 & .608 & -.068 & .116 \\
\hline \multirow[t]{2}{*}{ Brain region 10} & 1.00 & 2.00 & -.060 & .033 & .076 & -.127 & .007 \\
\hline & 2.00 & 1.00 & .060 & .033 & .076 & -.007 & .127 \\
\hline Brain region 11 & 1.00 & 2.00 & -.007 & .023 & .782 & -.054 & .041 \\
\hline
\end{tabular}




\begin{tabular}{lccccccc}
\hline & 2.00 & 1.00 & .007 & .023 & .782 & -.041 & .054 \\
\hline Brain region 12 & 1.00 & 2.00 & -.017 & .048 & .720 & -.114 & .079 \\
\cline { 2 - 7 } & 2.00 & 1.00 & .017 & .048 & .720 & -.079 & .114 \\
\hline \multirow{2}{*}{ Brain region 13 } & 1.00 & 2.00 & $-.063^{*}$ & .029 & .034 & -.122 & -.005 \\
\cline { 2 - 7 } & 2.00 & 1.00 & $.063^{*}$ & .029 & .034 & .005 & .122 \\
\hline
\end{tabular}

Based on estimated marginal means

a. Adjustment for multiple comparisons: Least Significant Difference (equivalent to no adjustments)

*. The mean difference is significant at the .05 level. 


\section{Table H4}

Means, Standard Errors and 95\% Confidence Intervals for Incentive Condition Effects

\begin{tabular}{|c|c|c|c|c|c|}
\hline \multirow[b]{2}{*}{ Brain region } & \multirow[b]{2}{*}{ Incentive cond } & \multirow[b]{2}{*}{ Mean } & \multirow[b]{2}{*}{ Std. Error } & \multicolumn{2}{|c|}{ 95\% Confidence Interval } \\
\hline & & & & Lower Bound & Upper Bound \\
\hline \multirow[t]{3}{*}{ Brain region 1} & 1 & -.227 & .025 & -.278 & -.176 \\
\hline & 2 & -.225 & .032 & -.289 & -.160 \\
\hline & 3 & -.146 & .027 & -.200 & -.093 \\
\hline \multirow[t]{3}{*}{ Brain region 2} & 1 & .037 & .023 & -.009 & .083 \\
\hline & 2 & .275 & .032 & .210 & .340 \\
\hline & 3 & .288 & .035 & .217 & .358 \\
\hline \multirow[t]{3}{*}{ Brain region 3} & 1 & -.154 & .021 & -.197 & -.111 \\
\hline & 2 & -.161 & .021 & -.203 & -.119 \\
\hline & 3 & -.119 & .022 & -.163 & -.076 \\
\hline \multirow[t]{3}{*}{ Brain region 4} & 1 & .054 & .025 & .003 & .104 \\
\hline & 2 & .266 & .034 & .197 & .335 \\
\hline & 3 & .284 & .035 & .213 & .355 \\
\hline \multirow[t]{3}{*}{ Brain region 5} & 1 & -.141 & .024 & -.190 & -.092 \\
\hline & 2 & -.176 & .023 & -.222 & -.130 \\
\hline & 3 & -.111 & .022 & -.155 & -.067 \\
\hline \multirow[t]{3}{*}{ Brain region 6} & 1 & -.096 & .020 & -.136 & -.056 \\
\hline & 2 & -.127 & .020 & -.167 & -.087 \\
\hline & 3 & -.099 & .018 & -.136 & -.062 \\
\hline \multirow[t]{3}{*}{ Brain region 7} & 1 & -.112 & .020 & -.152 & -.072 \\
\hline & 2 & -.117 & .021 & -.160 & -.075 \\
\hline & 3 & -.091 & .021 & -.134 & -.048 \\
\hline
\end{tabular}




\begin{tabular}{|c|c|c|c|c|c|}
\hline \multirow[t]{3}{*}{ Brain region 8} & 1 & -.113 & .024 & -.162 & -.063 \\
\hline & 2 & -.134 & .023 & -.179 & -.088 \\
\hline & 3 & -.079 & .023 & -.125 & -.034 \\
\hline \multirow[t]{3}{*}{ Brain region 9} & 1 & -.127 & .026 & -.181 & -.074 \\
\hline & 2 & -.170 & .028 & -.227 & -.114 \\
\hline & 3 & -.092 & .026 & -.144 & -.040 \\
\hline \multirow[t]{3}{*}{ Brain region 10} & 1 & -.119 & .020 & -.159 & -.079 \\
\hline & 2 & -.104 & .021 & -.146 & -.062 \\
\hline & 3 & -.095 & .019 & -.134 & -.057 \\
\hline \multirow[t]{3}{*}{ Brain region 11} & 1 & -.062 & .013 & -.088 & -.036 \\
\hline & 2 & -.066 & .013 & -.093 & -.039 \\
\hline & 3 & -.070 & .015 & -.101 & -.039 \\
\hline \multirow[t]{3}{*}{ Brain region 12} & 1 & -.152 & .027 & -.207 & -.098 \\
\hline & 2 & -.162 & .027 & -.216 & -.108 \\
\hline & 3 & -.097 & .027 & -.152 & -.042 \\
\hline \multirow[t]{3}{*}{ Brain region 13} & 1 & -.096 & .020 & -.137 & -.056 \\
\hline & 2 & -.124 & .023 & -.169 & -.078 \\
\hline & 3 & -.071 & .020 & -.112 & -.030 \\
\hline
\end{tabular}




\section{Table H5}

Mean Difference, Standard Error, P-Value and 95\% Confidence Interval for Incentive Condition Effects

\begin{tabular}{|c|c|c|c|c|c|c|c|}
\hline & & & Mean Difference & & & $95 \%$ Confidence Inte & al for Difference ${ }^{a}$ \\
\hline Brain region & (I) Incentive cond & (J) Incentive cond & $(\mathrm{I}-\mathrm{J})$ & Std. Error & Sig. ${ }^{a}$ & Lower Bound & Upper Bound \\
\hline \multirow[t]{6}{*}{ Brain region 1} & 1 & 2 & -.003 & .024 & .917 & -.051 & .046 \\
\hline & & 3 & $-.081^{*}$ & .023 & .001 & -.126 & -.035 \\
\hline & 2 & 1 & .003 & .024 & .917 & -.046 & .051 \\
\hline & & 3 & $-.078^{*}$ & .021 & .001 & -.121 & -.036 \\
\hline & 3 & 1 & $.081^{*}$ & .023 & .001 & .035 & .126 \\
\hline & & 2 & $.078^{*}$ & .021 & .001 & .036 & .121 \\
\hline \multirow[t]{6}{*}{ Brain region 2} & 1 & 2 & $-.238^{*}$ & .026 & .000 & -.290 & -.185 \\
\hline & & 3 & $-.251^{*}$ & .024 & .000 & -.300 & -.202 \\
\hline & 2 & 1 & $.238^{*}$ & .026 & .000 & .185 & .290 \\
\hline & & 3 & -.013 & .018 & .475 & -.051 & .024 \\
\hline & 3 & 1 & $.251^{*}$ & .024 & .000 & .202 & .300 \\
\hline & & 2 & .013 & .018 & .475 & -.024 & .051 \\
\hline \multirow[t]{6}{*}{ Brain region 3} & 1 & 2 & .007 & .019 & .696 & -.031 & .046 \\
\hline & & 3 & -.035 & .019 & .073 & -.072 & .003 \\
\hline & 2 & 1 & -.007 & .019 & .696 & -.046 & .031 \\
\hline & & 3 & $-.042^{*}$ & .020 & .039 & -.082 & -.002 \\
\hline & 3 & 1 & .035 & .019 & .073 & -.003 & .072 \\
\hline & & 2 & $.042^{*}$ & .020 & .039 & .002 & .082 \\
\hline \multirow[t]{3}{*}{ Brain region 4} & 1 & 2 & $-.212^{*}$ & .024 & .000 & -.261 & -.164 \\
\hline & & 3 & $-.230^{*}$ & .022 & .000 & -.275 & -.186 \\
\hline & 2 & 1 & $.212^{*}$ & .024 & .000 & .164 & .261 \\
\hline
\end{tabular}




\begin{tabular}{|c|c|c|c|c|c|c|c|}
\hline & & 3 & -.018 & .018 & .325 & -.054 & .018 \\
\hline & 3 & 1 & $.230^{*}$ & .022 & .000 & .186 & .275 \\
\hline & & 2 & .018 & .018 & .325 & -.018 & .054 \\
\hline \multirow[t]{6}{*}{ Brain region 5} & 1 & 2 & .035 & .021 & .101 & -.007 & .077 \\
\hline & & 3 & -.030 & .021 & .150 & -.072 & .011 \\
\hline & 2 & 1 & -.035 & .021 & .101 & -.077 & .007 \\
\hline & & 3 & $-.065^{*}$ & .020 & .002 & -.106 & -.025 \\
\hline & 3 & 1 & .030 & .021 & .150 & -.011 & .072 \\
\hline & & 2 & $.065^{*}$ & .020 & .002 & .025 & .106 \\
\hline \multirow[t]{6}{*}{ Brain region 6} & 1 & 2 & .031 & .024 & .203 & -.017 & .078 \\
\hline & & 3 & .003 & .019 & .878 & -.035 & .041 \\
\hline & 2 & 1 & -.031 & .024 & .203 & -.078 & .017 \\
\hline & & 3 & -.028 & .018 & .130 & -.064 & .008 \\
\hline & 3 & 1 & -.003 & .019 & .878 & -.041 & .035 \\
\hline & & 2 & .028 & .018 & .130 & -.008 & .064 \\
\hline \multirow[t]{6}{*}{ Brain region 7} & 1 & 2 & .006 & .019 & .768 & -.033 & .044 \\
\hline & & 3 & -.021 & .019 & .278 & -.058 & .017 \\
\hline & 2 & 1 & -.006 & .019 & .768 & -.044 & .033 \\
\hline & & 3 & -.026 & .018 & .153 & -.062 & .010 \\
\hline & 3 & 1 & .021 & .019 & .278 & -.017 & .058 \\
\hline & & 2 & .026 & .018 & .153 & -.010 & .062 \\
\hline \multirow[t]{5}{*}{ Brain region 8} & 1 & 2 & .021 & .025 & .403 & -.029 & .071 \\
\hline & & 3 & -.033 & .019 & .079 & -.071 & .004 \\
\hline & 2 & 1 & -.021 & .025 & .403 & -.071 & .029 \\
\hline & & 3 & $-.054^{*}$ & .027 & .046 & -.108 & -.001 \\
\hline & 3 & 1 & .033 & .019 & .079 & -.004 & .071 \\
\hline
\end{tabular}




\begin{tabular}{|c|c|c|c|c|c|c|c|}
\hline & & 2 & $.054^{*}$ & .027 & .046 & .001 & .108 \\
\hline \multirow[t]{6}{*}{ Brain region 9} & 1 & 2 & .043 & .027 & .116 & -.011 & .097 \\
\hline & & 3 & -.036 & .026 & .177 & -.088 & .017 \\
\hline & 2 & 1 & -.043 & .027 & .116 & -.097 & .011 \\
\hline & & 3 & $-.078^{*}$ & .020 & .000 & -.118 & -.039 \\
\hline & 3 & 1 & .036 & .026 & .177 & -.017 & .088 \\
\hline & & 2 & $.078^{*}$ & .020 & .000 & .039 & .118 \\
\hline \multirow[t]{6}{*}{ Brain region 10} & 1 & 2 & -.015 & .019 & .435 & -.054 & .024 \\
\hline & & 3 & -.024 & .021 & .262 & -.066 & .019 \\
\hline & 2 & 1 & .015 & .019 & .435 & -.024 & .054 \\
\hline & & 3 & -.009 & .018 & .621 & -.044 & .027 \\
\hline & 3 & 1 & .024 & .021 & .262 & -.019 & .066 \\
\hline & & 2 & .009 & .018 & .621 & -.027 & .044 \\
\hline \multirow[t]{6}{*}{ Brain region 11} & 1 & 2 & .004 & .011 & .685 & -.017 & .026 \\
\hline & & 3 & .008 & .014 & .567 & -.020 & .037 \\
\hline & 2 & 1 & -.004 & .011 & .685 & -.026 & .017 \\
\hline & & 3 & .004 & .013 & .780 & -.023 & .030 \\
\hline & 3 & 1 & -.008 & .014 & .567 & -.037 & .020 \\
\hline & & 2 & -.004 & .013 & .780 & -.030 & .023 \\
\hline \multirow[t]{6}{*}{ Brain region 12} & 1 & 2 & .010 & .023 & .664 & -.035 & .055 \\
\hline & & 3 & $-.055^{*}$ & .023 & .022 & -.103 & -.008 \\
\hline & 2 & 1 & -.010 & .023 & .664 & -.055 & .035 \\
\hline & & 3 & $-.065^{*}$ & .020 & .002 & -.105 & -.025 \\
\hline & 3 & 1 & $.055^{*}$ & .023 & .022 & .008 & .103 \\
\hline & & 2 & $.065^{*}$ & .020 & .002 & .025 & .105 \\
\hline Brain region 13 & 1 & 2 & .027 & .029 & .350 & -.031 & .086 \\
\hline
\end{tabular}




\begin{tabular}{llllllll}
\hline & 3 & -.025 & .024 & .289 & -.073 & .022 \\
\hline & 1 & -.027 & .029 & .350 & -.086 & .031 \\
& 1 & 3 & -.053 & .027 & .056 & -.107 & .001 \\
\hline & 1 & .025 & .024 & .289 & -.022 & .073 \\
& 2 & .053 & .027 & .056 & -.001 & .107 \\
\hline
\end{tabular}

Based on estimated marginal means

a. Adjustment for multiple comparisons: Least Significant Difference (equivalent to no adjustments).

*. The mean difference is significant at the .05 level. 


\section{Table H6}

Means, Standard Errors and 95\% Confidence Intervals for Accuracy Effects

\begin{tabular}{|c|c|c|c|c|c|}
\hline \multirow[b]{2}{*}{ Brain region } & \multirow[b]{2}{*}{ Accuracy } & \multirow[b]{2}{*}{ Mean } & \multirow[b]{2}{*}{ Std. Error } & \multicolumn{2}{|c|}{ 95\% Confidence Interval } \\
\hline & & & & Lower Bound & Upper Bound \\
\hline \multirow[t]{2}{*}{ Brain region 1} & 1 & -.189 & .024 & -.238 & -.140 \\
\hline & 2 & -.209 & .028 & -.267 & -.152 \\
\hline \multirow[t]{2}{*}{ Brain region 2} & 1 & .200 & .029 & .142 & .258 \\
\hline & 2 & .200 & .028 & .144 & .256 \\
\hline \multirow[t]{2}{*}{ Brain region 3} & 1 & -.129 & .019 & -.166 & -.091 \\
\hline & 2 & -.161 & .024 & -.209 & -.113 \\
\hline \multirow[t]{2}{*}{ Brain region 4} & 1 & .205 & .030 & .144 & .266 \\
\hline & 2 & .197 & .030 & .137 & .258 \\
\hline \multirow[t]{2}{*}{ Brain region 5} & 1 & -.137 & .021 & -.178 & -.095 \\
\hline & 2 & -.149 & .021 & -.191 & -.106 \\
\hline \multirow[t]{2}{*}{ Brain region 6} & 1 & -.105 & .017 & -.138 & -.072 \\
\hline & 2 & -.110 & .018 & -.145 & -.074 \\
\hline \multirow[t]{2}{*}{ Brain region 7} & 1 & -.114 & .021 & -.155 & -.072 \\
\hline & 2 & -.100 & .019 & -.138 & -.062 \\
\hline \multirow[t]{2}{*}{ Brain region 8} & 1 & -.093 & .019 & -.132 & -.054 \\
\hline & 2 & -.124 & .022 & -.167 & -.081 \\
\hline \multirow[t]{2}{*}{ Brain region 9} & 1 & -.101 & .026 & -.153 & -.049 \\
\hline & 2 & -.159 & .026 & -.211 & -.107 \\
\hline \multirow[t]{2}{*}{ Brain region 10} & 1 & -.106 & .017 & -.141 & -.072 \\
\hline & 2 & -.106 & .018 & -.143 & -.069 \\
\hline Brain region 11 & 1 & -.078 & .012 & -.103 & -.052 \\
\hline
\end{tabular}




\begin{tabular}{llllll}
\hline & & & & & \\
& & -.055 & .015 & -.084 & -.025 \\
\hline Brain region 12 & 1 & -.108 & .026 & -.160 & -.057 \\
& 2 & -.166 & .026 & -.217 & -.114 \\
\hline Brain region 13 & 1 & -.079 & .017 & -.113 & -.045 \\
& 2 & -.115 & .018 & -.150 & -.079 \\
\hline
\end{tabular}




\section{Table H7}

Mean Difference, Standard Error, P-Value and 95\% Confidence Interval for Accuracy Effects

\begin{tabular}{|c|c|c|c|c|c|c|c|}
\hline \multirow[b]{2}{*}{ Brain region } & \multirow[b]{2}{*}{ (I) Accuracy } & \multicolumn{3}{|c|}{ Mean Difference } & \multirow[b]{2}{*}{ Sig. ${ }^{a}$} & \multicolumn{2}{|c|}{ 95\% Confidence Interval for Difference ${ }^{a}$} \\
\hline & & (J) Accuracy & $(\mathrm{I}-\mathrm{J})$ & Std. Error & & Lower Bound & Upper Bound \\
\hline \multirow[t]{2}{*}{ Brain region 1} & 1 & 2 & .020 & .018 & .272 & -.016 & .057 \\
\hline & 2 & 1 & -.020 & .018 & .272 & -.057 & .016 \\
\hline \multirow[t]{2}{*}{ Brain region 2} & 1 & 2 & .000 & .014 & .984 & -.028 & .029 \\
\hline & 2 & 1 & .000 & .014 & .984 & -.029 & .028 \\
\hline \multirow[t]{2}{*}{ Brain region 3} & 1 & 2 & .032 & .022 & .156 & -.013 & .077 \\
\hline & 2 & 1 & -.032 & .022 & .156 & -.077 & .013 \\
\hline \multirow[t]{2}{*}{ Brain region 4} & 1 & 2 & .008 & .015 & .606 & -.022 & .038 \\
\hline & 2 & 1 & -.008 & .015 & .606 & -.038 & .022 \\
\hline \multirow[t]{2}{*}{ Brain region 5} & 1 & 2 & .012 & .015 & .433 & -.018 & .042 \\
\hline & 2 & 1 & -.012 & .015 & .433 & -.042 & .018 \\
\hline \multirow[t]{2}{*}{ Brain region 6} & 1 & 2 & .005 & .015 & .758 & -.026 & .036 \\
\hline & 2 & 1 & -.005 & .015 & .758 & -.036 & .026 \\
\hline \multirow[t]{2}{*}{ Brain region 7} & 1 & 2 & -.014 & .017 & .420 & -.048 & .021 \\
\hline & 2 & 1 & .014 & .017 & .420 & -.021 & .048 \\
\hline \multirow[t]{2}{*}{ Brain region 8} & 1 & 2 & .031 & .016 & .066 & -.002 & .064 \\
\hline & 2 & 1 & -.031 & .016 & .066 & -.064 & .002 \\
\hline \multirow[t]{2}{*}{ Brain region 9} & 1 & 2 & $.058^{*}$ & .024 & .021 & .009 & .106 \\
\hline & 2 & 1 & $-.058^{*}$ & .024 & .021 & -.106 & -.009 \\
\hline \multirow[t]{2}{*}{ Brain region 10} & 1 & 2 & .000 & .013 & .974 & -.027 & .026 \\
\hline & 2 & 1 & .000 & .013 & .974 & -.026 & .027 \\
\hline Brain region 11 & 1 & 2 & -.023 & .014 & .105 & -.050 & .005 \\
\hline
\end{tabular}




\begin{tabular}{lccccccr}
\hline & 2 & 1 & .023 & .014 & .105 & -.005 & .050 \\
\hline \multirow{2}{*}{ Brain region 12 } & 1 & 2 & $.057^{*}$ & .019 & .004 & .020 & .095 \\
\cline { 2 - 8 } & 2 & 1 & $-.057^{*}$ & .019 & .004 & -.095 & -.020 \\
\hline \multirow{2}{*}{ Brain region 13 } & 1 & 2 & .036 & .019 & .062 & -.002 & .073 \\
\cline { 2 - 7 } & 2 & 1 & -.036 & .019 & .062 & -.073 & .002 \\
\hline
\end{tabular}

Based on estimated marginal means

a. Adjustment for multiple comparisons: Least Significant Difference (equivalent to no adjustments)

*. The mean difference is significant at the .05 level. 


\section{Table H8}

Means, Standard Errors and 95\% Confidence Intervals for Group x Incentive Condition Effects

\begin{tabular}{|c|c|c|c|c|c|c|}
\hline \multirow[b]{2}{*}{ Brain region } & \multirow[b]{2}{*}{ Group } & \multirow[b]{2}{*}{ Incentive cond } & \multirow[b]{2}{*}{ Mean } & \multirow[b]{2}{*}{ Std. Error } & \multicolumn{2}{|c|}{ 95\% Confidence Interval } \\
\hline & & & & & Lower Bound & Upper Bound \\
\hline \multirow[t]{6}{*}{ Brain region 1} & 1.00 & 1 & -.299 & .036 & -.371 & -.228 \\
\hline & & 2 & -.283 & .045 & -.374 & -.192 \\
\hline & & 3 & -.161 & .038 & -.237 & -.085 \\
\hline & 2.00 & 1 & -.155 & .036 & -.227 & -.083 \\
\hline & & 2 & -.166 & .045 & -.258 & -.075 \\
\hline & & 3 & -.132 & .038 & -.207 & -.056 \\
\hline \multirow[t]{6}{*}{ Brain region 2} & 1.00 & 1 & -.002 & .032 & -.068 & .063 \\
\hline & & 2 & .243 & .046 & .152 & .335 \\
\hline & & 3 & .263 & .050 & .164 & .363 \\
\hline & 2.00 & 1 & .076 & .032 & .011 & .142 \\
\hline & & 2 & .306 & .046 & .214 & .398 \\
\hline & & 3 & .313 & .050 & .213 & .412 \\
\hline \multirow[t]{6}{*}{ Brain region 3} & 1.00 & 1 & -.190 & .030 & -.251 & -.130 \\
\hline & & 2 & -.192 & .030 & -.251 & -.132 \\
\hline & & 3 & -.130 & .031 & -.192 & -.068 \\
\hline & 2.00 & 1 & -.118 & .030 & -.178 & -.057 \\
\hline & & 2 & -.131 & .030 & -.190 & -.072 \\
\hline & & 3 & -.109 & .031 & -.171 & -.047 \\
\hline \multirow[t]{3}{*}{ Brain region 4} & 1.00 & 1 & .023 & .035 & -.048 & .094 \\
\hline & & 2 & .249 & .048 & .152 & .346 \\
\hline & & 3 & .277 & .050 & .176 & .377 \\
\hline
\end{tabular}




\begin{tabular}{|c|c|c|c|c|c|c|}
\hline & 2.00 & 1 & .084 & .035 & .013 & .156 \\
\hline & & 2 & .283 & .048 & .185 & .380 \\
\hline & & 3 & .291 & .050 & .190 & .391 \\
\hline \multirow[t]{6}{*}{ Brain region 5} & 1.00 & 1 & -.169 & .034 & -.238 & -.100 \\
\hline & & 2 & -.192 & .032 & -.258 & -.127 \\
\hline & & 3 & -.110 & .031 & -.172 & -.048 \\
\hline & 2.00 & 1 & -.113 & .034 & -.182 & -.044 \\
\hline & & 2 & -.159 & .032 & -.225 & -.094 \\
\hline & & 3 & -.111 & .031 & -.173 & -.049 \\
\hline \multirow[t]{6}{*}{ Brain region 6} & 1.00 & 1 & -.133 & .028 & -.189 & -.077 \\
\hline & & 2 & -.161 & .028 & -.218 & -.104 \\
\hline & & 3 & -.104 & .026 & -.156 & -.052 \\
\hline & 2.00 & 1 & -.059 & .028 & -.115 & -.003 \\
\hline & & 2 & -.092 & .028 & -.149 & -.036 \\
\hline & & 3 & -.094 & .026 & -.146 & -.042 \\
\hline \multirow[t]{6}{*}{ Brain region 7} & 1.00 & 1 & -.168 & .028 & -.225 & -.112 \\
\hline & & 2 & -.166 & .030 & -.226 & -.106 \\
\hline & & 3 & -.115 & .030 & -.176 & -.054 \\
\hline & 2.00 & 1 & -.055 & .028 & -.111 & .001 \\
\hline & & 2 & -.069 & .030 & -.129 & -.009 \\
\hline & & 3 & -.067 & .030 & -.128 & -.006 \\
\hline \multirow[t]{5}{*}{ Brain region 8} & 1.00 & 1 & -.134 & .035 & -.204 & -.065 \\
\hline & & 2 & -.126 & .032 & -.190 & -.061 \\
\hline & & 3 & -.069 & .032 & -.133 & -.004 \\
\hline & 2.00 & 1 & -.091 & .035 & -.160 & -.021 \\
\hline & & 2 & -.142 & .032 & -.206 & -.077 \\
\hline
\end{tabular}




\begin{tabular}{|c|c|c|c|c|c|c|}
\hline & & 3 & -.090 & .032 & -.154 & -.026 \\
\hline \multirow[t]{6}{*}{ Brain region 9} & 1.00 & 1 & -.160 & .037 & -.235 & -.084 \\
\hline & & 2 & -.163 & .040 & -.243 & -.083 \\
\hline & & 3 & -.103 & .036 & -.176 & -.029 \\
\hline & 2.00 & 1 & -.095 & .037 & -.171 & -.020 \\
\hline & & 2 & -.178 & .040 & -.258 & -.098 \\
\hline & & 3 & -.081 & .036 & -.154 & -.008 \\
\hline \multirow[t]{6}{*}{ Brain region 10} & 1.00 & 1 & -.159 & .028 & -.216 & -.102 \\
\hline & & 2 & -.135 & .029 & -.194 & -.075 \\
\hline & & 3 & -.114 & .027 & -.169 & -.060 \\
\hline & 2.00 & 1 & -.079 & .028 & -.136 & -.022 \\
\hline & & 2 & -.073 & .029 & -.132 & -.014 \\
\hline & & 3 & -.076 & .027 & -.130 & -.021 \\
\hline \multirow[t]{6}{*}{ Brain region 11} & 1.00 & 1 & -.087 & .018 & -.123 & -.051 \\
\hline & & 2 & -.068 & .019 & -.106 & -.030 \\
\hline & & 3 & -.053 & .022 & -.097 & -.009 \\
\hline & 2.00 & 1 & -.037 & .018 & -.073 & .000 \\
\hline & & 2 & -.065 & .019 & -.103 & -.027 \\
\hline & & 3 & -.087 & .022 & -.131 & -.043 \\
\hline \multirow[t]{6}{*}{ Brain region 12} & 1.00 & 1 & -.177 & .038 & -.255 & -.100 \\
\hline & & 2 & -.154 & .038 & -.230 & -.078 \\
\hline & & 3 & -.106 & .039 & -.184 & -.028 \\
\hline & 2.00 & 1 & -.127 & .038 & -.204 & -.050 \\
\hline & & 2 & -.170 & .038 & -.247 & -.094 \\
\hline & & 3 & -.088 & .039 & -.166 & -.010 \\
\hline Brain region 13 & 1.00 & 1 & -.119 & .028 & -.176 & -.061 \\
\hline
\end{tabular}




\begin{tabular}{cccccc}
\hline & & & & & \\
& 2 & -.137 & .032 & -.202 & -.073 \\
& 3 & -.130 & .029 & -.188 & -.072 \\
\hline \multirow{2}{2}{.00} & 1 & -.074 & .028 & -.131 & -.016 \\
& 2 & -.110 & .032 & -.175 & -.045 \\
& 3 & -.012 & .029 & -.070 & .046 \\
\hline
\end{tabular}




\section{Table H9}

Mean Difference, Standard Error, P-Value and 95\% Confidence Interval for Incentive Condition Effects by Group

\begin{tabular}{|c|c|c|c|c|c|c|c|c|}
\hline \multirow[b]{2}{*}{ Brain region } & \multirow[b]{2}{*}{ Group } & \multirow[b]{2}{*}{ (I) Incentive cond } & \multirow[b]{2}{*}{ (J) Incentive cond } & \multicolumn{3}{|l|}{ Mean Difference } & \multicolumn{2}{|c|}{ 95\% Confidence Interval for Difference } \\
\hline & & & & $(\mathrm{I}-\mathrm{J})$ & Std. Error & Sig. ${ }^{\text {a }}$ & Lower Bound & Upper Bound \\
\hline \multirow[t]{12}{*}{ Brain region 1} & 1.00 & 1 & 2 & -.017 & .034 & .625 & -.085 & .052 \\
\hline & & & 3 & $-.138^{*}$ & .032 & .000 & -.202 & -.074 \\
\hline & & 2 & 1 & .017 & .034 & .625 & -.052 & .085 \\
\hline & & & 3 & $-.121^{*}$ & .030 & .000 & -.181 & -.061 \\
\hline & & 3 & 1 & $.138^{*}$ & .032 & .000 & .074 & .202 \\
\hline & & & 2 & $.121^{*}$ & .030 & .000 & .061 & .181 \\
\hline & 2.00 & 1 & 2 & .012 & .034 & .732 & -.057 & .080 \\
\hline & & & 3 & -.023 & .032 & .472 & -.087 & .041 \\
\hline & & 2 & 1 & -.012 & .034 & .732 & -.080 & .057 \\
\hline & & & 3 & -.035 & .030 & .248 & -.095 & .025 \\
\hline & & 3 & 1 & .023 & .032 & .472 & -.041 & .087 \\
\hline & & & 2 & .035 & .030 & .248 & -.025 & .095 \\
\hline \multirow[t]{9}{*}{ Brain region 2} & 1.00 & 1 & 2 & $-.246^{*}$ & .037 & .000 & -.320 & -.171 \\
\hline & & & 3 & $-.266^{*}$ & .035 & .000 & -.335 & -.196 \\
\hline & & 2 & 1 & $.246^{*}$ & .037 & .000 & .171 & .320 \\
\hline & & & 3 & -.020 & .026 & .448 & -.073 & .033 \\
\hline & & 3 & 1 & $.266^{*}$ & .035 & .000 & .196 & .335 \\
\hline & & & 2 & .020 & .026 & .448 & -.033 & .073 \\
\hline & 2.00 & 1 & 2 & $-.230^{*}$ & .037 & .000 & -.304 & -.155 \\
\hline & & & 3 & $-.236^{*}$ & .035 & .000 & -.306 & -.167 \\
\hline & & 2 & 1 & $.230^{*}$ & .037 & .000 & .155 & .304 \\
\hline
\end{tabular}




\begin{tabular}{|c|c|c|c|c|c|c|c|c|}
\hline & & & 3 & -.007 & .026 & .800 & -.059 & .046 \\
\hline & & 3 & 1 & $.236^{*}$ & .035 & .000 & .167 & .306 \\
\hline & & & 2 & .007 & .026 & .800 & -.046 & .059 \\
\hline \multirow[t]{12}{*}{ Brain region 3} & 1.00 & 1 & 2 & .001 & .027 & .958 & -.053 & .055 \\
\hline & & & 3 & $-.060^{*}$ & .027 & .028 & -.114 & -.007 \\
\hline & & 2 & 1 & -.001 & .027 & .958 & -.055 & .053 \\
\hline & & & 3 & $-.062^{*}$ & .028 & .032 & -.118 & -.006 \\
\hline & & 3 & 1 & $.060^{*}$ & .027 & .028 & .007 & .114 \\
\hline & & & 2 & $.062^{*}$ & .028 & .032 & .006 & .118 \\
\hline & 2.00 & 1 & 2 & .013 & .027 & .618 & -.041 & .068 \\
\hline & & & 3 & -.009 & .027 & .744 & -.062 & .045 \\
\hline & & 2 & 1 & -.013 & .027 & .618 & -.068 & .041 \\
\hline & & & 3 & -.022 & .028 & .429 & -.078 & .034 \\
\hline & & 3 & 1 & .009 & .027 & .744 & -.045 & .062 \\
\hline & & & 2 & .022 & .028 & .429 & -.034 & .078 \\
\hline \multirow[t]{11}{*}{ Brain region 4} & 1.00 & 1 & 2 & $-.226^{*}$ & .034 & .000 & -.295 & -.158 \\
\hline & & & 3 & $-.254^{*}$ & .031 & .000 & -.317 & -.191 \\
\hline & & 2 & 1 & $.226^{*}$ & .034 & .000 & .158 & .295 \\
\hline & & & 3 & -.028 & .025 & .284 & -.079 & .024 \\
\hline & & 3 & 1 & $.254^{*}$ & .031 & .000 & .191 & .317 \\
\hline & & & 2 & .028 & .025 & .284 & -.024 & .079 \\
\hline & 2.00 & 1 & 2 & $-.198^{*}$ & .034 & .000 & -.267 & -.130 \\
\hline & & & 3 & $-.206^{*}$ & .031 & .000 & -.269 & -.144 \\
\hline & & 2 & 1 & $.198^{*}$ & .034 & .000 & .130 & .267 \\
\hline & & & 3 & -.008 & .025 & .749 & -.059 & .043 \\
\hline & & 3 & 1 & $.206^{*}$ & .031 & .000 & .144 & .269 \\
\hline
\end{tabular}




\begin{tabular}{|c|c|c|c|c|c|c|c|c|}
\hline & & & 2 & .008 & .025 & .749 & -.043 & .059 \\
\hline \multirow[t]{12}{*}{ Brain region 5} & 1.00 & 1 & 2 & .024 & .030 & .429 & -.036 & .083 \\
\hline & & & 3 & $-.059^{*}$ & .029 & .050 & -.117 & .000 \\
\hline & & 2 & 1 & -.024 & .030 & .429 & -.083 & .036 \\
\hline & & & 3 & $-.082^{*}$ & .028 & .006 & -.139 & -.025 \\
\hline & & 3 & 1 & $.059^{*}$ & .029 & .050 & .000 & .117 \\
\hline & & & 2 & $.082^{*}$ & .028 & .006 & .025 & .139 \\
\hline & 2.00 & 1 & 2 & .047 & .030 & .123 & -.013 & .106 \\
\hline & & & 3 & -.002 & .029 & .956 & -.060 & .057 \\
\hline & & 2 & 1 & -.047 & .030 & .123 & -.106 & .013 \\
\hline & & & 3 & -.048 & .028 & .097 & -.105 & .009 \\
\hline & & 3 & 1 & .002 & .029 & .956 & -.057 & .060 \\
\hline & & & 2 & .048 & .028 & .097 & -.009 & .105 \\
\hline \multirow[t]{12}{*}{ Brain region 6} & 1.00 & 1 & 2 & .028 & .033 & .405 & -.039 & .096 \\
\hline & & & 3 & -.029 & .027 & .287 & -.083 & .025 \\
\hline & & 2 & 1 & -.028 & .033 & .405 & -.096 & .039 \\
\hline & & & 3 & $-.057^{*}$ & .025 & .029 & -.108 & -.006 \\
\hline & & 3 & 1 & .029 & .027 & .287 & -.025 & .083 \\
\hline & & & 2 & $.057^{*}$ & .025 & .029 & .006 & .108 \\
\hline & 2.00 & 1 & 2 & .033 & .033 & .330 & -.034 & .100 \\
\hline & & & 3 & .035 & .027 & .202 & -.019 & .089 \\
\hline & & 2 & 1 & -.033 & .033 & .330 & -.100 & .034 \\
\hline & & & 3 & .002 & .025 & .940 & -.049 & .053 \\
\hline & & 3 & 1 & -.035 & .027 & .202 & -.089 & .019 \\
\hline & & & 2 & -.002 & .025 & .940 & -.053 & .049 \\
\hline Brain region 7 & 1.00 & 1 & 2 & -.002 & .027 & .928 & -.057 & .052 \\
\hline
\end{tabular}




\begin{tabular}{|c|c|c|c|c|c|c|c|c|}
\hline & & & 3 & -.053 & .026 & .050 & -.106 & .000 \\
\hline & & \multirow[t]{2}{*}{2} & 1 & .002 & .027 & .928 & -.052 & .057 \\
\hline & & & 3 & -.051 & .025 & .052 & -.102 & .001 \\
\hline & & \multirow[t]{2}{*}{3} & 1 & .053 & .026 & .050 & .000 & .106 \\
\hline & & & 2 & .051 & .025 & .052 & -.001 & .102 \\
\hline & \multirow[t]{6}{*}{2.00} & \multirow[t]{2}{*}{1} & 2 & .014 & .027 & .612 & -.041 & .068 \\
\hline & & & 3 & .012 & .026 & .648 & -.041 & .065 \\
\hline & & \multirow[t]{2}{*}{2} & 1 & -.014 & .027 & .612 & -.068 & .041 \\
\hline & & & 3 & -.002 & .025 & .949 & -.053 & .050 \\
\hline & & \multirow[t]{2}{*}{3} & 1 & -.012 & .026 & .648 & -.065 & .041 \\
\hline & & & 2 & .002 & .025 & .949 & -.050 & .053 \\
\hline \multirow[t]{12}{*}{ Brain region 8} & \multirow[t]{6}{*}{1.00} & \multirow[t]{2}{*}{1} & 2 & -.009 & .035 & .805 & -.080 & .062 \\
\hline & & & 3 & $-.066^{*}$ & .026 & .016 & -.119 & -.013 \\
\hline & & \multirow[t]{2}{*}{2} & 1 & .009 & .035 & .805 & -.062 & .080 \\
\hline & & & 3 & -.057 & .038 & .136 & -.133 & .019 \\
\hline & & \multirow[t]{2}{*}{3} & 1 & $.066^{*}$ & .026 & .016 & .013 & .119 \\
\hline & & & 2 & .057 & .038 & .136 & -.019 & .133 \\
\hline & \multirow[t]{6}{*}{2.00} & \multirow[t]{2}{*}{1} & 2 & .051 & .035 & .156 & -.020 & .122 \\
\hline & & & 3 & -.001 & .026 & .972 & -.054 & .052 \\
\hline & & \multirow[t]{2}{*}{2} & 1 & -.051 & .035 & .156 & -.122 & .020 \\
\hline & & & 3 & -.052 & .038 & .174 & -.127 & .024 \\
\hline & & \multirow[t]{2}{*}{3} & 1 & .001 & .026 & .972 & -.052 & .054 \\
\hline & & & 2 & .052 & .038 & .174 & -.024 & .127 \\
\hline \multirow[t]{3}{*}{ Brain region 9} & \multirow[t]{3}{*}{1.00} & \multirow[t]{2}{*}{1} & 2 & .003 & .038 & .935 & -.073 & .079 \\
\hline & & & 3 & -.057 & .037 & .126 & -.131 & .017 \\
\hline & & 2 & 1 & -.003 & .038 & .935 & -.079 & .073 \\
\hline
\end{tabular}




\begin{tabular}{|c|c|c|c|c|c|c|c|c|}
\hline & & & 3 & $-.060^{*}$ & .028 & .035 & -.116 & -.004 \\
\hline & & \multirow[t]{2}{*}{3} & 1 & .057 & .037 & .126 & -.017 & .131 \\
\hline & & & 2 & $.060^{*}$ & .028 & .035 & .004 & .116 \\
\hline & \multirow[t]{6}{*}{2.00} & \multirow[t]{2}{*}{1} & 2 & $.083^{*}$ & .038 & .034 & .006 & .159 \\
\hline & & & 3 & -.014 & .037 & .705 & -.088 & .060 \\
\hline & & \multirow[t]{2}{*}{2} & 1 & $-.083^{*}$ & .038 & .034 & -.159 & -.006 \\
\hline & & & 3 & $-.097^{*}$ & .028 & .001 & -.153 & -.041 \\
\hline & & \multirow[t]{2}{*}{3} & 1 & .014 & .037 & .705 & -.060 & .088 \\
\hline & & & 2 & $.097^{*}$ & .028 & .001 & .041 & .153 \\
\hline \multirow[t]{12}{*}{ Brain region 10} & \multirow[t]{6}{*}{1.00} & \multirow[t]{2}{*}{1} & 2 & -.024 & .027 & .377 & -.079 & .031 \\
\hline & & & 3 & -.045 & .030 & .140 & -.105 & .015 \\
\hline & & \multirow[t]{2}{*}{2} & 1 & .024 & .027 & .377 & -.031 & .079 \\
\hline & & & 3 & -.020 & .025 & .415 & -.070 & .030 \\
\hline & & \multirow[t]{2}{*}{3} & 1 & .045 & .030 & .140 & -.015 & .105 \\
\hline & & & 2 & .020 & .025 & .415 & -.030 & .070 \\
\hline & \multirow[t]{6}{*}{2.00} & \multirow[t]{2}{*}{1} & 2 & -.006 & .027 & .825 & -.061 & .049 \\
\hline & & & 3 & -.003 & .030 & .917 & -.063 & .057 \\
\hline & & \multirow[t]{2}{*}{2} & 1 & .006 & .027 & .825 & -.049 & .061 \\
\hline & & & 3 & .003 & .025 & .907 & -.047 & .053 \\
\hline & & \multirow[t]{2}{*}{3} & 1 & .003 & .030 & .917 & -.057 & .063 \\
\hline & & & 2 & -.003 & .025 & .907 & -.053 & .047 \\
\hline \multirow[t]{5}{*}{ Brain region 11} & \multirow[t]{5}{*}{1.00} & \multirow[t]{2}{*}{1} & 2 & -.019 & .015 & .216 & -.050 & .012 \\
\hline & & & 3 & -.034 & .020 & .091 & -.075 & .006 \\
\hline & & \multirow[t]{2}{*}{2} & 1 & .019 & .015 & .216 & -.012 & .050 \\
\hline & & & 3 & -.015 & .019 & .419 & -.053 & .022 \\
\hline & & 3 & 1 & .034 & .020 & .091 & -.006 & .075 \\
\hline
\end{tabular}




\begin{tabular}{|c|c|c|c|c|c|c|c|c|}
\hline & & & 2 & .015 & .019 & .419 & -.022 & .053 \\
\hline & 2.00 & 1 & 2 & .028 & .015 & .073 & -.003 & .059 \\
\hline & & & 3 & $.051^{*}$ & .020 & .014 & .011 & .091 \\
\hline & & 2 & 1 & -.028 & .015 & .073 & -.059 & .003 \\
\hline & & & 3 & .023 & .019 & .232 & -.015 & .060 \\
\hline & & 3 & 1 & $-.051^{*}$ & .020 & .014 & -.091 & -.011 \\
\hline & & & 2 & -.023 & .019 & .232 & -.060 & .015 \\
\hline \multirow[t]{12}{*}{ Brain region 12} & 1.00 & 1 & 2 & -.024 & .032 & .461 & -.088 & .040 \\
\hline & & & 3 & $-.072^{*}$ & .033 & .035 & -.138 & -.005 \\
\hline & & 2 & 1 & .024 & .032 & .461 & -.040 & .088 \\
\hline & & & 3 & -.048 & .028 & .095 & -.105 & .009 \\
\hline & & 3 & 1 & $.072^{*}$ & .033 & .035 & .005 & .138 \\
\hline & & & 2 & .048 & .028 & .095 & -.009 & .105 \\
\hline & 2.00 & 1 & 2 & .043 & .032 & .180 & -.021 & .108 \\
\hline & & & 3 & -.039 & .033 & .241 & -.106 & .027 \\
\hline & & 2 & 1 & -.043 & .032 & .180 & -.108 & .021 \\
\hline & & & 3 & $-.083^{*}$ & .028 & .005 & -.139 & -.026 \\
\hline & & 3 & 1 & .039 & .033 & .241 & -.027 & .106 \\
\hline & & & 2 & $.083^{*}$ & .028 & .005 & .026 & .139 \\
\hline \multirow[t]{7}{*}{ Brain region 13} & 1.00 & 1 & 2 & .019 & .041 & .650 & -.064 & .101 \\
\hline & & & 3 & .011 & .034 & .747 & -.057 & .078 \\
\hline & & 2 & 1 & -.019 & .041 & .650 & -.101 & .064 \\
\hline & & & 3 & -.008 & .038 & .839 & -.084 & .069 \\
\hline & & 3 & 1 & -.011 & .034 & .747 & -.078 & .057 \\
\hline & & & 2 & .008 & .038 & .839 & -.069 & .084 \\
\hline & 2.00 & 1 & 2 & .036 & .041 & .384 & -.046 & .118 \\
\hline
\end{tabular}




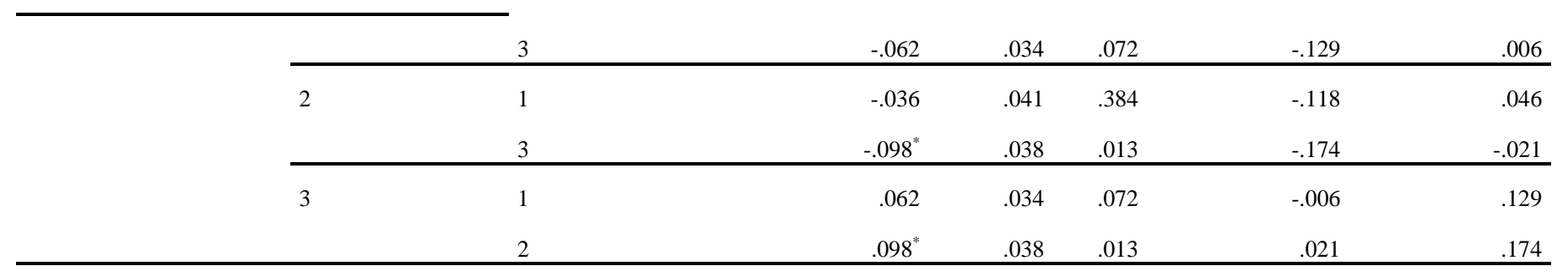

Based on estimated marginal means

a. Adjustment for multiple comparisons: Least Significant Difference (equivalent to no adjustments).

*. The mean difference is significant at the .05 level. 


\section{Table H10}

Mean Difference, Standard Error, P-Value and 95\% Confidence Interval for Group Effects by Incentive Condition

\begin{tabular}{|c|c|c|c|c|c|c|c|c|}
\hline & & & & Mean Difference & & & 95\% Confidence Inte & al for Difference ${ }^{a}$ \\
\hline Brain region & Incentive cond & (I) Group & (J) Group & $(\mathrm{I}-\mathrm{J})$ & Std. Error & Sig. ${ }^{\text {a }}$ & Lower Bound & Upper Bound \\
\hline \multirow[t]{6}{*}{ Brain region 1} & 1 & 1.00 & 2.00 & $-.145^{*}$ & .050 & .006 & -.246 & -.043 \\
\hline & & 2.00 & 1.00 & $.145^{*}$ & .050 & .006 & .043 & .246 \\
\hline & 2 & 1.00 & 2.00 & -.116 & .064 & .076 & -.245 & .013 \\
\hline & & 2.00 & 1.00 & .116 & .064 & .076 & -.013 & .245 \\
\hline & 3 & 1.00 & 2.00 & -.030 & .053 & .579 & -.137 & .078 \\
\hline & & 2.00 & 1.00 & . 030 & .053 & .579 & -.078 & .137 \\
\hline \multirow[t]{6}{*}{ Brain region 2} & 1 & 1.00 & 2.00 & -.079 & .046 & .093 & -.171 & .014 \\
\hline & & 2.00 & 1.00 & .079 & .046 & .093 & -.014 & .171 \\
\hline & 2 & 1.00 & 2.00 & -.063 & .064 & .337 & -.192 & .067 \\
\hline & & 2.00 & 1.00 & .063 & .064 & .337 & -.067 & .192 \\
\hline & 3 & 1.00 & 2.00 & -.049 & .070 & .486 & -.190 & .092 \\
\hline & & 2.00 & 1.00 & .049 & .070 & .486 & -.092 & .190 \\
\hline \multirow[t]{6}{*}{ Brain region 3} & 1 & 1.00 & 2.00 & -.073 & .043 & .094 & -.158 & .013 \\
\hline & & 2.00 & 1.00 & .073 & .043 & .094 & -.013 & .158 \\
\hline & 2 & 1.00 & 2.00 & -.061 & .042 & .154 & -.145 & .023 \\
\hline & & 2.00 & 1.00 & .061 & .042 & .154 & -.023 & .145 \\
\hline & 3 & 1.00 & 2.00 & -.021 & .043 & .630 & -.108 & .066 \\
\hline & & 2.00 & 1.00 & .021 & .043 & .630 & -.066 & .108 \\
\hline \multirow[t]{3}{*}{ Brain region 4} & 1 & 1.00 & 2.00 & -.062 & .050 & .226 & -.162 & .039 \\
\hline & & 2.00 & 1.00 & .062 & .050 & .226 & -.039 & .162 \\
\hline & 2 & 1.00 & 2.00 & -.033 & .068 & .627 & -.171 & .104 \\
\hline
\end{tabular}




\begin{tabular}{|c|c|c|c|c|c|c|c|c|}
\hline & & 2.00 & 1.00 & .033 & .068 & .627 & -.104 & .171 \\
\hline & 3 & 1.00 & 2.00 & -.014 & .071 & .844 & -.156 & .128 \\
\hline & & 2.00 & 1.00 & .014 & .071 & .844 & -.128 & .156 \\
\hline \multirow[t]{6}{*}{ Brain region 5} & 1 & 1.00 & 2.00 & -.056 & .048 & .254 & -.153 & .041 \\
\hline & & 2.00 & 1.00 & .056 & .048 & .254 & -.041 & .153 \\
\hline & 2 & 1.00 & 2.00 & -.033 & .046 & .474 & -.125 & .059 \\
\hline & & 2.00 & 1.00 & .033 & .046 & .474 & -.059 & .125 \\
\hline & 3 & 1.00 & 2.00 & .001 & .044 & .979 & -.086 & .089 \\
\hline & & 2.00 & 1.00 & -.001 & .044 & .979 & -.089 & .086 \\
\hline \multirow[t]{6}{*}{ Brain region 6} & 1 & 1.00 & 2.00 & -.074 & .039 & .068 & -.153 & .006 \\
\hline & & 2.00 & 1.00 & .074 & .039 & .068 & -.006 & .153 \\
\hline & 2 & 1.00 & 2.00 & -.069 & .040 & .090 & -.149 & .011 \\
\hline & & 2.00 & 1.00 & .069 & .040 & .090 & -.011 & .149 \\
\hline & 3 & 1.00 & 2.00 & -.010 & .037 & .791 & -.084 & .064 \\
\hline & & 2.00 & 1.00 & .010 & .037 & .791 & -.064 & .084 \\
\hline \multirow[t]{6}{*}{ Brain region 7} & 1 & 1.00 & 2.00 & $-.113^{*}$ & .040 & .006 & -.193 & -.034 \\
\hline & & 2.00 & 1.00 & $.113^{*}$ & .040 & .006 & .034 & .193 \\
\hline & 2 & 1.00 & 2.00 & $-.097^{*}$ & .042 & .025 & -.181 & -.013 \\
\hline & & 2.00 & 1.00 & $.097^{*}$ & .042 & .025 & .013 & .181 \\
\hline & 3 & 1.00 & 2.00 & -.048 & .043 & .271 & -.134 & .038 \\
\hline & & 2.00 & 1.00 & .048 & .043 & .271 & -.038 & .134 \\
\hline \multirow[t]{5}{*}{ Brain region 8} & 1 & 1.00 & 2.00 & -.043 & .049 & .379 & -.142 & .055 \\
\hline & & 2.00 & 1.00 & .043 & .049 & .379 & -.055 & .142 \\
\hline & 2 & 1.00 & 2.00 & .016 & .045 & .720 & -.075 & .107 \\
\hline & & 2.00 & 1.00 & -.016 & .045 & .720 & -.107 & .075 \\
\hline & 3 & 1.00 & 2.00 & .022 & .045 & .636 & -.069 & .113 \\
\hline
\end{tabular}




\begin{tabular}{|c|c|c|c|c|c|c|c|c|}
\hline & & 2.00 & 1.00 & -.022 & .045 & .636 & -.113 & .069 \\
\hline \multirow[t]{6}{*}{ Brain region 9} & 1 & 1.00 & 2.00 & -.065 & .053 & .229 & -.171 & .042 \\
\hline & & 2.00 & 1.00 & .065 & .053 & .229 & -.042 & .171 \\
\hline & 2 & 1.00 & 2.00 & .015 & .056 & .791 & -.099 & .129 \\
\hline & & 2.00 & 1.00 & -.015 & .056 & .791 & -.129 & .099 \\
\hline & 3 & 1.00 & 2.00 & -.021 & .051 & .680 & -.125 & .082 \\
\hline & & 2.00 & 1.00 & .021 & .051 & .680 & -.082 & .125 \\
\hline \multirow[t]{6}{*}{ Brain region 10} & 1 & 1.00 & 2.00 & -.080 & .040 & .051 & -.160 & .000 \\
\hline & & 2.00 & 1.00 & .080 & .040 & .051 & .000 & .160 \\
\hline & 2 & 1.00 & 2.00 & -.062 & .042 & .146 & -.146 & .022 \\
\hline & & 2.00 & 1.00 & .062 & .042 & .146 & -.022 & .146 \\
\hline & 3 & 1.00 & 2.00 & -.038 & .038 & .321 & -.115 & .039 \\
\hline & & 2.00 & 1.00 & .038 & .038 & .321 & -.039 & .115 \\
\hline \multirow[t]{6}{*}{ Brain region 11} & 1 & 1.00 & 2.00 & -.051 & .025 & .052 & -.102 & .001 \\
\hline & & 2.00 & 1.00 & .051 & .025 & .052 & -.001 & .102 \\
\hline & 2 & 1.00 & 2.00 & -.003 & .027 & .897 & -.057 & .050 \\
\hline & & 2.00 & 1.00 & .003 & .027 & .897 & -.050 & .057 \\
\hline & 3 & 1.00 & 2.00 & .034 & .031 & .268 & -.027 & .096 \\
\hline & & 2.00 & 1.00 & -.034 & .031 & .268 & -.096 & .027 \\
\hline \multirow[t]{6}{*}{ Brain region 12} & 1 & 1.00 & 2.00 & -.050 & .054 & .358 & -.160 & .059 \\
\hline & & 2.00 & 1.00 & .050 & .054 & .358 & -.059 & .160 \\
\hline & 2 & 1.00 & 2.00 & .017 & .053 & .756 & -.091 & .124 \\
\hline & & 2.00 & 1.00 & -.017 & .053 & .756 & -.124 & .091 \\
\hline & 3 & 1.00 & 2.00 & -.018 & .055 & .744 & -.128 & .092 \\
\hline & & 2.00 & 1.00 & .018 & .055 & .744 & -.092 & .128 \\
\hline Brain region 13 & 1 & 1.00 & 2.00 & -.045 & .040 & .270 & -.126 & .036 \\
\hline
\end{tabular}




\begin{tabular}{lcccccccc}
\hline & 2.00 & 1.00 & .045 & .040 & .270 & -.036 & .126 \\
\cline { 2 - 8 } & 1.00 & 2.00 & -.028 & .046 & .547 & -.119 & .064 \\
\cline { 2 - 8 } & 2.00 & 1.00 & .028 & .046 & .547 & -.064 & .119 \\
\cline { 2 - 8 } & 1.00 & 2.00 & $-.118^{*}$ & .041 & .006 & -.200 & -.036 \\
\cline { 2 - 8 } & 2.00 & 1.00 & $.118^{*}$ & .041 & .006 & .036 & .200 \\
\hline
\end{tabular}

Based on estimated marginal means

*. The mean difference is significant at the .05 level.

a. Adjustment for multiple comparisons: Least Significant Difference (equivalent to no adjustments). 


\section{Table H11}

Means, Standard Errors and 95\% Confidence Intervals for Group x Accuracy Effects

\begin{tabular}{|c|c|c|c|c|c|c|}
\hline \multirow[b]{2}{*}{ Brain region } & \multirow[b]{2}{*}{ Group } & \multirow[b]{2}{*}{ Accuracy } & \multirow[b]{2}{*}{ Mean } & \multirow[b]{2}{*}{ Std. Error } & \multicolumn{2}{|c|}{ 95\% Confidence Interval } \\
\hline & & & & & Lower Bound & Upper Bound \\
\hline \multirow[t]{4}{*}{ Brain region 1} & 1.00 & 1 & -.246 & .035 & -.316 & -.177 \\
\hline & & 2 & -.250 & .040 & -.331 & -.168 \\
\hline & 2.00 & 1 & -.132 & .035 & -.202 & -.063 \\
\hline & & 2 & -.169 & .040 & -.250 & -.088 \\
\hline \multirow[t]{4}{*}{ Brain region 2} & 1.00 & 1 & .155 & .041 & .074 & .237 \\
\hline & & 2 & .181 & .039 & .102 & .260 \\
\hline & 2.00 & 1 & .245 & .041 & .163 & .327 \\
\hline & & 2 & .218 & .039 & .139 & .298 \\
\hline \multirow[t]{4}{*}{ Brain region 3} & 1.00 & 1 & -.154 & .026 & -.207 & -.101 \\
\hline & & 2 & -.187 & .034 & -.255 & -.119 \\
\hline & 2.00 & 1 & -.103 & .026 & -.156 & -.051 \\
\hline & & 2 & -.135 & .034 & -.202 & -.067 \\
\hline \multirow[t]{4}{*}{ Brain region 4} & 1.00 & 1 & .174 & .043 & .088 & .260 \\
\hline & & 2 & .192 & .043 & .106 & .278 \\
\hline & 2.00 & 1 & .236 & .043 & .150 & .323 \\
\hline & & 2 & .202 & .043 & .116 & .288 \\
\hline \multirow[t]{4}{*}{ Brain region 5} & 1.00 & 1 & -.158 & .029 & -.217 & -.099 \\
\hline & & 2 & -.157 & .030 & -.217 & -.096 \\
\hline & 2.00 & 1 & -.115 & .029 & -.175 & -.056 \\
\hline & & 2 & -.140 & .030 & -.201 & -.080 \\
\hline Brain region 6 & 1.00 & 1 & -.138 & .023 & -.186 & -.091 \\
\hline
\end{tabular}




\begin{tabular}{|c|c|c|c|c|c|c|}
\hline & & 2 & -.127 & .025 & -.177 & -.076 \\
\hline & 2.00 & 1 & -.071 & .023 & -.118 & -.024 \\
\hline & & 2 & -.092 & .025 & -.143 & -.042 \\
\hline \multirow[t]{4}{*}{ Brain region 7} & 1.00 & 1 & -.170 & .029 & -.228 & -.112 \\
\hline & & 2 & -.130 & .027 & -.183 & -.076 \\
\hline & 2.00 & 1 & -.058 & .029 & -.116 & .001 \\
\hline & & 2 & -.070 & .027 & -.124 & -.017 \\
\hline \multirow[t]{4}{*}{ Brain region 8} & 1.00 & 1 & -.108 & .027 & -.163 & -.053 \\
\hline & & 2 & -.111 & .031 & -.172 & -.050 \\
\hline & 2.00 & 1 & -.078 & .027 & -.134 & -.023 \\
\hline & & 2 & -.137 & .031 & -.198 & -.076 \\
\hline \multirow[t]{4}{*}{ Brain region 9} & 1.00 & 1 & -.132 & .037 & -.206 & -.058 \\
\hline & & 2 & -.151 & .036 & -.225 & -.078 \\
\hline & 2.00 & 1 & -.070 & .037 & -.144 & .004 \\
\hline & & 2 & -.166 & .036 & -.240 & -.093 \\
\hline \multirow[t]{4}{*}{ Brain region 10} & 1.00 & 1 & -.146 & .024 & -.195 & -.097 \\
\hline & & 2 & -.126 & .026 & -.179 & -.074 \\
\hline & 2.00 & 1 & -.067 & .024 & -.116 & -.018 \\
\hline & & 2 & -.085 & .026 & -.138 & -.033 \\
\hline \multirow[t]{4}{*}{ Brain region 11} & 1.00 & 1 & -.087 & .018 & -.122 & -.051 \\
\hline & & 2 & -.052 & .021 & -.094 & -.010 \\
\hline & 2.00 & 1 & -.068 & .018 & -.104 & -.033 \\
\hline & & 2 & -.058 & .021 & -.099 & -.016 \\
\hline \multirow[t]{3}{*}{ Brain region 12} & 1.00 & 1 & -.136 & .036 & -.209 & -.063 \\
\hline & & 2 & -.155 & .036 & -.228 & -.082 \\
\hline & 2.00 & 1 & -.081 & .036 & -.153 & -.008 \\
\hline
\end{tabular}




\begin{tabular}{|c|c|c|c|c|c|c|}
\hline & & 2 & -.176 & .036 & -.250 & -.103 \\
\hline \multirow[t]{4}{*}{ Brain region 13} & 1.00 & 1 & -.118 & .024 & -.166 & -.070 \\
\hline & & 2 & -.139 & .025 & -.189 & -.089 \\
\hline & 2.00 & 1 & -.040 & .024 & -.088 & .008 \\
\hline & & 2 & -.090 & .025 & -.140 & -.040 \\
\hline
\end{tabular}




\section{Table H12}

Mean Difference, Standard Error, P-Value and 95\% Confidence Interval for Accuracy Effects by Group

\begin{tabular}{|c|c|c|c|c|c|c|c|c|}
\hline \multirow[b]{2}{*}{ Brain region } & \multirow[b]{2}{*}{ Group } & \multirow[b]{2}{*}{ (I) Accuracy } & \multirow[b]{2}{*}{ (J) Accuracy } & \multirow{2}{*}{$\begin{array}{c}\text { Mean Difference } \\
(\mathrm{I}-\mathrm{J}) \\
\end{array}$} & \multirow[b]{2}{*}{ Std. Error } & \multirow[b]{2}{*}{ Sig. ${ }^{\text {a }}$} & \multicolumn{2}{|c|}{ 95\% Confidence Interval for Difference } \\
\hline & & & & & & & Lower Bound & Upper Bound \\
\hline \multirow{4}{*}{ Brain region 1} & 1.00 & 1 & 2 & .003 & .026 & .895 & -.048 & .055 \\
\hline & & 2 & 1 & -.003 & .026 & .895 & -.055 & .048 \\
\hline & 2.00 & 1 & 2 & .037 & .026 & .157 & -.015 & .088 \\
\hline & & 2 & 1 & -.037 & .026 & .157 & -.088 & .015 \\
\hline \multirow[t]{4}{*}{ Brain region 2} & 1.00 & 1 & 2 & -.026 & .020 & .208 & -.066 & .015 \\
\hline & & 2 & 1 & .026 & .020 & .208 & -.015 & .066 \\
\hline & 2.00 & 1 & 2 & .026 & .020 & .198 & -.014 & .067 \\
\hline & & 2 & 1 & -.026 & .020 & .198 & -.067 & .014 \\
\hline \multirow[t]{4}{*}{ Brain region 3} & 1.00 & 1 & 2 & .033 & .032 & .300 & -.030 & .097 \\
\hline & & 2 & 1 & -.033 & .032 & .300 & -.097 & .030 \\
\hline & 2.00 & 1 & 2 & .031 & .032 & .326 & -.032 & .095 \\
\hline & & 2 & 1 & -.031 & .032 & .326 & -.095 & .032 \\
\hline \multirow[t]{4}{*}{ Brain region 4} & 1.00 & 1 & 2 & -.018 & .021 & .389 & -.061 & .024 \\
\hline & & 2 & 1 & .018 & .021 & .389 & -.024 & .061 \\
\hline & 2.00 & 1 & 2 & .034 & .021 & .116 & -.009 & .077 \\
\hline & & 2 & 1 & -.034 & .021 & .116 & -.077 & .009 \\
\hline \multirow[t]{4}{*}{ Brain region 5} & 1.00 & 1 & 2 & -.001 & .021 & .959 & -.044 & .042 \\
\hline & & 2 & 1 & .001 & .021 & .959 & -.042 & .044 \\
\hline & 2.00 & 1 & 2 & .025 & .021 & .247 & -.018 & .068 \\
\hline & & 2 & 1 & -.025 & .021 & .247 & -.068 & .018 \\
\hline Brain region 6 & 1.00 & 1 & 2 & -.012 & .022 & .597 & -.056 & .032 \\
\hline
\end{tabular}




\begin{tabular}{|c|c|c|c|c|c|c|c|c|}
\hline & & 2 & 1 & .012 & .022 & .597 & -.032 & .056 \\
\hline & 2.00 & 1 & 2 & .021 & .022 & .337 & -.023 & .065 \\
\hline & & 2 & 1 & -.021 & .022 & .337 & -.065 & .023 \\
\hline \multirow[t]{4}{*}{ Brain region 7} & 1.00 & 1 & 2 & -.040 & .024 & .102 & -.089 & .008 \\
\hline & & 2 & 1 & .040 & .024 & .102 & -.008 & .089 \\
\hline & 2.00 & 1 & 2 & .013 & .024 & .605 & -.036 & .061 \\
\hline & & 2 & 1 & -.013 & .024 & .605 & -.061 & .036 \\
\hline \multirow[t]{4}{*}{ Brain region 8} & 1.00 & 1 & 2 & .003 & .023 & .895 & -.044 & .050 \\
\hline & & 2 & 1 & -.003 & .023 & .895 & -.050 & .044 \\
\hline & 2.00 & 1 & 2 & $.059^{*}$ & .023 & .015 & .012 & .106 \\
\hline & & 2 & 1 & $-.059^{*}$ & .023 & .015 & -.106 & -.012 \\
\hline \multirow[t]{4}{*}{ Brain region 9} & 1.00 & 1 & 2 & .019 & .034 & .575 & -.049 & .088 \\
\hline & & 2 & 1 & -.019 & .034 & .575 & -.088 & .049 \\
\hline & 2.00 & 1 & 2 & $.096^{*}$ & .034 & .007 & .027 & .165 \\
\hline & & 2 & 1 & $-.096^{*}$ & .034 & .007 & -.165 & -.027 \\
\hline \multirow[t]{4}{*}{ Brain region 10} & 1.00 & 1 & 2 & -.019 & .019 & .309 & -.057 & .018 \\
\hline & & 2 & 1 & .019 & .019 & .309 & -.018 & .057 \\
\hline & 2.00 & 1 & 2 & .018 & .019 & .331 & -.019 & .056 \\
\hline & & 2 & 1 & -.018 & .019 & .331 & -.056 & .019 \\
\hline \multirow[t]{4}{*}{ Brain region 11} & 1.00 & 1 & 2 & -.035 & .019 & .080 & -.074 & .004 \\
\hline & & 2 & 1 & .035 & .019 & .080 & -.004 & .074 \\
\hline & 2.00 & 1 & 2 & -.011 & .019 & .588 & -.050 & .029 \\
\hline & & 2 & 1 & .011 & .019 & .588 & -.029 & .050 \\
\hline \multirow[t]{3}{*}{ Brain region 12} & 1.00 & 1 & 2 & .019 & .026 & .479 & -.034 & .072 \\
\hline & & 2 & 1 & -.019 & .026 & .479 & -.072 & .034 \\
\hline & 2.00 & 1 & 2 & $.096^{*}$ & .026 & .001 & .043 & .149 \\
\hline
\end{tabular}




\begin{tabular}{lccccccccc}
\hline & 2 & 1 & $-.096^{*}$ & .026 & .001 & -.149 & -.043 \\
\hline \multirow{2}{*}{ Brain region 13 } & \multirow{2}{*}{1.00} & 1 & 2 & .021 & .026 & .428 & -.032 & .074 \\
\cline { 2 - 9 } & & 2 & 1 & -.021 & .026 & .428 & -.074 & .032 \\
\cline { 2 - 9 } & \multirow{2}{*}{2.00} & 1 & 2 & .050 & .026 & .063 & -.003 & .103 \\
\cline { 2 - 8 } & 2 & 1 & -.050 & .026 & .063 & -.103 & .003 \\
\hline
\end{tabular}

Based on estimated marginal means

a. Adjustment for multiple comparisons: Least Significant Difference (equivalent to no adjustments)

*. The mean difference is significant at the .05 level. 


\section{Table H13}

\section{Mean Difference, Standard Error, P-Value and 95\% Confidence Interval for Group Effects by Accuracy}

\begin{tabular}{|c|c|c|c|c|c|c|c|c|}
\hline \multirow[b]{2}{*}{ Brain region } & \multirow[b]{2}{*}{ Accuracy } & \multirow[b]{2}{*}{ (I) Group } & \multirow[b]{2}{*}{ (J) Group } & \multirow{2}{*}{$\begin{array}{c}\text { Mean Difference } \\
(\mathrm{I}-\mathrm{J}) \\
\end{array}$} & \multirow[b]{2}{*}{ Std. Error } & \multirow[b]{2}{*}{ Sig. ${ }^{a}$} & \multicolumn{2}{|c|}{ 95\% Confidence Interval for Difference ${ }^{a}$} \\
\hline & & & & & & & Lower Bound & Upper Bound \\
\hline \multirow[t]{4}{*}{ Brain region 1} & 1 & 1.00 & 2.00 & $-.114^{*}$ & .049 & .024 & -.212 & -.015 \\
\hline & & 2.00 & 1.00 & $.114^{*}$ & .049 & .024 & .015 & .212 \\
\hline & 2 & 1.00 & 2.00 & -.080 & .057 & .166 & -.195 & .034 \\
\hline & & 2.00 & 1.00 & .080 & .057 & .166 & -.034 & .195 \\
\hline \multirow[t]{4}{*}{ Brain region 2} & 1 & 1.00 & 2.00 & -.089 & .057 & .126 & -.205 & .026 \\
\hline & & 2.00 & 1.00 & .089 & .057 & .126 & -.026 & .205 \\
\hline & 2 & 1.00 & 2.00 & -.037 & .056 & .505 & -.150 & .075 \\
\hline & & 2.00 & 1.00 & .037 & .056 & .505 & -.075 & .150 \\
\hline \multirow[t]{4}{*}{ Brain region 3} & 1 & 1.00 & 2.00 & -.051 & .037 & .180 & -.125 & .024 \\
\hline & & 2.00 & 1.00 & .051 & .037 & .180 & -.024 & .125 \\
\hline & 2 & 1.00 & 2.00 & -.052 & .048 & .277 & -.148 & .043 \\
\hline & & 2.00 & 1.00 & .052 & .048 & .277 & -.043 & .148 \\
\hline \multirow[t]{4}{*}{ Brain region 4} & 1 & 1.00 & 2.00 & -.063 & .061 & .307 & -.184 & .059 \\
\hline & & 2.00 & 1.00 & .063 & .061 & .307 & -.059 & .184 \\
\hline & 2 & 1.00 & 2.00 & -.010 & .060 & .868 & -.132 & .111 \\
\hline & & 2.00 & 1.00 & .010 & .060 & .868 & -.111 & .132 \\
\hline \multirow[t]{4}{*}{ Brain region 5} & 1 & 1.00 & 2.00 & -.042 & .042 & .314 & -.126 & .041 \\
\hline & & 2.00 & 1.00 & .042 & .042 & .314 & -.041 & .126 \\
\hline & 2 & 1.00 & 2.00 & -.016 & .043 & .705 & -.102 & .070 \\
\hline & & 2.00 & 1.00 & .016 & .043 & .705 & -.070 & .102 \\
\hline Brain region 6 & 1 & 1.00 & 2.00 & $-.067^{*}$ & .033 & .048 & -.134 & -.001 \\
\hline
\end{tabular}




\begin{tabular}{|c|c|c|c|c|c|c|c|c|}
\hline & & 2.00 & 1.00 & $.067^{*}$ & .033 & .048 & .001 & .134 \\
\hline & 2 & 1.00 & 2.00 & -.034 & .036 & .341 & -.106 & .037 \\
\hline & & 2.00 & 1.00 & .034 & .036 & .341 & -.037 & .106 \\
\hline \multirow[t]{4}{*}{ Brain region 7} & 1 & 1.00 & 2.00 & $-.113^{*}$ & .041 & .009 & -.195 & -.030 \\
\hline & & 2.00 & 1.00 & $.113^{*}$ & .041 & .009 & .030 & .195 \\
\hline & 2 & 1.00 & 2.00 & -.059 & .038 & .121 & -.135 & .016 \\
\hline & & 2.00 & 1.00 & .059 & .038 & .121 & -.016 & .135 \\
\hline \multirow[t]{4}{*}{ Brain region 8} & 1 & 1.00 & 2.00 & -.030 & .039 & .448 & -.108 & .048 \\
\hline & & 2.00 & 1.00 & .030 & .039 & .448 & -.048 & .108 \\
\hline & 2 & 1.00 & 2.00 & .026 & .043 & .549 & -.061 & .113 \\
\hline & & 2.00 & 1.00 & -.026 & .043 & .549 & -.113 & .061 \\
\hline \multirow[t]{4}{*}{ Brain region 9} & 1 & 1.00 & 2.00 & -.062 & .052 & .237 & -.166 & .042 \\
\hline & & 2.00 & 1.00 & .062 & .052 & .237 & -.042 & .166 \\
\hline & 2 & 1.00 & 2.00 & .015 & .052 & .776 & -.089 & .119 \\
\hline & & 2.00 & 1.00 & -.015 & .052 & .776 & -.119 & .089 \\
\hline \multirow[t]{4}{*}{ Brain region 10} & 1 & 1.00 & 2.00 & $-.079^{*}$ & .034 & .027 & -.148 & -.010 \\
\hline & & 2.00 & 1.00 & $.079^{*}$ & .034 & .027 & .010 & .148 \\
\hline & 2 & 1.00 & 2.00 & -.041 & .037 & .269 & -.115 & .033 \\
\hline & & 2.00 & 1.00 & .041 & .037 & .269 & -.033 & .115 \\
\hline \multirow[t]{4}{*}{ Brain region 11} & 1 & 1.00 & 2.00 & -.019 & .025 & .458 & -.069 & .032 \\
\hline & & 2.00 & 1.00 & .019 & .025 & .458 & -.032 & .069 \\
\hline & 2 & 1.00 & 2.00 & .006 & .029 & .850 & -.053 & .065 \\
\hline & & 2.00 & 1.00 & -.006 & .029 & .850 & -.065 & .053 \\
\hline \multirow[t]{3}{*}{ Brain region 12} & 1 & 1.00 & 2.00 & -.056 & .051 & .282 & -.159 & .047 \\
\hline & & 2.00 & 1.00 & .056 & .051 & .282 & -.047 & .159 \\
\hline & 2 & 1.00 & 2.00 & .021 & .052 & .683 & -.083 & .125 \\
\hline
\end{tabular}




\begin{tabular}{lcccccccc}
\hline & & 2.00 & 1.00 & -.021 & .052 & .683 & -.125 & .083 \\
\hline \multirow{2}{*}{ Brain region 13 } & 1 & 1.00 & 2.00 & $-.078^{*}$ & .034 & .026 & -.146 & -.010 \\
\cline { 2 - 8 } & 2.00 & 1.00 & $.078^{*}$ & .034 & .026 & .010 & .146 \\
\cline { 2 - 8 } & \multirow{2}{*}{2} & 1.00 & 2.00 & -.049 & .035 & .170 & -.120 & .022 \\
\cline { 2 - 8 } & 2.00 & 1.00 & .049 & .035 & .170 & -.022 & .120 \\
\hline
\end{tabular}

Based on estimated marginal means

*. The mean difference is significant at the .05 level.

a. Adjustment for multiple comparisons: Least Significant Difference (equivalent to no adjustments). 


\section{Table H14}

Means, Standard Errors and 95\% Confidence Intervals for Incentive Condition x Accuracy Effects

\begin{tabular}{|c|c|c|c|c|c|c|}
\hline \multirow[b]{2}{*}{ Brain region } & \multirow[b]{2}{*}{ Incentive cond } & \multirow[b]{2}{*}{ Accuracy } & \multirow[b]{2}{*}{ Mean } & \multirow[b]{2}{*}{ Std. Error } & \multicolumn{2}{|c|}{ 95\% Confidence Interval } \\
\hline & & & & & Lower Bound & Upper Bound \\
\hline \multirow[t]{6}{*}{ Brain region 1} & 1 & 1 & -.203 & .028 & -.260 & -.146 \\
\hline & & 2 & -.251 & .030 & -.312 & -.191 \\
\hline & 2 & 1 & -.219 & .029 & -.277 & -.161 \\
\hline & & 2 & -.230 & .043 & -.317 & -.143 \\
\hline & 3 & 1 & -.146 & .025 & -.197 & -.095 \\
\hline & & 2 & -.147 & .033 & -.214 & -.080 \\
\hline \multirow[t]{6}{*}{ Brain region 2} & 1 & 1 & .050 & .028 & -.006 & .106 \\
\hline & & 2 & .024 & .025 & -.026 & .074 \\
\hline & 2 & 1 & .258 & .033 & .192 & .325 \\
\hline & & 2 & .291 & .036 & .218 & .364 \\
\hline & 3 & 1 & .292 & .036 & .220 & .363 \\
\hline & & 2 & .284 & .038 & .207 & .362 \\
\hline \multirow[t]{6}{*}{ Brain region 3} & 1 & 1 & -.147 & .021 & -.189 & -.105 \\
\hline & & 2 & -.161 & .030 & -.221 & -.100 \\
\hline & 2 & 1 & -.132 & .022 & -.177 & -.088 \\
\hline & & 2 & -.190 & .031 & -.253 & -.128 \\
\hline & 3 & 1 & -.107 & .025 & -.157 & -.057 \\
\hline & & 2 & -.132 & .028 & -.188 & -.075 \\
\hline \multirow[t]{3}{*}{ Brain region 4} & 1 & 1 & .069 & .030 & .010 & .129 \\
\hline & & 2 & .038 & .028 & -.017 & .094 \\
\hline & 2 & 1 & .260 & .035 & .190 & .330 \\
\hline
\end{tabular}




\begin{tabular}{|c|c|c|c|c|c|c|}
\hline & & 2 & .272 & .037 & .198 & .347 \\
\hline & 3 & 1 & .286 & .035 & .216 & .357 \\
\hline & & 2 & .281 & .038 & .204 & .358 \\
\hline \multirow[t]{6}{*}{ Brain region 5} & 1 & 1 & -.130 & .024 & -.178 & -.082 \\
\hline & & 2 & -.152 & .029 & -.210 & -.094 \\
\hline & 2 & 1 & -.182 & .023 & -.229 & -.135 \\
\hline & & 2 & -.170 & .029 & -.228 & -.112 \\
\hline & 3 & 1 & -.098 & .025 & -.149 & -.048 \\
\hline & & 2 & -.124 & .028 & -.179 & -.068 \\
\hline \multirow[t]{6}{*}{ Brain region 6} & 1 & 1 & -.092 & .020 & -.131 & -.053 \\
\hline & & 2 & -.100 & .028 & -.157 & -.043 \\
\hline & 2 & 1 & -.131 & .022 & -.175 & -.087 \\
\hline & & 2 & -.122 & .025 & -.172 & -.072 \\
\hline & 3 & 1 & -.091 & .020 & -.131 & -.051 \\
\hline & & 2 & -.107 & .023 & -.154 & -.060 \\
\hline \multirow[t]{6}{*}{ Brain region 7} & 1 & 1 & -.099 & .024 & -.148 & -.051 \\
\hline & & 2 & -.124 & .022 & -.169 & -.079 \\
\hline & 2 & 1 & -.140 & .024 & -.189 & -.092 \\
\hline & & 2 & -.095 & .026 & -.146 & -.043 \\
\hline & 3 & 1 & -.102 & .025 & -.153 & -.051 \\
\hline & & 2 & -.081 & .027 & -.135 & -.027 \\
\hline \multirow[t]{5}{*}{ Brain region 8} & 1 & 1 & -.100 & .024 & -.148 & -.052 \\
\hline & & 2 & -.125 & .032 & -.189 & -.061 \\
\hline & 2 & 1 & -.110 & .025 & -.160 & -.060 \\
\hline & & 2 & -.158 & .036 & -.230 & -.086 \\
\hline & 3 & 1 & -.069 & .023 & -.116 & -.022 \\
\hline
\end{tabular}




\begin{tabular}{|c|c|c|c|c|c|c|}
\hline & & 2 & -.089 & .028 & -.145 & -.033 \\
\hline \multirow[t]{6}{*}{ Brain region 9} & 1 & 1 & -.096 & .030 & -.156 & -.036 \\
\hline & & 2 & -.158 & .030 & -.218 & -.099 \\
\hline & 2 & 1 & -.136 & .025 & -.187 & -.086 \\
\hline & & 2 & -.204 & .045 & -.294 & -.114 \\
\hline & 3 & 1 & -.071 & .032 & -.135 & -.006 \\
\hline & & 2 & -.113 & .032 & -.178 & -.049 \\
\hline \multirow[t]{6}{*}{ Brain region 10} & 1 & 1 & -.119 & .024 & -.166 & -.071 \\
\hline & & 2 & -.119 & .025 & -.169 & -.069 \\
\hline & 2 & 1 & -.092 & .022 & -.136 & -.048 \\
\hline & & 2 & -.116 & .026 & -.169 & -.063 \\
\hline & 3 & 1 & -.108 & .021 & -.150 & -.066 \\
\hline & & 2 & -.082 & .024 & -.129 & -.035 \\
\hline \multirow[t]{6}{*}{ Brain region 11} & 1 & 1 & -.068 & .015 & -.098 & -.039 \\
\hline & & 2 & -.055 & .020 & -.096 & -.015 \\
\hline & 2 & 1 & -.077 & .016 & -.108 & -.045 \\
\hline & & 2 & -.056 & .017 & -.091 & -.021 \\
\hline & 3 & 1 & -.087 & .017 & -.121 & -.054 \\
\hline & & 2 & -.053 & .020 & -.092 & -.013 \\
\hline \multirow[t]{6}{*}{ Brain region 12} & 1 & 1 & -.118 & .028 & -.174 & -.062 \\
\hline & & 2 & -.187 & .033 & -.253 & -.120 \\
\hline & 2 & 1 & -.133 & .031 & -.195 & -.070 \\
\hline & & 2 & -.192 & .029 & -.250 & -.133 \\
\hline & 3 & 1 & -.075 & .032 & -.138 & -.011 \\
\hline & & 2 & -.119 & .035 & -.190 & -.048 \\
\hline Brain region 13 & 1 & 1 & -.060 & .021 & -.102 & -.018 \\
\hline
\end{tabular}




\begin{tabular}{|c|c|c|c|c|c|}
\hline & 2 & -.132 & 年. & -.184 & -.080 \\
\hline \multirow[t]{2}{*}{2} & 1 & -.113 & . 024. & -.162 & -.064 \\
\hline & 2 & -.134 & .037. & -209 & -.060 \\
\hline \multirow[t]{2}{*}{3} & 1 & -.064 & . 023. & -.110 & -.018 \\
\hline & 2 & -.078 & .030 & -.138 & -.017 \\
\hline
\end{tabular}




\section{Table H15}

Mean Difference, Standard Error, P-Value and 95\% Confidence Interval for Incentive Condition Effects by Accuracy

\begin{tabular}{|c|c|c|c|c|c|c|c|c|}
\hline \multirow[t]{2}{*}{ Brain region } & \multirow[t]{2}{*}{ Accuracy } & \multirow[t]{2}{*}{ (I) Incentive cond } & \multirow[t]{2}{*}{ (J) Incentive cond } & \multirow{2}{*}{$\begin{array}{c}\text { Mean Difference } \\
(\mathrm{I}-\mathrm{J}) \\
\end{array}$} & \multirow[b]{2}{*}{ Std. Error } & \multirow[b]{2}{*}{ Sig. ${ }^{a}$} & \multicolumn{2}{|c|}{ 95\% Confidence Interval for Difference } \\
\hline & & & & & & & Lower Bound & Upper Bound \\
\hline \multirow[t]{12}{*}{ Brain region 1} & 1 & 1 & 2 & .016 & .023 & .469 & -.029 & .062 \\
\hline & & & 3 & $-.057^{*}$ & .022 & .013 & -.101 & -.013 \\
\hline & & 2 & 1 & -.016 & .023 & .469 & -.062 & .029 \\
\hline & & & 3 & $-.073^{*}$ & .022 & .002 & -.118 & -.029 \\
\hline & & 3 & 1 & $.057^{*}$ & .022 & .013 & .013 & .101 \\
\hline & & & 2 & $.073^{*}$ & .022 & .002 & .029 & .118 \\
\hline & 2 & 1 & 2 & -.021 & .043 & .623 & -.109 & .066 \\
\hline & & & 3 & $-.104^{*}$ & .037 & .008 & -.179 & -.029 \\
\hline & & 2 & 1 & .021 & .043 & .623 & -.066 & .109 \\
\hline & & & 3 & $-.083^{*}$ & .033 & .016 & -.149 & -.016 \\
\hline & & 3 & 1 & $.104^{*}$ & .037 & .008 & .029 & .179 \\
\hline & & & 2 & $.083^{*}$ & .033 & .016 & .016 & .149 \\
\hline \multirow[t]{9}{*}{ Brain region 2} & 1 & 1 & 2 & $-.208^{*}$ & .026 & .000 & -.260 & -.156 \\
\hline & & & 3 & $-.242^{*}$ & .029 & .000 & -.300 & -.183 \\
\hline & & 2 & 1 & $.208^{*}$ & .026 & .000 & .156 & .260 \\
\hline & & & 3 & -.033 & .021 & .118 & -.075 & .009 \\
\hline & & 3 & 1 & $.242^{*}$ & .029 & .000 & .183 & .300 \\
\hline & & & 2 & .033 & .021 & .118 & -.009 & .075 \\
\hline & 2 & 1 & 2 & $-.267^{*}$ & .037 & .000 & -.341 & -.192 \\
\hline & & & 3 & $-.260^{*}$ & .033 & .000 & -.326 & -.195 \\
\hline & & 2 & 1 & $.267^{*}$ & .037 & .000 & .192 & .341 \\
\hline
\end{tabular}




\begin{tabular}{|c|c|c|c|c|c|c|c|c|}
\hline & & & 3 & .006 & .028 & .821 & -.051 & .064 \\
\hline & & 3 & 1 & $.260^{*}$ & .033 & .000 & .195 & .326 \\
\hline & & & 2 & -.006 & .028 & .821 & -.064 & .051 \\
\hline \multirow[t]{12}{*}{ Brain region 3} & 1 & 1 & 2 & -.015 & .020 & .450 & -.054 & .024 \\
\hline & & & 3 & -.040 & .025 & .114 & -.090 & .010 \\
\hline & & 2 & 1 & .015 & .020 & .450 & -.024 & .054 \\
\hline & & & 3 & -.025 & .023 & .287 & -.072 & .022 \\
\hline & & 3 & 1 & .040 & .025 & .114 & -.010 & .090 \\
\hline & & & 2 & .025 & .023 & .287 & -.022 & .072 \\
\hline & 2 & 1 & 2 & .030 & .035 & .395 & -.040 & .100 \\
\hline & & & 3 & -.029 & .031 & .361 & -.092 & .034 \\
\hline & & 2 & 1 & -.030 & .035 & .395 & -.100 & .040 \\
\hline & & & 3 & $-.059^{*}$ & .026 & .029 & -.111 & -.006 \\
\hline & & 3 & 1 & .029 & .031 & .361 & -.034 & .092 \\
\hline & & & 2 & $.059^{*}$ & .026 & .029 & .006 & .111 \\
\hline \multirow[t]{11}{*}{ Brain region 4} & 1 & 1 & 2 & $-.191^{*}$ & .026 & .000 & -.243 & -.138 \\
\hline & & & 3 & $-.217^{*}$ & .026 & .000 & -.270 & -.164 \\
\hline & & 2 & 1 & $.191^{*}$ & .026 & .000 & .138 & .243 \\
\hline & & & 3 & -.027 & .019 & .156 & -.064 & .011 \\
\hline & & 3 & 1 & $.217^{*}$ & .026 & .000 & .164 & .270 \\
\hline & & & 2 & .027 & .019 & .156 & -.011 & .064 \\
\hline & 2 & 1 & 2 & $-.234^{*}$ & .032 & .000 & -.299 & -.169 \\
\hline & & & 3 & $-.243^{*}$ & .029 & .000 & -.302 & -.184 \\
\hline & & 2 & 1 & $.234^{*}$ & .032 & .000 & .169 & .299 \\
\hline & & & 3 & -.009 & .026 & .728 & -.061 & .043 \\
\hline & & 3 & 1 & $.243^{*}$ & .029 & .000 & .184 & .302 \\
\hline
\end{tabular}




\begin{tabular}{|c|c|c|c|c|c|c|c|c|}
\hline & & & 2 & .009 & .026 & .728 & -.043 & .061 \\
\hline \multirow[t]{12}{*}{ Brain region 5} & 1 & 1 & 2 & $.052^{*}$ & .020 & .013 & .011 & .092 \\
\hline & & & 3 & -.032 & .022 & .149 & -.076 & .012 \\
\hline & & 2 & 1 & $-.052^{*}$ & .020 & .013 & -.092 & -.011 \\
\hline & & & 3 & $-.084^{*}$ & .021 & .000 & -.126 & -.042 \\
\hline & & 3 & 1 & .032 & .022 & .149 & -.012 & .076 \\
\hline & & & 2 & $.084^{*}$ & .021 & .000 & .042 & .126 \\
\hline & 2 & 1 & 2 & .018 & .034 & .593 & -.050 & .087 \\
\hline & & & 3 & -.028 & .033 & .394 & -.095 & .038 \\
\hline & & 2 & 1 & -.018 & .034 & .593 & -.087 & .050 \\
\hline & & & 3 & -.047 & .031 & .134 & -.108 & .015 \\
\hline & & 3 & 1 & .028 & .033 & .394 & -.038 & .095 \\
\hline & & & 2 & .047 & .031 & .134 & -.015 & .108 \\
\hline \multirow[t]{12}{*}{ Brain region 6} & 1 & 1 & 2 & $.039^{*}$ & .019 & .047 & .001 & .078 \\
\hline & & & 3 & -.001 & .022 & .974 & -.045 & .043 \\
\hline & & 2 & 1 & $-.039^{*}$ & .019 & .047 & -.078 & -.001 \\
\hline & & & 3 & -.040 & .021 & .065 & -.082 & .003 \\
\hline & & 3 & 1 & .001 & .022 & .974 & -.043 & .045 \\
\hline & & & 2 & .040 & .021 & .065 & -.003 & .082 \\
\hline & 2 & 1 & 2 & .022 & .038 & .565 & -.054 & .098 \\
\hline & & & 3 & .007 & .029 & .822 & -.052 & .065 \\
\hline & & 2 & 1 & -.022 & .038 & .565 & -.098 & .054 \\
\hline & & & 3 & -.015 & .027 & .571 & -.070 & .039 \\
\hline & & 3 & 1 & -.007 & .029 & .822 & -.065 & .052 \\
\hline & & & 2 & .015 & .027 & .571 & -.039 & .070 \\
\hline Brain region 7 & 1 & 1 & 2 & $.041^{*}$ & .020 & .043 & .001 & .080 \\
\hline
\end{tabular}




\begin{tabular}{|c|c|c|c|c|c|c|c|c|}
\hline & & & 3 & .002 & .026 & .927 & -.049 & .054 \\
\hline & & \multirow[t]{2}{*}{2} & 1 & $-.041^{*}$ & .020 & .043 & -.080 & -.001 \\
\hline & & & 3 & -.038 & .024 & .110 & -.086 & .009 \\
\hline & & \multirow[t]{2}{*}{3} & 1 & -.002 & .026 & .927 & -.054 & .049 \\
\hline & & & 2 & .038 & .024 & .110 & -.009 & .086 \\
\hline & \multirow[t]{6}{*}{2} & 1 & 2 & -.030 & .029 & .315 & -.088 & .029 \\
\hline & & & 3 & -.043 & .029 & .144 & -.102 & .015 \\
\hline & & 2 & 1 & .030 & .029 & .315 & -.029 & .088 \\
\hline & & & 3 & -.014 & .027 & .611 & -.068 & .041 \\
\hline & & 3 & 1 & .043 & .029 & .144 & -.015 & .102 \\
\hline & & & 2 & .014 & .027 & .611 & -.041 & .068 \\
\hline \multirow[t]{12}{*}{ Brain region 8} & \multirow[t]{6}{*}{1} & 1 & 2 & .010 & .023 & .669 & -.037 & .057 \\
\hline & & & 3 & -.030 & .025 & .228 & -.080 & .020 \\
\hline & & 2 & 1 & -.010 & .023 & .669 & -.057 & .037 \\
\hline & & & 3 & -.040 & .025 & .117 & -.091 & .011 \\
\hline & & 3 & 1 & .030 & .025 & .228 & -.020 & .080 \\
\hline & & & 2 & .040 & .025 & .117 & -.011 & .091 \\
\hline & \multirow[t]{6}{*}{2} & 1 & 2 & .032 & .049 & .515 & -.066 & .130 \\
\hline & & & 3 & -.036 & .028 & .194 & -.092 & .019 \\
\hline & & 2 & 1 & -.032 & .049 & .515 & -.130 & .066 \\
\hline & & & 3 & -.068 & .043 & .120 & -.155 & .018 \\
\hline & & 3 & 1 & .036 & .028 & .194 & -.019 & .092 \\
\hline & & & 2 & .068 & .043 & .120 & -.018 & .155 \\
\hline \multirow[t]{3}{*}{ Brain region 9} & \multirow[t]{3}{*}{1} & 1 & 2 & .040 & .022 & .079 & -.005 & .084 \\
\hline & & & 3 & -.026 & .024 & .278 & -.074 & .022 \\
\hline & & 2 & 1 & -.040 & .022 & .079 & -.084 & .005 \\
\hline
\end{tabular}




\begin{tabular}{|c|c|c|c|c|c|c|c|c|}
\hline & & & 3 & $-.066^{*}$ & .024 & .010 & -.115 & -.017 \\
\hline & & \multirow[t]{2}{*}{3} & 1 & .026 & .024 & .278 & -.022 & .074 \\
\hline & & & 2 & $.066^{*}$ & .024 & .010 & .017 & .115 \\
\hline & \multirow[t]{6}{*}{2} & \multirow[t]{2}{*}{1} & 2 & .046 & .051 & .376 & -.057 & .149 \\
\hline & & & 3 & -.045 & .043 & .304 & -.133 & .042 \\
\hline & & \multirow[t]{2}{*}{2} & 1 & -.046 & .051 & .376 & -.149 & .057 \\
\hline & & & 3 & $-.091^{*}$ & .035 & .012 & -.161 & -.021 \\
\hline & & \multirow[t]{2}{*}{3} & 1 & .045 & .043 & .304 & -.042 & .133 \\
\hline & & & 2 & $.091^{*}$ & .035 & .012 & .021 & .161 \\
\hline \multirow[t]{12}{*}{ Brain region 10} & \multirow[t]{6}{*}{1} & \multirow[t]{2}{*}{1} & 2 & -.027 & .023 & .254 & -.073 & .020 \\
\hline & & & 3 & -.011 & .027 & .695 & -.065 & .043 \\
\hline & & \multirow[t]{2}{*}{2} & 1 & .027 & .023 & .254 & -.020 & .073 \\
\hline & & & 3 & .016 & .022 & .475 & -.029 & .061 \\
\hline & & \multirow[t]{2}{*}{3} & 1 & .011 & .027 & .695 & -.043 & .065 \\
\hline & & & 2 & -.016 & .022 & .475 & -.061 & .029 \\
\hline & \multirow[t]{6}{*}{2} & \multirow[t]{2}{*}{1} & 2 & -.004 & .031 & .909 & -.067 & .060 \\
\hline & & & 3 & -.037 & .030 & .225 & -.098 & .024 \\
\hline & & \multirow[t]{2}{*}{2} & 1 & .004 & .031 & .909 & -.060 & .067 \\
\hline & & & 3 & -.034 & .025 & .188 & -.084 & .017 \\
\hline & & \multirow[t]{2}{*}{3} & 1 & .037 & .030 & .225 & -.024 & .098 \\
\hline & & & 2 & .034 & .025 & .188 & -.017 & .084 \\
\hline \multirow[t]{5}{*}{ Brain region 11} & 1 & \multirow[t]{2}{*}{1} & 2 & .008 & .016 & .612 & -.024 & .041 \\
\hline & & & 3 & .019 & .016 & .256 & -.014 & .052 \\
\hline & & \multirow[t]{2}{*}{2} & 1 & -.008 & .016 & .612 & -.041 & .024 \\
\hline & & & 3 & .011 & .017 & .539 & -.024 & .045 \\
\hline & & 3 & 1 & -.019 & .016 & .256 & -.052 & .014 \\
\hline
\end{tabular}




\begin{tabular}{|c|c|c|c|c|c|c|c|c|}
\hline & & & 2 & -.011 & .017 & .539 & -.045 & .024 \\
\hline & \multirow[t]{6}{*}{2} & \multirow[t]{2}{*}{1} & 2 & .001 & .022 & .980 & -.043 & .044 \\
\hline & & & 3 & -.003 & .023 & .909 & -.048 & .043 \\
\hline & & \multirow[t]{2}{*}{2} & 1 & -.001 & .022 & .980 & -.044 & .043 \\
\hline & & & 3 & -.003 & .019 & .867 & -.041 & .034 \\
\hline & & \multirow[t]{2}{*}{3} & 1 & .003 & .023 & .909 & -.043 & .048 \\
\hline & & & 2 & .003 & .019 & .867 & -.034 & .041 \\
\hline \multirow[t]{12}{*}{ Brain region 12} & \multirow[t]{6}{*}{1} & \multirow[t]{2}{*}{1} & 2 & .015 & .025 & .557 & -.035 & .065 \\
\hline & & & 3 & -.043 & .031 & .174 & -.106 & .020 \\
\hline & & \multirow[t]{2}{*}{2} & 1 & -.015 & .025 & .557 & -.065 & .035 \\
\hline & & & 3 & $-.058^{*}$ & .027 & .041 & -.113 & -.003 \\
\hline & & \multirow[t]{2}{*}{3} & 1 & .043 & .031 & .174 & -.020 & .106 \\
\hline & & & 2 & $.058^{*}$ & .027 & .041 & .003 & .113 \\
\hline & \multirow[t]{6}{*}{2} & \multirow[t]{2}{*}{1} & 2 & .005 & .037 & .892 & -.070 & .080 \\
\hline & & & 3 & -.068 & .034 & .051 & -.136 & .000 \\
\hline & & \multirow[t]{2}{*}{2} & 1 & -.005 & .037 & .892 & -.080 & .070 \\
\hline & & & 3 & $-.073^{*}$ & .031 & .025 & -.136 & -.010 \\
\hline & & \multirow[t]{2}{*}{3} & 1 & .068 & .034 & .051 & .000 & .136 \\
\hline & & & 2 & $.073^{*}$ & .031 & .025 & .010 & .136 \\
\hline \multirow[t]{7}{*}{ Brain region 13} & \multirow[t]{6}{*}{1} & \multirow[t]{2}{*}{1} & 2 & $.053^{*}$ & .025 & .043 & .002 & .103 \\
\hline & & & 3 & .004 & .022 & .869 & -.041 & .048 \\
\hline & & \multirow[t]{2}{*}{2} & 1 & $-.053^{*}$ & .025 & .043 & -.103 & -.002 \\
\hline & & & 3 & -.049 & .031 & .116 & -.110 & .013 \\
\hline & & \multirow[t]{2}{*}{3} & 1 & -.004 & .022 & .869 & -.048 & .041 \\
\hline & & & 2 & .049 & .031 & .116 & -.013 & .110 \\
\hline & 2 & 1 & 2 & .002 & .050 & .966 & -.099 & .104 \\
\hline
\end{tabular}




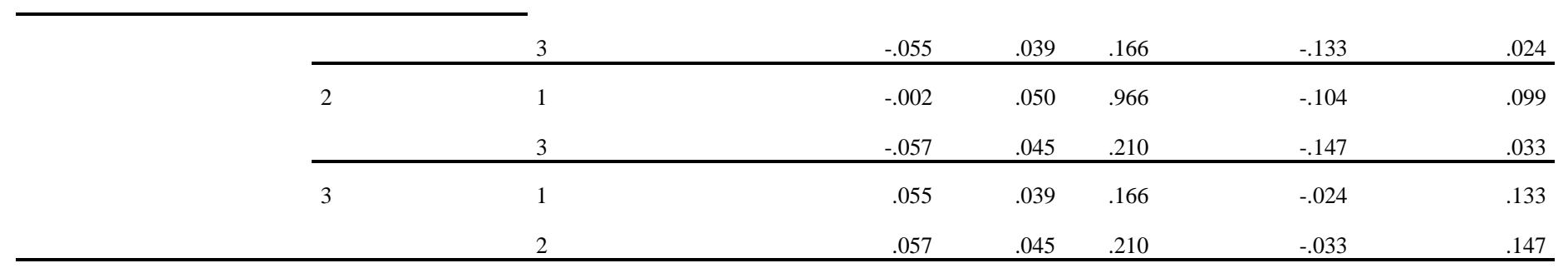

Based on estimated marginal means

a. Adjustment for multiple comparisons: Least Significant Difference (equivalent to no adjustments)

*. The mean difference is significant at the .05 level. 


\section{Table H16}

Mean Difference, Standard Error, P-Value and 95\% Confidence Interval for Accuracy Effects by Incentive Condition

\begin{tabular}{|c|c|c|c|c|c|c|c|c|}
\hline \multirow[t]{2}{*}{ Brain region } & \multirow[t]{2}{*}{ Incentive cond } & \multirow[t]{2}{*}{ (I) Accuracy } & \multirow[t]{2}{*}{ (J) Accuracy } & \multirow{2}{*}{$\begin{array}{c}\text { Mean Difference } \\
(\mathrm{I}-\mathrm{J}) \\
\end{array}$} & \multirow[b]{2}{*}{ Std. Error } & \multirow[b]{2}{*}{ Sig. ${ }^{\text {a }}$} & \multicolumn{2}{|c|}{ 95\% Confidence Interval for Difference ${ }^{a}$} \\
\hline & & & & & & & Lower Bound & Upper Bound \\
\hline \multirow[t]{6}{*}{ Brain region 1} & 1 & 1 & 2 & .049 & .030 & .108 & -.011 & .108 \\
\hline & & 2 & 1 & -.049 & .030 & .108 & -.108 & .011 \\
\hline & 2 & 1 & 2 & .011 & .036 & .772 & -.063 & .084 \\
\hline & & 2 & 1 & -.011 & .036 & .772 & -.084 & .063 \\
\hline & 3 & 1 & 2 & .001 & .025 & .961 & -.050 & .052 \\
\hline & & 2 & 1 & -.001 & .025 & .961 & -.052 & .050 \\
\hline \multirow[t]{6}{*}{ Brain region 2} & 1 & 1 & 2 & .026 & .026 & .317 & -.026 & .078 \\
\hline & & 2 & 1 & -.026 & .026 & .317 & -.078 & .026 \\
\hline & 2 & 1 & 2 & -.032 & .026 & .216 & -.084 & .020 \\
\hline & & 2 & 1 & .032 & .026 & .216 & -.020 & .084 \\
\hline & 3 & 1 & 2 & .007 & .024 & .763 & -.041 & .055 \\
\hline & & 2 & 1 & -.007 & .024 & .763 & -.055 & .041 \\
\hline \multirow[t]{6}{*}{ Brain region 3} & 1 & 1 & 2 & .014 & .029 & .645 & -.045 & .073 \\
\hline & & 2 & 1 & -.014 & .029 & .645 & -.073 & .045 \\
\hline & 2 & 1 & 2 & .058 & .034 & .096 & -.011 & .127 \\
\hline & & 2 & 1 & -.058 & .034 & .096 & -.127 & .011 \\
\hline & 3 & 1 & 2 & .025 & .031 & .423 & -.037 & .086 \\
\hline & & 2 & 1 & -.025 & .031 & .423 & -.086 & .037 \\
\hline \multirow[t]{3}{*}{ Brain region 4} & 1 & 1 & 2 & .031 & .028 & .272 & -.025 & .086 \\
\hline & & 2 & 1 & -.031 & .028 & .272 & -.086 & .025 \\
\hline & 2 & 1 & 2 & -.013 & .022 & .572 & -.057 & .032 \\
\hline
\end{tabular}




\begin{tabular}{|c|c|c|c|c|c|c|c|c|}
\hline & & 2 & 1 & .013 & .022 & .572 & -.032 & .057 \\
\hline & \multirow[t]{2}{*}{3} & 1 & 2 & .005 & .021 & .806 & -.037 & .047 \\
\hline & & 2 & 1 & -.005 & .021 & .806 & -.047 & .037 \\
\hline \multirow[t]{6}{*}{ Brain region 5} & 1 & 1 & 2 & .022 & .021 & .301 & -.020 & .064 \\
\hline & & 2 & 1 & -.022 & .021 & .301 & -.064 & .020 \\
\hline & 2 & 1 & 2 & -.012 & .026 & .650 & -.063 & .040 \\
\hline & & 2 & 1 & .012 & .026 & .650 & -.040 & .063 \\
\hline & 3 & 1 & 2 & .025 & .030 & .399 & -.035 & .086 \\
\hline & & 2 & 1 & -.025 & .030 & .399 & -.086 & .035 \\
\hline \multirow[t]{6}{*}{ Brain region 6} & 1 & 1 & 2 & .008 & .028 & .776 & -.049 & .065 \\
\hline & & 2 & 1 & -.008 & .028 & .776 & -.065 & .049 \\
\hline & 2 & 1 & 2 & -.009 & .024 & .713 & -.058 & .040 \\
\hline & & 2 & 1 & .009 & .024 & .713 & -.040 & .058 \\
\hline & 3 & 1 & 2 & .015 & .023 & .509 & -.031 & .062 \\
\hline & & 2 & 1 & -.015 & .023 & .509 & -.062 & .031 \\
\hline \multirow[t]{6}{*}{ Brain region 7} & 1 & 1 & 2 & .025 & .024 & .308 & -.024 & .073 \\
\hline & & 2 & 1 & -.025 & .024 & .308 & -.073 & .024 \\
\hline & 2 & 1 & 2 & -.046 & .027 & .099 & -.100 & .009 \\
\hline & & 2 & 1 & .046 & .027 & .099 & -.009 & .100 \\
\hline & 3 & 1 & 2 & -.021 & .030 & .482 & -.081 & .039 \\
\hline & & 2 & 1 & .021 & .030 & .482 & -.039 & .081 \\
\hline \multirow[t]{5}{*}{ Brain region 8} & 1 & 1 & 2 & .026 & .028 & .361 & -.030 & .081 \\
\hline & & 2 & 1 & -.026 & .028 & .361 & -.081 & .030 \\
\hline & 2 & 1 & 2 & .048 & .042 & .263 & -.037 & .132 \\
\hline & & 2 & 1 & -.048 & .042 & .263 & -.132 & .037 \\
\hline & 3 & 1 & 2 & .020 & .024 & .423 & -.029 & .069 \\
\hline
\end{tabular}




\begin{tabular}{|c|c|c|c|c|c|c|c|c|}
\hline & & 2 & 1 & -.020 & .024 & .423 & -.069 & .029 \\
\hline \multirow[t]{6}{*}{ Brain region 9} & 1 & 1 & 2 & $.062^{*}$ & .027 & .025 & .008 & .116 \\
\hline & & 2 & 1 & $-.062^{*}$ & .027 & .025 & -.116 & -.008 \\
\hline & 2 & 1 & 2 & .068 & .046 & .141 & -.024 & .160 \\
\hline & & 2 & 1 & -.068 & .046 & .141 & -.160 & .024 \\
\hline & 3 & 1 & 2 & .043 & .039 & .275 & -.035 & .121 \\
\hline & & 2 & 1 & -.043 & .039 & .275 & -.121 & .035 \\
\hline \multirow[t]{6}{*}{ Brain region 10} & 1 & 1 & 2 & .001 & .027 & .978 & -.054 & .056 \\
\hline & & 2 & 1 & -.001 & .027 & .978 & -.056 & .054 \\
\hline & 2 & 1 & 2 & .024 & .025 & .338 & -.026 & .074 \\
\hline & & 2 & 1 & -.024 & .025 & .338 & -.074 & .026 \\
\hline & 3 & 1 & 2 & -.026 & .023 & .266 & -.072 & .020 \\
\hline & & 2 & 1 & .026 & .023 & .266 & -.020 & .072 \\
\hline \multirow[t]{6}{*}{ Brain region 11} & 1 & 1 & 2 & -.013 & .024 & .595 & -.062 & .036 \\
\hline & & 2 & 1 & .013 & .024 & .595 & -.036 & .062 \\
\hline & 2 & 1 & 2 & -.021 & .019 & .288 & -.060 & .018 \\
\hline & & 2 & 1 & .021 & .019 & .288 & -.018 & .060 \\
\hline & 3 & 1 & 2 & -.034 & .020 & .085 & -.074 & .005 \\
\hline & & 2 & 1 & .034 & .020 & .085 & -.005 & .074 \\
\hline \multirow[t]{6}{*}{ Brain region 12} & 1 & 1 & 2 & $.069^{*}$ & .028 & .018 & .012 & .125 \\
\hline & & 2 & 1 & $-.069^{*}$ & .028 & .018 & -.125 & -.012 \\
\hline & 2 & 1 & 2 & $.059^{*}$ & .027 & .035 & .004 & .114 \\
\hline & & 2 & 1 & $-.059^{*}$ & .027 & .035 & -.114 & -.004 \\
\hline & 3 & 1 & 2 & .044 & .038 & .259 & -.033 & .121 \\
\hline & & 2 & 1 & -.044 & .038 & .259 & -.121 & .033 \\
\hline Brain region 13 & 1 & 1 & 2 & $.072^{*}$ & .024 & .005 & .023 & .121 \\
\hline
\end{tabular}




\begin{tabular}{lccccccccc}
\hline & 2 & 1 & $-.072^{*}$ & .024 & .005 & -.121 & -.023 \\
\cline { 2 - 8 } & 2 & 1 & 2 & .021 & .043 & .621 & -.065 & .108 \\
\cline { 2 - 8 } & 2 & 1 & -.021 & .043 & .621 & -.108 & .065 \\
\cline { 2 - 8 } & 1 & 2 & .013 & .034 & .696 & -.055 & .082 \\
\cline { 2 - 8 } & 2 & 1 & -.013 & .034 & .696 & -.082 & .055 \\
\hline
\end{tabular}

Based on estimated marginal means

a. Adjustment for multiple comparisons: Least Significant Difference (equivalent to no adjustments)

*. The mean difference is significant at the .05 level. 


\section{Table H17}

Means, Standard Errors and 95\% Confidence Intervals for Group x Incentive Condition x Accuracy Effects

\begin{tabular}{|c|c|c|c|c|c|c|c|}
\hline \multirow[t]{2}{*}{ Brain region } & \multirow[t]{2}{*}{ Group } & \multirow[t]{2}{*}{ Incentive cond } & \multirow[t]{2}{*}{ Accuracy } & \multirow[b]{2}{*}{ Mean } & \multirow[b]{2}{*}{ Std. Error } & \multicolumn{2}{|c|}{ 95\% Confidence Interval } \\
\hline & & & & & & Lower Bound & Upper Bound \\
\hline \multirow[t]{12}{*}{ Brain region 1} & 1.00 & 1 & 1 & -.277 & .040 & -.358 & -.197 \\
\hline & & & 2 & -.322 & .043 & -.408 & -.236 \\
\hline & & 2 & 1 & -.284 & .041 & -.366 & -.201 \\
\hline & & & 2 & -.282 & .061 & -.405 & -.158 \\
\hline & & 3 & 1 & -.177 & .036 & -.249 & -.105 \\
\hline & & & 2 & -.145 & .047 & -.240 & -.051 \\
\hline & 2.00 & 1 & 1 & -.128 & .040 & -.209 & -.048 \\
\hline & & & 2 & -.181 & .043 & -.267 & -.095 \\
\hline & & 2 & 1 & -.155 & .041 & -.237 & -.072 \\
\hline & & & 2 & -.178 & .061 & -.302 & -.055 \\
\hline & & 3 & 1 & -.114 & .036 & -.186 & -.042 \\
\hline & & & 2 & -.149 & .047 & -.243 & -.054 \\
\hline \multirow[t]{8}{*}{ Brain region 2} & 1.00 & 1 & 1 & -.007 & .039 & -.086 & .072 \\
\hline & & & 2 & .003 & .035 & -.068 & .073 \\
\hline & & 2 & 1 & .227 & .047 & .133 & .321 \\
\hline & & & 2 & .259 & .051 & .156 & .363 \\
\hline & & 3 & 1 & .246 & .050 & .145 & .347 \\
\hline & & & 2 & .281 & .054 & .172 & .390 \\
\hline & 2.00 & 1 & 1 & .107 & .039 & .028 & .186 \\
\hline & & & 2 & .045 & .035 & -.025 & .116 \\
\hline
\end{tabular}




\begin{tabular}{|c|c|c|c|c|c|c|c|}
\hline & & \multirow[t]{2}{*}{2} & 1 & .290 & .047 & .196 & .383 \\
\hline & & & 2 & .322 & .051 & .219 & .426 \\
\hline & & \multirow[t]{2}{*}{3} & 1 & .337 & .050 & .236 & .439 \\
\hline & & & 2 & .288 & .054 & .178 & .397 \\
\hline \multirow[t]{12}{*}{ Brain region 3} & \multirow[t]{6}{*}{1.00} & \multirow[t]{2}{*}{1} & 1 & -.192 & .030 & -.252 & -.133 \\
\hline & & & 2 & -.188 & .042 & -.273 & -.103 \\
\hline & & \multirow[t]{2}{*}{2} & 1 & -.160 & .031 & -.223 & -.097 \\
\hline & & & 2 & -.223 & .044 & -.312 & -.135 \\
\hline & & \multirow[t]{2}{*}{3} & 1 & -.110 & .035. & -.181 & -.039 \\
\hline & & & 2 & -.150 & .040 & -.229 & -.070 \\
\hline & \multirow[t]{6}{*}{2.00} & \multirow[t]{2}{*}{1} & 1 & -.102 & .030 & -.161 & -.042 \\
\hline & & & 2 & -.133 & .042 & -.218 & -.048 \\
\hline & & \multirow[t]{2}{*}{2} & 1 & -.105 & .031 & -.168 & -.042 \\
\hline & & & 2 & -.158 & .044 & -.246 & -.069 \\
\hline & & \multirow[t]{2}{*}{3} & 1 & -.104 & .035 & -.175 & -.033 \\
\hline & & & 2 & -.114 & .040 & -.193 & -.034 \\
\hline \multirow[t]{10}{*}{ Brain region 4} & \multirow[t]{6}{*}{1.00} & \multirow[t]{2}{*}{1} & 1 & .025. & .042 & -.059 & .109 \\
\hline & & & 2 & .021 & .039 & -.058 & .099 \\
\hline & & \multirow[t]{2}{*}{2} & 1 & .237 & .049 & .138 & .336 \\
\hline & & & 2 & . 261 & .052 & .156 & .367 \\
\hline & & \multirow[t]{2}{*}{3} & 1 & 259 & .050 & .159. & .359 \\
\hline & & & 2 & .295 & .054 & .185 & .404 \\
\hline & \multirow[t]{4}{*}{2.00} & \multirow[t]{2}{*}{1} & 1 & .113 & .042 & .029 & 197 \\
\hline & & & 2 & .056 & .039 & -.023 & .135 \\
\hline & & \multirow[t]{2}{*}{2} & 1 & . 282 & .049. & .183 & .382 \\
\hline & & & 2 & .283 & .052 & .178 & .388 \\
\hline
\end{tabular}




\begin{tabular}{|c|c|c|c|c|c|c|c|}
\hline & & 3 & 1 & .314 & .050 & .213 & .414 \\
\hline & & & 2 & .268 & .054 & .159 & .377 \\
\hline \multirow[t]{12}{*}{ Brain region 5} & 1.00 & 1 & 1 & -.157 & .034 & -.224 & -.089 \\
\hline & & & 2 & -.181 & .041 & -.263 & -.099 \\
\hline & & 2 & 1 & -.195 & .033 & -.262 & -.129 \\
\hline & & & 2 & -.190 & .041 & -.272 & -.108 \\
\hline & & 3 & 1 & -.121 & .035 & -.192 & -.050 \\
\hline & & & 2 & -.099 & .039 & -.178 & -.021 \\
\hline & 2.00 & 1 & 1 & -.103 & .034 & -.171 & -.036 \\
\hline & & & 2 & -.123 & .041 & -.205 & -.041 \\
\hline & & 2 & 1 & -.168 & .033 & -.235 & -.102 \\
\hline & & & 2 & -.151 & .041 & -.233 & -.069 \\
\hline & & 3 & 1 & -.075 & .035 & -.146 & -.004 \\
\hline & & & 2 & -.148 & .039 & -.226 & -.069 \\
\hline \multirow[t]{12}{*}{ Brain region 6} & 1.00 & 1 & 1 & -.140 & .028 & -.195 & -.084 \\
\hline & & & 2 & -.126 & .040 & -.206 & -.046 \\
\hline & & 2 & 1 & -.161 & .031 & -.223 & -.099 \\
\hline & & & 2 & -.161 & .035 & -.232 & -.090 \\
\hline & & 3 & 1 & -.115 & .028 & -.171 & -.058 \\
\hline & & & 2 & -.093 & .033 & -.160 & -.027 \\
\hline & 2.00 & 1 & 1 & -.044 & .028 & -.100 & .011 \\
\hline & & & 2 & -.074 & .040 & -.154 & .006 \\
\hline & & 2 & 1 & -.101 & .031 & -.163 & -.039 \\
\hline & & & 2 & -.083 & .035 & -.154 & -.012 \\
\hline & & 3 & 1 & -.068 & .028 & -.125 & -.011 \\
\hline & & & 2 & -.120 & .033 & -.187 & -.054 \\
\hline
\end{tabular}




\begin{tabular}{|c|c|c|c|c|c|c|c|}
\hline \multirow[t]{12}{*}{ Brain region 7} & 1.00 & 1 & 1 & -.174 & .034 & -.242 & -.105 \\
\hline & & & 2 & -.163 & .031 & -.226 & -.100 \\
\hline & & 2 & 1 & -.195 & .034 & -.263 & -.126 \\
\hline & & & 2 & -.137 & .036 & -.210 & -.064 \\
\hline & & 3 & 1 & -.142 & .036 & -.214 & -.070 \\
\hline & & & 2 & -.088 & .038 & -.165 & -.012 \\
\hline & 2.00 & 1 & 1 & -.025 & .034 & -.093 & .043 \\
\hline & & & 2 & -.085 & .031 & -.149 & -.022 \\
\hline & & 2 & 1 & -.086 & .034 & -.155 & -.017 \\
\hline & & & 2 & -.052 & .036 & -.125 & .021 \\
\hline & & 3 & 1 & -.062 & .036 & -.133 & .010 \\
\hline & & & 2 & -.073 & .038 & -.149 & .003 \\
\hline \multirow[t]{12}{*}{ Brain region 8} & 1.00 & 1 & 1 & -.148 & .034 & -.215 & -.080 \\
\hline & & & 2 & -.121 & .045 & -.212 & -.030 \\
\hline & & 2 & 1 & -.118 & .035 & -.190 & -.047 \\
\hline & & & 2 & -.133 & .051 & -.235 & -.031 \\
\hline & & 3 & 1 & -.058 & .033 & -.124 & .009 \\
\hline & & & 2 & -.079 & .039 & -.158 & .000 \\
\hline & 2.00 & 1 & 1 & -.052 & .034 & -.120 & .016 \\
\hline & & & 2 & -.130 & .045 & -.220 & -.039 \\
\hline & & 2 & 1 & -.102 & .035 & -.173 & -.030 \\
\hline & & & 2 & -.182 & .051 & -.284 & -.080 \\
\hline & & 3 & 1 & -.081 & .033 & -.147 & -.015 \\
\hline & & & 2 & -.099 & .039 & -.178 & -.020 \\
\hline \multirow[t]{2}{*}{ Brain region 9} & 1.00 & 1 & 1 & -.135 & .042 & -.219 & -.050 \\
\hline & & & 2 & -.185 & .042 & -.269 & -.101 \\
\hline
\end{tabular}




\begin{tabular}{|c|c|c|c|c|c|c|c|}
\hline & & \multirow[t]{2}{*}{2} & 1 & -.146 & .035 & -.217 & -.075 \\
\hline & & & 2 & -.180 & .063 & -.307 & -.052 \\
\hline & & \multirow[t]{2}{*}{3} & 1 & -.116 & .046 & -.208 & -.024 \\
\hline & & & 2 & -.089 & .045 & -.181 & .002 \\
\hline & \multirow[t]{6}{*}{2.00} & \multirow[t]{2}{*}{1} & 1 & -.058 & .042 & -.143 & .026 \\
\hline & & & 2 & -.132 & .042 & -.216 & -.048 \\
\hline & & \multirow[t]{2}{*}{2} & 1 & -.127 & .035 & -.198 & -.055 \\
\hline & & & 2 & -.229 & .063 & -.356 & -.102 \\
\hline & & \multirow[t]{2}{*}{3} & 1 & -.025 & .046 & -.117 & .067 \\
\hline & & & 2 & -.137 & .045 & -.229 & -.046 \\
\hline \multirow[t]{12}{*}{ Brain region 10} & \multirow[t]{6}{*}{1.00} & \multirow[t]{2}{*}{1} & 1 & -.163 & .033 & -.230 & -.096 \\
\hline & & & 2 & -.155 & .035 & -.225 & -.084 \\
\hline & & \multirow[t]{2}{*}{2} & 1 & -.134 & .031 & -.197 & -.072 \\
\hline & & & 2 & -.135 & .037 & -.210 & -.060 \\
\hline & & \multirow[t]{2}{*}{3} & 1 & -.139 & .030 & -.199 & -.079 \\
\hline & & & 2 & -.089 & .033 & -.156 & -.022 \\
\hline & \multirow[t]{6}{*}{2.00} & \multirow[t]{2}{*}{1} & 1 & -.074 & .033 & -.141 & -.007 \\
\hline & & & 2 & -.084 & .035 & -.155 & -.013 \\
\hline & & \multirow[t]{2}{*}{2} & 1 & -.049 & .031 & -.112 & .013 \\
\hline & & & 2 & -.096 & .037 & -.171 & -.022 \\
\hline & & \multirow[t]{2}{*}{3} & 1 & -.077 & .030 & -.137 & -.017 \\
\hline & & & 2 & -.075 & .033 & -.142 & -.008 \\
\hline \multirow[t]{4}{*}{ Brain region 11} & \multirow[t]{4}{*}{1.00} & \multirow[t]{2}{*}{1} & 1 & -.100 & .021 & -.142 & -.058 \\
\hline & & & 2 & -.074 & .028 & -.131 & -.017 \\
\hline & & \multirow[t]{2}{*}{2} & 1 & -.081 & .022 & -.126 & -.037 \\
\hline & & & 2 & -.055 & .024 & -.104 & -.006 \\
\hline
\end{tabular}




\begin{tabular}{|c|c|c|c|c|c|c|c|}
\hline & & \multirow[t]{2}{*}{3} & 1 & -.079 & .024 & -.127 & -.032 \\
\hline & & & 2 & -.026 & .028 & -.082 & .030 \\
\hline & \multirow[t]{6}{*}{2.00} & \multirow[t]{2}{*}{1} & 1 & -.037 & .021 & -.079 & .005 \\
\hline & & & 2 & -.036 & .028 & -.094 & .021 \\
\hline & & \multirow[t]{2}{*}{2} & 1 & -.072 & .022 & -.117 & -.028 \\
\hline & & & 2 & -.057 & .024 & -.106 & -.008 \\
\hline & & \multirow[t]{2}{*}{3} & 1 & -.095 & .024 & -.143 & -.048 \\
\hline & & & 2 & -.079 & .028 & -.135 & -.023 \\
\hline \multirow[t]{12}{*}{ Brain region 12} & \multirow[t]{6}{*}{1.00} & \multirow[t]{2}{*}{1} & 1 & -.163 & .039 & -.243 & -.084 \\
\hline & & & 2 & -.192 & .047 & -.285 & -.098 \\
\hline & & \multirow[t]{2}{*}{2} & 1 & -.140 & .044 & -.229 & -.052 \\
\hline & & & 2 & -.167 & .041 & -.249 & -.085 \\
\hline & & \multirow[t]{2}{*}{3} & 1 & -.105 & .045. & -.195 & -.015 \\
\hline & & & 2 & -.106 & .050 & -.207 & -.006 \\
\hline & \multirow[t]{6}{*}{2.00} & \multirow[t]{2}{*}{1} & 1 & -.073 & .039 & -.152 & .007 \\
\hline & & & 2 & -.181 & .047 & -.275 & -.088 \\
\hline & & \multirow[t]{2}{*}{2} & 1 & -.125 & .044 & -.213 & -.036 \\
\hline & & & 2 & -.216 & .041 & -.298 & -.134 \\
\hline & & \multirow[t]{2}{*}{3} & 1 & -.044 & .045 & -.134 & .045 \\
\hline & & & 2 & -.131 & .050 & -.231 & -.031 \\
\hline \multirow[t]{6}{*}{ Brain region 13} & \multirow[t]{6}{*}{1.00} & \multirow[t]{2}{*}{1} & 1 & -.101 & .030 & -.161 & -.042 \\
\hline & & & 2 & -.136 & .037 & -.210 & -.063 \\
\hline & & \multirow[t]{2}{*}{2} & 1 & -.141 & .034 & -.210 & -.072 \\
\hline & & & 2 & -.134 & .052 & -.239 & -.028 \\
\hline & & \multirow[t]{2}{*}{3} & 1 & -.112 & .032 & -.177 & -.047 \\
\hline & & & 2 & -.147 & .042 & -.232 & -.062 \\
\hline
\end{tabular}




\begin{tabular}{ccccccc}
\hline \multirow{2}{*}{2.00} & 1 & 1 & -.020 & .030 & -.079 & .040 \\
& & 2 & -.128 & .037 & -.202 & -.054 \\
\cline { 2 - 7 } & 2 & 1 & -.085 & .034 & -.154 & -.016 \\
& & 2 & -.135 & .052 & -.240 & -.029 \\
\cline { 2 - 7 } & 3 & 1 & -.016 & .032 & -.081 & .049 \\
& 2 & -.008 & .042 & -.093 & .077 \\
\hline
\end{tabular}




\section{Appendix I}

\section{Means and Significance Tests for All Main and Interaction Effects During the Stop Stimulus Period}

The tables in this Appendix contain names of brain regions and levels of variables and that have been abbreviated for formatting purposes. The abbreviations and the brain regions, groups, level of accuracy and incentive conditions they represent are set out below.

\section{Labels in the Tables and the Brain Regions They Represent}

\begin{tabular}{|c|c|}
\hline Label in table & Brain region \\
\hline Brain region 1 & $\begin{array}{l}\text { Right cingulate gyrus / Bilateral medial frontal gyrus / } \\
\text { Right superior and middle frontal gyrus }\end{array}$ \\
\hline Brain region 2 & Right supramarginal gyrus \\
\hline Brain region 3 & Right lingual gyrus \\
\hline Brain region 4 & Left lingual gyrus \\
\hline Brain region 5 & Left precentral gyrus / Left BA9 (DLPFC) \\
\hline Brain region 6 & Left inferior parietal lobule \\
\hline Brain region 7 & Left caudate (genu/tail) \\
\hline Brain region 8 & Left caudate (head) \\
\hline Brain region 9 & $\begin{array}{l}\text { Right anterior cingulate / Bilateral anterior cingulate (ventral } \\
\text { or rostral region) }\end{array}$ \\
\hline Brain region 10 & Right caudate (genu/tail) \\
\hline Brain region 11 & Left culmen \\
\hline Brain region 12 & Left superior frontal gyrus (and Left Superior medial gyrus) \\
\hline Brain region 13 & Left superior occipital gyrus / Left BA19 \\
\hline Brain region 14 & Left supramarginal gyrus \\
\hline Brain region 15 & Right middle temporal gyrus \\
\hline Brain region 16 & Left cuneus \\
\hline Brain region 17 & Left posterior cingulate / Left Precuneus \\
\hline Brain region 18 & Left middle temporal gyrus \\
\hline
\end{tabular}




\begin{tabular}{|c|c|}
\hline Brain region 19 & Right parahippocampal gyrus \\
\hline Brain region 20 & Right medial frontal gyrus / more Bilateral medial frontal gyrus \\
\hline Brain region 21 & Right superior temporal gyrus \\
\hline Brain region 22 & Right brain (brainstem) \\
\hline Brain region 23 & Right middle temporal gyrus / Right middle occipital gyrus \\
\hline Brain region 24 & $\begin{array}{l}\text { Right superior temporal gyrus / Right middle temporal (on border } \\
\text { between them) }\end{array}$ \\
\hline Brain region 25 & Left uvula \\
\hline
\end{tabular}

Labels in the Tables Referencing Low Trait Anxiety and High Trait Anxiety Groups

\begin{tabular}{cl}
\hline Group label & Group \\
\hline 1.00 & Low trait anxiety \\
2.00 & High trait anxiety \\
\hline
\end{tabular}

Labels in the Tables Referencing Accuracy

\begin{tabular}{cl}
\hline Accuracy label & Accuracy \\
\hline 1.00 & Correct \\
2.00 & Error \\
\hline
\end{tabular}

Labels in the Tables Referencing Incentive Condition

\begin{tabular}{cl}
\hline Incentive condition label & Incentive condition \\
\hline 1.00 & Neutral condition \\
2.00 & Punishment condition \\
3.00 & Reward condition \\
\hline
\end{tabular}

Note: In the tables that follow Incentive condition is abbreviated to Incentive cond 
Table I1

F-Values, Degrees of Freedom, and Levels of Significance for Regions of Event-Related BOLD Activity During Stop Stimulus Period

\begin{tabular}{|c|c|c|c|c|c|}
\hline \multirow{2}{*}{$\begin{array}{l}\text { Condition } \\
\text { Group }\end{array}$} & \multirow{2}{*}{$\begin{array}{l}\text { Brain region } \\
\text { Brain region } 1\end{array}$} & \multicolumn{2}{|c|}{$\mathrm{df}$} & \multirow{2}{*}{$\begin{array}{l}F \\
.645\end{array}$} & \multirow{2}{*}{$\begin{array}{l}\text { Sig. } \\
.426\end{array}$} \\
\hline & & 1 & 46 & & \\
\hline & Brain region 2 & 1 & 46 & .915 & .344 \\
\hline & Brain region 3 & 1 & 46 & .924 & .341 \\
\hline & Brain region 4 & 1 & 46 & .578 & .451 \\
\hline & Brain region 5 & 1 & 46 & .422 & .519 \\
\hline & Brain region 6 & 1 & 46 & 2.623 & .112 \\
\hline & Brain region 7 & 1 & 46 & 4.301 & .044 \\
\hline & Brain region 8 & 1 & 46 & 3.694 & .061 \\
\hline & Brain region 9 & 1 & 46 & .150 & .700 \\
\hline & Brain region 10 & 1 & 46 & 1.362 & .249 \\
\hline & Brain region 11 & 1 & 46 & .144 & .706 \\
\hline & Brain region 12 & 1 & 46 & 4.600 & .037 \\
\hline & Brain region 13 & 1 & 46 & .900 & .348 \\
\hline & Brain region 14 & 1 & 46 & .791 & .378 \\
\hline & Brain region 15 & 1 & 46 & .323 & .572 \\
\hline & Brain region 16 & 1 & 46 & 4.287 & .044 \\
\hline & Brain region 17 & 1 & 46 & 2.099 & .154 \\
\hline & Brain region 18 & 1 & 46 & 1.071 & .306 \\
\hline & Brain region 19 & 1 & 46 & .822 & .369 \\
\hline & Brain region 20 & 1 & 46 & 1.392 & .244 \\
\hline & Brain region 21 & 1 & 46 & 1.800 & .186 \\
\hline & Brain region 22 & 1 & 46 & .237 & .628 \\
\hline & Brain region 23 & 1 & 46 & 1.155 & .288 \\
\hline
\end{tabular}




\begin{tabular}{|c|c|c|c|c|c|}
\hline & Brain region 24 & 1 & 46 & .886 & .351 \\
\hline & Brain region 25 & 1 & 46 & 2.677 & .109 \\
\hline \multirow[t]{25}{*}{ Incentive cond } & Brain region 1 & 2 & 92 & 1.019 & .365 \\
\hline & Brain region 2 & 2 & 92 & .046 & .955 \\
\hline & Brain region 3 & 2 & 92 & 1.724 & 184 \\
\hline & Brain region 4 & 2 & 92 & 2.141 & .123 \\
\hline & Brain region 5 & 2 & 92 & .800 & .452 \\
\hline & Brain region 6 & 2 & 92 & 1.751 & .179 \\
\hline & Brain region 7 & 2 & 92 & .518 & .598 \\
\hline & Brain region 8 & 2 & 92 & 1.003 & .371 \\
\hline & Brain region 9 & 2 & 92 & .172 & .842 \\
\hline & Brain region 10 & 2 & 92 & 1.736 & .182 \\
\hline & Brain region 11 & 2 & 92 & 5.489 & .006 \\
\hline & Brain region 12 & 2 & 92 & 1.122 & .330 \\
\hline & Brain region 13 & 2 & 92 & 2.469 & .090 \\
\hline & Brain region 14 & 2 & 92 & .509 & .603 \\
\hline & Brain region 15 & 2 & 92 & .299 & .743 \\
\hline & Brain region 16 & 2 & 92 & 1.909 & .154 \\
\hline & Brain region 17 & 2 & 92 & .764 & .469 \\
\hline & Brain region 18 & 2 & 92 & .231 & .794 \\
\hline & Brain region 19 & 2 & 92 & .855 & .429 \\
\hline & Brain region 20 & 2 & 92 & .204 & .816 \\
\hline & Brain region 21 & 2 & 92 & .605 & .548 \\
\hline & Brain region 22 & 2 & 92 & 2.319 & .104 \\
\hline & Brain region 23 & 2 & 92 & 1.033 & .360 \\
\hline & Brain region 24 & 2 & 92 & .029 & .971 \\
\hline & Brain region 25 & 2 & 92 & 1.099 & .337 \\
\hline Incentive cond $\mathrm{x}$ group & Brain region 1 & 2 & 92 & 1.876 & .159 \\
\hline
\end{tabular}




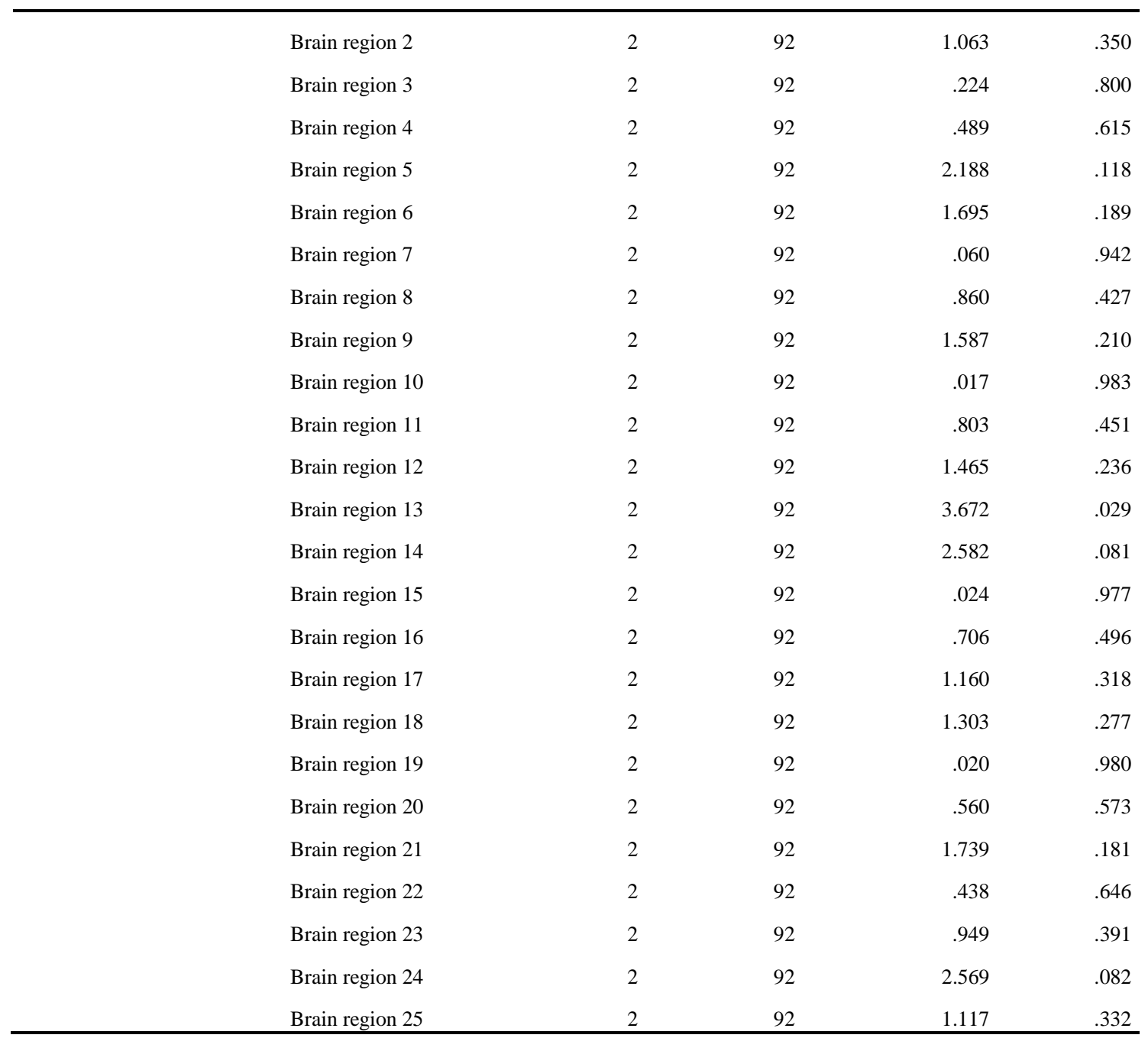


Table I2

Means, Standard Errors and 95\% Confidence Intervals for Group Effects

\begin{tabular}{|c|c|c|c|c|c|}
\hline \multirow[b]{2}{*}{ Brain region } & \multirow[b]{2}{*}{ Group } & \multirow[b]{2}{*}{ Mean } & \multirow[b]{2}{*}{ Std. Error } & \multicolumn{2}{|c|}{ 95\% Confidence Interval } \\
\hline & & & & Lower Bound & Upper Bound \\
\hline \multirow[t]{2}{*}{ Brain region 1} & 1.00 & .197 & .029 & .139 & .255 \\
\hline & 2.00 & .164 & .029 & .106 & .222 \\
\hline \multirow[t]{2}{*}{ Brain region 2} & 1.00 & .225 & .033 & .158 & .291 \\
\hline & 2.00 & .180 & .033 & .113 & 247 \\
\hline \multirow[t]{2}{*}{ Brain region 3} & 1.00 & .156 & .026 & .103 & .209 \\
\hline & 2.00 & .192 & .026 & .139 & .244 \\
\hline \multirow[t]{2}{*}{ Brain region 4} & 1.00 & .155 & .024 & .107 & .203 \\
\hline & 2.00 & .180 & .024 & .133 & .228 \\
\hline \multirow[t]{2}{*}{ Brain region 5} & 1.00 & .168 & .027 & .115 & .222 \\
\hline & 2.00 & .144 & .027 & .090 & 197 \\
\hline \multirow[t]{2}{*}{ Brain region 6} & 1.00 & .214 & .034 & .146 & .282 \\
\hline & 2.00 & .137 & .034 & .069 & .205 \\
\hline \multirow[t]{2}{*}{ Brain region 7} & 1.00 & -.095 & .015 & -.125 & -.064 \\
\hline & 2.00 & -.051 & .015 & -.081 & -.020 \\
\hline \multirow[t]{2}{*}{ Brain region 8} & 1.00 & -.135 & .024 & -.184 & -.087 \\
\hline & 2.00 & -.070 & .024 & -.118 & -.022 \\
\hline \multirow[t]{2}{*}{ Brain region 9} & 1.00 & -.091 & .033 & -.157 & -.025 \\
\hline & 2.00 & -.109 & .033 & -.174 & -.043 \\
\hline \multirow[t]{2}{*}{ Brain region 10} & 1.00 & -.087 & .017 & -.121 & -.052 \\
\hline & 2.00 & -.058 & .017 & -.093 & -.024 \\
\hline Brain region 11 & 1.00 & .166 & .034 & .098 & .235 \\
\hline
\end{tabular}




\begin{tabular}{|c|c|c|c|c|c|}
\hline & 2.00 & .148 & .034 & .080 & .216 \\
\hline \multirow[t]{2}{*}{ Brain region 12} & 1.00 & -.169 & .031 & -.231 & -.106 \\
\hline & 2.00 & -.074 & .031 & -.137 & -.012 \\
\hline \multirow[t]{2}{*}{ Brain region 13} & 1.00 & -.129 & .035 & -.200 & -.059 \\
\hline & 2.00 & -.083 & .035 & -.153 & -.012 \\
\hline \multirow[t]{2}{*}{ Brain region 14} & 1.00 & .208 & .039 & .129 & .288 \\
\hline & 2.00 & .159 & .039 & .079 & .238 \\
\hline \multirow[t]{2}{*}{ Brain region 15} & 1.00 & -.091 & .028 & -.148 & -.033 \\
\hline & 2.00 & -.113 & .028 & -.171 & -.056 \\
\hline \multirow[t]{2}{*}{ Brain region 16} & 1.00 & -.275 & .049 & -.374 & -.177 \\
\hline & 2.00 & -.132 & .049 & -.231 & -.034 \\
\hline \multirow[t]{2}{*}{ Brain region 17} & 1.00 & -.117 & .043 & -.204 & -.030 \\
\hline & 2.00 & -.205 & .043 & -.292 & -.118 \\
\hline \multirow[t]{2}{*}{ Brain region 18} & 1.00 & -.056 & .026 & -.109 & -.003 \\
\hline & 2.00 & -.095 & .026 & -.147 & -.042 \\
\hline \multirow[t]{2}{*}{ Brain region 19} & 1.00 & .105 & .023 & .060 & .151 \\
\hline & 2.00 & .076 & .023 & .031 & .122 \\
\hline \multirow[t]{2}{*}{ Brain region 20} & 1.00 & -.204 & .062 & -.328 & -.080 \\
\hline & 2.00 & -.101 & .062 & -.225 & .023 \\
\hline \multirow[t]{2}{*}{ Brain region 21} & 1.00 & .114 & .029 & .056 & .171 \\
\hline & 2.00 & .168 & .029 & .110 & .225 \\
\hline \multirow[t]{2}{*}{ Brain region 22} & 1.00 & .103 & .025 & .052 & .154 \\
\hline & 2.00 & . 086 & .025 & .035 & .137. \\
\hline \multirow[t]{2}{*}{ Brain region 23} & 1.00 & -.061 & .031 & -.123 & .002 \\
\hline & 2.00 & -.108 & .031 & -.170 & -.045 \\
\hline Brain region 24 & 1.00 & .116 & .032 & .051 & .181 \\
\hline
\end{tabular}




\begin{tabular}{llllll}
\hline & 2.00 & .159 & .032 & .094 & .224 \\
\hline Brain region 25 & 1.00 & .225 & .042 & .141 & .309 \\
& 2.00 & .129 & .042 & .045 & .213 \\
\hline
\end{tabular}


Table I3

Mean Difference, Standard Error, P-Value and 95\% Confidence Interval for Low Trait Anxiety and High Trait Anxiety Groups for Each Effect

\begin{tabular}{|c|c|c|c|c|c|c|c|}
\hline \multirow[b]{2}{*}{ Brain region } & \multirow[b]{2}{*}{ (I) Group } & \multirow{2}{*}{\multicolumn{2}{|c|}{$\begin{array}{c}\text { Mean Difference } \\
(\mathrm{I}-\mathrm{J}) \\
\end{array}$}} & \multirow[b]{2}{*}{ Std. Error } & \multirow[b]{2}{*}{ Sig. ${ }^{\text {a }}$} & \multicolumn{2}{|c|}{ 95\% Confidence Interval for Difference } \\
\hline & & & & & & Lower Bound & Upper Bound \\
\hline \multirow[t]{2}{*}{ Brain region 1} & 1.00 & 2.00 & .033 & .041 & .426 & -.049 & .115 \\
\hline & 2.00 & 1.00 & -.033 & .041 & .426 & -.115 & .049 \\
\hline \multirow[t]{2}{*}{ Brain region 2} & 1.00 & 2.00 & .045 & .047 & .344 & -.049 & .139 \\
\hline & 2.00 & 1.00 & -.045 & .047 & .344 & -.139 & .049 \\
\hline \multirow[t]{2}{*}{ Brain region 3} & 1.00 & 2.00 & -.036 & .037 & .341 & -.110 & .039 \\
\hline & 2.00 & 1.00 & .036 & .037 & .341 & -.039 & .110 \\
\hline \multirow[t]{2}{*}{ Brain region 4} & 1.00 & 2.00 & -.025 & .034 & .451 & -.093 & .042 \\
\hline & 2.00 & 1.00 & .025 & .034 & .451 & -.042 & .093 \\
\hline \multirow[t]{2}{*}{ Brain region 5} & 1.00 & 2.00 & .024 & .038 & .519 & -.051 & .100 \\
\hline & 2.00 & 1.00 & -.024 & .038 & .519 & -.100 & .051 \\
\hline \multirow[t]{2}{*}{ Brain region 6} & 1.00 & 2.00 & .077 & .048 & .112 & -.019 & .173 \\
\hline & 2.00 & 1.00 & -.077 & .048 & .112 & -.173 & .019 \\
\hline \multirow[t]{2}{*}{ Brain region 7} & 1.00 & 2.00 & $-.044^{*}$ & .021 & .044 & -.087 & -.001 \\
\hline & 2.00 & 1.00 & $.044^{*}$ & .021 & .044 & .001 & .087 \\
\hline \multirow[t]{2}{*}{ Brain region 8} & 1.00 & 2.00 & -.065 & .034 & .061 & -.133 & .003 \\
\hline & 2.00 & 1.00 & .065 & .034 & .061 & -.003 & .133 \\
\hline \multirow[t]{2}{*}{ Brain region 9} & 1.00 & 2.00 & .018 & .046 & .700 & -.075 & .111 \\
\hline & 2.00 & 1.00 & -.018 & .046 & .700 & -.111 & .075 \\
\hline \multirow[t]{2}{*}{ Brain region 10} & 1.00 & 2.00 & -.028 & .024 & .249 & -.077 & .021 \\
\hline & 2.00 & 1.00 & .028 & .024 & .249 & -.021 & .077 \\
\hline Brain region 11 & 1.00 & 2.00 & .018 & .048 & .706 & -.079 & .115 \\
\hline
\end{tabular}




\begin{tabular}{|c|c|c|c|c|c|c|c|}
\hline & 2.00 & 1.00 & -.018 & .048 & .706 & -.115 & .079 \\
\hline \multirow[t]{2}{*}{ Brain region 12} & 1.00 & 2.00 & $-.094^{*}$ & .044 & .037 & -.183 & -.006 \\
\hline & 2.00 & 1.00 & $.094^{*}$ & .044 & .037 & .006 & .183 \\
\hline \multirow[t]{2}{*}{ Brain region 13} & 1.00 & 2.00 & -.047 & .049 & .348 & -.146 & .052 \\
\hline & 2.00 & 1.00 & .047 & .049 & .348 & -.052 & .146 \\
\hline \multirow[t]{2}{*}{ Brain region 14} & 1.00 & 2.00 & .050 & .056 & .378 & -.063 & .162 \\
\hline & 2.00 & 1.00 & -.050 & .056 & .378 & -.162 & .063 \\
\hline \multirow[t]{2}{*}{ Brain region 15} & 1.00 & 2.00 & .023 & .040 & .572 & -.058 & .104 \\
\hline & 2.00 & 1.00 & -.023 & .040 & .572 & -.104 & .058 \\
\hline \multirow[t]{2}{*}{ Brain region 16} & 1.00 & 2.00 & $-.143^{*}$ & .069 & .044 & -.282 & -.004 \\
\hline & 2.00 & 1.00 & $.143^{*}$ & .069 & .044 & .004 & .282 \\
\hline \multirow[t]{2}{*}{ Brain region 17} & 1.00 & 2.00 & .089 & .061 & .154 & -.035 & .212 \\
\hline & 2.00 & 1.00 & -.089 & .061 & .154 & -.212 & .035 \\
\hline \multirow[t]{2}{*}{ Brain region 18} & 1.00 & 2.00 & .038 & .037 & .306 & -.036 & .113 \\
\hline & 2.00 & 1.00 & -.038 & .037 & .306 & -.113 & .036 \\
\hline \multirow[t]{2}{*}{ Brain region 19} & 1.00 & 2.00 & .029 & .032 & .369 & -.035 & .094 \\
\hline & 2.00 & 1.00 & -.029 & .032 & .369 & -.094 & .035 \\
\hline \multirow[t]{2}{*}{ Brain region 20} & 1.00 & 2.00 & -.103 & .087 & .244 & -.279 & .073 \\
\hline & 2.00 & 1.00 & .103 & .087 & .244 & -.073 & .279 \\
\hline \multirow[t]{2}{*}{ Brain region 21} & 1.00 & 2.00 & -.054 & .040 & .186 & -.136 & .027 \\
\hline & 2.00 & 1.00 & .054 & .040 & .186 & -.027 & .136 \\
\hline \multirow[t]{2}{*}{ Brain region 22} & 1.00 & 2.00 & .018 & .036 & .628 & -.055 & .090 \\
\hline & 2.00 & 1.00 & -.018 & .036 & .628 & -.090 & .055 \\
\hline \multirow[t]{2}{*}{ Brain region 23} & 1.00 & 2.00 & .047 & .044 & .288 & -.041 & .135 \\
\hline & 2.00 & 1.00 & -.047 & .044 & .288 & -.135 & .041 \\
\hline Brain region 24 & 1.00 & 2.00 & -.043 & .046 & .351 & -.135 & .049 \\
\hline
\end{tabular}




\begin{tabular}{lccccccc}
\hline & 2.00 & 1.00 & .043 & .046 & .351 & -.049 & .135 \\
\hline \multirow{2}{*}{ Brain region 25 } & 1.00 & 2.00 & .096 & .059 & .109 & -.022 & .215 \\
\cline { 2 - 7 } & 2.00 & 1.00 & -.096 & .059 & .109 & -.215 & .022 \\
\hline
\end{tabular}

Based on estimated marginal means

a. Adjustment for multiple comparisons: Least Significant Difference (equivalent to no adjustments).

*. The mean difference is significant at the .05 level. 
Table I4

Means, Standard Errors and 95\% Confidence Intervals for Incentive Condition Effects

\begin{tabular}{|c|c|c|c|c|c|}
\hline \multirow[b]{2}{*}{ Brain region } & \multirow[b]{2}{*}{ Incentive cond } & \multirow[b]{2}{*}{ Mean } & \multirow[b]{2}{*}{ Std. Error } & \multicolumn{2}{|c|}{ 95\% Confidence Interval } \\
\hline & & & & Lower Bound & Upper Bound \\
\hline \multirow[t]{3}{*}{ Brain region 1} & 1 & .169 & .023 & .123 & .214 \\
\hline & 2 & .193 & .022 & .149 & .237 \\
\hline & 3 & .180 & .023 & .134 & .227 \\
\hline \multirow[t]{3}{*}{ Brain region 2} & 1 & .199 & .026 & .148 & .251 \\
\hline & 2 & .202 & .026 & .150 & .255 \\
\hline & 3 & .205 & .025 & .154 & .256 \\
\hline \multirow[t]{3}{*}{ Brain region 3} & 1 & .152 & .022 & .107 & .197 \\
\hline & 2 & .187 & .023 & .141 & .233 \\
\hline & 3 & .182 & .020 & .141 & .223 \\
\hline \multirow[t]{3}{*}{ Brain region 4} & 1 & .145 & .021 & .102 & .188 \\
\hline & 2 & .178 & .021 & .137 & .220 \\
\hline & 3 & .180 & .018 & .144 & .216 \\
\hline \multirow[t]{3}{*}{ Brain region 5} & 1 & .162 & .022 & .118 & .207 \\
\hline & 2 & .162 & .021 & .120 & .205 \\
\hline & 3 & .143 & .021 & .102 & .185 \\
\hline \multirow[t]{3}{*}{ Brain region 6} & 1 & .155 & .027 & .101 & .209 \\
\hline & 2 & .194 & .027 & .139 & .248 \\
\hline & 3 & .178 & .026 & .125 & .231 \\
\hline \multirow[t]{3}{*}{ Brain region 7} & 1 & -.083 & .015 & -.113 & -.052 \\
\hline & 2 & -.069 & .012 & -.093 & -.045 \\
\hline & 3 & -.067 & .016 & -.099 & -.034 \\
\hline
\end{tabular}




\begin{tabular}{|c|c|c|c|c|c|}
\hline \multirow[t]{3}{*}{ Brain region 8} & 1 & -.122 & .025 & -.172 & -.073 \\
\hline & 2 & -.095 & .019 & -.135 & -.056 \\
\hline & 3 & -.090 & .021 & -.134 & -.047 \\
\hline \multirow[t]{3}{*}{ Brain region 9} & 1 & -.100 & .032 & -.165 & -.035 \\
\hline & 2 & -.108 & .025 & -.157 & -.058 \\
\hline & 3 & -.092 & .025 & -.143 & -.041 \\
\hline \multirow[t]{3}{*}{ Brain region 10} & 1 & -.090 & .018 & -.126 & -.054 \\
\hline & 2 & -.075 & .015 & -.105 & -.045 \\
\hline & 3 & -.053 & .018 & -.088 & -.017 \\
\hline \multirow[t]{3}{*}{ Brain region 11} & 1 & .112 & .030 & .052 & .173 \\
\hline & 2 & .193 & .028 & .136 & .249 \\
\hline & 3 & .167 & .025 & .115 & .218 \\
\hline \multirow[t]{3}{*}{ Brain region 12} & 1 & -.093 & .033 & -.160 & -.026 \\
\hline & 2 & -.137 & .025 & -.188 & -.086 \\
\hline & 3 & -.134 & .028 & -.190 & -.079 \\
\hline \multirow[t]{3}{*}{ Brain region 13} & 1 & -.071 & .027 & -.125 & -.016 \\
\hline & 2 & -.109 & .024 & -.158 & -.061 \\
\hline & 3 & -.138 & .038 & -.214 & -.062 \\
\hline \multirow[t]{3}{*}{ Brain region 14} & 1 & .197 & .034 & .129 & .265 \\
\hline & 2 & .172 & .030 & .112 & .232 \\
\hline & 3 & .182 & .030 & .121 & .243 \\
\hline \multirow[t]{3}{*}{ Brain region 15} & 1 & -.100 & .025 & -.149 & -.050 \\
\hline & 2 & -.110 & .023 & -.156 & -.065 \\
\hline & 3 & -.096 & .021 & -.139 & -.054 \\
\hline \multirow[t]{2}{*}{ Brain region 16} & 1 & -.246 & .046 & -.340 & -.153 \\
\hline & 2 & -.201 & .042 & -.286 & -.116 \\
\hline
\end{tabular}




\begin{tabular}{|c|c|c|c|c|c|}
\hline & 3 & -.164 & .038 & -.240 & -.088 \\
\hline \multirow[t]{3}{*}{ Brain region 17} & 1 & -.136 & .041 & -.218 & -.054 \\
\hline & 2 & -.165 & .031 & -.228 & -.102 \\
\hline & 3 & -.182 & .040 & -.262 & -.101 \\
\hline \multirow[t]{3}{*}{ Brain region 18} & 1 & -.083 & .025 & -.134 & -.033 \\
\hline & 2 & -.067 & .022 & -.112 & -.022 \\
\hline & 3 & -.076 & .022 & -.121 & -.031 \\
\hline \multirow[t]{3}{*}{ Brain region 19} & 1 & .075 & .022 & .030 & .120 \\
\hline & 2 & .105 & .019 & .067 & .144 \\
\hline & 3 & .092 & .021 & .049 & .135 \\
\hline \multirow[t]{3}{*}{ Brain region 20} & 1 & -.149 & .058 & -.266 & -.031 \\
\hline & 2 & -.175 & .052 & -.279 & -.071 \\
\hline & 3 & -.134 & .063 & -.261 & -.007 \\
\hline \multirow[t]{3}{*}{ Brain region 21} & 1 & .128 & .021 & .086 & .171 \\
\hline & 2 & .147 & .026 & .094 & .199 \\
\hline & 3 & .147 & .022 & .103 & .191 \\
\hline \multirow[t]{3}{*}{ Brain region 22} & 1 & .061 & .021 & .019 & .103 \\
\hline & 2 & .121 & .026 & .069 & .172 \\
\hline & 3 & .102 & .027 & .049 & .155 \\
\hline \multirow[t]{3}{*}{ Brain region 23} & 1 & -.073 & .031 & -.135 & -.010 \\
\hline & 2 & -.069 & .026 & -.121 & -.018 \\
\hline & 3 & -.110 & .029 & -.168 & -.053 \\
\hline \multirow[t]{3}{*}{ Brain region 24} & 1 & .136 & .025 & .086 & .187 \\
\hline & 2 & .141 & .024 & .092 & .190 \\
\hline & 3 & .136 & .028 & .079 & .193 \\
\hline Brain region 25 & 1 & .154 & .036 & .083 & .226 \\
\hline
\end{tabular}




\begin{tabular}{lllll}
\hline 2 & .191 & .031 & .128 & .254 \\
3 & .186 & .032 & .120 & .251 \\
\hline
\end{tabular}


Table I5

Mean Difference, Standard Error, P-Value and 95\% Confidence Interval for Incentive Condition Effects

\begin{tabular}{|c|c|c|c|c|c|c|c|}
\hline \multirow[b]{2}{*}{ Brain region } & \multirow[b]{2}{*}{ (I) Incentive cond } & \multicolumn{4}{|c|}{ Mean Difference } & \multicolumn{2}{|c|}{ 95\% Confidence Interval for Difference ${ }^{a}$} \\
\hline & & (J) Incentive cond & $(\mathrm{I}-\mathrm{J})$ & Std. Error & Sig. ${ }^{a}$ & Lower Bound & Upper Bound \\
\hline \multirow[t]{6}{*}{ Brain region 1} & 1 & 2 & -.024 & .018 & .180 & -.060 & .012 \\
\hline & & 3 & -.011 & .016 & .488 & -.044 & .021 \\
\hline & 2 & 1 & .024 & .018 & .180 & -.012 & .060 \\
\hline & & 3 & .013 & .017 & .450 & -.021 & .047 \\
\hline & 3 & 1 & .011 & .016 & .488 & -.021 & .044 \\
\hline & & 2 & -.013 & .017 & .450 & -.047 & .021 \\
\hline \multirow[t]{6}{*}{ Brain region 2} & 1 & 2 & -.003 & .020 & .882 & -.043 & .037 \\
\hline & & 3 & -.006 & .017 & .753 & -.040 & .029 \\
\hline & 2 & 1 & .003 & .020 & .882 & -.037 & .043 \\
\hline & & 3 & -.003 & .017 & .884 & -.037 & .032 \\
\hline & 3 & 1 & .006 & .017 & .753 & -.029 & .040 \\
\hline & & 2 & .003 & .017 & .884 & -.032 & .037 \\
\hline \multirow[t]{6}{*}{ Brain region 3} & 1 & 2 & -.035 & .023 & .127 & -.080 & .010 \\
\hline & & 3 & -.030 & .022 & .176 & -.073 & .014 \\
\hline & 2 & 1 & .035 & .023 & .127 & -.010 & .080 \\
\hline & & 3 & .005 & .016 & .741 & -.027 & .038 \\
\hline & 3 & 1 & .030 & .022 & .176 & -.014 & .073 \\
\hline & & 2 & -.005 & .016 & .741 & -.038 & .027 \\
\hline \multirow[t]{3}{*}{ Brain region 4} & 1 & 2 & -.033 & .021 & .111 & -.075 & .008 \\
\hline & & 3 & -.035 & .020 & .086 & -.075 & .005 \\
\hline & 2 & 1 & .033 & .021 & .111 & -.008 & .075 \\
\hline
\end{tabular}




\begin{tabular}{|c|c|c|c|c|c|c|c|}
\hline & & 3 & -.002 & .017 & .926 & -.035 & .032 \\
\hline & 3 & 1 & .035 & .020 & .086 & -.005 & .075 \\
\hline & & 2 & .002 & .017 & .926 & -.032 & .035 \\
\hline \multirow[t]{6}{*}{ Brain region 5} & 1 & 2 & .000 & .019 & 999 & -.038 & .038 \\
\hline & & 3 & .019 & .014 & .180 & -.009 & .047 \\
\hline & 2 & 1 & .000 & .019 & .999 & -.038 & .038 \\
\hline & & 3 & .019 & .019 & .315 & -.019 & .057 \\
\hline & 3 & 1 & -.019 & .014 & .180 & -.047 & .009 \\
\hline & & 2 & -.019 & .019 & .315 & -.057 & .019 \\
\hline \multirow[t]{6}{*}{ Brain region 6} & 1 & 2 & -.039 & .020 & .064 & -.079 & .002 \\
\hline & & 3 & -.023 & .021 & .285 & -.066 & .020 \\
\hline & 2 & 1 & .039 & .020 & .064 & -.002 & .079 \\
\hline & & 3 & .016 & .021 & .456 & -.026 & .057 \\
\hline & 3 & 1 & .023 & .021 & .285 & -.020 & .066 \\
\hline & & 2 & -.016 & .021 & .456 & -.057 & .026 \\
\hline \multirow[t]{6}{*}{ Brain region 7} & 1 & 2 & -.014 & .018 & .441 & -.050 & .022 \\
\hline & & 3 & -.016 & .017 & .355 & -.051 & .019 \\
\hline & 2 & 1 & .014 & .018 & .441 & -.022 & .050 \\
\hline & & 3 & -.002 & .016 & .883 & -.036 & .031 \\
\hline & 3 & 1 & .016 & .017 & .355 & -.019 & .051 \\
\hline & & 2 & .002 & .016 & .883 & -.031 & .036 \\
\hline \multirow[t]{5}{*}{ Brain region 8} & 1 & 2 & -.027 & .024 & .276 & -.076 & .022 \\
\hline & & 3 & -.032 & .026 & .224 & -.084 & .020 \\
\hline & 2 & 1 & .027 & .024 & .276 & -.022 & .076 \\
\hline & & 3 & -.005 & .022 & .822 & -.050 & .040 \\
\hline & 3 & 1 & .032 & .026 & .224 & -.020 & .084 \\
\hline
\end{tabular}




\begin{tabular}{|c|c|c|c|c|c|c|c|}
\hline & & 2 & .005 & .022 & .822 & -.040 & .050 \\
\hline \multirow[t]{6}{*}{ Brain region 9} & 1 & 2 & .007 & .027 & .783 & -.047 & .062 \\
\hline & & 3 & -.008 & .031 & .795 & -.071 & .055 \\
\hline & 2 & 1 & -.007 & .027 & .783 & -.062 & .047 \\
\hline & & 3 & -.016 & .021 & .452 & -.057 & .026 \\
\hline & 3 & 1 & .008 & .031 & .795 & -.055 & .071 \\
\hline & & 2 & .016 & .021 & .452 & -.026 & .057 \\
\hline \multirow[t]{6}{*}{ Brain region 10} & 1 & 2 & -.014 & .022 & .517 & -.059 & .030 \\
\hline & & 3 & -.037 & .020 & .066 & -.077 & .003 \\
\hline & 2 & 1 & .014 & .022 & .517 & -.030 & .059 \\
\hline & & 3 & -.023 & .018 & .219 & -.059 & .014 \\
\hline & 3 & 1 & .037 & .020 & .066 & -.003 & .077 \\
\hline & & 2 & .023 & .018 & .219 & -.014 & .059 \\
\hline \multirow[t]{6}{*}{ Brain region 11} & 1 & 2 & $-.080^{*}$ & .026 & .004 & -.133 & -.027 \\
\hline & & 3 & $-.054^{*}$ & .025 & .038 & -.105 & -.003 \\
\hline & 2 & 1 & $.080^{*}$ & .026 & .004 & .027 & .133 \\
\hline & & 3 & .026 & .022 & .252 & -.019 & .071 \\
\hline & 3 & 1 & $.054^{*}$ & .025 & .038 & .003 & .105 \\
\hline & & 2 & -.026 & .022 & .252 & -.071 & .019 \\
\hline \multirow[t]{6}{*}{ Brain region 12} & 1 & 2 & .044 & .035 & .223 & -.028 & .115 \\
\hline & & 3 & .041 & .036 & .255 & -.031 & .113 \\
\hline & 2 & 1 & -.044 & .035 & .223 & -.115 & .028 \\
\hline & & 3 & -.003 & .026 & .920 & -.056 & .050 \\
\hline & 3 & 1 & -.041 & .036 & .255 & -.113 & .031 \\
\hline & & 2 & .003 & .026 & .920 & -.050 & .056 \\
\hline Brain region 13 & 1 & 2 & .038 & .021 & .076 & -.004 & .081 \\
\hline
\end{tabular}




\begin{tabular}{|c|c|c|c|c|c|c|c|}
\hline & & 3 & .067 & .038 & .081 & -.009 & .143 \\
\hline & \multirow[t]{2}{*}{2} & 1 & -.038 & .021 & .076 & -.081 & .004 \\
\hline & & 3 & .029 & .030 & .341 & -.032 & .089 \\
\hline & \multirow[t]{2}{*}{3} & 1 & -.067 & .038 & .081 & -.143 & .009 \\
\hline & & 2 & -.029 & .030 & .341 & -.089 & .032 \\
\hline \multirow[t]{6}{*}{ Brain region 14} & \multirow[t]{2}{*}{1} & 2 & .025 & .025 & .319 & -.025 & .075 \\
\hline & & 3 & .015 & .025 & .561 & -.036 & .065 \\
\hline & \multirow[t]{2}{*}{2} & 1 & -.025 & .025 & .319 & -.075 & .025 \\
\hline & & 3 & -.010 & .025 & .678 & -.061 & .040 \\
\hline & \multirow[t]{2}{*}{3} & 1 & -.015 & .025 & .561 & -.065 & .036 \\
\hline & & 2 & .010 & .025 & .678 & -.040 & .061 \\
\hline \multirow[t]{6}{*}{ Brain region 15} & \multirow[t]{2}{*}{1} & 2 & .010 & .017 & .550 & -.024 & .045 \\
\hline & & 3 & -.003 & .021 & .877 & -.045 & .039 \\
\hline & \multirow[t]{2}{*}{2} & 1 & -.010 & .017 & .550 & -.045 & .024 \\
\hline & & 3 & -.014 & .017 & .426 & -.048 & .021 \\
\hline & \multirow[t]{2}{*}{3} & 1 & .003 & .021 & .877 & -.039 & .045 \\
\hline & & 2 & .014 & .017 & .426 & -.021 & .048 \\
\hline \multirow[t]{6}{*}{ Brain region 16} & \multirow[t]{2}{*}{1} & 2 & -.045 & .044 & .307 & -.134 & .043 \\
\hline & & 3 & -.082 & .044 & .071 & -.171 & .007 \\
\hline & \multirow[t]{2}{*}{2} & 1 & .045 & .044 & .307 & -.043 & .134 \\
\hline & & 3 & -.037 & .037 & .335 & -.112 & .039 \\
\hline & \multirow[t]{2}{*}{3} & 1 & .082 & .044 & .071 & -.007 & .171 \\
\hline & & 2 & .037 & .037 & .335 & -.039 & .112 \\
\hline \multirow[t]{3}{*}{ Brain region 17} & \multirow[t]{2}{*}{1} & 2 & .029 & .036 & .412 & -.042 & .101 \\
\hline & & 3 & .046 & .042 & .277 & -.038 & .130 \\
\hline & 2 & 1 & -.029 & .036 & .412 & -.101 & .042 \\
\hline
\end{tabular}




\begin{tabular}{|c|c|c|c|c|c|c|c|}
\hline & & 3 & .017 & .035 & .641 & -.055 & .088 \\
\hline & 3 & 1 & -.046 & .042 & .277 & -.130 & .038 \\
\hline & & 2 & -.017 & .035 & .641 & -.088 & .055 \\
\hline \multirow[t]{6}{*}{ Brain region 18} & 1 & 2 & -.017 & .023 & .485 & -.064 & .031 \\
\hline & & 3 & -.008 & .025 & .767 & -.058 & .043 \\
\hline & 2 & 1 & .017 & .023 & .485 & -.031 & .064 \\
\hline & & 3 & .009 & .024 & .712 & -.040 & .058 \\
\hline & 3 & 1 & .008 & .025 & .767 & -.043 & .058 \\
\hline & & 2 & -.009 & .024 & .712 & -.058 & .040 \\
\hline \multirow[t]{6}{*}{ Brain region 19} & 1 & 2 & -.030 & .022 & .176 & -.075 & .014 \\
\hline & & 3 & -.017 & .024 & .463 & -.065 & .030 \\
\hline & 2 & 1 & .030 & .022 & .176 & -.014 & .075 \\
\hline & & 3 & .013 & .024 & .596 & -.036 & .062 \\
\hline & 3 & 1 & .017 & .024 & .463 & -.030 & .065 \\
\hline & & 2 & -.013 & .024 & .596 & -.062 & .036 \\
\hline \multirow[t]{6}{*}{ Brain region 20} & 1 & 2 & .026 & .052 & .615 & -.079 & .132 \\
\hline & & 3 & -.015 & .079 & .851 & -.174 & .144 \\
\hline & 2 & 1 & -.026 & .052 & .615 & -.132 & .079 \\
\hline & & 3 & -.041 & .063 & .512 & -.168 & .085 \\
\hline & 3 & 1 & .015 & .079 & .851 & -.144 & .174 \\
\hline & & 2 & .041 & .063 & .512 & -.085 & .168 \\
\hline \multirow[t]{5}{*}{ Brain region 21} & 1 & 2 & -.018 & .018 & .303 & -.054 & .017 \\
\hline & & 3 & -.019 & .021 & .361 & -.060 & .022 \\
\hline & 2 & 1 & .018 & .018 & .303 & -.017 & .054 \\
\hline & & 3 & -.001 & .021 & .977 & -.042 & .041 \\
\hline & 3 & 1 & .019 & .021 & .361 & -.022 & .060 \\
\hline
\end{tabular}




\begin{tabular}{|c|c|c|c|c|c|c|c|}
\hline & & 2 & .001 & .021 & .977 & -.041 & .042 \\
\hline \multirow[t]{6}{*}{ Brain region 22} & 1 & 2 & $-.060^{*}$ & .030 & .048 & -.120 & .000 \\
\hline & & 3 & -.041 & .033 & .213 & -.107 & .025 \\
\hline & 2 & 1 & $.060^{*}$ & .030 & .048 & .000 & .120 \\
\hline & & 3 & .019 & .022 & .404 & -.026 & .064 \\
\hline & 3 & 1 & .041 & .033 & .213 & -.025 & .107 \\
\hline & & 2 & -.019 & .022 & .404 & -.064 & .026 \\
\hline \multirow[t]{6}{*}{ Brain region 23} & 1 & 2 & -.003 & .032 & .917 & -.068 & .062 \\
\hline & & 3 & .038 & .033 & .260 & -.029 & .104 \\
\hline & 2 & 1 & .003 & .032 & .917 & -.062 & .068 \\
\hline & & 3 & .041 & .029 & .171 & -.018 & .100 \\
\hline & 3 & 1 & -.038 & .033 & .260 & -.104 & .029 \\
\hline & & 2 & -.041 & .029 & .171 & -.100 & .018 \\
\hline \multirow[t]{6}{*}{ Brain region 24} & 1 & 2 & -.004 & .019 & .820 & -.042 & .034 \\
\hline & & 3 & .000 & .021 & .983 & -.042 & .043 \\
\hline & 2 & 1 & .004 & .019 & .820 & -.034 & .042 \\
\hline & & 3 & .005 & .025 & .847 & -.045 & .054 \\
\hline & 3 & 1 & .000 & .021 & .983 & -.043 & .042 \\
\hline & & 2 & -.005 & .025 & .847 & -.054 & .045 \\
\hline \multirow[t]{6}{*}{ Brain region 25} & 1 & 2 & -.036 & .028 & .209 & -.093 & .021 \\
\hline & & 3 & -.031 & .028 & .273 & -.088 & .025 \\
\hline & 2 & 1 & .036 & .028 & .209 & -.021 & .093 \\
\hline & & 3 & .005 & .022 & .824 & -.040 & .050 \\
\hline & 3 & 1 & .031 & .028 & .273 & -.025 & .088 \\
\hline & & 2 & -.005 & .022 & .824 & -.050 & .040 \\
\hline
\end{tabular}

Based on estimated marginal means 
a. Adjustment for multiple comparisons: Least Significant Difference (equivalent to no adjustments)

*. The mean difference is significant at the .05 level. 
Table I6

Means, Standard Errors and 95\% Confidence Intervals for Group x Incentive Condition Effects

\begin{tabular}{|c|c|c|c|c|c|c|}
\hline \multirow[b]{2}{*}{ Brain region } & \multirow[b]{2}{*}{ Group } & \multirow[b]{2}{*}{ Incentive cond } & \multirow[b]{2}{*}{ Mean } & \multirow[b]{2}{*}{ Std. Error } & \multicolumn{2}{|c|}{ 95\% Confidence Interval } \\
\hline & & & & & Lower Bound & Upper Bound \\
\hline \multirow[t]{6}{*}{ Brain region 1} & 1.00 & 1 & .192 & .032 & .128 & .257 \\
\hline & & 2 & .221 & .031 & .158 & .284 \\
\hline & & 3 & .178 & .033 & .112 & .244 \\
\hline & 2.00 & 1 & .145 & .032 & .081 & .210 \\
\hline & & 2 & .165 & .031 & .102 & .228 \\
\hline & & 3 & .183 & .033 & .117 & .249 \\
\hline \multirow[t]{6}{*}{ Brain region 2} & 1.00 & 1 & .218 & .036 & .145 & .292 \\
\hline & & 2 & .240 & .037 & .165 & .314 \\
\hline & & 3 & .216 & .036 & .145 & .288 \\
\hline & 2.00 & 1 & .181 & .036 & .107 & .254 \\
\hline & & 2 & .165 & .037 & .091 & .240 \\
\hline & & 3 & .194 & .036 & .122 & .265 \\
\hline \multirow[t]{6}{*}{ Brain region 3} & 1.00 & 1 & .133 & .032 & .069 & .197 \\
\hline & & 2 & .177 & .032 & .111 & .242 \\
\hline & & 3 & .158 & .029 & .099 & .216 \\
\hline & 2.00 & 1 & .171 & .032 & .107 & .235 \\
\hline & & 2 & .198 & .032 & .133 & .263 \\
\hline & & 3 & .206 & .029 & .148 & .264 \\
\hline \multirow[t]{3}{*}{ Brain region 4} & 1.00 & 1 & .140 & .030 & .079 & .201 \\
\hline & & 2 & .168 & .029 & .109 & .227 \\
\hline & & 3 & .157 & .025 & .106 & 208 \\
\hline
\end{tabular}




\begin{tabular}{|c|c|c|c|c|c|c|}
\hline & 2.00 & 1 & .150 & .030 & .089 & .210 \\
\hline & & 2 & .189 & .029 & .130 & .248 \\
\hline & & 3 & .203 & .025 & .152 & .254 \\
\hline \multirow[t]{6}{*}{ Brain region 5} & 1.00 & 1 & .183 & .031 & .120 & .246 \\
\hline & & 2 & .187 & .030 & .127 & .246 \\
\hline & & 3 & .134 & .029 & .076 & .193 \\
\hline & 2.00 & 1 & .141 & .031 & .078 & .204 \\
\hline & & 2 & .138 & .030 & .078 & .198 \\
\hline & & 3 & .152 & .029 & .093 & .211 \\
\hline \multirow[t]{6}{*}{ Brain region 6} & 1.00 & 1 & .180 & .038 & .104 & .256 \\
\hline & & 2 & .254 & .038 & .177 & .331 \\
\hline & & 3 & .209 & .037 & .134 & .284 \\
\hline & 2.00 & 1 & .131 & .038 & .055 & .207 \\
\hline & & 2 & .133 & .038 & .056 & .210 \\
\hline & & 3 & .147 & .037 & .072 & .222 \\
\hline \multirow[t]{6}{*}{ Brain region 7} & 1.00 & 1 & -.103 & .022 & -.146 & -.059 \\
\hline & & 2 & -.094 & .017 & -.129 & -.060 \\
\hline & & 3 & -.088 & .023 & -.134 & -.042 \\
\hline & 2.00 & 1 & -.063 & .022 & -.106 & -.020 \\
\hline & & 2 & -.043 & .017 & -.078 & -.009 \\
\hline & & 3 & -.045 & .023 & -.091 & .001 \\
\hline \multirow[t]{5}{*}{ Brain region 8} & 1.00 & 1 & -.162 & .035 & -.233 & -.092 \\
\hline & & 2 & -.110 & .028 & -.165 & -.054 \\
\hline & & 3 & -.134 & .030 & -.195 & -.073 \\
\hline & 2.00 & 1 & -.082 & .035 & -.153 & -.012 \\
\hline & & 2 & -.081 & .028 & -.137 & -.026 \\
\hline
\end{tabular}




\begin{tabular}{|c|c|c|c|c|c|c|}
\hline & & 3 & -.047 & .030 & -.108 & .014 \\
\hline \multirow[t]{6}{*}{ Brain region 9} & 1.00 & 1 & -.069 & .046 & -.161 & .023 \\
\hline & & 2 & -.096 & .035 & -.166 & -.026 \\
\hline & & 3 & -.108 & .036 & -.180 & -.035 \\
\hline & 2.00 & 1 & -.131 & .046 & -.224 & -.039 \\
\hline & & 2 & -.119 & .035 & -.189 & -.049 \\
\hline & & 3 & -.076 & .036 & -.149 & -.004 \\
\hline \multirow[t]{6}{*}{ Brain region 10} & 1.00 & 1 & -.106 & .025 & -.157 & -.055 \\
\hline & & 2 & -.088 & .021 & -.130 & -.046 \\
\hline & & 3 & -.067 & .025 & -.117 & -.017 \\
\hline & 2.00 & 1 & -.074 & .025 & -.124 & -.023 \\
\hline & & 2 & -.063 & .021 & -.105 & -.021 \\
\hline & & 3 & -.039 & .025 & -.088 & .011 \\
\hline \multirow[t]{6}{*}{ Brain region 11} & 1.00 & 1 & .104 & .042 & .018 & .189 \\
\hline & & 2 & .213 & .040 & .133 & .293 \\
\hline & & 3 & .182 & .036 & .110 & .255 \\
\hline & 2.00 & 1 & .121 & .042 & .036 & .206 \\
\hline & & 2 & .172 & .040 & .092 & .252 \\
\hline & & 3 & .151 & .036 & .078 & .223 \\
\hline \multirow[t]{6}{*}{ Brain region 12} & 1.00 & 1 & -.111 & .047 & -.206 & -.017 \\
\hline & & 2 & -.186 & .036 & -.259 & -.114 \\
\hline & & 3 & -.208 & .039 & -.287 & -.130 \\
\hline & 2.00 & 1 & -.075 & .047 & -.170 & .020 \\
\hline & & 2 & -.088 & .036 & -.160 & -.016 \\
\hline & & 3 & -.060 & .039 & -.139 & .019 \\
\hline Brain region 13 & 1.00 & 1 & -.065 & .038 & -.142 & .013 \\
\hline
\end{tabular}




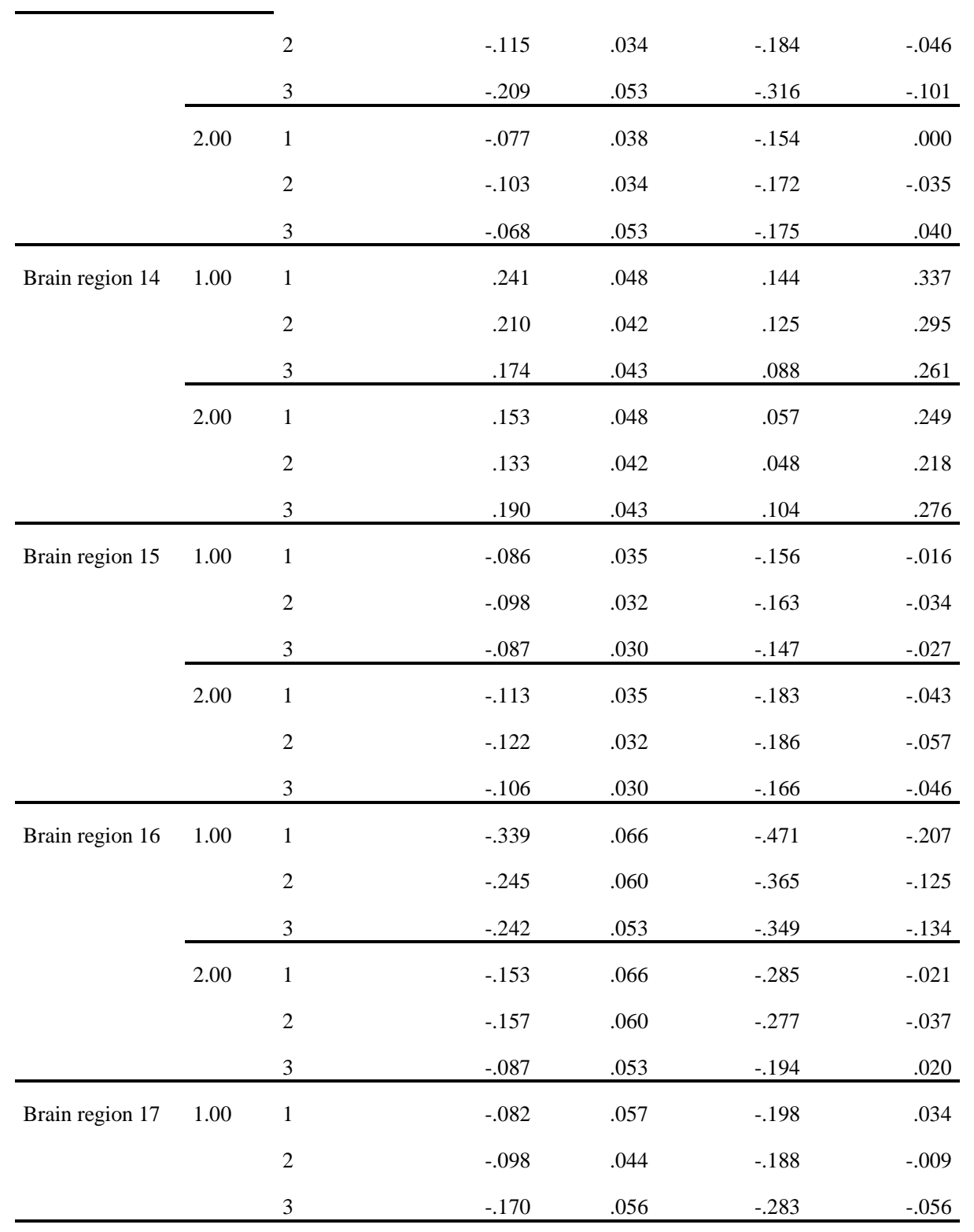




\begin{tabular}{|c|c|c|c|c|c|c|}
\hline & 2.00 & 1 & -.190 & .057 & -.305 & -.074 \\
\hline & & 2 & -.232 & .044 & -.322 & -.143 \\
\hline & & 3 & -.194 & .056 & -.308 & -.080 \\
\hline \multirow[t]{6}{*}{ Brain region 18} & 1.00 & 1 & -.060 & .035 & -.131 & .011 \\
\hline & & 2 & -.031 & .032 & -.094 & .033 \\
\hline & & 3 & -.078 & .032 & -.142 & -.015 \\
\hline & 2.00 & 1 & -.107 & .035 & -.178 & -.036 \\
\hline & & 2 & -.103 & .032 & -.167 & -.039 \\
\hline & & 3 & -.074 & .032 & -.137 & -.010 \\
\hline \multirow[t]{6}{*}{ Brain region 19} & 1.00 & 1 & .091 & .031 & .028 & .154 \\
\hline & & 2 & .121 & .027 & .067 & .175 \\
\hline & & 3 & .104 & .030 & .044 & .165 \\
\hline & 2.00 & 1 & .059 & .031 & -.004 & .122 \\
\hline & & 2 & .090 & .027 & .035 & .144 \\
\hline & & 3 & .081 & .030 & .020 & .141 \\
\hline \multirow[t]{6}{*}{ Brain region 20} & 1.00 & 1 & -.163 & .082 & -.329 & .004 \\
\hline & & 2 & -.233 & .073 & -.380 & -.087 \\
\hline & & 3 & -.216 & .089 & -.395 & -.037 \\
\hline & 2.00 & 1 & -.135 & .082 & -.301 & .031 \\
\hline & & 2 & -.117 & .073 & -.264 & .030 \\
\hline & & 3 & -.051 & .089 & -.231 & .128 \\
\hline \multirow[t]{5}{*}{ Brain region 21} & 1.00 & 1 & .111 & .030 & .051 & .172 \\
\hline & & 2 & .131 & .037 & .056 & .205 \\
\hline & & 3 & .099 & .031 & .037 & .161 \\
\hline & 2.00 & 1 & .145 & .030 & .085 & .206 \\
\hline & & 2 & .163 & .037 & .088 & .237 \\
\hline
\end{tabular}




\begin{tabular}{|c|c|c|c|c|c|c|}
\hline & & 3 & .196 & .031 & .133 & .258 \\
\hline \multirow[t]{6}{*}{ Brain region 22} & 1.00 & 1 & .054 & .029 & -.005 & .113 \\
\hline & & 2 & .136 & .036 & .063 & .208 \\
\hline & & 3 & .120 & .037 & .045 & .196 \\
\hline & 2.00 & 1 & .067 & .029 & .008 & .126 \\
\hline & & 2 & .106 & .036 & .033 & .179 \\
\hline & & 3 & .084 & .037 & .008 & .159 \\
\hline \multirow[t]{6}{*}{ Brain region 23} & 1.00 & 1 & -.038 & .044 & -.126 & .051 \\
\hline & & 2 & -.032 & .036 & -.105 & .040 \\
\hline & & 3 & -.112 & .040 & -.193 & -.031 \\
\hline & 2.00 & 1 & -.108 & .044 & -.196 & -.019 \\
\hline & & 2 & -.106 & .036 & -.179 & -.034 \\
\hline & & 3 & -.109 & .040 & -.190 & -.027 \\
\hline \multirow[t]{6}{*}{ Brain region 24} & 1.00 & 1 & .133 & .035 & .061 & .204 \\
\hline & & 2 & .129 & .034 & .060 & .199 \\
\hline & & 3 & .087 & .040 & .006 & .167 \\
\hline & 2.00 & 1 & .140 & .035 & .069 & .211 \\
\hline & & 2 & .152 & .034 & .083 & .221 \\
\hline & & 3 & .185 & .040 & .105 & .266 \\
\hline \multirow[t]{6}{*}{ Brain region 25} & 1.00 & 1 & .181 & .051 & .080 & .283 \\
\hline & & 2 & .257 & .044 & .168 & .346 \\
\hline & & 3 & .237 & .046 & .145 & .329 \\
\hline & 2.00 & 1 & .128 & .051 & .026 & .229 \\
\hline & & 2 & .125 & .044 & .036 & .213 \\
\hline & & 3 & .134 & .046 & .042 & .226 \\
\hline
\end{tabular}


Table I7

Mean Difference, Standard Error, P-Value and 95\% Confidence Interval for Incentive Condition Effects by Group

\begin{tabular}{|c|c|c|c|c|c|c|}
\hline \multirow[b]{2}{*}{ Brain region } & \multirow[b]{2}{*}{ Group } & \multirow[b]{2}{*}{ Incentive cond } & \multirow[b]{2}{*}{ Mean } & \multirow[b]{2}{*}{ Std. Error } & \multicolumn{2}{|c|}{ 95\% Confidence Interval } \\
\hline & & & & & Lower Bound & Upper Bound \\
\hline \multirow[t]{6}{*}{ Brain region 1} & 1.00 & 1 & .192 & .032 & .128 & .257 \\
\hline & & 2 & .221 & .031 & .158 & .284 \\
\hline & & 3 & .178 & .033 & .112 & .244 \\
\hline & 2.00 & 1 & .145 & .032 & .081 & .210 \\
\hline & & 2 & .165 & .031 & .102 & .228 \\
\hline & & 3 & .183 & .033 & .117 & .249 \\
\hline \multirow[t]{6}{*}{ Brain region 2} & 1.00 & 1 & .218 & .036 & .145 & .292 \\
\hline & & 2 & .240 & .037 & .165 & .314 \\
\hline & & 3 & .216 & .036 & .145 & .288 \\
\hline & 2.00 & 1 & .181 & .036 & .107 & .254 \\
\hline & & 2 & .165 & .037 & .091 & .240 \\
\hline & & 3 & .194 & .036 & .122 & .265 \\
\hline \multirow[t]{6}{*}{ Brain region 3} & 1.00 & 1 & .133 & .032 & .069 & .197 \\
\hline & & 2 & .177 & .032 & .111 & .242 \\
\hline & & 3 & .158 & .029 & .099 & .216 \\
\hline & 2.00 & 1 & .171 & .032 & .107 & .235 \\
\hline & & 2 & .198 & .032 & .133 & .263 \\
\hline & & 3 & .206 & .029 & .148 & .264 \\
\hline \multirow[t]{3}{*}{ Brain region 4} & 1.00 & 1 & .140 & .030 & .079 & .201 \\
\hline & & 2 & .168 & .029 & .109 & .227 \\
\hline & & 3 & .157 & .025 & .106 & .208 \\
\hline
\end{tabular}




\begin{tabular}{|c|c|c|c|c|c|c|}
\hline & 2.00 & 1 & .150 & .030 & .089 & .210 \\
\hline & & 2 & .189 & .029 & .130 & .248 \\
\hline & & 3 & .203 & .025 & .152 & .254 \\
\hline \multirow[t]{6}{*}{ Brain region 5} & 1.00 & 1 & .183 & .031 & .120 & .246 \\
\hline & & 2 & .187 & .030 & .127 & .246 \\
\hline & & 3 & .134 & .029 & .076 & .193 \\
\hline & 2.00 & 1 & .141 & .031 & .078 & .204 \\
\hline & & 2 & .138 & .030 & .078 & .198 \\
\hline & & 3 & .152 & .029 & .093 & .211 \\
\hline \multirow[t]{6}{*}{ Brain region 6} & 1.00 & 1 & .180 & .038 & .104 & .256 \\
\hline & & 2 & .254 & .038 & .177 & .331 \\
\hline & & 3 & .209 & .037 & .134 & .284 \\
\hline & 2.00 & 1 & .131 & .038 & .055 & .207 \\
\hline & & 2 & .133 & .038 & .056 & .210 \\
\hline & & 3 & .147 & .037 & .072 & .222 \\
\hline \multirow[t]{6}{*}{ Brain region 7} & 1.00 & 1 & -.103 & .022 & -.146 & -.059 \\
\hline & & 2 & -.094 & .017 & -.129 & -.060 \\
\hline & & 3 & -.088 & .023 & -.134 & -.042 \\
\hline & 2.00 & 1 & -.063 & .022 & -.106 & -.020 \\
\hline & & 2 & -.043 & .017 & -.078 & -.009 \\
\hline & & 3 & -.045 & .023 & -.091 & .001 \\
\hline \multirow[t]{5}{*}{ Brain region 8} & 1.00 & 1 & -.162 & .035 & -.233 & -.092 \\
\hline & & 2 & -.110 & .028 & -.165 & -.054 \\
\hline & & 3 & -.134 & .030 & -.195 & -.073 \\
\hline & 2.00 & 1 & -.082 & .035 & -.153 & -.012 \\
\hline & & 2 & -.081 & .028 & -.137 & -.026 \\
\hline
\end{tabular}




\begin{tabular}{|c|c|c|c|c|c|c|}
\hline & & 3 & -.047 & .030 & -.108 & .014 \\
\hline \multirow[t]{6}{*}{ Brain region 9} & 1.00 & 1 & -.069 & .046 & -.161 & .023 \\
\hline & & 2 & -.096 & .035 & -.166 & -.026 \\
\hline & & 3 & -.108 & .036 & -.180 & -.035 \\
\hline & 2.00 & 1 & -.131 & .046 & -.224 & -.039 \\
\hline & & 2 & -.119 & .035 & -.189 & -.049 \\
\hline & & 3 & -.076 & .036 & -.149 & -.004 \\
\hline \multirow[t]{6}{*}{ Brain region 10} & 1.00 & 1 & -.106 & .025 & -.157 & -.055 \\
\hline & & 2 & -.088 & .021 & -.130 & -.046 \\
\hline & & 3 & -.067 & .025 & -.117 & -.017 \\
\hline & 2.00 & 1 & -.074 & .025 & -.124 & -.023 \\
\hline & & 2 & -.063 & .021 & -.105 & -.021 \\
\hline & & 3 & -.039 & .025 & -.088 & .011 \\
\hline \multirow[t]{6}{*}{ Brain region 11} & 1.00 & 1 & .104 & .042 & .018 & .189 \\
\hline & & 2 & .213 & .040 & .133 & .293 \\
\hline & & 3 & .182 & .036 & .110 & .255 \\
\hline & 2.00 & 1 & .121 & .042 & .036 & .206 \\
\hline & & 2 & .172 & .040 & .092 & .252 \\
\hline & & 3 & .151 & .036 & .078 & .223 \\
\hline \multirow[t]{6}{*}{ Brain region 12} & 1.00 & 1 & -.111 & .047 & -.206 & -.017 \\
\hline & & 2 & -.186 & .036 & -.259 & -.114 \\
\hline & & 3 & -.208 & .039 & -.287 & -.130 \\
\hline & 2.00 & 1 & -.075 & .047 & -.170 & .020 \\
\hline & & 2 & -.088 & .036 & -.160 & -.016 \\
\hline & & 3 & -.060 & .039 & -.139 & .019 \\
\hline Brain region 13 & 1.00 & 1 & -.065 & .038 & -.142 & .013 \\
\hline
\end{tabular}




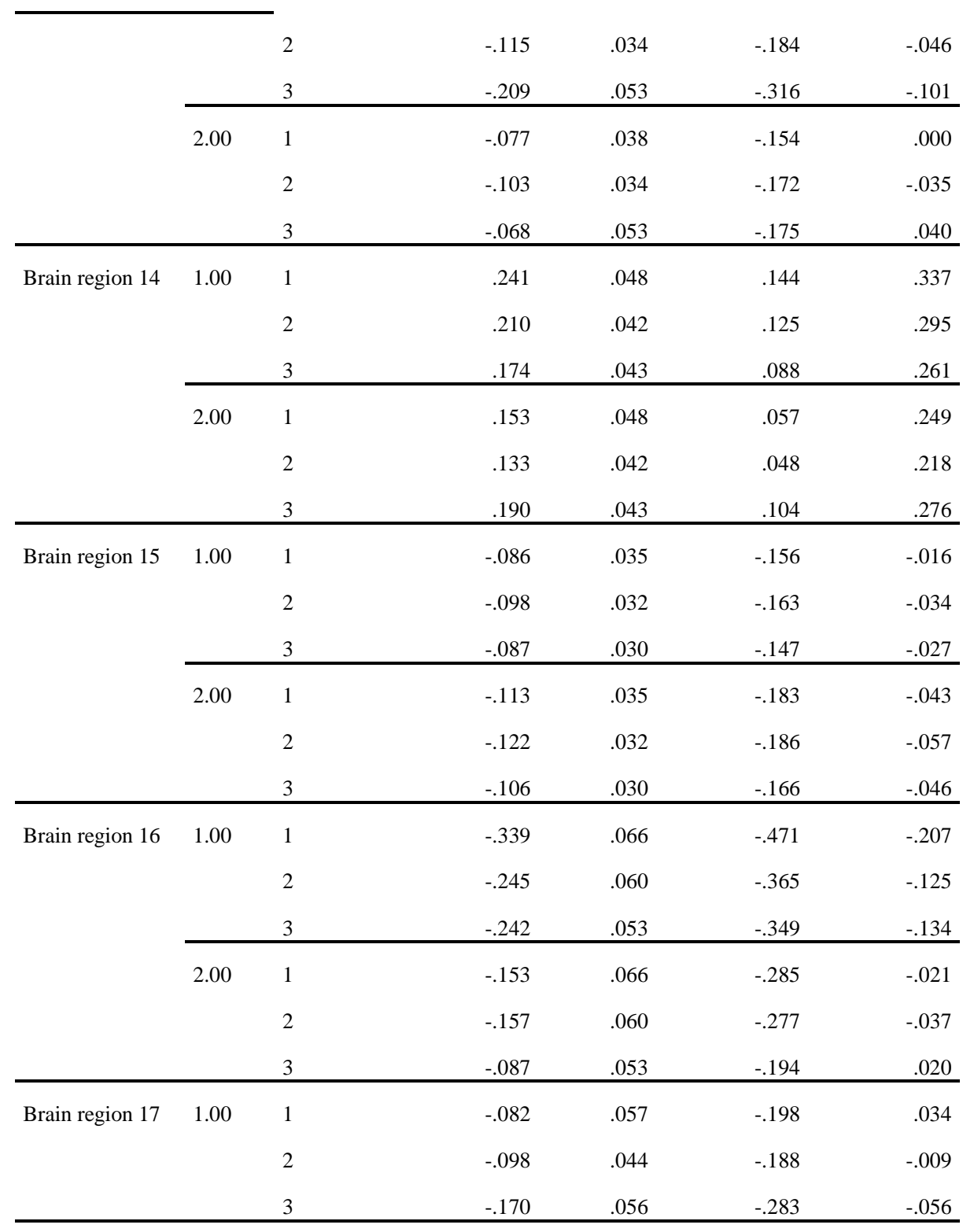




\begin{tabular}{|c|c|c|c|c|c|c|}
\hline & 2.00 & 1 & -.190 & .057 & -.305 & -.074 \\
\hline & & 2 & -.232 & .044 & -.322 & -.143 \\
\hline & & 3 & -.194 & .056 & -.308 & -.080 \\
\hline \multirow[t]{6}{*}{ Brain region 18} & 1.00 & 1 & -.060 & .035 & -.131 & .011 \\
\hline & & 2 & -.031 & .032 & -.094 & .033 \\
\hline & & 3 & -.078 & .032 & -.142 & -.015 \\
\hline & 2.00 & 1 & -.107 & .035 & -.178 & -.036 \\
\hline & & 2 & -.103 & .032 & -.167 & -.039 \\
\hline & & 3 & -.074 & .032 & -.137 & -.010 \\
\hline \multirow[t]{6}{*}{ Brain region 19} & 1.00 & 1 & .091 & .031 & .028 & .154 \\
\hline & & 2 & .121 & .027 & .067 & .175 \\
\hline & & 3 & .104 & .030 & .044 & .165 \\
\hline & 2.00 & 1 & .059 & .031 & -.004 & .122 \\
\hline & & 2 & .090 & .027 & .035 & .144 \\
\hline & & 3 & .081 & .030 & .020 & .141 \\
\hline \multirow[t]{6}{*}{ Brain region 20} & 1.00 & 1 & -.163 & .082 & -.329 & .004 \\
\hline & & 2 & -.233 & .073 & -.380 & -.087 \\
\hline & & 3 & -.216 & .089 & -.395 & -.037 \\
\hline & 2.00 & 1 & -.135 & .082 & -.301 & .031 \\
\hline & & 2 & -.117 & .073 & -.264 & .030 \\
\hline & & 3 & -.051 & .089 & -.231 & .128 \\
\hline \multirow[t]{5}{*}{ Brain region 21} & 1.00 & 1 & .111 & .030 & .051 & .172 \\
\hline & & 2 & .131 & .037 & .056 & .205 \\
\hline & & 3 & .099 & .031 & .037 & .161 \\
\hline & 2.00 & 1 & .145 & .030 & .085 & .206 \\
\hline & & 2 & .163 & .037 & .088 & .237 \\
\hline
\end{tabular}




\begin{tabular}{|c|c|c|c|c|c|c|}
\hline & & 3 & .196 & .031 & .133 & .258 \\
\hline \multirow[t]{6}{*}{ Brain region 22} & 1.00 & 1 & .054 & .029 & -.005 & .113 \\
\hline & & 2 & .136 & .036 & .063 & .208 \\
\hline & & 3 & .120 & .037 & .045 & .196 \\
\hline & 2.00 & 1 & .067 & .029 & .008 & .126 \\
\hline & & 2 & .106 & .036 & .033 & .179 \\
\hline & & 3 & .084 & .037 & .008 & .159 \\
\hline \multirow[t]{6}{*}{ Brain region 23} & 1.00 & 1 & -.038 & .044 & -.126 & .051 \\
\hline & & 2 & -.032 & .036 & -.105 & .040 \\
\hline & & 3 & -.112 & .040 & -.193 & -.031 \\
\hline & 2.00 & 1 & -.108 & .044 & -.196 & -.019 \\
\hline & & 2 & -.106 & .036 & -.179 & -.034 \\
\hline & & 3 & -.109 & .040 & -.190 & -.027 \\
\hline \multirow[t]{6}{*}{ Brain region 24} & 1.00 & 1 & .133 & .035 & .061 & .204 \\
\hline & & 2 & .129 & .034 & .060 & .199 \\
\hline & & 3 & .087 & .040 & .006 & .167 \\
\hline & 2.00 & 1 & .140 & .035 & .069 & .211 \\
\hline & & 2 & .152 & .034 & .083 & .221 \\
\hline & & 3 & .185 & .040 & .105 & .266 \\
\hline \multirow[t]{6}{*}{ Brain region 25} & 1.00 & 1 & .181 & .051 & .080 & .283 \\
\hline & & 2 & .257 & .044 & .168 & .346 \\
\hline & & 3 & .237 & .046 & .145 & .329 \\
\hline & 2.00 & 1 & .128 & .051 & .026 & .229 \\
\hline & & 2 & .125 & .044 & .036 & .213 \\
\hline & & 3 & .134 & .046 & .042 & .226 \\
\hline
\end{tabular}


Table I8

Mean Difference, Standard Error, P-Value and 95\% Confidence Interval for Group Effects by Incentive Condition

\begin{tabular}{|c|c|c|c|c|c|c|c|c|}
\hline \multirow[b]{2}{*}{ Brain region } & \multirow[b]{2}{*}{ Incentive cond } & \multirow[b]{2}{*}{ (I) Group } & \multirow[b]{2}{*}{ (J) Group } & \multirow{2}{*}{$\begin{array}{c}\text { Mean Difference } \\
(\mathrm{I}-\mathrm{J}) \\
\end{array}$} & \multirow[b]{2}{*}{ Std. Error } & \multirow[b]{2}{*}{ Sig. ${ }^{\text {a }}$} & \multicolumn{2}{|c|}{ 95\% Confidence Interval for Difference } \\
\hline & & & & & & & Lower Bound & Upper Bound \\
\hline \multirow[t]{6}{*}{ Brain region 1} & 1 & 1.00 & 2.00 & .047 & .045 & .304 & -.044 & .138 \\
\hline & & 2.00 & 1.00 & -.047 & .045 & .304 & -.138 & .044 \\
\hline & 2 & 1.00 & 2.00 & .056 & .044 & 209 & -.033 & .145 \\
\hline & & 2.00 & 1.00 & -.056 & .044 & .209 & -.145 & .033 \\
\hline & 3 & 1.00 & 2.00 & -.005 & .046 & .916 & -.098 & .088 \\
\hline & & 2.00 & 1.00 & .005 & .046 & .916 & -.088 & .098 \\
\hline \multirow[t]{6}{*}{ Brain region 2} & 1 & 1.00 & 2.00 & .038 & .051 & .468 & -.066 & .141 \\
\hline & & 2.00 & 1.00 & -.038 & .051 & .468 & -.141 & .066 \\
\hline & 2 & 1.00 & 2.00 & .074 & .052 & .162 & -.031 & .179 \\
\hline & & 2.00 & 1.00 & -.074 & .052 & .162 & -.179 & .031 \\
\hline & 3 & 1.00 & 2.00 & .023 & .050 & .656 & -.079 & .124 \\
\hline & & 2.00 & 1.00 & -.023 & .050 & .656 & -.124 & .079 \\
\hline \multirow[t]{6}{*}{ Brain region 3} & 1 & 1.00 & 2.00 & -.038 & .045 & .406 & -.128 & .053 \\
\hline & & 2.00 & 1.00 & .038 & .045 & .406 & -.053 & .128 \\
\hline & 2 & 1.00 & 2.00 & -.021 & .046 & .645 & -.113 & .071 \\
\hline & & 2.00 & 1.00 & .021 & .046 & .645 & -.071 & .113 \\
\hline & 3 & 1.00 & 2.00 & -.048 & .041 & .245 & -.130 & .034 \\
\hline & & 2.00 & 1.00 & .048 & .041 & .245 & -.034 & .130 \\
\hline \multirow[t]{3}{*}{ Brain region 4} & 1 & 1.00 & 2.00 & -.009 & .043 & .828 & -.095 & .077 \\
\hline & & 2.00 & 1.00 & .009 & .043 & .828 & -.077 & .095 \\
\hline & 2 & 1.00 & 2.00 & -.021 & .041 & .619 & -.104 & .063 \\
\hline
\end{tabular}




\begin{tabular}{|c|c|c|c|c|c|c|c|c|}
\hline & & 2.00 & 1.00 & .021 & .041 & .619 & -.063 & .104 \\
\hline & \multirow[t]{2}{*}{3} & 1.00 & 2.00 & -.046 & .036 & .204 & -.119 & .026 \\
\hline & & 2.00 & 1.00 & .046 & .036 & .204 & -.026 & .119 \\
\hline \multirow[t]{6}{*}{ Brain region 5} & \multirow[t]{2}{*}{1} & 1.00 & 2.00 & .042 & .044 & .346 & -.047 & .131 \\
\hline & & 2.00 & 1.00 & -.042 & .044 & .346 & -.131 & .047 \\
\hline & \multirow[t]{2}{*}{2} & 1.00 & 2.00 & .049 & .042 & .255 & -.036 & .133 \\
\hline & & 2.00 & 1.00 & -.049 & .042 & .255 & -.133 & .036 \\
\hline & \multirow[t]{2}{*}{3} & 1.00 & 2.00 & -.017 & .041 & .675 & -.101 & .066 \\
\hline & & 2.00 & 1.00 & .017 & .041 & .675 & -.066 & .101 \\
\hline \multirow[t]{6}{*}{ Brain region 6} & \multirow[t]{2}{*}{1} & 1.00 & 2.00 & .049 & .053 & .363 & -.058 & .157 \\
\hline & & 2.00 & 1.00 & -.049 & .053 & .363 & -.157 & .058 \\
\hline & \multirow[t]{2}{*}{2} & 1.00 & 2.00 & $.121^{*}$ & .054 & .030 & .012 & .229 \\
\hline & & 2.00 & 1.00 & $-.121^{*}$ & .054 & .030 & -.229 & -.012 \\
\hline & \multirow[t]{2}{*}{3} & 1.00 & 2.00 & .062 & .053 & .245 & -.044 & .168 \\
\hline & & 2.00 & 1.00 & -.062 & .053 & .245 & -.168 & .044 \\
\hline \multirow[t]{6}{*}{ Brain region 7} & \multirow[t]{2}{*}{1} & 1.00 & 2.00 & -.040 & .030 & .201 & -.101 & .022 \\
\hline & & 2.00 & 1.00 & .040 & .030 & .201 & -.022 & .101 \\
\hline & \multirow[t]{2}{*}{2} & 1.00 & 2.00 & $-.051^{*}$ & .024 & .040 & -.100 & -.002 \\
\hline & & 2.00 & 1.00 & $.051^{*}$ & .024 & .040 & .002 & .100 \\
\hline & \multirow[t]{2}{*}{3} & 1.00 & 2.00 & -.043 & .032 & .195 & -.108 & .023 \\
\hline & & 2.00 & 1.00 & .043 & .032 & .195 & -.023 & .108 \\
\hline \multirow[t]{5}{*}{ Brain region 8} & \multirow[t]{2}{*}{1} & 1.00 & 2.00 & -.080 & .049 & .111 & -.179 & .019 \\
\hline & & 2.00 & 1.00 & .080 & .049 & .111 & -.019 & .179 \\
\hline & \multirow[t]{2}{*}{2} & 1.00 & 2.00 & -.029 & .039 & .465 & -.107 & .050 \\
\hline & & 2.00 & 1.00 & .029 & .039 & .465 & -.050 & .107 \\
\hline & 3 & 1.00 & 2.00 & $-.087^{*}$ & .043 & .049 & -.173 & .000 \\
\hline
\end{tabular}




\begin{tabular}{|c|c|c|c|c|c|c|c|c|}
\hline & & 2.00 & 1.00 & $.087^{*}$ & .043 & .049 & .000 & .173 \\
\hline \multirow[t]{6}{*}{ Brain region 9} & 1 & 1.00 & 2.00 & .063 & .065 & .339 & -.068 & .193 \\
\hline & & 2.00 & 1.00 & -.063 & .065 & .339 & -.193 & .068 \\
\hline & 2 & 1.00 & 2.00 & .023 & .049 & .645 & -.076 & .122 \\
\hline & & 2.00 & 1.00 & -.023 & .049 & .645 & -.122 & .076 \\
\hline & 3 & 1.00 & 2.00 & -.032 & .051 & .536 & -.134 & .071 \\
\hline & & 2.00 & 1.00 & .032 & .051 & .536 & -.071 & .134 \\
\hline \multirow[t]{6}{*}{ Brain region 10} & 1 & 1.00 & 2.00 & -.032 & .036 & .372 & -.104 & .040 \\
\hline & & 2.00 & 1.00 & .032 & .036 & .372 & -.040 & .104 \\
\hline & 2 & 1.00 & 2.00 & -.025 & .030 & .408 & -.084 & .035 \\
\hline & & 2.00 & 1.00 & .025 & .030 & .408 & -.035 & .084 \\
\hline & 3 & 1.00 & 2.00 & -.028 & .035 & .424 & -.099 & .042 \\
\hline & & 2.00 & 1.00 & .028 & .035 & .424 & -.042 & .099 \\
\hline \multirow[t]{6}{*}{ Brain region 11} & 1 & 1.00 & 2.00 & -.017 & .060 & .772 & -.138 & .103 \\
\hline & & 2.00 & 1.00 & .017 & .060 & .772 & -.103 & .138 \\
\hline & 2 & 1.00 & 2.00 & .041 & .056 & .472 & -.073 & .155 \\
\hline & & 2.00 & 1.00 & -.041 & .056 & .472 & -.155 & .073 \\
\hline & 3 & 1.00 & 2.00 & .031 & .051 & .543 & -.071 & .134 \\
\hline & & 2.00 & 1.00 & -.031 & .051 & .543 & -.134 & .071 \\
\hline \multirow[t]{6}{*}{ Brain region 12} & 1 & 1.00 & 2.00 & -.036 & .067 & .590 & -.170 & .098 \\
\hline & & 2.00 & 1.00 & .036 & .067 & .590 & -.098 & .170 \\
\hline & 2 & 1.00 & 2.00 & -.098 & .051 & .059 & -.201 & .004 \\
\hline & & 2.00 & 1.00 & .098 & .051 & .059 & -.004 & .201 \\
\hline & 3 & 1.00 & 2.00 & $-.148^{*}$ & .055 & .010 & -.260 & -.037 \\
\hline & & 2.00 & 1.00 & $.148^{*}$ & .055 & .010 & .037 & .260 \\
\hline Brain region 13 & 1 & 1.00 & 2.00 & .012 & .054 & .825 & -.097 & .121 \\
\hline
\end{tabular}




\begin{tabular}{|c|c|c|c|c|c|c|c|c|}
\hline & & 2.00 & 1.00 & -.012 & .054 & .825 & -.121 & .097 \\
\hline & \multirow[t]{2}{*}{2} & 1.00 & 2.00 & -.012 & .048 & .812 & -.109 & .086 \\
\hline & & 2.00 & 1.00 & .012 & .048 & .812 & -.086 & .109 \\
\hline & \multirow[t]{2}{*}{3} & 1.00 & 2.00 & -.141 & .076 & .069 & -.293 & .011 \\
\hline & & 2.00 & 1.00 & .141 & .076 & .069 & -.011 & .293 \\
\hline \multirow[t]{6}{*}{ Brain region 14} & \multirow[t]{2}{*}{1} & 1.00 & 2.00 & .088 & .068 & .202 & -.049 & .224 \\
\hline & & 2.00 & 1.00 & -.088 & .068 & .202 & -.224 & .049 \\
\hline & \multirow[t]{2}{*}{2} & 1.00 & 2.00 & .077 & .060 & .205 & -.043 & .197 \\
\hline & & 2.00 & 1.00 & -.077 & .060 & .205 & -.197 & .043 \\
\hline & \multirow[t]{2}{*}{3} & 1.00 & 2.00 & -.016 & .061 & .798 & -.138 & .106 \\
\hline & & 2.00 & 1.00 & .016 & .061 & .798 & -.106 & .138 \\
\hline \multirow[t]{6}{*}{ Brain region 15} & \multirow[t]{2}{*}{1} & 1.00 & 2.00 & .027 & .049 & .592 & -.072 & .125 \\
\hline & & 2.00 & 1.00 & -.027 & .049 & .592 & -.125 & .072 \\
\hline & \multirow[t]{2}{*}{2} & 1.00 & 2.00 & .024 & .045 & .605 & -.067 & .115 \\
\hline & & 2.00 & 1.00 & -.024 & .045 & .605 & -.115 & .067 \\
\hline & \multirow[t]{2}{*}{3} & 1.00 & 2.00 & .019 & .042 & .661 & -.066 & .103 \\
\hline & & 2.00 & 1.00 & -.019 & .042 & .661 & -.103 & .066 \\
\hline \multirow[t]{6}{*}{ Brain region 16} & \multirow[t]{2}{*}{1} & 1.00 & 2.00 & -.186 & .093 & .051 & -.373 & .001 \\
\hline & & 2.00 & 1.00 & .186 & .093 & .051 & -.001 & .373 \\
\hline & \multirow[t]{2}{*}{2} & 1.00 & 2.00 & -.088 & .084 & .300 & -.258 & .081 \\
\hline & & 2.00 & 1.00 & .088 & .084 & .300 & -.081 & .258 \\
\hline & \multirow[t]{2}{*}{3} & 1.00 & 2.00 & $-.155^{*}$ & .075 & .046 & -.306 & -.003 \\
\hline & & 2.00 & 1.00 & $.155^{*}$ & .075 & .046 & .003 & .306 \\
\hline \multirow[t]{3}{*}{ Brain region 17} & \multirow[t]{2}{*}{1} & 1.00 & 2.00 & .108 & .081 & .192 & -.056 & .271 \\
\hline & & 2.00 & 1.00 & -.108 & .081 & .192 & -.271 & .056 \\
\hline & 2 & 1.00 & 2.00 & $.134^{*}$ & .063 & .038 & .008 & .261 \\
\hline
\end{tabular}




\begin{tabular}{|c|c|c|c|c|c|c|c|c|}
\hline & & 2.00 & 1.00 & $-.134^{*}$ & .063 & .038 & -.261 & -.008 \\
\hline & 3 & 1.00 & 2.00 & .024 & .080 & .764 & -.137 & .185 \\
\hline & & 2.00 & 1.00 & -.024 & .080 & .764 & -.185 & .137 \\
\hline \multirow[t]{6}{*}{ Brain region 18} & 1 & 1.00 & 2.00 & .047 & .050 & .347 & -.053 & .148 \\
\hline & & 2.00 & 1.00 & -.047 & .050 & .347 & -.148 & .053 \\
\hline & 2 & 1.00 & 2.00 & .072 & .045 & .113 & -.018 & .162 \\
\hline & & 2.00 & 1.00 & -.072 & .045 & .113 & -.162 & .018 \\
\hline & 3 & 1.00 & 2.00 & -.005 & .045 & .917 & -.095 & .085 \\
\hline & & 2.00 & 1.00 & .005 & .045 & .917 & -.085 & .095 \\
\hline \multirow[t]{6}{*}{ Brain region 19} & 1 & 1.00 & 2.00 & .032 & .044 & .477 & -.058 & .121 \\
\hline & & 2.00 & 1.00 & -.032 & .044 & .477 & -.121 & .058 \\
\hline & 2 & 1.00 & 2.00 & .032 & .038 & .410 & -.045 & .108 \\
\hline & & 2.00 & 1.00 & -.032 & .038 & .410 & -.108 & .045 \\
\hline & 3 & 1.00 & 2.00 & .024 & .043 & .582 & -.062 & .110 \\
\hline & & 2.00 & 1.00 & -.024 & .043 & .582 & -.110 & .062 \\
\hline \multirow[t]{6}{*}{ Brain region 20} & 1 & 1.00 & 2.00 & -.028 & .117 & .813 & -.263 & .207 \\
\hline & & 2.00 & 1.00 & .028 & .117 & .813 & -.207 & .263 \\
\hline & 2 & 1.00 & 2.00 & -.116 & .103 & .265 & -.324 & .091 \\
\hline & & 2.00 & 1.00 & .116 & .103 & .265 & -.091 & .324 \\
\hline & 3 & 1.00 & 2.00 & -.165 & .126 & .197 & -.418 & .089 \\
\hline & & 2.00 & 1.00 & .165 & .126 & .197 & -.089 & .418 \\
\hline \multirow[t]{5}{*}{ Brain region 21} & 1 & 1.00 & 2.00 & -.034 & .042 & .427 & -.120 & .051 \\
\hline & & 2.00 & 1.00 & .034 & .042 & .427 & -.051 & .120 \\
\hline & 2 & 1.00 & 2.00 & -.032 & .052 & .542 & -.137 & .073 \\
\hline & & 2.00 & 1.00 & .032 & .052 & .542 & -.073 & .137 \\
\hline & 3 & 1.00 & 2.00 & $-.097^{*}$ & .044 & .032 & -.184 & -.009 \\
\hline
\end{tabular}




\begin{tabular}{|c|c|c|c|c|c|c|c|c|}
\hline & & 2.00 & 1.00 & $.097^{*}$ & .044 & .032 & .009 & .184 \\
\hline \multirow[t]{6}{*}{ Brain region 22} & 1 & 1.00 & 2.00 & -.013 & .042 & .754 & -.097 & .071 \\
\hline & & 2.00 & 1.00 & .013 & .042 & .754 & -.071 & .097 \\
\hline & 2 & 1.00 & 2.00 & .029 & .051 & .568 & -.073 & .132 \\
\hline & & 2.00 & 1.00 & -.029 & .051 & .568 & -.132 & .073 \\
\hline & 3 & 1.00 & 2.00 & .036 & .053 & .497 & -.070 & .143 \\
\hline & & 2.00 & 1.00 & -.036 & .053 & .497 & -.143 & .070 \\
\hline \multirow[t]{6}{*}{ Brain region 23} & 1 & 1.00 & 2.00 & .070 & .062 & .264 & -.055 & .195 \\
\hline & & 2.00 & 1.00 & -.070 & .062 & .264 & -.195 & .055 \\
\hline & 2 & 1.00 & 2.00 & .074 & .051 & .155 & -.029 & .177 \\
\hline & & 2.00 & 1.00 & -.074 & .051 & .155 & -.177 & .029 \\
\hline & 3 & 1.00 & 2.00 & -.003 & .057 & .955 & -.118 & .112 \\
\hline & & 2.00 & 1.00 & .003 & .057 & .955 & -.112 & .118 \\
\hline \multirow[t]{6}{*}{ Brain region 24} & 1 & 1.00 & 2.00 & -.007 & .050 & .888 & -.108 & .094 \\
\hline & & 2.00 & 1.00 & .007 & .050 & .888 & -.094 & .108 \\
\hline & 2 & 1.00 & 2.00 & -.023 & .049 & .644 & -.121 & .075 \\
\hline & & 2.00 & 1.00 & .023 & .049 & .644 & -.075 & .121 \\
\hline & 3 & 1.00 & 2.00 & -.099 & .057 & .088 & -.213 & .015 \\
\hline & & 2.00 & 1.00 & .099 & .057 & .088 & -.015 & .213 \\
\hline \multirow[t]{6}{*}{ Brain region 25} & 1 & 1.00 & 2.00 & .054 & .072 & .454 & -.090 & .198 \\
\hline & & 2.00 & 1.00 & -.054 & .072 & .454 & -.198 & .090 \\
\hline & 2 & 1.00 & 2.00 & $.132^{*}$ & .062 & .040 & .007 & .258 \\
\hline & & 2.00 & 1.00 & $-.132^{*}$ & .062 & .040 & -.258 & -.007 \\
\hline & 3 & 1.00 & 2.00 & .103 & .065 & .119 & -.027 & .234 \\
\hline & & 2.00 & 1.00 & -.103 & .065 & .119 & -.234 & .027 \\
\hline
\end{tabular}

Based on estimated marginal means 
a. Adjustment for multiple comparisons: Least Significant Difference (equivalent to no adjustments)

*. The mean difference is significant at the .05 level. 


\section{Appendix J}

\section{Means and Significance Tests for All Main and Interaction Effects During the Feedback Period}

The tables in this Appendix contain names of brain regions and levels of variables that have been abbreviated for formatting purposes. The abbreviations and the brain regions, groups, level of accuracy and incentive conditions they represent are set out below.

Labels in the Tables and the Brain Regions They Represent

\begin{tabular}{|c|c|}
\hline Label in table & Brain region \\
\hline Brain region 1 & Left fusiform gyrus and Left lingual gyrus \\
\hline Brain region 2 & Right lingual gyrus and Right fusiform gyrus \\
\hline Brain region 3 & Left cingulate gyrus \\
\hline Brain region 4 & Right cingulate gyrus \\
\hline Brain region 5 & Left medial frontal gyrus / Left BA6 / Left SMA \\
\hline Brain region 6 & Right middle frontal gyrus / BA10 \\
\hline Brain region 7 & Right inferior parietal lobule / BA40 \\
\hline Brain region 8 & Right anterior cingulate / Right BA32 (dACC) \\
\hline Brain region 9 & Right medial frontal gyrus / Right superior frontal gyrus \\
\hline Brain region 10 & Left inferior frontal gyrus (P. triangularis BA45) \\
\hline Brain region 11 & Right insula \\
\hline Brain region 12 & Right cerebellar tonsil \\
\hline Brain region 13 & Right thalamus \\
\hline Brain region 14 & Right superior frontal gyrus / Right middle frontal gyrus \\
\hline Brain region 15 & Left thalamus - Left ventral lateral nucleus \\
\hline Brain region 16 & Left thalamus \\
\hline Brain region 17 & Left lentiform nucleus and Left putamen \\
\hline Brain region 18 & Right culmen \\
\hline
\end{tabular}


Brain region 19 Left culmen / Left fusiform gyrus

Brain region $20 \quad$ Right angular gyrus

Brain region $21 \quad$ Left precentral gyrus and Left BA4 (PMC)

Brain region 22 Right cingulate gyrus / Bilateral cingulate

Labels in the Tables Referencing Low Trait Anxiety and High Trait Anxiety Groups

\begin{tabular}{cl}
\hline Group label & Group \\
\hline 1.00 & Low trait anxiety \\
2.00 & High trait anxiety \\
\hline
\end{tabular}

Labels in the Tables Referencing Accuracy

\begin{tabular}{cl}
\hline Accuracy label & Accuracy \\
\hline 1.00 & Correct \\
2.00 & Error \\
\hline
\end{tabular}

Labels in the Tables Referencing Incentive Condition

\begin{tabular}{cl}
\hline Incentive condition label & Incentive condition \\
\hline 1.00 & Neutral condition \\
2.00 & Punishment condition \\
3.00 & Reward condition \\
\hline
\end{tabular}

Note: In the tables that follow Incentive condition is abbreviated to Incentive cond 
Table J1

F-Values, Degrees of Freedom, and Levels of Significance for Regions of Event-Related BOLD Activity During Feedback Period

\begin{tabular}{|c|c|c|c|c|c|}
\hline Condition & Brain region & & & $F$ & Sig. \\
\hline \multirow[t]{22}{*}{ Group } & Brain region 1 & 1 & 46 & .529 & .471 \\
\hline & Brain region 2 & 1 & 46 & .051 & .823 \\
\hline & Brain region 3 & 1 & 46 & 1.906 & .174 \\
\hline & Brain region 4 & 1 & 46 & 2.342 & .133 \\
\hline & Brain region 5 & 1 & 46 & 1.121 & .295 \\
\hline & Brain region 6 & 1 & 46 & .782 & .381 \\
\hline & Brain region 7 & 1 & 46 & .881 & .353 \\
\hline & Brain region 8 & 1 & 46 & .066 & .798 \\
\hline & Brain region 9 & 1 & 46 & .048 & .827 \\
\hline & Brain region 10 & 1 & 46 & .054 & .817 \\
\hline & Brain region 11 & 1 & 46 & .000 & .982 \\
\hline & Brain region 12 & 1 & 46 & 1.060 & .309 \\
\hline & Brain region 13 & 1 & 46 & .027 & .870 \\
\hline & Brain region 14 & 1 & 46 & .011 & .917 \\
\hline & Brain region 15 & 1 & 46 & .188 & .667 \\
\hline & Brain region 16 & 1 & 46 & 1.680 & .201 \\
\hline & Brain region 17 & 1 & 46 & .149 & .701 \\
\hline & Brain region 18 & 1 & 46 & 2.271 & .139 \\
\hline & Brain region 19 & 1 & 46 & 2.430 & .126 \\
\hline & Brain region 20 & 1 & 46 & .144 & .706 \\
\hline & Brain region 21 & 1 & 46 & 1.024 & .317 \\
\hline & Brain region 22 & 1 & 46 & 1.887 & .176 \\
\hline Incentive cond & Brain region 1 & 2 & 92 & .023 & .977 \\
\hline
\end{tabular}




\begin{tabular}{|c|c|c|c|c|c|}
\hline & Brain region 2 & 2 & 92 & .192 & .826 \\
\hline & Brain region 3 & 2 & 92 & 1.670 & .194 \\
\hline & Brain region 4 & 2 & 92 & 1.006 & .370 \\
\hline & Brain region 5 & 2 & 92 & 7.371 & .001 \\
\hline & Brain region 6 & 2 & 92 & 6.907 & .002 \\
\hline & Brain region 7 & 2 & 92 & 10.363 & .000 \\
\hline & Brain region 8 & 2 & 92 & 5.020 & .009 \\
\hline & Brain region 9 & 2 & 92 & 4.888 & .010 \\
\hline & Brain region 10 & 2 & 92 & .317 & .729 \\
\hline & Brain region 11 & 2 & 92 & .136 & .873 \\
\hline & Brain region 12 & 2 & 92 & .939 & .395 \\
\hline & Brain region 13 & 2 & 92 & .522 & .595 \\
\hline & Brain region 14 & 2 & 92 & 14.987 & .000 \\
\hline & Brain region 15 & 2 & 92 & .110 & .896 \\
\hline & Brain region 16 & 2 & 92 & .367 & .694 \\
\hline & Brain region 17 & 2 & 92 & .790 & .457 \\
\hline & Brain region 18 & 2 & 92 & .434 & .649 \\
\hline & Brain region 19 & 2 & 92 & .133 & .876 \\
\hline & Brain region 20 & 2 & 92 & 9.606 & .000 \\
\hline & Brain region 21 & 2 & 92 & 6.233 & .003 \\
\hline & Brain region 22 & 2 & 92 & 3.164 & .047 \\
\hline \multirow[t]{7}{*}{ Incentive cond $\mathrm{x}$ group } & Brain region 1 & 2 & 92 & .365 & .695 \\
\hline & Brain region 2 & 2 & 92 & .409 & .665 \\
\hline & Brain region 3 & 2 & 92 & .423 & .656 \\
\hline & Brain region 4 & 2 & 92 & 1.323 & .271 \\
\hline & Brain region 5 & 2 & 92 & .079 & .924 \\
\hline & Brain region 6 & 2 & 92 & 1.509 & .227 \\
\hline & Brain region 7 & 2 & 92 & 2.188 & .118 \\
\hline
\end{tabular}




\begin{tabular}{|c|c|c|c|c|c|}
\hline & Brain region 8 & 2 & 92 & .239 & .788 \\
\hline & Brain region 9 & 2 & 92 & .569 & .568 \\
\hline & Brain region 10 & 2 & 92 & 1.021 & .364 \\
\hline & Brain region 11 & 2 & 92 & .257 & .774 \\
\hline & Brain region 12 & 2 & 92 & 1.123 & .330 \\
\hline & Brain region 13 & 2 & 92 & 1.067 & .348 \\
\hline & Brain region 14 & 2 & 92 & 3.738 & .027 \\
\hline & Brain region 15 & 2 & 92 & .184 & .832 \\
\hline & Brain region 16 & 2 & 92 & .472 & .625 \\
\hline & Brain region 17 & 2 & 92 & .398 & .673 \\
\hline & Brain region 18 & 2 & 92 & .137 & .872 \\
\hline & Brain region 19 & 2 & 92 & .020 & .980 \\
\hline & Brain region 20 & 2 & 92 & 2.451 & .092 \\
\hline & Brain region 21 & 2 & 92 & .705 & .497 \\
\hline & Brain region 22 & 2 & 92 & .022 & .978 \\
\hline \multirow[t]{13}{*}{ Accuracy } & Brain region 1 & 1 & 46 & .676 & .415 \\
\hline & Brain region 2 & 1 & 46 & 1.286 & .263 \\
\hline & Brain region 3 & 1 & 46 & 10.438 & .002 \\
\hline & Brain region 4 & 1 & 46 & 22.645 & .000 \\
\hline & Brain region 5 & 1 & 46 & 7.574 & .008 \\
\hline & Brain region 6 & 1 & 46 & 1.510 & .225 \\
\hline & Brain region 7 & 1 & 46 & .179 & .674 \\
\hline & Brain region 8 & 1 & 46 & 5.167 & .028 \\
\hline & Brain region 9 & 1 & 46 & 3.981 & .052 \\
\hline & Brain region 10 & 1 & 46 & 3.657 & .062 \\
\hline & Brain region 11 & 1 & 46 & .061 & .806 \\
\hline & Brain region 12 & 1 & 46 & 3.259 & .078 \\
\hline & Brain region 13 & 1 & 46 & .478 & .493 \\
\hline
\end{tabular}




\begin{tabular}{|c|c|c|c|c|c|}
\hline & Brain region 14 & 1 & 46 & .114 & .737 \\
\hline & Brain region 15 & 1 & 46 & .016 & .901 \\
\hline & Brain region 16 & 1 & 46 & 5.594 & .022 \\
\hline & Brain region 17 & 1 & 46 & 2.253 & .140 \\
\hline & Brain region 18 & 1 & 46 & .006 & .940 \\
\hline & Brain region 19 & 1 & 46 & .024 & .879 \\
\hline & Brain region 20 & 1 & 46 & .046 & .831 \\
\hline & Brain region 21 & 1 & 46 & 9.520 & .003 \\
\hline & Brain region 22 & 1 & 46 & .882 & .352 \\
\hline \multirow[t]{19}{*}{ Accuracy $\mathrm{x}$ group } & Brain region 1 & 1 & 46 & .546 & .464 \\
\hline & Brain region 2 & 1 & 46 & 2.147 & .150 \\
\hline & Brain region 3 & 1 & 46 & 1.195 & .280 \\
\hline & Brain region 4 & 1 & 46 & 1.902 & .175 \\
\hline & Brain region 5 & 1 & 46 & .028 & .868 \\
\hline & Brain region 6 & 1 & 46 & .000 & .988 \\
\hline & Brain region 7 & 1 & 46 & .303 & .584 \\
\hline & Brain region 8 & 1 & 46 & .717 & .402 \\
\hline & Brain region 9 & 1 & 46 & .012 & .912 \\
\hline & Brain region 10 & 1 & 46 & .207 & .651 \\
\hline & Brain region 11 & 1 & 46 & 7.433 & .009 \\
\hline & Brain region 12 & 1 & 46 & 4.895 & .032 \\
\hline & Brain region 13 & 1 & 46 & 1.043 & .313 \\
\hline & Brain region 14 & 1 & 46 & 1.973 & .167 \\
\hline & Brain region 15 & 1 & 46 & .891 & .350 \\
\hline & Brain region 16 & 1 & 46 & .415 & .522 \\
\hline & Brain region 17 & 1 & 46 & .805 & .374 \\
\hline & Brain region 18 & 1 & 46 & .631 & .431 \\
\hline & Brain region 19 & 1 & 46 & .916 & .344 \\
\hline
\end{tabular}




\begin{tabular}{|c|c|c|c|c|c|}
\hline & Brain region 20 & 1 & 46 & .361 & .551 \\
\hline & Brain region 21 & 1 & 46 & .315 & .578 \\
\hline & Brain region 22 & 1 & 46 & .001 & .977 \\
\hline \multirow[t]{22}{*}{ Incentive cond $\mathrm{x}$ accuracy } & Brain region 1 & 2 & 92 & .116 & .890 \\
\hline & Brain region 2 & 2 & 92 & .025 & .976 \\
\hline & Brain region 3 & 2 & 92 & 3.653 & .030 \\
\hline & Brain region 4 & 2 & 92 & 4.170 & .018 \\
\hline & Brain region 5 & 2 & 92 & 1.227 & .298 \\
\hline & Brain region 6 & 2 & 92 & 3.189 & .046 \\
\hline & Brain region 7 & 2 & 92 & 1.200 & .306 \\
\hline & Brain region 8 & 2 & 92 & .351 & .705 \\
\hline & Brain region 9 & 2 & 92 & .605 & .548 \\
\hline & Brain region 10 & 2 & 92 & 2.656 & .076 \\
\hline & Brain region 11 & 2 & 92 & 1.947 & .149 \\
\hline & Brain region 12 & 2 & 92 & 1.846 & .164 \\
\hline & Brain region 13 & 2 & 92 & .015 & .985 \\
\hline & Brain region 14 & 2 & 92 & 1.671 & .194 \\
\hline & Brain region 15 & 2 & 92 & .392 & .677 \\
\hline & Brain region 16 & 2 & 92 & .953 & .389 \\
\hline & Brain region 17 & 2 & 92 & .606 & .548 \\
\hline & Brain region 18 & 2 & 92 & .798 & .453 \\
\hline & Brain region 19 & 2 & 92 & .674 & .512 \\
\hline & Brain region 20 & 2 & 92 & 1.059 & .351 \\
\hline & Brain region 21 & 2 & 92 & 2.299 & .106 \\
\hline & Brain region 22 & 2 & 92 & .337 & .715 \\
\hline \multirow[t]{3}{*}{ Incentive cond $\mathrm{x}$ accuracy $\mathrm{x}$ group } & Brain region 1 & 2 & 92 & .349 & .706 \\
\hline & Brain region 2 & 2 & 92 & .900 & .410 \\
\hline & Brain region 3 & 2 & 92 & .275 & .760 \\
\hline
\end{tabular}




\begin{tabular}{lllll}
\hline Brain region 4 & 2 & 92 & .497 & .610 \\
Brain region 5 & 2 & 92 & 2.123 & .126 \\
Brain region 6 & 2 & 92 & 1.032 & .361 \\
Brain region 7 & 2 & 92 & 1.591 & .209 \\
Brain region 8 & 2 & 92 & 1.035 & .359 \\
Brain region 9 & 2 & 92 & .925 & .400 \\
Brain region 10 & 2 & 92 & 2.782 & .067 \\
Brain region 11 & 2 & 92 & .893 & .413 \\
Brain region 12 & 2 & 92 & 1.926 & .152 \\
Brain region 13 & 2 & 92 & .232 & .793 \\
Brain region 14 & 2 & 92 & 1.158 & .319 \\
Brain region 15 & 2 & 92 & 2.447 & .092 \\
Brain region 16 & 2 & .962 & .386 \\
Brain region 17 & 2 & 92 & .618 & .541 \\
Brain region 18 & 2 & 92 & .197 & .821 \\
Brain region 19 & 2 & 92 & .770 & .466 \\
Brain region 20 & 2 & .503 & .606 \\
Brain region 21 & 2 & 92 & 1.441 & .242 \\
Brain region 22 & 2 & 92 & .315 & .731 \\
\hline
\end{tabular}


Table J2

Means, Standard Errors and 95\% Confidence Intervals for Group Effects

\begin{tabular}{|c|c|c|c|c|c|}
\hline \multirow[b]{2}{*}{ Brain region } & \multirow[b]{2}{*}{ Group } & \multirow[b]{2}{*}{ Mean } & \multirow[b]{2}{*}{ Std. Error } & \multicolumn{2}{|c|}{ 95\% Confidence Interval } \\
\hline & & & & Lower Bound & Upper Bound \\
\hline \multirow[t]{2}{*}{ Brain region 1} & 1.00 & .242 & .040 & .162 & .321 \\
\hline & 2.00 & .201 & .040 & .121 & .281 \\
\hline \multirow[t]{2}{*}{ Brain region 2} & 1.00 & .190 & .037 & .115 & .265 \\
\hline & 2.00 & .202 & .037 & .127 & .277 \\
\hline \multirow[t]{2}{*}{ Brain region 3} & 1.00 & .063 & .016 & .031 & .096 \\
\hline & 2.00 & .032 & .016 & -.001 & .064 \\
\hline \multirow[t]{2}{*}{ Brain region 4} & 1.00 & .062 & .016 & .030 & .095 \\
\hline & 2.00 & .028 & .016 & -.004 & .060 \\
\hline \multirow[t]{2}{*}{ Brain region 5} & 1.00 & -.128 & .027 & -.182 & -.074 \\
\hline & 2.00 & -.168 & .027 & -.222 & -.114 \\
\hline \multirow[t]{2}{*}{ Brain region 6} & 1.00 & .225 & .037 & .150 & .299 \\
\hline & 2.00 & .178 & .037 & .103 & .253 \\
\hline \multirow[t]{2}{*}{ Brain region 7} & 1.00 & .299 & .043 & .212 & .386 \\
\hline & 2.00 & .241 & .043 & .154 & .328 \\
\hline \multirow[t]{2}{*}{ Brain region 8} & 1.00 & .100 & .032 & .035 & .164 \\
\hline & 2.00 & .111 & .032 & .047 & .175 \\
\hline \multirow[t]{2}{*}{ Brain region 9} & 1.00 & .107 & .022 & .062 & .152 \\
\hline & 2.00 & .100 & .022 & .056 & .145 \\
\hline \multirow[t]{2}{*}{ Brain region 10} & 1.00 & .162 & .032 & .097 & .227 \\
\hline & 2.00 & .151 & .032 & .086 & .217 \\
\hline Brain region 11 & 1.00 & .107 & .024 & .058 & .155 \\
\hline
\end{tabular}




\begin{tabular}{cccccc}
\hline & & & & & \\
& & & & & \\
Brain region 12 & 1.00 & .194 & .040 & .112 & .275 \\
& 2.00 & .135 & .040 & .053 & .216 \\
\hline Brain region 13 & 1.00 & -.119 & .035 & -.190 & -.049 \\
& 2.00 & -.111 & .035 & -.181 & -.041 \\
\hline Brain region 14 & 1.00 & .217 & .038 & .140 & .294 \\
& 2.00 & .211 & .038 & .134 & .289 \\
\hline Brain region 15 & 1.00 & -.090 & .032 & -.154 & -.026 \\
& 2.00 & -.110 & .032 & -.174 & -.045 \\
\hline Brain region 16 & 1.00 & .362 & .124 & .111 & .612 \\
& 2.00 & .134 & .124 & -.117 & .384 \\
\hline Brain region 17 & 1.00 & .058 & .034 & -.010 & .126 \\
& 2.00 & .040 & .034 & -.028 & .108 \\
\hline Brain region 18 & 1.00 & .113 & .031 & .049 & .176 \\
& 2.00 & .046 & .031 & -.018 & .109 \\
\hline Brain region 19 & 1.00 & .144 & .038 & .067 & .221 \\
& 2.00 & .059 & .038 & -.018 & .136 \\
\hline Brain region 20 & 1.00 & .234 & .051 & .131 & .337 \\
\hline Brain region 21 & 1.00 & -.043 & .027 & -.097 & .010 \\
& 2.00 & -.081 & .027 & -.135 & -.028 \\
\hline Brain region 22 & 1.00 & .256 & .060 & .134 & .377 \\
& 2.00 & .138 & .060 & .017 & .260 \\
\hline & & & & & .159 \\
\hline
\end{tabular}


Table J3

Mean Difference, Standard Error, P-Value and 95\% Confidence Interval for Low Trait Anxiety and High Trait Anxiety Groups for Each Effect

\begin{tabular}{|c|c|c|c|c|c|c|c|}
\hline \multirow[b]{2}{*}{ Brain region } & \multirow[b]{2}{*}{ (I) Group } & \multirow[b]{2}{*}{ (J) Group } & \multirow{2}{*}{$\begin{array}{c}\text { Mean Difference } \\
(\mathrm{I}-\mathrm{J}) \\
\end{array}$} & \multirow[b]{2}{*}{ Std. Error } & \multirow[b]{2}{*}{ Sig. ${ }^{a}$} & \multicolumn{2}{|c|}{ 95\% Confidence Interval for Difference } \\
\hline & & & & & & Lower Bound & Upper Bound \\
\hline \multirow[t]{2}{*}{ Brain region 1} & 1.00 & 2.00 & .041 & .056 & .471 & -.072 & .153 \\
\hline & 2.00 & 1.00 & -.041 & .056 & .471 & -.153 & .072 \\
\hline \multirow[t]{2}{*}{ Brain region 2} & 1.00 & 2.00 & -.012 & .053 & .823 & -.118 & .094 \\
\hline & 2.00 & 1.00 & .012 & .053 & .823 & -.094 & .118 \\
\hline \multirow[t]{2}{*}{ Brain region 3} & 1.00 & 2.00 & .032 & .023 & .174 & -.015 & .078 \\
\hline & 2.00 & 1.00 & -.032 & .023 & .174 & -.078 & .015 \\
\hline \multirow{2}{*}{ Brain region 4} & 1.00 & 2.00 & .035 & .023 & .133 & -.011 & .080 \\
\hline & 2.00 & 1.00 & -.035 & .023 & .133 & -.080 & .011 \\
\hline \multirow[t]{2}{*}{ Brain region 5} & 1.00 & 2.00 & .040 & .038 & .295 & -.036 & .116 \\
\hline & 2.00 & 1.00 & -.040 & .038 & .295 & -.116 & .036 \\
\hline \multirow[t]{2}{*}{ Brain region 6} & 1.00 & 2.00 & .046 & .052 & .381 & -.059 & .152 \\
\hline & 2.00 & 1.00 & -.046 & .052 & .381 & -.152 & .059 \\
\hline \multirow[t]{2}{*}{ Brain region 7} & 1.00 & 2.00 & .057 & .061 & .353 & -.066 & .181 \\
\hline & 2.00 & 1.00 & -.057 & .061 & .353 & -.181 & .066 \\
\hline \multirow[t]{2}{*}{ Brain region 8} & 1.00 & 2.00 & -.012 & .045 & .798 & -.102 & .079 \\
\hline & 2.00 & 1.00 & .012 & .045 & .798 & -.079 & .102 \\
\hline \multirow[t]{2}{*}{ Brain region 9} & 1.00 & 2.00 & .007 & .032 & .827 & -.057 & .070 \\
\hline & 2.00 & 1.00 & -.007 & .032 & .827 & -.070 & .057 \\
\hline \multirow[t]{2}{*}{ Brain region 10} & 1.00 & 2.00 & .011 & .046 & .817 & -.082 & .103 \\
\hline & 2.00 & 1.00 & -.011 & .046 & .817 & -.103 & .082 \\
\hline Brain region 11 & 1.00 & 2.00 & -.001 & .034 & .982 & -.070 & .068 \\
\hline
\end{tabular}




\begin{tabular}{|c|c|c|c|c|c|c|c|}
\hline & 2.00 & 1.00 & .001 & .034 & .982 & -.068 & .070 \\
\hline \multirow[t]{2}{*}{ Brain region 12} & 1.00 & 2.00 & .059 & .057 & .309 & -.056 & .174 \\
\hline & 2.00 & 1.00 & -.059 & .057 & .309 & -.174 & .056 \\
\hline \multirow[t]{2}{*}{ Brain region 13} & 1.00 & 2.00 & -.008 & .050 & .870 & -.108 & .092 \\
\hline & 2.00 & 1.00 & .008 & .050 & .870 & -.092 & .108 \\
\hline \multirow[t]{2}{*}{ Brain region 14} & 1.00 & 2.00 & .006 & .054 & .917 & -.104 & .115 \\
\hline & 2.00 & 1.00 & -.006 & .054 & .917 & -.115 & .104 \\
\hline \multirow[t]{2}{*}{ Brain region 15} & 1.00 & 2.00 & .020 & .045 & .667 & -.071 & .111 \\
\hline & 2.00 & 1.00 & -.020 & .045 & .667 & -.111 & .071 \\
\hline \multirow[t]{2}{*}{ Brain region 16} & 1.00 & 2.00 & .228 & .176 & .201 & -.126 & .582 \\
\hline & 2.00 & 1.00 & -.228 & .176 & .201 & -.582 & .126 \\
\hline \multirow[t]{2}{*}{ Brain region 17} & 1.00 & 2.00 & .018 & .048 & .701 & -.078 & .114 \\
\hline & 2.00 & 1.00 & -.018 & .048 & .701 & -.114 & .078 \\
\hline \multirow[t]{2}{*}{ Brain region 18} & 1.00 & 2.00 & .067 & .045 & .139 & -.023 & .157 \\
\hline & 2.00 & 1.00 & -.067 & .045 & .139 & -.157 & .023 \\
\hline \multirow[t]{2}{*}{ Brain region 19} & 1.00 & 2.00 & .084 & .054 & .126 & -.025 & .193 \\
\hline & 2.00 & 1.00 & -.084 & .054 & .126 & -.193 & .025 \\
\hline \multirow[t]{2}{*}{ Brain region 20} & 1.00 & 2.00 & -.027 & .072 & .706 & -.173 & .118 \\
\hline & 2.00 & 1.00 & .027 & .072 & .706 & -.118 & .173 \\
\hline \multirow[t]{2}{*}{ Brain region 21} & 1.00 & 2.00 & .038 & .038 & .317 & -.038 & .114 \\
\hline & 2.00 & 1.00 & -.038 & .038 & .317 & -.114 & .038 \\
\hline \multirow[t]{2}{*}{ Brain region 22} & 1.00 & 2.00 & .117 & .085 & .176 & -.055 & .289 \\
\hline & 2.00 & 1.00 & -.117 & .085 & .176 & -.289 & .055 \\
\hline
\end{tabular}

Based on estimated marginal means

a. Adjustment for multiple comparisons: Least Significant Difference (equivalent to no adjustments). 
Table J4

Means, Standard Errors and 95\% Confidence Intervals for Incentive Condition Effects

\begin{tabular}{|c|c|c|c|c|c|}
\hline \multirow[b]{2}{*}{ Brain region } & \multirow[b]{2}{*}{ Incentive cond } & \multirow[b]{2}{*}{ Mean } & \multirow[b]{2}{*}{ Std. Error } & \multicolumn{2}{|c|}{ 95\% Confidence Interval } \\
\hline & & & & Lower Bound & Upper Bound \\
\hline \multirow[t]{3}{*}{ Brain region 1} & 1 & .220 & .025 & .169 & .271 \\
\hline & 2 & .219 & .039 & .142 & .297 \\
\hline & 3 & .225 & .031 & .162 & .287 \\
\hline \multirow[t]{3}{*}{ Brain region 2} & 1 & .206 & .027 & .152 & .259 \\
\hline & 2 & .190 & .037 & .115 & .265 \\
\hline & 3 & .192 & .028 & .136 & .248 \\
\hline \multirow[t]{3}{*}{ Brain region 3} & 1 & .061 & .011 & .038 & .084 \\
\hline & 2 & .044 & .016 & .012 & .077 \\
\hline & 3 & .037 & .014 & .009 & .065 \\
\hline \multirow[t]{3}{*}{ Brain region 4} & 1 & .055 & .012 & .030 & .079 \\
\hline & 2 & .045 & .015 & .014 & .076 \\
\hline & 3 & .036 & .013 & .009 & .063 \\
\hline \multirow[t]{3}{*}{ Brain region 5} & 1 & -.093 & .017 & -.128 & -.058 \\
\hline & 2 & -.160 & .033 & -.226 & -.095 \\
\hline & 3 & -.191 & .020 & -.231 & -.151 \\
\hline \multirow[t]{3}{*}{ Brain region 6} & 1 & .150 & .026 & .098 & .203 \\
\hline & 2 & .209 & .029 & .152 & .267 \\
\hline & 3 & .244 & .035 & .174 & .315 \\
\hline \multirow[t]{3}{*}{ Brain region 7} & 1 & .202 & .028 & .145 & .259 \\
\hline & 2 & .264 & .037 & .189 & .339 \\
\hline & 3 & .344 & .040 & .264 & .425 \\
\hline
\end{tabular}




\begin{tabular}{|c|c|c|c|c|c|}
\hline \multirow[t]{3}{*}{ Brain region 8} & 1 & .076 & .021 & .033 & .119 \\
\hline & 2 & .100 & .028 & .043 & .156 \\
\hline & 3 & .141 & .027 & .087 & .194 \\
\hline \multirow[t]{3}{*}{ Brain region 9} & 1 & .081 & .015 & .051 & .111 \\
\hline & 2 & .101 & .017 & .067 & .135 \\
\hline & 3 & .130 & .022 & .086 & .174 \\
\hline \multirow[t]{3}{*}{ Brain region 10} & 1 & .145 & .025 & .096 & .195 \\
\hline & 2 & .165 & .025 & .115 & .216 \\
\hline & 3 & .159 & .032 & .095 & .223 \\
\hline \multirow[t]{3}{*}{ Brain region 11} & 1 & .105 & .018 & .070 & .140 \\
\hline & 2 & .104 & .024 & .056 & .151 \\
\hline & 3 & .113 & .019 & .075 & .151 \\
\hline \multirow[t]{3}{*}{ Brain region 12} & 1 & .165 & .030 & .106 & .225 \\
\hline & 2 & .145 & .037 & .071 & .218 \\
\hline & 3 & .182 & .031 & .119 & .246 \\
\hline \multirow[t]{3}{*}{ Brain region 13} & 1 & -.100 & .024 & -.149 & -.052 \\
\hline & 2 & -.133 & .041 & -.217 & -.050 \\
\hline & 3 & -.112 & .025 & -.163 & -.061 \\
\hline \multirow[t]{3}{*}{ Brain region 14} & 1 & .155 & .030 & .095 & .214 \\
\hline & 2 & .200 & .029 & .143 & .258 \\
\hline & 3 & .287 & .034 & .220 & .355 \\
\hline \multirow[t]{3}{*}{ Brain region 15} & 1 & -.101 & .022 & -.146 & -.056 \\
\hline & 2 & -.092 & .037 & -.166 & -.018 \\
\hline & 3 & -.107 & .027 & -.161 & -.053 \\
\hline \multirow[t]{2}{*}{ Brain region 16} & 1 & .236 & .082 & .071 & .402 \\
\hline & 2 & .221 & .109 & .002 & .439 \\
\hline
\end{tabular}




\begin{tabular}{llllll}
\hline & 3 & .286 & .105 & .075 & .497 \\
\hline Brain region 17 & 1 & .067 & .023 & .021 & .112 \\
& 2 & .049 & .035 & -.021 & .120 \\
& 3 & .031 & .028 & -.024 & .086 \\
\hline Brain region 18 & 1 & .094 & .026 & .042 & .147 \\
& 2 & .063 & .035 & -.008 & .135 \\
& 3 & .080 & .026 & .029 & .132 \\
\hline Brain region 19 & 1 & .092 & .024 & .044 & .140 \\
& 2 & .105 & .041 & .022 & .188 \\
& 3 & .107 & .031 & .044 & .171 \\
\hline Brain region 20 & 1 & .212 & .035 & .142 & .282 \\
& 2 & .216 & .041 & .135 & .298 \\
& 3 & .315 & .042 & .231 & .400 \\
\hline Brain region 21 & 1 & -.009 & .017 & -.043 & .025 \\
& 2 & -.094 & .031 & -.156 & -.032 \\
& 3 & -.083 & .023 & -.129 & -.037 \\
\hline Brain region 22 & 1 & .119 & .044 & .031 & .207 \\
& 2 & .221 & .067 & .087 & .355 \\
& 3 & .251 & .046 & .158 & .344 \\
\hline
\end{tabular}


Table J5

Mean Difference, Standard Error, P-Value and 95\% Confidence Interval for Incentive Condition Effects

\begin{tabular}{|c|c|c|c|c|c|c|c|}
\hline & & & Mean Difference & & & $95 \%$ Confidence Inte & al for Difference ${ }^{a}$ \\
\hline Brain region & (I) Incentive cond & (J) Incentive cond & $(\mathrm{I}-\mathrm{J})$ & Std. Error & Sig. ${ }^{a}$ & Lower Bound & Upper Bound \\
\hline \multirow[t]{6}{*}{ Brain region 1} & 1 & 2 & .001 & .030 & .982 & -.059 & .061 \\
\hline & & 3 & -.005 & .018 & .794 & -.041 & .031 \\
\hline & 2 & 1 & -.001 & .030 & .982 & -.061 & .059 \\
\hline & & 3 & -.005 & .032 & .868 & -.070 & .059 \\
\hline & 3 & 1 & .005 & .018 & .794 & -.031 & .041 \\
\hline & & 2 & .005 & .032 & .868 & -.059 & .070 \\
\hline \multirow[t]{6}{*}{ Brain region 2} & 1 & 2 & .016 & .031 & .606 & -.046 & .079 \\
\hline & & 3 & .014 & .020 & .500 & -.027 & .055 \\
\hline & 2 & 1 & -.016 & .031 & .606 & -.079 & .046 \\
\hline & & 3 & -.002 & .032 & .942 & -.066 & .061 \\
\hline & 3 & 1 & -.014 & .020 & .500 & -.055 & .027 \\
\hline & & 2 & .002 & .032 & .942 & -.061 & .066 \\
\hline \multirow[t]{6}{*}{ Brain region 3} & 1 & 2 & .017 & .014 & .246 & -.012 & .045 \\
\hline & & 3 & $.024^{*}$ & .012 & .045 & .001 & .048 \\
\hline & 2 & 1 & -.017 & .014 & .246 & -.045 & .012 \\
\hline & & 3 & .007 & .014 & .608 & -.022 & .037 \\
\hline & 3 & 1 & $-.024^{*}$ & .012 & .045 & -.048 & -.001 \\
\hline & & 2 & -.007 & .014 & .608 & -.037 & .022 \\
\hline \multirow[t]{3}{*}{ Brain region 4} & 1 & 2 & .010 & .015 & .502 & -.019 & .039 \\
\hline & & 3 & .019 & .011 & .089 & -.003 & .041 \\
\hline & 2 & 1 & -.010 & .015 & .502 & -.039 & .019 \\
\hline
\end{tabular}




\begin{tabular}{|c|c|c|c|c|c|c|c|}
\hline & & 3 & .009 & .014 & .528 & -.020 & .038 \\
\hline & 3 & 1 & -.019 & .011 & .089 & -.041 & .003 \\
\hline & & 2 & -.009 & .014 & .528 & -.038 & .020 \\
\hline \multirow[t]{6}{*}{ Brain region 5} & 1 & 2 & $.067^{*}$ & .031 & .036 & .005 & .130 \\
\hline & & 3 & $.098^{*}$ & .020 & .000 & .058 & .138 \\
\hline & 2 & 1 & $-.067^{*}$ & .031 & .036 & -.130 & -.005 \\
\hline & & 3 & .030 & .026 & .247 & -.022 & .083 \\
\hline & 3 & 1 & $-.098^{*}$ & .020 & .000 & -.138 & -.058 \\
\hline & & 2 & -.030 & .026 & .247 & -.083 & .022 \\
\hline \multirow[t]{6}{*}{ Brain region 6} & 1 & 2 & $-.059^{*}$ & .024 & .018 & -.108 & -.010 \\
\hline & & 3 & $-.094^{*}$ & .027 & .001 & -.149 & -.039 \\
\hline & 2 & 1 & $.059^{*}$ & .024 & .018 & .010 & .108 \\
\hline & & 3 & -.035 & .025 & .173 & -.086 & .016. \\
\hline & 3 & 1 & $.094^{*}$ & .027 & .001 & .039 & .149 \\
\hline & & 2 & .035 & .025 & .173 & -.016 & .086 \\
\hline \multirow[t]{6}{*}{ Brain region 7} & 1 & 2 & -.062 & .034 & .077 & -.130 & .007 \\
\hline & & 3 & $-.142^{*}$ & .032 & .000 & -.206 & -.078 \\
\hline & 2 & 1 & .062 & .034 & .077 & -.007 & .130 \\
\hline & & 3 & $-.081^{*}$ & .028 & .006 & -.137 & -.025 \\
\hline & 3 & 1 & $.142^{*}$ & .032 & .000 & .078 & .206 \\
\hline & & 2 & $.081^{*}$ & .028 & .006 & .025 & .137 \\
\hline \multirow[t]{5}{*}{ Brain region 8} & 1 & 2 & -.024 & .022 & .281 & -.068 & .020 \\
\hline & & 3 & $-.065^{*}$ & .019 & .001 & -.102 & -.028 \\
\hline & 2 & 1 & .024 & .022 & .281 & -.020 & .068 \\
\hline & & 3 & -.041 & .022 & .064 & -.085 & .003 \\
\hline & 3 & 1 & $.065^{*}$ & .019 & .001 & .028 & .102 \\
\hline
\end{tabular}




\begin{tabular}{|c|c|c|c|c|c|c|c|}
\hline & & 2 & .041 & .022 & .064 & -.003 & .085 \\
\hline \multirow[t]{6}{*}{ Brain region 9} & 1 & 2 & -.020 & .013 & .130 & -.046 & .006 \\
\hline & & 3 & $-.049^{*}$ & .016 & .004 & -.081 & -.017 \\
\hline & 2 & 1 & .020 & .013 & .130 & -.006 & .046 \\
\hline & & 3 & -.029 & .018 & .114 & -.065 & .007 \\
\hline & 3 & 1 & $.049^{*}$ & .016 & .004 & .017 & .081 \\
\hline & & 2 & .029 & .018 & .114 & -.007 & .065 \\
\hline \multirow[t]{6}{*}{ Brain region 10} & 1 & 2 & -.020 & .025 & .430 & -.071 & .031 \\
\hline & & 3 & -.014 & .029 & .634 & -.073 & .045 \\
\hline & 2 & 1 & .020 & .025 & .430 & -.031 & .071 \\
\hline & & 3 & .006 & .023 & .791 & -.040 & .052 \\
\hline & 3 & 1 & .014 & .029 & .634 & -.045 & .073 \\
\hline & & 2 & -.006 & .023 & .791 & -.052 & .040 \\
\hline \multirow[t]{6}{*}{ Brain region 11} & 1 & 2 & .001 & .020 & .944 & -.040 & .042 \\
\hline & & 3 & -.008 & .017 & .658 & -.042 & .027 \\
\hline & 2 & 1 & -.001 & .020 & .944 & -.042 & .040 \\
\hline & & 3 & -.009 & .018 & .625 & -.046 & .028 \\
\hline & 3 & 1 & .008 & .017 & .658 & -.027 & .042 \\
\hline & & 2 & .009 & .018 & .625 & -.028 & .046 \\
\hline \multirow[t]{6}{*}{ Brain region 12} & 1 & 2 & .020 & .026 & .437 & -.032 & .073 \\
\hline & & 3 & -.017 & .021 & .420 & -.059 & .025 \\
\hline & 2 & 1 & -.020 & .026 & .437 & -.073 & .032 \\
\hline & & 3 & -.037 & .034 & .271 & -.105 & .030 \\
\hline & 3 & 1 & .017 & .021 & .420 & -.025 & .059 \\
\hline & & 2 & .037 & .034 & .271 & -.030 & .105 \\
\hline Brain region 13 & 1 & 2 & .033 & .033 & .324 & -.034 & .100 \\
\hline
\end{tabular}




\begin{tabular}{|c|c|c|c|c|c|c|c|}
\hline & & 3 & .011 & .027 & .677 & -.043 & .066 \\
\hline & \multirow[t]{2}{*}{2} & 1 & -.033 & .033 & .324 & -.100 & .034 \\
\hline & & 3 & -.022 & .038 & .566 & -.098 & .054 \\
\hline & \multirow[t]{2}{*}{3} & 1 & -.011 & .027 & .677 & -.066 & .043 \\
\hline & & 2 & .022 & .038 & .566 & -.054 & .098 \\
\hline \multirow[t]{6}{*}{ Brain region 14} & \multirow[t]{2}{*}{1} & 2 & -.046 & .023 & .055 & -.093 & .001 \\
\hline & & 3 & $-.133^{*}$ & .025 & .000 & -.184 & -.082 \\
\hline & \multirow[t]{2}{*}{2} & 1 & .046 & .023 & .055 & -.001 & .093 \\
\hline & & 3 & $-.087^{*}$ & .025 & .001 & -.138 & -.036 \\
\hline & \multirow[t]{2}{*}{3} & 1 & $.133^{*}$ & .025 & .000 & .082 & .184 \\
\hline & & 2 & $.087^{*}$ & .025 & .001 & .036 & .138 \\
\hline \multirow[t]{6}{*}{ Brain region 15} & \multirow[t]{2}{*}{1} & 2 & -.009 & .032 & .781 & -.074 & .056 \\
\hline & & 3 & .006 & .026 & .823 & -.047 & .059 \\
\hline & \multirow[t]{2}{*}{2} & 1 & .009 & .032 & .781 & -.056 & .074 \\
\hline & & 3 & .015 & .037 & .687 & -.059 & .089 \\
\hline & \multirow[t]{2}{*}{3} & 1 & -.006 & .026 & .823 & -.059 & .047 \\
\hline & & 2 & -.015 & .037 & .687 & -.089 & .059 \\
\hline \multirow[t]{6}{*}{ Brain region 16} & \multirow[t]{2}{*}{1} & 2 & .016 & .081 & .845 & -.147 & .178 \\
\hline & & 3 & -.050 & .064 & .440 & -.178 & .079 \\
\hline & \multirow[t]{2}{*}{2} & 1 & -.016 & .081 & .845 & -.178 & .147 \\
\hline & & 3 & -.065 & .092 & .481 & -.251 & .120 \\
\hline & \multirow[t]{2}{*}{3} & 1 & .050 & .064 & .440 & -.079 & .178 \\
\hline & & 2 & .065 & .092 & .481 & -.120 & .251 \\
\hline \multirow[t]{3}{*}{ Brain region 17} & \multirow[t]{2}{*}{1} & 2 & .017 & .033 & .601 & -.049 & .084 \\
\hline & & 3 & .036 & .023 & .132 & -.011 & .082 \\
\hline & 2 & 1 & -.017 & .033 & .601 & -.084 & .049 \\
\hline
\end{tabular}




\begin{tabular}{|c|c|c|c|c|c|c|c|}
\hline & & 3 & .018 & .028 & .516 & -.038 & .074 \\
\hline & 3 & 1 & -.036 & .023 & .132 & -.082 & .011 \\
\hline & & 2 & -.018 & .028 & .516 & -.074 & .038 \\
\hline \multirow[t]{6}{*}{ Brain region 18} & 1 & 2 & .031 & .038 & .424 & -.046 & .108 \\
\hline & & 3 & .014 & .019 & .468 & -.024 & .052 \\
\hline & 2 & 1 & -.031 & .038 & .424 & -.108 & .046 \\
\hline & & 3 & -.017 & .038 & .661 & -.094 & .060 \\
\hline & 3 & 1 & -.014 & .019 & .468 & -.052 & .024 \\
\hline & & 2 & .017 & .038 & .661 & -.060 & .094 \\
\hline \multirow[t]{6}{*}{ Brain region 19} & 1 & 2 & -.013 & .037 & .718 & -.088 & .061 \\
\hline & & 3 & -.016 & .025 & .543 & -.067 & .036 \\
\hline & 2 & 1 & .013 & .037 & .718 & -.061 & .088 \\
\hline & & 3 & -.002 & .035 & .951 & -.072 & .068 \\
\hline & 3 & 1 & .016 & .025 & .543 & -.036 & .067 \\
\hline & & 2 & .002 & .035 & .951 & -.068 & .072 \\
\hline \multirow[t]{6}{*}{ Brain region 20} & 1 & 2 & -.004 & .029 & .890 & -.061 & .053 \\
\hline & & 3 & $-.103^{*}$ & .027 & .000 & -.158 & -.048 \\
\hline & 2 & 1 & .004 & .029 & .890 & -.053 & .061 \\
\hline & & 3 & $-.099^{*}$ & .024 & .000 & -.148 & -.051 \\
\hline & 3 & 1 & $.103^{*}$ & .027 & .000 & .048 & .158 \\
\hline & & 2 & $.099^{*}$ & .024 & .000 & .051 & .148 \\
\hline \multirow[t]{5}{*}{ Brain region 21} & 1 & 2 & $.086^{*}$ & .031 & .009 & .023 & .148 \\
\hline & & 3 & $.075^{*}$ & .020 & .001 & .034 & .115 \\
\hline & 2 & 1 & $-.086^{*}$ & .031 & .009 & -.148 & -.023 \\
\hline & & 3 & -.011 & .027 & .679 & -.065 & .042 \\
\hline & 3 & 1 & $-.075^{*}$ & .020 & .001 & -.115 & -.034 \\
\hline
\end{tabular}




\begin{tabular}{|c|c|c|c|c|c|c|c|}
\hline & & 2 & .011 & .027 & .679 & -.042 & .065 \\
\hline \multirow[t]{6}{*}{ Brain region 22} & 1 & 2 & -.102 & .051 & .052 & -.205 & .001 \\
\hline & & 3 & $-.132^{*}$ & .040 & .002 & -.212 & -.052 \\
\hline & 2 & 1 & .102 & .051 & .052 & -.001 & .205 \\
\hline & & 3 & -.030 & .070 & .671 & -.171 & .111 \\
\hline & 3 & 1 & $.132^{*}$ & .040 & .002 & .052 & .212 \\
\hline & & 2 & .030 & .070 & .671 & -.111 & .171 \\
\hline
\end{tabular}

Based on estimated marginal means

a. Adjustment for multiple comparisons: Least Significant Difference (equivalent to no adjustments).

*. The mean difference is significant at the .05 level. 
Table J6

Means, Standard Errors and 95\% Confidence Intervals for Accuracy Effects

\begin{tabular}{|c|c|c|c|c|c|}
\hline \multirow[b]{2}{*}{ Brain region } & \multirow[b]{2}{*}{ Accuracy } & \multirow[b]{2}{*}{ Mean } & \multirow[b]{2}{*}{ Std. Error } & \multicolumn{2}{|c|}{ 95\% Confidence Interval } \\
\hline & & & & Lower Bound & Upper Bound \\
\hline \multirow[t]{2}{*}{ Brain region 1} & 1 & .234 & .025 & .183 & .285 \\
\hline & 2 & .209 & .037 & .134 & .284 \\
\hline \multirow[t]{2}{*}{ Brain region 2} & 1 & .212 & .023 & .166 & .258 \\
\hline & 2 & .180 & .036 & .108 & .251 \\
\hline \multirow[t]{2}{*}{ Brain region 3} & 1 & .071 & .010 & .050 & .091 \\
\hline & 2 & .024 & .016 & -.008 & .057 \\
\hline \multirow[t]{2}{*}{ Brain region 4} & 1 & .080 & .011 & .058 & .102 \\
\hline & 2 & .011 & .016 & -.021 & .042 \\
\hline \multirow[t]{2}{*}{ Brain region 5} & 1 & -.109 & .019 & -.148 & -.071 \\
\hline & 2 & -.187 & .027 & -.242 & -.131 \\
\hline \multirow[t]{2}{*}{ Brain region 6} & 1 & .183 & .029 & .125 & .241 \\
\hline & 2 & .219 & .031 & .157 & .282 \\
\hline \multirow[t]{2}{*}{ Brain region 7} & 1 & .277 & .031 & .214 & .340 \\
\hline & 2 & .263 & .037 & .189 & .338 \\
\hline \multirow[t]{2}{*}{ Brain region 8} & 1 & .135 & .026 & .082 & .187 \\
\hline & 2 & .076 & .026 & .024 & .128 \\
\hline \multirow[t]{2}{*}{ Brain region 9} & 1 & .118 & .017 & .084 & .151 \\
\hline & 2 & .090 & .018 & .054 & .126 \\
\hline \multirow[t]{2}{*}{ Brain region 10} & 1 & .130 & .023 & .084 & .176 \\
\hline & 2 & .183 & .030 & .122 & .244 \\
\hline Brain region 11 & 1 & .110 & .014 & .082 & .138 \\
\hline
\end{tabular}




\begin{tabular}{|c|c|c|c|c|c|}
\hline & 2 & .105 & .024 & .055 & .154 \\
\hline \multirow[t]{2}{*}{ Brain region 12} & 1 & .193 & .027 & .139 & .248 \\
\hline & 2 & .135 & .038 & .059 & .211 \\
\hline \multirow[t]{2}{*}{ Brain region 13} & 1 & -.104 & .026 & -.156 & -.052 \\
\hline & 2 & -.126 & .033 & -.193 & -.060 \\
\hline \multirow[t]{2}{*}{ Brain region 14} & 1 & .219 & .027 & .163 & .274 \\
\hline & 2 & .210 & .033 & .144 & .275 \\
\hline \multirow[t]{2}{*}{ Brain region 15} & 1 & -.102 & .023 & -.147 & -.057 \\
\hline & 2 & -.098 & .033 & -.164 & -.032 \\
\hline \multirow[t]{2}{*}{ Brain region 16} & 1 & .370 & .098 & .173 & .566 \\
\hline & 2 & .126 & .106 & -.088 & .339 \\
\hline \multirow[t]{2}{*}{ Brain region 17} & 1 & .073 & .025 & .023 & .123 \\
\hline & 2 & .025 & .032 & -.039 & .089 \\
\hline \multirow[t]{2}{*}{ Brain region 18} & 1 & .078 & .017 & .043 & .112 \\
\hline & 2 & .080 & .036 & .009 & .152 \\
\hline \multirow[t]{2}{*}{ Brain region 19} & 1 & . 104 & .025 & .053 & .155 \\
\hline & 2 & .099 & .036 & .026 & .172 \\
\hline \multirow[t]{2}{*}{ Brain region 20} & 1 & .252 & .035 & .182 & .322 \\
\hline & 2 & .244 & .047 & .150 & .338 \\
\hline \multirow[t]{2}{*}{ Brain region 21} & 1 & -.013 & .018 & -.049 & .024 \\
\hline & 2 & -.112 & .030 & -.172 & -.052 \\
\hline \multirow[t]{2}{*}{ Brain region 22} & 1 & .169 & .039 & .091 & .247 \\
\hline & 2 & .225 & .062 & .099 & .350 \\
\hline
\end{tabular}


Table $\mathbf{J 7}$

Mean Difference, Standard Error, P-Value and 95\% Confidence Interval for Accuracy Effects

\begin{tabular}{|c|c|c|c|c|c|c|c|}
\hline \multirow[b]{2}{*}{ Brain region } & \multirow[b]{2}{*}{ (I) Accuracy } & \multirow{2}{*}{\multicolumn{2}{|c|}{$\begin{array}{c}\text { Mean Difference } \\
(\mathrm{I}-\mathrm{J}) \\
\end{array}$}} & \multirow[b]{2}{*}{ Std. Error } & \multirow[b]{2}{*}{ Sig. ${ }^{\text {a }}$} & \multicolumn{2}{|c|}{ 95\% Confidence Interval for Difference ${ }^{a}$} \\
\hline & & & & & & Lower Bound & Upper Bound \\
\hline \multirow[t]{2}{*}{ Brain region 1} & 1 & 2 & .025 & .030 & .415 & -.036 & .086 \\
\hline & 2 & 1 & -.025 & .030 & .415 & -.086 & .036 \\
\hline \multirow[t]{2}{*}{ Brain region 2} & 1 & 2 & .033 & .029 & .263 & -.025 & .090 \\
\hline & 2 & 1 & -.033 & .029 & .263 & -.090 & .025 \\
\hline \multirow[t]{2}{*}{ Brain region 3} & 1 & 2 & $.046^{*}$ & .014 & .002 & .017 & .075 \\
\hline & 2 & 1 & $-.046^{*}$ & .014 & .002 & -.075 & -.017 \\
\hline \multirow[t]{2}{*}{ Brain region 4} & 1 & 2 & $.069^{*}$ & .015 & .000 & .040 & .098 \\
\hline & 2 & 1 & $-.069^{*}$ & .015 & .000 & -.098 & -.040 \\
\hline \multirow[t]{2}{*}{ Brain region 5} & 1 & 2 & $.077^{*}$ & .028 & .008 & .021 & .134 \\
\hline & 2 & 1 & $-.077^{*}$ & .028 & .008 & -.134 & -.021 \\
\hline \multirow[t]{2}{*}{ Brain region 6} & 1 & 2 & -.036 & .029 & .225 & -.095 & .023 \\
\hline & 2 & 1 & .036 & .029 & .225 & -.023 & .095 \\
\hline \multirow[t]{2}{*}{ Brain region 7} & 1 & 2 & .013 & .031 & .674 & -.050 & .077 \\
\hline & 2 & 1 & -.013 & .031 & .674 & -.077 & .050 \\
\hline \multirow[t]{2}{*}{ Brain region 8} & 1 & 2 & $.059^{*}$ & .026 & .028 & .007 & .111 \\
\hline & 2 & 1 & $-.059^{*}$ & .026 & .028 & -.111 & -.007 \\
\hline \multirow[t]{2}{*}{ Brain region 9} & 1 & 2 & .028 & .014 & .052 & .000 & .056 \\
\hline & 2 & 1 & -.028 & .014 & .052 & -.056 & .000 \\
\hline \multirow[t]{2}{*}{ Brain region 10} & 1 & 2 & -.053 & .028 & .062 & -.108 & .003 \\
\hline & 2 & 1 & .053 & .028 & .062 & -.003 & .108 \\
\hline Brain region 11 & 1 & 2 & .005 & .021 & .806 & -.036 & .047 \\
\hline
\end{tabular}




\begin{tabular}{|c|c|c|c|c|c|c|c|}
\hline & 2 & 1 & -.005 & .021 & .806 & -.047 & .036 \\
\hline \multirow[t]{2}{*}{ Brain region 12} & 1 & 2 & .059 & .032 & .078 & -.007 & .124 \\
\hline & 2 & 1 & -.059 & .032 & .078 & -.124 & .007 \\
\hline \multirow[t]{2}{*}{ Brain region 13} & 1 & 2 & .022 & .032 & .493 & -.043 & .088 \\
\hline & 2 & 1 & -.022 & .032 & .493 & -.088 & .043 \\
\hline \multirow[t]{2}{*}{ Brain region 14} & 1 & 2 & .009 & .026 & .737 & -.044 & .061 \\
\hline & 2 & 1 & -.009 & .026 & .737 & -.061 & .044 \\
\hline \multirow[t]{2}{*}{ Brain region 15} & 1 & 2 & -.004 & .033 & .901 & -.071 & .063 \\
\hline & 2 & 1 & .004 & .033 & .901 & -.063 & .071 \\
\hline \multirow[t]{2}{*}{ Brain region 16} & 1 & 2 & $.244^{*}$ & .103 & .022 & .036 & .451 \\
\hline & 2 & 1 & $-.244^{*}$ & .103 & .022 & -.451 & -.036 \\
\hline \multirow[t]{2}{*}{ Brain region 17} & 1 & 2 & .047 & .032 & .140 & -.016 & .111 \\
\hline & 2 & 1 & -.047 & .032 & .140 & -.111 & .016 \\
\hline \multirow[t]{2}{*}{ Brain region 18} & 1 & 2 & -.003 & .034 & .940 & -.070 & .065 \\
\hline & 2 & 1 & .003 & .034 & .940 & -.065 & .070 \\
\hline \multirow[t]{2}{*}{ Brain region 19} & 1 & 2 & .005 & .032 & .879 & -.059 & .068 \\
\hline & 2 & 1 & -.005 & .032 & .879 & -.068 & .059 \\
\hline \multirow[t]{2}{*}{ Brain region 20} & 1 & 2 & .009 & .040 & .831 & -.071 & .089 \\
\hline & 2 & 1 & -.009 & .040 & .831 & -.089 & .071 \\
\hline \multirow[t]{2}{*}{ Brain region 21} & 1 & 2 & $.099^{*}$ & .032 & .003 & .034 & .164 \\
\hline & 2 & 1 & $-.099^{*}$ & .032 & .003 & -.164 & -.034 \\
\hline \multirow[t]{2}{*}{ Brain region 22} & 1 & 2 & -.056 & .059 & .352 & -.174 & .063 \\
\hline & 2 & 1 & .056 & .059 & .352 & -.063 & .174 \\
\hline
\end{tabular}

Based on estimated marginal means

a. Adjustment for multiple comparisons: Least Significant Difference (equivalent to no adjustments)

*. The mean difference is significant at the .05 level. 
Table J8

Means, Standard Errors and 95\% Confidence Intervals for Group x Incentive Condition Effects

\begin{tabular}{|c|c|c|c|c|c|c|}
\hline \multirow[b]{2}{*}{ Brain region } & \multirow[b]{2}{*}{ Group } & \multirow[b]{2}{*}{ Incentive cond } & \multirow[b]{2}{*}{ Mean } & \multirow[b]{2}{*}{ Std. Error } & \multicolumn{2}{|c|}{ 95\% Confidence Interval } \\
\hline & & & & & Lower Bound & Upper Bound \\
\hline \multirow[t]{6}{*}{ Brain region 1} & 1.00 & 1 & .254 & .036 & .182 & .326 \\
\hline & & 2 & .233 & .055 & .123 & .343 \\
\hline & & 3 & .239 & .044 & .150 & .327 \\
\hline & 2.00 & 1 & 186 & .036 & .114 & .258 \\
\hline & & 2 & .206 & .055 & .096 & .316 \\
\hline & & 3 & .211 & .044 & .122 & .299 \\
\hline \multirow[t]{6}{*}{ Brain region 2} & 1.00 & 1 & .214 & .038 & .138 & .290 \\
\hline & & 2 & .173 & .053 & .067 & .280 \\
\hline & & 3 & .182 & .039 & .103 & .261 \\
\hline & 2.00 & 1 & .197 & .038 & .121 & .273 \\
\hline & & 2 & .206 & .053 & .100 & .312 \\
\hline & & 3 & .202 & .039 & .123 & .281 \\
\hline \multirow[t]{6}{*}{ Brain region 3} & 1.00 & 1 & .083 & .016 & .051 & .116 \\
\hline & & 2 & .054 & .023 & .008 & .100 \\
\hline & & 3 & .053 & .019 & .014 & .092 \\
\hline & 2.00 & 1 & .039 & .016 & .006 & .071 \\
\hline & & 2 & .035 & .023 & -.011 & .081 \\
\hline & & 3 & .021 & .019 & -.018 & .060 \\
\hline \multirow[t]{3}{*}{ Brain region 4} & 1.00 & 1 & .076 & .017 & .042 & .110 \\
\hline & & 2 & .050 & .022 & .006 & .093 \\
\hline & & 3 & .061 & .019 & .023 & .099 \\
\hline
\end{tabular}




\begin{tabular}{|c|c|c|c|c|c|c|}
\hline & 2.00 & 1 & .033 & .017 & -.001 & .068 \\
\hline & & 2 & .040 & .022 & -.004 & .083 \\
\hline & & 3 & .010 & .019 & -.028 & .048 \\
\hline \multirow[t]{6}{*}{ Brain region 5} & 1.00 & 1 & -.076 & .025 & -.126 & -.027 \\
\hline & & 2 & -.134 & .046 & -.227 & -.042 \\
\hline & & 3 & -.173 & .028 & -.229 & -.116 \\
\hline & 2.00 & 1 & -.109 & .025 & -.159 & -.059 \\
\hline & & 2 & -.186 & .046 & -.279 & -.093 \\
\hline & & 3 & -.209 & .028 & -.265 & -.152 \\
\hline \multirow[t]{6}{*}{ Brain region 6} & 1.00 & 1 & .199 & .037 & .125 & .273 \\
\hline & & 2 & .218 & .041 & .137 & .300 \\
\hline & & 3 & .257 & .050 & .157 & .356 \\
\hline & 2.00 & 1 & .102 & .037 & .028 & .175 \\
\hline & & 2 & .201 & .041 & .119 & .282 \\
\hline & & 3 & .232 & .050 & .132 & .332 \\
\hline \multirow[t]{6}{*}{ Brain region 7} & 1.00 & 1 & .268 & .040 & .188 & .349 \\
\hline & & 2 & .277 & .053 & .171 & .384 \\
\hline & & 3 & .350 & .057 & .236 & .464 \\
\hline & 2.00 & 1 & .136 & .040 & .055 & .216 \\
\hline & & 2 & .250 & .053 & .144 & .356 \\
\hline & & 3 & .338 & .057 & .224 & .452 \\
\hline \multirow[t]{5}{*}{ Brain region 8} & 1.00 & 1 & .074 & .030 & .013 & .135 \\
\hline & & 2 & .085 & .040 & .005 & .166 \\
\hline & & 3 & .139 & .038 & .064 & .215 \\
\hline & 2.00 & 1 & .078 & .030 & .017 & .138 \\
\hline & & 2 & .114 & .040 & .033 & 194 \\
\hline
\end{tabular}




\begin{tabular}{|c|c|c|c|c|c|c|}
\hline & & 3 & .142 & .038 & .066 & .218 \\
\hline \multirow[t]{6}{*}{ Brain region 9} & 1.00 & 1 & .093 & .021 & .051 & .135 \\
\hline & & 2 & .097 & .024 & .048 & .145 \\
\hline & & 3 & .132 & .031 & .070 & .195 \\
\hline & 2.00 & 1 & .068 & .021 & .026 & .111 \\
\hline & & 2 & .105 & .024 & .057 & .154 \\
\hline & & 3 & .127 & .031 & .065 & .190 \\
\hline \multirow[t]{6}{*}{ Brain region 10} & 1.00 & 1 & .171 & .035 & .101 & .241 \\
\hline & & 2 & .156 & .036 & .084 & .227 \\
\hline & & 3 & .159 & .045 & .068 & .250 \\
\hline & 2.00 & 1 & .119 & .035 & .049 & .189 \\
\hline & & 2 & .175 & .036 & .103 & .246 \\
\hline & & 3 & .159 & .045 & .069 & .250 \\
\hline \multirow[t]{6}{*}{ Brain region 11} & 1.00 & 1 & .111 & .025 & .062 & .161 \\
\hline & & 2 & .097 & .034 & .029 & .164 \\
\hline & & 3 & .112 & .027 & .059 & .166 \\
\hline & 2.00 & 1 & .099 & .025 & .049 & .149 \\
\hline & & 2 & .111 & .034 & .043 & .178 \\
\hline & & 3 & .113 & .027 & .059 & .167 \\
\hline \multirow[t]{6}{*}{ Brain region 12} & 1.00 & 1 & .206 & .042 & .121 & .290 \\
\hline & & 2 & .151 & .052 & .047 & .255 \\
\hline & & 3 & .225 & .044 & .135 & .314 \\
\hline & 2.00 & 1 & .125 & .042 & .041 & .210 \\
\hline & & 2 & .139 & .052 & .035 & .243 \\
\hline & & 3 & .140 & .044 & .050 & .229 \\
\hline Brain region 13 & 1.00 & 1 & -.094 & .034 & -.163 & -.026 \\
\hline
\end{tabular}




\begin{tabular}{|c|c|c|c|c|c|c|}
\hline & & 2 & -.165 & .058 & -.283 & -.047 \\
\hline & & 3 & -.098 & .036 & -.170 & -.026 \\
\hline & 2.00 & 1 & -.106 & .034 & -.175 & -.038 \\
\hline & & 2 & -.102 & .058 & -.220 & .016 \\
\hline & & 3 & -.125 & .036 & -.197 & -.053 \\
\hline \multirow[t]{6}{*}{ Brain region 14} & 1.00 & 1 & .195 & .042 & .111 & .280 \\
\hline & & 2 & .177 & .040 & .096 & .259 \\
\hline & & 3 & .278 & .048 & .183 & .374 \\
\hline & 2.00 & 1 & .114 & .042 & .029 & 198 \\
\hline & & 2 & .224 & .040 & .142 & .305 \\
\hline & & 3 & .296 & .048 & .201 & .392 \\
\hline \multirow[t]{6}{*}{ Brain region 15} & 1.00 & 1 & -.094 & .032 & -.158 & -.031 \\
\hline & & 2 & -.071 & .052 & -.176 & .034 \\
\hline & & 3 & -.105 & .038 & -.181 & -.029 \\
\hline & 2.00 & 1 & -.108 & .032 & -.171 & -.044 \\
\hline & & 2 & -.113 & .052 & -.218 & -.008 \\
\hline & & 3 & -.109 & .038 & -.185 & -.033 \\
\hline \multirow[t]{6}{*}{ Brain region 16} & 1.00 & 1 & .394 & .116 & .160 & .628 \\
\hline & & 2 & .321 & .154 & .012 & .631 \\
\hline & & 3 & .370 & .148 & .071 & .668 \\
\hline & 2.00 & 1 & .079 & .116 & -.155 & .313 \\
\hline & & 2 & .120 & .154 & -.190 & .429 \\
\hline & & 3 & .202 & .148 & -.096 & .501 \\
\hline \multirow[t]{3}{*}{ Brain region 17} & 1.00 & 1 & .089 & .032 & .024 & .154 \\
\hline & & 2 & .046 & .050 & -.053 & .146 \\
\hline & & 3 & .039 & .039 & -.039 & .118 \\
\hline
\end{tabular}




\begin{tabular}{|c|c|c|c|c|c|c|}
\hline & 2.00 & 1 & .044 & .032 & -.020 & .109 \\
\hline & & 2 & .052 & .050 & -.048 & .152 \\
\hline & & 3 & .023 & .039 & -.056 & .101 \\
\hline \multirow[t]{6}{*}{ Brain region 18} & 1.00 & 1 & .137 & .037 & .063 & .211 \\
\hline & & 2 & .089 & .050 & -.012 & .190 \\
\hline & & 3 & .113 & .036 & .040 & .185 \\
\hline & 2.00 & 1 & .051 & .037 & -.023 & .126 \\
\hline & & 2 & .038 & .050 & -.063 & .139 \\
\hline & & 3 & .048 & .036 & -.025 & .120 \\
\hline \multirow[t]{6}{*}{ Brain region 19} & 1.00 & 1 & .134 & .034 & .066 & .203 \\
\hline & & 2 & .151 & .058 & .033 & .268 \\
\hline & & 3 & .146 & .044 & .057 & .236 \\
\hline & 2.00 & 1 & .050 & .034 & -.019 & .118 \\
\hline & & 2 & .060 & .058 & -.057 & .177 \\
\hline & & 3 & .069 & .044 & -.021 & .158 \\
\hline \multirow[t]{6}{*}{ Brain region 20} & 1.00 & 1 & .225 & .049 & .126 & .324 \\
\hline & & 2 & .207 & .057 & .092 & .323 \\
\hline & & 3 & .270 & .059 & .150 & .390 \\
\hline & 2.00 & 1 & .199 & .049 & .100 & .298 \\
\hline & & 2 & .225 & .057 & .110 & .340 \\
\hline & & 3 & .361 & .059 & .241 & .480 \\
\hline \multirow[t]{5}{*}{ Brain region 21} & 1.00 & 1 & .028 & .024 & -.020 & .076 \\
\hline & & 2 & -.086 & .044 & -.174 & .001 \\
\hline & & 3 & -.071 & .032 & -.136 & -.006 \\
\hline & 2.00 & 1 & -.046 & .024 & -.094 & .002 \\
\hline & & 2 & -.102 & .044 & -.190 & -.015 \\
\hline
\end{tabular}




\begin{tabular}{lllllll}
\hline & & & & & & \\
& & & 095 & .032 & -.160 & -.031 \\
\hline Brain region 22 & 1.00 & 1 & .172 & .062 & .047 & .297 \\
& & 2 & .280 & .094 & .090 & .469 \\
& & 3 & .316 & .065 & .184 & .447 \\
\cline { 2 - 7 } & 2.00 & 1 & .066 & .062 & -.059 & .191 \\
& & 2 & .163 & .094 & -.027 & .352 \\
& & .187 & .065 & .055 & .318 \\
\hline
\end{tabular}


Table J9

Mean Difference, Standard Error, P-Value and 95\% Confidence Interval for Incentive Condition Effects by Group

\begin{tabular}{|c|c|c|c|c|c|c|c|c|}
\hline \multirow[b]{2}{*}{ Brain region } & \multirow[b]{2}{*}{ Group } & \multirow[b]{2}{*}{ (I) Incentive cond } & \multicolumn{3}{|c|}{ Mean Difference } & \multirow[b]{2}{*}{ Sig. ${ }^{a}$} & \multicolumn{2}{|c|}{ 95\% Confidence Interval for Difference ${ }^{a}$} \\
\hline & & & (J) Incentive cond & $(\mathrm{I}-\mathrm{J})$ & Std. Error & & Lower Bound & Upper Bound \\
\hline \multirow[t]{12}{*}{ Brain region 1} & 1.00 & 1 & 2 & .021 & .042 & .618 & -.064 & .106 \\
\hline & & & 3 & .015 & .025 & .551 & -.036 & .066 \\
\hline & & 2 & 1 & -.021 & .042 & .618 & -.106 & .064 \\
\hline & & & 3 & -.006 & .045 & .894 & -.097 & .085 \\
\hline & & 3 & 1 & -.015 & .025 & .551 & -.066 & .036 \\
\hline & & & 2 & .006 & .045 & .894 & -.085 & .097 \\
\hline & 2.00 & 1 & 2 & -.020 & .042 & .640 & -.105 & .065 \\
\hline & & & 3 & -.025 & .025 & .336 & -.075 & .026 \\
\hline & & 2 & 1 & .020 & .042 & .640 & -.065 & .105 \\
\hline & & & 3 & -.005 & .045 & .919 & -.096 & .087 \\
\hline & & 3 & 1 & .025 & .025 & .336 & -.026 & .075 \\
\hline & & & 2 & .005 & .045 & .919 & -.087 & .096 \\
\hline \multirow[t]{9}{*}{ Brain region 2} & 1.00 & 1 & 2 & .041 & .044 & .359 & -.048 & .129 \\
\hline & & & 3 & .032 & .029 & .271 & -.026 & .090 \\
\hline & & 2 & 1 & -.041 & .044 & .359 & -.129 & .048 \\
\hline & & & 3 & -.009 & .045 & .847 & -.099 & .081 \\
\hline & & 3 & 1 & -.032 & .029 & .271 & -.090 & .026 \\
\hline & & & 2 & .009 & .045 & .847 & -.081 & .099 \\
\hline & 2.00 & 1 & 2 & -.008 & .044 & .849 & -.097 & .080 \\
\hline & & & 3 & -.004 & .029 & .879 & -.062 & .053 \\
\hline & & 2 & 1 & .008 & .044 & .849 & -.080 & .097 \\
\hline
\end{tabular}




\begin{tabular}{|c|c|c|c|c|c|c|c|c|}
\hline & & & 3 & .004 & .045 & .929 & -.086 & .094 \\
\hline & & 3 & 1 & .004 & .029 & .879 & -.053 & .062 \\
\hline & & & 2 & -.004 & .045 & .929 & -.094 & .086 \\
\hline \multirow[t]{12}{*}{ Brain region 3} & 1.00 & 1 & 2 & .029 & .020 & .154 & -.011 & .069 \\
\hline & & & 3 & .031 & .017 & .070 & -.003 & .064 \\
\hline & & 2 & 1 & -.029 & .020 & .154 & -.069 & .011 \\
\hline & & & 3 & .002 & .020 & .938 & -.040 & .043 \\
\hline & & 3 & 1 & -.031 & .017 & .070 & -.064 & .003 \\
\hline & & & 2 & -.002 & .020 & .938 & -.043 & .040 \\
\hline & 2.00 & 1 & 2 & .004 & .020 & .834 & -.036 & .044 \\
\hline & & & 3 & .018 & .017 & .293 & -.016 & .051 \\
\hline & & 2 & 1 & -.004 & .020 & .834 & -.044 & .036 \\
\hline & & & 3 & .013 & .020 & .518 & -.028 & .055 \\
\hline & & 3 & 1 & -.018 & .017 & .293 & -.051 & .016 \\
\hline & & & 2 & -.013 & .020 & .518 & -.055 & .028 \\
\hline \multirow[t]{11}{*}{ Brain region 4} & 1.00 & 1 & 2 & .026 & .021 & .212 & -.015 & .068 \\
\hline & & & 3 & .015 & .015 & .348 & -.016 & .045 \\
\hline & & 2 & 1 & -.026 & .021 & .212 & -.068 & .015 \\
\hline & & & 3 & -.011 & .020 & .569 & -.052 & .029 \\
\hline & & 3 & 1 & -.015 & .015 & .348 & -.045 & .016 \\
\hline & & & 2 & .011 & .020 & .569 & -.029 & .052 \\
\hline & 2.00 & 1 & 2 & -.006 & .021 & .758 & -.048 & .035 \\
\hline & & & 3 & .023 & .015 & .138 & -.008 & .054 \\
\hline & & 2 & 1 & .006 & .021 & .758 & -.035 & .048 \\
\hline & & & 3 & .030 & .020 & .148 & -.011 & .070 \\
\hline & & 3 & 1 & -.023 & .015 & .138 & -.054 & .008 \\
\hline
\end{tabular}




\begin{tabular}{|c|c|c|c|c|c|c|c|c|}
\hline & & & 2 & -.030 & .020 & .148 & -.070 & .011 \\
\hline \multirow[t]{12}{*}{ Brain region 5} & 1.00 & 1 & 2 & .058 & .044 & .196 & -.031 & .146 \\
\hline & & & 3 & $.096^{*}$ & .028 & .001 & .039 & .153 \\
\hline & & 2 & 1 & -.058 & .044 & .196 & -.146 & .031 \\
\hline & & & 3 & .039 & .037 & .299 & -.035 & .112 \\
\hline & & 3 & 1 & $-.096^{*}$ & .028 & .001 & -.153 & -.039 \\
\hline & & & 2 & -.039 & .037 & .299 & -.112 & .035 \\
\hline & 2.00 & 1 & 2 & .077 & .044 & .087 & -.012 & .166 \\
\hline & & & 3 & $.099^{*}$ & .028 & .001 & .042 & . 157. \\
\hline & & 2 & 1 & -.077 & .044 & .087 & -.166 & .012 \\
\hline & & & 3 & .022 & .037 & .545 & -.051 & .096 \\
\hline & & 3 & 1 & $-.099^{*}$ & .028 & .001 & -.157 & -.042 \\
\hline & & & 2 & -.022 & .037 & .545 & -.096 & .051 \\
\hline \multirow[t]{12}{*}{ Brain region 6} & 1.00 & 1 & 2 & -.019 & .034 & .581 & -.088 & .050 \\
\hline & & & 3 & -.057 & .038 & .143 & -.135 & .020 \\
\hline & & 2 & 1 & .019 & .034 & .581 & -.050 & .088 \\
\hline & & & 3 & -.038 & .036 & .287 & -.110 & .033 \\
\hline & & 3 & 1 & .057 & .038 & .143 & -.020 & .135 \\
\hline & & & 2 & .038 & .036 & .287 & -.033 & .110 \\
\hline & 2.00 & 1 & 2 & $-.099^{*}$ & .034 & .006 & -.168 & -.030 \\
\hline & & & 3 & $-.131^{*}$ & .038 & .001 & -.208 & -.053 \\
\hline & & 2 & 1 & $.099^{*}$ & .034 & .006 & .030 & .168 \\
\hline & & & 3 & -.031 & .036 & .383 & -.103 & .040 \\
\hline & & 3 & 1 & $.131^{*}$ & .038 & .001 & .053 & .208 \\
\hline & & & 2 & .031 & .036 & .383 & -.040 & .103 \\
\hline Brain region 7 & 1.00 & 1 & 2 & -.009 & .048 & .852 & -.106 & .088 \\
\hline
\end{tabular}




\begin{tabular}{|c|c|c|c|c|c|c|c|c|}
\hline & & & 3 & -.082 & .045 & .076 & -.173 & .009 \\
\hline & & \multirow[t]{2}{*}{2} & 1 & .009 & .048 & .852 & -.088 & .106 \\
\hline & & & 3 & -.073 & .039 & .069 & -.152 & .006 \\
\hline & & \multirow[t]{2}{*}{3} & 1 & .082 & .045 & .076 & -.009 & .173 \\
\hline & & & 2 & .073 & .039 & .069 & -.006 & .152 \\
\hline & \multirow[t]{6}{*}{2.00} & \multirow[t]{2}{*}{1} & 2 & $-.114^{*}$ & .048 & .022 & -.211 & -.017 \\
\hline & & & 3 & $-.202^{*}$ & .045 & .000 & -.293 & -.112 \\
\hline & & \multirow[t]{2}{*}{2} & 1 & $.114^{*}$ & .048 & .022 & .017 & .211 \\
\hline & & & 3 & $-.088^{*}$ & .039 & .029 & -.167 & -.009 \\
\hline & & \multirow[t]{2}{*}{3} & 1 & $.202^{*}$ & .045 & .000 & .112 & .293 \\
\hline & & & 2 & $.088^{*}$ & .039 & .029 & .009 & .167 \\
\hline \multirow[t]{12}{*}{ Brain region 8} & \multirow[t]{6}{*}{1.00} & \multirow[t]{2}{*}{1} & 2 & -.012 & .031 & .709 & -.074 & .051 \\
\hline & & & 3 & $-.065^{*}$ & .026 & .016 & -.118 & -.013 \\
\hline & & \multirow[t]{2}{*}{2} & 1 & .012 & .031 & .709 & -.051 & .074 \\
\hline & & & 3 & -.054 & .031 & .087 & -.116 & .008 \\
\hline & & \multirow[t]{2}{*}{3} & 1 & $.065^{*}$ & .026 & .016 & .013 & .118 \\
\hline & & & 2 & .054 & .031 & .087 & -.008 & .116 \\
\hline & \multirow[t]{6}{*}{2.00} & \multirow[t]{2}{*}{1} & 2 & -.036 & .031 & .249 & -.098 & .026 \\
\hline & & & 3 & $-.065^{*}$ & .026 & .018 & -.117 & -.012 \\
\hline & & \multirow[t]{2}{*}{2} & 1 & .036 & .031 & .249 & -.026 & .098 \\
\hline & & & 3 & -.029 & .031 & .358 & -.091 & .033 \\
\hline & & \multirow[t]{2}{*}{3} & 1 & $.065^{*}$ & .026 & .018 & .012 & .117 \\
\hline & & & 2 & .029 & .031 & .358 & -.033 & .091 \\
\hline \multirow[t]{3}{*}{ Brain region 9} & \multirow[t]{3}{*}{1.00} & \multirow[t]{2}{*}{1} & 2 & -.003 & .018 & .854 & -.041 & .034 \\
\hline & & & 3 & -.039 & .023 & .088 & -.085 & .006 \\
\hline & & 2 & 1 & .003 & .018 & .854 & -.034 & .041 \\
\hline
\end{tabular}




\begin{tabular}{|c|c|c|c|c|c|c|c|c|}
\hline & & & 3 & -.036 & .025 & .164 & -.087 & .015 \\
\hline & & \multirow[t]{2}{*}{3} & 1 & .039 & .023 & .088 & -.006 & .085 \\
\hline & & & 2 & .036 & .025 & .164 & -.015 & .087 \\
\hline & \multirow[t]{6}{*}{2.00} & \multirow[t]{2}{*}{1} & 2 & -.037 & .018 & .052 & -.074 & .000 \\
\hline & & & 3 & $-.059^{*}$ & .023 & .012 & -.104 & -.013 \\
\hline & & \multirow[t]{2}{*}{2} & 1 & .037 & .018 & .052 & .000 & .074 \\
\hline & & & 3 & -.022 & .025 & .391 & -.073 & .029 \\
\hline & & \multirow[t]{2}{*}{3} & 1 & $.059^{*}$ & .023 & .012 & .013 & .104 \\
\hline & & & 2 & .022 & .025 & .391 & -.029 & .073 \\
\hline \multirow[t]{12}{*}{ Brain region 10} & \multirow[t]{6}{*}{1.00} & \multirow[t]{2}{*}{1} & 2 & .016 & .036 & .664 & -.056 & .087 \\
\hline & & & 3 & .012 & .041 & .769 & -.071 & .095 \\
\hline & & \multirow[t]{2}{*}{2} & 1 & -.016 & .036 & .664 & -.087 & .056 \\
\hline & & & 3 & -.003 & .032 & .917 & -.068 & .061 \\
\hline & & \multirow[t]{2}{*}{3} & 1 & -.012 & .041 & .769 & -.095 & .071 \\
\hline & & & 2 & .003 & .032 & .917 & -.061 & .068 \\
\hline & \multirow[t]{6}{*}{2.00} & \multirow[t]{2}{*}{1} & 2 & -.056 & .036 & .125 & -.127 & .016 \\
\hline & & & 3 & -.040 & .041 & .335 & -.123 & .043 \\
\hline & & \multirow[t]{2}{*}{2} & 1 & .056 & .036 & .125 & -.016 & .127 \\
\hline & & & 3 & .015 & .032 & .633 & -.049 & .080 \\
\hline & & \multirow[t]{2}{*}{3} & 1 & .040 & .041 & .335 & -.043 & .123 \\
\hline & & & 2 & -.015 & .032 & .633 & -.080 & .049 \\
\hline \multirow[t]{5}{*}{ Brain region 11} & \multirow[t]{5}{*}{1.00} & \multirow[t]{2}{*}{1} & 2 & .015 & .029 & .609 & -.043 & .073 \\
\hline & & & 3 & -.001 & .024 & .965 & -.050 & .048 \\
\hline & & \multirow[t]{2}{*}{2} & 1 & -.015 & .029 & 609 & -.073 & .043 \\
\hline & & & 3 & -.016 & .026 & .544 & -.068 & .036 \\
\hline & & 3 & 1 & .001 & .024 & .965 & -.048 & .050 \\
\hline
\end{tabular}




\begin{tabular}{|c|c|c|c|c|c|c|c|c|}
\hline & & & 2 & .016 & .026 & .544 & -.036 & .068 \\
\hline & \multirow[t]{6}{*}{2.00} & \multirow[t]{2}{*}{1} & 2 & -.012 & .029 & .680 & -.070 & .046 \\
\hline & & & 3 & -.014 & .024 & .561 & -.063 & .035 \\
\hline & & \multirow[t]{2}{*}{2} & 1 & .012 & .029 & .680 & -.046 & .070 \\
\hline & & & 3 & -.002 & .026 & .932 & -.055 & .050 \\
\hline & & \multirow[t]{2}{*}{3} & 1 & .014 & .024 & .561 & -.035 & .063 \\
\hline & & & 2 & .002 & .026 & .932 & -.050 & .055 \\
\hline \multirow[t]{12}{*}{ Brain region 12} & \multirow[t]{6}{*}{1.00} & \multirow[t]{2}{*}{1} & 2 & .055 & .037 & .145 & -.020 & .129 \\
\hline & & & 3 & -.019 & .029 & .520 & -.078 & .040 \\
\hline & & \multirow[t]{2}{*}{2} & 1 & -.055 & .037 & .145 & -.129 & .020 \\
\hline & & & 3 & -.074 & .047 & .126 & -.169 & .022 \\
\hline & & \multirow[t]{2}{*}{3} & 1 & .019 & .029 & .520 & -.040 & .078 \\
\hline & & & 2 & .074 & .047 & .126 & -.022 & .169 \\
\hline & \multirow[t]{6}{*}{2.00} & \multirow[t]{2}{*}{1} & 2 & -.014 & .037 & .709 & -.088 & .060 \\
\hline & & & 3 & -.015 & .029 & .618 & -.074 & .044 \\
\hline & & \multirow[t]{2}{*}{2} & 1 & .014 & .037 & .709 & -.060 & .088 \\
\hline & & & 3 & -.001 & .047 & .985 & -.096 & .095 \\
\hline & & \multirow[t]{2}{*}{3} & 1 & .015 & .029 & .618 & -.044 & .074 \\
\hline & & & 2 & .001 & .047 & .985 & -.095 & .096 \\
\hline \multirow[t]{7}{*}{ Brain region 13} & \multirow[t]{6}{*}{1.00} & \multirow[t]{2}{*}{1} & 2 & .071 & .047 & .140 & -.024 & .165 \\
\hline & & & 3 & .004 & .038 & .922 & -.073 & .081 \\
\hline & & \multirow[t]{2}{*}{2} & 1 & -.071 & .047 & .140 & -.165 & .024 \\
\hline & & & 3 & -.067 & .054 & .218 & -.175 & .041 \\
\hline & & \multirow[t]{2}{*}{3} & 1 & -.004 & .038 & .922 & -.081 & .073 \\
\hline & & & 2 & .067 & .054 & .218 & -.041 & .175 \\
\hline & 2.00 & 1 & 2 & -.004 & .047 & .928 & -.099 & .090 \\
\hline
\end{tabular}




\begin{tabular}{|c|c|c|c|c|c|c|c|c|}
\hline & & & 3 & .019 & .038 & .624 & -.058 & .096 \\
\hline & & 2 & 1 & .004 & .047 & .928 & -.090 & .099 \\
\hline & & & 3 & .023 & .054 & .667 & -.085 & .131 \\
\hline & & 3 & 1 & -.019 & .038 & .624 & -.096 & .058 \\
\hline & & & 2 & -.023 & .054 & .667 & -.131 & .085 \\
\hline \multirow[t]{12}{*}{ Brain region 14} & 1.00 & 1 & 2 & .018 & .033 & .583 & -.048 & .085 \\
\hline & & & 3 & $-.083^{*}$ & .036 & .024 & -.155 & -.011 \\
\hline & & 2 & 1 & -.018 & .033 & .583 & -.085 & .048 \\
\hline & & & 3 & $-.101^{*}$ & .036 & .007 & -.173 & -.029 \\
\hline & & 3 & 1 & $.083^{*}$ & .036 & .024 & .011 & .155 \\
\hline & & & 2 & $.101^{*}$ & .036 & .007 & .029 & .173 \\
\hline & 2.00 & 1 & 2 & $-.110^{*}$ & .033 & .002 & -.177 & -.044 \\
\hline & & & 3 & $-.182^{*}$ & .036 & .000 & -.254 & -.111 \\
\hline & & 2 & 1 & $.110^{*}$ & .033 & .002 & .044 & .177 \\
\hline & & & 3 & $-.072^{*}$ & .036 & .049 & -.144 & .000 \\
\hline & & 3 & 1 & $.182^{*}$ & .036 & .000 & .111 & .254 \\
\hline & & & 2 & $.072^{*}$ & .036 & .049 & .000 & .144 \\
\hline \multirow[t]{9}{*}{ Brain region 15} & 1.00 & 1 & 2 & -.023 & .046 & .618 & -.115 & .069 \\
\hline & & & 3 & .011 & .037 & .774 & -.064 & .086 \\
\hline & & 2 & 1 & .023 & .046 & .618 & -.069 & .115 \\
\hline & & & 3 & .034 & .052 & .520 & -.071 & .139 \\
\hline & & 3 & 1 & -.011 & .037 & .774 & -.086 & .064 \\
\hline & & & 2 & -.034 & .052 & .520 & -.139 & .071 \\
\hline & 2.00 & 1 & 2 & .005 & .046 & .915 & -.087 & .097 \\
\hline & & & 3 & .001 & .037 & .977 & -.074 & .076 \\
\hline & & 2 & 1 & -.005 & .046 & .915 & -.097 & .087 \\
\hline
\end{tabular}




\begin{tabular}{|c|c|c|c|c|c|c|c|c|}
\hline & & & 3 & -.004 & .052 & .942 & -.109 & .101 \\
\hline & & 3 & 1 & -.001 & .037 & .977 & -.076 & .074 \\
\hline & & & 2 & .004 & .052 & .942 & -.101 & .109 \\
\hline \multirow[t]{12}{*}{ Brain region 16} & 1.00 & 1 & 2 & .073 & .114 & .528 & -.157 & .302 \\
\hline & & & 3 & .024 & .090 & .787 & -.157 & .206 \\
\hline & & 2 & 1 & -.073 & .114 & .528 & -.302 & .157 \\
\hline & & & 3 & -.048 & .130 & .713 & -.310 & .214 \\
\hline & & 3 & 1 & -.024 & .090 & .787 & -.206 & .157 \\
\hline & & & 2 & .048 & .130 & .713 & -.214 & .310 \\
\hline & 2.00 & 1 & 2 & -.041 & .114 & .722 & -.271 & .189 \\
\hline & & & 3 & -.124 & .090 & .177 & -.305 & .058 \\
\hline & & 2 & 1 & .041 & .114 & .722 & -.189 & .271 \\
\hline & & & 3 & -.083 & .130 & .528 & -.345 & .179 \\
\hline & & 3 & 1 & .124 & .090 & .177 & -.058 & .305 \\
\hline & & & 2 & .083 & .130 & .528 & -.179 & .345 \\
\hline \multirow[t]{11}{*}{ Brain region 17} & 1.00 & 1 & 2 & .043 & .047 & .367 & -.051 & .136 \\
\hline & & & 3 & .049 & .033 & .139 & -.017 & .115 \\
\hline & & 2 & 1 & -.043 & .047 & .367 & -.136 & .051 \\
\hline & & & 3 & .007 & .039 & .863 & -.072 & .086 \\
\hline & & 3 & 1 & -.049 & .033 & .139 & -.115 & .017 \\
\hline & & & 2 & -.007 & .039 & .863 & -.086 & .072 \\
\hline & 2.00 & 1 & 2 & -.008 & .047 & .867 & -.102 & .086 \\
\hline & & & 3 & .022 & .033 & .512 & -.044 & .088 \\
\hline & & 2 & 1 & .008 & .047 & .867 & -.086 & .102 \\
\hline & & & 3 & .030 & .039 & .456 & -.050 & .109 \\
\hline & & 3 & 1 & -.022 & .033 & .512 & -.088 & .044 \\
\hline
\end{tabular}




\begin{tabular}{|c|c|c|c|c|c|c|c|c|}
\hline & & & 2 & -.030 & .039 & .456 & -.109 & .050 \\
\hline \multirow[t]{12}{*}{ Brain region 18} & 1.00 & 1 & 2 & .048 & .054 & .378 & -.061 & .157 \\
\hline & & & 3 & .024 & .027 & .375 & -.030 & .078 \\
\hline & & 2 & 1 & -.048 & .054 & .378 & -.157 & .061 \\
\hline & & & 3 & -.024 & .054 & .660 & -.134 & .085 \\
\hline & & 3 & 1 & -.024 & .027 & .375 & -.078 & .030 \\
\hline & & & 2 & .024 & .054 & .660 & -.085 & .134 \\
\hline & 2.00 & 1 & 2 & .014 & .054 & .803 & -.095 & .122 \\
\hline & & & 3 & .004 & .027 & .891 & -.050 & .058 \\
\hline & & 2 & 1 & -.014 & .054 & .803 & -.122 & .095 \\
\hline & & & 3 & -.010 & .054 & .857 & -.119 & .100 \\
\hline & & 3 & 1 & -.004 & .027 & .891 & -.058 & .050 \\
\hline & & & 2 & .010 & .054 & .857 & -.100 & .119 \\
\hline \multirow[t]{12}{*}{ Brain region 19} & 1.00 & 1 & 2 & -.016 & .052 & .755 & -.121 & .089 \\
\hline & & & 3 & -.012 & .036 & .739 & -.084 & .060 \\
\hline & & 2 & 1 & .016 & .052 & .755 & -.089 & .121 \\
\hline & & & 3 & .004 & .049 & .930 & -.095 & .103 \\
\hline & & 3 & 1 & .012 & .036 & .739 & -.060 & .084 \\
\hline & & & 2 & -.004 & .049 & .930 & -.103 & .095 \\
\hline & 2.00 & 1 & 2 & -.010 & .052 & .842 & -.115 & .095 \\
\hline & & & 3 & -.019 & .036 & .597 & -.091 & .053 \\
\hline & & 2 & 1 & .010 & .052 & .842 & -.095 & .115 \\
\hline & & & 3 & -.009 & .049 & .861 & -.108 & .090 \\
\hline & & 3 & 1 & .019 & .036 & .597 & -.053 & .091 \\
\hline & & & 2 & .009 & .049 & .861 & -.090 & .108 \\
\hline Brain region 20 & 1.00 & 1 & 2 & .018 & .040 & .656 & -.063 & .099 \\
\hline
\end{tabular}




\begin{tabular}{|c|c|c|c|c|c|c|c|c|}
\hline & & & 3 & -.045 & .039 & .253 & -.123 & .033 \\
\hline & & \multirow[t]{2}{*}{2} & 1 & -.018 & .040 & .656 & -.099 & .063 \\
\hline & & & 3 & -.063 & .034 & .071 & -.131 & .006 \\
\hline & & \multirow[t]{2}{*}{3} & 1 & .045 & .039 & .253 & -.033 & .123 \\
\hline & & & 2 & .063 & .034 & .071 & -.006 & .131 \\
\hline & \multirow[t]{6}{*}{2.00} & \multirow[t]{2}{*}{1} & 2 & -.026 & .040 & .522 & -.107 & .055 \\
\hline & & & 3 & $-.162^{*}$ & .039 & .000 & -.240 & -.084 \\
\hline & & \multirow[t]{2}{*}{2} & 1 & .026 & .040 & .522 & -.055 & .107 \\
\hline & & & 3 & $-.136^{*}$ & .034 & .000 & -.204 & -.067 \\
\hline & & \multirow[t]{2}{*}{3} & 1 & $.162^{*}$ & .039 & .000 & .084 & .240 \\
\hline & & & 2 & $.136^{*}$ & .034 & .000 & .067 & .204 \\
\hline \multirow[t]{12}{*}{ Brain region 21} & \multirow[t]{6}{*}{1.00} & \multirow[t]{2}{*}{1} & 2 & $.115^{*}$ & .044 & .012 & .026 & .203 \\
\hline & & & 3 & $.099^{*}$ & .029 & .001 & .042 & .157 \\
\hline & & \multirow[t]{2}{*}{2} & 1 & $-.115^{*}$ & .044 & .012 & -.203 & -.026 \\
\hline & & & 3 & -.015 & .038 & .689 & -.091 & .061 \\
\hline & & \multirow[t]{2}{*}{3} & 1 & $-.099^{*}$ & .029 & .001 & -.157 & -.042 \\
\hline & & & 2 & .015 & .038 & .689 & -.061 & .091 \\
\hline & \multirow[t]{6}{*}{2.00} & \multirow[t]{2}{*}{1} & 2 & .057 & .044 & .204 & -.032 & .145 \\
\hline & & & 3 & .050 & .029 & .089 & -.008 & .107 \\
\hline & & \multirow[t]{2}{*}{2} & 1 & -.057 & .044 & .204 & -.145 & .032 \\
\hline & & & 3 & -.007 & .038 & .852 & -.083 & .069 \\
\hline & & \multirow[t]{2}{*}{3} & 1 & -.050 & .029 & .089 & -.107 & .008 \\
\hline & & & 2 & .007 & .038 & .852 & -.069 & .083 \\
\hline \multirow[t]{3}{*}{ Brain region 22} & \multirow[t]{3}{*}{1.00} & \multirow[t]{2}{*}{1} & 2 & -.108 & .072 & .143 & -.253 & .038 \\
\hline & & & 3 & $-.144^{*}$ & .056 & .014 & -.257 & -.031 \\
\hline & & 2 & 1 & .108 & .072 & .143 & -.038 & .253 \\
\hline
\end{tabular}




\begin{tabular}{|c|c|c|c|c|c|c|c|}
\hline & & 3 & -.036 & .099 & .718 & -.235 & .163 \\
\hline & \multirow[t]{2}{*}{3} & 1 & $.144^{*}$ & .056 & .014 & .031 & .257 \\
\hline & & 2 & .036 & .099 & .718 & -.163 & .235 \\
\hline \multirow[t]{6}{*}{2.00} & 1 & 2 & -.097 & .072 & .188 & -.242 & .049 \\
\hline & & 3 & $-.120^{*}$ & .056 & .037 & -.233 & -.007 \\
\hline & 2 & 1 & .097 & .072 & .188 & -.049 & .242 \\
\hline & & 3 & -.024 & .099 & .810 & -.223 & .175 \\
\hline & 3 & 1 & $.120^{*}$ & .056 & .037 & .007 & .233 \\
\hline & & 2 & .024 & .099 & .810 & -.175 & .223 \\
\hline
\end{tabular}

Based on estimated marginal means

a. Adjustment for multiple comparisons: Least Significant Difference (equivalent to no adjustments).

*. The mean difference is significant at the .05 level. 


\section{Table J10}

Mean Difference, Standard Error, P-Value and 95\% Confidence Interval for Group Effects by Incentive Condition

\begin{tabular}{|c|c|c|c|c|c|c|c|c|}
\hline & & & & Mean Difference & & & $95 \%$ Confidence Inte & al for Difference ${ }^{\mathrm{a}}$ \\
\hline Brain region & Incentive cond & (I) Group & (J) Group & $(\mathrm{I}-\mathrm{J})$ & Std. Error & Sig. ${ }^{a}$ & Lower Bound & Upper Bound \\
\hline \multirow[t]{6}{*}{ Brain region 1} & 1 & 1.00 & 2.00 & .068 & .050 & .187 & -.034 & .169 \\
\hline & & 2.00 & 1.00 & -.068 & .050 & 187 & -.169 & .034 \\
\hline & 2 & 1.00 & 2.00 & .027 & .077 & .733 & -.129 & .182 \\
\hline & & 2.00 & 1.00 & -.027 & .077 & .733 & -.182 & .129 \\
\hline & 3 & 1.00 & 2.00 & .028 & .062 & .655 & -.097 & .153 \\
\hline & & 2.00 & 1.00 & -.028 & .062 & .655 & -.153 & .097 \\
\hline \multirow[t]{6}{*}{ Brain region 2} & 1 & 1.00 & 2.00 & .017 & .053 & .757 & -.091 & .124 \\
\hline & & 2.00 & 1.00 & -.017 & .053 & .757 & -.124 & .091 \\
\hline & 2 & 1.00 & 2.00 & -.032 & .075 & .666 & -.182 & .118 \\
\hline & & 2.00 & 1.00 & .032 & .075 & .666 & -.118 & .182 \\
\hline & 3 & 1.00 & 2.00 & -.020 & .055 & .723 & -.131 & .092 \\
\hline & & 2.00 & 1.00 & .020 & .055 & .723 & -.092 & .131 \\
\hline \multirow[t]{6}{*}{ Brain region 3} & 1 & 1.00 & 2.00 & .044 & .023 & .059 & -.002 & .091 \\
\hline & & 2.00 & 1.00 & -.044 & .023 & .059 & -.091 & .002 \\
\hline & 2 & 1.00 & 2.00 & .020 & .032 & .546 & -.045 & .085 \\
\hline & & 2.00 & 1.00 & -.020 & .032 & .546 & -.085 & .045 \\
\hline & 3 & 1.00 & 2.00 & .031 & .027 & .259 & -.024 & .087 \\
\hline & & 2.00 & 1.00 & -.031 & .027 & .259 & -.087 & .024 \\
\hline \multirow[t]{3}{*}{ Brain region 4} & 1 & 1.00 & 2.00 & .042 & .024 & .085 & -.006 & .091 \\
\hline & & 2.00 & 1.00 & -.042 & .024 & .085 & -.091 & .006 \\
\hline & 2 & 1.00 & 2.00 & .010 & .031 & .744 & -.052 & .072 \\
\hline
\end{tabular}




\begin{tabular}{|c|c|c|c|c|c|c|c|c|}
\hline & & 2.00 & 1.00 & -.010 & .031 & .744 & -.072 & .052 \\
\hline & \multirow[t]{2}{*}{3} & 1.00 & 2.00 & .051 & .027 & .062 & -.003 & .105 \\
\hline & & 2.00 & 1.00 & -.051 & .027 & .062 & -.105 & .003 \\
\hline \multirow[t]{6}{*}{ Brain region 5} & \multirow[t]{2}{*}{1} & 1.00 & 2.00 & .033 & .035 & .355 & -.038 & .103 \\
\hline & & 2.00 & 1.00 & -.033 & .035 & .355 & -.103 & .038 \\
\hline & \multirow[t]{2}{*}{2} & 1.00 & 2.00 & .052 & .065 & .429 & -.079 & .183 \\
\hline & & 2.00 & 1.00 & -.052 & .065 & .429 & -.183 & .079 \\
\hline & \multirow[t]{2}{*}{3} & 1.00 & 2.00 & .036 & .040 & .371 & -.044 & .116 \\
\hline & & 2.00 & 1.00 & -.036 & .040 & .371 & -.116 & .044 \\
\hline \multirow[t]{6}{*}{ Brain region 6} & \multirow[t]{2}{*}{1} & 1.00 & 2.00 & .098 & .052 & .066 & -.007 & .202 \\
\hline & & 2.00 & 1.00 & -.098 & .052 & .066 & -.202 & .007 \\
\hline & \multirow[t]{2}{*}{2} & 1.00 & 2.00 & .017 & .057 & .763 & -.098 & .133 \\
\hline & & 2.00 & 1.00 & -.017 & .057 & .763 & -.133 & .098 \\
\hline & \multirow[t]{2}{*}{3} & 1.00 & 2.00 & .024 & .070 & .730 & -.117 & .165 \\
\hline & & 2.00 & 1.00 & -.024 & .070 & .730 & -.165 & .117 \\
\hline \multirow[t]{6}{*}{ Brain region 7} & \multirow[t]{2}{*}{1} & 1.00 & 2.00 & $.133^{*}$ & .057 & .023 & .019 & .246 \\
\hline & & 2.00 & 1.00 & $-.133^{*}$ & .057 & .023 & -.246 & -.019 \\
\hline & \multirow[t]{2}{*}{2} & 1.00 & 2.00 & .028 & .075 & .713 & -.123 & .178 \\
\hline & & 2.00 & 1.00 & -.028 & .075 & .713 & -.178 & .123 \\
\hline & \multirow[t]{2}{*}{3} & 1.00 & 2.00 & .012 & .080 & .880 & -.149 & .173 \\
\hline & & 2.00 & 1.00 & -.012 & .080 & .880 & -.173 & .149 \\
\hline \multirow[t]{5}{*}{ Brain region 8} & \multirow[t]{2}{*}{1} & 1.00 & 2.00 & $\begin{array}{r}-.004 \\
\end{array}$ & .043 & .930 & -.090 & .082 \\
\hline & & 2.00 & 1.00 & .004 & .043 & .930 & -.082 & .090 \\
\hline & \multirow[t]{2}{*}{2} & 1.00 & 2.00 & -.028 & .056 & .619 & -.142 & .085 \\
\hline & & 2.00 & 1.00 & .028 & .056 & .619 & -.085 & .142 \\
\hline & 3 & 1.00 & 2.00 & -.003 & .053 & .957 & -.110 & .104 \\
\hline
\end{tabular}




\begin{tabular}{|c|c|c|c|c|c|c|c|c|}
\hline & & 2.00 & 1.00 & .003 & .053 & .957 & -.104 & .110 \\
\hline \multirow[t]{6}{*}{ Brain region 9} & 1 & 1.00 & 2.00 & .025 & .030 & .411 & -.035 & .084 \\
\hline & & 2.00 & 1.00 & -.025 & .030 & .411 & -.084 & .035 \\
\hline & 2 & 1.00 & 2.00 & -.009 & .034 & .797 & -.078 & .060 \\
\hline & & 2.00 & 1.00 & .009 & .034 & .797 & -.060 & .078 \\
\hline & 3 & 1.00 & 2.00 & .005 & .044 & .909 & -.083 & .093 \\
\hline & & 2.00 & 1.00 & -.005 & .044 & .909 & -.093 & .083 \\
\hline \multirow[t]{6}{*}{ Brain region 10} & 1 & 1.00 & 2.00 & .052 & .049 & .296 & -.047 & .151 \\
\hline & & 2.00 & 1.00 & -.052 & .049 & .296 & -.151 & .047 \\
\hline & 2 & 1.00 & 2.00 & -.019 & .050 & .703 & -.120 & .082 \\
\hline & & 2.00 & 1.00 & .019 & .050 & .703 & -.082 & .120 \\
\hline & 3 & 1.00 & 2.00 & .000 & .064 & .994 & -.129 & .128 \\
\hline & & 2.00 & 1.00 & .000 & .064 & .994 & -.128 & .129 \\
\hline \multirow[t]{6}{*}{ Brain region 11} & 1 & 1.00 & 2.00 & .013 & .035 & .722 & -.058 & .083 \\
\hline & & 2.00 & 1.00 & -.013 & .035 & .722 & -.083 & .058 \\
\hline & 2 & 1.00 & 2.00 & -.014 & .047 & .765 & -.110 & .081 \\
\hline & & 2.00 & 1.00 & .014 & .047 & .765 & -.081 & .110 \\
\hline & 3 & 1.00 & 2.00 & -.001 & .038 & .988 & -.076 & .075 \\
\hline & & 2.00 & 1.00 & .001 & .038 & .988 & -.075 & .076 \\
\hline \multirow[t]{6}{*}{ Brain region 12} & 1 & 1.00 & 2.00 & .080 & .059 & .182 & -.039 & .200 \\
\hline & & 2.00 & 1.00 & -.080 & .059 & .182 & -.200 & .039 \\
\hline & 2 & 1.00 & 2.00 & .012 & .073 & .873 & -.135 & .159 \\
\hline & & 2.00 & 1.00 & -.012 & .073 & .873 & -.159 & .135 \\
\hline & 3 & 1.00 & 2.00 & .085 & .063 & .185 & -.042 & .211 \\
\hline & & 2.00 & 1.00 & -.085 & .063 & .185 & -.211 & .042 \\
\hline Brain region 13 & 1 & 1.00 & 2.00 & .012 & .048 & .807 & -.085 & .109 \\
\hline
\end{tabular}




\begin{tabular}{|c|c|c|c|c|c|c|c|c|}
\hline & & 2.00 & 1.00 & -.012 & .048 & .807 & -.109 & .085 \\
\hline & \multirow[t]{2}{*}{2} & 1.00 & 2.00 & -.063 & .083 & .449 & -.230 & .103 \\
\hline & & 2.00 & 1.00 & .063 & .083 & .449 & -.103 & .230 \\
\hline & \multirow[t]{2}{*}{3} & 1.00 & 2.00 & .027 & .051 & .598 & -.075 & .129 \\
\hline & & 2.00 & 1.00 & -.027 & .051 & .598 & -.129 & .075 \\
\hline \multirow[t]{6}{*}{ Brain region 14} & \multirow[t]{2}{*}{1} & 1.00 & 2.00 & .082 & .059 & .175 & -.038 & .201 \\
\hline & & 2.00 & 1.00 & -.082 & .059 & .175 & -.201 & .038 \\
\hline & \multirow[t]{2}{*}{2} & 1.00 & 2.00 & -.047 & .057 & .417 & -.162 & .068 \\
\hline & & 2.00 & 1.00 & .047 & .057 & .417 & -.068 & .162 \\
\hline & \multirow[t]{2}{*}{3} & 1.00 & 2.00 & -.018 & .067 & .793 & -.153 & .118 \\
\hline & & 2.00 & 1.00 & .018 & .067 & .793 & -.118 & .153 \\
\hline \multirow[t]{6}{*}{ Brain region 15} & \multirow[t]{2}{*}{1} & 1.00 & 2.00 & .014 & .045 & .763 & -.076 & .103 \\
\hline & & 2.00 & 1.00 & -.014 & .045 & .763 & -.103 & .076 \\
\hline & \multirow[t]{2}{*}{2} & 1.00 & 2.00 & .042 & .074 & .577 & -.107 & .190 \\
\hline & & 2.00 & 1.00 & -.042 & .074 & .577 & -.190 & .107 \\
\hline & \multirow[t]{2}{*}{3} & 1.00 & 2.00 & .004 & .053 & .943 & -.104 & .111 \\
\hline & & 2.00 & 1.00 & -.004 & .053 & .943 & -.111 & .104 \\
\hline \multirow[t]{6}{*}{ Brain region 16} & \multirow[t]{2}{*}{1} & 1.00 & 2.00 & .315 & .164 & .062 & -.016 & .646 \\
\hline & & 2.00 & 1.00 & -.315 & .164 & .062 & -.646 & .016 \\
\hline & \multirow[t]{2}{*}{2} & 1.00 & 2.00 & .202 & .217 & .358 & -.236 & .640 \\
\hline & & 2.00 & 1.00 & -.202 & .217 & .358 & -.640 & .236 \\
\hline & \multirow[t]{2}{*}{3} & 1.00 & 2.00 & .167 & .210 & .429 & -.255 & .589 \\
\hline & & 2.00 & 1.00 & -.167 & .210 & .429 & -.589 & .255 \\
\hline \multirow[t]{3}{*}{ Brain region 17} & \multirow[t]{2}{*}{1} & 1.00 & 2.00 & .044 & .046 & .334 & -.047 & .136 \\
\hline & & 2.00 & 1.00 & -.044 & .046 & .334 & -.136 & .047 \\
\hline & 2 & 1.00 & 2.00 & -.006 & .070 & .933 & -.147 & .135 \\
\hline
\end{tabular}




\begin{tabular}{|c|c|c|c|c|c|c|c|c|}
\hline & & 2.00 & 1.00 & .006 & .070 & .933 & -.135 & .147 \\
\hline & \multirow[t]{2}{*}{3} & 1.00 & 2.00 & .017 & .055 & .762 & -.094 & .128 \\
\hline & & 2.00 & 1.00 & -.017 & .055 & .762 & -.128 & .094 \\
\hline \multirow[t]{6}{*}{ Brain region 18} & 1 & 1.00 & 2.00 & .085 & .052 & .108 & -.019 & .190 \\
\hline & & 2.00 & 1.00 & -.085 & .052 & .108 & -.190 & .019 \\
\hline & 2 & 1.00 & 2.00 & .051 & .071 & .477 & -.092 & .194 \\
\hline & & 2.00 & 1.00 & -.051 & .071 & .477 & -.194 & .092 \\
\hline & 3 & 1.00 & 2.00 & .065 & .051 & .209 & -.038 & .168 \\
\hline & & 2.00 & 1.00 & -.065 & .051 & .209 & -.168 & .038 \\
\hline \multirow[t]{6}{*}{ Brain region 19} & 1 & 1.00 & 2.00 & .085 & .048 & .084 & -.012 & .181 \\
\hline & & 2.00 & 1.00 & -.085 & .048 & .084 & -.181 & .012 \\
\hline & 2 & 1.00 & 2.00 & .091 & .083 & .278 & -.075 & .257 \\
\hline & & 2.00 & 1.00 & -.091 & .083 & .278 & -.257 & .075 \\
\hline & 3 & 1.00 & 2.00 & .078 & .063 & .223 & -.049 & .204 \\
\hline & & 2.00 & 1.00 & -.078 & .063 & .223 & -.204 & .049 \\
\hline \multirow[t]{6}{*}{ Brain region 20} & 1 & 1.00 & 2.00 & .026 & .070 & .706 & -.114 & .166 \\
\hline & & 2.00 & 1.00 & -.026 & .070 & .706 & -.166 & .114 \\
\hline & 2 & 1.00 & 2.00 & -.018 & .081 & .828 & -.181 & .145 \\
\hline & & 2.00 & 1.00 & .018 & .081 & .828 & -.145 & .181 \\
\hline & 3 & 1.00 & 2.00 & -.091 & .084 & .287 & -.260 & .079 \\
\hline & & 2.00 & 1.00 & .091 & .084 & .287 & -.079 & .260 \\
\hline \multirow[t]{5}{*}{ Brain region 21} & 1 & 1.00 & 2.00 & $.074^{*}$ & .034 & .034 & .006 & .142 \\
\hline & & 2.00 & 1.00 & $-.074^{*}$ & .034 & .034 & -.142 & -.006 \\
\hline & 2 & 1.00 & 2.00 & .016 & .062 & .796 & -.108 & .140 \\
\hline & & 2.00 & 1.00 & -.016 & .062 & .796 & -.140 & .108 \\
\hline & 3 & 1.00 & 2.00 & .024 & .046 & .599 & -.068 & .116 \\
\hline
\end{tabular}




\begin{tabular}{llccccccc}
\hline & 2.00 & 1.00 & -.024 & .046 & .599 & -.116 & .068 \\
\hline \multirow{2}{*}{ Brain region 22 } & 1 & 1.00 & 2.00 & .106 & .088 & .234 & -.071 & .282 \\
\cline { 2 - 8 } & 2.00 & 1.00 & -.106 & .088 & .234 & -.282 & .071 \\
\cline { 2 - 8 } & 2 & 1.00 & 2.00 & .117 & .133 & .385 & -.151 & .385 \\
\cline { 2 - 8 } & 2.00 & 1.00 & -.117 & .133 & .385 & -.385 & .151 \\
\cline { 2 - 8 } & \multirow{2}{*}{3} & 1.00 & 2.00 & .129 & .092 & .169 & -.057 & .315 \\
\cline { 2 - 8 } & 2.00 & 1.00 & -.129 & .092 & .169 & -.315 & .057 \\
\hline
\end{tabular}

Based on estimated marginal means

a. Adjustment for multiple comparisons: Least Significant Difference (equivalent to no adjustments)

*. The mean difference is significant at the .05 level. 
Table J11

Means, Standard Errors and 95\% Confidence Intervals for Group x Accuracy Effects

\begin{tabular}{|c|c|c|c|c|c|c|}
\hline \multirow[b]{2}{*}{ Brain region } & \multirow[b]{2}{*}{ Group } & \multirow[b]{2}{*}{ Accuracy } & \multirow[b]{2}{*}{ Mean } & \multirow[b]{2}{*}{ Std. Error } & \multicolumn{2}{|c|}{ 95\% Confidence Interval } \\
\hline & & & & & Lower Bound & Upper Bound \\
\hline \multirow[t]{4}{*}{ Brain region 1} & 1.00 & 1 & .266 & .036 & .194 & .337 \\
\hline & & 2 & .218 & .053 & .112 & .324 \\
\hline & 2.00 & 1 & .202 & .036 & .130 & .274 \\
\hline & & 2 & .200 & .053 & .094 & .306 \\
\hline \multirow[t]{4}{*}{ Brain region 2} & 1.00 & 1 & .227 & .033 & .162 & .293 \\
\hline & & 2 & .153 & .050 & .051 & .254 \\
\hline & 2.00 & 1 & .197 & .033 & .131 & .263 \\
\hline & & 2 & .206 & .050 & .105 & .308 \\
\hline \multirow[t]{4}{*}{ Brain region 3} & 1.00 & 1 & .094 & .015 & .065 & .124 \\
\hline & & 2 & .032 & .023 & -.014 & .078 \\
\hline & 2.00 & 1 & .047 & .015 & .017 & .076 \\
\hline & & 2 & .016 & .023 & -.030 & .062 \\
\hline \multirow[t]{4}{*}{ Brain region 4} & 1.00 & 1 & .107 & .015 & .076 & .138 \\
\hline & & 2 & .018 & .022 & -.026 & .062 \\
\hline & 2.00 & 1 & .052 & .015 & .021 & .083 \\
\hline & & 2 & .003 & .022 & -.041 & .048 \\
\hline \multirow[t]{4}{*}{ Brain region 5} & 1.00 & 1 & -.087 & .027 & -.141 & -.033 \\
\hline & & 2 & -.169 & .039 & -.247 & -.091 \\
\hline & 2.00 & 1 & -.132 & .027 & -.186 & -.077 \\
\hline & & 2 & -.204 & .039 & -.282 & -.126 \\
\hline Brain region 6 & 1.00 & 1 & .206 & .041 & .124 & .289 \\
\hline
\end{tabular}




\begin{tabular}{|c|c|c|c|c|c|c|}
\hline & & 2 & .243 & .044 & .154 & .332 \\
\hline & 2.00 & 1 & .160 & .041 & .078 & .243 \\
\hline & & 2 & .196 & .044 & .107 & .285 \\
\hline \multirow[t]{4}{*}{ Brain region 7} & 1.00 & 1 & .314 & .044 & .225 & .403 \\
\hline & & 2 & .283 & .053 & .178 & .389 \\
\hline & 2.00 & 1 & .239 & .044 & .150 & .329 \\
\hline & & 2 & .243 & .053 & .137 & .349 \\
\hline \multirow[t]{4}{*}{ Brain region 8} & 1.00 & 1 & .140 & .037 & .066 & .214 \\
\hline & & 2 & .059 & .037 & -.015 & .133 \\
\hline & 2.00 & 1 & .130 & .037 & .056 & .203 \\
\hline & & 2 & .093 & .037 & .019 & .167 \\
\hline \multirow[t]{4}{*}{ Brain region 9} & 1.00 & 1 & .120 & .024 & .073 & .168 \\
\hline & & 2 & .094 & .025 & .044 & .145 \\
\hline & 2.00 & 1 & .115 & .024 & .068 & .162 \\
\hline & & 2 & .086 & .025 & .035 & .136 \\
\hline \multirow[t]{4}{*}{ Brain region 10} & 1.00 & 1 & .142 & .032 & .077 & .206 \\
\hline & & 2 & .182 & .043 & .096 & .268 \\
\hline & 2.00 & 1 & .119 & .032 & .054 & .183 \\
\hline & & 2 & .184 & .043 & .097 & .270 \\
\hline \multirow[t]{4}{*}{ Brain region 11} & 1.00 & 1 & .137 & .020 & .098 & .177 \\
\hline & & 2 & .076 & .035 & .007 & .146 \\
\hline & 2.00 & 1 & .082 & .020 & .042 & .122 \\
\hline & & 2 & .133 & .035 & .063 & .203 \\
\hline \multirow[t]{3}{*}{ Brain region 12} & 1.00 & 1 & .259 & .038 & .182 & .336 \\
\hline & & 2 & .128 & .054 & .020 & .236 \\
\hline & 2.00 & 1 & .128 & .038 & .051 & .205 \\
\hline
\end{tabular}




\begin{tabular}{|c|c|c|c|c|c|c|}
\hline & & 2 & .141 & .054 & .033 & .249 \\
\hline \multirow[t]{4}{*}{ Brain region 13} & 1.00 & 1 & -.091 & .037 & -.165 & -.018 \\
\hline & & 2 & -.147 & .047 & -.241 & -.053 \\
\hline & 2.00 & 1 & -.116 & .037 & -.190 & -.043 \\
\hline & & 2 & -.106 & .047 & -.199 & -.012 \\
\hline \multirow[t]{4}{*}{ Brain region 14} & 1.00 & 1 & .240 & .039 & .162 & .318 \\
\hline & & 2 & .194 & .046 & .101 & .287 \\
\hline & 2.00 & 1 & .197 & .039 & .119 & .276 \\
\hline & & 2 & .225 & .046 & .132 & .318 \\
\hline \multirow[t]{4}{*}{ Brain region 15} & 1.00 & 1 & -.076 & .032 & -.141 & -.012 \\
\hline & & 2 & -.104 & .046 & -.197 & -.011 \\
\hline & 2.00 & 1 & -.128 & .032 & -.192 & -.063 \\
\hline & & 2 & -.092 & .046 & -.185 & .001 \\
\hline \multirow[t]{4}{*}{ Brain region 16} & 1.00 & 1 & .517 & .138 & .239 & .795 \\
\hline & & 2 & .207 & .150 & -.095 & .509 \\
\hline & 2.00 & 1 & .222 & .138 & -.056 & .500 \\
\hline & & 2 & .045 & .150 & -.257 & .347 \\
\hline \multirow[t]{4}{*}{ Brain region 17} & 1.00 & 1 & .096 & .035 & .025 & .167 \\
\hline & & 2 & .020 & .045 & -.070 & .111 \\
\hline & 2.00 & 1 & .049 & .035 & -.022 & .120 \\
\hline & & 2 & .030 & .045 & -.061 & .121 \\
\hline \multirow[t]{4}{*}{ Brain region 18} & 1.00 & 1 & .098 & .024 & .049 & .147 \\
\hline & & 2 & .127 & .050 & .026 & .229 \\
\hline & 2.00 & 1 & .058 & .024 & .009 & .106 \\
\hline & & 2 & .034 & .050 & -.068 & .135 \\
\hline Brain region 19 & 1.00 & 1 & .131 & .036 & .059 & .203 \\
\hline
\end{tabular}




\begin{tabular}{|c|c|c|c|c|c|c|}
\hline & & 2 & .156 & .051 & .053 & .260 \\
\hline & \multirow[t]{2}{*}{2.00} & 1 & .077 & .036 & .005 & 149 \\
\hline & & 2 & .042 & .051 & -.062 & .145 \\
\hline \multirow[t]{4}{*}{ Brain region 20} & 1.00 & 1 & .250 & .049 & .151 & .349 \\
\hline & & 2 & .218 & .066 & .085 & .351 \\
\hline & 2.00 & 1 & .254 & . 049 & .155 & .353 \\
\hline & & 2 & .269 & .066 & .136 & .402 \\
\hline \multirow[t]{4}{*}{ Brain region 21} & 1.00 & 1 & -.003 & .026 & -.054 & .049 \\
\hline & & 2 & -.084 & .042 & -.169 & .001 \\
\hline & 2.00 & 1 & -.023 & .026 & -.074 & .029 \\
\hline & & 2 & -.140 & .042 & -.225 & -.055 \\
\hline \multirow[t]{4}{*}{ Brain region 22} & 1.00 & 1 & .227 & .055 & .117 & .338 \\
\hline & & 2 & .284 & . 088 & 107 & .462 \\
\hline & 2.00 & 1 & .112 & .055 & .001 & .222 \\
\hline & & 2 & .165 & .088 & -.012 & .343 \\
\hline
\end{tabular}




\section{Table J12}

Mean Difference, Standard Error, P-Value and 95\% Confidence Interval for Accuracy Effects by Group

\begin{tabular}{|c|c|c|c|c|c|c|c|c|}
\hline & & & & Mean Difference & & & 95\% Confidence Inte & al for Difference ${ }^{a}$ \\
\hline Brain region & Group & (I) Accuracy & (J) Accuracy & $(\mathrm{I}-\mathrm{J})$ & Std. Error & Sig. ${ }^{a}$ & Lower Bound & Upper Bound \\
\hline \multirow[t]{4}{*}{ Brain region 1} & 1.00 & 1 & 2 & .048 & .043 & .275 & -.039 & .134 \\
\hline & & 2 & 1 & -.048 & .043 & .275 & -.134 & .039 \\
\hline & 2.00 & 1 & 2 & .003 & .043 & .953 & -.084 & .089 \\
\hline & & 2 & 1 & -.003 & .043 & .953 & -.089 & .084 \\
\hline \multirow[t]{4}{*}{ Brain region 2} & 1.00 & 1 & 2 & .075 & .041 & .073 & -.007 & .157 \\
\hline & & 2 & 1 & -.075 & .041 & .073 & -.157 & .007 \\
\hline & 2.00 & 1 & 2 & -.010 & .041 & .816 & -.091 & .072 \\
\hline & & 2 & 1 & .010 & . 041 & .816 & -.072 & .091 \\
\hline \multirow[t]{4}{*}{ Brain region 3} & 1.00 & 1 & 2 & $.062^{*}$ & .020 & .004 & .021 & .103 \\
\hline & & 2 & 1 & $-.062^{*}$ & .020 & .004 & -.103 & -.021 \\
\hline & 2.00 & 1 & 2 & .031 & .020 & .137 & -.010 & .072 \\
\hline & & 2 & 1 & -.031 & .020 & .137 & -.072 & .010 \\
\hline \multirow[t]{4}{*}{ Brain region 4} & 1.00 & 1 & 2 & $.089^{*}$ & .021 & .000 & .048 & .131 \\
\hline & & 2 & 1 & $-.089^{*}$ & .021 & .000 & -.131 & -.048 \\
\hline & 2.00 & 1 & 2 & $.049^{*}$ & .021 & .021 & .008 & .091 \\
\hline & & 2 & 1 & $-.049^{*}$ & .021 & .021 & -.091 & -.008 \\
\hline \multirow[t]{4}{*}{ Brain region 5} & 1.00 & 1 & 2 & $.082^{*}$ & .040 & .045 & .002 & .162 \\
\hline & & 2 & 1 & $-.082^{*}$ & .040 & .045 & -.162 & -.002 \\
\hline & 2.00 & 1 & 2 & .073 & .040 & .074 & -.007 & .152 \\
\hline & & 2 & 1 & -.073 & .040 & .074 & -.152 & .007 \\
\hline Brain region 6 & 1.00 & 1 & 2 & -.037 & .042 & .384 & -.120 & .047 \\
\hline
\end{tabular}




\begin{tabular}{|c|c|c|c|c|c|c|c|c|}
\hline & & 2 & 1 & .037 & .042 & .384 & -.047 & .120 \\
\hline & 2.00 & 1 & 2 & -.036 & .042 & .395 & -.119 & .048 \\
\hline & & 2 & 1 & .036 & .042 & .395 & -.048 & .119 \\
\hline \multirow[t]{4}{*}{ Brain region 7} & 1.00 & 1 & 2 & .031 & .045 & .494 & -.059 & .120 \\
\hline & & 2 & 1 & -.031 & .045 & .494 & -.120 & .059 \\
\hline & 2.00 & 1 & 2 & -.004 & .045 & .929 & -.094 & .086 \\
\hline & & 2 & 1 & .004 & .045 & .929 & -.086 & .094 \\
\hline \multirow[t]{4}{*}{ Brain region 8} & 1.00 & 1 & 2 & $.080^{*}$ & .036 & .032 & .007 & .154 \\
\hline & & 2 & 1 & $-.080^{*}$ & .036 & .032 & -.154 & -.007 \\
\hline & 2.00 & 1 & 2 & .037 & .036 & .318 & -.037 & .110 \\
\hline & & 2 & 1 & -.037 & .036 & .318 & -.110 & .037 \\
\hline \multirow[t]{4}{*}{ Brain region 9} & 1.00 & 1 & 2 & .026 & .020 & .189 & -.013 & .066 \\
\hline & & 2 & 1 & -.026 & .020 & .189 & -.066 & .013 \\
\hline & 2.00 & 1 & 2 & .029 & .020 & .143 & -.010 & .069 \\
\hline & & 2 & 1 & -.029 & .020 & .143 & -.069 & .010 \\
\hline \multirow[t]{4}{*}{ Brain region 10} & 1.00 & 1 & 2 & -.040 & .039 & .308 & -.119 & .038 \\
\hline & & 2 & 1 & .040 & .039 & .308 & -.038 & .119 \\
\hline & 2.00 & 1 & 2 & -.065 & .039 & .101 & -.144 & .013 \\
\hline & & 2 & 1 & .065 & .039 & .101 & -.013 & .144 \\
\hline \multirow[t]{4}{*}{ Brain region 11} & 1.00 & 1 & 2 & $.061^{*}$ & .029 & .041 & .003 & .120 \\
\hline & & 2 & 1 & $-.061^{*}$ & .029 & .041 & -.120 & -.003 \\
\hline & 2.00 & 1 & 2 & -.051 & .029 & .086 & -.110 & .008 \\
\hline & & 2 & 1 & .051 & .029 & .086 & -.008 & .110 \\
\hline \multirow[t]{3}{*}{ Brain region 12} & 1.00 & 1 & 2 & $.131^{*}$ & .046 & .007 & .038 & .223 \\
\hline & & 2 & 1 & $-.131^{*}$ & .046 & .007 & -.223 & -.038 \\
\hline & 2.00 & 1 & 2 & -.013 & .046 & .775 & -.106 & .079 \\
\hline
\end{tabular}




\begin{tabular}{|c|c|c|c|c|c|c|c|c|}
\hline & & 2 & 1 & .013 & .046 & .775 & -.079 & .106 \\
\hline \multirow[t]{4}{*}{ Brain region 13} & 1.00 & 1 & 2 & .056 & .046 & .232 & -.037 & .148 \\
\hline & & 2 & 1 & -.056 & .046 & .232 & -.148 & .037 \\
\hline & 2.00 & 1 & 2 & -.011 & .046 & .817 & -.103 & .082 \\
\hline & & 2 & 1 & .011 & .046 & .817 & -.082 & .103 \\
\hline \multirow[t]{4}{*}{ Brain region 14} & 1.00 & 1 & 2 & .045 & .037 & .224 & -.029 & .119 \\
\hline & & 2 & 1 & -.045 & .037 & .224 & -.119 & .029 \\
\hline & 2.00 & 1 & 2 & -.028 & .037 & .455 & -.102 & .046 \\
\hline & & 2 & 1 & .028 & .037 & .455 & -.046 & .102 \\
\hline \multirow[t]{4}{*}{ Brain region 15} & 1.00 & 1 & 2 & .027 & .047 & .566 & -.068 & .122 \\
\hline & & 2 & 1 & -.027 & .047 & .566 & -.122 & .068 \\
\hline & 2.00 & 1 & 2 & -.036 & .047 & .453 & -.131 & .059 \\
\hline & & 2 & 1 & .036 & .047 & .453 & -.059 & .131 \\
\hline \multirow[t]{4}{*}{ Brain region 16} & 1.00 & 1 & 2 & $.310^{*}$ & .146 & .039 & .017 & .604 \\
\hline & & 2 & 1 & $-.310^{*}$ & .146 & .039 & -.604 & -.017 \\
\hline & 2.00 & 1 & 2 & .177 & .146 & .230 & -.116 & .471 \\
\hline & & 2 & 1 & -.177 & .146 & .230 & -.471 & .116 \\
\hline \multirow[t]{4}{*}{ Brain region 17} & 1.00 & 1 & 2 & .076 & .045 & .097 & -.014 & .166 \\
\hline & & 2 & 1 & -.076 & .045 & .097 & -.166 & .014 \\
\hline & 2.00 & 1 & 2 & .019 & .045 & .671 & -.071 & .109 \\
\hline & & 2 & 1 & -.019 & .045 & .671 & -.109 & .071 \\
\hline \multirow[t]{4}{*}{ Brain region 18} & 1.00 & 1 & 2 & -.029 & .048 & .541 & -.125 & .067 \\
\hline & & 2 & 1 & .029 & .048 & .541 & -.067 & .125 \\
\hline & 2.00 & 1 & 2 & .024 & .048 & .614 & -.072 & .120 \\
\hline & & 2 & 1 & -.024 & .048 & .614 & -.120 & .072 \\
\hline Brain region 19 & 1.00 & 1 & 2 & -.025 & .045 & .573 & -.115 & .064 \\
\hline
\end{tabular}




\begin{tabular}{|c|c|c|c|c|c|c|c|c|}
\hline & & 2 & 1 & .025 & .045 & .573 & -.064 & .115 \\
\hline & 2.00 & 1 & 2 & .035 & .045 & .436 & -.055 & .125 \\
\hline & & 2 & 1 & -.035 & .045 & .436 & -.125 & .055 \\
\hline \multirow[t]{4}{*}{ Brain region 20} & 1.00 & 1 & 2 & .032 & .056 & .567 & -.081 & .146 \\
\hline & & 2 & 1 & -.032 & .056 & .567 & -.146 & .081 \\
\hline & 2.00 & 1 & 2 & -.015 & .056 & .786 & -.128 & .098 \\
\hline & & 2 & 1 & .015 & .056 & .786 & -.098 & .128 \\
\hline \multirow[t]{4}{*}{ Brain region 21} & 1.00 & 1 & 2 & .081 & .045 & .081 & -.010 & .173 \\
\hline & & 2 & 1 & -.081 & .045 & .081 & -.173 & .010 \\
\hline & 2.00 & 1 & 2 & $.117^{*}$ & .045 & .013 & .026 & .209 \\
\hline & & 2 & 1 & $-.117^{*}$ & .045 & .013 & -.209 & -.026 \\
\hline \multirow[t]{4}{*}{ Brain region 22} & 1.00 & 1 & 2 & -.057 & .084 & .497 & -.225 & .111 \\
\hline & & 2 & 1 & .057 & .084 & .497 & -.111 & .225 \\
\hline & 2.00 & 1 & 2 & -.054 & .084 & .523 & -.222 & .114 \\
\hline & & 2 & 1 & .054 & .084 & .523 & -.114 & .222 \\
\hline
\end{tabular}

Based on estimated marginal means

a. Adjustment for multiple comparisons: Least Significant Difference (equivalent to no adjustments).

*. The mean difference is significant at the .05 level. 


\section{Table J13}

Mean Difference, Standard Error, P-Value and 95\% Confidence Interval for Group Effects by Accuracy

\begin{tabular}{|c|c|c|c|c|c|c|c|c|}
\hline & & & & Mean Difference & & & 95\% Confidence Inte & al for Difference ${ }^{a}$ \\
\hline Brain region & Accuracy & (I) Group & (J) Group & $(\mathrm{I}-\mathrm{J})$ & Std. Error & Sig. ${ }^{\text {a }}$ & Lower Bound & Upper Bound \\
\hline \multirow[t]{4}{*}{ Brain region 1} & 1 & 1.00 & 2.00 & .063 & .051 & .217 & -.038 & .165 \\
\hline & & 2.00 & 1.00 & -.063 & .051 & .217 & -.165 & .038 \\
\hline & 2 & 1.00 & 2.00 & .018 & .075 & .809 & -.132 & .168 \\
\hline & & 2.00 & 1.00 & -.018 & .075 & .809 & -.168 & .132 \\
\hline \multirow[t]{4}{*}{ Brain region 2} & 1 & 1.00 & 2.00 & .030 & .046 & .514 & -.062 & .123 \\
\hline & & 2.00 & 1.00 & -.030 & .046 & .514 & -.123 & .062 \\
\hline & 2 & 1.00 & 2.00 & -.054 & .071 & .452 & -.197 & .089 \\
\hline & & 2.00 & 1.00 & .054 & .071 & .452 & -.089 & .197 \\
\hline \multirow[t]{4}{*}{ Brain region 3} & 1 & 1.00 & 2.00 & $.047^{*}$ & .021 & .027 & .006 & .089 \\
\hline & & 2.00 & 1.00 & $-.047^{*}$ & .021 & .027 & -.089 & -.006 \\
\hline & 2 & 1.00 & 2.00 & .016 & .032 & .620 & -.049 & .081 \\
\hline & & 2.00 & 1.00 & -.016 & .032 & .620 & -.081 & .049 \\
\hline \multirow[t]{4}{*}{ Brain region 4} & 1 & 1.00 & 2.00 & $.055^{*}$ & .022 & .016 & .011 & .098 \\
\hline & & 2.00 & 1.00 & $-.055^{*}$ & .022 & .016 & -.098 & -.011 \\
\hline & 2 & 1.00 & 2.00 & .014 & .031 & .644 & -.048 & .077 \\
\hline & & 2.00 & 1.00 & -.014 & .031 & .644 & -.077 & .048 \\
\hline \multirow[t]{4}{*}{ Brain region 5} & 1 & 1.00 & 2.00 & .045 & .038 & .246 & -.032 & .121 \\
\hline & & 2.00 & 1.00 & -.045 & .038 & .246 & -.121 & .032 \\
\hline & 2 & 1.00 & 2.00 & .035 & .055 & .521 & -.075 & .146 \\
\hline & & 2.00 & 1.00 & -.035 & .055 & .521 & -.146 & .075 \\
\hline Brain region 6 & 1 & 1.00 & 2.00 & .046 & .058 & .430 & -.070 & .162 \\
\hline
\end{tabular}




\begin{tabular}{|c|c|c|c|c|c|c|c|c|}
\hline & & 2.00 & 1.00 & -.046 & .058 & .430 & -.162 & .070 \\
\hline & 2 & 1.00 & 2.00 & .047 & .062 & .457 & -.079 & .173 \\
\hline & & 2.00 & 1.00 & -.047 & .062 & .457 & -.173 & .079 \\
\hline \multirow[t]{4}{*}{ Brain region 7} & 1 & 1.00 & 2.00 & .075 & .063 & .240 & -.052 & .201 \\
\hline & & 2.00 & 1.00 & -.075 & .063 & .240 & -.201 & .052 \\
\hline & 2 & 1.00 & 2.00 & .040 & .074 & .592 & -.110 & .190 \\
\hline & & 2.00 & 1.00 & -.040 & .074 & .592 & -.190 & .110 \\
\hline \multirow[t]{4}{*}{ Brain region 8} & 1 & 1.00 & 2.00 & .010 & .052 & .845 & -.094 & .115 \\
\hline & & 2.00 & 1.00 & -.010 & .052 & .845 & -.115 & .094 \\
\hline & 2 & 1.00 & 2.00 & -.033 & .052 & .524 & -.138 & .071 \\
\hline & & 2.00 & 1.00 & .033 & .052 & .524 & -.071 & .138 \\
\hline \multirow[t]{4}{*}{ Brain region 9} & 1 & 1.00 & 2.00 & .005 & .033 & .872 & -.062 & .072 \\
\hline & & 2.00 & 1.00 & -.005 & .033 & .872 & -.072 & .062 \\
\hline & 2 & 1.00 & 2.00 & .008 & .036 & .813 & -.063 & .080 \\
\hline & & 2.00 & 1.00 & -.008 & .036 & .813 & -.080 & .063 \\
\hline \multirow[t]{4}{*}{ Brain region 10} & 1 & 1.00 & 2.00 & .023 & .045 & .611 & -.068 & .115 \\
\hline & & 2.00 & 1.00 & -.023 & .045 & .611 & -.115 & .068 \\
\hline & 2 & 1.00 & 2.00 & -.002 & .061 & .976 & -.124 & .120 \\
\hline & & 2.00 & 1.00 & .002 & .061 & .976 & -.120 & .124 \\
\hline \multirow[t]{4}{*}{ Brain region 11} & 1 & 1.00 & 2.00 & .055 & .028 & .054 & -.001 & .112 \\
\hline & & 2.00 & 1.00 & -.055 & .028 & .054 & -.112 & .001 \\
\hline & 2 & 1.00 & 2.00 & -.057 & .049 & .251 & -.155 & .042 \\
\hline & & 2.00 & 1.00 & .057 & .049 & .251 & -.042 & .155 \\
\hline \multirow[t]{3}{*}{ Brain region 12} & 1 & 1.00 & 2.00 & $.131^{*}$ & .054 & .020 & .022 & .240 \\
\hline & & 2.00 & 1.00 & $-.131^{*}$ & .054 & .020 & -.240 & -.022 \\
\hline & 2 & 1.00 & 2.00 & -.013 & .076 & .865 & -.166 & .140 \\
\hline
\end{tabular}




\begin{tabular}{|c|c|c|c|c|c|c|c|c|}
\hline & & 2.00 & 1.00 & .013 & .076 & .865 & -.140 & .166 \\
\hline \multirow[t]{4}{*}{ Brain region 13} & 1 & 1.00 & 2.00 & .025 & .052 & .631 & -.079 & .129 \\
\hline & & 2.00 & 1.00 & -.025 & .052 & .631 & -.129 & .079 \\
\hline & 2 & 1.00 & 2.00 & -.041 & .066 & .534 & -.174 & .091 \\
\hline & & 2.00 & 1.00 & .041 & .066 & .534 & -.091 & .174 \\
\hline \multirow[t]{4}{*}{ Brain region 14} & 1 & 1.00 & 2.00 & .042 & .055 & .446 & -.068 & .153 \\
\hline & & 2.00 & 1.00 & -.042 & .055 & .446 & -.153 & .068 \\
\hline & 2 & 1.00 & 2.00 & -.031 & .065 & .639 & -.162 & .100 \\
\hline & & 2.00 & 1.00 & .031 & .065 & .639 & -.100 & .162 \\
\hline \multirow[t]{4}{*}{ Brain region 15} & 1 & 1.00 & 2.00 & .051 & .045 & .263 & -.040 & .142 \\
\hline & & 2.00 & 1.00 & -.051 & .045 & .263 & -.142 & .040 \\
\hline & 2 & 1.00 & 2.00 & -.012 & .065 & .856 & -.144 & .120 \\
\hline & & 2.00 & 1.00 & .012 & .065 & .856 & -.120 & .144 \\
\hline \multirow[t]{4}{*}{ Brain region 16} & 1 & 1.00 & 2.00 & .295 & .195 & .139 & -.099 & .688 \\
\hline & & 2.00 & 1.00 & -.295 & .195 & .139 & -.688 & .099 \\
\hline & 2 & 1.00 & 2.00 & .162 & .212 & .450 & -.265 & .589 \\
\hline & & 2.00 & 1.00 & -.162 & .212 & .450 & -.589 & .265 \\
\hline \multirow[t]{4}{*}{ Brain region 17} & 1 & 1.00 & 2.00 & .047 & .050 & .353 & -.054 & .147 \\
\hline & & 2.00 & 1.00 & -.047 & .050 & .353 & -.147 & .054 \\
\hline & 2 & 1.00 & 2.00 & -.010 & .064 & .877 & -.138 & .118 \\
\hline & & 2.00 & 1.00 & .010 & .064 & .877 & -.118 & .138 \\
\hline \multirow[t]{4}{*}{ Brain region 18} & 1 & 1.00 & 2.00 & .040 & .034 & .244 & -.029 & .109 \\
\hline & & 2.00 & 1.00 & -.040 & .034 & .244 & -.109 & .029 \\
\hline & 2 & 1.00 & 2.00 & .094 & .071 & .194 & -.049 & .237 \\
\hline & & 2.00 & 1.00 & -.094 & .071 & .194 & -.237 & .049 \\
\hline Brain region 19 & 1 & 1.00 & 2.00 & .054 & .050 & .289 & -.047 & .156 \\
\hline
\end{tabular}




\begin{tabular}{|c|c|c|c|c|c|c|c|c|}
\hline & & 2.00 & 1.00 & -.054 & .050 & .289 & -.156 & .047 \\
\hline & \multirow[t]{2}{*}{2} & 1.00 & 2.00 & .114 & .073 & .122 & -.032 & .261 \\
\hline & & 2.00 & 1.00 & -.114 & .073 & .122 & -.261 & .032 \\
\hline \multirow[t]{4}{*}{ Brain region 20} & 1 & 1.00 & 2.00 & -.003 & .070 & .960 & -.143 & .137 \\
\hline & & 2.00 & 1.00 & .003 & .070 & .960 & -.137 & .143 \\
\hline & 2 & 1.00 & 2.00 & -.051 & .094 & .586 & -.239 & .137 \\
\hline & & 2.00 & 1.00 & .051 & .094 & .586 & -.137 & .239 \\
\hline \multirow[t]{4}{*}{ Brain region 21} & 1 & 1.00 & 2.00 & .020 & .036 & .586 & -.053 & .093 \\
\hline & & 2.00 & 1.00 & -.020 & .036 & .586 & -.093 & .053 \\
\hline & 2 & 1.00 & 2.00 & .056 & .060 & .353 & -.064 & .176 \\
\hline & & 2.00 & 1.00 & -.056 & .060 & .353 & -.176 & .064 \\
\hline \multirow[t]{4}{*}{ Brain region 22} & 1 & 1.00 & 2.00 & .116 & .078 & .144 & -.041 & .272 \\
\hline & & 2.00 & 1.00 & -.116 & .078 & .144 & -.272 & .041 \\
\hline & 2 & 1.00 & 2.00 & .119 & .125 & .345 & -.132 & .370 \\
\hline & & 2.00 & 1.00 & -.119 & .125 & .345 & -.370 & .132 \\
\hline
\end{tabular}

Based on estimated marginal means

a. Adjustment for multiple comparisons: Least Significant Difference (equivalent to no adjustments).

*. The mean difference is significant at the .05 level. 


\section{Table J14}

Means, Standard Errors and 95\% Confidence Intervals for Incentive Condition x Accuracy Effects

\begin{tabular}{|c|c|c|c|c|c|c|}
\hline \multirow[b]{2}{*}{ Brain region } & \multirow[b]{2}{*}{ Incentive cond } & \multirow[b]{2}{*}{ Accuracy } & \multirow[b]{2}{*}{ Mean } & \multirow[b]{2}{*}{ Std. Error } & \multicolumn{2}{|c|}{ 95\% Confidence Interval } \\
\hline & & & & & Lower Bound & Upper Bound \\
\hline \multirow[t]{6}{*}{ Brain region 1} & 1 & 1 & .234 & .027 & .180 & .287 \\
\hline & & 2 & .206 & .030 & .147 & .266 \\
\hline & 2 & 1 & .238 & .027 & .183 & .293 \\
\hline & & 2 & .201 & .066 & .067 & .334 \\
\hline & 3 & 1 & .230 & .030 & .170 & .290 \\
\hline & & 2 & .220 & .040 & .140 & .299 \\
\hline \multirow[t]{6}{*}{ Brain region 2} & 1 & 1 & .224 & .029 & .166 & .282 \\
\hline & & 2 & .188 & .032 & .123 & .252 \\
\hline & 2 & 1 & .202 & .027 & .147 & .258 \\
\hline & & 2 & .177 & .062 & .052 & .302 \\
\hline & 3 & 1 & .210 & .027 & .156 & .263 \\
\hline & & 2 & .174 & .036 & .102 & .247 \\
\hline \multirow[t]{6}{*}{ Brain region 3} & 1 & 1 & .070 & .012 & .045 & .094 \\
\hline & & 2 & .052 & .016 & .020 & .085 \\
\hline & 2 & 1 & .087 & .011 & .064 & .109 \\
\hline & & 2 & .002 & .027 & -.053 & .057 \\
\hline & 3 & 1 & .055 & .015 & .026 & .085 \\
\hline & & 2 & .018 & .017 & -.017 & .054 \\
\hline \multirow[t]{3}{*}{ Brain region 4} & 1 & 1 & .077 & .015 & .046 & .107 \\
\hline & & 2 & .033 & .016 & .001 & .064 \\
\hline & 2 & 1 & .101 & .012 & .076 & .125 \\
\hline
\end{tabular}




\begin{tabular}{|c|c|c|c|c|c|c|}
\hline & & 2 & -.011 & .025 & -.061 & .039 \\
\hline & 3 & 1 & .062 & .015 & .031 & .093 \\
\hline & & 2 & .010 & .017 & -.024 & .043 \\
\hline \multirow[t]{6}{*}{ Brain region 5} & 1 & 1 & -.069 & .022 & -.113 & -.024 \\
\hline & & 2 & -.117 & .025 & -.167 & -.066 \\
\hline & 2 & 1 & -.102 & .026 & -.153 & -.050 \\
\hline & & 2 & -.219 & .053 & -.325 & -.112 \\
\hline & 3 & 1 & -.157 & .020 & -.197 & -.117 \\
\hline & & 2 & -.224 & .028 & -.280 & -.168 \\
\hline \multirow[t]{6}{*}{ Brain region 6} & 1 & 1 & .157 & .031 & .095 & .218 \\
\hline & & 2 & .144 & .033 & .078 & .210 \\
\hline & 2 & 1 & .196 & .032 & .132 & .259 \\
\hline & & 2 & .223 & .038 & .147 & .300 \\
\hline & 3 & 1 & .198 & .036 & .125 & .271 \\
\hline & & 2 & .291 & .043 & .204 & .378 \\
\hline \multirow[t]{6}{*}{ Brain region 7} & 1 & 1 & .231 & .035 & .160 & .301 \\
\hline & & 2 & .174 & .035 & .103 & .244 \\
\hline & 2 & 1 & .270 & .033 & .203 & .336 \\
\hline & & 2 & .258 & .053 & .150 & .365 \\
\hline & 3 & 1 & .330 & .039 & .252 & .408 \\
\hline & & 2 & .359 & .052 & .254 & .463 \\
\hline \multirow[t]{5}{*}{ Brain region 8} & 1 & 1 & .108 & .026 & .055 & .160 \\
\hline & & 2 & .044 & .024 & -.005 & .092 \\
\hline & 2 & 1 & .136 & .030 & .077 & .196 \\
\hline & & 2 & .063 & .040 & -.019 & .144 \\
\hline & 3 & 1 & .160 & .033 & .094 & .226 \\
\hline
\end{tabular}




\begin{tabular}{|c|c|c|c|c|c|c|}
\hline & & 2 & .121 & .032 & .058 & .185 \\
\hline \multirow[t]{6}{*}{ Brain region 9} & 1 & 1 & .099 & .015 & .070 & .128 \\
\hline & & 2 & .063 & .019 & .025 & .101 \\
\hline & 2 & 1 & .120 & .021 & .077 & .163 \\
\hline & & 2 & .082 & .023 & .035 & .128 \\
\hline & 3 & 1 & .134 & .022 & .089 & .179 \\
\hline & & 2 & .126 & .026 & .073 & .178 \\
\hline \multirow[t]{6}{*}{ Brain region 10} & 1 & 1 & .136 & .026 & .084 & .187 \\
\hline & & 2 & .155 & .032 & .090 & .219 \\
\hline & 2 & 1 & .154 & .028 & .097 & .210 \\
\hline & & 2 & .177 & .035 & .106 & .247 \\
\hline & 3 & 1 & .101 & .034 & .032 & .170 \\
\hline & & 2 & .217 & .044 & .129 & .306 \\
\hline \multirow[t]{6}{*}{ Brain region 11} & 1 & 1 & .096 & .018 & .060 & .131 \\
\hline & & 2 & .114 & .023 & .069 & .160 \\
\hline & 2 & 1 & .126 & .018 & .090 & .161 \\
\hline & & 2 & .082 & .039 & .004 & .160 \\
\hline & 3 & 1 & .108 & .017 & .074 & .142 \\
\hline & & 2 & .118 & .027 & .063 & .173 \\
\hline \multirow[t]{6}{*}{ Brain region 12} & 1 & 1 & .184 & .032 & .120 & .247 \\
\hline & & 2 & .147 & .036 & .074 & .220 \\
\hline & 2 & 1 & .203 & .030 & .142 & .264 \\
\hline & & 2 & .087 & .058 & -.031 & .204 \\
\hline & 3 & 1 & .194 & .030 & .132 & .255 \\
\hline & & 2 & .171 & .043 & .085 & .257 \\
\hline Brain region 13 & 1 & 1 & -.092 & .030 & -.153 & -.030 \\
\hline
\end{tabular}




\begin{tabular}{|c|c|c|c|c|c|c|}
\hline & & 2 & -.109 & .033 & -.175 & -.043 \\
\hline & \multirow[t]{2}{*}{2} & 1 & -.119 & .035 & -.190 & -.048 \\
\hline & & 2 & -.148 & .065 & -.279 & -.017 \\
\hline & \multirow[t]{2}{*}{3} & 1 & -.101 & .025 & -.150 & -.051 \\
\hline & & 2 & -.122 & .036 & -.195 & -.049 \\
\hline \multirow[t]{6}{*}{ Brain region 14} & \multirow[t]{2}{*}{1} & 1 & .178 & .031 & .115 & .240 \\
\hline & & 2 & .131 & .039 & .053 & .210 \\
\hline & \multirow[t]{2}{*}{2} & 1 & .213 & .028 & .157 & .269 \\
\hline & & 2 & .188 & .044 & .100 & .276 \\
\hline & \multirow[t]{2}{*}{3} & 1 & .265 & .034 & .198 & .333 \\
\hline & & 2 & .310 & .041 & .226 & .393 \\
\hline \multirow[t]{6}{*}{ Brain region 15} & \multirow[t]{2}{*}{1} & 1 & -.111 & .027 & -.165 & -.057 \\
\hline & & 2 & -.090 & .030 & -.151 & -.030 \\
\hline & \multirow[t]{2}{*}{2} & 1 & -.079 & .030 & -.140 & -.019 \\
\hline & & 2 & -.104 & .061 & -.226 & .018 \\
\hline & \multirow[t]{2}{*}{3} & 1 & -.115 & .027 & -.170 & -.061 \\
\hline & & 2 & -.099 & .039 & -.176 & -.021 \\
\hline \multirow[t]{6}{*}{ Brain region 16} & \multirow[t]{2}{*}{1} & 1 & .385 & .098 & .188 & .581 \\
\hline & & 2 & .088 & .102 & -.117 & .293 \\
\hline & \multirow[t]{2}{*}{2} & 1 & .386 & .103 & .179 & .592 \\
\hline & & 2 & .055 & .170 & -.287 & .397 \\
\hline & \multirow[t]{2}{*}{3} & 1 & .338 & .135 & .066 & .611 \\
\hline & & 2 & .234 & .114 & .004 & .463 \\
\hline \multirow[t]{3}{*}{ Brain region 17} & \multirow[t]{2}{*}{1} & 1 & .074 & .031 & .012 & .136 \\
\hline & & 2 & .059 & .027 & .005 & .113 \\
\hline & 2 & 1 & .082 & .030 & .022 & .142 \\
\hline
\end{tabular}




\begin{tabular}{|c|c|c|c|c|c|c|}
\hline & & 2 & .016 & .054 & -.092 & .125 \\
\hline & 3 & 1 & .062 & .030 & .002 & .122 \\
\hline & & 2 & .000 & .039 & -.078 & .078 \\
\hline \multirow[t]{6}{*}{ Brain region 18} & 1 & 1 & .096 & .024 & .048 & .144 \\
\hline & & 2 & .092 & .040 & .013 & .172 \\
\hline & 2 & 1 & .081 & .025 & .031 & .131 \\
\hline & & 2 & .045 & .066 & -.089 & .179 \\
\hline & 3 & 1 & .057 & .025 & .005 & .108 \\
\hline & & 2 & .104 & .035 & .033 & .175 \\
\hline \multirow[t]{6}{*}{ Brain region 19} & 1 & 1 & .111 & .028 & .054 & .168 \\
\hline & & 2 & .073 & .032 & .007 & .138 \\
\hline & 2 & 1 & .115 & .034 & .046 & .183 \\
\hline & & 2 & .096 & .069 & -.043 & .236 \\
\hline & 3 & 1 & .086 & .036 & .014 & .158 \\
\hline & & 2 & .129 & .039 & .050 & .207 \\
\hline \multirow[t]{6}{*}{ Brain region 20} & 1 & 1 & .226 & .036 & .154 & .298 \\
\hline & & 2 & .198 & .050 & .098 & .299 \\
\hline & 2 & 1 & .234 & .038 & .157 & .312 \\
\hline & & 2 & .198 & .057 & .084 & .312 \\
\hline & 3 & 1 & .296 & .039 & .218 & .374 \\
\hline & & 2 & .335 & .057 & . 220 & . 450 \\
\hline \multirow[t]{5}{*}{ Brain region 21} & 1 & 1 & .011 & .027 & -.044 & .065 \\
\hline & & 2 & -.028 & .027 & -.082 & .025 \\
\hline & 2 & 1 & -.017 & .022 & -.061 & .026 \\
\hline & & 2 & -.172 & .055 & -.281 & -.062 \\
\hline & 3 & 1 & -.031 & .023 & -.077 & .015 \\
\hline
\end{tabular}




\begin{tabular}{|c|c|c|c|c|c|c|}
\hline & & 2 & -.136 & .034 & -.203 & -.068 \\
\hline \multirow[t]{6}{*}{ Brain region 22} & 1 & 1 & .115 & .050 & .013 & .216 \\
\hline & & 2 & .123 & .053 & .017 & .230 \\
\hline & 2 & 1 & .195 & .044 & 105 & .284 \\
\hline & & 2 & .247 & .123 & -.001 & .496 \\
\hline & 3 & 1 & .198 & .052 & .094 & .303 \\
\hline & & 2 & .304 & .064 & .176 & .432 \\
\hline
\end{tabular}




\section{Table J15}

Mean Difference, Standard Error, P-Value and 95\% Confidence Interval for Incentive Condition Effects by Accuracy

\begin{tabular}{|c|c|c|c|c|c|c|c|c|}
\hline \multirow[b]{2}{*}{ Brain region } & \multirow[b]{2}{*}{ Accuracy } & \multirow[b]{2}{*}{ (I) Incentive cond } & \multirow[b]{2}{*}{ (J) Incentive cond } & \multirow{2}{*}{$\begin{array}{c}\text { Mean Difference } \\
(\mathrm{I}-\mathrm{J}) \\
\end{array}$} & \multirow[b]{2}{*}{ Std. Error } & \multirow[b]{2}{*}{ Sig. ${ }^{\text {a }}$} & \multicolumn{2}{|c|}{ 95\% Confidence Interval for Difference } \\
\hline & & & & & & & Lower Bound & Upper Bound \\
\hline \multirow[t]{12}{*}{ Brain region 1} & 1 & 1 & 2 & -.004 & .021 & .839 & -.046 & .037 \\
\hline & & & 3 & .004 & .020 & .849 & -.037 & .045 \\
\hline & & 2 & 1 & .004 & .021 & .839 & -.037 & .046 \\
\hline & & & 3 & .008 & .021 & .701 & -.034 & .050 \\
\hline & & 3 & 1 & -.004 & .020 & .849 & -.045 & .037 \\
\hline & & & 2 & -.008 & .021 & .701 & -.050 & .034 \\
\hline & 2 & 1 & 2 & .006 & .059 & .925 & -.112 & .124 \\
\hline & & & 3 & -.013 & .030 & .662 & -.074 & .047 \\
\hline & & 2 & 1 & -.006 & .059 & .925 & -.124 & .112 \\
\hline & & & 3 & -.019 & .060 & .757 & -.140 & .103 \\
\hline & & 3 & 1 & .013 & .030 & .662 & -.047 & .074 \\
\hline & & & 2 & .019 & .060 & .757 & -.103 & .140 \\
\hline \multirow[t]{9}{*}{ Brain region 2} & 1 & 1 & 2 & .022 & .029 & .458 & -.036 & .080 \\
\hline & & & 3 & .014 & .029 & .622 & -.043 & .072 \\
\hline & & 2 & 1 & -.022 & .029 & .458 & -.080 & .036 \\
\hline & & & 3 & -.007 & .022 & .741 & -.052 & .037 \\
\hline & & 3 & 1 & -.014 & .029 & .622 & -.072 & .043 \\
\hline & & & 2 & .007 & .022 & .741 & -.037 & .052 \\
\hline & 2 & 1 & 2 & .011 & .056 & .851 & -.103 & .124 \\
\hline & & & 3 & .013 & .029 & .647 & -.045 & .072 \\
\hline & & 2 & 1 & -.011 & .056 & .851 & -.124 & .103 \\
\hline
\end{tabular}




\begin{tabular}{|c|c|c|c|c|c|c|c|c|}
\hline & & & 3 & .003 & .055 & .961 & -.109 & .114 \\
\hline & & 3 & 1 & -.013 & .029 & .647 & -.072 & .045 \\
\hline & & & 2 & -.003 & .055 & .961 & -.114 & .109 \\
\hline \multirow[t]{12}{*}{ Brain region 3} & 1 & 1 & 2 & -.017 & .013 & .195 & -.044 & .009 \\
\hline & & & 3 & .014 & .012 & .243 & -.010 & .038 \\
\hline & & 2 & 1 & .017 & .013 & .195 & -.009 & .044 \\
\hline & & & 3 & $.032^{*}$ & .013 & .023 & .005 & .059 \\
\hline & & 3 & 1 & -.014 & .012 & .243 & -.038 & .010 \\
\hline & & & 2 & $-.032^{*}$ & .013 & .023 & -.059 & -.005 \\
\hline & 2 & 1 & 2 & .051 & .025 & .052 & .000 & .102 \\
\hline & & & 3 & .034 & .018 & .071 & -.003 & .071 \\
\hline & & 2 & 1 & -.051 & .025 & .052 & -.102 & .000 \\
\hline & & & 3 & -.017 & .025 & .507 & -.067 & .033 \\
\hline & & 3 & 1 & -.034 & .018 & .071 & -.071 & .003 \\
\hline & & & 2 & .017 & .025 & .507 & -.033 & .067 \\
\hline \multirow[t]{11}{*}{ Brain region 4} & 1 & 1 & 2 & -.024 & .016 & .153 & -.057 & .009 \\
\hline & & & 3 & .014 & .014 & .320 & -.015 & .044 \\
\hline & & 2 & 1 & .024 & .016 & .153 & -.009 & .057 \\
\hline & & & 3 & $.038^{*}$ & .017 & .028 & .004 & .072 \\
\hline & & 3 & 1 & -.014 & .014 & .320 & -.044 & .015 \\
\hline & & & 2 & $-.038^{*}$ & .017 & .028 & -.072 & -.004 \\
\hline & 2 & 1 & 2 & .044 & .023 & .066 & -.003 & .090 \\
\hline & & & 3 & .023 & .016 & .161 & -.010 & .056 \\
\hline & & 2 & 1 & -.044 & .023 & .066 & -.090 & .003 \\
\hline & & & 3 & -.020 & .022 & .350 & -.064 & .023 \\
\hline & & 3 & 1 & -.023 & .016 & .161 & -.056 & .010 \\
\hline
\end{tabular}




\begin{tabular}{|c|c|c|c|c|c|c|c|c|}
\hline & & & 2 & .020 & .022 & .350 & -.023 & .064 \\
\hline \multirow[t]{12}{*}{ Brain region 5} & 1 & 1 & 2 & .033 & .022 & .150 & -.012 & .078 \\
\hline & & & 3 & $.088^{*}$ & .022 & .000 & .044 & .133 \\
\hline & & 2 & 1 & -.033 & .022 & .150 & -.078 & .012 \\
\hline & & & 3 & $.055^{*}$ & .019 & .006 & .017 & .094 \\
\hline & & 3 & 1 & $-.088^{*}$ & .022 & .000 & -.133 & -.044 \\
\hline & & & 2 & $-.055^{*}$ & .019 & .006 & -.094 & -.017 \\
\hline & 2 & 1 & 2 & .102 & .053 & .062 & -.005 & .210 \\
\hline & & & 3 & $.108^{*}$ & .030 & .001 & .048 & .167 \\
\hline & & 2 & 1 & -.102 & .053 & .062 & -.210 & .005 \\
\hline & & & 3 & .005 & .046 & .906 & -.087 & .098 \\
\hline & & 3 & 1 & $-.108^{*}$ & .030 & .001 & -.167 & -.048 \\
\hline & & & 2 & -.005 & .046 & .906 & -.098 & .087 \\
\hline \multirow[t]{12}{*}{ Brain region 6} & 1 & 1 & 2 & -.039 & .024 & .120 & -.088 & .010 \\
\hline & & & 3 & -.041 & .028 & .150 & -.097 & .015 \\
\hline & & 2 & 1 & .039 & .024 & .120 & -.010 & .088 \\
\hline & & & 3 & -.002 & .029 & .939 & -.061 & .057 \\
\hline & & 3 & 1 & .041 & .028 & .150 & -.015 & .097 \\
\hline & & & 2 & .002 & .029 & .939 & -.057 & .061 \\
\hline & 2 & 1 & 2 & $-.079^{*}$ & .039 & .046 & -.157 & -.001 \\
\hline & & & 3 & $-.147^{*}$ & .041 & .001 & -.229 & -.065 \\
\hline & & 2 & 1 & $.079^{*}$ & .039 & .046 & .001 & .157 \\
\hline & & & 3 & -.067 & .034 & .057 & -.137 & .002 \\
\hline & & 3 & 1 & $.147^{*}$ & .041 & .001 & .065 & .229 \\
\hline & & & 2 & .067 & .034 & .057 & -.002 & .137 \\
\hline Brain region 7 & 1 & 1 & 2 & -.039 & .027 & .154 & -.093 & .015 \\
\hline
\end{tabular}




\begin{tabular}{|c|c|c|c|c|c|c|c|c|}
\hline & & & 3 & $-.099^{*}$ & .034 & .005 & -.168 & -.031 \\
\hline & & \multirow[t]{2}{*}{2} & 1 & .039 & .027 & .154 & -.015 & .093 \\
\hline & & & 3 & $-.060^{*}$ & .026 & .026 & -.113 & -.008 \\
\hline & & \multirow[t]{2}{*}{3} & 1 & $.099^{*}$ & .034 & .005 & .031 & .168 \\
\hline & & & 2 & $.060^{*}$ & .026 & .026 & .008 & .113 \\
\hline & \multirow[t]{6}{*}{2} & 1 & 2 & -.084 & .057 & .144 & -.198 & .030 \\
\hline & & & 3 & $-.185^{*}$ & .046 & .000 & -.277 & -.093 \\
\hline & & 2 & 1 & .084 & .057 & .144 & -.030 & .198 \\
\hline & & & 3 & -.101 & .051 & .056 & -.204 & .003 \\
\hline & & 3 & 1 & $.185^{*}$ & .046 & .000 & .093 & .277 \\
\hline & & & 2 & .101 & .051 & .056 & -.003 & .204 \\
\hline \multirow[t]{12}{*}{ Brain region 8} & \multirow[t]{6}{*}{1} & \multirow[t]{2}{*}{1} & 2 & -.029 & .024 & .240 & -.077 & .020 \\
\hline & & & 3 & $-.052^{*}$ & .026 & .048 & -.104 & .000 \\
\hline & & \multirow[t]{2}{*}{2} & 1 & .029 & .024 & .240 & -.020 & .077 \\
\hline & & & 3 & -.024 & .024 & .326 & -.072 & .024 \\
\hline & & \multirow[t]{2}{*}{3} & 1 & $.052^{*}$ & .026 & .048 & .000 & .104 \\
\hline & & & 2 & .024 & .024 & .326 & -.024 & .072 \\
\hline & \multirow[t]{6}{*}{2} & \multirow[t]{2}{*}{1} & 2 & -.019 & .041 & .641 & -.101 & .063 \\
\hline & & & 3 & $-.078^{*}$ & .030 & .012 & -.137 & -.018 \\
\hline & & \multirow[t]{2}{*}{2} & 1 & .019 & .041 & .641 & -.063 & .101 \\
\hline & & & 3 & -.059 & .032 & .074 & -.123 & .006 \\
\hline & & \multirow[t]{2}{*}{3} & 1 & $.078^{*}$ & .030 & .012 & .018 & .137 \\
\hline & & & 2 & .059 & .032 & .074 & -.006 & .123 \\
\hline \multirow[t]{3}{*}{ Brain region 9} & \multirow[t]{3}{*}{1} & \multirow[t]{2}{*}{1} & 2 & -.022 & .017 & .208 & -.056 & .012 \\
\hline & & & 3 & -.035 & .018 & .055 & -.071 & .001 \\
\hline & & 2 & 1 & .022 & .017 & .208 & -.012 & .056 \\
\hline
\end{tabular}




\begin{tabular}{|c|c|c|c|c|c|c|c|c|}
\hline & & & 3 & -.014 & .020 & .489 & -.053 & .026 \\
\hline & & \multirow[t]{2}{*}{3} & 1 & .035 & .018 & .055 & -.001 & .071 \\
\hline & & & 2 & .014 & .020 & .489 & -.026 & .053 \\
\hline & \multirow[t]{6}{*}{2} & \multirow[t]{2}{*}{1} & 2 & -.019 & .024 & .433 & -.066 & .029 \\
\hline & & & 3 & $-.063^{*}$ & .022 & .007 & -.107 & -.018 \\
\hline & & \multirow[t]{2}{*}{2} & 1 & .019 & .024 & .433 & -.029 & .066 \\
\hline & & & 3 & -.044 & .029 & .135 & -.102 & .014 \\
\hline & & \multirow[t]{2}{*}{3} & 1 & $.063^{*}$ & .022 & .007 & .018 & .107 \\
\hline & & & 2 & .044 & .029 & .135 & -.014 & .102 \\
\hline \multirow[t]{12}{*}{ Brain region 10} & \multirow[t]{6}{*}{1} & \multirow[t]{2}{*}{1} & 2 & -.018 & .026 & .501 & -.071 & .035 \\
\hline & & & 3 & .035 & .038 & .359 & -.041 & .111 \\
\hline & & \multirow[t]{2}{*}{2} & 1 & .018 & .026 & .501 & -.035 & .071 \\
\hline & & & 3 & .053 & .033 & .114 & -.013 & .119 \\
\hline & & \multirow[t]{2}{*}{3} & 1 & -.035 & .038 & .359 & -.111 & .041 \\
\hline & & & 2 & -.053 & .033 & .114 & -.119 & .013 \\
\hline & \multirow[t]{6}{*}{2} & \multirow[t]{2}{*}{1} & 2 & -.022 & .038 & .561 & -.098 & .054 \\
\hline & & & 3 & -.063 & .041 & .135 & -.146 & .020 \\
\hline & & \multirow[t]{2}{*}{2} & 1 & .022 & .038 & .561 & -.054 & .098 \\
\hline & & & 3 & -.041 & .033 & .229 & -.108 & .027 \\
\hline & & \multirow[t]{2}{*}{3} & 1 & .063 & .041 & .135 & -.020 & .146 \\
\hline & & & 2 & .041 & .033 & .229 & -.027 & .108 \\
\hline \multirow[t]{5}{*}{ Brain region 11} & \multirow[t]{5}{*}{1} & \multirow[t]{2}{*}{1} & 2 & -.030 & .018 & .099 & -.065 & .006 \\
\hline & & & 3 & -.012 & .020 & .546 & -.052 & .028 \\
\hline & & \multirow[t]{2}{*}{2} & 1 & .030 & .018 & .099 & -.006 & .065 \\
\hline & & & 3 & .018 & .017 & .288 & -.016 & .051 \\
\hline & & 3 & 1 & .012 & .020 & .546 & -.028 & .052 \\
\hline
\end{tabular}




\begin{tabular}{|c|c|c|c|c|c|c|c|c|}
\hline & & & 2 & -.018 & .017 & .288 & -.051 & .016 \\
\hline & \multirow[t]{6}{*}{2} & \multirow[t]{2}{*}{1} & 2 & .033 & .036 & .373 & -.040 & .106 \\
\hline & & & 3 & -.003 & .025 & .897 & -.053 & .046 \\
\hline & & \multirow[t]{2}{*}{2} & 1 & -.033 & .036 & .373 & -.106 & .040 \\
\hline & & & 3 & -.036 & .031 & .252 & -.098 & .026 \\
\hline & & \multirow[t]{2}{*}{3} & 1 & .003 & .025 & .897 & -.046 & .053 \\
\hline & & & 2 & .036 & .031 & .252 & -.026 & .098 \\
\hline \multirow[t]{12}{*}{ Brain region 12} & \multirow[t]{6}{*}{1} & \multirow[t]{2}{*}{1} & 2 & -.019 & .022 & .376 & -.063 & .024 \\
\hline & & & 3 & -.010 & .025 & .697 & -.060 & .041 \\
\hline & & \multirow[t]{2}{*}{2} & 1 & .019 & .022 & .376 & -.024 & .063 \\
\hline & & & 3 & .010 & .029 & .743 & -.049 & .068 \\
\hline & & \multirow[t]{2}{*}{3} & 1 & .010 & .025 & .697 & -.041 & .060 \\
\hline & & & 2 & -.010 & .029 & .743 & -.068 & .049 \\
\hline & \multirow[t]{6}{*}{2} & \multirow[t]{2}{*}{1} & 2 & .060 & .053 & .260 & -.046 & .167 \\
\hline & & & 3 & -.024 & .032 & .456 & -.089 & .040 \\
\hline & & \multirow[t]{2}{*}{2} & 1 & -.060 & .053 & .260 & -.167 & .046 \\
\hline & & & 3 & -.084 & .054 & .123 & -.192 & .024 \\
\hline & & \multirow[t]{2}{*}{3} & 1 & .024 & .032 & .456 & -.040 & .089 \\
\hline & & & 2 & .084 & .054 & .123 & -.024 & .192 \\
\hline \multirow[t]{7}{*}{ Brain region 13} & \multirow[t]{6}{*}{1} & \multirow[t]{2}{*}{1} & 2 & .028 & .030 & .352 & -.032 & .087 \\
\hline & & & 3 & .009 & .027 & .739 & -.046 & .064 \\
\hline & & \multirow[t]{2}{*}{2} & 1 & -.028 & .030 & .352 & -.087 & .032 \\
\hline & & & 3 & -.019 & .026 & .482 & -.071 & .034 \\
\hline & & \multirow[t]{2}{*}{3} & 1 & -.009 & .027 & .739 & -.064 & .046 \\
\hline & & & 2 & .019 & .026 & .482 & -.034 & .071 \\
\hline & 2 & 1 & 2 & .039 & .063 & .541 & -.088 & .165 \\
\hline
\end{tabular}




\begin{tabular}{|c|c|c|c|c|c|c|c|c|}
\hline & & & 3 & .013 & .041 & .743 & -.068 & .095 \\
\hline & & 2 & 1 & -.039 & .063 & .541 & -.165 & .088 \\
\hline & & & 3 & -.025 & .067 & .711 & -.161 & .110 \\
\hline & & 3 & 1 & -.013 & .041 & .743 & -.095 & .068 \\
\hline & & & 2 & .025 & .067 & .711 & -.110 & .161 \\
\hline \multirow[t]{12}{*}{ Brain region 14} & 1 & 1 & 2 & -.035 & .024 & .154 & -.084 & .014 \\
\hline & & & 3 & $-.087^{*}$ & .026 & .002 & -.140 & -.035 \\
\hline & & 2 & 1 & .035 & .024 & .154 & -.014 & .084 \\
\hline & & & 3 & $-.052^{*}$ & .023 & .029 & -.099 & -.006 \\
\hline & & 3 & 1 & $.087^{*}$ & .026 & .002 & .035 & .140 \\
\hline & & & 2 & $.052^{*}$ & .023 & .029 & .006 & .099 \\
\hline & 2 & 1 & 2 & -.057 & .045 & .210 & -.147 & .033 \\
\hline & & & 3 & $-.178^{*}$ & .042 & .000 & -.264 & -.093 \\
\hline & & 2 & 1 & .057 & .045 & .210 & -.033 & .147 \\
\hline & & & 3 & $-.121^{*}$ & .045 & .010 & -.213 & -.030 \\
\hline & & 3 & 1 & $.178^{*}$ & .042 & .000 & .093 & .264 \\
\hline & & & 2 & $.121^{*}$ & .045 & .010 & .030 & .213 \\
\hline \multirow[t]{9}{*}{ Brain region 15} & 1 & 1 & 2 & -.032 & .026 & .221 & -.084 & .020 \\
\hline & & & 3 & .004 & .031 & .907 & -.059 & .066 \\
\hline & & 2 & 1 & .032 & .026 & .221 & -.020 & .084 \\
\hline & & & 3 & .036 & .030 & .237 & -.024 & .095 \\
\hline & & 3 & 1 & -.004 & .031 & .907 & -.066 & .059 \\
\hline & & & 2 & -.036 & .030 & .237 & -.095 & .024 \\
\hline & 2 & 1 & 2 & .014 & .060 & .820 & -.107 & .135 \\
\hline & & & 3 & .008 & .035 & .814 & -.062 & .079 \\
\hline & & 2 & 1 & -.014 & .060 & .820 & -.135 & .107 \\
\hline
\end{tabular}




\begin{tabular}{|c|c|c|c|c|c|c|c|c|}
\hline & & & 3 & -.006 & .061 & .928 & -.128 & .117 \\
\hline & & 3 & 1 & -.008 & .035 & .814 & -.079 & .062 \\
\hline & & & 2 & .006 & .061 & .928 & -.117 & .128 \\
\hline \multirow[t]{12}{*}{ Brain region 16} & 1 & 1 & 2 & -.001 & .085 & .989 & -.172 & .170 \\
\hline & & & 3 & .046 & .102 & .651 & -.158 & .251 \\
\hline & & 2 & 1 & .001 & .085 & .989 & -.170 & .172 \\
\hline & & & 3 & .047 & .109 & .664 & -.171 & .266 \\
\hline & & 3 & 1 & -.046 & .102 & .651 & -.251 & .158 \\
\hline & & & 2 & -.047 & .109 & .664 & -.266 & .171 \\
\hline & 2 & 1 & 2 & .033 & .142 & .818 & -.254 & .320 \\
\hline & & & 3 & -.145 & .087 & .102 & -.321 & .030 \\
\hline & & 2 & 1 & -.033 & .142 & .818 & -.320 & .254 \\
\hline & & & 3 & -.178 & .166 & .288 & -.512 & .156 \\
\hline & & 3 & 1 & .145 & .087 & .102 & -.030 & .321 \\
\hline & & & 2 & .178 & .166 & .288 & -.156 & .512 \\
\hline \multirow[t]{11}{*}{ Brain region 17} & 1 & 1 & 2 & -.008 & .034 & .811 & -.077 & .060 \\
\hline & & & 3 & .012 & .025 & .639 & -.038 & .061 \\
\hline & & 2 & 1 & .008 & .034 & .811 & -.060 & .077 \\
\hline & & & 3 & .020 & .029 & .494 & -.038 & .078 \\
\hline & & 3 & 1 & -.012 & .025 & .639 & -.061 & .038 \\
\hline & & & 2 & -.020 & .029 & .494 & -.078 & .038 \\
\hline & 2 & 1 & 2 & .043 & .052 & .414 & -.062 & .148 \\
\hline & & & 3 & .059 & .040 & .140 & -.020 & .139 \\
\hline & & 2 & 1 & -.043 & .052 & .414 & -.148 & .062 \\
\hline & & & 3 & .016 & .044 & .711 & -.073 & .106 \\
\hline & & 3 & 1 & -.059 & .040 & .140 & -.139 & .020 \\
\hline
\end{tabular}




\begin{tabular}{|c|c|c|c|c|c|c|c|c|}
\hline & & & 2 & -.016 & .044 & .711 & -.106 & .073 \\
\hline \multirow[t]{12}{*}{ Brain region 18} & 1 & 1 & 2 & .015 & .029 & .612 & -.043 & .072 \\
\hline & & & 3 & .039 & .027 & .149 & -.015 & .093 \\
\hline & & 2 & 1 & -.015 & .029 & .612 & -.072 & .043 \\
\hline & & & 3 & .025 & .037 & .506 & -.049 & .098 \\
\hline & & 3 & 1 & -.039 & .027 & .149 & -.093 & .015 \\
\hline & & & 2 & -.025 & .037 & .506 & -.098 & .049 \\
\hline & 2 & 1 & 2 & .047 & .070 & .501 & -.093 & .187 \\
\hline & & & 3 & -.011 & .032 & .725 & -.076 & .053 \\
\hline & & 2 & 1 & -.047 & .070 & .501 & -.187 & .093 \\
\hline & & & 3 & -.058 & .067 & .386 & -.193 & .076 \\
\hline & & 3 & 1 & .011 & .032 & .725 & -.053 & .076 \\
\hline & & & 2 & .058 & .067 & .386 & -.076 & .193 \\
\hline \multirow[t]{12}{*}{ Brain region 19} & 1 & 1 & 2 & -.004 & .034 & .917 & -.072 & .065 \\
\hline & & & 3 & .025 & .042 & .556 & -.059 & .109 \\
\hline & & 2 & 1 & .004 & .034 & .917 & -.065 & .072 \\
\hline & & & 3 & .028 & .034 & .410 & -.040 & .097 \\
\hline & & 3 & 1 & -.025 & .042 & .556 & -.109 & .059 \\
\hline & & & 2 & -.028 & .034 & .410 & -.097 & .040 \\
\hline & 2 & 1 & 2 & -.023 & .069 & .739 & -.163 & .116 \\
\hline & & & 3 & -.056 & .033 & .100 & -.123 & .011 \\
\hline & & 2 & 1 & .023 & .069 & .739 & -.116 & .163 \\
\hline & & & 3 & -.033 & .066 & .623 & -.165 & .100 \\
\hline & & 3 & 1 & .056 & .033 & .100 & -.011 & .123 \\
\hline & & & 2 & .033 & .066 & .623 & -.100 & .165 \\
\hline Brain region 20 & 1 & 1 & 2 & -.008 & .024 & .732 & -.057 & .041 \\
\hline
\end{tabular}




\begin{tabular}{|c|c|c|c|c|c|c|c|c|}
\hline & & & 3 & $-.070^{*}$ & .027 & .012 & -.124 & -.016 \\
\hline & & \multirow[t]{2}{*}{2} & 1 & .008 & .024 & .732 & -.041 & .057 \\
\hline & & & 3 & $-.062^{*}$ & .024 & .014 & -.110 & -.013 \\
\hline & & \multirow[t]{2}{*}{3} & 1 & $.070^{*}$ & .027 & .012 & .016 & .124 \\
\hline & & & 2 & $.062^{*}$ & .024 & .014 & .013 & .110 \\
\hline & \multirow[t]{6}{*}{2} & 1 & 2 & .000 & .055 & .994 & -.110 & .111 \\
\hline & & & 3 & $-.137^{*}$ & .049 & .008 & -.236 & -.037 \\
\hline & & 2 & 1 & .000 & .055 & .994 & -.111 & .110 \\
\hline & & & 3 & $-.137^{*}$ & .042 & .002 & -.221 & -.053 \\
\hline & & 3 & 1 & $.137^{*}$ & .049 & .008 & .037 & .236 \\
\hline & & & 2 & $.137^{*}$ & .042 & .002 & .053 & .221 \\
\hline \multirow[t]{12}{*}{ Brain region 21} & \multirow[t]{6}{*}{1} & 1 & 2 & .028 & .027 & .317 & -.027 & .083 \\
\hline & & & 3 & .042 & .032 & .201 & -.023 & .106 \\
\hline & & 2 & 1 & -.028 & .027 & .317 & -.083 & .027 \\
\hline & & & 3 & .014 & .019 & .473 & -.025 & .052 \\
\hline & & 3 & 1 & -.042 & .032 & .201 & -.106 & .023 \\
\hline & & & 2 & -.014 & .019 & .473 & -.052 & .025 \\
\hline & \multirow[t]{6}{*}{2} & 1 & 2 & $.144^{*}$ & .054 & .011 & .034 & .253 \\
\hline & & & 3 & $.107^{*}$ & .031 & .001 & .045 & .170 \\
\hline & & 2 & 1 & $-.144^{*}$ & .054 & .011 & -.253 & -.034 \\
\hline & & & 3 & -.036 & .050 & .475 & -.137 & .065 \\
\hline & & 3 & 1 & $-.107^{*}$ & .031 & .001 & -.170 & -.045 \\
\hline & & & 2 & .036 & .050 & .475 & -.065 & .137 \\
\hline \multirow[t]{3}{*}{ Brain region 22} & \multirow[t]{3}{*}{1} & 1 & 2 & -.080 & .048 & .103 & -.177 & .017 \\
\hline & & & 3 & -.083 & .047 & .083 & -.178 & .011 \\
\hline & & 2 & 1 & .080 & .048 & .103 & -.017 & .177 \\
\hline
\end{tabular}




\begin{tabular}{|c|c|c|c|c|c|c|c|}
\hline & & 3 & -.003 & .060 & .956 & -.123 & .117 \\
\hline & \multirow[t]{2}{*}{3} & 1 & .083 & .047 & .083 & -.011 & .178 \\
\hline & & 2 & .003 & .060 & .956 & -.117 & .123 \\
\hline \multirow[t]{6}{*}{2} & 1 & 2 & -.124 & .107 & .251 & -.339 & .091 \\
\hline & & 3 & $-.181^{*}$ & .053 & .001 & -.287 & -.075 \\
\hline & 2 & 1 & .124 & .107 & .251 & -.091 & .339 \\
\hline & & 3 & -.057 & .131 & .667 & -.320 & .207 \\
\hline & 3 & 1 & $.181^{*}$ & .053 & .001 & .075 & .287 \\
\hline & & 2 & .057. & .131 & .667 & -.207 & .320 \\
\hline
\end{tabular}

Based on estimated marginal means

a. Adjustment for multiple comparisons: Least Significant Difference (equivalent to no adjustments).

*. The mean difference is significant at the .05 level. 


\section{Table J16}

Mean Difference, Standard Error, P-Value and 95\% Confidence Interval for Accuracy Effects by Incentive Condition

\begin{tabular}{|c|c|c|c|c|c|c|c|c|}
\hline & & & & Mean Difference & & & 95\% Confidence Inte & al for Difference ${ }^{a}$ \\
\hline Brain region & Incentive cond & (I) Accuracy & (J) Accuracy & $(\mathrm{I}-\mathrm{J})$ & Std. Error & Sig. ${ }^{\text {a }}$ & Lower Bound & Upper Bound \\
\hline \multirow[t]{6}{*}{ Brain region 1} & 1 & 1 & 2 & .027 & .025 & .277 & -.023 & .078 \\
\hline & & 2 & 1 & -.027 & .025 & .277 & -.078 & .023 \\
\hline & 2 & 1 & 2 & .037 & .065 & .571 & -.094 & .169 \\
\hline & & 2 & 1 & -.037 & .065 & .571 & -.169 & .094 \\
\hline & 3 & 1 & 2 & .010 & .033 & .754 & -.056 & .077 \\
\hline & & 2 & 1 & -.010 & .033 & .754 & -.077 & .056 \\
\hline \multirow[t]{6}{*}{ Brain region 2} & 1 & 1 & 2 & .036 & .030 & .224 & -.023 & .096 \\
\hline & & 2 & 1 & -.036 & .030 & .224 & -.096 & .023 \\
\hline & 2 & 1 & 2 & .026 & .060 & .674 & -.096 & .147 \\
\hline & & 2 & 1 & -.026 & .060 & .674 & -.147 & .096 \\
\hline & 3 & 1 & 2 & .036 & .031 & .252 & -.026 & .098 \\
\hline & & 2 & 1 & -.036 & .031 & .252 & -.098 & .026 \\
\hline \multirow[t]{6}{*}{ Brain region 3} & 1 & 1 & 2 & .017 & .018 & .339 & -.018 & .053 \\
\hline & & 2 & 1 & -.017 & .018 & .339 & -.053 & .018 \\
\hline & 2 & 1 & 2 & $.085^{*}$ & .026 & .002 & .032 & .138 \\
\hline & & 2 & 1 & $-.085^{*}$ & .026 & .002 & -.138 & -.032 \\
\hline & 3 & 1 & 2 & $.037^{*}$ & .017 & .033 & .003 & .071 \\
\hline & & 2 & 1 & $-.037^{*}$ & .017 & .033 & -.071 & -.003 \\
\hline \multirow[t]{3}{*}{ Brain region 4} & 1 & 1 & 2 & $.044^{*}$ & .019 & .025 & .006 & .082 \\
\hline & & 2 & 1 & $-.044^{*}$ & .019 & .025 & -.082 & -.006 \\
\hline & 2 & 1 & 2 & $.111^{*}$ & .025 & .000 & .062 & .161 \\
\hline
\end{tabular}




\begin{tabular}{|c|c|c|c|c|c|c|c|c|}
\hline & & 2 & 1 & $-.111^{*}$ & .025 & .000 & -.161 & -.062 \\
\hline & \multirow[t]{2}{*}{3} & 1 & 2 & $.053^{*}$ & .018 & .005 & .017 & .089 \\
\hline & & 2 & 1 & $-.053^{*}$ & .018 & .005 & -.089 & -.017 \\
\hline \multirow[t]{6}{*}{ Brain region 5} & 1 & 1 & 2 & .048 & .032 & .139 & -.016 & .112 \\
\hline & & 2 & 1 & -.048 & .032 & .139 & -.112 & .016 \\
\hline & 2 & 1 & 2 & $.117^{*}$ & .052 & .028 & .013 & .221 \\
\hline & & 2 & 1 & $-.117^{*}$ & .052 & .028 & -.221 & -.013 \\
\hline & 3 & 1 & 2 & $.067^{*}$ & .028 & .020 & .011 & .123 \\
\hline & & 2 & 1 & $-.067^{*}$ & .028 & .020 & -.123 & -.011 \\
\hline \multirow[t]{6}{*}{ Brain region 6} & 1 & 1 & 2 & .013 & .036 & .729 & -.060 & .086 \\
\hline & & 2 & 1 & -.013 & .036 & .729 & -.086 & .060 \\
\hline & 2 & 1 & 2 & -.028 & .040 & .492 & -.109 & .053 \\
\hline & & 2 & 1 & .028 & .040 & .492 & -.053 & .109 \\
\hline & 3 & 1 & 2 & $-.093^{*}$ & .038 & .018 & -.169 & -.017 \\
\hline & & 2 & 1 & $.093^{*}$ & .038 & .018 & .017 & .169 \\
\hline \multirow[t]{6}{*}{ Brain region 7} & 1 & 1 & 2 & .057 & .042 & .177 & -.027 & .141 \\
\hline & & 2 & 1 & -.057 & .042 & .177 & -.141 & .027 \\
\hline & 2 & 1 & 2 & .012 & .048 & .808 & -.085 & .109 \\
\hline & & 2 & 1 & -.012 & .048 & .808 & -.109 & .085 \\
\hline & 3 & 1 & 2 & -.029 & .045 & .522 & -.119 & .061 \\
\hline & & 2 & 1 & .029 & .045 & .522 & -.061 & .119 \\
\hline \multirow[t]{5}{*}{ Brain region 8} & 1 & 1 & 2 & $.064^{*}$ & .026 & .020 & .011 & .117 \\
\hline & & 2 & 1 & $-.064^{*}$ & .026 & .020 & -.117 & -.011 \\
\hline & 2 & 1 & 2 & .073 & .043 & .094 & -.013 & .160 \\
\hline & & 2 & 1 & -.073 & .043 & .094 & -.160 & .013 \\
\hline & 3 & 1 & 2 & .039 & .036 & .291 & -.034 & .111 \\
\hline
\end{tabular}




\begin{tabular}{|c|c|c|c|c|c|c|c|c|}
\hline & & 2 & 1 & -.039 & .036 & .291 & -.111 & .034 \\
\hline \multirow[t]{6}{*}{ Brain region 9} & 1 & 1 & 2 & $.036^{*}$ & .016 & .031 & .004 & .068 \\
\hline & & 2 & 1 & $-.036^{*}$ & .016 & .031 & -.068 & -.004 \\
\hline & 2 & 1 & 2 & .039 & .029 & .184 & -.019 & .097 \\
\hline & & 2 & 1 & -.039 & .029 & .184 & -.097 & .019 \\
\hline & 3 & 1 & 2 & .008 & .021 & .683 & -.033 & .050 \\
\hline & & 2 & 1 & -.008 & .021 & .683 & -.050 & .033 \\
\hline \multirow[t]{6}{*}{ Brain region 10} & 1 & 1 & 2 & -.019 & .031 & .545 & -.081 & .043 \\
\hline & & 2 & 1 & .019 & .031 & .545 & -.043 & .081 \\
\hline & 2 & 1 & 2 & -.023 & .039 & .556 & -.100 & .055 \\
\hline & & 2 & 1 & .023 & .039 & .556 & -.055 & .100 \\
\hline & 3 & 1 & 2 & $-.117^{*}$ & .046 & .016 & -.210 & -.023 \\
\hline & & 2 & 1 & $.117^{*}$ & .046 & .016 & .023 & .210 \\
\hline \multirow[t]{6}{*}{ Brain region 11} & 1 & 1 & 2 & -.019 & .020 & .357 & -.059 & .022 \\
\hline & & 2 & 1 & .019 & .020 & .357 & -.022 & .059 \\
\hline & 2 & 1 & 2 & .044 & .037 & .248 & -.031 & .119 \\
\hline & & 2 & 1 & -.044 & .037 & .248 & -.119 & .031 \\
\hline & 3 & 1 & 2 & -.010 & .025 & .700 & -.061 & .041 \\
\hline & & 2 & 1 & .010 & .025 & .700 & -.041 & .061 \\
\hline \multirow[t]{6}{*}{ Brain region 12} & 1 & 1 & 2 & .037 & .034 & .279 & -.031 & .104 \\
\hline & & 2 & 1 & -.037 & .034 & .279 & -.104 & .031 \\
\hline & 2 & 1 & 2 & $.117^{*}$ & .057 & .048 & .001 & .232 \\
\hline & & 2 & 1 & $-.117^{*}$ & .057 & .048 & -.232 & -.001 \\
\hline & 3 & 1 & 2 & .023 & .039 & .565 & -.056 & .101 \\
\hline & & 2 & 1 & -.023 & .039 & .565 & -.101 & .056 \\
\hline Brain region 13 & 1 & 1 & 2 & .017 & .041 & .674 & -.065 & .100 \\
\hline
\end{tabular}




\begin{tabular}{|c|c|c|c|c|c|c|c|c|}
\hline & & 2 & 1 & -.017 & .041 & .674 & -.100 & .065 \\
\hline & \multirow[t]{2}{*}{2} & 1 & 2 & .028 & .064 & .662 & -.101 & .157 \\
\hline & & 2 & 1 & -.028 & .064 & .662 & -.157 & .101 \\
\hline & \multirow[t]{2}{*}{3} & 1 & 2 & .022 & .036 & .549 & -.051 & .094 \\
\hline & & 2 & 1 & -.022 & .036 & .549 & -.094 & .051 \\
\hline \multirow[t]{6}{*}{ Brain region 14} & \multirow[t]{2}{*}{1} & 1 & 2 & .046 & .038 & .235 & -.031 & .124 \\
\hline & & 2 & 1 & -.046 & .038 & .235 & -.124 & .031 \\
\hline & \multirow[t]{2}{*}{2} & 1 & 2 & .025 & .046 & .594 & -.067 & .116 \\
\hline & & 2 & 1 & -.025 & .046 & .594 & -.116 & .067 \\
\hline & \multirow[t]{2}{*}{3} & 1 & 2 & -.044 & .034 & .196 & -.113 & .024 \\
\hline & & 2 & 1 & .044 & .034 & .196 & -.024 & .113 \\
\hline \multirow[t]{6}{*}{ Brain region 15} & \multirow[t]{2}{*}{1} & 1 & 2 & -.021 & .035 & .557 & -.092 & .050 \\
\hline & & 2 & 1 & .021 & .035 & .557 & -.050 & .092 \\
\hline & \multirow[t]{2}{*}{2} & 1 & 2 & .025 & .061 & .687 & -.098 & .148 \\
\hline & & 2 & 1 & -.025 & .061 & .687 & -.148 & .098 \\
\hline & \multirow[t]{2}{*}{3} & 1 & 2 & -.016 & .040 & .685 & -.097 & .064 \\
\hline & & 2 & 1 & .016 & .040 & .685 & -.064 & .097 \\
\hline \multirow[t]{6}{*}{ Brain region 16} & \multirow[t]{2}{*}{1} & 1 & 2 & $.296^{*}$ & .113 & .012 & .069 & .524 \\
\hline & & 2 & 1 & $-.296^{*}$ & .113 & .012 & -.524 & -.069 \\
\hline & \multirow[t]{2}{*}{2} & 1 & 2 & .331 & .177 & .069 & -.027 & .688 \\
\hline & & 2 & 1 & -.331 & .177 & .069 & -.688 & .027 \\
\hline & \multirow[t]{2}{*}{3} & 1 & 2 & .105 & .137 & .448 & -.171 & .380 \\
\hline & & 2 & 1 & -.105 & .137 & .448 & -.380 & .171 \\
\hline \multirow[t]{3}{*}{ Brain region 17} & \multirow[t]{2}{*}{1} & 1 & 2 & .015 & .036 & .687 & -.058 & .087 \\
\hline & & 2 & 1 & -.015 & .036 & .687 & -.087 & .058 \\
\hline & 2 & 1 & 2 & .066 & .051 & .207 & -.038 & .169 \\
\hline
\end{tabular}




\begin{tabular}{|c|c|c|c|c|c|c|c|c|}
\hline & & 2 & 1 & -.066 & .051 & .207 & -.169 & .038 \\
\hline & \multirow[t]{2}{*}{3} & 1 & 2 & .062 & .042 & .146 & -.023 & .147 \\
\hline & & 2 & 1 & -.062 & .042 & .146 & -.147 & .023 \\
\hline \multirow[t]{6}{*}{ Brain region 18} & 1 & 1 & 2 & .003 & .040 & .932 & -.076 & .083 \\
\hline & & 2 & 1 & -.003 & .040 & .932 & -.083 & .076 \\
\hline & 2 & 1 & 2 & .036 & .071 & .615 & -.107 & .179 \\
\hline & & 2 & 1 & -.036 & .071 & .615 & -.179 & .107 \\
\hline & 3 & 1 & 2 & -.047 & .034 & .178 & -.116 & .022 \\
\hline & & 2 & 1 & .047 & .034 & .178 & -.022 & .116 \\
\hline \multirow[t]{6}{*}{ Brain region 19} & 1 & 1 & 2 & .038 & .038 & .316 & -.038 & .114 \\
\hline & & 2 & 1 & -.038 & .038 & .316 & -.114 & .038 \\
\hline & 2 & 1 & 2 & .019 & .072 & .797 & -.126 & .163 \\
\hline & & 2 & 1 & -.019 & .072 & .797 & -.163 & .126 \\
\hline & 3 & 1 & 2 & -.042 & .041 & .303 & -.124 & .040 \\
\hline & & 2 & 1 & .042 & .041 & .303 & -.040 & .124 \\
\hline \multirow[t]{6}{*}{ Brain region 20} & 1 & 1 & 2 & .028 & .052 & .595 & -.077 & .132 \\
\hline & & 2 & 1 & -.028 & .052 & .595 & -.132 & .077 \\
\hline & 2 & 1 & 2 & .037 & .053 & .491 & -.069 & .142 \\
\hline & & 2 & 1 & -.037 & .053 & .491 & -.142 & .069 \\
\hline & 3 & 1 & 2 & -.039 & .050 & .440 & -.139 & .061 \\
\hline & & 2 & 1 & .039 & .050 & .440 & -.061 & .139 \\
\hline \multirow[t]{5}{*}{ Brain region 21} & 1 & 1 & 2 & .039 & .042 & .358 & -.045 & .123 \\
\hline & & 2 & 1 & -.039 & .042 & .358 & -.123 & .045 \\
\hline & 2 & 1 & 2 & $.154^{*}$ & .055 & .008 & .043 & .266 \\
\hline & & 2 & 1 & $-.154^{*}$ & .055 & .008 & -.266 & -.043 \\
\hline & 3 & 1 & 2 & $.104^{*}$ & .035 & .005 & .034 & .175 \\
\hline
\end{tabular}




\begin{tabular}{|c|c|c|c|c|c|c|c|c|}
\hline & & 2 & 1 & $-.104^{*}$ & .035 & .005 & -.175 & -.034 \\
\hline \multirow[t]{6}{*}{ Brain region 22} & 1 & 1 & 2 & -.008 & .055 & .880 & -.119 & .102 \\
\hline & & 2 & 1 & .008 & .055 & .880 & -.102 & .119 \\
\hline & 2 & 1 & 2 & -.052 & .129 & .686 & -.312 & .207 \\
\hline & & 2 & 1 & .052 & .129 & .686 & -.207 & .312 \\
\hline & 3 & 1 & 2 & -.106 & .070 & .140 & -.248 & .036 \\
\hline & & 2 & 1 & .106 & .070 & .140 & -.036 & .248 \\
\hline
\end{tabular}

Based on estimated marginal means

a. Adjustment for multiple comparisons: Least Significant Difference (equivalent to no adjustments)

*. The mean difference is significant at the .05 level. 


\section{Table J17}

Means, Standard Errors and 95\% Confidence Intervals for Group x Incentive Condition x Accuracy Effects

\begin{tabular}{|c|c|c|c|c|c|c|c|}
\hline \multirow[b]{2}{*}{ Brain region } & \multirow[b]{2}{*}{ Group } & \multirow[b]{2}{*}{ Incentive cond } & \multirow[b]{2}{*}{ Accuracy } & \multirow[b]{2}{*}{ Mean } & \multirow[b]{2}{*}{ Std. Error } & \multicolumn{2}{|c|}{ 95\% Confidence Interval } \\
\hline & & & & & & Lower Bound & Upper Bound \\
\hline \multirow[t]{12}{*}{ Brain region 1} & 1.00 & 1 & 1 & .285 & .038 & .209 & .361 \\
\hline & & & 2 & .223 & .042 & .138 & .307 \\
\hline & & 2 & 1 & .270 & .038 & .193 & .347 \\
\hline & & & 2 & .195 & .094 & .007 & .384 \\
\hline & & 3 & 1 & .242 & .042 & .156 & .327 \\
\hline & & & 2 & .236 & .056 & .123 & .349 \\
\hline & 2.00 & 1 & 1 & .183 & .038 & .107 & .258 \\
\hline & & & 2 & .190 & .042 & .106 & .274 \\
\hline & & 2 & 1 & .206 & .038 & .129 & .283 \\
\hline & & & 2 & .206 & .094 & .018 & .395 \\
\hline & & 3 & 1 & .218 & .042 & .133 & .303 \\
\hline & & & 2 & .203 & .056 & .090 & .316 \\
\hline \multirow[t]{9}{*}{ Brain region 2} & 1.00 & 1 & 1 & .268 & .041 & .186 & .351 \\
\hline & & & 2 & .160 & .045 & .069 & .251 \\
\hline & & 2 & 1 & .213 & .039 & .135 & .291 \\
\hline & & & 2 & .134 & .088 & -.043 & .311 \\
\hline & & 3 & 1 & .201 & .038 & .125 & .276 \\
\hline & & & 2 & .164 & .051 & .061 & .266 \\
\hline & 2.00 & 1 & 1 & .180 & .041 & .097 & .262 \\
\hline & & & 2 & .215 & .045 & .124 & .306 \\
\hline & & 2 & 1 & .192 & .039 & .114 & .270 \\
\hline
\end{tabular}




\begin{tabular}{|c|c|c|c|c|c|c|c|}
\hline & & & 2 & .220 & .088 & .043 & .396 \\
\hline & & 3 & 1 & .219 & .038 & .143 & .295 \\
\hline & & & 2 & .185 & .051 & .082 & .287 \\
\hline \multirow[t]{12}{*}{ Brain region 3} & 1.00 & 1 & 1 & .100 & .017 & .065 & .135 \\
\hline & & & 2 & .067 & .023 & .020 & .113 \\
\hline & & 2 & 1 & .109 & .016 & .077 & .141 \\
\hline & & & 2 & -.001 & .039 & -.078 & .077 \\
\hline & & 3 & 1 & .074 & .021 & .033 & .115 \\
\hline & & & 2 & .031 & .025 & -.019 & .081 \\
\hline & 2.00 & 1 & 1 & .039 & .017 & .004 & .074 \\
\hline & & & 2 & .038 & .023 & -.008 & .085 \\
\hline & & 2 & 1 & .065 & .016 & .033 & .096 \\
\hline & & & 2 & .005 & .039 & -.073 & .082 \\
\hline & & 3 & 1 & .037 & .021 & -.005 & .078 \\
\hline & & & 2 & .006 & .025 & -.044 & .056 \\
\hline \multirow[t]{11}{*}{ Brain region 4} & 1.00 & 1 & 1 & .110 & .021 & .067 & .153 \\
\hline & & & 2 & .042 & .022 & -.003 & .086 \\
\hline & & 2 & 1 & .121 & .017 & .085 & .156 \\
\hline & & & 2 & -.021 & .035 & -.092 & .050 \\
\hline & & 3 & 1 & .091 & .022 & .047 & .134 \\
\hline & & & 2 & .032 & .024 & -.016 & .080 \\
\hline & 2.00 & 1 & 1 & .043 & .021 & .001 & .086 \\
\hline & & & 2 & .024 & .022 & -.021 & .068 \\
\hline & & 2 & 1 & .080 & .017 & .045 & .116 \\
\hline & & & 2 & -.001 & .035 & -.072 & .070 \\
\hline & & 3 & 1 & .034 & .022 & -.010 & .077 \\
\hline
\end{tabular}




\begin{tabular}{|c|c|c|c|c|c|c|c|}
\hline & & & 2 & -.013 & .024 & -.061 & .035 \\
\hline \multirow[t]{12}{*}{ Brain region 5} & 1.00 & 1 & 1 & -.026 & .031 & -.089 & .037 \\
\hline & & & 2 & -.127 & .035 & -.198 & -.056 \\
\hline & & 2 & 1 & -.075 & .036 & -.148 & -.003 \\
\hline & & & 2 & -.193 & .075 & -.344 & -.042 \\
\hline & & 3 & 1 & -.159 & .028 & -.216 & -.102 \\
\hline & & & 2 & -.186 & .039 & -.265 & -.107 \\
\hline & 2.00 & 1 & 1 & -.112 & .031 & -.175 & -.049 \\
\hline & & & 2 & -.106 & .035 & -.177 & -.035 \\
\hline & & 2 & 1 & -.128 & .036 & -.201 & -.055 \\
\hline & & & 2 & -.244 & .075 & -.395 & -.094 \\
\hline & & 3 & 1 & -.155 & .028 & -.212 & -.098 \\
\hline & & & 2 & -.262 & .039 & -.341 & -.183 \\
\hline \multirow[t]{12}{*}{ Brain region 6} & 1.00 & 1 & 1 & .199 & .043 & .112 & .286 \\
\hline & & & 2 & .199 & .046 & .106 & .292 \\
\hline & & 2 & 1 & .221 & .045 & .131 & .311 \\
\hline & & & 2 & .215 & .054 & .106 & .324 \\
\hline & & 3 & 1 & .199 & .051 & .095 & .302 \\
\hline & & & 2 & .314 & .061 & .192 & .437 \\
\hline & 2.00 & 1 & 1 & .114 & .043 & .027 & .201 \\
\hline & & & 2 & .089 & .046 & -.004 & .182 \\
\hline & & 2 & 1 & .170 & .045 & .080 & .260 \\
\hline & & & 2 & .232 & .054 & .123 & .340 \\
\hline & & 3 & 1 & .197 & .051 & .094 & .300 \\
\hline & & & 2 & .267 & .061 & .145 & .390 \\
\hline Brain region 7 & 1.00 & 1 & 1 & .287 & .050 & .186 & .387 \\
\hline
\end{tabular}




\begin{tabular}{|c|c|c|c|c|c|c|c|}
\hline & & & 2 & .250 & .049 & .151 & .350 \\
\hline & & \multirow[t]{2}{*}{2} & 1 & .320 & .046 & .226 & .413 \\
\hline & & & 2 & .235 & .076 & .083 & .387 \\
\hline & & \multirow[t]{2}{*}{3} & 1 & .336 & .055 & .226 & .446 \\
\hline & & & 2 & .365 & .074 & .217 & .513 \\
\hline & \multirow[t]{6}{*}{2.00} & \multirow[t]{2}{*}{1} & 1 & .175 & .050 & .075 & .275 \\
\hline & & & 2 & .097 & .049 & -.003 & .197 \\
\hline & & \multirow[t]{2}{*}{2} & 1 & .219 & .046 & .126 & .313 \\
\hline & & & 2 & .281 & .076 & .128 & .433 \\
\hline & & \multirow[t]{2}{*}{3} & 1 & .324 & .055 & .214 & .434 \\
\hline & & & 2 & .353 & .074 & .205 & .500 \\
\hline \multirow[t]{12}{*}{ Brain region 8} & \multirow[t]{6}{*}{1.00} & \multirow[t]{2}{*}{1} & 1 & .124 & .037 & .050 & .198 \\
\hline & & & 2 & .024 & .034 & -.045 & .092 \\
\hline & & \multirow[t]{2}{*}{2} & 1 & .144 & .042 & .060 & .228 \\
\hline & & & 2 & .027 & .057 & -.088 & .142 \\
\hline & & \multirow[t]{2}{*}{3} & 1 & .152 & .046 & .059 & .245 \\
\hline & & & 2 & .127 & .045 & .037 & .217 \\
\hline & \multirow[t]{6}{*}{2.00} & \multirow[t]{2}{*}{1} & 1 & .092 & .037 & .017 & .166 \\
\hline & & & 2 & .064 & .034 & -.005 & .132 \\
\hline & & \multirow[t]{2}{*}{2} & 1 & .129 & .042 & .045 & .213 \\
\hline & & & 2 & .098 & .057 & -.017 & .214 \\
\hline & & \multirow[t]{2}{*}{3} & 1 & .168 & .046 & .075 & .261 \\
\hline & & & 2 & .116 & .045 & .026 & .206 \\
\hline \multirow[t]{3}{*}{ Brain region 9} & \multirow[t]{3}{*}{1.00} & \multirow[t]{2}{*}{1} & 1 & .115 & .021 & .074 & .156 \\
\hline & & & 2 & .071 & .027 & .017 & .125 \\
\hline & & 2 & 1 & .122 & .030 & .061 & .183 \\
\hline
\end{tabular}




\begin{tabular}{|c|c|c|c|c|c|c|c|}
\hline & & & 2 & .071 & .033 & .005 & .137 \\
\hline & & \multirow[t]{2}{*}{3} & 1 & .124 & .031 & .061 & .187 \\
\hline & & & 2 & .141 & .037 & .066 & .215 \\
\hline & \multirow[t]{6}{*}{2.00} & \multirow[t]{2}{*}{1} & 1 & .082 & .021 & .041 & .124 \\
\hline & & & 2 & .054 & .027 & .000 & .109 \\
\hline & & \multirow[t]{2}{*}{2} & 1 & .118 & .030 & .057 & .179 \\
\hline & & & 2 & .092 & .033 & .026 & .158 \\
\hline & & \multirow[t]{2}{*}{3} & 1 & .144 & .031 & .081 & .207 \\
\hline & & & 2 & .110 & .037 & .036 & .185 \\
\hline \multirow[t]{12}{*}{ Brain region 10} & \multirow[t]{6}{*}{1.00} & \multirow[t]{2}{*}{1} & 1 & .162 & .036 & .089 & .235 \\
\hline & & & 2 & .180 & .045 & .089 & .271 \\
\hline & & \multirow[t]{2}{*}{2} & 1 & .181 & .040 & .101 & .261 \\
\hline & & & 2 & .130 & .049 & .031 & .229 \\
\hline & & \multirow[t]{2}{*}{3} & 1 & .082 & .048 & -.015 & .180 \\
\hline & & & 2 & .236 & .062 & .111 & .361 \\
\hline & \multirow[t]{6}{*}{2.00} & \multirow[t]{2}{*}{1} & 1 & .110 & .036 & .037 & .182 \\
\hline & & & 2 & .129 & .045 & .038 & .220 \\
\hline & & \multirow[t]{2}{*}{2} & 1 & .126 & .040 & .047 & .206 \\
\hline & & & 2 & .223 & .049 & .124 & .323 \\
\hline & & \multirow[t]{2}{*}{3} & 1 & .120 & .048 & .022 & .217 \\
\hline & & & 2 & .199 & .062 & .074 & .324 \\
\hline \multirow[t]{5}{*}{ Brain region 11} & \multirow[t]{5}{*}{1.00} & \multirow[t]{2}{*}{1} & 1 & .140 & .025 & .090 & .190 \\
\hline & & & 2 & .083 & .032 & .019 & .147 \\
\hline & & \multirow[t]{2}{*}{2} & 1 & .149 & .025 & .098 & .200 \\
\hline & & & 2 & .044 & .055 & -.066 & .155 \\
\hline & & 3 & 1 & .123 & .024 & .075 & .172 \\
\hline
\end{tabular}




\begin{tabular}{|c|c|c|c|c|c|c|c|}
\hline & & & 2 & .102 & .039 & .024 & .179 \\
\hline & 2.00 & 1 & 1 & .051 & .025 & .002 & .101 \\
\hline & & & 2 & .146 & .032 & .082 & .210 \\
\hline & & 2 & 1 & .102 & .025 & .051 & .153 \\
\hline & & & 2 & .119 & .055 & .009 & .230 \\
\hline & & 3 & 1 & .093 & .024 & .044 & .141 \\
\hline & & & 2 & .134 & .039 & .056 & .211 \\
\hline \multirow[t]{12}{*}{ Brain region 12} & 1.00 & 1 & 1 & .262 & .045 & .172 & .352 \\
\hline & & & 2 & .149 & .051 & .045 & .253 \\
\hline & & 2 & 1 & .270 & .043 & .184 & .356 \\
\hline & & & 2 & .032 & .083 & -.134 & .198 \\
\hline & & 3 & 1 & .245 & .043 & .158 & .332 \\
\hline & & & 2 & .204 & .060 & .083 & .326 \\
\hline & 2.00 & 1 & 1 & .105 & .045 & .015 & .195 \\
\hline & & & 2 & .145 & .051 & .041 & .249 \\
\hline & & 2 & 1 & .137 & .043 & .051 & .223 \\
\hline & & & 2 & .141 & .083 & -.025 & .307 \\
\hline & & 3 & 1 & .142 & .043 & .056 & .229 \\
\hline & & & 2 & .138 & .060 & .016 & .259 \\
\hline \multirow[t]{7}{*}{ Brain region 13} & 1.00 & 1 & 1 & -.057 & .043 & -.144 & .029 \\
\hline & & & 2 & -.131 & .046 & -.225 & -.038 \\
\hline & & 2 & 1 & -.137 & .050 & -.237 & -.037 \\
\hline & & & 2 & -.193 & .092 & -.378 & -.008 \\
\hline & & 3 & 1 & -.080 & .035 & -.150 & -.010 \\
\hline & & & 2 & -.117 & .051 & -.220 & -.013 \\
\hline & 2.00 & 1 & 1 & -.126 & .043 & -.213 & -.039 \\
\hline
\end{tabular}




\begin{tabular}{|c|c|c|c|c|c|c|c|}
\hline & & & 2 & -.087 & .046 & -.180 & .007 \\
\hline & & 2 & 1 & -.102 & .050 & -.202 & -.001 \\
\hline & & & 2 & -.102 & .092 & -.287 & .083 \\
\hline & & 3 & 1 & -.122 & .035 & -.192 & -.052 \\
\hline & & & 2 & -.128 & .051 & -.232 & -.025 \\
\hline \multirow[t]{12}{*}{ Brain region 14} & 1.00 & 1 & 1 & .222 & .044 & .133 & .310 \\
\hline & & & 2 & .169 & .055 & .058 & .281 \\
\hline & & 2 & 1 & .230 & .039 & .151 & .309 \\
\hline & & & 2 & .124 & .062 & .000 & .248 \\
\hline & & 3 & 1 & .267 & .047 & .172 & .363 \\
\hline & & & 2 & .290 & .058 & .172 & .407 \\
\hline & 2.00 & 1 & 1 & .134 & .044 & .046 & .222 \\
\hline & & & 2 & .094 & .055 & -.018 & .205 \\
\hline & & 2 & 1 & .196 & .039 & .116 & .275 \\
\hline & & & 2 & .252 & .062 & .128 & .376 \\
\hline & & 3 & 1 & .263 & .047 & .167 & .358 \\
\hline & & & 2 & .330 & .058 & .212 & .447 \\
\hline \multirow[t]{9}{*}{ Brain region 15} & 1.00 & 1 & 1 & -.055 & .038 & -.132 & .021 \\
\hline & & & 2 & -.133 & .042 & -.219 & -.048 \\
\hline & & 2 & 1 & -.071 & .043 & -.157 & .014 \\
\hline & & & 2 & -.071 & .086 & -.243 & .102 \\
\hline & & 3 & 1 & -.103 & .038 & -.180 & -.025 \\
\hline & & & 2 & -.107 & .055 & -.217 & .003 \\
\hline & 2.00 & 1 & 1 & -.168 & .038 & -.244 & -.091 \\
\hline & & & 2 & -.048 & .042 & -.133 & .038 \\
\hline & & 2 & 1 & -.087 & .043 & -.173 & -.002 \\
\hline
\end{tabular}




\begin{tabular}{|c|c|c|c|c|c|c|c|}
\hline & & & 2 & -.138 & .086 & -.310 & .035 \\
\hline & & 3 & 1 & -.128 & .038 & -.205 & -.050 \\
\hline & & & 2 & -.090 & .055 & -.200 & .020 \\
\hline \multirow[t]{12}{*}{ Brain region 16} & 1.00 & 1 & 1 & .617 & .138 & .339 & .895 \\
\hline & & & 2 & .171 & .144 & -.118 & .461 \\
\hline & & 2 & 1 & .549 & .145 & .257 & .841 \\
\hline & & & 2 & .094 & .240 & -.390 & .578 \\
\hline & & 3 & 1 & .385 & .192 & -.001 & .770 \\
\hline & & & 2 & .354 & .161 & .030 & .679 \\
\hline & 2.00 & 1 & 1 & .152 & .138 & -.126 & .431 \\
\hline & & & 2 & .005 & .144 & -.285 & .295 \\
\hline & & 2 & 1 & .223 & .145 & -.069 & .515 \\
\hline & & & 2 & .017 & .240 & -.467 & .500 \\
\hline & & 3 & 1 & .292 & .192 & -.094 & .677 \\
\hline & & & 2 & .113 & .161 & -.211 & .437 \\
\hline \multirow[t]{11}{*}{ Brain region 17} & 1.00 & 1 & 1 & .126 & .044 & .038 & .214 \\
\hline & & & 2 & .051 & .038 & -.025 & .128 \\
\hline & & 2 & 1 & .089 & .042 & .005 & .173 \\
\hline & & & 2 & .003 & .076 & -.150 & .156 \\
\hline & & 3 & 1 & .073 & .042 & -.012 & .158 \\
\hline & & & 2 & .006 & .055 & -.105 & .117 \\
\hline & 2.00 & 1 & 1 & .021 & .044 & -.067 & .109 \\
\hline & & & 2 & .067 & .038 & -.009 & .144 \\
\hline & & 2 & 1 & .075 & .042 & -.009 & .159 \\
\hline & & & 2 & .029 & .076 & -.124 & .182 \\
\hline & & 3 & 1 & .051 & .042 & -.034 & 137 \\
\hline
\end{tabular}




\begin{tabular}{|c|c|c|c|c|c|c|c|}
\hline & & & 2 & -.006 & .055 & -.117 & .104 \\
\hline \multirow[t]{12}{*}{ Brain region 18} & 1.00 & 1 & 1 & .136 & .034 & .068 & .204 \\
\hline & & & 2 & .137 & .056 & .025 & .250 \\
\hline & & 2 & 1 & .092 & .035 & .021 & .162 \\
\hline & & & 2 & .086 & .094 & -.103 & .275 \\
\hline & & 3 & 1 & .066 & .036 & -.006 & .139 \\
\hline & & & 2 & .159 & .050 & .059 & .259 \\
\hline & 2.00 & 1 & 1 & .055 & .034 & -.013 & .123 \\
\hline & & & 2 & .047 & .056 & -.065 & .160 \\
\hline & & 2 & 1 & .071 & .035 & .000 & .141 \\
\hline & & & 2 & .005 & .094 & -.185 & .194 \\
\hline & & 3 & 1 & .047 & .036 & -.026 & .120 \\
\hline & & & 2 & .048 & .050 & -.052 & .149 \\
\hline \multirow[t]{12}{*}{ Brain region 19} & 1.00 & 1 & 1 & .163 & .040 & .083 & .244 \\
\hline & & & 2 & .105 & .046 & .013 & .197 \\
\hline & & 2 & 1 & .138 & .048 & .040 & .235 \\
\hline & & & 2 & .164 & .098 & -.034 & .361 \\
\hline & & 3 & 1 & .092 & .051 & -.010 & .194 \\
\hline & & & 2 & .200 & .055 & .089 & .311 \\
\hline & 2.00 & 1 & 1 & .059 & .040 & -.022 & .139 \\
\hline & & & 2 & .041 & .046 & -.052 & .133 \\
\hline & & 2 & 1 & .092 & .048 & -.006 & .189 \\
\hline & & & 2 & .028 & .098 & -.169 & .226 \\
\hline & & 3 & 1 & .080 & .051 & -.021 & .182 \\
\hline & & & 2 & .057 & .055 & -.054 & .168 \\
\hline Brain region 20 & 1.00 & 1 & 1 & .239 & .051 & .137 & .341 \\
\hline
\end{tabular}




\begin{tabular}{|c|c|c|c|c|c|c|c|}
\hline & & & 2 & .212 & .071 & .070 & .354 \\
\hline & & \multirow[t]{2}{*}{2} & 1 & .234 & .054 & .125 & .343 \\
\hline & & & 2 & .180 & .080 & .019 & .341 \\
\hline & & \multirow[t]{2}{*}{3} & 1 & .278 & .055 & .168 & .389 \\
\hline & & & 2 & .262 & .081 & .099 & .425 \\
\hline & \multirow[t]{6}{*}{2.00} & \multirow[t]{2}{*}{1} & 1 & .213 & .051 & .111 & .315 \\
\hline & & & 2 & .185 & .071 & .043 & .327 \\
\hline & & \multirow[t]{2}{*}{2} & 1 & .235 & .054 & .126 & .344 \\
\hline & & & 2 & .215 & .080 & .054 & .376 \\
\hline & & \multirow[t]{2}{*}{3} & 1 & .314 & .055 & .203 & .424 \\
\hline & & & 2 & .408 & .081 & .245 & .570 \\
\hline \multirow[t]{12}{*}{ Brain region 21} & \multirow[t]{6}{*}{1.00} & \multirow[t]{2}{*}{1} & 1 & .063 & .038 & -.014 & .140 \\
\hline & & & 2 & -.007 & .038 & -.083 & .069 \\
\hline & & \multirow[t]{2}{*}{2} & 1 & -.022 & .030 & -.084 & .039 \\
\hline & & & 2 & -.150 & .077 & -.306 & .005 \\
\hline & & \multirow[t]{2}{*}{3} & 1 & -.049 & .032 & -.113 & .016 \\
\hline & & & 2 & -.094 & .048 & -.190 & .002 \\
\hline & \multirow[t]{6}{*}{2.00} & \multirow[t]{2}{*}{1} & 1 & -.042 & .038 & -.119 & .035 \\
\hline & & & 2 & -.049 & .038 & -.125 & .026 \\
\hline & & \multirow[t]{2}{*}{2} & 1 & -.012 & .030 & -.073 & .049 \\
\hline & & & 2 & -.193 & .077 & -.348 & -.038 \\
\hline & & \multirow[t]{2}{*}{3} & 1 & -.013 & .032 & -.078 & .051 \\
\hline & & & 2 & -.177 & .048 & -.273 & -.081 \\
\hline \multirow[t]{3}{*}{ Brain region 22} & \multirow[t]{3}{*}{1.00} & \multirow[t]{2}{*}{1} & 1 & .179 & .071 & .036 & .323 \\
\hline & & & 2 & .165 & .075 & .014 & .316 \\
\hline & & 2 & 1 & .267 & .063 & .141 & .394 \\
\hline
\end{tabular}




\begin{tabular}{|c|c|c|c|c|c|c|}
\hline & & 2 & .292 & .174 & -.059 & .643 \\
\hline & \multirow[t]{2}{*}{3} & 1 & .235 & .073 & .087 & .382 \\
\hline & & 2 & .396 & .090 & .215 & .578 \\
\hline \multirow[t]{6}{*}{2.00} & 1 & 1 & .051 & .071 & -.093 & .194 \\
\hline & & 2 & .082 & .075 & -.069 & .233 \\
\hline & 2 & 1 & .122 & .063 & -.004 & 249 \\
\hline & & 2 & .203 & .174 & -.148 & .554 \\
\hline & 3 & 1 & .162 & .073 & .014 & .309 \\
\hline & & 2 & .211 & .090 & .030 & .393 \\
\hline
\end{tabular}




\section{University Library}

\section{- MINERVA}

\section{A gateway to Melbourne's research publications}

Minerva Access is the Institutional Repository of The University of Melbourne

Author/s:

Levis, Bianca

Title:

The effect of reward and punishment on inhibitory control in anxiety

Date:

2017

Persistent Link:

http://hdl.handle.net/11343/197725

Terms and Conditions:

Terms and Conditions: Copyright in works deposited in Minerva Access is retained by the copyright owner. The work may not be altered without permission from the copyright owner. Readers may only download, print and save electronic copies of whole works for their own personal non-commercial use. Any use that exceeds these limits requires permission from the copyright owner. Attribution is essential when quoting or paraphrasing from these works. 